\begin{tabular}{|c|c|}
\hline $\begin{array}{l}\text { 2. To: (Receiving organization) } \\
\text { Distribution }\end{array}$ & $\begin{array}{l}\text { 3. Fron: (Originating Organization) } \\
\text { SNF Storage Projects }\end{array}$ \\
\hline $\begin{array}{l}\text { 5. Proj./Prog./Dept./Div.: } \\
\text { SNF Project/W- } 443\end{array}$ & $\begin{array}{l}\text { 6. Design Authority/ Design Agent/Cog. } \\
\text { Engr.: } \\
\text { A. T. Kee }\end{array}$ \\
\hline
\end{tabular}

8. Originator Remarks: are suratar

The attached documents is being submitted for release.

Design review comments are documented in

HNF-SD-SNF-DRCR-001.

11. Receiver Remarks: 11A. Design Baseline Document? [X] Yes [] No

4. Related EDT No.:

620084

7. Purchase order No.:

MJK-SPX-452727

9. Equip./Component No.:

NA

10. System/Bldg./Facility:

$\mathrm{K}$ Basins $/ 212 \mathrm{H}$

13. Permit/Permit Application No.: NA

14. Required Response Date: $2 / 5 / 97$

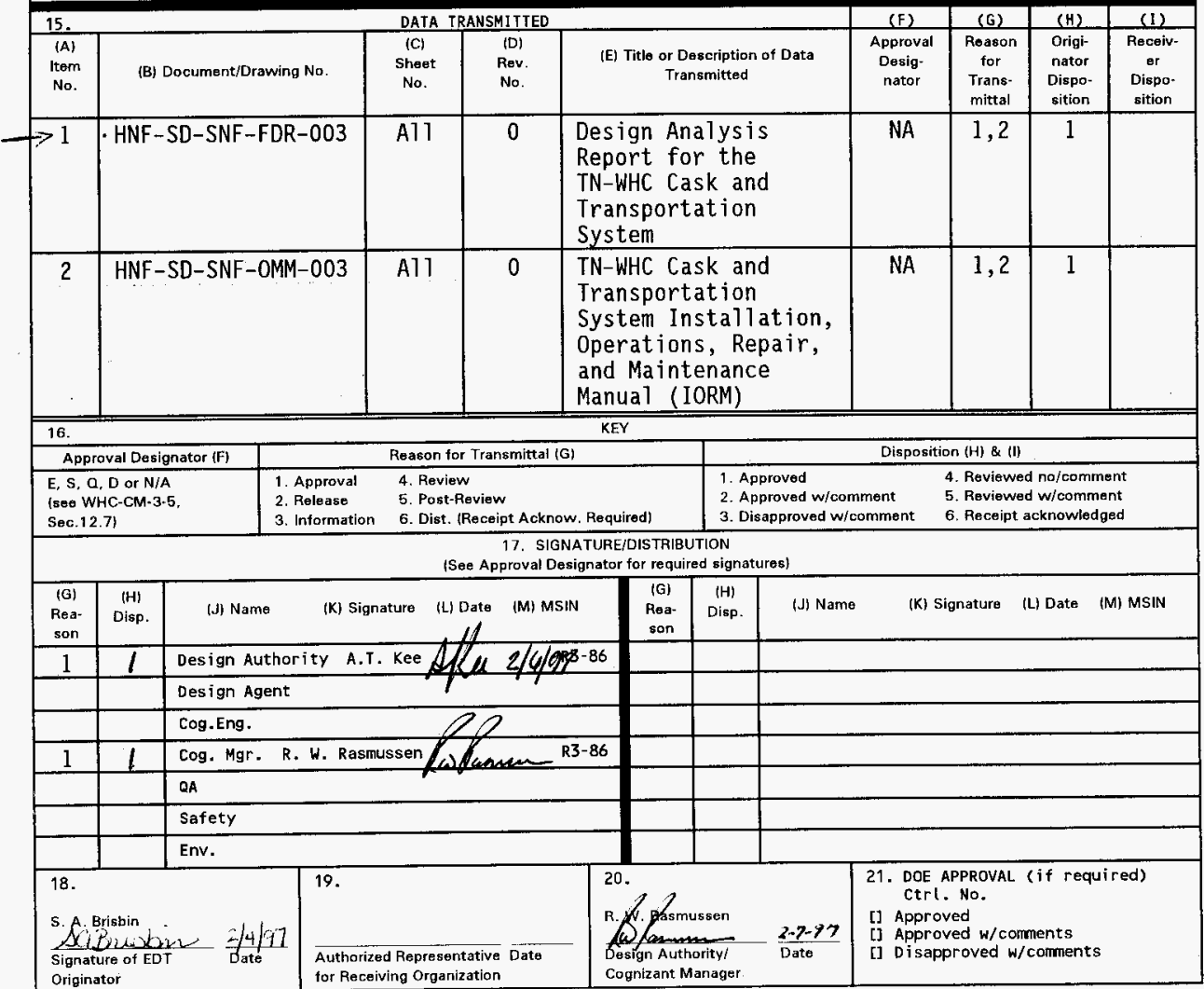




\title{
Design Analysis Report for the TN-WHC Cask and Transportation System
}

\author{
S. A. Brisbin
}

Duke Engineering and Services Hanford, Richland, WA 99352

U.S. Department of Energy Contract DE-AC06-96RL13200
EDT/ECN: 620085
Org Code: 2T240
UC: 600
Charge Code: LF026
B\&R Code: EW7040000
Total Pages: 658

Key Words: Design analysis, TN-WHC cask, cask and transportation system, conveyance

Abstract: This document presents the evaluation of the Spent Nuclear Fue] Cask and Transportation System. The system design was developed by Transnuclear, Inc. and its team members NAC International, Nelson Manufacturing, Precision Components Corporation, and Numatec, Inc. The cask is designated the TN-WHC cask. This report describes the design features and presents preliminary analyses performed to size critical dimensions of the system while meeting the requirements of the performance specification.

Photo Copied materials in this document have been reviewed and $i t$ has been determined that is NOT copyrighted.

TRADEMARK DISCLAIMER. Reference herein to any specific commercial product, process, or service by trade name, trademark, manufacturer, or otherwise, does not necessarily constitute or imply its endorsement, recommendation, or favoring by the United States Government or any agency thereof or its contractors or subcontractors.

Printed in the United States of America. To obtain copies of this document, contact: Document Control Services, P.0. Box 950, Mailstop H6-08, Richland WA 99352, Phone (509) 372-2420;

Fax (509) 376-4989.
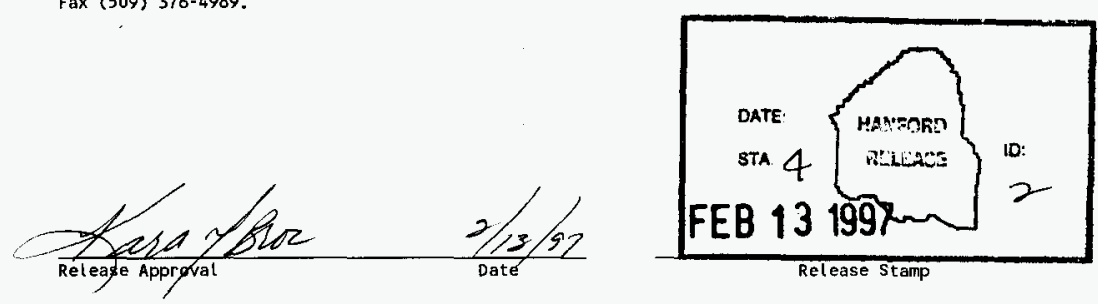

Approved for Public Release 
HNF-SD-SNF-FDR-003 Rev. 0

E-15166

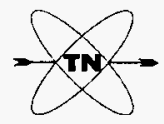

DESIGN ANALYSIS REPORT

FOR THE

TN-WHC CASK

AND

\section{TRANSPORTATION SYSTEM}

PROJECT 3035

Item no. 8

WHC Specification no. WHC-S-0396

DE\&SH Purchase Order no. MJK-SPX-452727

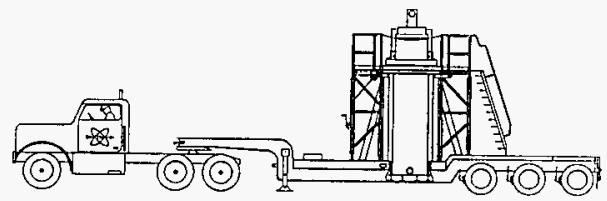

Transnuclear Inc.

Four Skyline Drive

Hawthorne, NY 10532 
Final Design Analysis Report

for the

TN-WHC Cask and Transportation System

Initial Issue:

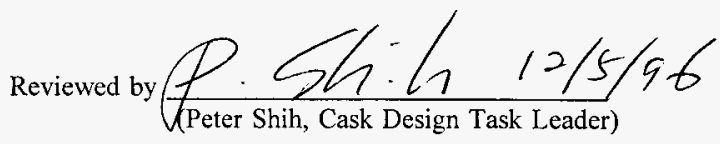

Reviewed by $\frac{12 / 5 / 41}{\text { (Michael Mason, Chief Engineer) }}$

Reviewed by $\frac{\text { Senu Sueura 12/5/96 }}{\text { (Glenn Guerra, Project Manager) }}$

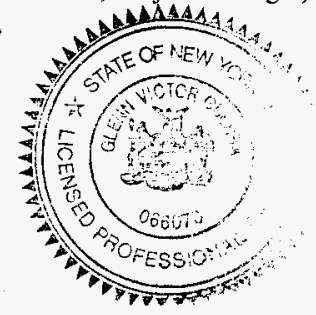




\section{TABLE OF CONTENTS}

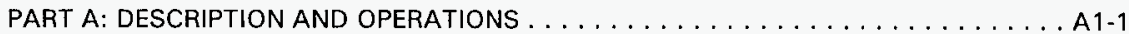

A1.0 INTRODUCTION .......................... 1 1-1

A1.1 GENERAL INFORMATION $\ldots \ldots \ldots \ldots \ldots \ldots \ldots \ldots \ldots$ A $1-1$

A1.2 SYSTEM DESCRIPTION $\ldots \ldots \ldots \ldots \ldots \ldots \ldots \ldots \ldots \ldots$ A $1-1$

A1.2.1 Cask ........................ 1 1-3

A1.2.2 Cask Lifting Attachment $\ldots \ldots \ldots \ldots \ldots \ldots \ldots \ldots$ A1-3

A1.2.3 Conveyance ...................A1-6

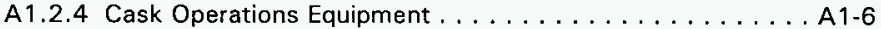

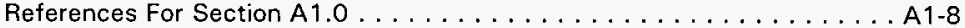

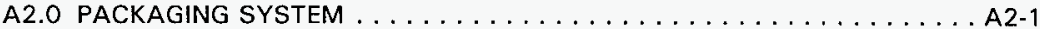

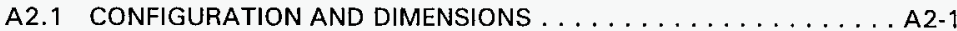

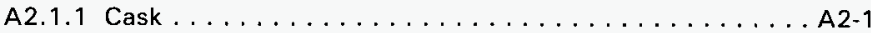

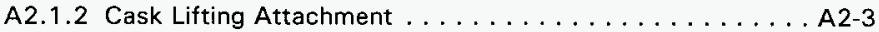

A2.1.3 Conveyance ...................A2-3

A2.1.4 Cask Operations Equipment . . . . . . . . . . A2-4

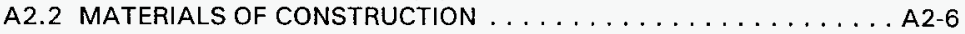

A2.2.1 Cask ...................... A2-6

A2.2.2 Cask Lifting Attachment . . . . . . . . . . . A2-6

A2.2.3 Conveyance ..................... A2-6

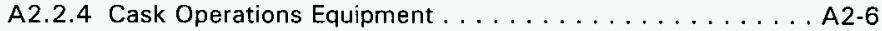

A2.3 MECHANICAL PROPERTIES OF MATERIALS $\ldots \ldots \ldots \ldots \ldots \ldots$ A2-7

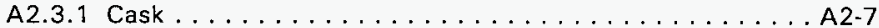

A2.3.2 Cask Lifting Attachment . . . . . . . . . . . . . A2-8

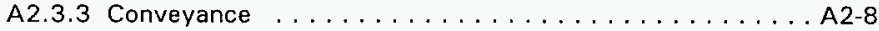

A2.3.4 Cask Operations Equipment . . . . . . . . . . . . . . A2-9

A2.4 DESIGN AND FABRICATION METHODS $\ldots \ldots \ldots \ldots \ldots \ldots \ldots$ A2-9

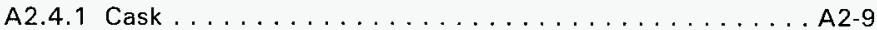

A2.4.2 Cask Lifting Attachment ............... A2-10

A2.4.3 Conveyance ..................... A2-10

A2.4.4 Cask Operations Equipment . . . . . . . . . . . A2-10

A2.5 WEIGHTS AND CENTER OF GRAVITY $\ldots \ldots \ldots \ldots \ldots$ A2-11

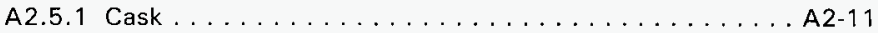

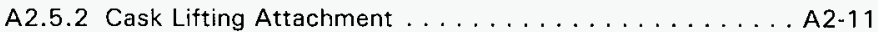

A2.5.3 Conveyance .................. A2-11

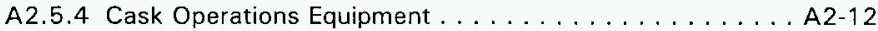

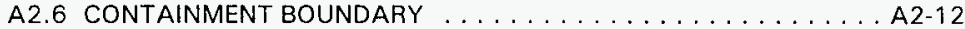

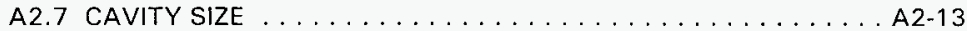

A2.8 HEAT DISSIPATION $\ldots \ldots \ldots \ldots \ldots \ldots \ldots \ldots \ldots \ldots$ A2-13

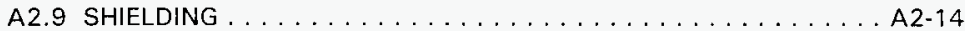

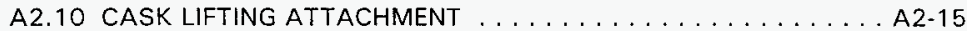

A2.11 TIEDOWN ATTACHMENTS $\ldots \ldots \ldots \ldots \ldots \ldots \ldots \ldots$ A2-15

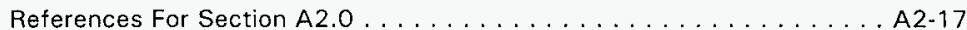

A3.0 PACKAGE CONTENTS $\ldots \ldots \ldots \ldots \ldots \ldots \ldots \ldots \ldots \ldots \ldots$ A3-1

A3.1 GENERAL DESCRIPTION ................... A3-1

A3.1.1 Physical Form $\ldots \ldots \ldots \ldots \ldots \ldots \ldots \ldots \ldots \ldots \ldots$ A3-1 
A3.1.2 MCO Weights and Dimensions . . . . . . . . . . . A3-1

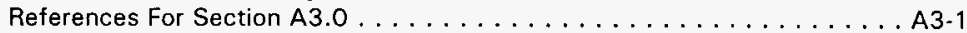

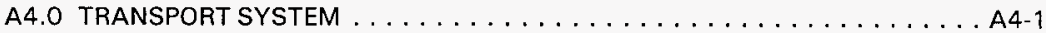

A4.1 TRANSPORTER . . . . . . . . . . . . . . . A4-1

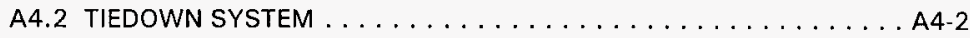

A4.3 SPECIAL TRANSFER REQUIREMENTS $\ldots \ldots \ldots \ldots \ldots \ldots \ldots$ A4-4

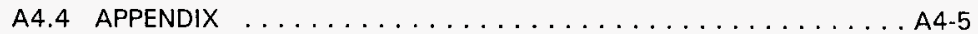

A5.0 ACCEPTANCE OF PACKAGING FOR USE $\ldots \ldots \ldots \ldots \ldots \ldots \ldots \ldots$ A5-1

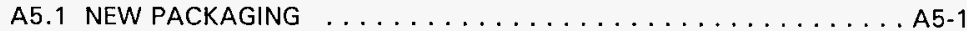

A5.1.1 Acceptance Requirements . . . . . . . . . . . . . A5-1

A5.1.2 Inspection and Testing . . . . . . . . . . . . A5-1

A5.2 ANNUAL INSPECTION REQUIREMENTS . . . . . . . . . . . . A5-2

A5.2.1 Acceptance Requirements . . . . . . . . . . . . . . A5-2

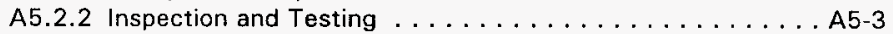

References For Section A5.0 . . . . . . . . . . . . . . A5-4

A6.0 OPERATING REQUIREMENTS . . . . . . . . . . . . . . . A6-1

A6.1 GENERAL REQUIREMENTS . . . . . . . . . . . . . A6-1

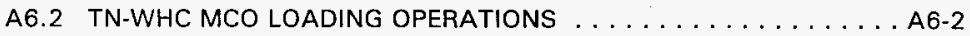

A6.3 CASK UNLOADING PROCEDURES . . . . . . . . . . . . . A6-6

A6.4 EMPTY PACKAGING PROCEDURES . . . . . . . . . . . . . A6-7

A6.5 CASK LOADING RADIATION DOSE ESTIMATES . . . . . . . . . . . A6-8

A6.6 LEAK TESTING TN-WHC CASK . . . . . . . . . . . . A6-10

A6.6.1 Containment System Periodic Verification Test . . . . . . . A6-10

A6.6.2 Containment System Assembly Verification Test . . . . . . A6-10

A7.0 QUALITY ASSURANCE REQUIREMENTS . . . . . . . . . . . . . . . A7-1

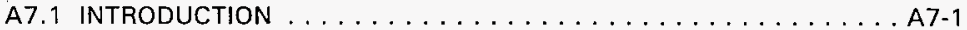

A7.2 GENERAL REQUIREMENTS ... . . . . . . . . . A7-1

A7.2.1 Organization . . . . . . . . . . . . . . A7-1

A7.2.2 Ouality Assurance Program . . . . . . . . . . . . A7 A 1

A7.2.3 Design Control . . . . . . . . . . . . . . . . A7-2

A7.2.4 Procurement Control . . . . . . . . . . . . . . . . A7-2

A7.2.5 Instructions, Procedures and Drawings . . . . . . . A A7-2

A7.2.6 Document Control . . . . . . . . . . . . . . . A7-2

A7.2.7 Control of Purchased Material, Equipment and Services . . A A7-2

A7.2.8 Identification and Control of Materials, Parts and

Components ........................

A7.2.9 Control of Special Processes . . . . . . . . . . . A7-3

A7.2.10 inspection . . . . . . . . . . . . . . A7-3

A7.2.11 Test Control ............................ . . .

A7.2.12 Control of Measuring and Test Equipment . . . . . . . A7-3

A7.2.13 Handling, Storage and Shipping . . . . . . . . . . . . A7-4

A7.2.14 Inspection, Test and Operating Status . . . . . . . . . . A A7-4

A7.2.15 Nonconforming Material, Parts or Components . . . . . . A7-4

A7.2.16 Corrective Action . . . . . . . . . . . . . . . A7-4

A7.2.17 Records ....................... A7-4

A7.2.18 Audits . . . . . . . . . . . . . . . . A A7-4

A8.0 MAINTENANCE $\ldots \ldots \ldots \ldots \ldots \ldots \ldots \ldots \ldots \ldots \ldots \ldots \ldots \ldots$

A8.1 GENERAL REQUIREMENTS $\ldots \ldots \ldots \ldots \ldots \ldots \ldots \ldots \ldots$. . . . . . . . . . . . . . .

A8.2 INSPECTION AND VERIFICATION SCHEDULES $\ldots \ldots \ldots \ldots \ldots \ldots . . . . . .$. 
A9.0 APPENDIX .......................... A9-1 A9.1 TN-WHC CASK AND TRANSPORTATION SYSTEM DRAWINGS . . A9.1-1 A9.2 CASK OPERATIONS EQUIPMENT ANALYSES $\ldots \ldots \ldots \ldots$ A9.2-1

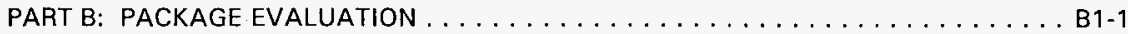

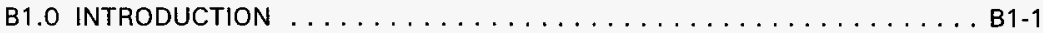

B1.1 SAFETY EVALUATION METHODOLOGY $\ldots \ldots \ldots \ldots \ldots \ldots$ B1-1

B1.2 EVALUATION SUMMARY AND CONCLUSIONS $\ldots \ldots \ldots \ldots \ldots$ B1-2

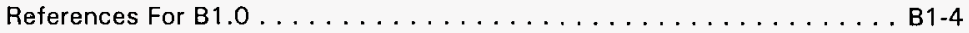

B2.0 CONTAINMENT EVALUATION $\ldots \ldots \ldots \ldots \ldots \ldots \ldots \ldots \ldots \ldots \ldots$ B2-1

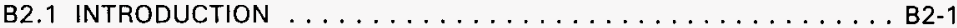

B2.2 CONTAINMENT SOURCE SPECIFICATION $\ldots \ldots \ldots \ldots \ldots \ldots$ B2-1

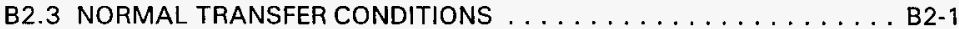

B2.3.1 Conditions to be Evaluated ............... B2-1

B2.3.2 Containment Acceptance Criteria . . . . . . . . . . B2-1

B2.3.3 Containment Model ................... B2-1

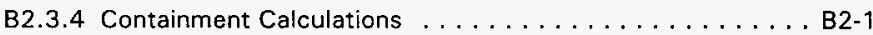

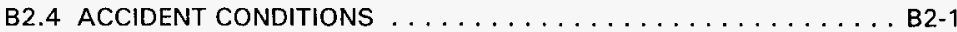

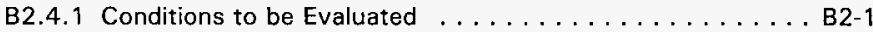

B2.4.2 Containment Acceptance Criteria . . . . . . . . . . . B2-1

B2.4.3 Containment Model ... . . . . . . . . . . . . . B2-1

B2.4.4 Containment Calculations ............... B2-1

B2.5 CONTAINMENT EVALUATION AND CONCLUSIONS . . . . . . B2-1

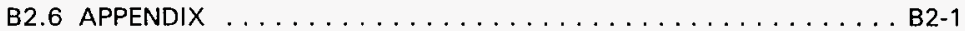

B3.0 SHIELDING EVALUATION $\ldots \ldots \ldots \ldots \ldots \ldots \ldots \ldots \ldots \ldots$ B3-1

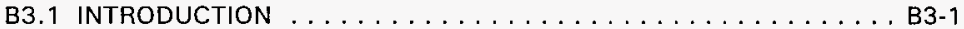

B3.2 DIRECT RADIATION SOURCE SPECIFICATION $\ldots \ldots \ldots \ldots \ldots$ B3-1

B3.2.1 Gamma Source . . . . . . . . . . . . . . . B3-1

B3.2.2 Neutron Source .................. B3-2

B3.3 SUMMARY OF SHIELDING PROPERTIES OF MATERIALS $\ldots \ldots \ldots$ B3-3

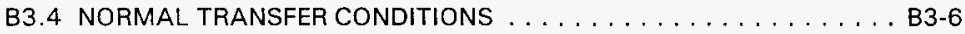

B3.4.1 Conditions to be Evaluated $\ldots \ldots \ldots \ldots \ldots \ldots \ldots$ B3-6

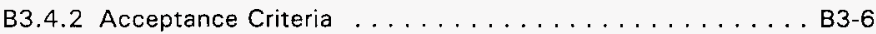

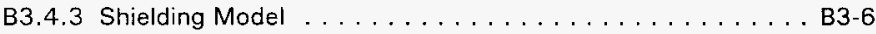

B3.4.4 Shielding Calculations $\ldots \ldots \ldots \ldots \ldots \ldots \ldots \ldots$ B3-7

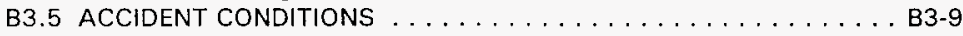

B3.5.1 Conditions To Be Evaluated . . . . . . . . . . . B3-9

B3.5.2 Acceptance Criteria . . . . . . . . . . . . . B3-9

B3.5.3 Shielding Model . . . . . . . . . . . . . . . . . B3-9

B3.5.4 Shielding Calculations ................ B3-9

B3.6 SHIELDING EVALUATION AND CONCLUSIONS . . . . . . . B3-9

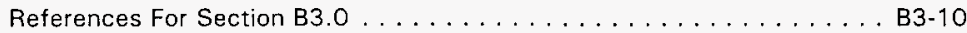

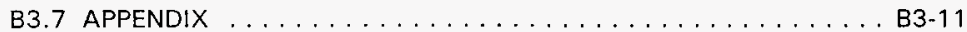

B4.0 STRUCTURAL EVALUATION $\ldots \ldots \ldots \ldots \ldots \ldots \ldots \ldots \ldots$ B4-1

B4.1 INTRODUCTION ...................... B4-1

B4.2 STRUCTURAL EVALUATION OF PACKAGE $\ldots \ldots \ldots \ldots \ldots$ B4-1

B4.2.1 Structural Design and Features . . . . . . . . . B B-1

B4.2.2 Mechanical Properties of Materials . . . . . . . . . B4-1

B4.2.3 Chemical and Galvanic Reaction ............. B4-2 
B4.2.4 Size of Package and Cavity ............... B4-2

B4.2.5 Weights and Center of Gravity . . . . . . . . . . B4-5

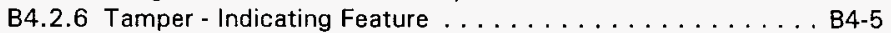

B4.2.7 Positive Closure ... . . . . . . . . . . . . . . B4-5

B4.2.8 Cask Lifting Attachments . . . . . . . . . . . . B4-5

B4.2.9 Brittle Fracture . . . . . . . . . . . . . . B4-5

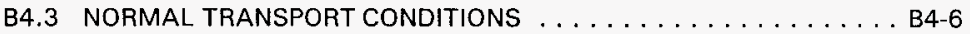

B4.3.1 Conditions To Be Evaluated . . . . . . . . . . . . . B4-11

B4.3.2 Acceptance Criteria . . . . . . . . . . . . . B B4-12

B4.3.3 Structural Model . . . . . . . . . . . . . . . . . . B4-15

B4.3.4 Initial Conditions . . . . . . . . . . . . . . . B4-15

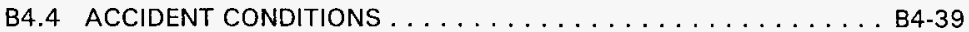

B4.4.1 Conditions To Be Evaluated . . . . . . . . . . . . . . B4-44

B4.4.2 Acceptance Criteria . . . . . . . . . . . . . . . . B4-44

B4.4.3 Structural Model . . . . . . . . . . . . . . . . . . B4-45

B4.4.4 Initial Conditions . . . . . . . . . . . . . . B4-45

References For Section B4.0 . . . . . . . . . . . . . . B4-65

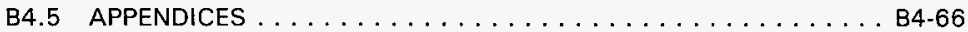

B5.0 THERMAL EVALUATION $\ldots \ldots \ldots \ldots \ldots \ldots \ldots \ldots \ldots \ldots \ldots \ldots$ B5-1

B5.1 INTRODUCTION . . . . . . . . . . . . . . . B5-1

B5.2 THERMAL SOURCE SPECIFICATION ................. B5-1

B5.2.1 Normal Conditions of Transport . . . . . . . . . . . . . B5-1

B5.2.2 Hypothetical Accident Conditions . . . . . . . . . . . B5-2

B5.3 SUMMARY OF THERMAL PROPERTIES OF MATERIALS . . . . . . . B5-2

B5.4 THERMAL EVALUATION FOR NORMAL TRANSFER CONDITIONS . . B5-4

B5.4.1 Conditions Evaluated . . . . . . . . . . . . . B5-4

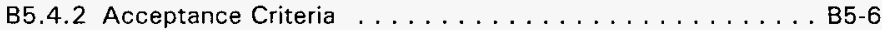

B5.4.3 Thermal Model . . . . . . . . . . . . . . . . B5-6

B5.4.4 Thermal Analysis . . . . . . . . . . . . . . . . . B5-7

B5.5 THERMAL EVALUATION FOR ACCIDENT CONDITIONS . . . . . . . . B5-8

B5.5.1 Conditions Evaluated . . . . . . . . . . . B5-8

B5.5.2 Acceptance Criteria . . . . . . . . . . . . . B5-9

B5.5.3 Thermal Model . . . . . . . . . . . . . . . . . . B5-9

B5.5.4 Thermal Analysis . . . . . . . . . . . . . . . . B5-10

B5.6 THERMAL EVALUATION AND CONCLUSIONS . . . . . . . . . . B5-11

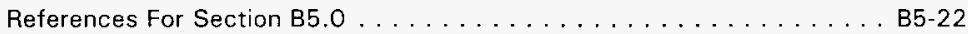

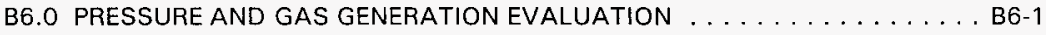

B6.1 GAS GENERATION . . . . . . . . . . . . . . . B6-1

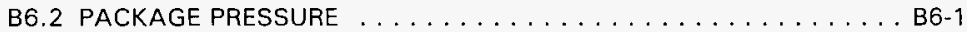

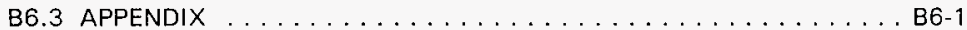

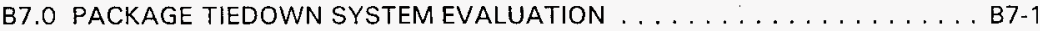

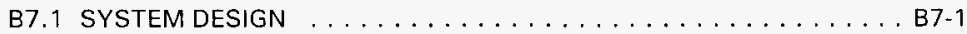

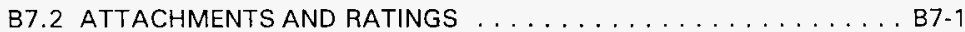

B7.2.1 Design Load Factors . . . . . . . . . . . . . . B7-1

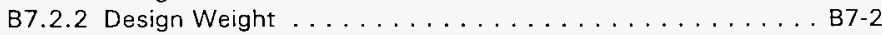

B7.2.3 Design Loadings . . . . . . . . . . . . . . . . B7-2

B7.2.4 Summary and Conclusions . . . . . . . . . B7-2

References For Section B7.0 . . . . . . . . . . . . B7-13 


\section{LIST OF TABLES}

Table A3.1-1 Weights and Dimensions of MCO Components . . . . . . . . . . . . A3-2

Table A6.5-1 Estimated Total Dose . . . . . . . . . . . . . . . . . . . . . . . . . . A6-9

Table B3.2-1 Gamma Source Spectra . . . . . . . . . . . . . . . . . . . B3-2

Table B3.2-2 Neutron Spectra . . . . . . . . . . . . . . . . . . B3-3

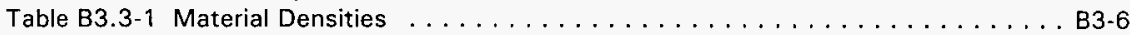

Table B3.4-1 Summary of Maximum Dose Rates - Cask Surface . . . . . . . . . . . . . . . B3-7

Table B3.4-2 Summary of Maximum Dose Rates - 2 meters from the Cask

Surface . . . . . . . . . . . . . . . . . . . . . B3-7

Table B4.2-1 Temperature Dependent Material Properties . . . . . . . . . . . . . . . . B4-2

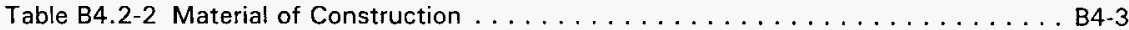

Table B4.3-1 Normal Conditions of Transport, TN-WHC Cask Performance Evaluation Overview . . . . . . . . . . . . . . . . . . . B4-8

Table B4.3-2 Normal Conditions of Transport, Individual Load Cases for Cask Body Analysis . . . . . . . . . . . . . . . . . . . . B B 9

Table B4.3-3 Summary of Load Combinations for Normal Conditions of

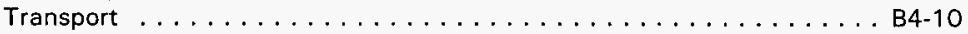

Table B4.3-4 Containment Structure/Fastener Allowable Stress . . . . . . . . . . . . . B4-14

Table B4.3-5 Cask Body Stresses Under Hot Environment . . . . . . . . . . . . . . B4-23

Table B4.3-6 Cask Body Stresses Under Cold Environment . . . . . . . . . . . . . . . B4-24

Table B4.3-7 Cask Body Stresses Under Hot Environment Vibrations . . . . . . . . . . B B-25

Table B4.3-8 Cask Body Stresses Under Cold Environment Vibrations . . . . . . . . . B4-26

Table B4.3-9 Cask Body Stresses Under Hot Environment 1 Foot Bottom End

Drop . . . . . . . . . . . . . . . . . . . . . . . . . B B4-27

Table B4.3-10 Cask Body Stresses Under Cold Environment 1 Foot Bottom End

Drop . . . . . . . . . . . . . . . . . . . . . . . . . B B4-28

Table B4.3-11 Cask Body Stresses Under Hot Environment 1 Foot Lid End Drop . . . . B4-29

Table B4.3-12 Cask Body Stresses Under Cold Environment 1 Foot Lid End

Drop . . . . . . . . . . . . . . . . . . . . . . . . . B4-30

Table B4.3-13 Cask Body Stresses Under Hot Environment 1 Foot Side Drop

(contact side)

Table B4.3-14 Cask Body Stresses Under Hot Environment 1 Foot Side Drop

(opp. contact side) . . . . . . . . . . . . . . . . .

Table B4.3-15 Cask Body Stresses Under Cold Environment 1 Foot Side Drop

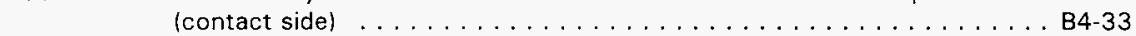

Table B4.3-16 Cask Body Stresses Under Cold Environment 1 Foot Side Drop

(opp. contact side) . . . . . . . . . . . . . . . . . . . . . . B4-34

Table B4.3-17 Cask Body Stresses Under Hot Environment 1 Foot Corner Drop (contact side)

Table B4.3-18 Cask Body Stresses Under Hot Environment 1 Foot Corner Drop

(opp. contact side)

Table B4.3-19 Cask Body Stresses Under Cold Environment 1 Foot Corner

Drop (contact side) . . . . . . . . . . . . . . . . . . . B4-37

Table B4.3-20 Cask Body Stresses Under Cold Environment 1 Foot Corner

Drop lopp. contact sidel . . . . . . . . . . . . . . . B4-38

Table B4.4-1 Accident Conditions of Transport, TN-WHC Performance

Evaluation Overview 


\section{HNF-SD-SNF-FDR-003 Rev. 0}

E-15166

Table B4.4-2 Accident Conditions of Transport, Individual Load Cases for Cask

Body Analysis . . . . . . . . . . . . . . . . . . . . . B B4-42

Table B4.4-3 Summary of Load Combinations for Accident Conditions of

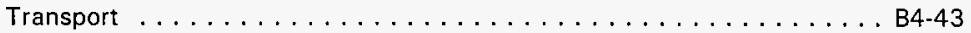

Table B4.4-4 Cask Body Stresses Under Hot Environment 30 Foot Bottom End

Drop . . . . . . . . . . . . . . . . . . . . . . . B4-53

Table B4.4-5 Cask Body Stresses Under Cold Environment 30 Foot Bottom End Drop . . . . . . . . . . . . . . . . . . . . . . . . B4-54

Table B4.4-6 Cask Body Stresses Under Hot Environment 30 Foot Lid End Drop

Table B4.4-7 Cask Body Stresses Under Cold Environment 30 Foot Lid End

Drop Condition . . . . . . . . . . . . . . . . . . B4-56

Table B4.4-8 Cask Body Stresses Under Hot Environment 30 Foot Side Drop

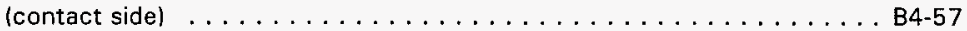

Table B4.4-9 Cask Body Stresses Under Hot Environment 30 Foot Side Drop (opp. contact side) . . . . . . . . . . . . . . . . . . . B4-58

Table B4.4-10 Cask Body Stresses Under Cold Environment 30 Foot Side Drop

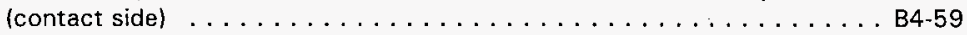

Table B4.4-11 Cask Body Stresses Under Cold Environment 30 Foot Side Drop lopp. contact side) . . . . . . . . . . . . . . . . . . . . B4-60

Table B4.4-12 Cask Body Stresses Under Hot Environ. 30 Foot Corner Drop (contact side) $\ldots \ldots \ldots \ldots \ldots \ldots \ldots \ldots \ldots \ldots \ldots$. . . . . . . . . . . . . .

Table B4.4-13 Cask Body Stresses Under Hot Environ. 30 Foot Corner Drop

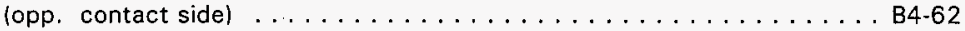

Table B4.4-14 Cask Body Stresses Under Cold Environ. 30 Foot Corner Drop (contact side)

Table B4.4-15 Cask Body Stresses Under Cold Environ. 30 Foot Corner Drop lopp. contact side) . . . . . . . . . . . . . . . . . B4-64

Table B5.4-1 Hanford Air Temperature . . . . . . . . . . . . . . . . . B5-5

Table B5.4-2 Maximum Solar Radiation Received $\left(B t u / h r-\mathrm{ft}^{2}\right) \ldots \ldots \ldots$. . . . . . B5-5

Table B7.2-1 Load Factors for Tiedown Systems . . . . . . . . . . . . . . . B7-1

Table B7.2-2 Summary of Tiedown System Stress Evaluation . . . . . . . . . . . . B7-3 


\section{LIST OF FIGURES}

Figure A1.2-1 TN-WHC Cask and Conveyance System . . . . . . . . . . . . A 1-2

Figure A1.2-2 General Arrangement of the TN-WHC Cask . . . . . . . . . . . . . . A1-4

Figure A1.2-3 TN-WHC Cask Lifting Attachment . . . . . . . . . . . . . A1-5

Figure A1.2-4 K-Basin Loadout Pit Operations Equipment . . . . . . . . . . . A 1-7

Figure A2.6-1 TN-WHC Cask Containment Boundary . . . . . . . . . . . . . A A2-16

Figure A3.1-1 Typical Arrangement of Spent Fuel in the MCO . . . . . . . . . . . . A3-3

Figure A4.2-1 TN-WHC Cask Tiedown System - Opened and Closed Positions . . . . . . A A4-3

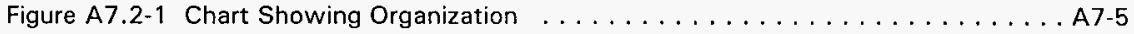

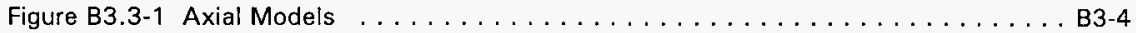

Figure B3.3-2 Radial Model $\ldots \ldots \ldots \ldots \ldots \ldots \ldots \ldots \ldots \ldots \ldots \ldots \ldots$

Figure B3.3-3 Drain Port Model . . . . . . . . . . . . . . . . . . . . . . B3-8

Figure B4.2-1 General Arrangement of TN-WHC CASK . . . . . . . . . . . . . . B4-4

Figure B4.3-1 Standard Stress Reporting Locations . . . . . . . . . . . . . . . . . . . B4-22

Figure B5.4.3-1 Normal Conditions Thermal Model . . . . . . . . . . . . . . . . . . B5-12

Figure B5.4.4-1 Normal Conditions Temperature History at Hottest MCO

Location . . . . . . . . . . . . . . . . . . . . . . . B5-13

Figure B5.4.4-2 Normal Conditions MCO and Cask Temp. Distribution -

Maximum MCO Temperature . . . . . . . . . . . . . . . . . . . . B5-14

Figure B5.4.4-3 Normal Conditions Cask Temperature Distribution - Maximum

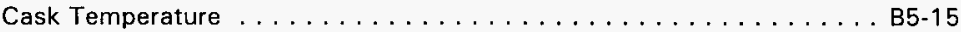

Figure B5.5.4-1 Accident Conditions Thermal Model . . . . . . . . . . . . . . . B5-16

Figure B5.5.4-2 Accident Conditions Temperature History at Hottest MCO

Location . . . . . . . . . . . . . . . . . . . . B5-17

Figure B5.5.4-3 Accident Conditions Temperature History at Top Corner

(Hottest Cask) . . . . . . . . . . . . . . . . . . . . 85-18

Figure B5.5.4-4 Accident Conditions MCO and Cask Temp. Distribution - Initial

Conditions . . . . . . . . . . . . . . . . . . . . . . . . . . B5-19

Figure B5.5.4-5 Accident Conditions MCO and Cask Temp. Distribution - End of

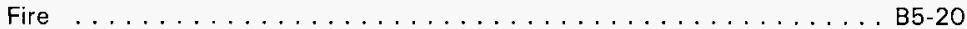

Figure B5.5.4-6 Accident Conditions $\mathrm{MCO}$ and Cask Temp. Distribution -

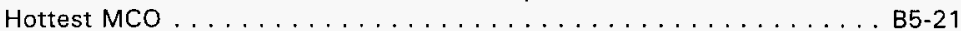

Figure B7.1-1 TN-WHC Cask Tiedown System - Opened and Closed Positions . . . . . . B7-5

Figure B7.2-1 TN-WHC Cask Transport Configuration . . . . . . . . . . . . . . . B7-6

Figure B7.2-2 Tiedown Loading Condition - $2 \mathrm{G}$ Vertical Up . . . . . . . . . . . . . B7-7

Figure B7.2-3 Tiedown Loading Condition - 3G Down . . . . . . . . . . . . . . . . B7-8

Figure B7.2-4 Tiedown Loading Condition - 1.5G Lateral . . . . . . . . . . . . . . . . . B7-9

Figure B7.2-5 Tiedown Loading Condition - 2G Longitudinal . . . . . . . . . . . . . . . . B7-10

Figure B7.2-6 Tiedown Loading Condition - 2G Longitudinal (aft) . . . . . . . . . . B7-11

Figure B7.2-7 Tiedown Loading Condition - 1G Static Deflection . . . . . . . . . . . . B7-12 


\title{
PART A: DESCRIPTION AND OPERATIONS
}

\author{
A1.0 INTRODUCTION
}

\section{A1.1 GENERAL INFORMATION}

This Design Analysis Report (DAR) presents the evaluation of the design of the cask and transportation system (including a unique operations support system) to perform the on-site transfer of Type B, Highway Route Controlled Quantity (HRCQ) fissile spent fuel. The design of the system has been developed by Transnuclear, Inc. and its team members consisting of NAC International, Nelson Manufacturing, Precision Components Corporation and Numatec, Inc. The cask is designated the TN-WHC. This DAR describes design features and presents preliminary analyses performed to size critical dimensions of the system while meeting the requirements of the Specification for SNF Path Forward Cask and Transportation System, Hanford Specification ${ }^{(1)}$.

The TN-WHC Cask and Transportation System will be used for safely packaging and transporting approximately 2,100 metric tons of unprocessed, spent nuclear fuel from the $105 \mathrm{~K}$ East and $\mathrm{K}$ West Basins to the $200 \mathrm{E}$ Area Canister Storage Building (CSB). Portions of the system will also be used for drying the spent fuel under cold vacuum conditions prior to placement in interim storage.

The spent nuclear fuel is currently stored underwater in the two K-Basins. The fuel elements will be placed in special baskets and stacked in the Multiple Canister Overpacks (MCO) that have been previously placed in the cask. The MCO shield plug will be installed on the MCO. The cask will be removed from the $\mathrm{K}$ Basin load out area and taken to the cold vacuum drying station. Here the MCO shield plug retaining ring will be installed on the $\mathrm{MCO}$, the water will be removed from the MCO and the cask prepared for transportation to the CSB. Shipments will occur exclusively on-site, specifically between the K-Basins and the CSB. Travel will be by road with one cask per trailer.

\section{A1.2 SYSTEM DESCRIPTION}

The Cask and Transportation System consists of a transport cask with a dedicated semi-trailer. It is designed to transport the cask in the vertical orientation. A single MCO loaded with spent fuel baskets will be transported from the K-Basins' Loadout Facility to the CSB. The System layout is shown on Figure A1.2-1. Unique operations equipment has been developed for the K-Basin 
HNF-SD-SNF-FDR-003 Rev. 0

E-15166

Figure A1.2-1 TN-WHC Cask and Conveyance System

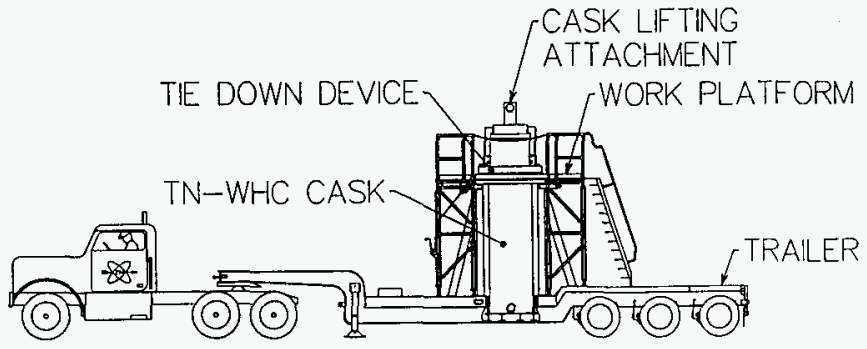


Loadout pit. The equipment has been engineered to:

- minimize cycle times

- achieve excellent control of contamination

- provide high margins of safety

- minimize waste generation

- minimize personnel exposure

- support ease of operations by facility personnel.

Descriptions of the cask, conveyance (trailer), cask lifting attachment and the operations equipment are provided below. Detailed design drawings are provided in Section A9.1 (Appendix).

\section{A1.2.1 Cask}

The cask consists of a body fabricated from stainless steel forging(s) and a bolted-on stainless steel lid with two welded on trunnions. The cask incorporates features for ease of loading, decontamination and routine handling. The design is engineered to minimize cask maintenance and maximize in-service time and for ease of fabricability to enhance project completion goals.

The overall dimensions of the cask are 170.25 inches long and 39.81 inches in diameter. The cask cavity has a length of 160.50 inches and a cavity ID of 25.19 inches. The general arrangement of the cask is depicted in Figure A1.2-2. The cask is designed to be lifted and placed in a vertical orientation only. Component terminology used in this DAR is also identified on Figure A1.2-2. The closure lid end is referred to as the top with the cask in the vertical orientation.

\section{A1.2.2 Cask Lifting Attachment}

The cask is lifted from the trailer and from the Cask Operations Equipment, and maneuvered by two trunnions welded on the lid. The layout of the attachment is shown on Figure A1.2-3. The lifting attachment is used for loading and unloading the cask from the conveyance system, for movement to and from the load out pit and the CSB, and for any other cask handling operations. It is designed to lift the cask vertically and move with the cask in the vertical orientation only. The lifting attachment structural members are constructed of stainless steel.

The lifting attachment consists of two trunnions which are welded to the cask lid by a set of brackets and gussets. The geometry and dimensions are shown in Figure A1.2.3. All components are made of 304 stainless steel. 
HNF-SD-SNF-FDR-003 Rev. 0

E-15166

Figure A1.2-2 General Arrangement of the TN-WHC Cask

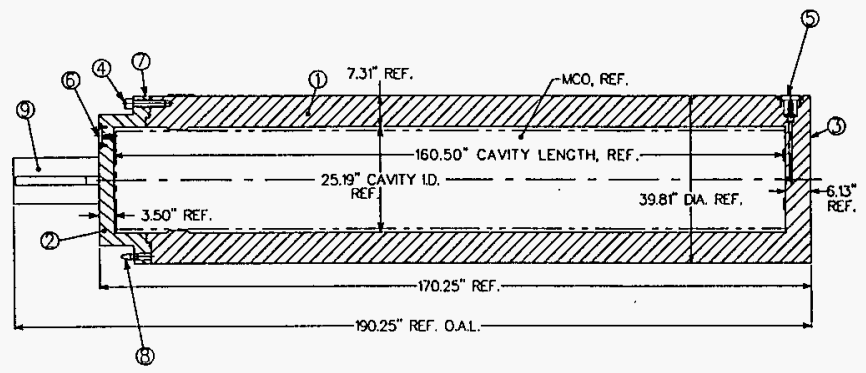

ITEM NO.

1
2
3
4
5
6
7
8
9
DESCRIPTION

Cask Body

Closure Lid

Bottom Plate

Lid Bolts

Drain Port

Vent Port

Bolting Flange

Lid Alignment Pin

Cask Lifting Attachment 
HNF-SD-SNF-FDR-003 Rev. 0

E-15166

Figure A1.2-3 TN-WHC Cask Lifting Attachment

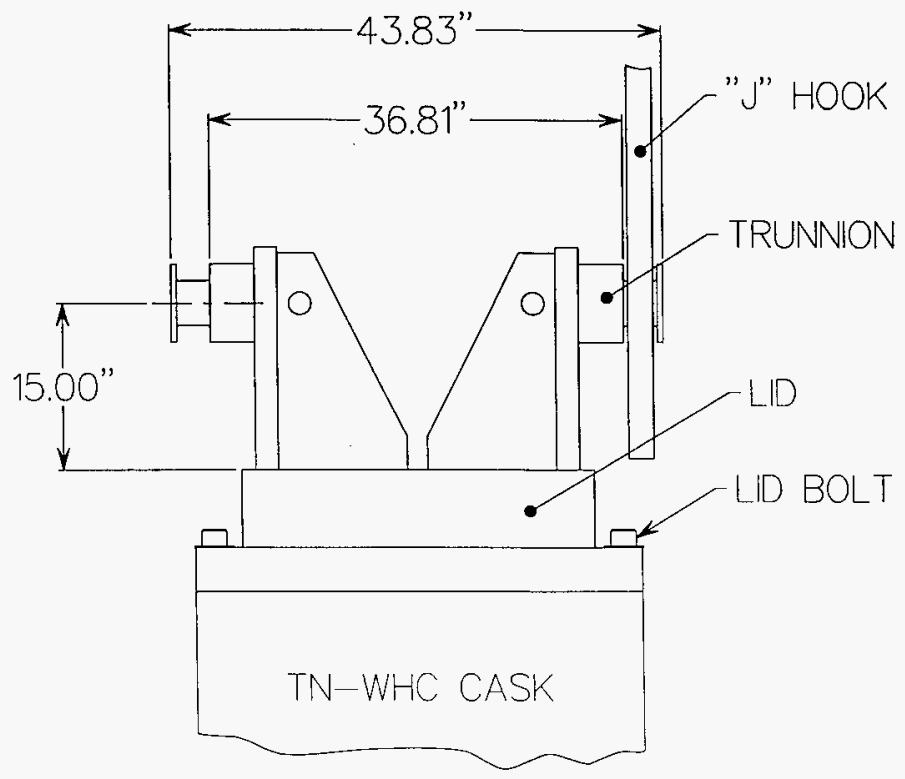




\section{A1.2.3 Conveyance}

The conveyance system is a semi-trailer which can be attached to a standard tractor. The trailer provides the necessary supports and attachment points for securing the cask in the vertical orientation.

\section{A1.2.4 Cask Operations Equipment}

The K-Basin Loadout Pit Operations Equipment for the TN-WHC cask has been designed to support the concept of "start clean, stay clean". An immersion pail with a sealing lid encloses the cask in a clean demineralized water cavity. The entire enclosed immersion pail, cask, MCO, and seal lid assembly is then lowered into the K-Basin Loadout Pit for loading of the MCO. Pneumatic seal contact surfaces between the immersion pail seal lid and immersion pail, and between the seal lid and $\mathrm{MCO}$, contain an internal immersion pail positive 2 psig relative pressure to external hydrostatic pressure during all in-pit operational sequences. Use of the sealed immersion pail precludes contamination of the exterior and interior surfaces of the cask.

The immersion pail is supported by a steel frame support structure extending from the floor of the loadout pit to the top of the loadout pit shield wall, or from lift slings and the facility crane, depending on the system's operational configuration. During immersion pail cask loading and unloading activities, the immersion pail is supported by the immersion pail support structure. The facility crane with slings is used to lower and raise the immersion pail from the pit side support structure to the pit floor for MCO loading. Worker access is provided to the immersion pail seal lid and system connections in the area opposite the fuel transfer canal during pre and post MCO loading by two worker platforms supported from the loadout pit shield wall. This loadout pit worker platform is provided for operational flexibility and not intended to be a required access point for normal operations. Normal operation of the immersion pail system is intended to be performed by operators from the main operations floor without the use of special tooling. The immersion pail support structure and worker platform are passive structures that, once installed prior to initiation of operations, do not need to be removed during the normal cask operations. Compressed air and demineralized water supply are required to support the MCO loading operation. Figure A1.2-4 presents the K-Basin Loadout Pit Operations Equipment as supported by both the in pool support structure and facility crane.

The immersion pail lid is handled by the facility crane during installation and removal for each loading cycle. The pail lid is lowered in place, seals are pressurized, and deionized water flow established. Seal integrity verification occurs at this point in the operation.

The immersion pail lid is fabricated of stainless steel to mitigate concerns about corrosion and abrasion. The lid is held in place through seal pressure, dead weight and four bolts to the main pail structure. The lid design limits seal crevasses and pool water entrapment. permits flushing of the seal surface prior to breaking the seal, and permits clean immersion pail water to flow from the seal boundary when seal pressure is removed. Each of these features supports ease of decontamination during the operation sequence. 
Figure A1.2-4 K-Basin Loadout Pit Operations Equipment

A. Support Structure Configuration

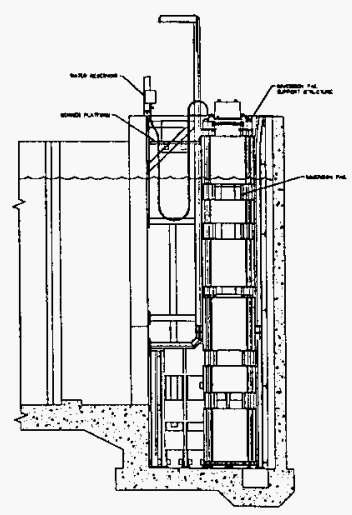

B. Crane Support Configuration

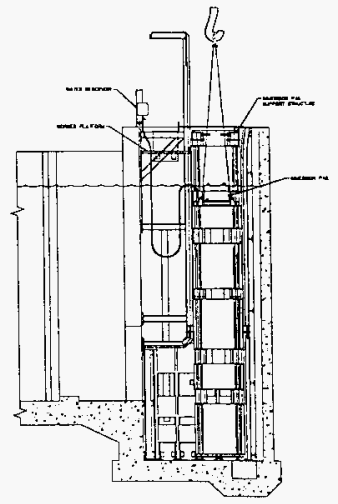


HNF-SD-SNF-FDR-003 Rev. $0 \quad$ E-15166

\section{References For Section A1.0}

1. Performance Specification For TN-WHC Cask And Transportation System, WHC-S-0396, Rev. 1, September 1995. 


\section{A2.0 PACKAGING SYSTEM}

The packaging system is the assembly of components necessary to ensure compliance with the materials containment and compliance requirements delineated in Hanford Specification ${ }^{(1)}$. The Cask and Transportation System consists of the cask with lifting attachment, the dedicated trailer with the tiedown system, and the operations equipment.

\section{A2.1 CONFIGURATION AND DIMENSIONS}

\section{A2.1.1 Cask}

The structure of the cask is a right circular cylinder with a bottom and a closure lid. The basic components of the cask are the cask body, closure lid and the lid bolts. The cask body consists of the cylindrical shell and the bottom plate. The closure lid is attached to the cask body with twelve 1.5 inch diameter bolts. Two lifting trunnions with brackets and gussets are welded to the top end of the lid and are a $180^{\circ}$ apart. Two penetrations into the containment are provided to support cask operations. One is located at the lid and the other is located in the cask bottom. The maximum gross weight of the loaded cask is 57,910 pounds including a payload of 18,950 pounds. The cask is transported in the vertical orientation with the lid end facing upward. During transport, the cask is supported on the trailer by an upper tiedown device and a lower cup shaped retainer.

The following sections provide a physical and functional description of each major component. Detailed drawings showing dimensions are provided in Section A9.1. A complete materials list is provided in Section A2.2. Reference to these drawings is made in the following physical description sections and in general, throughout this DAR. Fabrication of the cask will be performed in accordance with the design drawings.

\section{$\underline{\text { Cask Body }}$}

The cask body assembly is shown on drawing H-1-81535, sheet 1 of 5 and consists of a circular cylinder (shell) welded to a bottom plate. The stainless steel material for the shell and bottom plate is ASME SA-336 Type F304 or equivalent. The shell is 7.31 inches thick. The bottom plate is 6.13 inches thick. The overall length and diameter of the cask body is 170.25 inches and 40.57 inches respectively. The welds in the shell assembly (containment boundary welds) are full penetration welds.

Attachments and subassemblies associated with the cask body include:

- Lifting attachment on top of the lid.

- MCO standoffs on the inner surface of bottom plate. 
- Cask drain penetration through the bottom plate.

- Cask vent penetration through the lid.

- Lid alignment pins.

- Lid bolts and washers

\section{Closure Lid and Bolts}

The design of the closure lid is shown on drawing H-1-81535, sheet 4 of 5 . The closure lid is a 3.5 inch flanged plate made from ASME SA-336, Type F304 stainless steel or equivalent. This plate forms the lid portion of the containment boundary. The perimeter of the lid has 12 equally spaced holes for the closure bolts which are located on a 36.44 inch diameter bolt circle. The closure bolts are nominally 1.5 inch in diameter manufactured from ASME SA-479-XM19, hot rolled or equivalent material.

One dovetail seal groove is machined in the underside of the lid flange. A Butyl o-ring is installed in this seal groove. The o-ring provides the containment boundary function.

Two lid alignment pins are provided to ensure that the lid is properly aligned with respect to the cask body so that the lid bolts can be installed.

\section{Cask Lifting Attachment}

The lifting function is provided by a pair of lifting trunnions welded to the brackets and gussets. The brackets and gussets are welded to the top surface of the lid, $180^{\circ}$ apart. Detailed dimensions of the lifting attachment are shown on drawing H-1-81535, sheet 4 of 5 . This trunnion pair is sufficient for lifting a 30 ton loaded cask vertically.

\section{Containment Penetrations}

There are a total of two penetrations through the containment vessel, one located in the lid end (designated the Vent Port) and the other, in the cask bottom (designated the Drain Port). The penetrations are used to drain, dry, backfill and vent the containment boundary, or circulate warm water in the interspace between the cavity wall and the MCO. All penetrations are closed and sealed during transport. The configuration details of each penetration including seals, covers and bolts are shown on drawings $\mathrm{H}-1-81535$, sheets 3 of 5 , and 5 of 5 .

\section{$\underline{\text { Vent Port }}$}

The vent port is used for venting and back filling of the cask cavity. It is a direct penetration through the lid, 0.44 inch in diameter, with a threaded outer end to accept a quick-disconnect fitting.

The vent port is closed by a 6.0 inch diameter, 0.75 inch thick blind flange which is secured to the lid with four 0.5 inch diameter socket head cap screws. The penetration cover is recessed 
into the outer plate of the lid so that the outer surfaces are flush. A single Butyl o-ring is mounted in a dovetail groove machined in the underside of the penetration cover.

\section{Drain Port}

This penetration is located in the bottom of the cask body. Access to this penetration is located on the side of the cask body at the bottom plate. A 0.63 inch hole is drilled from the inside surface of the cavity bottom to the cask body side and includes a $90^{\circ}$ bend as shown on drawing $\mathrm{H}-1-81535$, sheet 3 of 5 . The drain port permits draining of the cask cavity with the TN-WHC cask in a vertical orientation. A quick-disconnect coupling is provided at this penetration. As on the vent port, a blind flange which maintains the containment boundary at this point is secured over the drain port by four bolts. A single butyl o-ring is located in the dovetail groove machined in the penetration cover.

\section{A2.1.2 Cask Lifting Attachment}

The lifting attachment for the cask is used for loading and unloading the cask from the conveyance system, for movement at the loadout pit and the CSB, and for any other cask handling operations. It is designed to lift the cask vertically with the cask in the vertical orientation only.

The lifting assembly consists of two trunnions which are attached to the cask lid by a set of brackets and gussets. The overall dimension of the lifting attachment is 43.83 inches. The design details are provided on drawing H-1-81535, sheet 4 of 5 .

\section{A2.1.3 Conveyance}

The design of the trailer is shown on drawings $\mathrm{H}-1-81555, \mathrm{H}-1-81556$, and H-1-81557. The basic components are:

- Main Beams

- Frame

- Deck

- Kingpin

- Landing Legs

- Axles

- Suspensions

- Wheels

- Tires

- Brakes

The major dimensions are as follow: 
- Overall length $40^{\prime}$, With the tractor approximately $54^{\prime}$

- Overall width $10^{\prime}$

- Overall height 17'-7" (loaded at top of the cask lifting attachment)

- Loaded deck height 3'

- Loaded ground clearance 12"

\section{A2.1.4 Cask Operations Equipment}

The K-Basin Loadout Pit Operations Equipment consists of an immersion pail, support structure and operator work platform. In addition to pit side equipment an MCO Cold Vacuum Drying (CVD) Lid is provided to support conditioning/drying of the MCO. Drawings of the individual equipment components and pail assembly showing dimensions and materials for the operations equipment, and drawings of the system general arrangement include the following:

H-1-81543 K-Basin Immersion Pail, Lift Beam Assembly

H-1-81544 K-Basin Immersion Pail

H-1-81545 K-Basin Immersion Pail Support Structure

H-1-81546 K-Basin Immersion Pail Seal Lid

H-1-81547 K-Basin Immersion Pail Assembly

H-1-81549 K-Basin MCO / Cask Cold Vacuum Drying Lid

H-1-81550 K-Basin Immersion Pail Ancillary Equipment

H-1-81551 K-Basin Immersion Pail Interface Control Drawing

H-1-81552 K-Basin Immersion Pail Lock Pin Guide Assembly

H-1-81553 K-Basin Immersion Pail Support Structure Assembly

H-1-81554 K-Basin Immersion Pail Support Equipment

The immersion pail is a thin walled circular cylinder 165.5 inches long with a sealed foam filled floatation cavity. Use of the floatation cavity in the immersion pail design permits the existing crane hardware to lift the pail, cask and filled MCO to the top of the loadout pit shield wall without challenging the crane load capacity. Locating the top of the immersion pail at the top of the loadout pit shield wall for operator access reduces cumulative operator dose supporting project ALARA objectives.

The immersion pail base plate carries the cask load to the pail wall which uniformly transmits the cask load in tension to the top shell ring structure and four immersion pail lifting lugs. The immersion pail lift lugs interface with the loadout pit immersion pail support structure or the 
crane lift slings. During system operation when the immersion pail is moving between support points the immersion pail lift lugs travel within a captured tracking provided by the support structure tub steel columns. The cross section envelop of the immersion pail is 42.75 inches $x$ 53.5 inches which transmits the cask load to the immersion pail structure base plate in bearing when the immersion pail and cask is located on the floor of the loadout pit for the MCO loading operation. The immersion pail support structure base plate is 48.06 inches $\mathrm{x} 56.87$ inches. These envelope dimensions permit pail operation without removal of existing rail structures mounted to the side of the loadout pit. Location control and guidance of the cask during insertion into the immersion pail is provided by a series of beveled, stepped concentric landings. Initial pail opening of 42 inches reduces to 40 inches over approximately 18 inches of axial cask travel.

The immersion pail loadout pit support structure is a steel frame two part structure approximately 320 inches high. Leveling feet are provided between each set of corner column tube steel supports for initial structure installation into the loadout pit. Solid foundation for the support structure is provided during the equipment installation process. Following initial location of the immersion pail, pit support structure grout is installed beneath the support structure base plate providing a solid load path from the support structure to the loadout pit floor. The immersion pail loadout pit support structure provides support for the empty MCO, cask and immersion pail during the task activities performed for maintaining a contamination free boundary prior to lowering the cask to the pit floor. Also, this structure provides support for the loaded system following fuel loading while performing activities for moving the contamination free cask to the cask transport trailer. Manually operated lock pin assemblies are mounted to the top of each of the immersion pail support structure corner columns. The lock pins are designed to be locked in the full open or full closed position.

Two worker platforms are suspended from opposite sides of the loadout pit shield wall to form a slotted work surface, permitting operator access to the immersion pail lid opposite the fuel transfer canal without the use of special tooling. Slotted access is maintained to provide MCO loading system flexibility permitting monorail cable access from the pool to the loadout pit during fuel loading of the MCO. Providing the slotted access permits system operation without movement of the work platform.

The immersion pail seal lid is a stainless steel weldment fitted with inflatable seals on the pail and MCO interface surfaces. The lid is sized to provide approximately 0.5 inch nominal clearance between the MCO and inside diameter of the seal lid. The seal lid and immersion pail boundary provides approximately 0.1 inch nominal clearance. Seal pressure of 45 psig activates the silicone seals which have been rated to withstand radiation environments of $150 \mathrm{Rem} / \mathrm{hr}$. Three lifting lugs are provided for moving the immersion pail lid to and from the immersion pail using slings and the facility crane. Vent port design captures the highest fluid elevation in the inner radius of the lid cavity and is located in the outboard section of the lid to permit easier access during operation. 


\section{A2.2 MATERIALS OF CONSTRUCTION}

\section{A2.2.1 Cask}

The materials of construction are summarized in the following table:

\section{Component}

Cask Shell

Bottom Plate

Closure Lid

Closure Bolts

O-Ring
Material

ASME SA-336, Type F304 or equivalent

ASME SA-336, Type F304 or equivalent

ASME SA-336, Type F304 or equivalent

ASME SA-479-XM19, hot rolled or equivalent

Butyl, Parker B612-70 or equivalent

Materials for other miscellaneous components are listed on drawings H-1-81535, sheets 1 to 5 . (Section A9.1).

\section{A2.2.2 Cask Lifting Attachment}

The lifting attachment structural members are constructed of stainless steel, ASME SA-182, Grade F304. Materials for other miscellaneous components are listed on drawing H-1-81535, sheet 4 of 5 .

\section{A2.2.3 Conveyance}

The materials of construction are summarized in the following table:

\begin{tabular}{||l|l||}
\hline \multicolumn{1}{|c|}{ Component } & \multicolumn{1}{c|}{ Material } \\
\hline Main Beams & A514 (T1) \\
\hline Frame & A-36 \\
\hline Kingpin & SAE-J700 \\
\hline
\end{tabular}

Materials for other miscellaneous components are indicated on drawings H-1-81555, H-1-81556, and H-1-81557.

\section{A2.2.4 Cask Operations Equipment}

The K-Basin Loadout Pit Operations Equipment consists of an immersion pail, loadout pit mounted support structure and loadout pit shield wall suspended work platform. In addition to the loadout pit operations equipment an MCO Cold Vacuum Drying Lid is provided to support conditioning/drying of the MCO. Materials of construction are listed on each component drawing for the respective component item number. The primary immersion pail structure is constructed from Type 304 stainless steel. The immersion pail support structure and loadout pit work 
platform are fabricated from Type A36 and Type A500 series carbon steel and coated to protect it against corrosion over the life of the system. The immersion pail lift beam is fabricated from Type 516 carbon steel and the sling storage box and the immersion pail positive pressure reservoir are made from Type 304 stainless steel. The lock pin is Type 17-4PH stainless steel in order to provide significant margin of safety when postulating a two support configuration with ANSI 14.6 load factors.

The immersion pail seal lid and the MCO conditioning lid are fabricated from Type 304 stainless steel.

\section{A2.3 MECHANICAL PROPERTIES OF MATERIALS}

\section{A2.3.1 Cask}

The mechanical properties of the structural materials used in the cask is shown on following table as a function of temperature. The materials are identified and procured by reference to ASME or corresponding ASTM specifications. The yield and ultimate strengths of the structural steels shown on table are the minimum values specified in the material specifications. The ASME $^{(2)}$ design stress intensity values $\left(\mathrm{S}_{\mathrm{m}}\right)$ for Class 1 components are used to establish allowable stresses for the elastic analyses performed for the cask. Stress intensity limits for the various stress categories are discussed in Sections B4.3 and B4.4. 
Temperature Dependent Material Properties

\begin{tabular}{|c|c|c|c|c|c|c|c|}
\hline Component & Material & $\begin{array}{l}\text { Temp. } \\
{ }^{\circ} \mathrm{F}\end{array}$ & $\begin{array}{c}\text { Ultimate } \\
\mathrm{S}_{\mathrm{u}}(\mathrm{ksi}) \\
\end{array}$ & $\begin{array}{c}\text { Yield } \\
\mathrm{S}_{\mathrm{y}}(\mathrm{ksi}) \\
\end{array}$ & $\begin{array}{l}\text { Allow, } \\
\mathrm{S}_{\mathrm{m}}(\mathrm{ksi})\end{array}$ & $\begin{array}{c}E \\
\left(E^{6} \mathrm{psi}\right) \\
\end{array}$ & $\begin{array}{c}\alpha^{*} \\
\left(\mathrm{E}^{-6}\right) \\
\end{array}$ \\
\hline \multirow{4}{*}{$\begin{array}{l}\text { Cask Body } \\
\text { and } \\
\text { Lid }\end{array}$} & \multirow{4}{*}{$\begin{array}{l}\text { SA-336 } \\
\text { Type F304 }\end{array}$} & 70 & 70 & 30 & 20 & 28.3 & 8.55 \\
\hline & & 200 & 66.2 & 25 & 20 & 27.6 & 8.79 \\
\hline & & 300 & 61.5 & 22.5 & 20 & 27.0 & 9.0 \\
\hline & & 400 & 60.0 & 20.7 & 18.7 & 26.5 & 9.19 \\
\hline \multirow[t]{4}{*}{ Lid Bolt } & \multirow{4}{*}{$\begin{array}{c}\text { SA-479 } \\
\text { XM19 } \\
\text { Hot Rolled }\end{array}$} & 70 & 135 & 105 & & 28.34 & 8.87 \\
\hline & & 200 & & & & 27.6 & 9.02 \\
\hline & & 300 & & & & 27.0 & 9.10 \\
\hline & & 400 & & & & 26.5 & 9.14 \\
\hline
\end{tabular}

* Mean Coefficient of Thermal Expansion (in/in- ${ }^{\circ} \mathrm{F}$ ) from $70^{\circ} \mathrm{F}$ to the Indicated Temperature.

\section{A2.3.2 Cask Lifting Attachment}

Mechanical properties of the material used in the cask lifting attachment are listed in the following table.

\begin{tabular}{||l|c|c|c|c||}
\hline \multicolumn{1}{|c|}{ Component } & Material & $\begin{array}{c}\text { Temp. } \\
{ }^{\circ} \mathrm{F}\end{array}$ & $\begin{array}{c}\text { Ultimate } \\
\mathrm{S}_{\mathrm{u}}(\mathrm{ksi})\end{array}$ & $\begin{array}{c}\text { Yield } \\
\mathrm{S}_{\mathrm{y}}(\mathrm{ksi})\end{array}$ \\
\hline Trunnion & SA-182 F304 & $70^{\circ}$ & 75 & 30 \\
\hline Bracket & Type 304 SS & $70^{\circ}$ & 75 & 30 \\
\hline Gusset & Type 304 SS & $70^{\circ}$ & 75 & 30 \\
\hline
\end{tabular}

The lifting attachment will be designed per ANSI N14.6 $6^{(3)}$ with a factor of safety of three to yield or five to ultimate, whichever is most restrictive.

\section{A2.3.3 Conveyance}

Mechanical properties of the material used in the trailer are listed in the following table. 
HNF-SD-SNF-FDR-003 Rev. $0 \quad$ E-15166

\begin{tabular}{||l|c|c|c|c||}
\hline \multicolumn{1}{|c|}{ Component } & Material & $\begin{array}{c}\text { Temp. } \\
{ }^{\circ} \mathrm{F}\end{array}$ & $\begin{array}{c}\text { Ultimate } \\
\mathrm{S}_{\mathrm{u}}(\mathrm{ksi})\end{array}$ & $\begin{array}{c}\text { Yield } \\
\mathrm{S}_{\mathrm{y}}(\mathrm{ksi})\end{array}$ \\
\hline Main Beams & $\begin{array}{c}\text { A-514 } \\
(\mathrm{T} 1)\end{array}$ & $70^{\circ}$ & 110 & 100 \\
\hline Frame & A-36 & $70^{\circ}$ & 58 & 36 \\
\hline
\end{tabular}

\section{A2.3.4 Cask Operations Equipment}

Cask operational equipment load path structures are fabricated from 500 series carbon steel, 17-4 PH stainless steel or Type 304 stainless steel. Yield strength and material ultimate strength for these materials at service temperatures equal to or less than $150^{\circ} \mathrm{F}$ are as follows:

$\underline{\text { Material }}$

Carbon Steel, ASTM A500, GR B

Type 304 Stainless Steel

17-4PH Stainless Steel
Yield Strength

(ksi)

46.0

30.0

100.1

\section{Ultimate Strength} (ksi)

58.0

75.0

135.0

\section{A2.4 DESIGN AND FABRICATION METHODS}

\section{A2.4.1 Cask}

\section{A2.4.1.1 Design}

The cask is designed to comply with all the requirements of Hanford Specification ${ }^{(1)}$ for normal and accident conditions. Part B of this DAR provides the details of the design analyses. The structural material selected is ASME/ASTM certified stainless steel (See design drawings, Section A9.1). Stainless steel has adequate resistance to the corrosive effects of materials (liquids, vapors, gases and solids) that it could come in contact with the cask during the cask's life cycle at the Hanford Nuclear Reservation site. The stainless steel material also minimizes contamination adhesion and chemical-galvanic reactions between the payload components and the cask. The material also complies with the material requirements identified in NUREG/CR$3854^{(4)}$. A minimum wall thickness of at least 3 -in. $(7.62-\mathrm{cm})$ of stainless steel has been provided as required by Hanford Specification ${ }^{(1)}$. The design considers avoiding potential contamination traps to the greatest extent practicable. 


\section{A2.4.1.2 Fabrication Methods}

Fabrication criteria for a Category I packaging, as delineated in NUREG/CR-3854 will be followed. Fabrication of the packaging will be performed in accordance with the ASME Code, Section III as required by NUREG/CR-3854.

The cask will be fabricated from stainless steel using proven manufacturing techniques for spent fuel transportation casks. The cask body will consist of one or more forged cylinders welded with a complete penetration weld to a bottom closure head forging. It is anticipated that the cask body welds will be narrow groove welds made primary by Gas Tungsten Arc Automatic Welding (GTAW) and the Submerged Arc Automatic Welding (SAW) process and welded from the outer diameter.

All welds and weld joints will be examined per the ASME Code, Section III. Welds will be inspected per ASME Section V by welders qualified to ASME Section IX. All containment welds will be radiographed per ASME requirements.

\section{A2.4.2 Cask Lifting Attachment}

The lifting Attachment is designed per ANSI N14.6 with a factor of safety of three to yield or five to ultimate, whichever is most restrictive. Fabrication of the lifting attachment will also be performed in accordance with the requirements of ANSI N14.6.

\section{A2.4.3 Conveyance}

The trailer will be designed per Hanford Specification ${ }^{(1)}$ with a factor of safety of two to yield. All welding will be in accordance with AWS D1.1.

\section{A2.4.4 Cask Operations Equipment}

The K-Basin Loadout Pit Operations Equipment consists of an immersion pail, immersion pail support structure, and loadout pit operations work platform. Safety related load path components are designed in accordance with ANSI N14.6 ${ }^{(3)}$.

To supplement the requirements of ANSI N14.6, applicable design criteria from the American Institute of Steel Construction (AISC) and ASME Boiler and Pressure Vessel Code have been adopted for evaluation of support structures for loads other than tension loading. 


\section{A2.5 WEIGHTS AND CENTER OF GRAVITY}

\section{A2.5.1 Cask}

The calculated gross weight of the cask (including contents) is 57,910 pounds. Approximate weights of major individual components or subassemblies are tabulated below:

$\begin{array}{ll}\text { Weight of Lid: } & 1,890 \mathrm{lbs} \\ \text { Weight of Shell: } & 34,300 \mathrm{lbs} \\ \text { Weight of Bottom: } & 2,270 \mathrm{lbs} \\ \text { Weight of Lifting Attachment: } & 500 \mathrm{lbs} \\ \text { Weight of Dry MCO: } & 18,950 \mathrm{lbs} \\ & \\ \text { Gross Cask Weight (Dry): } & 57,910 \mathrm{lbs}\end{array}$

The center of gravity of the unloaded packaging is located on the cylindrical axis at 82.29 inches from the outer bottom surface.

The center of gravity for a loaded packaging (Dry) is located on the cylindrical axis at approximately 83.6 inches from the outer bottom surface.

\section{A2.5.2 Cask Lifting Attachment}

The calculated gross weight of the lifting attachment is 500 pounds. Approximate weights of major individual components are tabulated below:

Trunnions: $120 \mathrm{lbs}$

Brackets: $250 \mathrm{lbs}$

Gussets: $\quad 130$ lbs

\section{A2.5.3 Conveyance}

The calculated gross weight of the trailer is $19,500 \mathrm{lbs}$. Approximate weights of the major individual components used for trailer design are tabulated below:

$\begin{array}{ll}\text { Tractor: } & 18,500 \# \\ \text { Trailer: } & 19,500 \# \\ \text { Cask \& Loaded MCO: } & 57,910 \# \text { (Dry), 59,370\# (Wet) } \\ \text { Platform: } & 2,500 \# \\ \text { Tie Down System: } & 5,000 \# \\ & \\ \text { Total gross weight } & 103,410 \# \text { (Dry), 104,870\# (Wet) }\end{array}$


The center of gravity of the loaded cask on the trailer is 104.27 " above the ground and is not exceed $120 \%$ of the axle track (axle track is $95 "$ ).

\section{A2.5.4 Cask Operations Equipment}

The K-Basin operations equipment components of interest relative to weight considerations include the immersion pail and immersion pail seal lid. These components represent the operational equipment which influence loading on the crane and loadout pit immersion pail support structure. Although the weight of these structures influence total load path loading, the design for the in-pit equipment incorporates buoyancy force of the submerged pail volume and retains full crane capacity for the loaded cask.

Summary of component and system weight loading:

$\begin{array}{ll}\text { Component } & \underline{\text { Pounds }} \\ \text { Pail } & 5,700(\#) \\ \text { Pail Lid } & 600(\#) \\ \text { Cask } & 36,570(\#) \\ \text { Cask Lid } & 1,890(\#) \\ \text { Cask Lifting Attachment } & 500(\#) \\ \text { MCO } & 1,900(\#) \\ \text { MCO Contents } & 15,685(\#) \\ \text { MCO Water } & 1,210(\#) \\ \text { MCO Shield } & 1,360(\#) \\ \text { MCO Loaded (wet) } & 20,160(\#) \\ & \\ \text { Pail Slings } & 400(\#) \\ \text { Pail Lift Beam } & 550(\#) \\ \text { Pail Water w/cask } & 850(\#) \\ \text { MCO/Cask Annulus Water } & 250(\#)\end{array}$

\section{A2.6 CONTAINMENT BOUNDARY}

The containment boundary consists of the cask body cylindrical shell, bottom plate and the lid. The lid bolts and seals are also part of the containment boundary as is the drain and vent cover plates, bolts and seals. The containment boundary is designed to be an ASME Section III, Subsection NB Class I component as applicable. The Subsection NB rules for materials, design, fabrication and examination are applied to all of the above components to the maximum practical extent. The containment boundary is shown on Figure A2.6-1. 
HNF-SD-SNF-FDR-003 Rev. $0 \quad$ E-15166

The acceptability of the containment boundary under the applied loads is based on the following criteria:

- ASME Code Design Stress Intensities

- Fatigue Failure to be Precluded

- Brittle Fracture to be Precluded

- Buckling to be Prevented

The values for material properties, design stress intensities $\left(S_{m}\right)$ and design fatigue curves for Class 1 components given in Part D of Section II of the ASME B\&PV Code shall be used for the containment boundary materials. Allowable stress levels for containment components are outlined in Table B4.3-4.

The design properties of other materials are based on industry-recognized specifications, or standards, or on appropriate test data.

\section{A2.7 CAVITY SIZE}

The basic structure of the cask cavity is a right circular cylinder. The cask cavity has a length of 160.50 inches and an internal diameter of 25.19 inches. A 4.0 inch land is located towards the top of the cavity. This land has an internal diameter of 24.25 inches. The cavity of the TN-WHC cask is depicted in Figure A2.6-1.

\section{A2.8 HEAT DISSIPATION}

The cask is designed to dissipate the design basis heat load of the payload while maintaining cask component temperatures within acceptable ranges. The thermal evaluation is provided in Section B5.0. The following is a summary of the heat to be dissipated during normal and accident conditions: 
HNF-SD-SNF-FDR-003 Rev. $0 \quad$ E-15166

Normal Conditions

\begin{tabular}{||l|l|l|l|l||}
\hline \hline Payload & $\begin{array}{l}\text { Surface Heat Flux } \\
\text { At MCO Sidewalls }\end{array}$ & $\begin{array}{l}\text { Surface } \\
\text { Heat Flux } \\
\text { At MCO } \\
\text { Top }\end{array}$ & $\begin{array}{l}\text { Surface Heat } \\
\text { Flux At } \\
\text { MCO } \\
\text { Bottom }\end{array}$ & $\begin{array}{l}\text { Total } \\
\text { Watts Per } \\
\text { MCO }\end{array}$ \\
\hline Maximum & 12.7 Watts $/ \mathrm{ft}^{2}$ & $\begin{array}{l}6.0 \\
\text { Watts } / \mathrm{ft}^{2}\end{array}$ & 0 & 950 \\
\hline Minimum & 0 & 0 & 0 & 0 \\
\hline
\end{tabular}

(The defined sidewall surface heat flux occurs over the lower $11.7 \mathrm{ft}$. of the MCO) Accident Conditions

\begin{tabular}{||c|l|l|c||}
\hline Payload & $\begin{array}{l}\text { Surface Heat Flux at } \\
\text { Center 28 in Section } \\
\text { of MCO Sidewall }\end{array}$ & $\begin{array}{l}\text { Surface Heat Flux at } \\
\text { Remainder of MCO } \\
\text { Sidewall }\end{array}$ & $\begin{array}{l}\text { Total Watts } \\
\text { Per MCO }\end{array}$ \\
\hline Maximum & 30 Watts $/ \mathrm{ft}^{2}$ & 12.7 Watts $/ \mathrm{ft}^{2}$ & 1200 \\
\hline Minimum & 0 & 0 & 0 \\
\hline
\end{tabular}

\section{A2.9 SHIELDING}

The most significant shielding design features of the cask are the thick walled forged stainless steel cask body and lid. The cask body has a minimum wall thickness of 7.25 inches and a minimum bottom thickness of 6.13 inches. Additional shielding is provided by the fuel assemblies, the baskets, and the MCO. The cask design does not included separate neutron shielding because of the relatively low neutron source term in the spent fuel.

The shielding analysis of the cask is performed using industry standard codes and conservative modeling assumptions. An evaluation of the shielding performance of the cask was performed assuming dry cask conditions. This evaluation is based on the maximum source term payload.

The bounding source term used for the shielding evaluation is the Mark IV fuel elements, $0.95 \mathrm{U}$ 235 irradiation to $16 \% \mathrm{Pu}-240$, thirteen years after discharge from the $\mathrm{N}$ reactor.

The gamma and neutron analyses are performed using the one dimensional SAS1 module of SCALE-4, with the $27 \mathrm{n}-18 \mathrm{~g}$ coupled cross-section library. This uses the codes XSDRNPM and 
XSDOSE to calculate surface flux and translate the flux into dose rates away from the cask surface. ANSI standard flux-to-dose factors, within SCALE-4, are used for the dose calculation at the selected points.

The method in which the cask and its contents are modeled for the shielding analysis and the shielding analysis results are described in the Section B3.0.

\section{A2.10 CASK LIFTING ATTACHMENT}

The lifting attachment for the cask are the two lifting trunnions, two brackets and two gussets. The trunnions, brackets and gussets are designed per ANSI N14.6 with a factor of safety of three to yield or five to ultimate, whichever is most restrictive.

\section{A2.11 TIEDOWN ATTACHMENTS}

The cask is held down by the cask hold down device which is part of the tiedown system. There are no tiedown attachments required at the cask. The tiedown system is designed to withstand the tiedown vertical $2 \mathrm{~g}$ loads. The cask tiedown system design and load evaluations are provided in Section B7.0. Detailed dimensions of the tiedown system are shown on drawings H-1-81539, sheets 1 to 3 . 
HNF-SD-SNF-FDR-003 Rev. 0

E-15166

Figure A2.6-1 TN-WHC Cask Containment Boundary

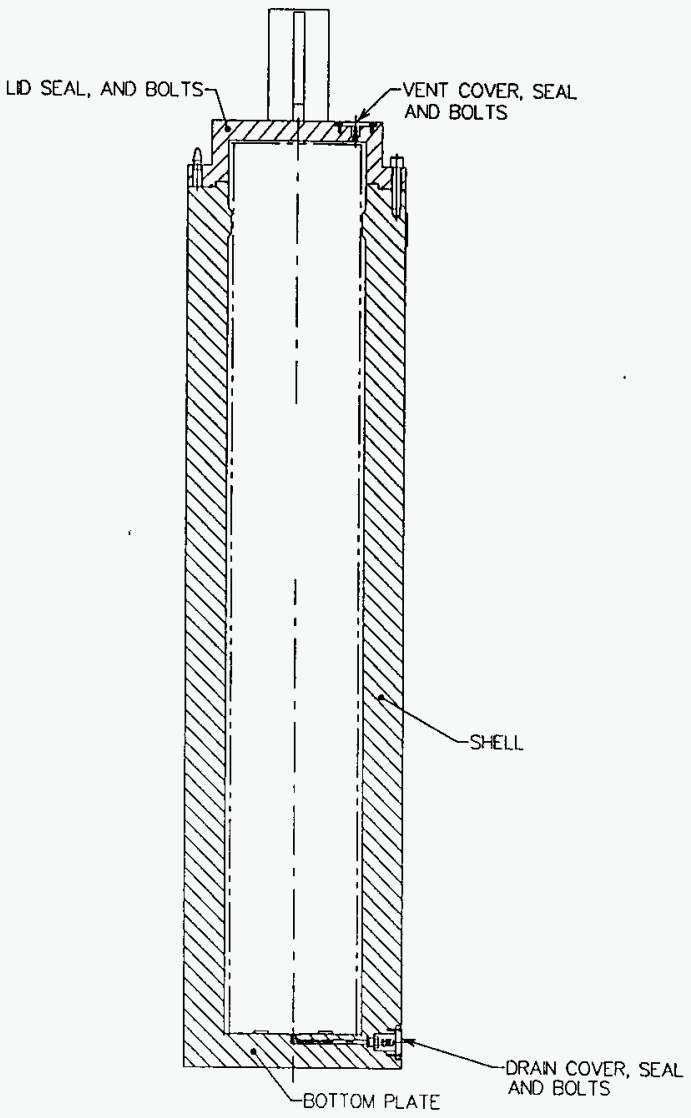




\section{References For Section A2.0}

1. Performance Specification For TN-WHC Cask And Transportation System, WHC-S-0396, Rev. 1, September 1995.

2. ASME Boiler and Pressure Vessel Code, Section III, Subsection NB, American Society of Mechanical Engineers, NY, 1992. (1992 Revision).

3. Special Lifting Devices for Shipping Containers Weighing 10,000 Pounds or More, ANSI N14.6, American National Standards Institute Inc., NY, 1993,

4. Fischer, L. E. and Lai, W., 1985, Fabrication Criteria for Shipping Containers, NUREG/CR-3854 UCRL-53544, Lawrence Livermore National Laboratory, Livermore, California. 
HNF-SD-SNF-FDR-003 Rev. 0 E-15166

\section{A3.0 PACKAGE CONTENTS}

\section{A3.1 GENERAL DESCRIPTION}

\section{A3.1.1 Physical Form}

The Cask contents consist of a Multiple Canister Overpack (MCO) which is the primary containment for the fuel elements. MCOs are $24 \mathrm{in.}(0.61 \mathrm{~m})$ outside diameter, stainless steel pipe slightly over $13 \mathrm{ft}(4 \mathrm{~m})$ long, with the metallic uranium fuel elements in baskets stacked inside (Figure A3.1-1). Approximately 400 of these fuel containers (MCOs) will be handled.

\section{A3.1.2 MCO Weights and Dimensions}

The approximate weights of the MCO components and some dimensions are supplied in Table A3.1-1.

References For Section A3.0

1. Performance Specification For TN-WHC Cask And Transportation System, WHC-S-0396, Rev. 1, September 1995. 
HNF-SD-SNF-FDR-003 Rev. $0 \quad$ E-15166

Table A3.1-1 Weights and Dimensions of MCO Components

\begin{tabular}{|l|c|}
\hline \multicolumn{1}{|c|}{ Payload configuration } & Figure 14 of Ref. 1 \\
\hline MCO length & Figure 15 of Ref. 1 \\
\hline MCO outer diameter & Figure 15 of Ref. 1 \\
\hline MCO wall thickness & Figure 15 of Ref. 1 \\
\hline Water - Kg (lb) & $551(1,210)$ \\
\hline $\begin{array}{l}\text { MCO w/o shield plug \& empty } \\
\text { Kg (lb) }\end{array}$ & $862(1,900)$ \\
\hline $\begin{array}{l}\text { MCO shield plug (30.5 cm thick) } \\
\text { Fully assembled - Kg (lb) }\end{array}$ & $616(1,360)$ \\
\hline \begin{tabular}{l} 
Zr Clad - Kg (lb) \\
\hline Fuel and Clad - Kg (lb)
\end{tabular} & $445(980)$ \\
\hline $\begin{array}{l}\text { E MK IV Baskets w/54 Mk IV } \\
\text { Elements per basket - Kg (lb) }\end{array}$ & $6,835(15,050)$ \\
\hline $\begin{array}{l}\text { Total flooded maximum MCO weight } \\
\text { Wet - Kg (lb) }\end{array}$ & $7,115(15,685)$ \\
\hline
\end{tabular}


HNF-SD-SNF-FDR-003 Rev. $0 \quad$ E-15166

Figure A3.1-1 Typical Arrangement of Spent Fuel in the MCO

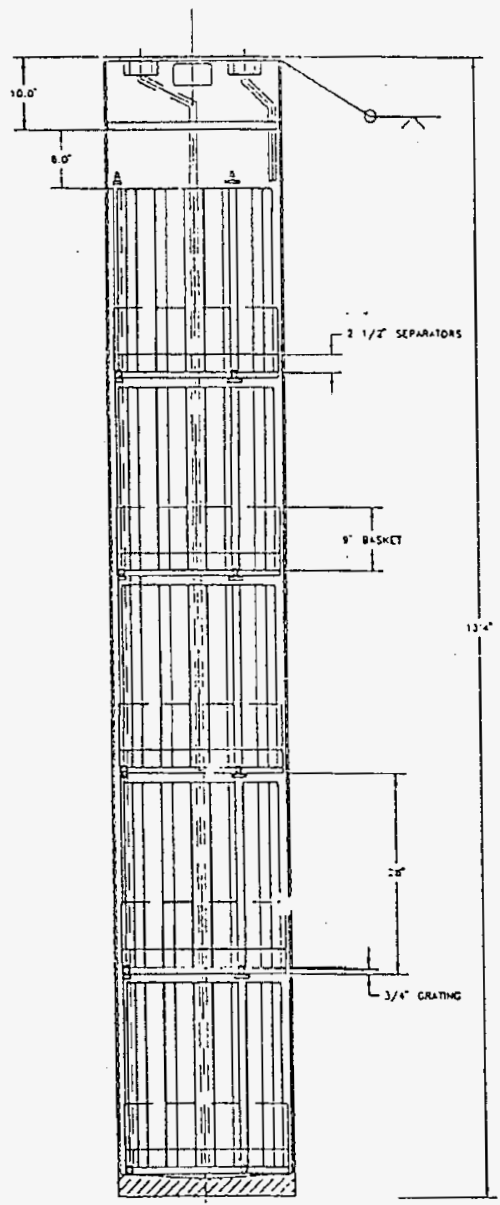




\section{A4.0 TRANSPORT SYSTEM}

\section{A4. 1 TRANSPORTER}

\section{System Components}

The design of the trailer is shown on drawings H-1-81555, H-1-81556, and H-1-81557. The basic components are:

- Main Beams

- Frame

- Deck

- Kingpin

- Landing Legs

- Axles

- Suspensions

- Wheels

- Tires

- Brakes

The major dimensions are as follow:

- Overall length $40^{\prime}$, With the tractor approximately $54^{\prime}$

- Overall width $10^{\prime}$

- Overall height $17^{\prime} 7^{\prime \prime}$ (loaded at top of cask lifting attachment)

- Loaded deck height $3^{\prime}$

- Loaded ground clearance $12^{\prime \prime}$

The trailer has three axles. The axles have a nominal capacity of $22,000 \mathrm{lbs}$ per axle. The axle track is $95^{\prime \prime}$. The trailer has three air ride suspensions. Each suspension has a rated capacity of $25,000 \#$. The tri-axle assembly will be controlled by a single automatic height control valve. A manual override system will also be provided to raise or lower the rear of the trailer as needed.

Landing legs is adequately braced to the frame of the trailer. A $38^{\prime \prime}$ square bearing pad is provided for each landing leg to accommodate the soil bearing restrictions. The pad will be placed under the legs on an as needed basis and not permanently attached to the legs.

275/R x 22.5 - Load Range H Bridgestone Tires stated rating on the side wall shall be $6,175 \#$ per tire. Actual load on the tire will not exceed $90 \%$ of the stated rating or exceed $600 \#$ per inch of the tire width. The loaded section for this tire is $11.9^{\prime \prime}$. 


\title{
Design Load Factor
}

The trailer is designed to meet the requirements of the Hanford Specification. The trailer system is capable of resisting the forces for road, as described below.

Design load requirements:

\author{
$2.5 \mathrm{G}$ down vertical \\ $2.0 \mathrm{G}$ up vertical \\ $1.5 \mathrm{G}$ lateral both directions \\ $2.0 \mathrm{G}$ fore \\ $1.5 \mathrm{G}$ aft \\ Parked \\ $1.5 \dot{\mathrm{G}}$ down vertical
}

The weight distribution ( $1 \mathrm{G}$ down) for the six axle combination shall be approximately 10,000 lbs on the steering axle, $37,250 \mathrm{lbs}$ on the tractor drive axles, and 57,620 lbs on the trailer triaxle assembly.

\section{A4.2 TIEDOWN SYSTEM}

The basic components of the system are:

- Frames and Beams

- Cask Tiedown Device

- Cask Hold Down Device

- Cask Support Device

The eight frames, two cross beams and four support braces (including the weld attachments of the vertical support frames to the support pads of the trailer) are the major components of the tiedown system. The loads from the cask are transmitted by the frames to the trailer. The cask tiedown device consists of two rotating flanges, six tiedown bolts, and two hold down pins. The cask hold down device consists of four hold down arms which bolted to the brackets of the cask tiedown device. The cask support device consists of one cylindrical cup, bottom plate, and fourteen (14) attachment bolts. A detailed design drawing for the cask tiedown system is provided in Section A9.1.

The cask is supported by the support device (cylindrical cup) at the bottom and secured by the tiedown device (two rotating flanges) at the top. The two rotating flanges can be opened and closed (by removing the six tiedown bolts and rotated from the hold down pins) for loading and unloading the cask. Figure A4.2-1 shows the opened and closed positions of the rotating flanges of the cask tiedown device. The cask is transported in the vertical orientation with the lid end facing up. 
Figure A4.2-1 TN-WHC Cask Tiedown System - Opened and Closed Positions
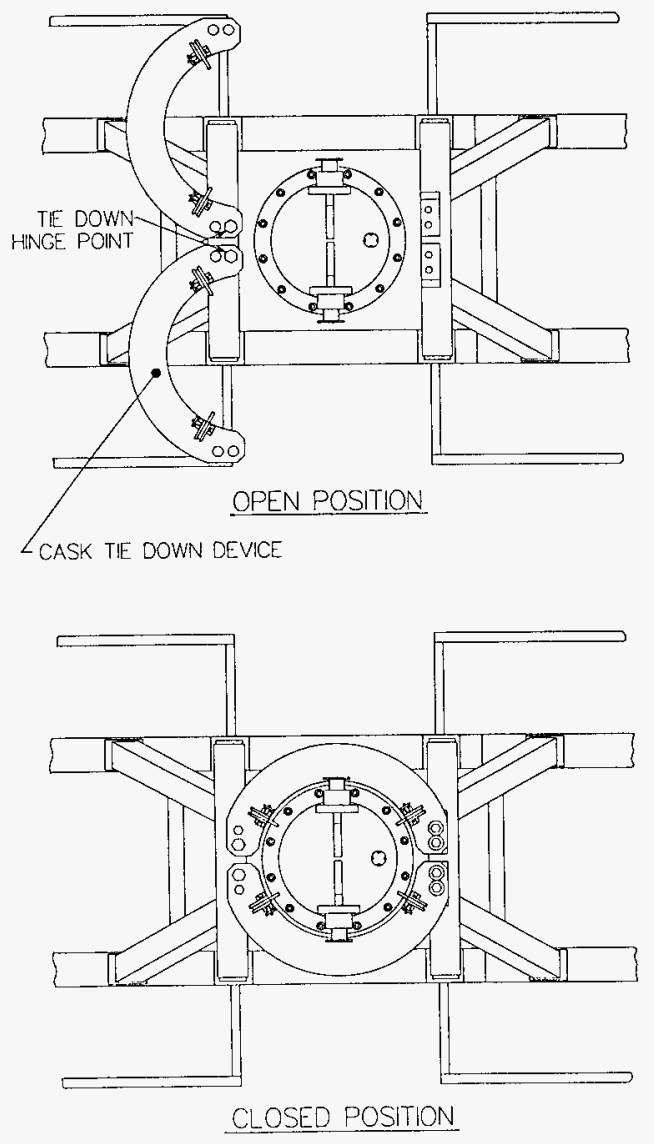


\section{A4.3 SPECIAL TRANSFER REQUIREMENTS}

None. 


\section{HNF-SD-SNF-FDR-003 ReV. ${ }^{\circ}$ E-15166}

\section{A4.4 APPENDIX}

Structural Analysis of the Trailer 


\section{TABLE OF CONTENTS}

\section{Section}

1. INTRODUCTION

2. SUMMARY OF RESULTS 2

3. COMPUTER MODEL AND ANALYSIS 3

4. LOADING CRITERIA 9

4.1 Trailer Weight 9

4.2 Wind Loading 9

4.3 Parked Loading 9

4. 4 Computer Load Cases 9

5. SUPPLEMENTAL CALCULATIONS

5.1 Maximum Main Beam Stress 11

5.2. Maximum Cross Beam and Other Structural stress 11

5.3 Loads on Seismic Lugs 12

5.4 Deflections 12

APPENDIX A - MEMBER PROPERTIES

APPENDIX C - COMPUTER OUTPUT

APPENDIX D - SEISMIC ANALYSIS DATA

APPENDIX E - CALCULATION OF OVERTURNING G LOAD

APPENDIX F - FATIGUE ANALYSIS 


\section{INTRODUCTION}

This report contains a very detailed computer analysis made of this trailer. A computer model which is virtually identical with the actual trailer construction has been developed and the various loading conditions applied. There were seven separate computer analyses made. See section 4.5 of this report for a listing of the load cases and their corresponding data. The computer output for all load cases is given in Appendix $\mathrm{C}$.

All analyses were made in a conservative manner. Thus, the results and conclusions contained in this report will give a safe view of the trailer's ability to withstand the specified loading.

The computer model used is shown in considerable detail in Figures 1 , 2, 3, and 4. The properties of the members are given in Appendix A. The allowable stresses used axe 50\% of the yield stress for all loading cases. All allowables are in accordance with the specification.
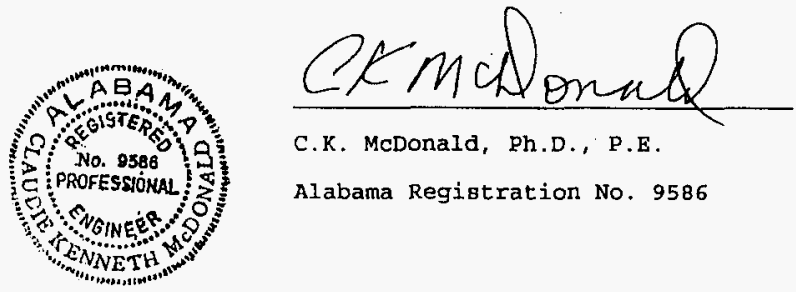

C.K. McDonald, Ph.D., P.E.

Alabama Registration No. 9586 


\section{SUMMARY OF RESULTS}

The maximum stresses are compared to the allowables below. See Appendix $C$ for the computer output for all loading conditions. The actual and allowable stresses are in psi.

Components

Maximum Main Beam Stress, psi

Maximum Cross Beam and Other Structural Stress, Psi

\section{Actual Allowable}

$$
43,384 \quad 50,000
$$

$16,828 \quad 18,000$

The main beam is $T 1$ steel with a minimum yield stress of 100,000 psi. All other structural is A-36 steel. 
E-15166

HNF-SD-SNF-FDR-003 Rev. O

\section{COMPUTER MODEL AND ANALYSIS}

The computer model for the trailer is shown in Figures 1, 2, 3, and 4 . The model contains approximately 5,000 nodes and 6,000 elements/members. The units used in this analysis are lbs. and inches.

The analysis was made utilizing the computer code ANSYS 5.3 operating on a Pentium 100 Megaherz computer.

See Appendix $\mathrm{C}$ for the computer output all load cases.

Modeling'a trailer, although appearing deceptively simple, presents some special modeling problems. This is because you are modeling an assemblege of plates and beams with a relatively course mesh. The purpose is to ascertain the overall structural integrity of the trailer, thus a coarse mesh is adequate. However, for a trailer, most of the beam type elements can only bend in one direction, the other direction is restrained by a continuous weld or closely spaced bolts. In some case, such as corners of the trailer, both directions are restrained from localized bending. Since the beams are only attached to the plates at nodes in the computer model, unrealistic bending stresses are sometimes predicted by the computer.

In this model, in order to eliminate these unrealistic bending stresses in some beam elements, the computer input is adjusted such that it does this without affecting the integrity and results of the model. This is done by setting the distance from the nuetral axis to near zero in the real constants for that element that cannot bend. This eliminates the stress without affecting the model. The reason that the value is set to near zero but not zero is because the computer will stop each time and question the zero and this is cumbersome in performing the analysis. 


\section{HNF-SD-SNF-FDR-003 Rev. 0}

The localized bending of the beams does not affect the overall structural integrity of the trailer which is maintained by the web plates and the floor deck. 


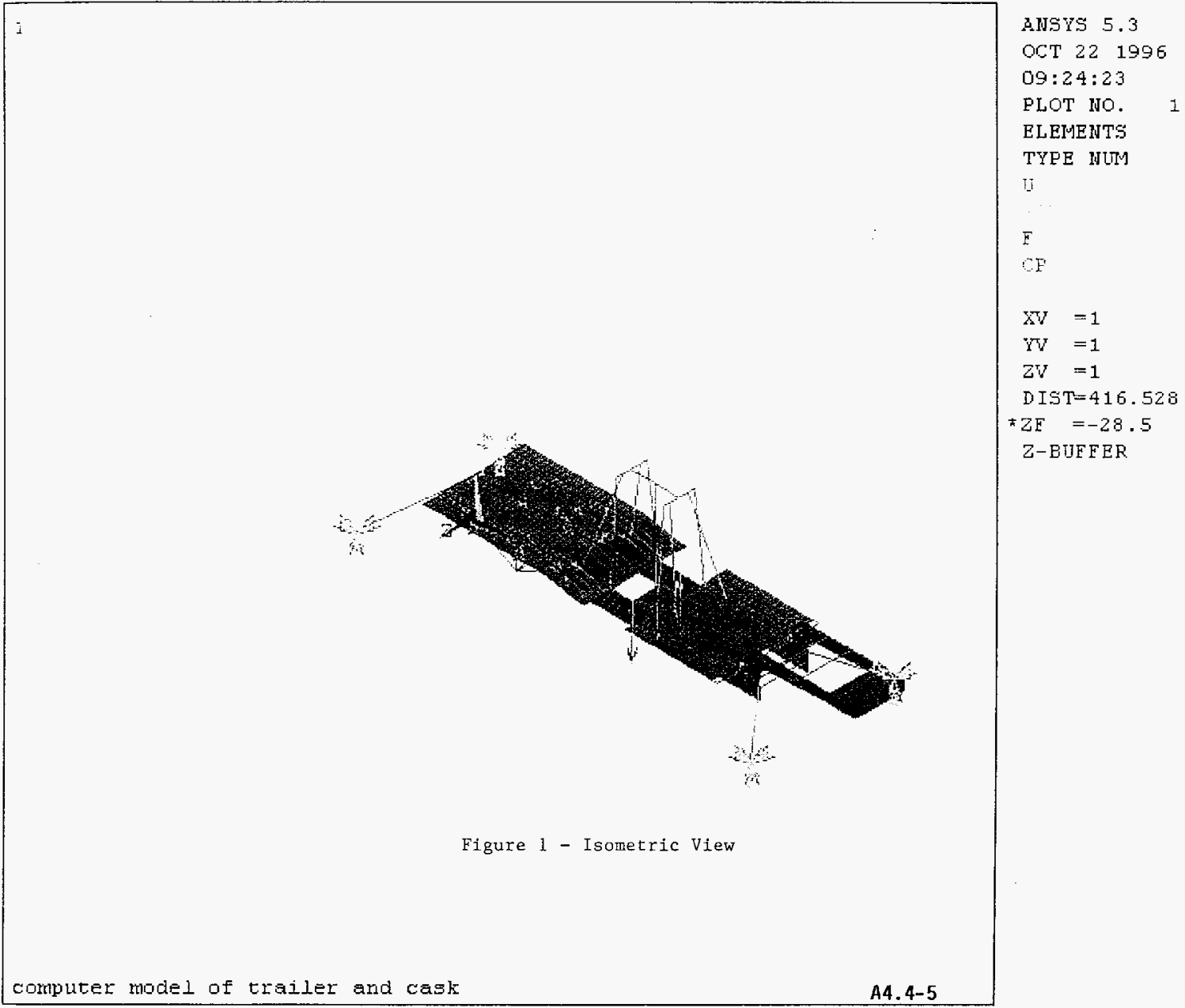




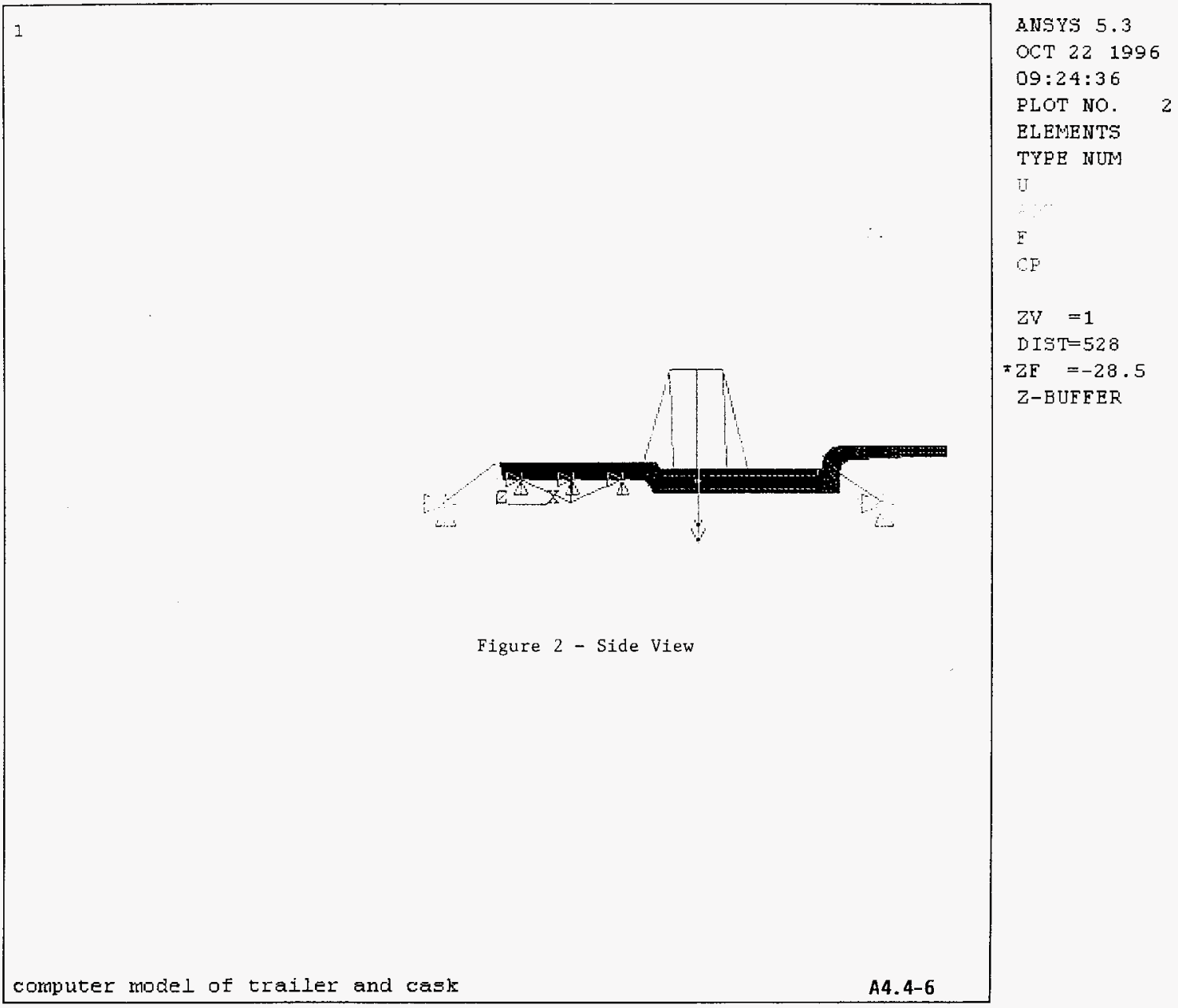

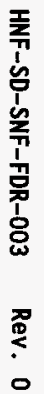




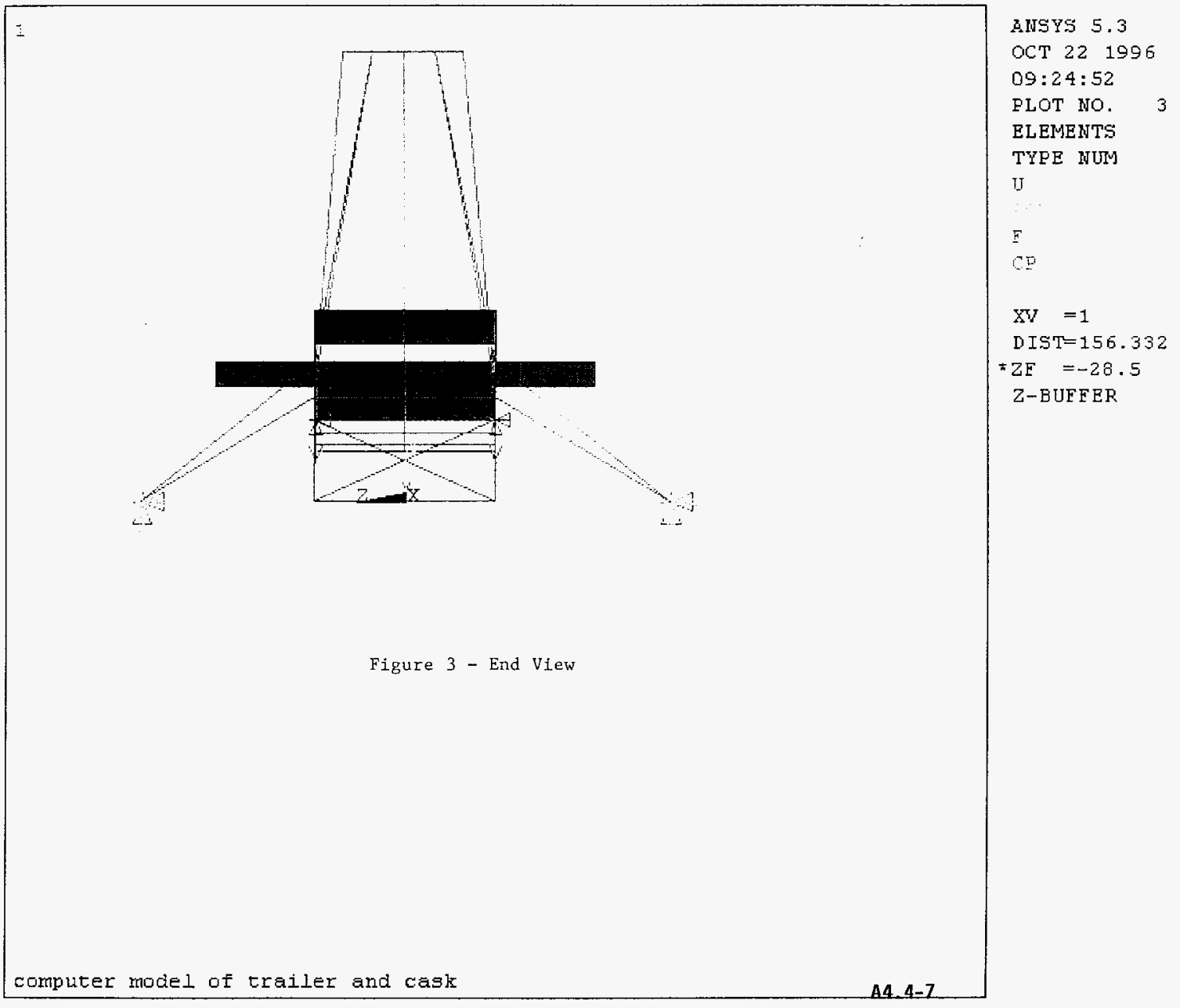




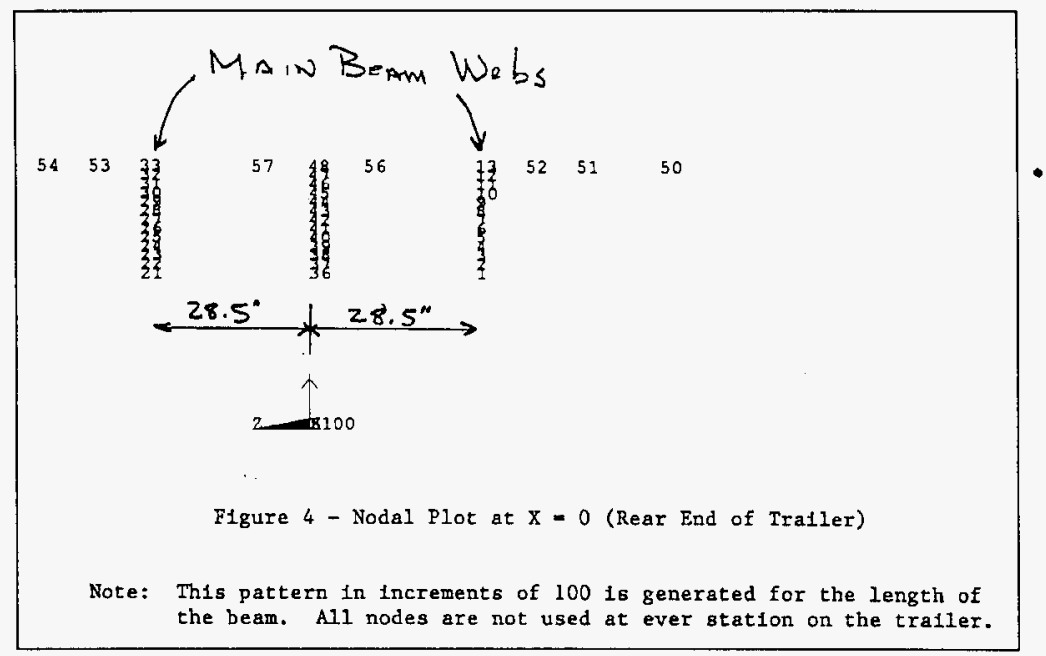

ANSYS 5.3

OCT 22,1996

$12: 14: 46$

PLOT NO. 1

NODES

NODE NUM

$\mathrm{CP}$

ACEL

$\mathrm{XV}=1$

DIST $=97.35$

$\star Z F=-28.5$

$\mathrm{Z}-\mathrm{BUFFER}$ 


\section{LOADING CRITERIA}

\subsection{Trailer Weight}

The total trailer weight used in the analysis is 86,100 1bs, including the cask and tie down system.

\subsection{Shock Loads}

The shock loads applied are $2.5 \mathrm{~g}$ downward, $2 \mathrm{~g}$ upward, $2 \mathrm{~g}$ fore, and $1.5 \mathrm{~g}$ aft. A load of $1.5 \mathrm{~g}$ lateral is specified but a load of $0.5 \mathrm{~g}$ lateral overturns the trailer when loaded, see reactions for the lateral load analysis, page C-25, to confirm this. The seismic loads also includes a lateral load when the trailer is tied down with the seismic struts. See Section 4.4 below for a detailed discussion of the computer loading.

\subsection{Parked Loading}

The parked shock factor is $1.5 \mathrm{~g}$ vertical.

\subsection{Computer Load Cases}

The above loadings are imposed by computer in the following load cases.

A) $2.5 \mathrm{~g}$ Vertical Down

The trailer is supported at the kingpin and on the rear axles.

B) 2.0g Vertical Up + Deadweight

The entire trailer will never see this load because it would leave the ground. However, an individual component could see it. Thus, to be 


\section{HNF-SD-SNF-FDR-003 Rev. 0}

conservative, the entire trailer is loaded with $2 \mathrm{~g}$ upward plus deadweight and the trailer is supported at the seismic struts, which is conservative.

C) $2.0 \mathrm{~g}$ Fore + Deadweight

The trailer is supported by the kingpin in the fore direction and by the kingpin and wheels in the vertical direction.

D) $1.5 \mathrm{~g} \mathrm{Aft}+$ Deadweight

The trailer is supported in the aft direction on the kingpin and vertically by the wheels and kingpin.

E) $1.5 \mathrm{~g}$ Parked (File PARK.DAT)

The trailer is supported by the wheels and landing gear.

F) Seismic + Deadweight

The trailer is supported entirely by the four seismic struts. This is conservative because the trailer is also supported vertical by a pad directly under the cask. The trailer is also supported laterally by friction on the pad and on the tires and landing gear (or tractor).

G) 1.5 Lateral + Deadweight

The maximum load that can be applied laterally without overturning the trailer is $0.5 \mathrm{~g}$. An additional lateral load is applied by seismic, as noted above. 


\section{SUPPLEMENTAL HAND CALCULATIONS}

\subsection{Maximum Main Beam Stress}

The main beam structal stresses are given on pages $\mathrm{C}-1$ through $\mathrm{C}-7$. The maximum stress is:

$$
S=43,384 \text { psi (page } C-4 \text { ) }
$$

The allowable stress for Tl steel which has a minimum yield of 100,000 psi, is 50,000 psi. In additional to the conventional structural stresses, which are maximum in the beam flanges by well known beam theory, the concentrated stresses in the beam were calculated by use of finite elements in the web. These stresses are given on pages C-15 through $\mathrm{C}-21$. The maximum concentrated stress is given on page C-17 and is 75,325 psi. This concentrated stress is only $75 \%$ of the yield stress and occurs at the 90 degree turn at the start of the gooseneck. Note that the plots on pages C-15 through 21 are of such scale that the location of the maximum cannot be seen. Therefore, to confirm the location of the maximum and to check the stresses at other locations.

\subsection{Maximum Stress in Cross Beams and Other Structural}

These stresses are given on pages $\mathrm{C}-8$ through $\mathrm{C}-14$. The maximum is:

$$
\mathrm{S}=16,828 \mathrm{psi} \text { (page } \mathrm{C}-14 \text { ) }
$$

The allowable for $\mathrm{A}-36$ steel is $.5(36000)=18,000$ psi 


\section{HNF-SD-SNF-FDR-003 Rev. 0}

\subsection{Loads on Seismic Strut Lugs}

The loads on the seismic struts are shown on page $\mathrm{C}-28$. The maximum loads for which the lugs on the trailer should be designed are:

$$
\begin{array}{ll}
\mathrm{FX}=43,982 \mathrm{lbs} & \text { (Longitudinal load on lug) } \\
\mathrm{FY}=35,185 \mathrm{lbs} & \text { (Vertical load on lug) } \\
\mathrm{Fz}=53,066 \mathrm{lbs} & \text { (Lateral load on lug) }
\end{array}
$$

\subsection{Deflections}

The deflections of the main beam along the $24 "$ deep span are given on pages $\mathrm{C}-22$ through $\mathrm{C}-28$. For convenience in determining the locations of these deflections the coordinates of the nodes are given on page C-29. The $\mathrm{x}$ dimension on the coordinates is the location rear to front, measured from the rear of the trailer. 
HNF-SD-SNF-FDR-003 Rev. 0

APPENDIX A

MEMBER PROPERTIES

A4.4-13 


$$
\text { HNF-SD-SNF-FDR-003 Rev. } 0
$$

E-15166

Appendix A- Member Properties

Real 1 Main Beam web

$$
t=1 / 2
$$

Real 2 Main Beam Flange Modeled As A Berm. Area is Adjusted to Account for Distance to Doter Fiber of Beam.

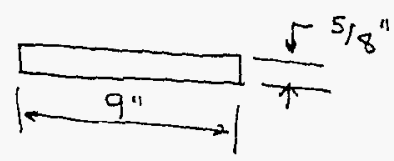

$$
\begin{aligned}
& I_{1}=\frac{.625(9)^{3}}{12}=37.97 \mathrm{in}^{4} \\
& I_{2}=\frac{9(.625)^{3}}{12}=.183 \mathrm{in}^{4} . \\
& I_{\text {Total of Beam }}=\frac{5(18.75)^{3}}{12}+2(.625)(9)(19.6875)^{2}=4635 \mathrm{in}^{4} \\
& \text { Using Compotes Mode) of web, } \\
& I=4635=\frac{.5(20)^{3}}{12}+A^{\prime}(20)^{2}(2) \\
& \left.A^{\prime}=\frac{4635-333}{800}=5.378 \mathrm{in}^{2} \text { (instead of .675(9)=5.625 } \mathrm{in}^{2}\right)
\end{aligned}
$$

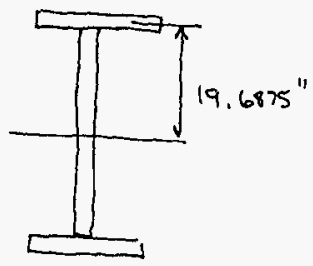

A 4.4-14 


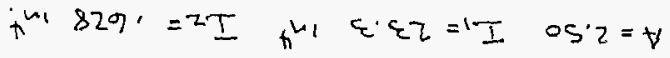

$$
\begin{aligned}
& \text { 1244040 } 5.8 \times 80-\overline{L 792 y} \\
& \text { int } 01 \text { it }=2 I \\
& h^{41} \sin \theta=1 I \\
& z^{u 1} \text { bo. } \varepsilon=\forall \\
& 29 n_{1} h_{1} \times \varepsilon x h-\frac{}{9 v_{2} y}
\end{aligned}
$$

$i^{4} 9200=2 I$

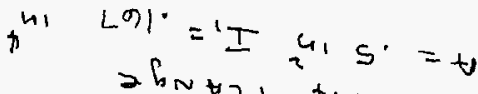

$$
\begin{aligned}
& 2 b_{N \rightarrow 7 g} x_{1 / 1} x_{2}-\overline{51+121}
\end{aligned}
$$

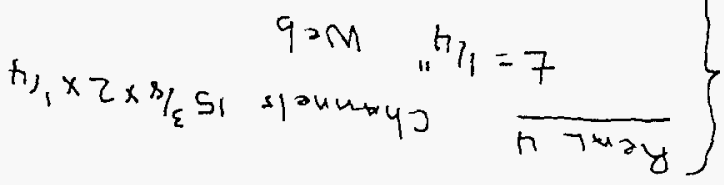

$$
\begin{aligned}
& i^{41} s 8.61=\frac{21}{(52 \cdot L) 527}=2 I \\
& \text { in }^{n} L \hbar 1^{\circ}=\frac{21}{(529) 52 \cdot L}=1 \mathrm{I} \\
& i^{n 1} L L \cdot h=(529) 75 z \cdot L=\forall \\
& \text { "S2.L }=4 p 10 \quad \text { "S/S }=7 \\
& \text { but mage wion } \frac{}{\varepsilon 7 \text { try }} \\
& z-\forall
\end{aligned}
$$




\section{A-3}

Renl 8

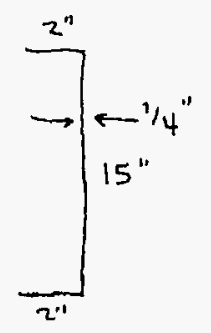

$$
\begin{gathered}
A=14.75(.25)+.25(1.875)^{(2)}=4.63 \mathrm{in}^{2} \\
I_{1}=\frac{.25(14.5)^{3}}{12}+2(2)(.25)(7.375)^{2}=117.91 \mathrm{n}^{4} \\
\bar{y}=\frac{4(.25)(.875)}{4.6 .3}=.1891 \\
I_{2}=\frac{2(.25)(2)^{3}}{12}+14.5(.25)(.189)^{2}=.46 \mathrm{in}^{4}
\end{gathered}
$$

ReAL 9 W6x25 Beam

$$
A=7.34 \mathrm{in}^{2} I_{1}=53.4 \mathrm{in}^{4} \quad I_{2}=17.1 \mathrm{in}^{4}
$$

Real 10

$$
t=1 / 2^{\prime \prime}
$$


HNF-SD-SNF-FDR-003

Rev. 0

E-15166

A- 4

ReaL $11 \quad 4 \times 8 \times 3 / 16$ Tubing

$$
A=4.27 \mathrm{in}^{2} \quad I_{1}=35.3 \mathrm{in}^{4} \quad I_{2}=12.0 \mathrm{in}^{4}
$$

Real ir $5 \times 3 \times 5 / 16$ Angle

$$
A=2.40 \mathrm{in}^{2} \quad I_{1}=6.26 \quad I_{2}=1.75 \mathrm{in}^{4}
$$

ReAl $13 C 5 \times 6.7$

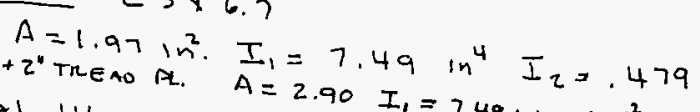

$+3^{\prime \prime}+2^{\prime \prime}$ Tr
ReAL 14

ReAL 14

$$
\begin{aligned}
& \left.A L 14 \quad C 10 \times 15.3=11.47 \mathrm{in}^{4} .97(.81)^{2}+.94(1.60)^{2}\right] \\
& A=4.49 \mathrm{in}^{2} \quad I_{1}=67.4 \mathrm{in}^{4} \quad I_{2}=2.28 \mathrm{in}^{4} \\
& A L 15 \\
& A=.938 \mathrm{in}^{2} \times 1 / 4 \text { Angle }
\end{aligned}
$$$$
A=.938 \mathrm{in}^{2} \quad I_{1}=I_{2}=.348 \mathrm{in}^{4}
$$

Real 16 tread plate

$$
t=3 / 16^{11}
$$

Rent 17 $178 \times 18$

$$
A=5.26 \mathrm{in}^{2} \quad I_{1}=61.9 \mathrm{in}^{4} \quad I_{2}=7.97 \mathrm{in}^{4}
$$

ReAL 18 Dummy members to simulate axles
Ans king ain

A4.4-17 
HNF-SD-SNF-FDR-003 Rev. 0

E-15166

A- 5

Real 19 web Stiffeners

$$
t=3 / 8^{\prime \prime}
$$

REAL $20 \quad w_{e b}+$ StIFFener

$$
t=1 / 2+3 / 8^{\prime \prime}=7 / 8^{\prime \prime}
$$

ReAl 21 Fig + Stiffener

$$
\begin{aligned}
& A=8.5 \quad I_{1}=\frac{8.5(1)^{3}}{12}=.708 \mathrm{in}^{4} \quad I_{2}=\frac{1(8.5)^{3}}{12}=51.17 \\
& \mathrm{in} 4^{4}
\end{aligned}
$$

Real 22 Web StIFFener

$$
\begin{aligned}
& 2-3 / 8^{\prime \prime} \times 4^{\prime \prime} \text { PLATES } \\
& A=2(.375)(4)=3 \mathrm{in}^{2} . \\
& I_{1}=\frac{2(.375)(4)^{3}}{12}=4 \mathrm{in}^{4} \quad I_{2}=\frac{2(4)(375)^{3}}{12}=.035 \\
& \mathrm{in}^{4} .
\end{aligned}
$$

A4.4-18 
E-15166

$$
\text { HNF-SD-SNF-FDR-003 }
$$

Rev. 0

$$
A-6
$$

Real 23

$$
\begin{aligned}
& 6 \times 6 \times \frac{1}{2} \text { Cask Sup pts } \\
& A=10.4 \mathrm{in}^{2} \quad I=50.5 \mathrm{in}^{4}
\end{aligned}
$$

ReAl 24

$$
\begin{aligned}
& 8 \times 8 \times \frac{1}{2} \text { CASk Soppts } \\
& A=14.4 \mathrm{in}^{2} \\
& I=32.9 \mathrm{in}^{4}
\end{aligned}
$$

ReAl 25

Real 24 Dummy Member to Represent Hold DOWN Device. Dummy Member to Represent
Cask 


\section{APPENDIX B}

(deleted) 
HNF-SD-SNF-FDR-003 Rev. 0

E-15166

\section{APPENDIX C}

\section{COMPUTER OUTPUT DATA}




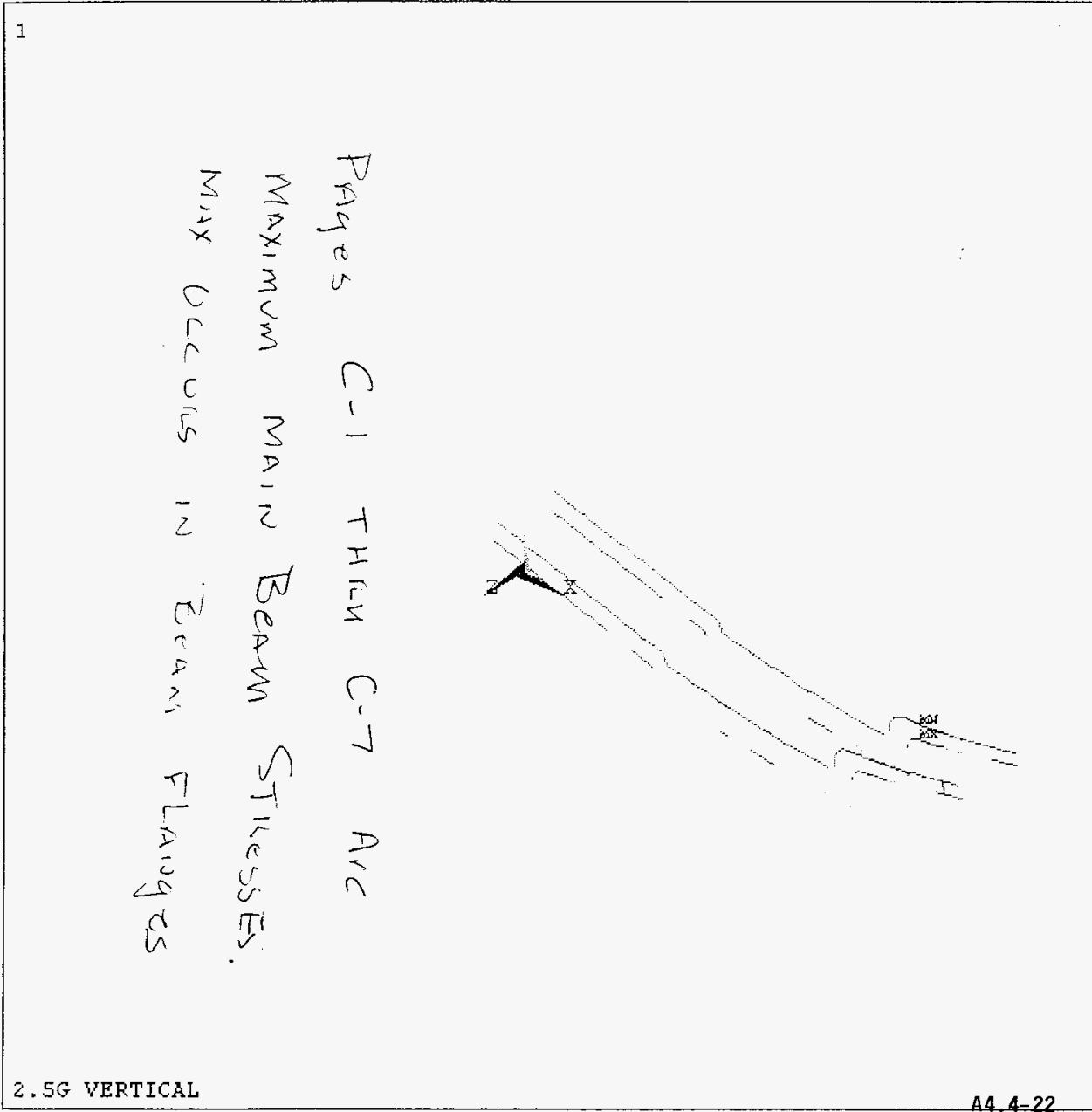

4N5YS 5.3

OCT $21 \quad 1996$

$23: 25: 19$

FLOT NO

ELEMENT SOLUTION $\mathrm{STEP}=1$

$S U B=1$

$\mathrm{TIME}=1$

53

(NOAVG)

TOP

$\mathrm{DWX}=1.731$

$5 \mathrm{IN} 1 \mathrm{IJ}=-36.351$

$5 \mathrm{MX}=37922$

\begin{tabular}{l}
$\square$ \\
$\square$ \\
$\square$ \\
\hline \\
$\square$ \\
$\square$ \\
$\square$ \\
$\square$
\end{tabular}

$-36351$

$-28098$

$-19846$

$-11593$

$-3341$

4912

13164

21417

29669

37922 


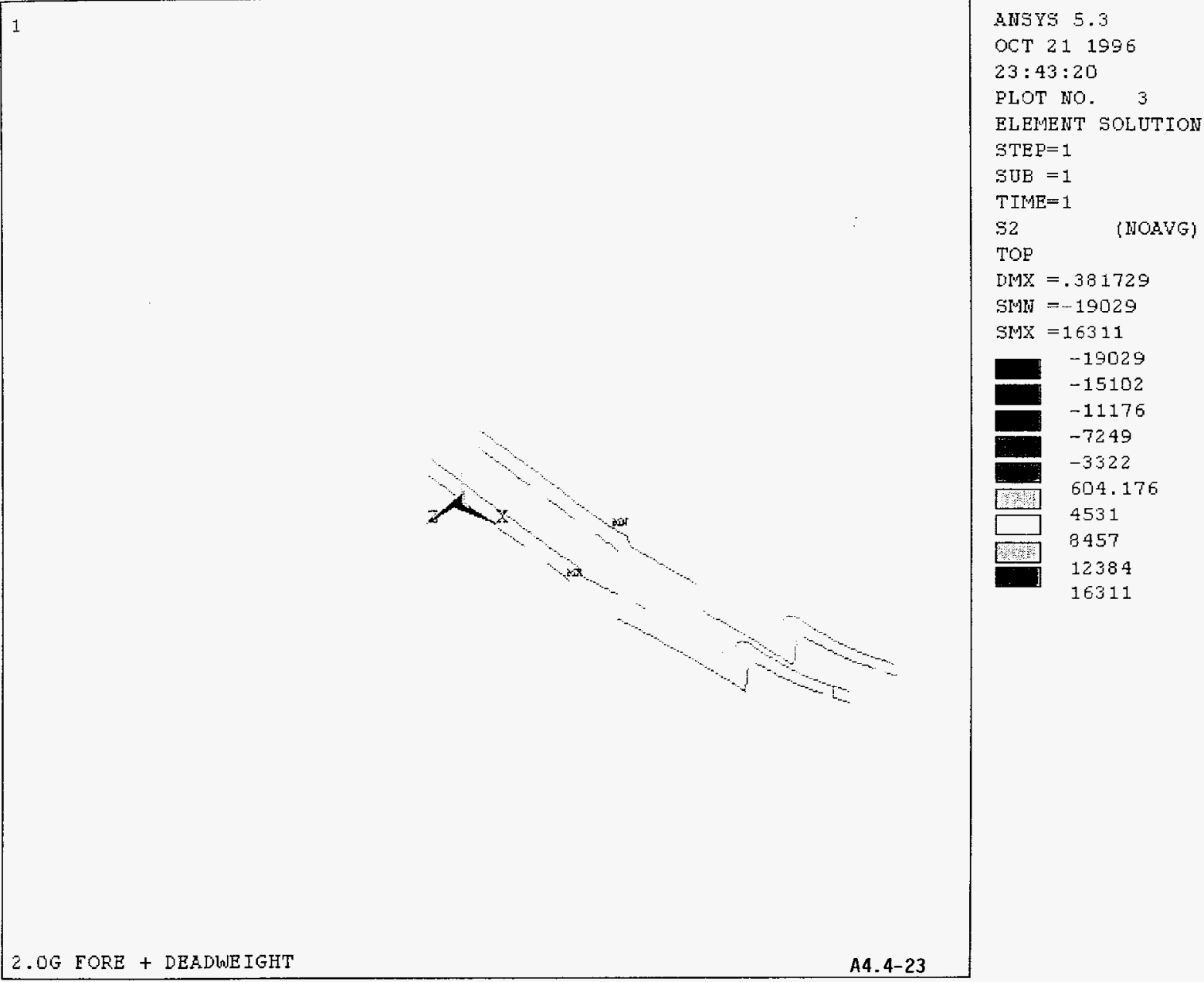


HNF-SD-SNF-FDR-003 Rev. 0

m in 0

产

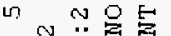

in $N$ मे

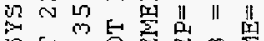

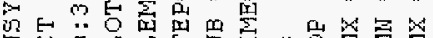

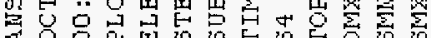

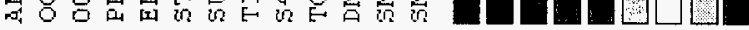

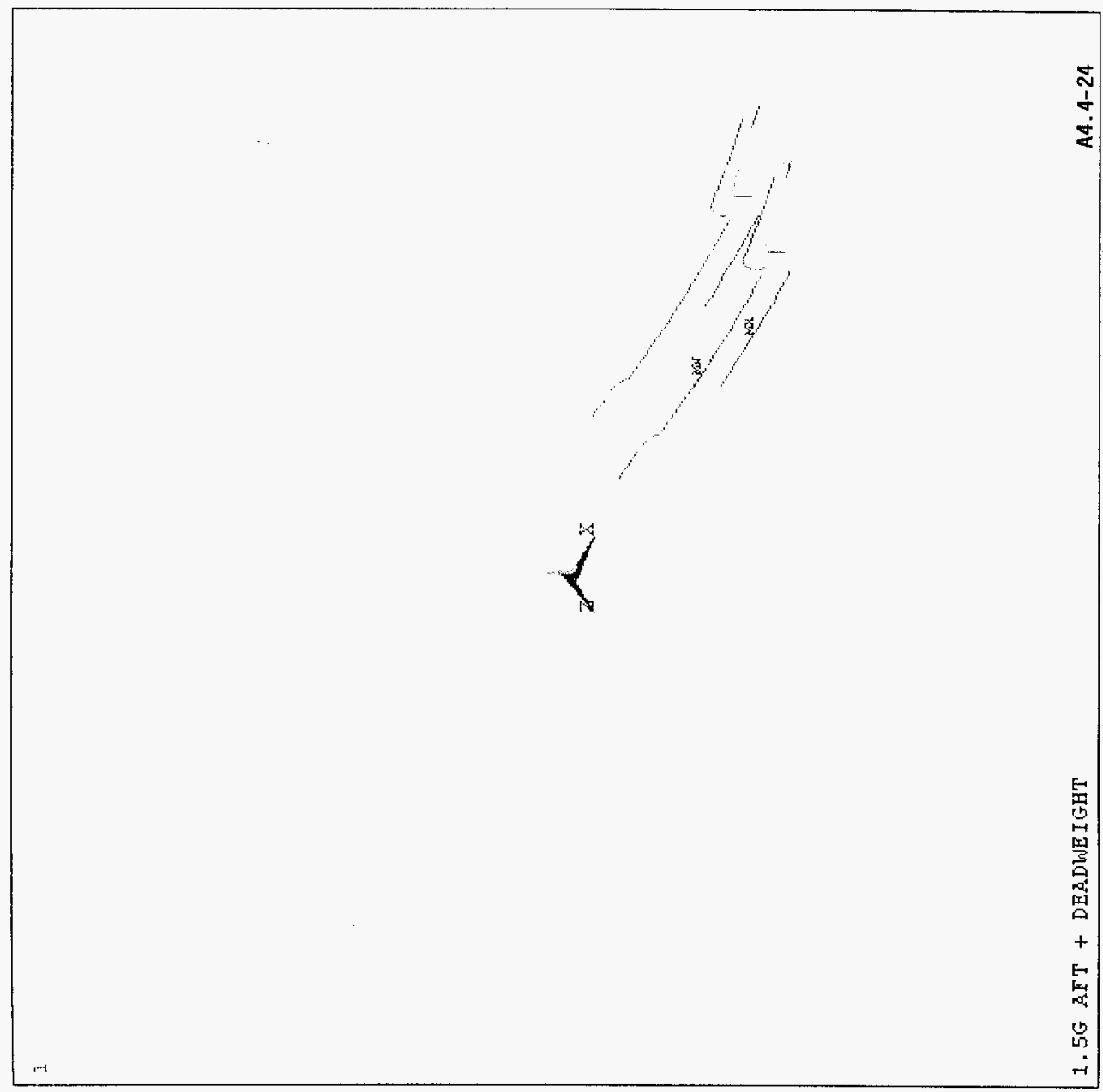




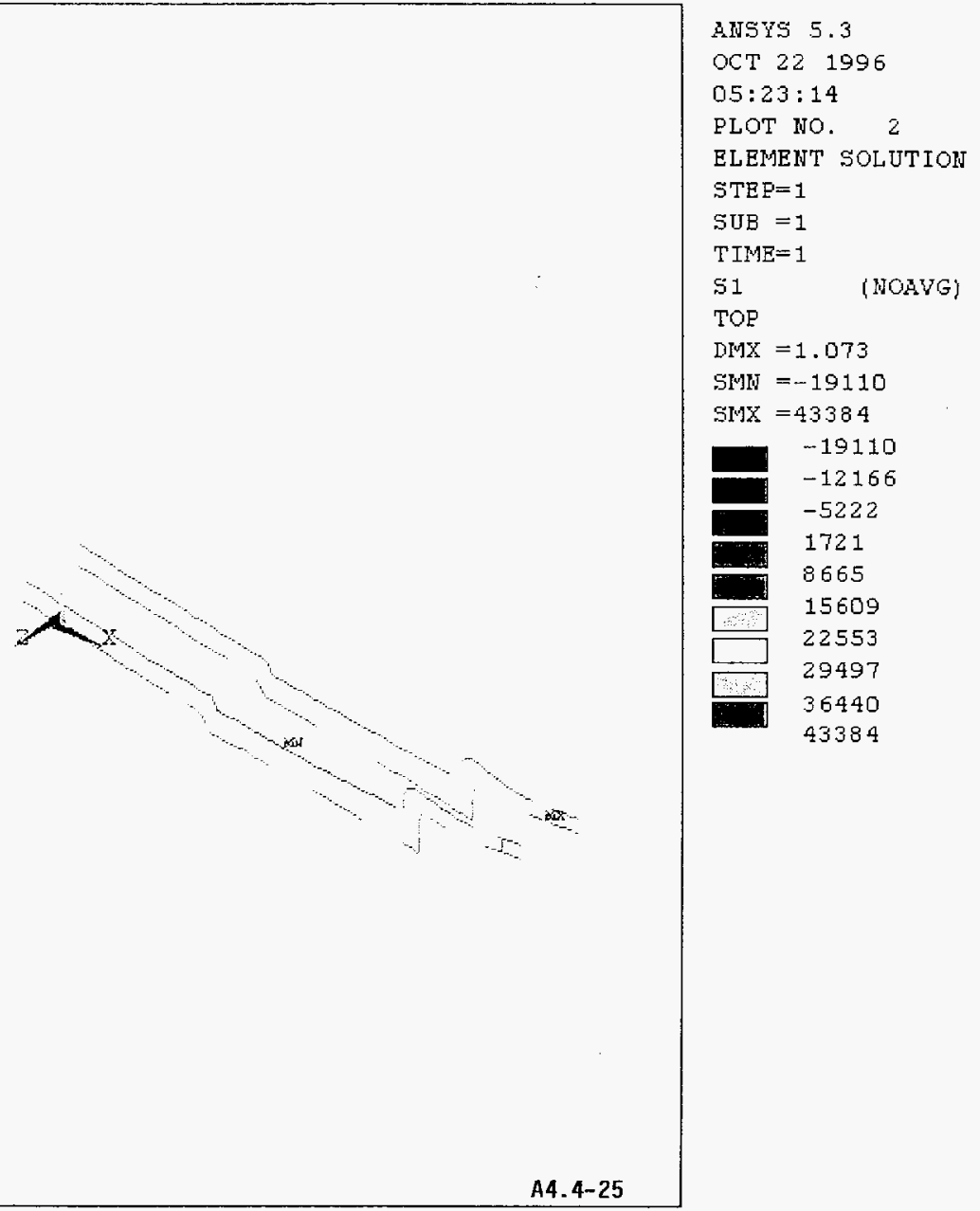



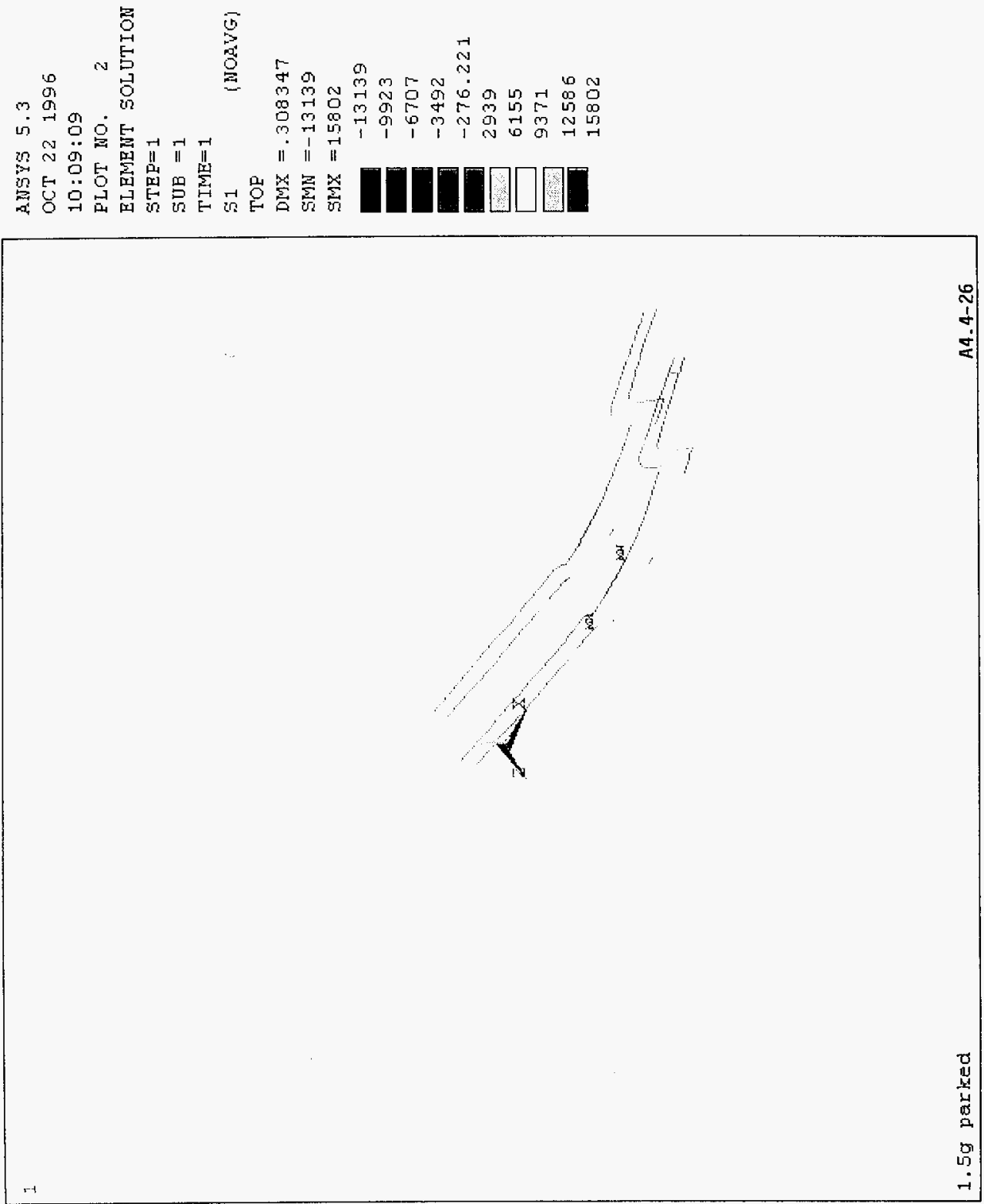


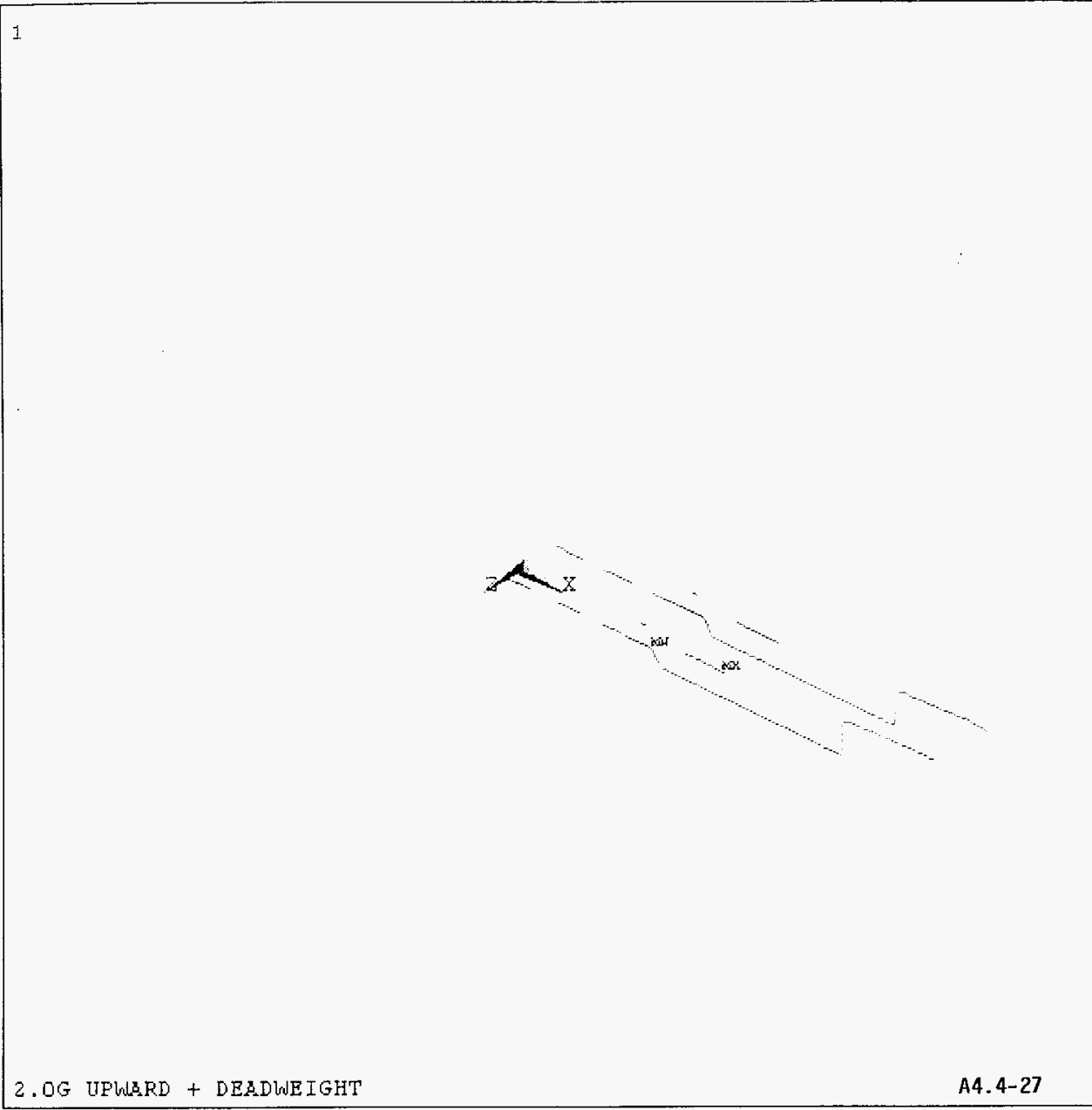

ANSYS 5.3

OCT $22 \quad 1996$

$11: 49: 16$

PLOT NO.

ELEMENT SOLUTION STEP $=1$

SUB $=1$

TIME $=1$

52

(HOAVG)

TOP

$\mathrm{DMX}=278003$

$S M N=-19938$

$5 M X=15628$

$-19938$

$-15986$

$-12034$

$-8082$

$-4131$

$-178.943$

3773

7725

11676

15628 


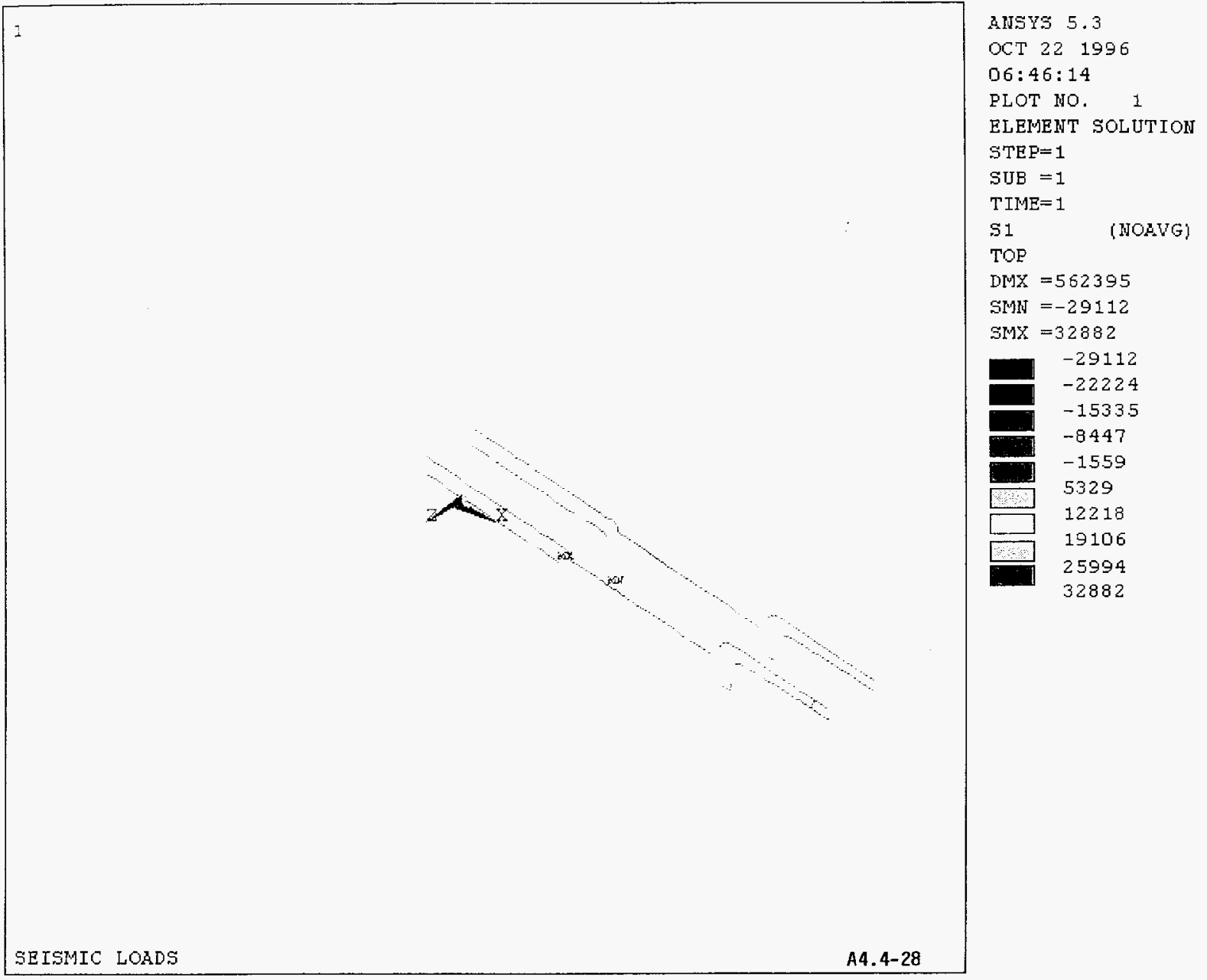


A.NSYS 5.3

OCT 211996

$22: 59: 40$

PLOT NO, 1

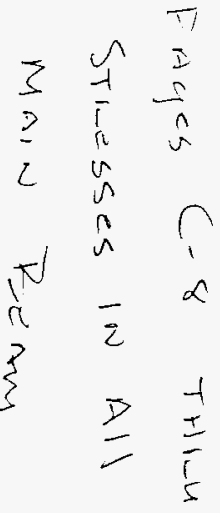

ELEMENT SOLUTION

STEP $=1$

SUB $=1$

TIME $=1$

53

(NOAVG)

$\mathrm{TOP}$

$\operatorname{DIMX}=1.731$

SMI $=-7807$

SMX $=16692$

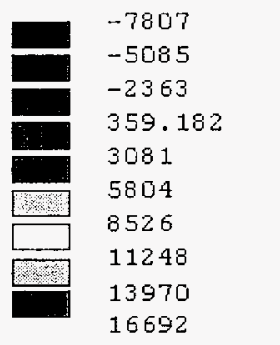

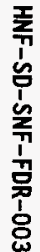

$\begin{array}{ll}0 & P \\ 3 & 1 \\ 5 & \end{array}$

$24 \times 2$

蛋

$=$
11
$x$
+1
+5

苞 


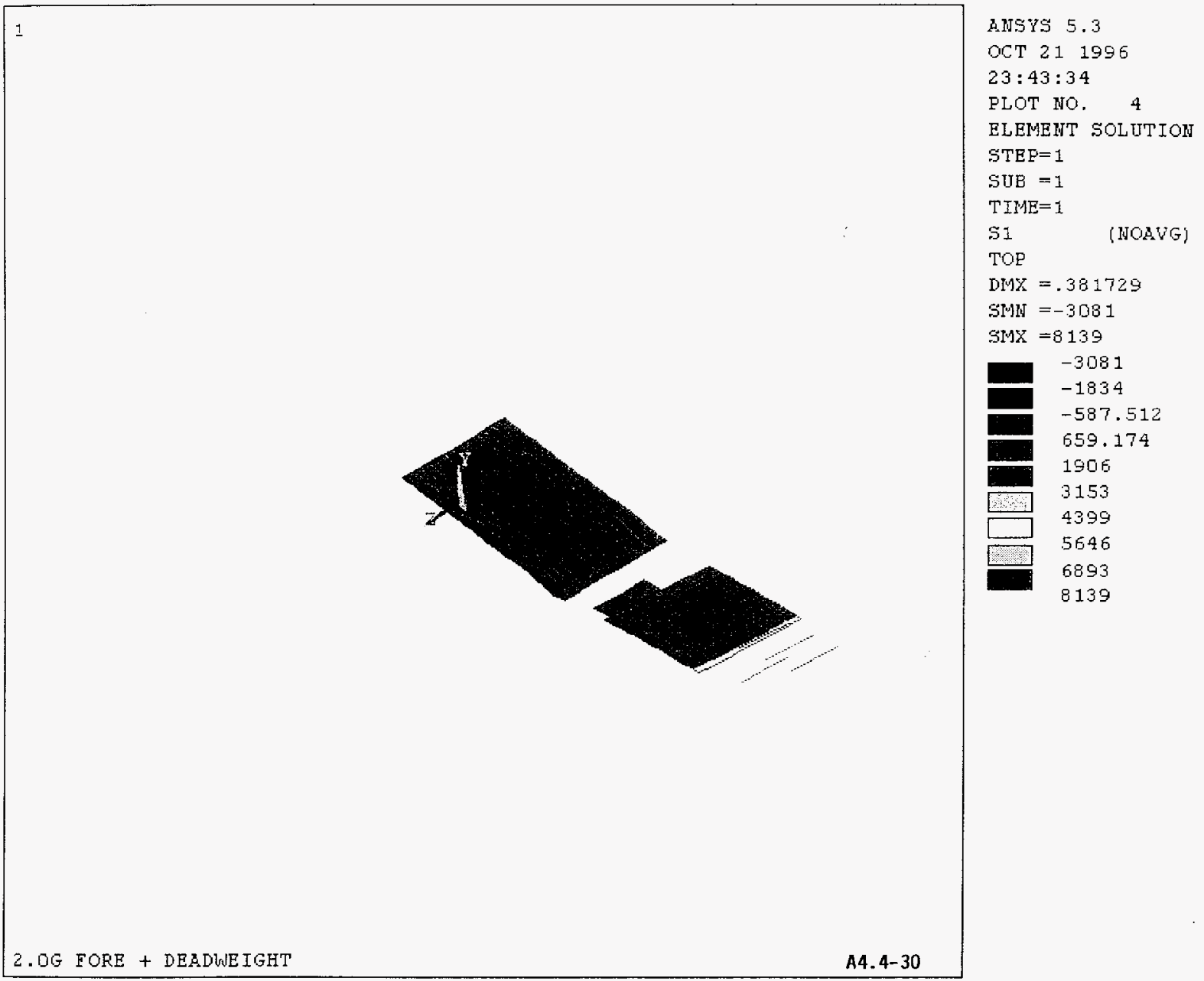

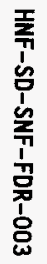

备

0

ํㅜㅇ 


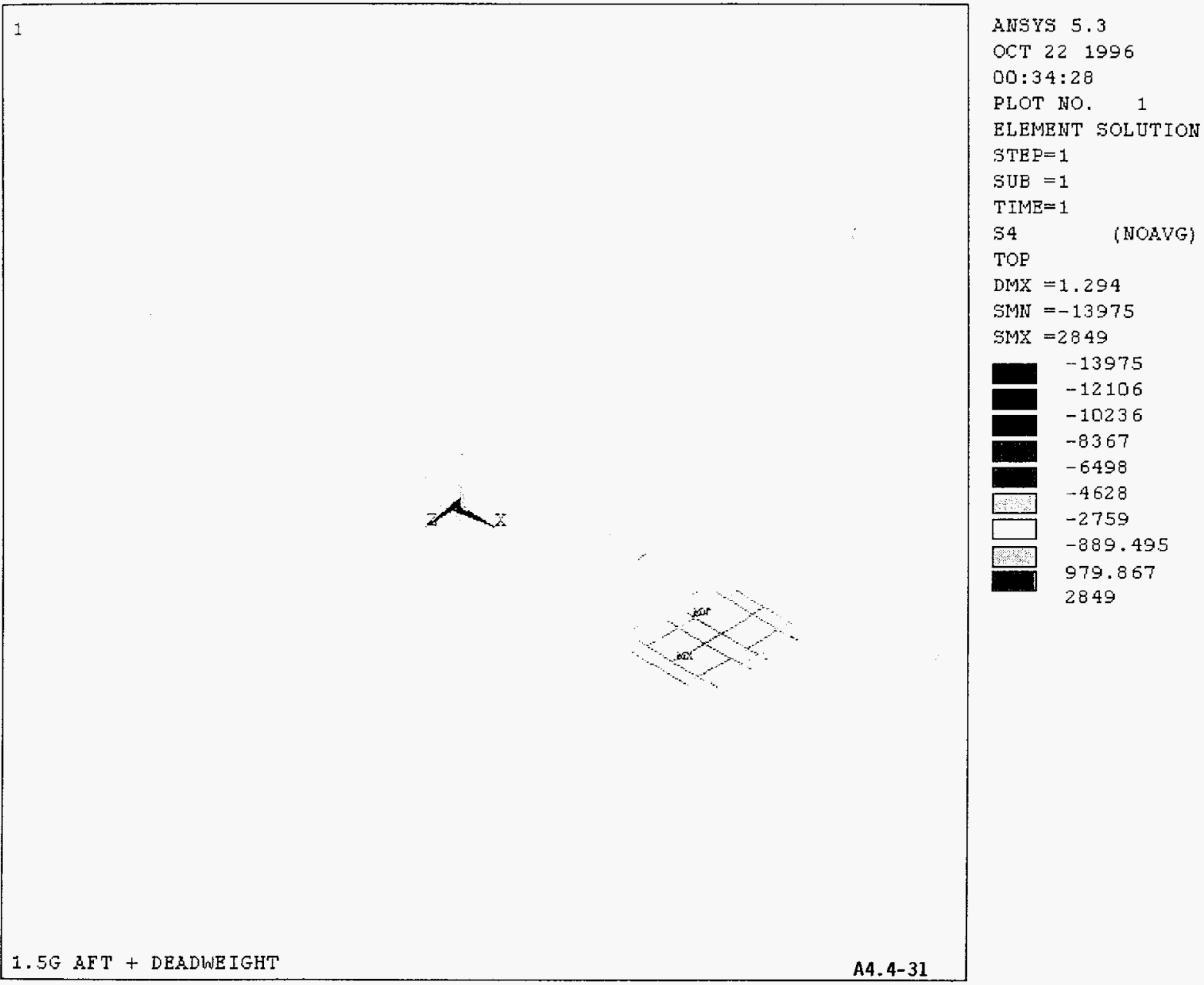




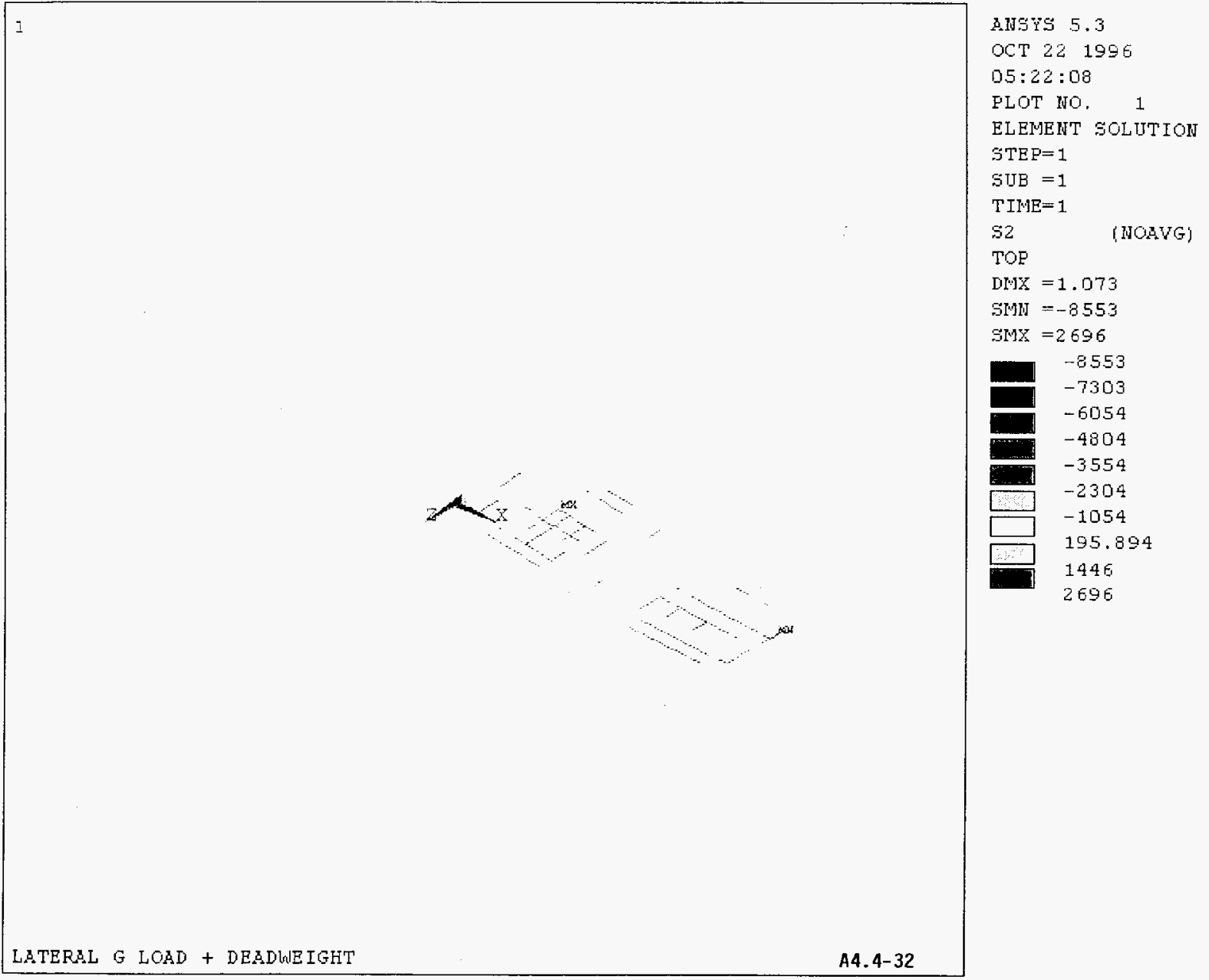




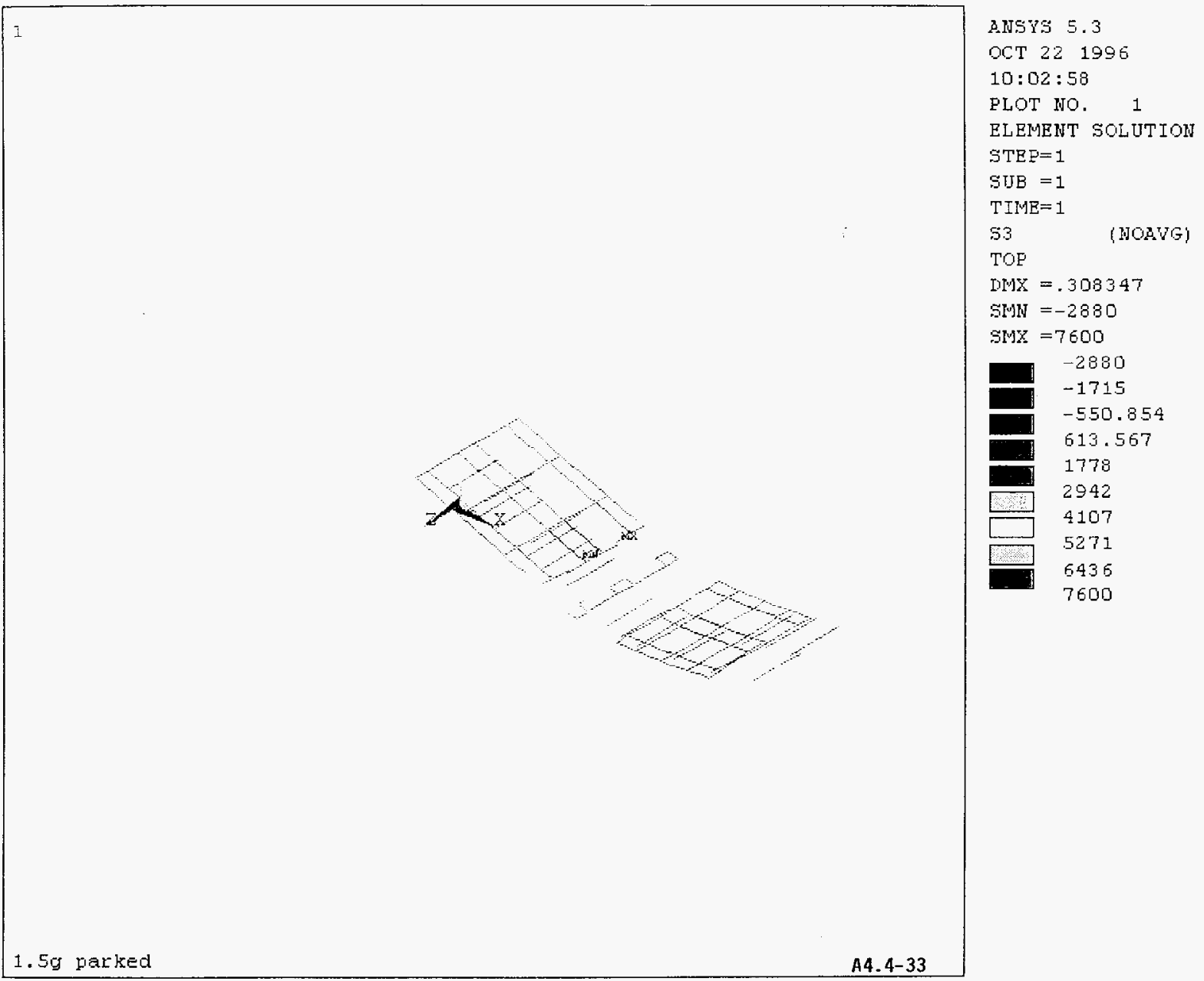

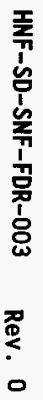

$T$
$\frac{1}{5}$
昜 


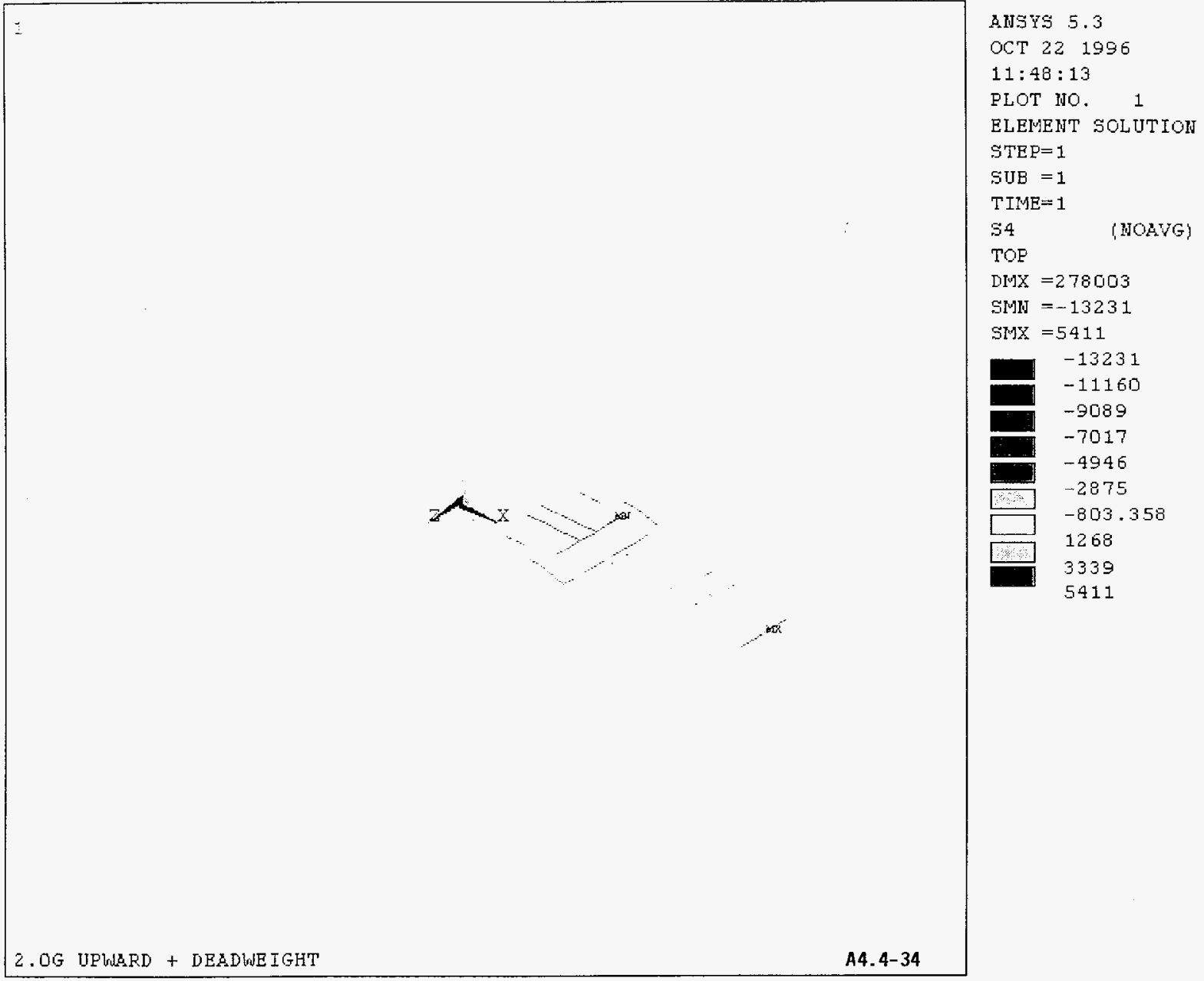




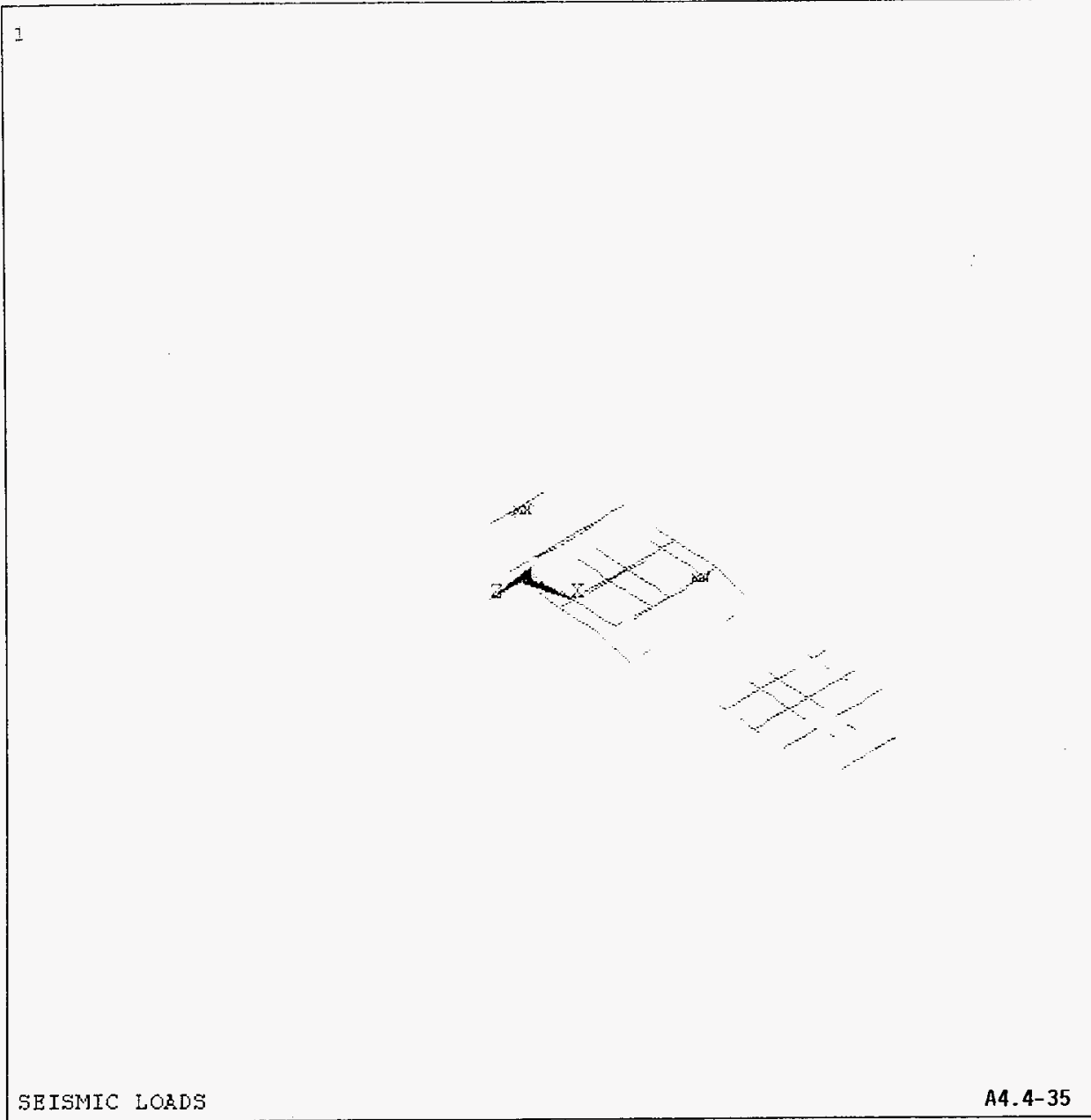

ANSYS 5.3

OCT 221996

D6:46:34

ELOT NO.

ELEMENT SOLUTION

STEP $=1$

$\mathrm{SUB}=1$

$T I M E=1$

54

(NOAVG)

TOP

LMX $=562395$

$5 M N=-16828$

$S M X=2226$

$-16828$

$-14711$

$-12594$

$-10477$

$-8359$

$-6242$

$-4125$

$-2008$

109.039

2226 
E-15166

HNF-SD-SNF-FDR-003 Rev. 0

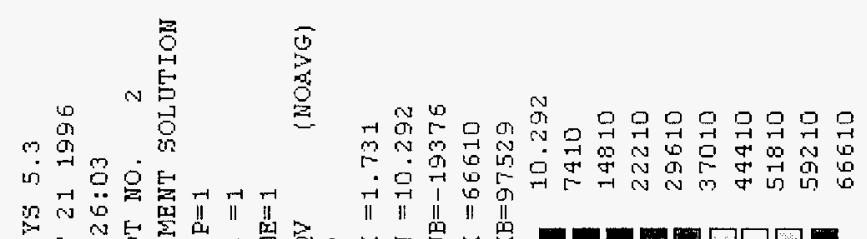

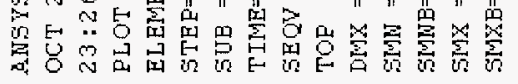

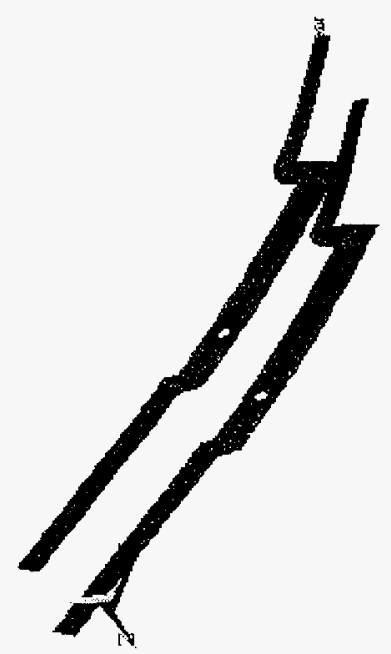

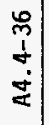

Pages C-15 Thru C-21 Are Maximum Stress Concentrations AT SHARP Corners in Main Beam Web.

-3
0
0
0
0
0
0
0
0
$n$
0 


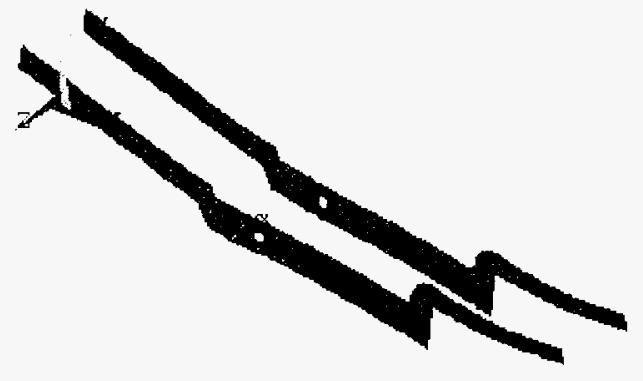

ANSTS 5.3

OCT 211996

$23: 44: 14$

PLOT HO. 5

ELEMENT SOLUTION

$S T E P=1$

SUB $=1$

TIME $=1$

SEQV

(MOAVG)

TOP

$\mathrm{DMX}=.381729$

$5{ }^{\prime} \mathrm{N}=6.785$

SMHB $=-18275$

SIMX $=4.5913$

$S M X B=78342$

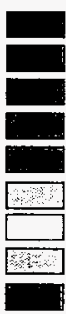

6.785

5108

10208

15309

20410

25510

30611

35712

40813

45913 


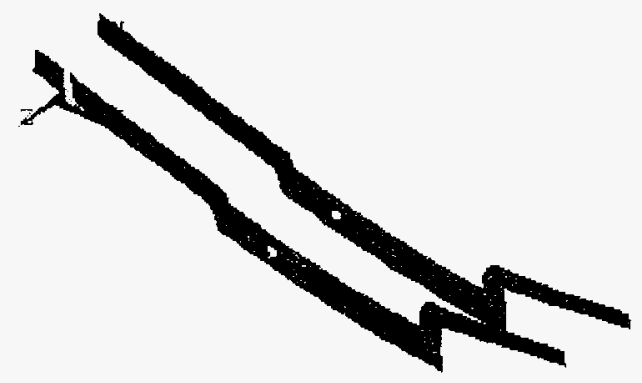

ANSYS 5.3

OCT 221996

$00: 35: 13$

FLOT NO.

ELEMENT SOLUTION

STEF $=1$

SUB $=1$

$\mathrm{TIME}=1$

SEQV

(NORVG)

TOP

$D M X=1.294$

SMN $=5.158$

$5 M N B=-24194$

$S M X=75325$

$5 \mathrm{MXB}=98654$

5.158

- 6374

16743

25112

33481

41850

50218

58587

66956

75325 


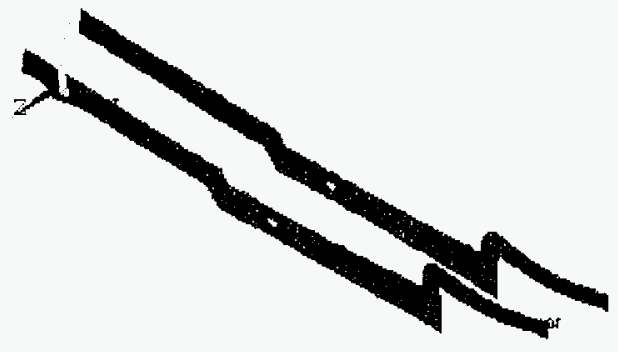

A.NSYS 5.3

OCT $22 \quad 1996$

$05: 24: 01$

PLOT NO. 3

ELEMENT SOLUTION

$S T E P=1$

$S U B=1$

TIME $=1$

SEQV

(NOSVG)

TOP

$D M X=1.073$

$5 \mathrm{MN}=69.35$

$S M N B=-27839$

$5 M X=60.538$

$5 M X B=82738$

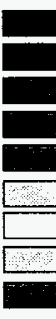

69.35

6788

13507

20226

26944

33663

40382

47101

53819

60538 


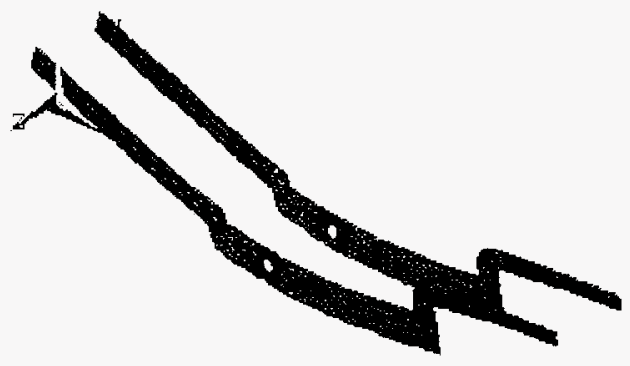

AHSYS 5.3

OCT $22 \quad 1996$

10:09:51

PLOT NO.

ELEMENT SOLUTION

STE $E=1$

$\mathrm{SUB}=1$

$T I M E=1$

SEQV

MOAVG)

TOP

$\mathrm{DMX}=.308347$

$5 \mathrm{MN}=5.789$

$5 M N B=-9.523$

$S M X=30679$

$S M X B=44374$

5.789

3414

6822

10230

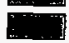

13638

17047

20455

23863

27271

30679 


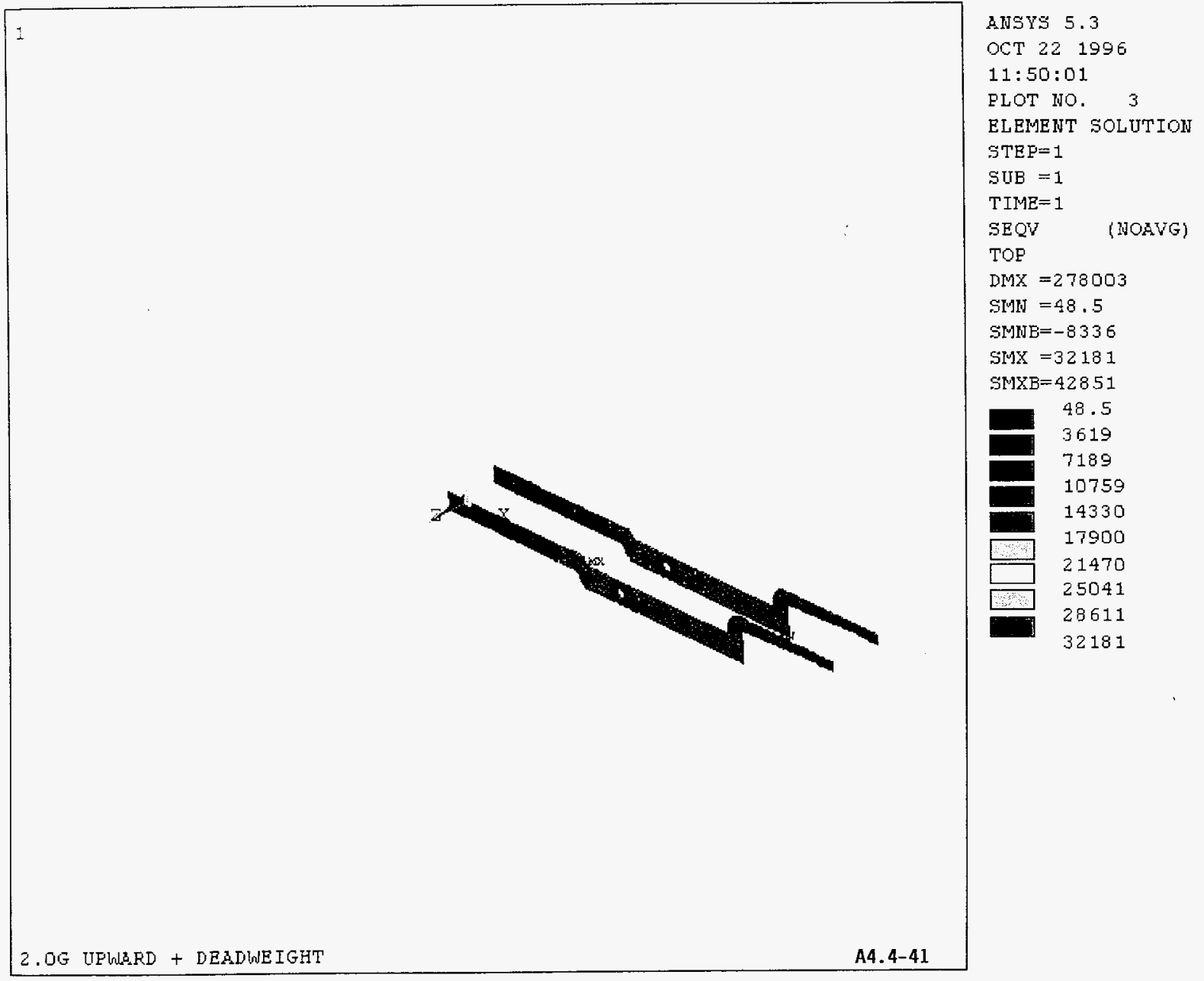




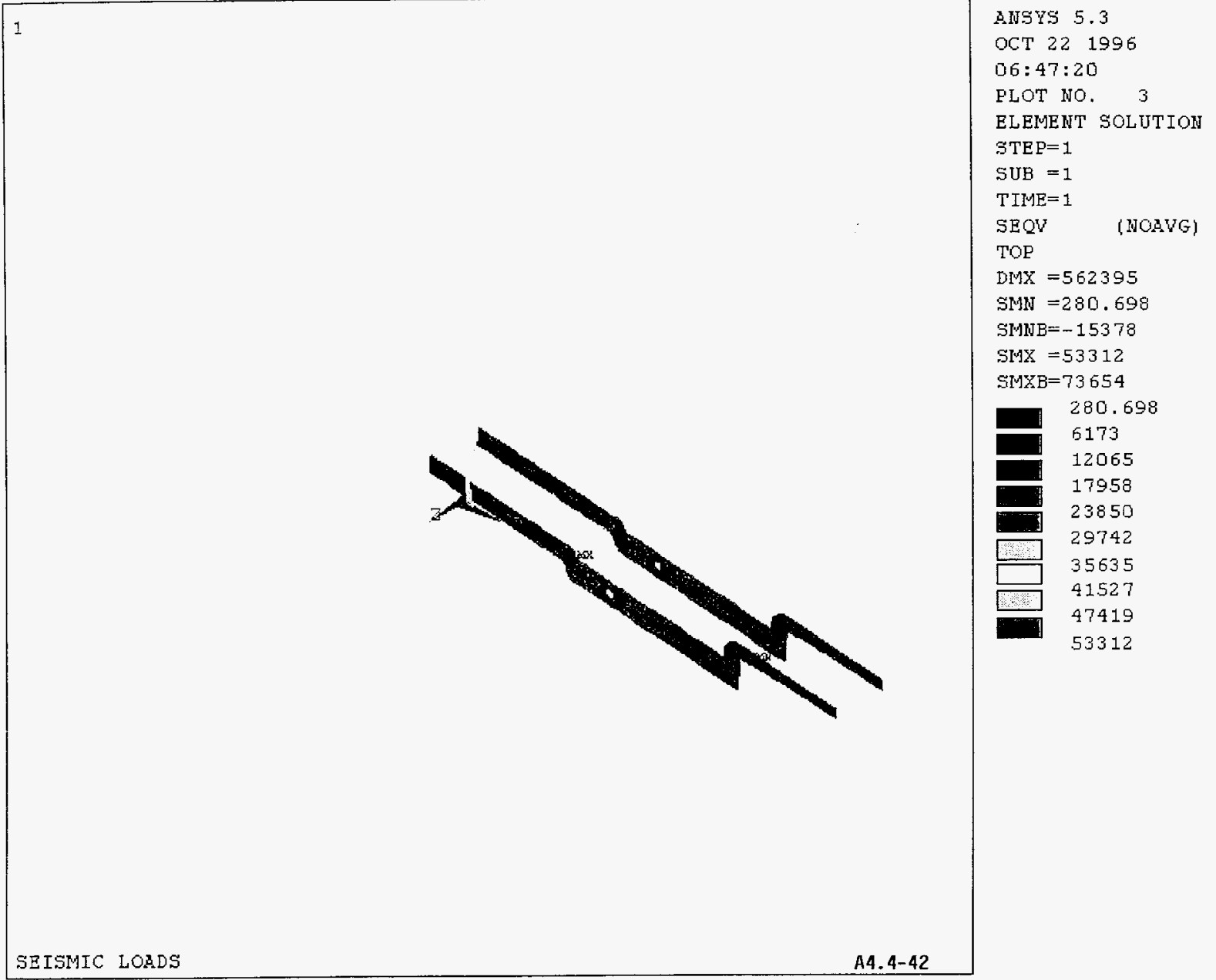




\section{HNF-SD-SNF-FDR-003 Rev. 0}

\section{$\mathrm{C}-22$}

PRINT F REACTION SOLUTIONS PER MOOE

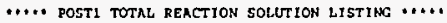

LOAD STEP-
TIME- $2.0000^{2}$ SUBSTEP- LOAD CASE $=0$

THE FOLLOHING $X, Y, z$ SOLUTIONS ARE IN GLODAL, COORDINATES

\begin{tabular}{|c|c|c|c|c|}
\hline $\begin{array}{l}\text { MOOE } \\
1315 \\
2335 \\
B 391\end{array}$ & $-.205158-05$ & $\begin{array}{r}F Y \\
72584 . \\
71626 . \\
72064 .\end{array}$ & $\begin{array}{c}F Z \\
-.99503 E-04 \\
.99605 E-04\end{array}$ & 2.5 \\
\hline
\end{tabular}

TOTAL VALUES

YAl.UB $\quad .10515 \mathrm{~B}-05 \quad .215275+06 \quad .101908-06$

/OUTPUT FILE - JOKE, DAT

NRSE YOR LABEL- $Y$ BETWEEN 36.000 AIT 36.000 XABS. 0.

TOLERAHCE= .160000

TIE FOLLOWIMG DEGRE OF FREEPOM RESUI.TS ARK IN GLOBAL COORDIMATES

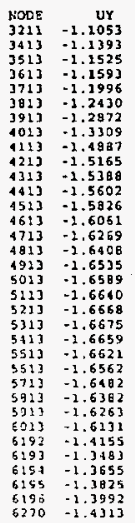

$\begin{array}{rr}077 & +1.4466 \\ 2772 & -1.4613\end{array}$

$5279-1.4754$

- ... fostl hoonl oegree of freedcil lisinhio . . .

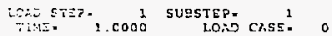

THF FELLONING DEGREE OF FREEDOI RESULTS AFE IZI GLOBAL CCOADIMATES

$\begin{array}{ccc}Y i=D Z & \text { UY } \\ 691 & 0.5019\end{array}$

$6451 \quad-1.6019$

$6.133 \cdot 1.6055$

Mi:TU: Fasot.UTE VATLES

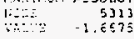


HNF-SD-SNF-FDR-003 Rev. 0

C- 23

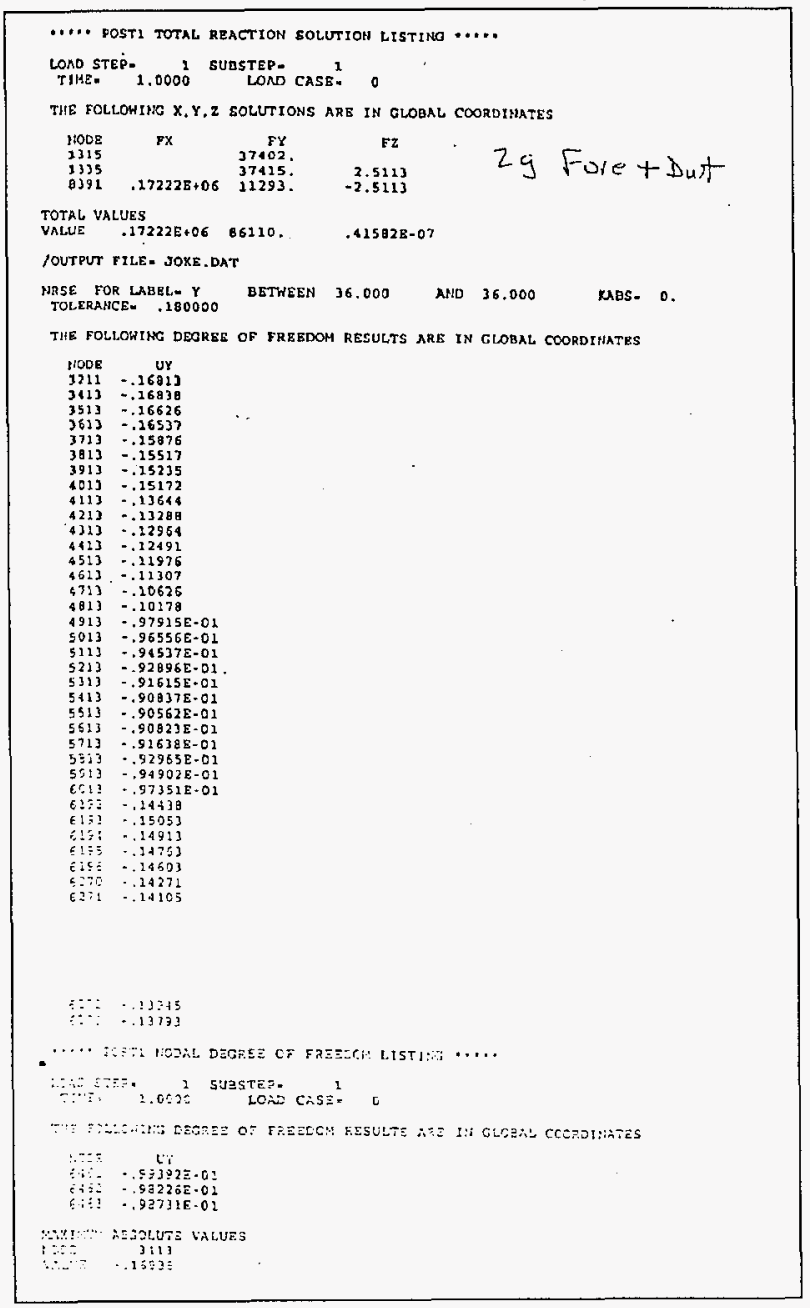




\section{HNF-SD-SNF-FDR-003 Rev. 0}

$\mathrm{C}-24$

THE FOLLOWING $X, Y, Z$ SOLUTIONS ARE IN GLOIAT, COORDINATES

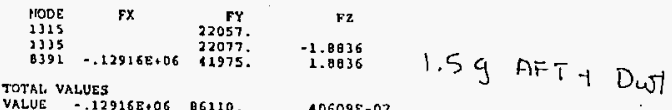

VALUE $-.12916 \mathrm{E}+06$ 06110. .406098-07

TIIE FOLLOWING DERREE OF FAEEDOM RESULTS ARE IN CLOBAL COORDIMTES

JODE UY

$3211-.64750$

$3413-.67125$

$3513=.60200$

$3613-.68747$

$3713-.72063$

$3013-.75374$

$3913-.78677$

1021 - 81734

$4113-93976$

421\} $=95189$

431] -9799 )

$4413=99648$

$4513-1.0180$

$4613-1.0394$

5013 - 1.008

(11) - 1.0939

$5213-1.097$

$531-1.0986$

5 sil -1.096

5513 -1.0956

$5713-3.0050$

$5713-1.0850$

5.3 -2.0672

$6013 \quad-1.0561$

$6192-06257$

-.ej09a

$6294-6410]$

$6195 \div 85706$

$\because 6599$.

$-89450$

$71-90685$

प? $272 \quad-91315$

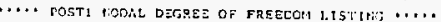

LORS SREP.
TIMIE 1.0000 SUGSTEP.
LOAD CASE:

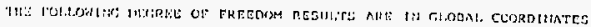

$$
\therefore: ; \quad-, 253)
$$

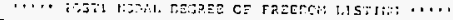

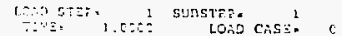

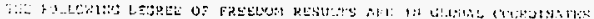

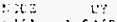

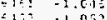

6.15: -1.0125

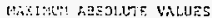

$\because 2, \quad-531\}$ 


\section{HNF-SD-SNF-FDR-003 Rev. 0}

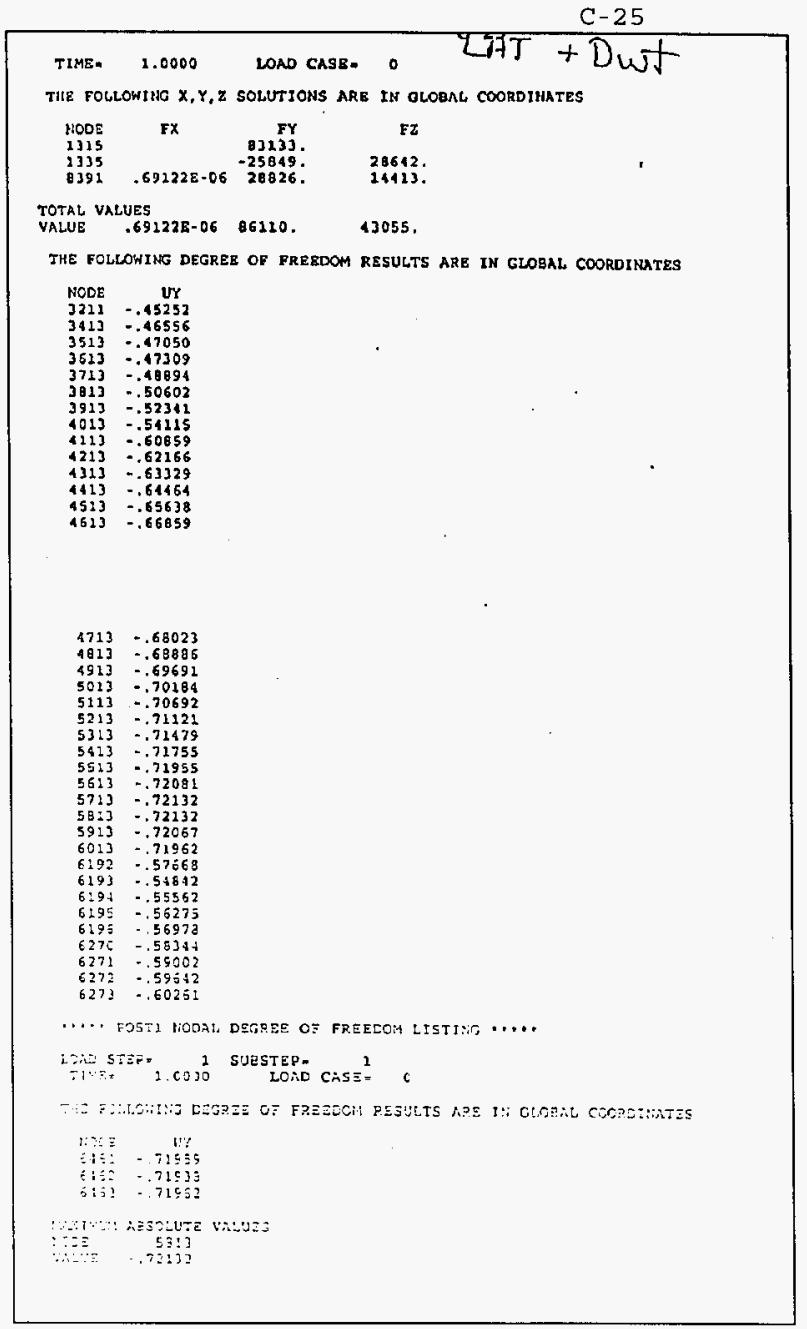


HNF-SD-SNF-FDR-003 Rev. 0

C -26

THE FOLLONING $x, y, z$ SOLUTIONS ARE IN GLOBNL COORDINATES

\begin{tabular}{|c|c|c|c|}
\hline $\begin{array}{l}11005 \\
1315 \\
1335 \\
6538 \\
6934\end{array}$ & $\begin{array}{c}8 X \\
-., 57881 E-06\end{array}$ & $\begin{array}{r}\text { PY } \\
35300 \\
34236 \\
29269 \\
30359\end{array}$ & $\begin{array}{c}\text { P2 } \\
-.78414 E-04 \\
.781795-04\end{array}$ \\
\hline & & $.12916 B+06$ & $.34632 \mathrm{E}-07$ \\
\hline
\end{tabular}

THE FOLLOHIN DEGREE OP FREEDOM RESULTS ARE IN CLOBAL COORDINATES

$\begin{array}{cc}\text { MODE } & \text { UY } \\ 3211 & \because .25608 \\ 3413 & \because 26173 \\ 3513 & -.26220\end{array}$

$3613 \quad-.26250$

$3733-.26250$

$3813=.26739$

$3913-.27088$

$4013 \quad-.27399$

$4113 \quad-.27133$

$4213 \quad-.26723$

$4323-.26064$

$4413 \quad-.25372$

$\begin{array}{ll}4513 & -24721 \\ 4613 & -.24124\end{array}$

$4713-.23438$

$4813-.22473$

$4913 \quad-.21452$

$5013=.20147$

$5113-.18853$

$5213 \quad-.17497$

$5313 \quad-.16077$

$5413 \quad-.24597$

$5513-.13063$

$5613 \quad .11482$

$5713-.99614 \mathrm{E}-02$

$5013-.02047 E-01$

$5913-651688-01$

$6013-.479748-01$

$6192-.27501$

$6193-.27452$

$6194 \quad-27486$

$6195-.27503$

$6196-.27513$

$6270-27470$

$6271 \quad-.274$

$6272 \quad \because 27348$

$6273-.27254$

... POSTI nODAC DEgREE of FREELOM LISTINC *...

LOAD STEP-
TLNEX 1.0000 SUESTEP-
LONO CASE

THE FOLLOWIYG DEGREE OF FREEDOH REEULTS AOE IR CLOBAL COORDIMATES

1:O0Z U.

$6461-.270855 .01$

$6.162-.410948-01$

$6+53 \quad-, 34013 \mathrm{E}-02$

Mativin hasocute values

$\begin{array}{rr}\text { HOOE } & 6196 \\ \text { VALLE } & -.27513\end{array}$ 
THE FOLLOWING $x, Y, z$ SOCUTHONS ARE IN GLOBAL COORDILATES

$\begin{array}{rccr}\text { NOOR } & F X & F Y & r F 7 \\ 3903 & & & -3072.6 \\ 1203 & & & 3216.0 \\ 9100 & 28567 . & -20494 . & -34467 . \\ 9101 & 28182 . & -20217 . & 34002 . \\ 9102 & -28214 . & -22571 . & -20472\end{array}$

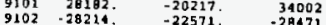

$9303.28533 . \quad-22827 . \quad 28793$.

TOTAL VALUES

VALUS $1.3722 \quad-76109 . \quad$.227578-01

fOUTPUS RILE: JOKB, DAT

TIMB = 2.0000 LOAD CASE- 0

THE POLLOHING DRGRE OF FREEDOM RESULTS ARE IN CLOBAL COORDINATES

NODE UY

$3411 \quad 3798.4$

35130379.5

36139200.0

37314123.

381319046

191323959.

401328892

411350225.

421355147.

431360070

441364993.

151369918

$1513 \quad 24839$.

$4713 \quad 75762$.

481384685

191369608.

501394531

51ג了 99454.

$5213.10438 \mathrm{R}+06$

$5313 \quad .109308+05$

$5413 \quad .11422 \mathrm{E}+06$

5613 . $119158+06$

$5613 \quad .124078+06$

$5713.128998+06$

$5813.13391 \mathrm{~B}+06$

$5913 \quad+136548+06$

$6013 \quad+143768+06$

619239558 .

$\begin{array}{ll}6193 & 31075 . \\ 6194 & 33150 .\end{array}$

619535292.

6195 J7425.

6270 4169.

627245958 .

$\begin{array}{ll}6272 & 45958 . \\ 6273 & 18091\end{array}$

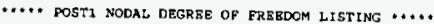

LOAD STEP.
TIME $=$ 1. COOO

THE FOLLONING DEGREE OF FRERTCM RESULTG ARB IN GLOBAC CODRDINATES NOOE $\quad \mathrm{YY}$

$6461+149508+06$

$6462+145578+06$

$6453 \quad .147598+06$

MAXIMUM ABSOLUTE VALUES

MODE 6461

VALUE .14950E+OG 
HNF-SD-SNF-FDR-003 Rev. 0

$\mathrm{C}-28$

TIIE FOLLOWIKG $X, Y, z$ SOLUTIONS ARE IN GLOBAL COORDINATES

$\begin{array}{rrrr}\text { NOOE } & F X & F Y & F 2 \\ 9100 & -43982 . & 31553, & 53066 . \\ 9101 & -43982 . & 31553 . & -53066 . \\ 9202 & 43981 . & 35185 . & 41361 . \\ 9103 & 43981 . & 35185 . & -44381 .\end{array}$

TOTAL VALUES

VALUE $-2.5872 \quad .133478 .06 \quad .674208 .03$ 
HNF-SD-SंNF-FDR-003 Rev. 0

E-15166

$C-29$

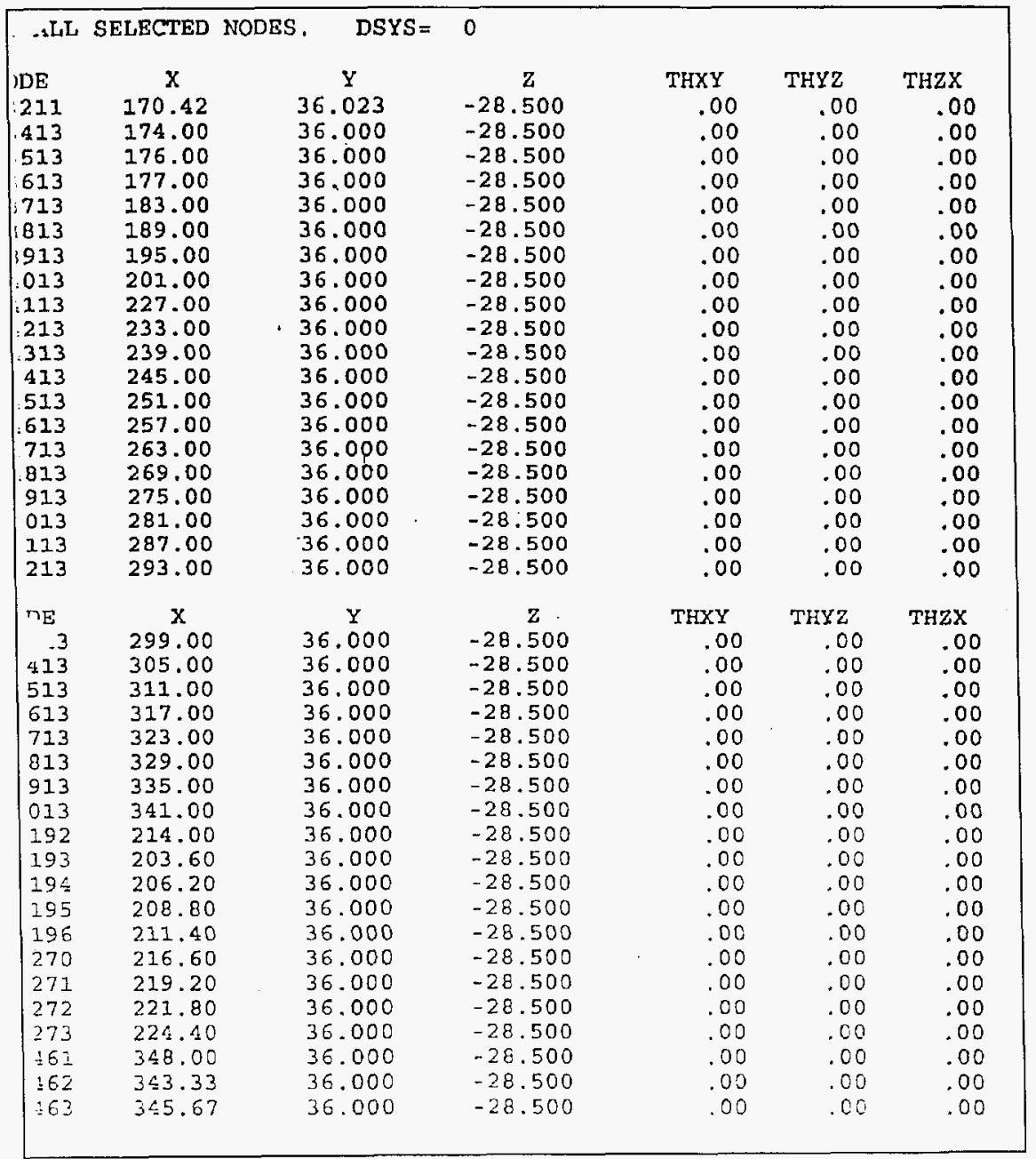


HNF-SD-SNF-FDR-003 Rev. 0 E-15166

\section{APPENDIX D}

SEISMIC ANALYSIS DATA 
APFENDIX D - SEISMIC ANALYSIS DATA

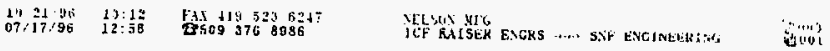
pinger key
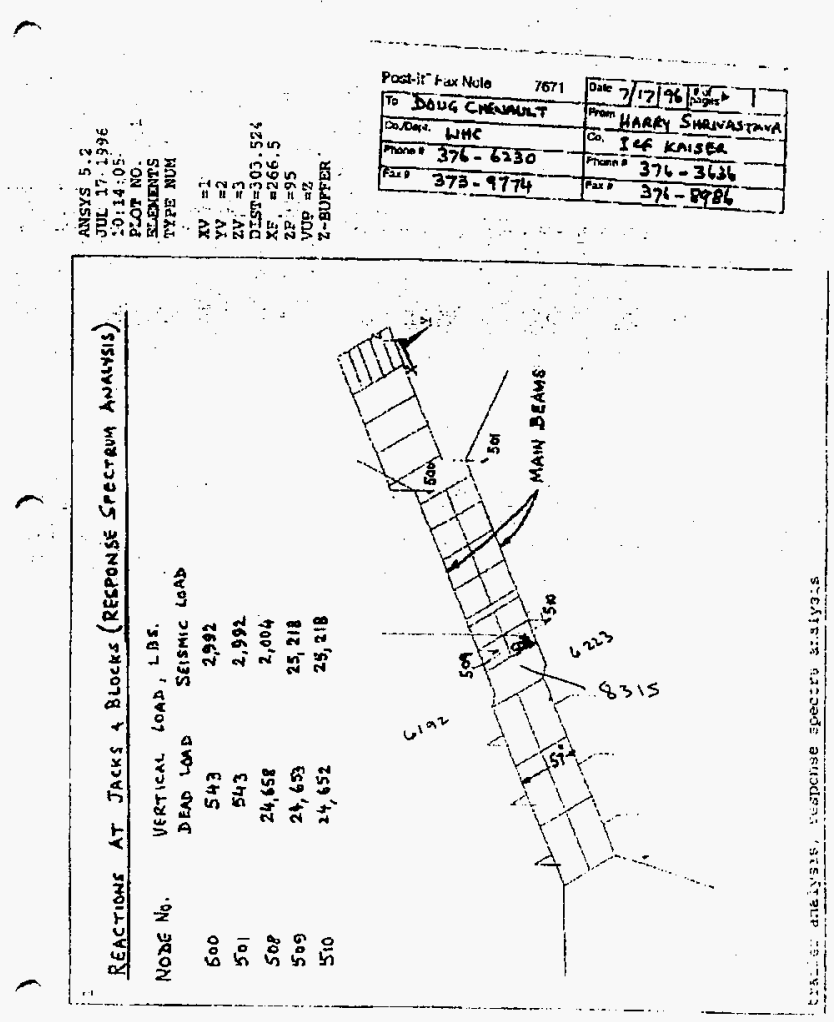


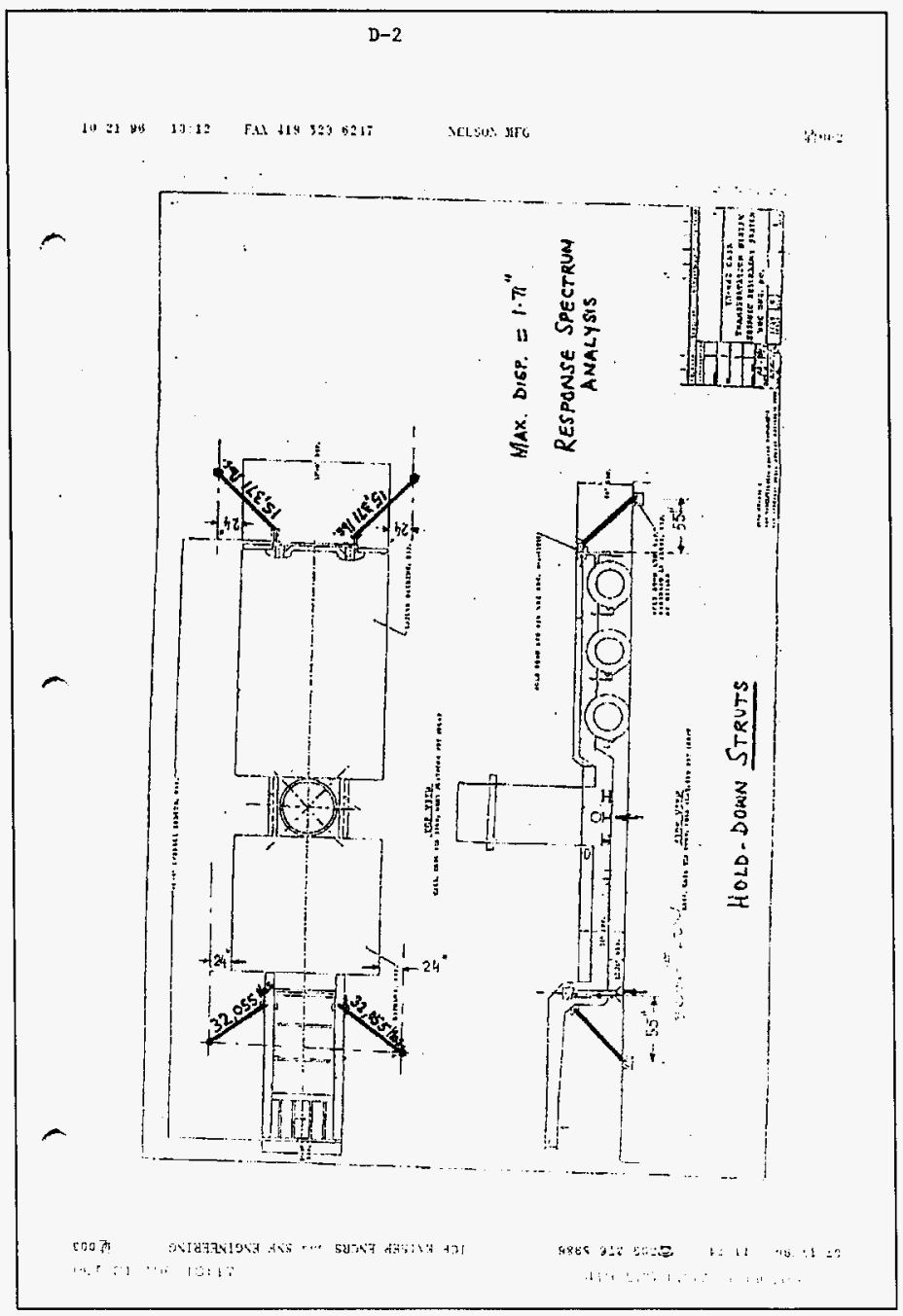




\section{APPENDIX E}

\section{CALCULATION OF OVERTURNING G LOAD}




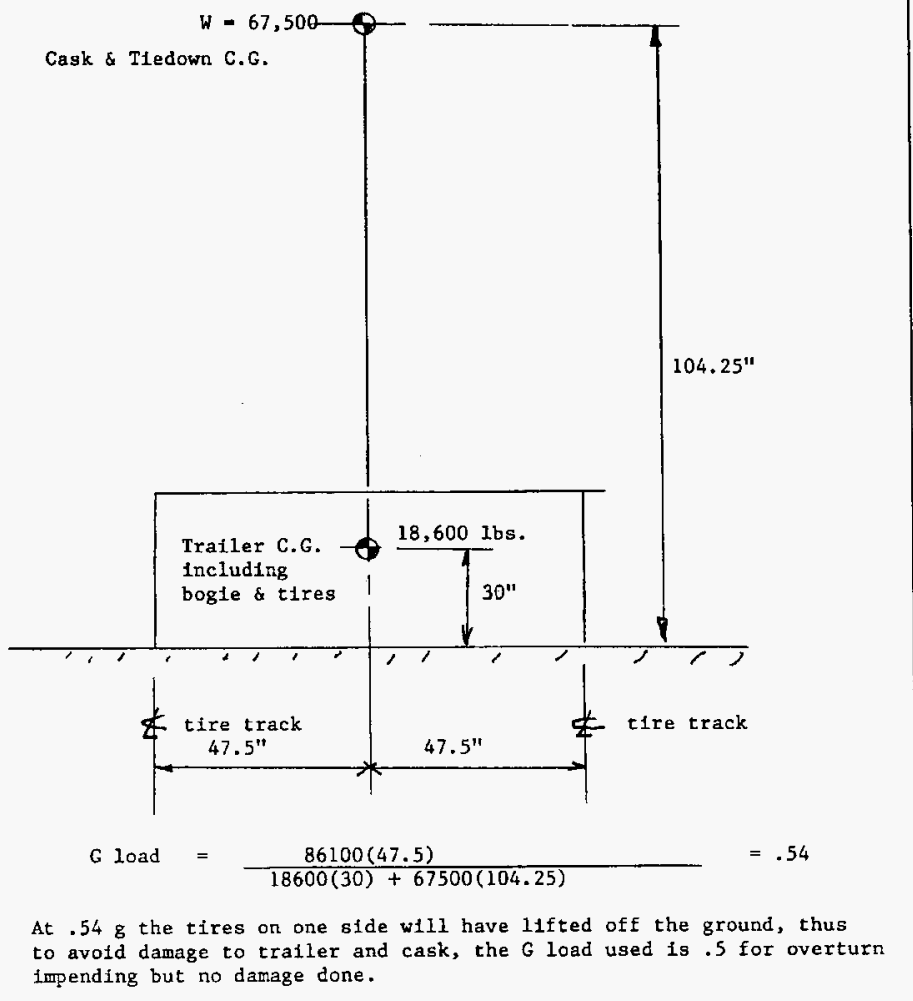




$$
\text { HNF-SD-SNF-FDR-003 Rev. O }
$$

\section{APPENDIX $F$}

\section{FATIGUE ANALYSIS}




\section{APPENDIX F - FATIGUE ANALYSIS}

A fatigue analysis is made, using 400 events for all loading cases except the deadload case, which can have an infinite number of occurances as shown in the following. Figure I-9.1 from ASME Section III Boiler and Pressure Vessel Code is used for the analysis.

The deadweight peak stress can be obtained by linear ratio from the 2.5 g vertical stress, page C-15. Thus, $S=(1 / 2.5)(66610)=26,644$ pai. One half of the total stress is the alternating stress, thus salt $=13,322 \mathrm{psi}$. From Fig I-9.1, the allowable cycles are infinite and the usage factor is zero for deadweight. The peak atress for all load cases given on pages C-16 through C-21 is conservatively assumed to be the alternating stress. The peak otress for $2.5 \mathrm{~g}$ vertical down is halved for the alternating stress since it is known that this is one directional stress.

The usage factors are:

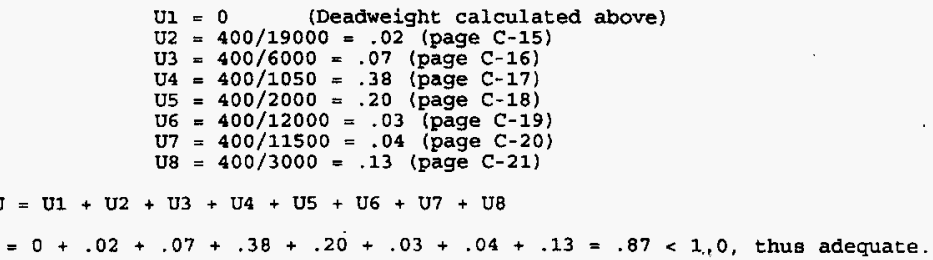


HNF-SD-SNF-FDR-003 Rev. $0 \quad$ E-15166

\section{A5.0 ACCEPTANCE OF PACKAGING FOR USE}

\section{A5.1 NEW PACKAGING}

\section{A5.1.1 Acceptance Requirements}

Acceptance of the Cask and Transportation System will based on inspection and tests that will be performed by the fabricators prior to shipment to the Hanford Nuclear Reservation site. These tests will be performed in accordance with written procedures prepared by the fabricators and approved by Transnuclear. In addition documentation packages will be submitted for acceptance. The documentation will be unit specific and demonstrate that the unit fulfills the final material, welding and dimensional requirements of the approved design drawings and the procurement specification, as required by the Hanford Specification.

\section{A5.1.2 Inspection and Testing}

\section{A5.1.2.1 Cask}

The inspection and testing requirements will satisfy the requirements of the Hanford Specification. All cask containment boundary welds will be radiographically inspected. The final surface of all welds will be liquid penetrant inspected. The fabrication drawings will show cask regions that require the following inspections:

a. Liquid Penetrant Inspection: This inspection will be performed in accordance with $\mathrm{ASME}^{(1)}$ Section V based upon the acceptance criteria from ASME Section III. Any indication of a defect will be removed, repaired and reinspected as required by the Hanford Specification.

b. Radiographic Inspection: Welds that require radiographic inspection will performed in accordance with the ASME Code, Section III. Any indication of a defect will be removed, repaired and reinspected as required by the Hanford Specification.

The following tests will be performed to satisfy the requirements of the Hanford Specification:

a. Leakage Rate Testing: Helium leakage rate tests will be performed to verify the leak tightness of the seals of the containment boundary of the cask. Leakage shall not exceed $1 \times 10^{-7} \mathrm{sec} / \mathrm{sec}$, air.

b. Pressure Testing: Each unit will be hydrostatically tested in accordance with the requirements of the Hanford Specification. The acceptance criteria is no evidence of leakage. 
After fabrication and prior to first use, a visual inspection shall be performed to verify that all accessible cask surfaces have no damage (cracks, surface discontinuities with depth exceeding minimum wall thickness requirements, etc.), and are free of grease, oil or other contamination. The sealing surfaces will be examined for cracks or scratches that may result in acceptable leakage. In general, a confirmation will be made that all cask components are in acceptable condition for use.

\section{A5.1.2.2 Cask Lifting Attachment}

The lifting attachment will be subjected to a load test of $150 \%$ of the maximum service load. A confirmation will be made that all components of the lifting attachment are in acceptable condition for use.

\section{A5.1.2.3 Conveyance}

Standard pre trip inspection shall be performed by the driver in accordance with CDL requirements. Annual inspection shall be performed in accordance with standard DOT specification.

Upon completion of the trailer fabrication and prior to the installation of the upper tiedown system, the trailer will have a static load test. It will be tested at $120 \%$ of the expected concentrated load.

\section{A5.1.2.4 Cask Operations Equipment}

The immersion pail and loadout pit support structure are both designed to meet the criteria of ANSI $14.6^{(2)}$. These structures will be subjected to a load test of $150 \%$ of the maximum service load, which is testing to 90,000 pounds.

Assembly testing will be performed at the fabricators facility for each of the component interface boundaries permitted to be evaluated prior to shipment.

\section{A5.2 ANNUAL INSPECTION REQUIREMENTS}

The annual inspection and testing requirements will be provided in the Installation, Operation, Repair, and Maintenance Manuals (IORM) for the System. Included in the manuals will be the inspection and testing to be performed, and the acceptance requirements.

\section{A5.2.1 Acceptance Requirements}

The annual inspection requirements for the cask is the visual inspection of the cask components including seals and sealing surfaces. Typically the replacement of the seal and 
bolts (depending on usage) occur at this time. The Containment Verification Leak Test is performed after the change of the seals using approved procedures. Acceptance requirements is the leakage rate of $1 \times 10^{-7} \mathrm{scc} / \mathrm{sec}$, air.

\section{A5.2.2 Inspection and Testing}

Load testing of the cask operational equipment will be required following any major maintenance or alteration to the load path structure, or if an incident occurred in which any of the load-bearing components may have been subjected to stresses substantially in excess of those for which the structure has been qualified by previous testing, or following an incident that may have caused permanent distortion of the load-bearing parts.

Inspection of the equipment including the cask lifting attachment and the cask will be performed by operating personnel for indications of damage or deformation prior to each use. Maintenance personnel, or other non-operating personnel, will be required to visually inspect load path components at intervals not to exceed three months in length for indications of damage or deformation.

The annual Containment System Verification Test is required to be performed on the cask in accordance with ANSI N14.5(3). 
References For Section A5.0

1. ASME Boiler and Pressure Vessel Code, American Society of Mechanical Engineers, NY, 1992.

2. Special Lifting Devices for Shipping Containers Weighing 10,000 Pounds or More, ANSI N14.6, American National Standards Institute Inc., NY, 1993.

3. Leakage Tests on Packages for Shipment of Radioactive Materials, ANSI N14.5, American National Standards Institute Inc., NY, 1987. 
HNF-SD-SNF-FDR-003 Rev. $0 \quad$ E-15166

\section{A6.0 OPERATING REQUIREMENTS}

\section{A6.1 GENERAL REQUIREMENTS}

The TN-WHC Transport Cask and the K-Basin Operational Equipment have been designed to facilitate the loading of fuel from the K-Basins into the Multi-Canister Overpacks (MCOs). The procedures provided in this Section provide the necessary steps associated with the operation of the TN-WHC Transport Cask and the K-Basin Operational Equipment. Integration of these procedures into site specific procedures will form the bases for system facility operation.

The Operational Equipment and procedures are based on the concept of "start clean, stay clean". This concept has been incorporated into the design of the K-Basin immersion pail, support structure and loadout pit work platform.

The immersion pail support structure is a welded steel structure which provides an operations guide system and support for the TN-WHC cask during loadout pit MCO loading operations. When loaded into the support structure at the operator access level, the cask is lowered into and sealed with the immersion pail system which precludes contamination of the cask's surfaces. This "start clean, stay clean" operational system thereby improves turnaround time and reduces operator dose rates by eliminating the need for extensive cask decontamination efforts following MCO loading operations.

As part of the operational procedures development, a radiation dose evaluation has been performed for the MCO loading operations. This evaluation provides a baseline for the development of site specific operating procedures which incorporate As Low As Reasonably Achievable (ALARA) principles to minimize the dose to operators during the MCO loading operations. 


\section{A6.2 TN-WHC MCO LOADING OPERATIONS}

The following operating procedures are provided to permit understanding of operations sequencing and generic task identification. Detailed system operating procedures are provided in the "Installation, Operation, Repair and Maintenance Manual, (IORM)".

\section{A6.2.1 Receiving Cask in $\mathrm{K}$ Basin}

1. Prepare cask receiving area for TN-WHC cask and trailer receipt.

2. Open door and back loaded cask trailer into facility.

3. Set tractor brakes, shut off tractor engine, change trailer override valve to manual, lower the rear of the trailer, set trailer brakes and install wheel chocks. Connect building air supply to the landing leg air supply glad hand, lower the landing legs until the trailer is lifted off the tractor.

4. Disconnect tractor electrical and air connections from trailer, release the fifth wheel and remove tractor from facility. Lower the front of the trailer until the trailer becomes relatively level.

5. Remove the four quick release locking pins from the vertical hold down devices and disengage the arms. Place the pins in the unlock position.

6. Align crane hooks with cask lifting trunnions and raise crane hoist to apply slight load on crane.

7. Remove the six tie-down bolts from the cask tie-down device. Place the bolts in the storage positions provided on the work platform.

8. Lower the removable trailer work platform from the working side of the trailer and disconnect cable. Relocate the safety chain.

9. Swing both sides of the tie-down device to the open position.

10. Raise the cask to clear the trailer.

11. Move the cask over the safe load path to the load out pit.

12. Align the cask with the immersion pail and lower into the pail until weight of cask is removed from crane hook. 


\section{A6.2.2 In-Pool Loading Operations}

1. Detorque the cask lid bolts in sequence indicated. Ensure all bolts have disengaged from cask. Note: Bolts are captive on the cask lid.

2. Slowly raise crane hoist to remove cask lid. Position cask lid in area where lid oring can be visually inspected for feathering, crack, etc.. If damage is noted reject cask and send for maintenance. If no damage is noted place cask lid in storage position. Take care to protect cask and lid sealing surfaces.

3. Retrieve immersion pail lid from storage location with crane and lifting slings, and place in position on top of immersion pail structure. Check alignment of bolt holes. (Note: Immersion pail lid sealing surfaces will now be mated to the external surface of the MCO and the inner edge of the immersion pail structure.) Release immersion pail lid and remove slings from crane. Store immersion pail slings.

4. Engage the four immersion pail lid fasteners and inflate the immersion pail seal.

5. Tighten the pail lid fasteners "hand tight" and inflate the MCO seal.

6. Connect immersion pail water vent line.

7. Fill MCO with processed water until within one inch of top of pail structure.

8. Fill pail with clean deionized water through the fill connection while venting from the pail lid vent line. When fill water discharges from vent line, disconnect vent line from valve quick disconnect, and configure water fill line to the immersion pail water control box. Observe pail and MCO seals for any water leakage, correct as required.

9. Install the immersion pail lift beam on the crane hooks. Retrieve pail system slings from storage location and connect pail system slings to crane.

10. Slowly raise pail system until the alignment lines on the immersion pail lifting lugs are exposed at the lock pin housing. Lift lock pin gate and retract lock pins until the pin gate can close again. This ensures full retraction.

11. Using the crane, slowly lower the immersion pail system to the bottom of the loadout pit. Remove slings from master link and secure sling hooks to the sling storage boxes. Position immersion pail lift beam and crane for MCO loading.

12. Perform MCO loading operations per MCO loading procedures.

13. Position the crane with immersion pail lift beam and reattach pail lifting slings to the master link. Lift the immersion pail to the water surface using the lifting slings. 
14. Rinse all surfaces free of possible fuel particulate with water at a hold point where the immersion pail lid is approximately 6 inches above the water surface.

Continue raising the immersion pail while rinsing the $\mathrm{MCO}$ and pail lid surfaces with clean deionized water to remove any residual contamination.

15. Slowly raise pail into the support structure guides until the pin engagement point is reached. Stop crane and engage immersion pail locking pins.

16. Slowly lower immersion pail until resting on locking pins.

17. Remove immersion pail lifting slings and lift beam from crane.

18. Complete $\mathrm{MCO}$ closure and transport preparation using $\mathrm{MCO}$ operations procedures.

\section{A6.2.3 Preparation of Loaded Cask for Transport}

1. Decontaminate MCO shield plug top surfaces and immersion pail lid, as required. Vent air from $\mathrm{MCO}$ and pail seals. Permit water to flow past seals to flush contamination. Isolate deionized water supply.

2. Survey the pail lid prior to removing the lid.

3. Disengage four pail lid fasteners and attach lifting slings from immersion pail lid to crane.

4. Lift immersion pail lid, place it in storage and disengage the lift beam from crane.

5. Inspect lid end of cask body for cleanliness. Attach the cask lid to the crane and align with alignment pins and slowly lower the lid onto the cask body. Remove any weight from crane hoist.

6. Disengage crane hook from cask lid. Torque cask lid bolts in appropriate sequence.

7. Raise cask from the immersion pail.

8. Verify cask surfaces are free of contamination. Monitor surface radiation dose rates. (Note: Take pail water sample to verify water cleanliness prior to next MCO loading cycle.)

9. Transfer the cask to the trailer. Lower cask on to the trailer. Reposition cask operations platform on trailer and engage cask tie-down system.

10. Disengage crane from the trunnions and move free of trailer. 
11. Perform cask and trailer final radiation and contamination surveys. Decontaminate as required.

12. Connect building air supply to the landing leg air supply glad hand. Open door and back tractor to front of trailer. Raise the front of the trailer to match the tractor fifth wheel and lock in place. Set tractor brake and shut off tractor engine.

13. Retract landing legs, change building air supply from landing leg to trailer air glad hand. Raise the rear of the trailer to defined location. Change the override valve to automatic, disconnect the building air supply and connect tractor air lines and electrical cable. Remove wheel chocks.

14. Release cask for transfer to CVD. 
HNF-SD-SNF-FDR-003 Rev. $0 \quad$ E-15166

\section{A6.3 CASK UNLOADING PROCEDURES}

Transfer of the MCO to the Cold Vacuum Drying facility and subsequent transfer to the Canister Storage Building dose not require movement of the transfer cask from the transfer trailer. Site specific operating procedures will be developed using trailer and cask general operating procedures outlined in Section 6.2 of this report and the Systems "Installation, Operation, Repair and Maintenance Manuals". MCO unloading procedures will be provided with supply of the MCO. 


\section{A6.4 EMPTY PACKAGING PROCEDURES}

Similar to the discussion provided in Section 6.3 the transfer cask is not intended to be moved from the transfer trailer during Cold Vacuum Drying, unloading the MCO in the Canister Storage Building and reloading the transfer cask with an empty MCO for the return to $105 \mathrm{~K}$ East and $105 \mathrm{~K}$ West Basins. Site specific operating procedures will be developed using trailer and cask general operating procedures outlined in Section 6.2 of this report and the Systems "Installation, Operation, Repair and Maintenance Manuals". Empty MCO loading procedures will be provided with supply of the MCO. 


\section{A6.5 CASK LOADING RADIATION DOSE ESTIMATES}

Table 6.5-1 provides the estimated total dose for operators loading the MCO at the K-Basins. These dose estimates have been prepared based on the cask loading procedures described in Section 6.2 and estimates of the number of operators and time required to perform specific cask loading operations. The background dose rates were provided by WHC. The cask dose rate estimates were provided by $T N$ based on the limiting $\mathrm{MCO}$ source terms. Cask dose rates were adjusted to reflect average source for integrated dose assessment. The factor representative of the average source has been defined as $42 \%$ of the design bases maximum source. 
HNF-SD-SNF-FDR-003 Rev. $0 \quad$ E-15166

Table A6.5-1 Estimated Total Dose

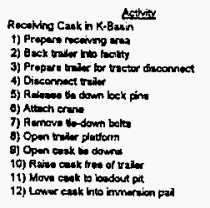

6.2.1 Tot

In Pool Laveding Openters

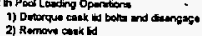

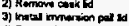

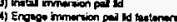

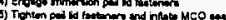

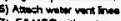

7) Fil MCO with water

b) Fil pal mol catk with waver

0) hrot pell it beren and ating

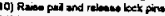

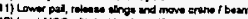

12) Lowc WCO with heet baskere (2)

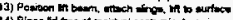

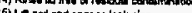

16)

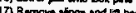

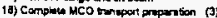

6.2.2 Toked

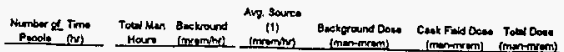

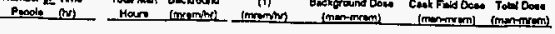

$\begin{array}{lll}2 & 1 & 2 \\ 1 & 0.25 & 0.25 \\ 2 & 0.25 & 0.5 \\ 1 & 0.25 & 0.25 \\ 1 & 0.1 & 0.1 \\ 2 & 0.25 & 0.5 \\ 2 & 0.4 & 0.5 \\ 1 & 0.1 & 0.1 \\ 2 & 0.1 & 0.2 \\ 2 & 0.2 & 0.1 \\ 1 & 0.2 & 0.2 \\ 1 & 0.2 & 0.2\end{array}$

$\begin{array}{ll}0 & 1 \\ 0 & 0.5 \\ 0 & 1 \\ 0 & 0.5 \\ 0 & 0.2 \\ 0 & 1 \\ 0 & 1.4 \\ 0 & 0.2 \\ 0 & 0.4 \\ 0 & 0.9 \\ 0 & 1 \\ 0 & 1\end{array}$

12.2

$\begin{array}{cccc}2 & 0.3 & 1 & 10 \\ 1 & 0.3 & 0.3 & 10 \\ 2 & 0.5 & 1 & 10 \\ 1 & 0.3 & 0.3 & 10 \\ 1 & 0.2 & 0.2 & 10 \\ 1 & 0.1 & 0.1 & 10 \\ 1 & 0.4 & 0.4 & 10 \\ 1 & 0.4 & 0.4 & 10 \\ 2 & 0.2 & 0.4 & 5 \\ 2 & 0.2 & 0.4 & 10 \\ 2 & 0.2 & 0.4 & 10 \\ 2 & 1 & 0.1 & 2 \\ 2 & 0.4 & 0.1 & 10 \\ 1 & 0.2 & 0.2 & 10 \\ 2 & 0.2 & 0.4 & 10 \\ 1 & 0.1 & 0.1 & 10 \\ 2 & 0.2 & 0.4 & 5 \\ 0 & 0 & 0 & 0\end{array}$

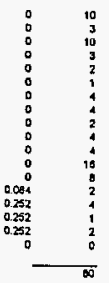

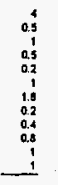

122 
HNF-SD-SNF-FDR-003 Rev. $0 . \quad$ E-15166

\section{A6.6 LEAK TESTING TN-WHC CASK}

A6.6.1 Containment System Periodic Verification Test

Method: Helium Mass Spectrometer Envelope, Pressurized Envelope Method

(ANSI 14.5, A3.10.2)

Equipment required: Helium Mass Spectrometer Leak Detector

Vacuum Pump

\section{Inspection of the Vent Port and Lid Seals}

a. Verify that the cask is dry.

b. Remove quick-disconnect from Vent Port and install cover.

c. Connect the vacuum pump/helium mass spectrometer to the Drain Port quickdisconnect. Evacuate the cask cavity.

d. Install plastic hood around the top of the cask to cover the lid and the Vent Port.

e. The plastic hood is purged and pressurized with helium to slightly above atmospheric pressure.

f. Perform leak test using the leak detector.

g. Acceptance criteria is $1 \times 10^{-7} \mathrm{std} \mathrm{cc} / \mathrm{sec}$, air.

\section{Inspection of Drain Port Seal}

a. Install quick-disconnect in Vent Port.

b. Remove quick-disconnect from Drain Port and install cover.

c. Connect the vacuum pump/helium mass spectrometer to the Drain Port quickdisconnect. Evacuate the cask cavity.

d. Install plastic bag to cover the Drain Port cover.

e. Purge bag with helium and pressurize to slightly above atmospheric pressure.

f. Perform leak test using the leak detector.

g. Acceptance criteria is $1 \times 10^{-7} \mathrm{std} \mathrm{cc} / \mathrm{sec}$, air.

\section{A6.6.2 Containment System Assembly Verification Test}

Method: $\quad$ Pressure Rise (ANSI 14.5, A3.5)

Equipment required: Vacuum pump

Vacuum bell

\section{Inspection of the Lid Seal}

a. Verify that the cask is dry.

b. Evacuate the cask cavity using the Vent Port quick-disconnect.

c. Perform leak test. 


\section{HNF-SD-SNF-FDR-003 Rev. $0 \quad$ E-15166}

d. Test sensitivity is $1 \times 10^{-3} \mathrm{std} \mathrm{cc} / \mathrm{sec}$, air.

e. Install Vent Port cover.

f. Backfill with the appropriate fill gas.

\section{Inspection of Vent and Drain Port Seals}
a. Place vacuum bell over Vent Port cover.
b. Evacuate the vacuum bell.
c. Perform leak test
d. Test sensitivity is $1 \times 10^{-3} \mathrm{std} \mathrm{cc} / \mathrm{sec}$, air.
e. Place vacuum bell over Drain Port cover.
f. Repeat steps b-d. 
HNF-SD-SNF-FDR-003 Rev. $0 \quad$ E-15166

\section{A7.0 QUALITY ASSURANCE REQUIREMENTS}

\section{A7.1 INTRODUCTION}

Transnuclear's Quality Assurance Program is applicable to the safety related items (WHC Safety Class I) and activities performed on this project.

TN's QA Program Manual, " Quality Assurance Program for Design, Fabrication, Assembly, Testing, Maintenance, Repair, Modification and Use of Packaging for Transport of Radioactive Materials, E-1473", was prepared and implemented to satisfy the criteria of Subpart $\mathrm{H}$ to 10CFR71. This QA Program has been approved by the USNRC. In addition, WHC has reviewed the program and concluded that it is acceptable for use on this project.

All Transnuclear cask components are designated as "safety-related" except quick-disconnects and associated seals, lubricants, and nameplates. Lifting attachments and other hardware associated with lifting the cask at the K-Basin sites are designated "safety-related". All other equipment (e.g. trailer, platform, pail) of the cask transportation system and operational equipment are non-safety related.

\section{A7.2 GENERAL REQUIREMENTS}

\section{A7.2.1 Organization}

The organizational line relationships within TN and between TN, WHC and a Major Participating Organization are identified in Figure A7.2-1. Dotted lines on the organization chart (Figure A7.2-1) indicate communication and solid lines indicate direction, as appropriate.

\section{A7.2.2 Quality Assurance Program}

Transnuclear will follow the requirements of their QA Program, E-1473 for all components that are designated safety-related. Major Participating Organizations, whose services are used during the course of this project, will develop and implement their own Quality Assurance programs, as approved by Transnuclear. 


\section{A7.2.3 Design Control}

TN has established measures to assure that regulatory requirements and packaging design have been or are correctly translated into drawings, specifications, procedures and instructions. Design inputs are documented and approved in accordance with QA procedures. Changes to design shall be identified, reviewed, approved and incorporated into the appropriate revision of design documents. These changes require the same review and approval as the original.

\section{A7.2.4 Procurement Control}

TN's safety related procurement documents shall identify which documents are to be prepared by a major participating organization and which documents are to be submitted to TN for review, information and/or approval. The procurement documents shall also specify which documents are to be retained, controlled and maintained by the major participating organizations for specified periods and which records shall be transmitted to TN prior to use of the packaging.

\section{A7.2.5 Instructions, Procedures and Drawings}

Instructions, procedures and drawings shall include quantitative and/or qualitative acceptance criteria to verify that activities affecting quality have been satisfactorily accomplished.

\section{A7.2.6 Document Control}

TN has established and implemented procedures to control the issuance of TN documents which prescribe activities affecting quality. These procedures define document control measures to assure adequate review, approval, release and distribution of original documents and subsequent revisions. Major participating organizations shall establish and implement document control procedures in accord with their approved QA program.

\section{A7.2.7 Control of Purchased Material, Equipment and Services}

Measures have been established and implemented to assure that all purchased material, equipment, and services conform to procurement documents. Major participating organizations shall provide objective evidence that safety-related components meet all quality requirements. 
HNF-SD-SNF-FDR-003 Rev. $0 \quad$ E-15166

\section{A7.2.8 Identification and Control of Materials, Parts and Components}

Measures have been established and implemented to identify and control materials, parts and components. These measures shall assure identification and control by appropriate means during the fabrication, installation and use of the material/part/component. This shall prevent the inadvertent use of incorrect or defective items. The identification and control of safetyrelated items shall be traceable through procurement, fabrication, inspection and test records.

\section{A7.2.9 Control of Special Processes}

Measures have been established and implemented for the control of special processes used in the fabrication, modification and repair of safety-related components. Special processes shall be performed in accordance with approved written procedures. Personnel who perform special processes shall be formally trained and qualified in accordance with applicable codes, standards or specifications.

\section{A7.2.10 Inspection}

Measures shall be established and implemented to inspect materials, parts, processes or other activities affecting quality to verify conformance with documented instructions, procedures, specifications, drawings, or other procurement documents. These inspections shall be performed by personnel other than those who performed the activity being inspected. Modifications and/or repairs to and replacement of safety related components shall be inspected in accordance with the original design and inspection requirements or approved alternatives.

\section{A7.2.11 Test Control}

A program has been established and implemented to perform required acceptance and operational tests, as identified in procurement documents and the application for component approval. The tests shall be performed by qualified personnel in accordance with approved written instructions, procedures, and/or checklists. Test results shall be documented and evaluated to demonstrate that the acceptance criteria has been met.

\section{A7.2.12 Control of Measuring and Test Equipment}

Major participating organizations are responsible for appropriate selection, calibration and control of measuring and test equipment. Measuring and test equipment used for verifying safety related characteristics shall have documented, valid relationships to nationally recognized standards. Instrumentation and test equipment supplied by TN for testing following loading operations shall be calibrated and controlled in accordance with QA procedures. 
HNF-SD-SNF-FDR-003 Rev. $0 \quad$ E-15166

\section{A7.2.13 Handling, Storage and Shipping}

TN documents shall identify handling, storage and shipping requirements. These shall include instructions for marking, special lift points, orientation for storage, shipping and preservation. Based on this, the major participating organizations shall invoke all required handling, storage and shipping requirements in accordance with their approved, written procedures.

\section{A7.2.14 Inspection, Test and Operating Status}

Major participating organizations shall implement their established systems for determining and identifying the acceptability of safety-related components regarding inspections and tests to be performed. Only those safety-related components which have met the required acceptance criteria shall be used. Nonconforming components shall be clearly identified to prevent inadvertent use.

\section{A7.2.15 Nonconforming Material, Parts or Components}

Measures shall be established and implemented to control materials, parts and components which do not conform to requirements to prevent their inadvertent use in subsequent manufacturing operations or during service. Nonconformance reports will be utilized for the procedural control of nonconformances.

\section{A7.2.16 Corrective Action}

TN personnel shall assess the need for corrective action based on audit findings, inspection reports, test reports, nonconformance reports, etc. as required by our QA procedures.

\section{A7.2.17 Records}

For each safety related component a program shall be established and implemented to assure that sufficient written records are maintained to furnish evidence of activities affecting quality.

\section{A7.2.18 Audits}

$\mathrm{TN}$ internal audits and audits of major participating organizations' activities shall be planned and conducted by the TN Project QA Engineer as required by QA procedures. Major participating organizations shall perform audits as required by their QA programs. 
Figure A7.2-1 Chart Showing Organization

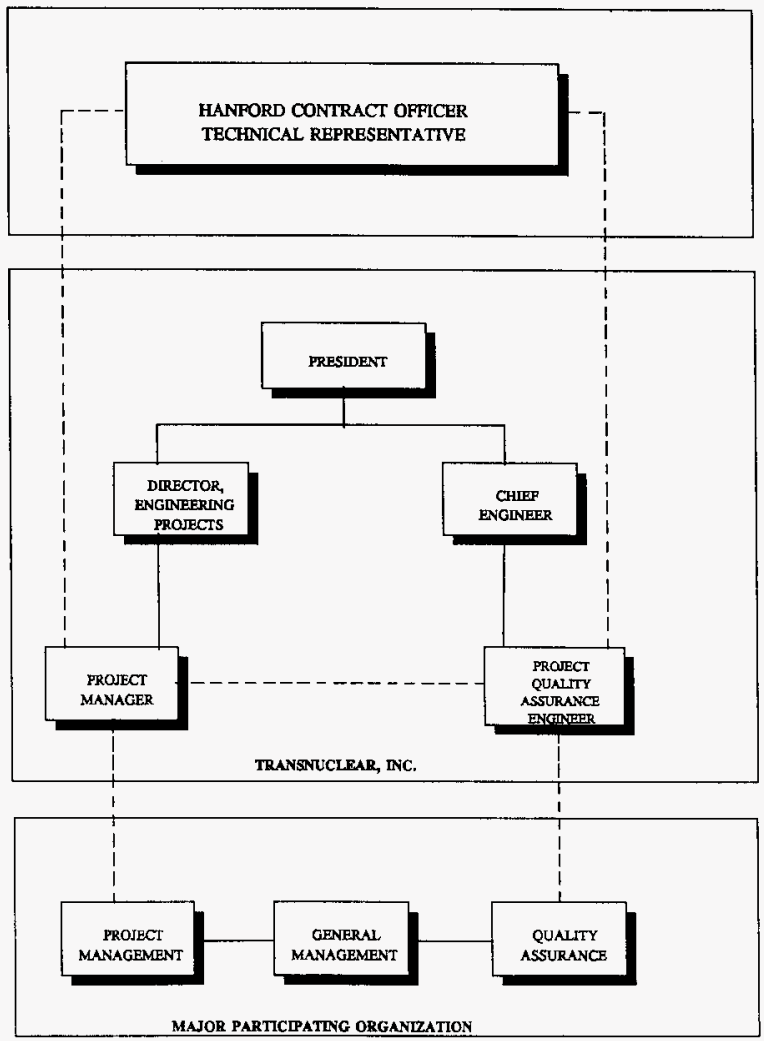




\section{A8.0 MAINTENANCE}

\section{A8.1 GENERAL REQUIREMENTS}

All manuals necessary for the operation and maintenance of the Cask and Transportation System will be provide at the time of delivery.

The general requirements for the cask would be the replacement of the seals on an annual basis. In addition, a visual inspection of all bolt holes is required to detect damage or galling. Replacement of damaged helicoils would occur at this time. A. Containment System Verification Leak Test is required whenever the seals are replaced.

The cask operations equipment for the K-Basin loadout pit includes few mechanical systems and requires little maintenance activity. Maintenance support is limited to that required as a result of system operations inspections performed prior to each cask loading cycle and other system inspections performed monthly, tri-monthly and annually. Components which may require maintenance include immersion pail seal lid seals; immersion pail seal lid fasteners; support structure lock pins; demineralized water reservoir seals, hoses, and connectors; air lines and connectors; and lift slings and pail attachments. Due to the integrity of the design and timited operational components maintenance support is minimal.

\section{A8.2 INSPECTION AND VERIFICATION SCHEDULES}

Installation, Operation, Repair, and Maintenance Manuals (IORM) will be provided with the delivery of the Cask and Transportation System. These manuals will contain the required inspection and verification schedules.

Periodic inspection requirements for the operational system are minimal and include the following:

Support inspection - Prior to each submersion cycle, the pail lifting slings and clevis pins are to be visually inspected for wear, frayed slings, or other signs of deterioration. If detected, part replacement is performed with the pail supported by the immersion pail support structure.

Immersion pail seal verification - When the immersion pail lid is in place between the pail and the MCO and seals are inflated, a positive pressure head of deionized water is applied to the contained space between the two seal boundaries. Loss of air pressure in the seal or evidence of water leakage around the seal provides evidence of seal failure. The immersion pail lid is removed to contaminated equipment repair and the spare immersion pail seal lid is substituted.

Immersion pail integrity - Visual inspection for leakage when the pail is supported by 
the immersion pail support structure can be performed by observing a lowering of the water elevation when the reservoir is isolated from the immersion pail. While such failure is highly unlikely, the inspection process is capable of detecting this condition so that repairs can be affected prior to significant deterioration.

Miscellaneous - Monthly inspection of painted surfaces, air fittings and water fittings is performed in addition to the normal daily observation by operating personnel. 


\section{HNF-SD-SNF-FDR-003 Rev. O E-15166}

\section{A9.0 APPENDIX}

A9.1 TN-WHC CASK AND TRANSPORTATION SYSTEM DRAWINGS

A9.2 CASK OPERATIONS EQUIPMENT ANALYSES 


\section{A9.1 TN-WHC CASK AND TRANSPORTATION SYSTEM DRAWINGS}

DRAWING/DOCUMENT NO.

WHC Dwg. No. H-1-81533

WHC Dwg. No. H-1-81534

WHC Dwg. No. H-1-81535

Sheet 1

Sheet 2

Sheet 3

Sheet 4

Sheet 5

WI-HC Dwg. No. H-1-81536

WHC Dwg. No. H-1-81537

WHC Dwg. No. H-1-81539

Sheet 1

Sheet 2

Sheet 3

WHC Dwg. No. H-1-81543

WHC Dwg. No. H-1-81544

WHC Dwg. No. H-1-81545

WHC Dwg. No. H-1-81546

WHC Dwg. No. H-1-81547

WHC Dwg. No. H-1-8148

WHC Dwg. No. H-1-81549
TITLE

TN-WHC Cask Transportation System, Arrangement Drawing

TN-WHC Cask Transportation System, Assembly Drawing

TN-WHC Cask Transportation System, Assembly and Parts List

TN-WHC Cask Transportation System, Cask Body and Parts List TN-WHC Cask Transportation System, Cask Body Details

TN-WHC Cask Transportation System, Lid

TN-WHC Cask Transportation System, Lid

TN-WHC Cask Name Plate

TN-WHC Cask Transportation System, Seismic Restraint System

TN-WHC Cask Transportation System, Tiedown System TN-WHC Cask Transportation System, Ticdown System Details

TN-WHC Cask Transportation System, Tiedown System Details

$K$ Basin Immersion Pail, Lift Beam assembly (2 Sheets) $\mathrm{K}$ Basin Immersion Pail, TN-WHC Transport Cask

K Basin Immersion Pail Support Structure (2 Sheets)

K-Basin Immersion Pail Seal Lid (2 Sheets)

K Basin Immersion Pail Assembly

K Basin Transport Trailer Work Platform (7 Sheets)

K Basin MCO/Cask CVD Lid (2 Sheets) 
HNF-SD-SNF-FDR-003 Rev. $0 \quad$ E-15166

WHC Dwg. No. H-1-81550

WHC Dwg. No. H-1-81551

WHC Dwg. No. H-1-81552

WHC Dwg. No. H-1-81553

WHC Dwg. No. H-1-81554

WHC Dwg. No. H-1-81555
K Basin Immersion Pail Ancillary Equipment

K Basin Immersion Pail Interface Control Drawing (2 Sheets)

$\mathrm{K}$ Basin Immersion Pail Lock Pin Guide Assembly K Basin Immersion Pail Support Structure Assembly

K Basin Immersion Pail Support Equipment (3 Sheets)

Transport Trailer Design (3 Sheets) 


\section{A9.2 CASK OPERATION EQUIPMENT ANALYSES}

Structural analyses of the cask operation equipment has been performed relative to ANSI 14.6 criteria for safety related components in the immersion pail and loadout pit support structure. AISC criteria has been employed for structural evaluations of the steel support structure in combination with the ANSI N14.6 load factors. In addition to analyses of the safety related load path cask operational equipment, the cold vacuum drying lid is evaluated to assure the $\mathrm{MCO}$ is contained during cold vacuum drying operations. Table A9.2-1 summarizes results for each of the safety related system operational load path components.

Component analyses performed for non-safety related components include evaluation of the system support structures, including the immersion pail lid, the loadout pit work platform and the trailer work platform. Structural analyses results for these components are summarized in Table A9.2.2.

The following discussion provides a brief description of each calculation package documenting structural evaluations of the operation equipment components.

Immersion Pail - Safety Related Support Load Path (Calculations 457-2003.2 and 2003.3)

Calculation Package 457-2003.2 evaluates the immersion pail against the lifting requirements defined in ANSI N14.6. The calculation includes a determination of the pail weight and buoyancy force, along with evaluations of the bottom plate, lifting lug, lug to shell welds, and primary shell. The design is based on a two (2) point lift load.

Two analysis calculation packages encompass the evaluation of the pail under stand resting conditions. Both calculation packages invoke the load factor requirements defined in ANSI N14.6. The calculation package scopes are delineated as follows:

- Calculation 457-2003.2 evaluates the immersion pail upper flange forging subjected to support structure loading conditions. The design is based on a normal condition four (4) point support load.

- Calculation 457-2003.3 analyzes the immersion pail upper flange forging subjected to support structure loading conditions. The analyses considers a two (2) point support load. A general purpose finite element program, ANSYS, Version 5.2, is used to evaluate this condition.

Additionally, Calculation 457-2003.2 documents the maximum lift load under various loading scenarios. These loading scenarios are summarized below: 
Load Condition

I
II
III
IV
V
Description

Setting Pail In Water In Support Stand Lowering Pail / Empty Cask To Pit Bottom

Raising Pail / Full Cask Off Bottom

Raising Pail / Full Cask To Stand Height

Raising Fully Loaded Cask From Pit

\section{$\underline{\text { Load (lbs) }}$}

9,096

36,680

53,730

57,877

59,820

Immersion Pail Support Structure - Safety Related Support Load Path (Calculation 457 2005.2)

Calculation Package 457-2005.2 evaluates the immersion pail support structure utilizing the load factor requirements defined in ANSI N14.6. Documented in the calculation package are evaluations of the pail support structure for composite frame buckling and corner post assembly buckling. Tie plates used to provide stability to the structure are sized and evaluated for compression loads. Bearing and shear stress evaluations were performed for the guide assembly and lock pin.

\section{Lift Beam - Safety Related Support Load Path (Calculation 457-2005.3)}

Calculation Package 457-2005.3 evaluates the lift beam utilizing the load factor requirements defined in ANSI N14.6. The evaluation documents the structural adequacy of the lift beam weldment and the eye plate.

Cold Vacuum Drying Lid - Safety Related Operations Pressure Loading (Calculation 457-2001.2)

Calculation Package 457-2001.2 evaluates the conditioning lid for internal pressure and lid lifting load conditions. The conditioning lid is used during the $\mathrm{MCO}$ vacuum drying operation in the conditioning facility immediately after the MCO is loaded. The internal pressure load is developed as a result of circulating hot water between the MCO outer surface and the inner surface of the cask. Once the cask is within the conditioning facility, the cask lid is removed and the conditioning lid installed. The lid is a " $\mathrm{Z}$ " shaped ring. The lower leg of the $\mathrm{Z}$ rests on the top of the cask. Three (3) lid bolts are used to join the conditioning lid to the cask. The upper $Z$ leg bears against the MCO top surface. The lid provides pressure boundary (two seals) and pressure restraint during MCO draining/drying in addition to providing additional shielding during the vacuum drying operation.

The lid is assessed utilizing a pressure loading of 60 psi. The lid bolting and the upper $\mathrm{Z}$ leg of the lid are influenced by pressure loading.

Additionally, the lid lifting lugs and welds are designed and evaluated to carry the lid weight loading. 
Immersion Pail Seal Lid - Non Safety Related (Calculation 457-2004.2)

Calculation Package 457-2004.2 evaluates the immersion pail lid for internal pressure and lid lifting load conditions. Maximum internal pressure of $5 \mathrm{psi}$ is developed by the positive pressure anti-contamination water system. Only the lid bolting and adjacent flange are influenced by a pressure loading of this level.

Additionally, the lid lifting lugs and welds are designed and evaluated to carry the lid weight loading only.

Loadout Pit Work Platform - Non Safety Related (Calculation 457-2007.2)

Calculation Package 457-2007.2 evaluates the loadout pit platform for design loads based on OSHA requirements defined in Title 29 of the Code Of Federal Regulations. Frame brace, weld, and wall plate stresses are limited to the acceptance criteria defined in the requirements of the AISC Code.

Transport Trailer Work Platform - Non Safety Related (Calculation 457-2002.2)

Calculation Package 457-2002.2 evaluates the transport trailer work platform for design loads based on OSHA requirements defined in Title 29 of the Code Of Federal Regulations.

Channels, support pipes, end caps, and end cap weld stresses are limited to the acceptance criteria defined in the requirements of the AISC Code.

The following Appendix Sections contain analyses calculation documentation.

A9.2.1 Immersion Pail Calculation 457-2003.2

A9.2.2 Immersion Pail Calculation 457-2003.3

A9.2.3 Support Structure Calculation 457-2005.2

A9.2.4 Lift Beam Calculation 457-2005.3

A9.2.5 Cold Vacuum Drying Lid Calculation 457-2001.2

A9.2.6 Seal Lid Calculation 457-2004.2

A9.2.7 Loadout Pit Work Platform 457-2007.2

A9.2.8 Trailer Work Platform 457-2002.2 
Table A9.2-1

K Basin Loadout Pit and Related Operation Equipment Safety Related Load Path Components

Summary of Stress Analysis

\begin{tabular}{|c|c|c|c|c|c|c|c|c|c|}
\hline $\begin{array}{l}\text { Calculation } \\
\text { No. }\end{array}$ & $\begin{array}{l}\text { Drawing } \\
\text { No. } / \\
\text { Component }\end{array}$ & $\begin{array}{l}\text { Item } \\
\text { No. }\end{array}$ & Ttem & $\begin{array}{l}\text { Applied } \\
\text { Load }\end{array}$ & Design Check & $\begin{array}{l}\text { Calculated } \\
\text { Loading }\end{array}$ & Állowable & Basis & M.S. \\
\hline \multirow{10}{*}{$\begin{array}{l}457-2003.2 \\
457-2003.3\end{array}$} & $457-101 /$ & $\mathrm{T}$ & Bottom Plate & $3 W$ & plate bending & $17.728 \mathrm{ksi}$ & $30 \mathrm{ksi}$ & NI4.6 & 0.41 \\
\hline & Immersion & 19,1 & $\begin{array}{l}\text { Pail shell to } \\
\text { bottom plate } \\
\text { interface }\end{array}$ & $\begin{array}{l}\mathrm{W}, \\
\text { pressure }\end{array}$ & $\begin{array}{l}\text { net tension } \\
\text { shell bending \& shear }\end{array}$ & $\begin{array}{l}3.6 \mathrm{ksi} \\
16.636 \mathrm{ksi}\end{array}$ & $\begin{array}{l}30 \mathrm{ksi} \\
30 \mathrm{ksi}\end{array}$ & N14.6 & $\begin{array}{l}0.88 \\
0.45\end{array}$ \\
\hline & \multirow[t]{8}{*}{ Pail } & $10-11$ & Tug to shell weld & $3 \mathrm{~W}$ & shear & 90.680 kips & 594 kips & N14.6 & 0.85 \\
\hline & & 10 & pail shell flange & $3 \mathrm{~W}$ & lifting - longitudinal bending & $24.970 \mathrm{ksi}$ & $30 \mathrm{ksi}$ & N14.6 & 0.17 \\
\hline & & 10 & $\begin{array}{l}\text { pail shell flange, } \\
\text { stiffening ring }\end{array}$ & $3 \mathrm{~W}$ & $\begin{array}{l}\text { lifting } \\
\text { circumferential bending }+ \text { torsion } \\
\text { circumferential shear }\end{array}$ & $\begin{array}{l}17.938 \mathrm{ksi} \\
0.53 \mathrm{ksi}\end{array}$ & $\begin{array}{l}30 \mathrm{ksi} \\
18 \mathrm{ksi} \\
\end{array}$ & N14.6 & $\begin{array}{l}0.40 \\
\text { large }\end{array}$ \\
\hline & & 10 & $\begin{array}{l}\text { pait shell flange, } \\
\text { stiffening ring }\end{array}$ & $3 \mathrm{~W}$ & $\begin{array}{l}\text { stand resting } \\
\text { bending + torsion ( } 4 \mathrm{pt} \mathrm{lift)} \\
\text { memb. + bending, Von Mises }(2 \mathrm{pt})\end{array}$ & $\begin{array}{l}17.104 \mathrm{ksi} \\
28.16 \mathrm{ksi}\end{array}$ & $\begin{array}{l}30 \mathrm{ksi} \\
30 \mathrm{ksi}\end{array}$ & N14.6 & $\begin{array}{l}0.43 \\
0.065\end{array}$ \\
\hline & & 11 & Jug plate & $3 \mathrm{~W}$ & bearing - lifting lug location & $35.771 \mathrm{ksi}$ & $75 \mathrm{ksi}$ & NI4.6 & 0.52 \\
\hline & & 11 & lug plate & $5 \mathrm{~W}$ & net tension - lifting lug location & $30.440 \mathrm{ksi}$ & $75 \mathrm{ksi}$ & N14.6 & 0.62 \\
\hline & & 11 & Tug plate & $5 \bar{W}$ & shear pull out - lifting lug location & $30.434 \mathrm{ksi}$ & $48 \mathrm{ksi}$ & N14.6 & 0.37 \\
\hline & & 11 & Tug plate & $3 \mathrm{~W}$ & bearing - guide assembly location & $60.453 \mathrm{ksi}$ & $75 \mathrm{ksi}$ & N14.6 & 0.19 \\
\hline \multirow[t]{3}{*}{$457-2005.2$} & \multirow{3}{*}{$\begin{array}{l}457-102 / \\
\text { Support } \\
\text { Structure }\end{array}$} & 98,99 & assembly & $5 \bar{W}$ & buckling & 300 kips & 1,064 kips & AISC & 0.72 \\
\hline & & 4,15 & tie plates & $5 W$ & compression & $0.48 \mathrm{ksi}$ & $0.694 \mathrm{ksi}$ & $\bar{A} I S C$ & 0.31 \\
\hline & & 5 & corner post & $5 W$ & buckling & 72.5 kips & $75.0 \mathrm{kips}$ & AISC & 0.03 \\
\hline \multirow[t]{2}{*}{$457-2005.2$} & \multirow{2}{*}{$\begin{array}{l}457-109 / \\
\text { Lock Pin }\end{array}$} & 4 & Tock pin & $5 \mathrm{~W}$ & double shear & $49 \mathrm{ksi}$ & $81 \mathrm{ksi}$ & AISC & 0.40 \\
\hline & & 10 & guide assembly & $5 \mathrm{~W}$ & bearing & $16 \mathrm{ksi}$ & $54 \mathrm{ksi}$ & $\mathrm{NC}$ & 0.70 \\
\hline \multirow[t]{2}{*}{$457-2005.3$} & \multirow[t]{2}{*}{$\begin{array}{l}457-112 / \\
\text { Lift Beam }\end{array}$} & 1 & $\begin{array}{l}\text { lift beam } \\
\text { weldment }\end{array}$ & $\begin{array}{l}3 W \\
3 W \\
5 W\end{array}$ & $\begin{array}{l}\text { beam bending } \\
\text { beam shear } \\
\text { trunnion shear }\end{array}$ & $\begin{array}{l}25.447 \mathrm{ksi} \\
2.769 \mathrm{ksi} \\
11.933 \mathrm{ksi}\end{array}$ & $\begin{array}{l}30 \cdot \mathrm{ksi} \\
18 \mathrm{ksi} \\
40.20 \mathrm{ksi}\end{array}$ & AISC & $\begin{array}{l}0.15 \\
0.85 \\
0.70\end{array}$ \\
\hline & & 10 & eye plate & $3 \mathrm{~W}$ & $\begin{array}{l}\text { tension } \\
\text { bearing }\end{array}$ & $\begin{array}{l}14.4 \mathrm{ksi} \\
10.65 \mathrm{ksi}\end{array}$ & $\begin{array}{l}30 \mathrm{ksi} \\
45 \mathrm{ksi}\end{array}$ & AISC & $\begin{array}{l}0.52 \\
0.76\end{array}$ \\
\hline \multirow[t]{3}{*}{$457-2001.2$} & \multirow{3}{*}{$\begin{array}{l}57-106 / \\
\text { Cold } \\
\text { Vacuum } \\
\text { Drying Lid }\end{array}$} & 1 & Tid flange & pressure & torsion & $5.031 \mathrm{ksi}$ & $16.7 \mathrm{ksi}$ & $\mathrm{NC}$ & 0.70 \\
\hline & & 2 & bolt & pressure & tension & $13.59 \mathrm{ksi}$ & $16.7 \mathrm{ksi}$ & $\mathrm{NC}$ & 0.19 \\
\hline & & 8 & Tifting lug & $3 \mathrm{~W}$ & $\begin{array}{l}\text { bearing } \\
\text { tension } \\
\text { shear }\end{array}$ & $\begin{array}{l}4.093 \mathrm{ksi} \\
4.093 \mathrm{ksi} \\
8.187 \mathrm{ksi}\end{array}$ & $\begin{array}{l}30 \mathrm{ksi} \\
30 \mathrm{ksi} \\
18 \mathrm{ksi}\end{array}$ & AISC & $\begin{array}{l}0.86 \\
0.86 \\
0.55\end{array}$ \\
\hline
\end{tabular}


Table A9.2-2

E-15166

K Basin Loadout Pit and Related Operation Equipment

Non-Safety Related Components

Summary of Stress Analysis

\begin{tabular}{|c|c|c|c|c|c|c|c|c|c|}
\hline $\begin{array}{c}\text { Calculation } \\
\text { No. }\end{array}$ & $\begin{array}{c}\text { Drawing } \\
\text { No./ } \\
\text { Component }\end{array}$ & $\begin{array}{l}\text { Item } \\
\text { No. }\end{array}$ & Item & $\begin{array}{l}\text { Applied } \\
\text { Load }\end{array}$ & Design Check & $\begin{array}{l}\text { Calculated } \\
\text { Loading }\end{array}$ & Aillowable & Basis & M.S. \\
\hline \multirow[t]{3}{*}{$457-2004.2$} & \multirow{3}{*}{$\begin{array}{l}457-103 / \\
\text { Seal Lid }\end{array}$} & 2 & Tid flange & pressure & bending & $8.936 \mathrm{ksi}$ & $25.05 \mathrm{ksi}$ & $\mathrm{NC}$ & 0.70 \\
\hline & & 20 & bolt & pressure & tension & $4.201 \mathrm{ksi}$ & $16.7 \mathrm{ksi}$ & $\mathrm{NC}$ & 0.75 \\
\hline & & 3 & Tifting lug & $3 W$ & $\begin{array}{l}\text { bearing } \\
\text { tension } \\
\text { shear }\end{array}$ & $\begin{array}{l}1.6 \mathrm{ksi} \\
1.6 \mathrm{ksi} \\
3.2 \mathrm{ksi}\end{array}$ & $\begin{array}{l}30 \mathrm{ksi} \\
30 \mathrm{ksi} \\
18 \mathrm{ksi}\end{array}$ & AISC & $\begin{array}{l}0.95 \\
0.95 \\
0.82\end{array}$ \\
\hline \multirow[t]{2}{*}{$457-2002.2$} & \multirow{2}{*}{$\begin{array}{l}457-105 / \\
\text { Trailer } \\
\text { Work } \\
\text { Platform }\end{array}$} & 24 & channel & $125 \mathrm{lbs} / \mathrm{ft}$ & $\begin{array}{l}\text { bending } \\
\text { shear }\end{array}$ & $\begin{array}{l}8.676 \mathrm{ksi} \\
0.820 \mathrm{ksi}\end{array}$ & $\begin{array}{l}21 \mathrm{ksi} \\
14 \mathrm{ksi}\end{array}$ & AISC & $\begin{array}{l}0.59 \\
0.94\end{array}$ \\
\hline & & 26 & plank hook & $591 \mathrm{lbs}$ & bearing & $1.182 \mathrm{ksi}$ & $35 \mathrm{ksi}$ & AISC & $0 . \overline{97}$ \\
\hline \multirow[t]{3}{*}{$457-2007.2$} & \multirow[t]{3}{*}{457.111} & $\begin{array}{l}4,5 \\
3\end{array}$ & $\begin{array}{l}\text { brace, extension, } \\
\text { brace }\end{array}$ & $2000 \mathrm{lbs}$ & $\begin{array}{l}\text { tube bending } \\
\text { shear }\end{array}$ & $\begin{array}{l}22.077 \mathrm{ksi} \\
2.0 \mathrm{ksi}\end{array}$ & $\begin{array}{l}27.6 \mathrm{ksi} \\
18.4 \mathrm{ksi}\end{array}$ & AISC & $\begin{array}{l}0.20 \\
0.89\end{array}$ \\
\hline & & $\begin{array}{l}5 \text { to } \\
9\end{array}$ & weld & $2000 \mathrm{lbs}$ & combined shear and torsion & $1,824 \mathrm{lbs} / \mathrm{in}$ & $2,545 \mathrm{lbs} / \mathrm{in}$ & AISC & $0 . \overline{28}$ \\
\hline & & $\begin{array}{l}9 \text { to } \\
7\end{array}$ & weld & $200 \overline{0} \mathrm{lbs}$ & shear & $0.133 \mathrm{ksi}$ & $14.4 \mathrm{ks}$ & AISC & $0 . \overline{99}$ \\
\hline
\end{tabular}


Appendix A9.2.1 Immersion Pail Calculation 457-2003.2

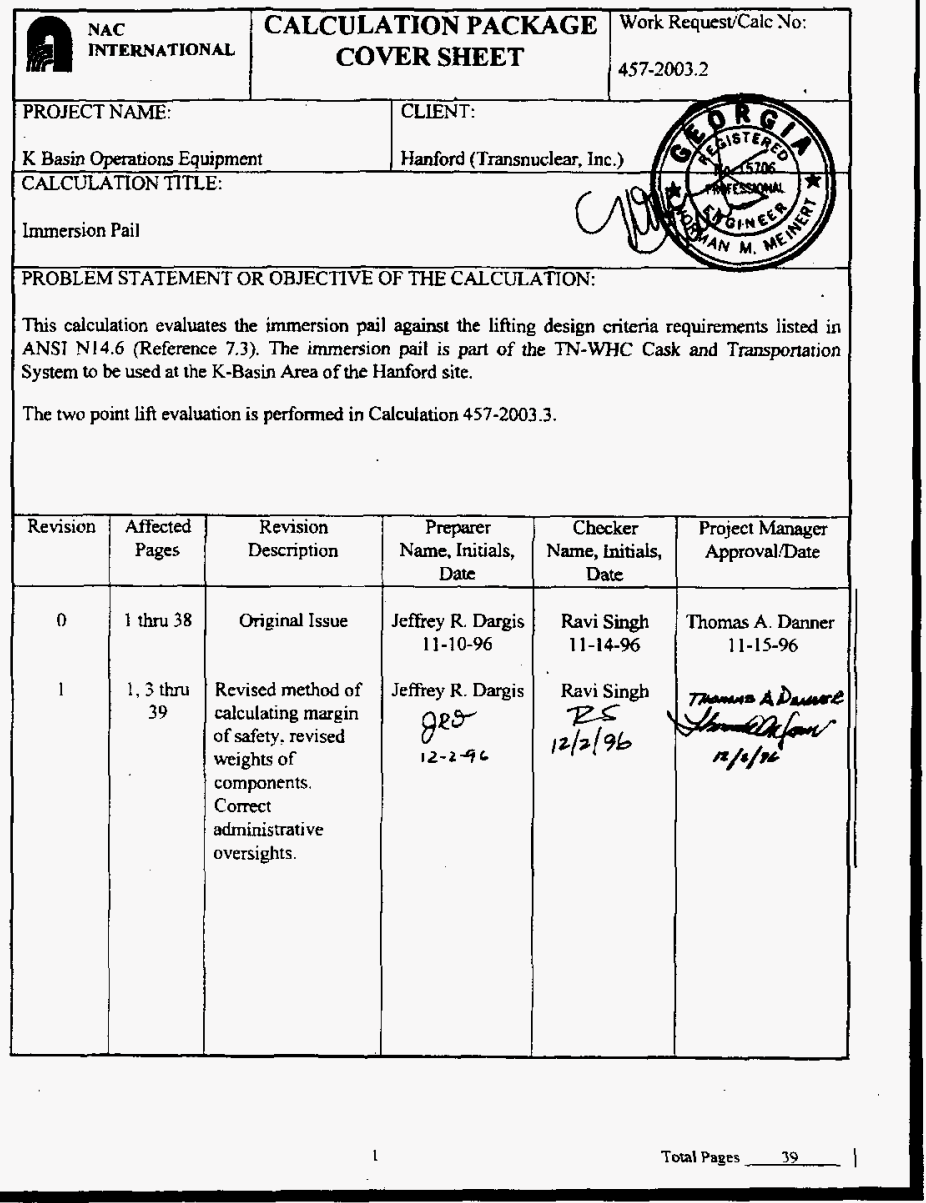


Appendix A9.2.1 Immersion Pail Calculation 457-2003.2 (Continued)

\section{INDEPENDENT DESIGN VERIFICATION CHECK SHEET}

Work Request/Calculation No: 457-2003.2 Revision 으

Scope of Analysis File: This calculation structurallu evaluates the immersion pail against: the lifting degign criteria requirements of ANSI N14.6.

Review Methodology: Check Of Calculations Allernate Analyses Other (Explain)

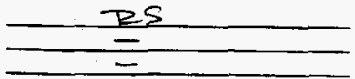

Confirm That The Work Request / Calculation Package Reviewed includes:

1. Statement of Purpose

2. Defined Method of Analysis

3. Listing of Assumptions

4. Detailed Analysis Record

5. Statement of Conclusions / Recommendations (if applicable)

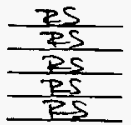

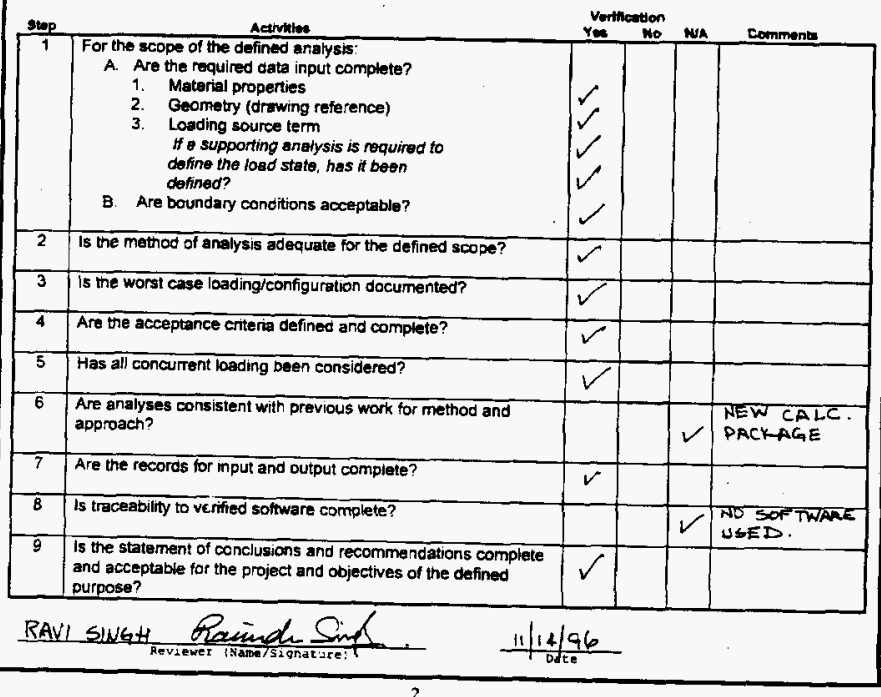


Appendix A9.2.1 Immersion Pail Calculation 457-2003.2 (Continued)

\section{INDEPENDENT DESIGN VERIFICATION CHECK SHEET}

Work RequestCalculation No: 457-2003.2 Revision 1

Scope Of Analysis File: This calculation structurally evaluates the irnmersion pail against the lifting design criteris requirements of ANSI N14.6.

Review Methodology: Check Of Calculations Alternate Analyses Other (Explain)

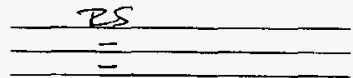

Confirm That The Work Request / Calculation Package Reviewed Includes:

1. Statement of Purpose

2. Defined Method of Analysis

3. Listing of Assumptions

4. Detailed Analysis Record

5. Statement of Conclusions / Recommendations (if applicable)

\begin{tabular}{|c|c|c|c|c|c|}
\hline Stap & Activitites & & tition & & \\
\hline 1 & $\begin{array}{l}\text { For the scope of the defined analysis: } \\
\text { A. Are the required data input complete? } \\
\text { 1. Material properties } \\
\text { 2. Geometry (drawing reference) } \\
\text { 3. Loading source term } \\
\text { If a supporting analysis is required to } \\
\text { dotine the loed stefe, has it been } \\
\text { defined? } \\
\text { B. Are boundary conditions acceptable? }\end{array}$ & $\begin{array}{l}\checkmark \\
\checkmark \\
\checkmark \\
\checkmark\end{array}$ & & & 年 \\
\hline$\overline{2}$ & Is the method of analysis adequate for the defined scope? & $\checkmark$ & & & \\
\hline 3 & Is the worst case loading/configuration documented? & $\checkmark$ & & & \\
\hline 4 & Are the acceptance criteria defined and complete? & $\checkmark$ & & & \\
\hline 5 & Has alf concurrent loading been considered? & $\checkmark$ & & & \\
\hline 6 & $\begin{array}{l}\text { Are analyses consistent with previous work for method and } \\
\text { approach? }\end{array}$ & 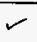 & & & \\
\hline 7 & Are the records for input and output complete? & $\checkmark$ & & & \\
\hline 8 & is traceability to verified software complete? & & & 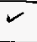 & $\begin{array}{l}\text { RO SOFTWRAE } \\
\text { USED. }\end{array}$ \\
\hline 9 & $\begin{array}{l}\text { Is the statement of conchusions and recommendations complete } \\
\text { and acceptable for the project and objectives of the defined } \\
\text { purpose? }\end{array}$ & $\checkmark$ & & & \\
\hline
\end{tabular}


Appendix A9.2.1 Immersion Pail Calculation 457-2003.2 (Continued)

\section{TABLE OF CONTENTS}

\begin{tabular}{|c|c|}
\hline Section & Description \\
\hline- & Calculation Package Cover Sheet \\
\hline$\ldots$ & Independent Design Verification Check Sheet \\
\hline- & Table of Contents \\
\hline 1.0 & Synopsis of Results \\
\hline 2.0 & Introduction/Purpose \\
\hline 3.0 & Method of Analysis \\
\hline 4.0 & 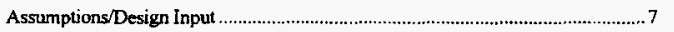 \\
\hline 5.0 & Analysis Derail \\
\hline 6.0 & Summary of Results / Conciusions \\
\hline 7.0 & 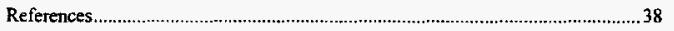 \\
\hline
\end{tabular}

\begin{tabular}{|c|c|c|c|}
\hline $\begin{array}{l}\text { NAC: } \\
\text { NATERNATHONAL }\end{array}$ & Performed by: $g R \theta$ & $\begin{array}{l}\text { Date: } \\
1 / 25-x\end{array}$ & $\begin{array}{l}\text { Calculation No } 457 \cdot 2003.2 \\
\text { Revision } 1\end{array}$ \\
\hline & Checked by: -28 & $11 / 25 / 96$ & Page 4 of 39 \\
\hline
\end{tabular}


Appendix A9.2.1 Immersion Pail Calculation 457-2003.2 (Continued)

Surnmary of Stress Analysis

\begin{tabular}{|c|c|c|c|c|c|c|c|}
\hline $\begin{array}{l}\text { Drawing } \\
\text { No. }\end{array}$ & $\begin{array}{l}\text { Iten } \\
\text { No. }\end{array}$ & Component & $\begin{array}{l}\text { Applied } \\
\text { Load }\end{array}$ & Design Check & $\begin{array}{l}\text { Calculated } \\
\text { Loading }\end{array}$ & Allowable & M.S. \\
\hline $457-101$ & 1 & $\begin{array}{l}\text { bottom } \\
\text { plate } \\
\end{array}$ & $3 \bar{W}$ & plate bending & $17,728 \mathrm{psi}$ & $30,000 \mathrm{psi}$ & 0.41 \\
\hline $457-101$ & 19,1 & $\begin{array}{l}\text { pail shell to } \\
\text { bottom } \\
\text { plate } \\
\text { interface }\end{array}$ & $\begin{array}{l}3 \mathrm{~W}, \\
\text { pressure }\end{array}$ & $\begin{array}{l}\text { net tension } \\
\text { shell bending } \\
\& \text { shear }\end{array}$ & $\begin{array}{l}3605 \text { psi } \\
16,636 \mathrm{psi}\end{array}$ & $\begin{array}{l}30,000 \mathrm{psi} \\
30,000 \mathrm{psi}\end{array}$ & $\begin{array}{l}0.88 \\
0.45\end{array}$ \\
\hline $457-101$ & \begin{tabular}{|l|}
10 to \\
11
\end{tabular} & $\begin{array}{l}\text { lug to shell } \\
\text { weld }\end{array}$ & $3 w$ & shear & 90.68 kips & $594 \mathrm{kips}$ & 0.85 \\
\hline $457-101$ & 10 & flange shell & $3 \mathrm{~W}$ & $\begin{array}{l}\text { lifting } \\
\text { longitudinal } \\
\text { bending }\end{array}$ & $24,970 \mathrm{psi}$ & $30,000 \mathrm{psi}$ & 0.17 \\
\hline $457-101$ & 10 & flange shell & $3 \vec{w}$ & $\begin{array}{l}\text { lifting } \\
\text { circumferential } \\
\text { bending }+ \\
\text { torsion } \\
\text { circumferential } \\
\text { shear }\end{array}$ & $\begin{array}{l}17,938 \mathrm{psi} \\
530 \mathrm{psi}\end{array}$ & $\begin{array}{l}30,000 \text { psi } \\
18,000 \mathrm{psi}\end{array}$ & large \\
\hline $457-101$ & 10 & flange shell & $3 w$ & $\begin{array}{l}\text { stand resting } \\
\text { bending + } \\
\text { torsion }\end{array}$ & 17,104 psi & $30,000 \mathrm{psi}$ & 0.43 \\
\hline $457-101$ & 11 & lug plate & $3 \mathrm{~W}$ & $\begin{array}{l}\text { bearing: } \\
\text { lifting lug } \\
\text { location }\end{array}$ & $35,771 \mathrm{psi}$ & $75,000 \mathrm{psi}$ & 0.52 \\
\hline $457-101$ & 11 & Iug plate & $5 \mathrm{~W}$ & $\begin{array}{l}\text { net tension: } \\
\text { lifting hug } \\
\text { location }\end{array}$ & 30,440 psi & $75,000 \mathrm{psi}$ & 0.62 \\
\hline $457-101$ & 11 & lug platc & $5 \bar{W}$ & $\begin{array}{l}\text { shear pull out: } \\
\text { lifting lug } \\
\text { location }\end{array}$ & $30,434 \mathrm{psi}$ & $48,000 \mathrm{psi}$ & 0.37 \\
\hline $457-101$ & 11 & lug plate & $3 \mathrm{~W}$ & $\begin{array}{l}\text { bearing: guide } \\
\text { assembly } \\
\text { location }\end{array}$ & $60, \overline{453} \mathrm{psi}$ & $75,000 \mathrm{psi}$ & 0.19 \\
\hline
\end{tabular}

The immersion pail design meets the criteria defined in Section 4.2 .

\begin{tabular}{|l|l|l|l|}
\hline $\begin{array}{l}\text { NaC } \\
\text { INTFivational }\end{array}$ & \begin{tabular}{l} 
Performed by: \\
\cline { 2 - 4 }
\end{tabular} & $\begin{array}{l}\text { Date: } \\
1 / 25-96\end{array}$ & $\begin{array}{l}\text { Calculation No. 457-2003.2 } \\
\text { Revision } 1\end{array}$ \\
\hline
\end{tabular}


Appendix A9.2.1 Immersion Pail Calculation 457-2003.2 (Continued)

\subsection{INTRODUCTION/ PURPOSE}

This calculation evaluates the immersion pail against the lifting design criteria requirements listed in Reference 7.3. The immersion pail is part of the TN-WHC Cask and Transportation System to be used at the K-Basin Area of the Hanford site. The immersion pail design was initially evaluated in Reference 7.1 .

3.0 METHOD OF ANALYSIS

Hand calculations using classic texthook solutions are used to structurally evaluate the immersion pail.

The design will be assessed using the load factors discussed in Section 4.2.5. The acceptanee criteria defined in Reference 7.3 limits the lifting induced tensile stresses to the lesser of:

(a) one-third material yield strength; or

(b) one-fifh the material ultimate strength.

Shear stresses will be limited to 0.6 times the tensile stress limits. Bearing stresses will be limited to $1.5 \mathrm{~S}_{\mathrm{y}}$ in accotdance with Reference 7.7 .4 .

The following evaluations are documented within this calculation.

- Component Weight Detemination;

- Buoyancy Force And Buoyancy Rate Determination;

- Maximum Lift Load Determination:

- Maximum Lug Load Determination;

- Bottom Plate Evaluation;

- Primary Inner Sheil Evaluation; and

- Lug Evaluation.

\begin{tabular}{|c|c|c|c|c|}
\hline $\begin{array}{l}\text { AAC } \\
\text { INTERNATIONAL }\end{array}$ & Performed by & geo & Date: & $\begin{array}{l}\text { Calculation Ro. } 457-2003.2 \\
\text { Revision I }\end{array}$ \\
\hline & Checked by: & $E S$ & Datis/20/96 & Page 6 of 39 \\
\hline
\end{tabular}


Appendix A9.2.1 Immersion Pail Calculation 457-2003.2 (Continued)

4.0 ASSLMPTIONS / DESIGN INPUTS

4.1 Assumptions

There are no unverified assumptions within this calculation.

\subsection{Design Criteria}

4.2.1 Tensile Stresses

Calculated tensile stress (based on load factor of 3 ) $\quad<S_{\text {yiew }}$

Calculated tensile stress (based on load factor of 5) $<S_{\text {utimmons }}$

\subsubsection{Shear Stresses}

Calculated shear stress (based on load factor of 3) $<0.6 \times \mathrm{S}_{\text {yiels }}$

Calculated shear stress (based on load factor of 5) $\quad<0.6 \times \mathrm{S}_{\text {wlimue }}$

4.2.3 Bearing stresses

Calculated bearing stress $\quad<1.5 \times \mathrm{S}_{\mathrm{y}} \quad$ (Ref. 7.7.4)

4.2.4 Material Properties

Poison's ratio:

Modulus of Elasticity:

Steel density:

Water density

Foam density:

$$
\begin{aligned}
v & =0.3 \\
E & =28.3 \times 10^{6} \mathrm{psi} \quad \quad \text { (Ref. 7.7.2) } \\
\rho_{\text {stce }} & =0.288 \mathrm{lbs} / \mathrm{in}^{3} . \\
\rho_{\text {water }} & =0.036 \mathrm{lbs} / \mathrm{in}^{3} . \\
\rho_{\text {fom }} & =0.00347 \mathrm{lbs} / \mathrm{in}^{3} . \quad \text { (Reference 7.6.1) }
\end{aligned}
$$

\subsubsection{Stress Design Checks}

All components evaluated within this calculation package are made of A240 Type

\begin{tabular}{|c|c|c|c|}
\hline ANTERNATIONAL & Performed by: $g R \theta$ & $\begin{array}{l}\text { Date: } \\
1 /-25-36\end{array}$ & $\begin{array}{l}\text { Calculation No. 457-2003.2 } \\
\text { Revision 1 }\end{array}$ \\
\hline & Checked by: $\mathrm{KS}$ & $\begin{array}{l}\text { Date: } \\
11 / 25 / 96\end{array}$ & Page 7 of 39. \\
\hline
\end{tabular}
304 stainless steel or or A533 carbon steel. Normalizing the material yield and ultimate strengths by the Reference 7.3 load factors yields the following (See Section 4.2 .1 for load factors):

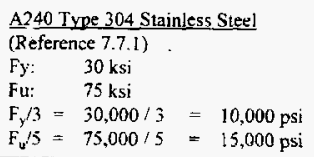


Appendix A9.2.1 Immersion Pail Calculation 457-2003.2 (Continued)

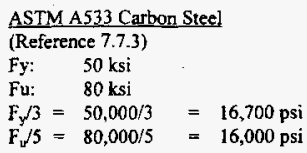

Based on the above comparisons of yield and ultimate allowables for the two (2) materials, comparing a factor (LF) of 3 against yield is more restrictive for A240, while comparing a factor (LF) of 5 against uttimate is more restrictive for A533. Only the limiting conditions are evaluated within this calculation package.

4.3 Desion Conditons

\subsubsection{Temperature} Ambient, $100^{\circ} \mathrm{F}$.

4.3.2 Lifting

Two Point Lift (set) condition is design controlling.

4.3.3 Pressure Effects

Intemal Pressure: $5 \mathrm{psi}$.

For a worst case condition, where pail loses intemal pressure:

$$
\begin{aligned}
\text { Hydrostatic Pressure at Pit Botion } & =\frac{62.4 \frac{\mathrm{lbs}}{\mathrm{f}^{3}} \times 25.75 \mathrm{ft}}{144 \frac{\mathrm{in}^{2}}{\mathrm{ft}^{2}}} \\
& =11.2 \mathrm{psi} \\
\text { Hoop Stress, Inner Shell }(11.2 \mathrm{psi}) & =\mathrm{P} \times \mathrm{r} / \mathrm{t} \\
& =11.2 \times 21.1875 / 0.375 \\
& =633 \mathrm{psi} \\
\text { Longitudinal Stress } & =400 \mathrm{p} \mathrm{Stress} / 2 \\
& =6.33 / 2 \\
& =317 \mathrm{psi}
\end{aligned}
$$

Both the pail hoop and longitudinal pressure stresses are insignificant. Pressure effects on the lid are addressed in a separate calculation.

\begin{tabular}{|l|l|l|l|}
\hline \multirow{2}{*}{$\begin{array}{l}\text { NAC } \\
\text { NTERvaIional }\end{array}$} & \begin{tabular}{l} 
Performed by: $g R \theta$ \\
\cline { 2 - 4 }
\end{tabular} & $\begin{array}{l}\text { Date: } \\
11-25-96\end{array}$ & $\begin{array}{l}\text { Calculation No. 457-2003.2 } \\
\text { Revision I }\end{array}$ \\
\cline { 2 - 4 } & Checked by: & $\begin{array}{l}\text { Date: } \\
11 / 25 / 96\end{array}$ & Page 8 of 39 \\
\hline
\end{tabular}


Appendix A9.2.1 Immersion Pail Calculation 457-2003.2 (Continued)

\subsection{Weights}

Cask weight (loaded, dry)

MCO weight (full, dry)

Cask lid weight

MCO cask annulus water weight

$\mathrm{MCO}$ water weight

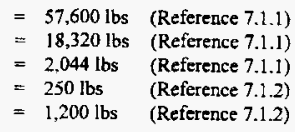

4.5 Elevations of the Fuel Pit (Reference 7.1.3)

Pit floor elevation: $\quad$. 25'-9"

Fuel floor elevation: $\quad$. 20'-9"

Load pit floor elevation: $\quad 0^{\prime}-0^{\prime \prime}$

Top of pit wall elevation: $\quad+2^{\prime}-0^{\prime \prime}$

Water elevation: $\quad$ - 4'.9"

4.6 Pail Dimensional Data (Reference 7.6.1)

\subsubsection{Inner Shell}

ID shell

$=42.0 \mathrm{in}$

thet

$=0.375 \mathrm{in}$

length shert-inner

$=163.5 \mathrm{in}$

length $_{\text {shelt }}(t=0.375$ in $)$

$=163.5-23=140.5$ in

$A_{\text {shelf }}(t=0.375$ in $)$

$=\pi \times\left[((42.0 / 2)+0.375)^{2}-(42.0 / 2)^{2}\right]$

$=49.922$ in $^{2}$

$V_{\text {pail }}$

$=\pi \times\left(\mathrm{ID}_{\text {shefl }} / 2\right)^{2} \times$ length $_{\text {shell }}$ (total)

$=\pi \times(42.0 / 2)^{2}$

$=226,520 \mathrm{in}^{2}$

$\mathrm{OD}_{\text {lange }} \quad=46.0 \mathrm{in}$

$t_{\text {flenge }}$

$=2.0 \mathrm{in}, 1.375 \mathrm{in}$ (shaved)

length $_{\text {Iange }}$

$\equiv 8.0 \mathrm{in}$

A nange $_{\text {nat }}$

$=\pi \times\left[(46.0 / 2)^{2}-(42.0 / 2)^{2}\right]$

$=276.460 \mathrm{in}^{2}$

ivt: NaC:

\begin{tabular}{|c|c|c|}
\hline Performed by: $g R \theta$ & $\begin{array}{l}\text { Date: } \\
11-25-x\end{array}$ & $\begin{array}{l}\text { Calculation No. 457.2003.2 } \\
\text { Revision I }\end{array}$ \\
\hline Checked by: $R 8$ & $\begin{array}{r}\text { Date; } \\
\text { II] }\end{array}$ & Page 9 of 39 \\
\hline
\end{tabular}


Appendix A9.2.1 Immersion Pail Calculation 457-2003.2 (Continued)

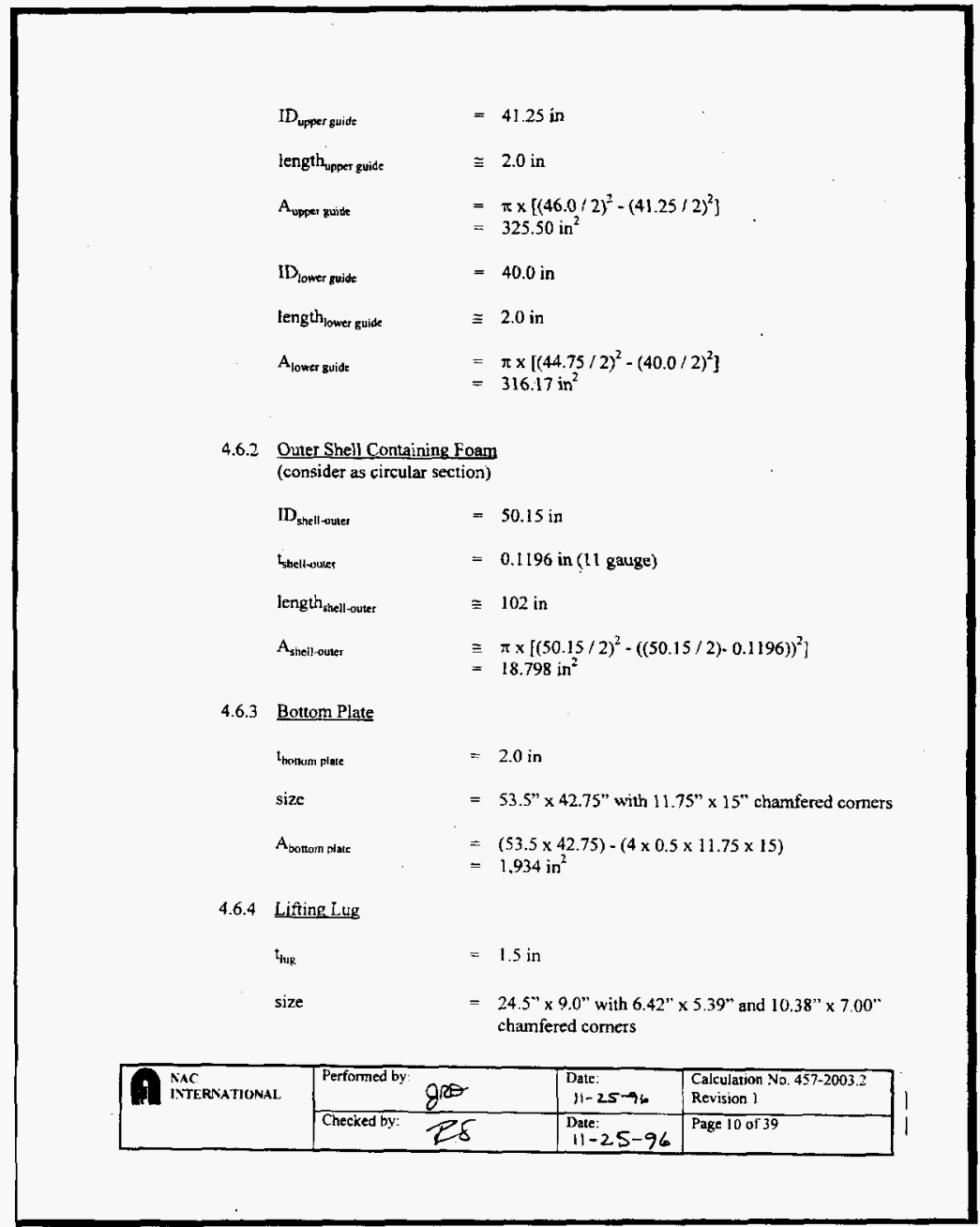


Appendix A9.2.1 Immersion Pail Calculation 457-2003.2 (Continued)

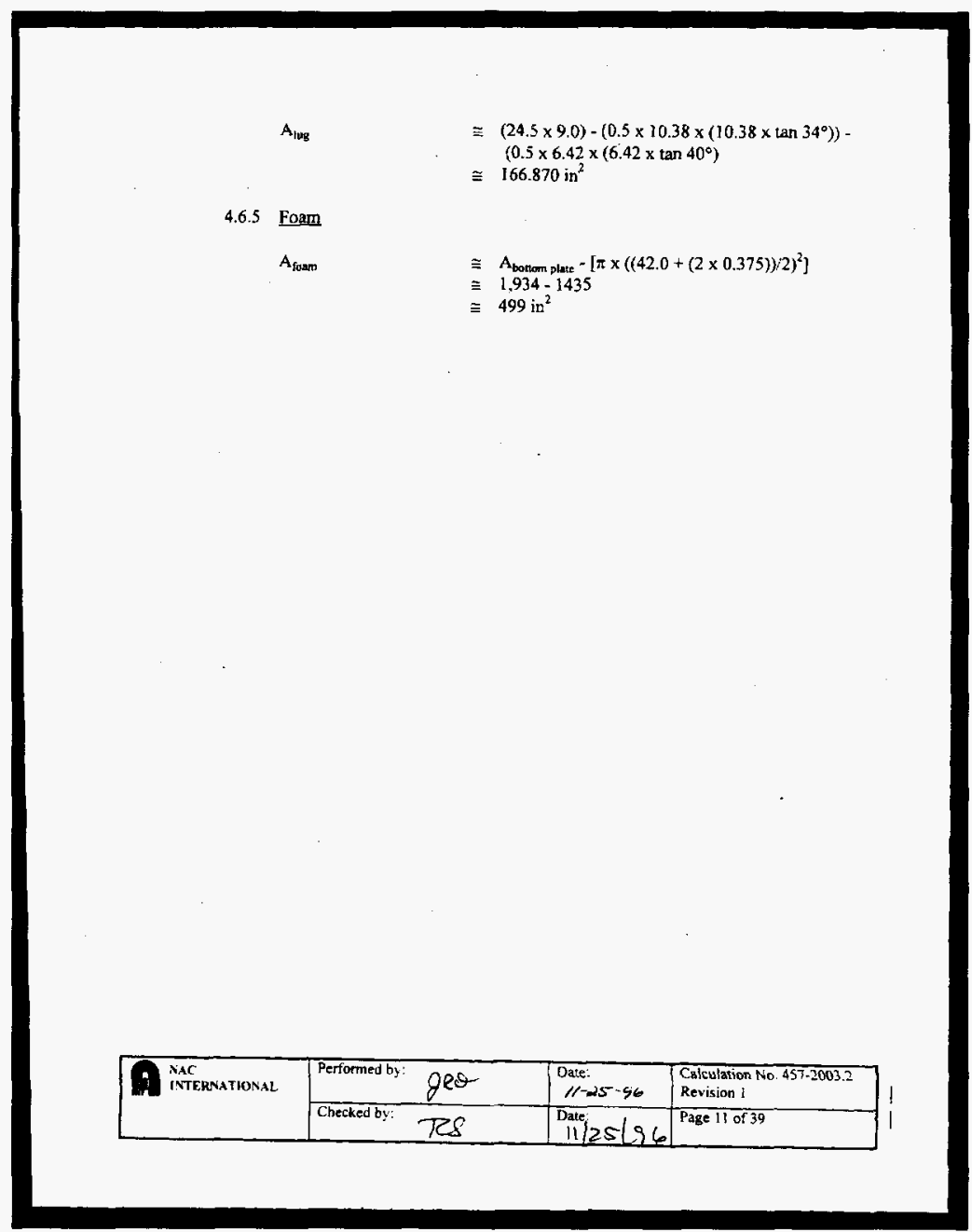


Appendix A9.2.1 Immersion Pail Calculation 457-2003.2 (Continued)

5.0 ANALYSIS DETAIL

\subsection{Component Weight Determination}

5.1.1 Pail

Inner Shell $(t=0.375$ in $) \quad=\rho_{\text {steel }} \times$ length $_{\text {shell }} \times A_{\text {shall }}$

$=0.288 \times 140.5 \times 49.922$

$=2020 \mathrm{lbs}$

Inner Sheil Flange

$=\rho_{\text {steci }} \times$ length $_{\text {flange }} \times$ A $_{\text {nange }}$

$=0.288 \times 8.0 \times 276.460$

$=637 \mathrm{lbs}$

Inner Shell Upper Guide

$=\rho_{\text {sleul }} \times$ length $_{\text {upper }} \times A_{\text {upper }}$

$=0.288 \times 2.0 \times 325.50$

$=188 \mathrm{lbs}$

Inner Shell Between Upper

And Lower Guides

$=\rho_{\text {skel }} \times$ length $\times A_{\text {upper }}$

$=0.288 \times 8.0 \quad \mathrm{x}$ $\left[\pi \times(44.75 / 2)^{2}-(42.0 / 2)^{2}\right]$

$=432 \mathrm{los}$

Inner Shell Lower Guide

$=\rho_{\text {seel }} \times$ length luwer $\times A_{\text {lower }}$

$=0.288 \times 2.0 \times 316.17$

$=182 \mathrm{lbs}$

Lugs

$=\rho_{\text {steel }} \times 4_{\text {lug }} \quad x \quad A_{\text {iug }} \quad x \quad 4$ lugs

$=0.288 \times 1.5 \times 166.870 \times 4$

$=288 \mathrm{lbs}$

Outer Shel!

$=\rho_{\text {stect }} \times$ length shell-auler $\times \mathbf{A}_{\text {shell-aulez }}$

$=0.288 \times 102.0 \times 18.798$

$=552 \mathrm{lbs}$

Foam

$=\rho_{\text {foam }} \times$ length $_{\text {shell-outer }} \times A_{\text {foam }}$

$=0.00347 \times 102$ $\times \quad 499$

$=177 \mathrm{lbs}$

Bottom Plate

$=P_{\text {steel }} \times t_{\text {bvitom plate }} \times A_{\text {bolem ptate }}$

$=0.288 \times 2 \times 1934$

$=1114 \mathrm{lbs}$

\begin{tabular}{|c|c|c|c|}
\hline NAC & Pefformed by: $Q R \theta$ & $\begin{array}{l}\text { Date: } \\
11-25-40\end{array}$ & $\begin{array}{l}\text { Calculation No, } 457-2003.2 \\
\text { Revision } 1\end{array}$ \\
\hline & Checked by: $P S$ & $11 / 25 / 96$ & Page 12 of 39 \\
\hline
\end{tabular}


Total Pail Weight $=5590 \mathrm{Jbs}=6000 \mathrm{lbs}$

(Note: Approximated weight on drawing (Reference 7.6.1) is 5.700 lbs.)

\subsubsection{Other Components}

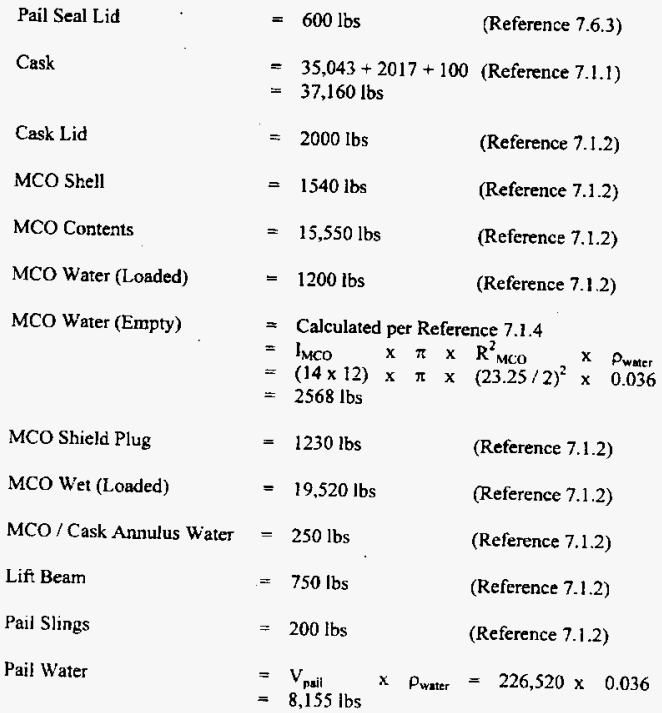

5.2 Buoyancy Force and Buoyancy Rate Determination

Displaced Volume (pail immersed) $=\left[\pi \times\left(O D_{\text {sheil-ouler }} / 2\right)^{2} \times i_{\text {shel-ouxer }}\right]+$ $\left[\pi \times\left(O D_{\text {shell-inner }} / 2\right)^{2} \times\left(l_{\text {shell-inner }}-l_{\text {shell-outer }}\right)\right]$

$=\left[\pi \times(50.39 / 2)^{2} \times 102\right]+$ $\left[\pi \times(42.75 / 2)^{2} \times(163.5-102)\right]$

$=291,688 \mathrm{in}^{3}$

\begin{tabular}{|l|l|}
\hline Perfomed by: & $\begin{array}{l}\text { Date } \\
11-25-46\end{array}$ \\
\hline Checked by: & $\begin{array}{c}\text { Date } \\
1 / 25 / 96\end{array}$ \\
\hline
\end{tabular}




\section{HNF-SD-SNF-FRD-003 Rev. 0}

Appendix A9.2.1 Immersion Pail Calculation 457-2003.2 (Continued)

Bubyant Force

Buoyant Rate

(above floatation interface)

(below floatation interface)
$=$ Displaced Volume $\times p_{\text {wher }}$

$=291.688 \times 0.036$

$=10,500 \mathrm{lbs}$

$=p_{\text {watke }} \times\left[\pi \times\left(O D_{\text {shell-inser }} / 2\right)^{2} \times\right.$

$\left.\left(l_{\text {shell-inner }}-l_{\text {shefl-outer }}\right)\right] /\left(l_{\text {shell-inner }}-l_{\text {shell-ouler }}\right)$

$=0.036 \times\left[\pi \times(42.75 / 2)^{2} \times(163.5-102)\right]$ !

$(163.5-102)$

$=51.67 \mathrm{lbs} / \mathrm{in}$

$=\rho_{\text {watr }} \times\left[\pi \times\left(O D_{\text {shelt-ouver }} / 2\right)^{2} \times I_{\text {shell-outer }}\right]^{\prime}$

$1_{\text {shell-owes }}$

$=0.036 \times\left[\pi \times(50.39 / 2)^{2} \times(102)\right] /$ (102)

$=71.79 \mathrm{lbs} / \mathrm{in}$

\subsection{Maximum Lift Load Delermination}

The maximum lift load will be determined as the enveloping condition for the following lift conditions:

Condition I: Setting pail in water on support stand.

Condition II: Lowering pail / empty cask to pit bottom.

Condition IlI: Raising pail / full cask off bottom.

Condition IV: Raising pail / full cask to stand height.

Condition V: Raising fully loaded cask from pit.

\subsubsection{Lift Load for Condition I: Setting Pail In Water On Support Stand}
Pai]
Pail Slings
$5,700 \mathrm{lbs}$
Lift Beam
$400 \mathrm{lbs}$
Pail Water (conservatively, consider
550 lbs
4 feet of water in pail,
$30 \%$ of $8155 \mathrm{lbs}$ )
2,446 lbs
Lift Load I
$9,096 \mathrm{lbs}$

INTERNATIONAL

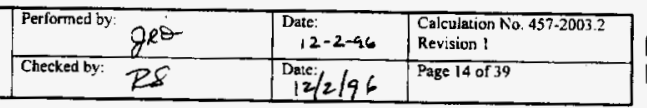


5.3.2 Lift Load for Condition II: Lowering Pail / Emply Cask To Pit Bottom

$\begin{array}{lr}\text { Pail } & 5,700 \mathrm{lbs} \\ \text { Pail Seal Lid } & 600 \mathrm{lbs} \\ \text { Pail Slings } & 400 \mathrm{lbs} \\ \text { Lift Beam } & 550 \mathrm{lbs} \\ \text { Cask } & 36,570 \mathrm{lbs} \\ \text { MCO / Cask Annulus Water } & 250 \mathrm{lbs} \\ \text { MCO Shell } & 1,900 \mathrm{lbs} \\ \text { MCO Water (Empty) } & 1,210 \mathrm{lbs} \\ \text { Buoyant Force } & -10,500 \mathrm{lbs} \\ \text { Lift Load Il } & \\ \end{array}$

5.3.3 Lift Load for Condition III: Raising Pail / Full Cask Off Bottom

Pail

Pail Seal Lid

5,700 lbs

Pail Slings

$600 \mathrm{lbs}$

Lift Beam

$400 \mathrm{lbs}$

Cask

MCO / Cask Annulus Water

MCO Wet (Loaded)

$550 \mathrm{lbs}$

$36,570 \mathrm{lbs}$

$250 \mathrm{lbs}$

Buoyant Force

$20,160 \mathrm{lbs}$

$-10,500 \mathrm{lbs}$

Lift Load III

$53,730 \mathrm{lbs}$

5.3.4 Lift Load for Condition IV: Raising Pail / Full Cask To Stand Height (Pail raised to floor elevation 1' $-6 "$ ", water level at -4 ' -9 ")

Pail

Pail Seal Lid

Pail Slings

Lift Beam

Cask

MCO / Cask Annulus Water

MCO Wet (Loaded)

Calculate Buoyant Force

Pail submerged $163.5^{\prime \prime}-75^{\prime \prime}=$

Buoyant Force $=88.5 \times 71,79=$

Lift Load IV
$5,700 \mathrm{lbs}$

$600 \mathrm{lbs}$

$400 \mathrm{lbs}$

$550 \mathrm{lbs}$

$36,570 \mathrm{lbs}$

$250 \mathrm{lbs}$

$20,160 \mathrm{lbs}$

88.5 in

$-6,353 \mathrm{lbs}$

$57.877 \mathrm{lbs}$

NAC INTERNATIONAL

\begin{tabular}{|l|l|l|}
\hline $\begin{array}{l}\text { Performed by: } \\
\text { Checked by: }\end{array}$ & $\begin{array}{l}\text { Dale: } \\
12-2-26\end{array}$ & $\begin{array}{l}\text { Calculation No. 457-2003.2 } \\
\text { Revision 1 }\end{array}$ \\
\hline T5 & $\begin{array}{c}\text { Date: } 1 / 2 / 96 \\
12 / 2 / 96\end{array}$ & Page 15 of 39 \\
\hline
\end{tabular}


HNF-SD-SNF-FRD-003

Rev. 0

Appendix A9.2.1 Immersion Pail Calculation 457-2003.2 (Continued)

\subsubsection{Lift Load for Condition V: Raising Fully Loaded Cask From Pit}

$\begin{array}{lr}\text { Cask } & 36,570 \mathrm{lbs} \\ \text { Cask Lid } & 1,890 \mathrm{lbs} \\ \text { MCO / Cask Annulus Water } & 250 \mathrm{lbs} \\ \text { MCO Wet (Loaded) } & 20,160 \mathrm{lbs} \\ \text { Pail Slings } & 400 \mathrm{lbs} \\ \text { Lift Beam } & 550 \mathrm{lbs}\end{array}$

Lift Load V $\quad 59,820$ lbs

\subsubsection{Maximum Lift Load}

The limiting lift load condition is Condition V, Raising Fully Loaded Cask From Pit. The lift load associated with Condition $V$ as determined in Section 5.3 .5 is 59,820 lbs.

The lift load used in the design of the immersion pail will be:

$P_{\text {lif }}=60,000 \mathrm{lbs}$.

\subsection{Lug Load Determination}

The Hanford crane configuration employs a hook design, all attached to a shackle located at the center of the lift beam. The lug-to-lug distance in one direction per Reference 7.6.1 is:

$1_{1}=44.5-\left(2 \times 4.5 \times \cos 38^{\circ}\right)$

$=37.41 \mathrm{in}$

The Jug-to-lug distance in the opposite direction per Reference 7.6 .1 is:

$l_{2}=34.77-\left(2 \times 4.5 \times \sin 38^{\circ}\right)$

$=28.6$ in

The sling is to be 16 feet long per Reference 7.6.5:

$I_{3}$

$$
=16 \mathrm{ft}
$$

The angle in the vertical plane with the hooks, $\theta$, is:

\begin{tabular}{|l|l|l|l|}
\hline \multirow{2}{*}{$\begin{array}{l}\text { NaC } \\
\text { INTERnational }\end{array}$} & \begin{tabular}{l} 
Peffomed by: $g R \theta$ \\
\cline { 2 - 4 }
\end{tabular} & $\begin{array}{l}\text { Date: } \\
12-2-96^{\circ}\end{array}$ & $\begin{array}{l}\text { Calculation No. 457-2003.2 } \\
\text { Revision J }\end{array}$ \\
\cline { 2 - 4 } & Checked by: & Date: $/ 2 / 96$ & Page 16 of 39 \\
\hline
\end{tabular}


HNF-SD-SNF-FRD-003 Rev. 0

Appendix A9.2.1 Immersion Pail Calculation 457-2003.2 (Continued)

5.3.5 Lift Load for Condition V: Raising Fully L,oaded Cask From Pit

$\begin{array}{lr}\text { Cask } & 36,570 \mathrm{lbs} \\ \text { Cask Lid } & 1,890 \mathrm{lbs} \\ \text { MCO / Cask Annulus Water } & 250 \mathrm{lbs} \\ \text { MCO Wet (Loaded) } & 20,160 \mathrm{lbs} \\ \text { Pail Slings } & 400 \mathrm{lbs} \\ \text { Lift Beam } & 550 \mathrm{lbs} \\ & \\ \text { Lift Load V } & 59,820 \mathrm{lbs}\end{array}$

\subsubsection{Maximum Lift Load}

The limiting lift load condition is Condition V, Raising Fully Loaded Cask From Pit. The lif load associated with Condition $\mathrm{V}$ as determined in Section 5.3.5 is $59,820 \mathrm{lbs}$

The lift load used in the design of the immersion pail will be:

$P_{\text {tift }}=60,000 \mathrm{lbs}$

\subsection{Lug Load Determination}

The Hanford crane configuration employs a hook design, all attached to a shackle located at the center of the lift beam. The lug-to-lug distance in one direction per Reference 7.6.1 is:

$l_{1}=44.5-\left(2 \times 4.5 \times \cos 38^{\circ}\right)$

$=37.41 \mathrm{in}$

The lug-to-lug distance in the opposite direction per Reference 7.6 .1 is:

$l_{2}=34.77-\left(2 \times 4.5 \times \sin 38^{\circ}\right)$

$=28.6$ in

The sling is to be 16 feet long per Reference 7.6.5:

$\mathrm{l}_{3}$

$=16 \mathrm{ft}$

The angle in the vertical plane with the hooks, $\theta$, is:

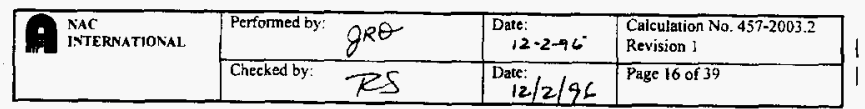


Appendix A9.2.1 Immersion Pail Calculation 457-2003.2 (Continued)

$$
\begin{aligned}
\mathrm{L}_{\text {lug }} & =\mathrm{LF} \times \mathrm{P}_{\text {liff }} /\left(2 \times \cos 7.04^{\circ}\right) \\
& =3 \times 60,000 /(2 \times 0.9925) \\
& \cong 90,680 \mathrm{lbs}
\end{aligned}
$$

\subsection{Bottom Plate Evaluation}

Treating the bottom plate as a simply supported plate along the radial location of the inner shell with uniform loading along its surface, the maximum radial and tangential plate stress is:

$$
\begin{aligned}
& \sigma_{,}=\sigma_{1}=\frac{3 \mathrm{~W}}{8 \pi \mathrm{m} \mathrm{t}^{2}}(3 \mathrm{~m}+1) \quad \text { (Reference 7.4.1) } \\
& \text { where: } \begin{aligned}
\mathrm{W} & =\mathrm{LF} \times \mathrm{P}_{\mathrm{lift}} \\
\mathrm{LF} & =\text { Load factor based on yield strength comparison } \\
& =3 \\
\mathrm{P}_{\mathrm{lift}} & =60,000 \mathrm{lbs} \\
\mathrm{m} & =\text { Reciprocal of Poisson's ratio } \\
& =1 / \mathrm{v}=1 / 0.3=3.33 \\
\mathrm{t} & =\text { plate thickness } \\
& =2.0 \text { in }
\end{aligned}
\end{aligned}
$$

\begin{tabular}{|c|c|c|c|c|}
\hline \multirow[t]{2}{*}{1 intervational } & Pefformed by & QRe & $\begin{array}{l}\text { Date: } \\
11+2-26\end{array}$ & $\begin{array}{l}\text { Calculation No. 457.2003.2 } \\
\text { Revision } 1\end{array}$ \\
\hline & Checked by: & 25 & Date $/ 25 / 96$ & Page 18 of 39 \\
\hline
\end{tabular}

Therefore:

$\sigma_{r}=\sigma_{?}=\frac{3(3 \times 60,000)}{8 \pi(3.33)(2)^{2}}(3(3.33)+1)$

$\sigma_{,}=\sigma_{1}=17,728 \mathrm{psi}$

Plate material is A240 Type 304. The safety margin is:

Safety Margin $=1-\frac{17.728}{30,000}=0.41$ 


\section{HNF-SD-SNF-FRD-003 Rev. 0}

Appendix A9.2.1 Immersion Pail Calculation 457-2003.2 (Continued)

5.6 Inner Shell Evaluation (Primary Shell)

\subsubsection{Net Tension Check}

The net tension stress on the minimum cross section, $t=0.375$ in, is:

$$
\begin{aligned}
\sigma_{\mathrm{t}} & =P_{\text {tat }} \times \mathrm{LF} / \mathrm{A}_{\text {stual }}(\mathrm{t}=0.375 \mathrm{in}) \\
& =60,000 \times 3 / 49.922 \\
& =3605 \mathrm{psi}
\end{aligned}
$$

Shell material is A240 Type 304. The safety margin is:

Safety Margin $=1-\frac{3,605}{30,000}=\mathbf{0 . 8 8}$

5.6.2 Inner Shell To Bottom Plate Interface

The inner shell to bottom plate interface will be assessed by comparing the rotational stiffness of a plate at the outer edge to the rotational stiffness of a cylinder with an applied end mornent.

The cylinder stiffness, $\mathrm{M}_{0} / \theta$, is determined from Reference 7.4 .2 , where:

$\theta=M_{n} / \lambda D$

where: $\quad \lambda=\sqrt{\frac{3\left(1-v^{2}\right)}{\mathrm{R}_{2}{ }^{2} \mathrm{t}^{2}}}$

and:

$$
\mathrm{D}=\frac{E \mathrm{t}^{3}}{12\left(1-\nu^{2}\right)}
$$

when: $\quad R=$ Outer radius of inner shell

$=(42.0+(2)(0.375)) / 2$

$=21.375 \mathrm{in}$

$v=0.3$

$E=28.3 \times 10^{6} \mathrm{psi}$

: = inner shell thickness

\begin{tabular}{|c|c|c|}
\hline Pefformed by: $g R$ & $\begin{array}{l}\text { Date: } \\
1 /-25-76\end{array}$ & $\begin{array}{l}\text { Calculation No. } 457-2003.2 \\
\text { Revision I }\end{array}$ \\
\hline Checked by: RS & Date: $11 / 25 / 76$ & Page 19 of 39 \\
\hline
\end{tabular}

$$
=0.375 \mathrm{in}
$$


Appendix A9.2.1 Immersion Pail Calculation 457-2003.2 (Continued)

$$
\begin{aligned}
& \text { Therefore: } \lambda=\sqrt[4]{\frac{3\left(1-(0.3)^{2}\right)}{(21.375)^{2}(0.375)^{2}}} \\
& =0.454 \mathrm{in} \\
& \mathrm{D}=\frac{\left(28.3 \times 10^{6}\right)(0.375)^{3}}{12\left(1-(0.3)^{2}\right)} \\
& =136,665 \\
& \mathrm{M}_{0} / \theta=\lambda \mathrm{D} \\
& =0.454(136,665) \\
& =62,046 \mathrm{in}-\mathrm{lb} / \mathrm{rad}
\end{aligned}
$$

The plate stiffness, $M_{0} / \theta$, is determined from Reference 7.4.3, where:

$$
\begin{aligned}
\theta & =\frac{12(\mathrm{~m}-1) \mathrm{Ma}}{\mathrm{E} \mathrm{t}^{3}} \\
& =\frac{12(\mathrm{~m}-1) \mathrm{Ma}}{\mathrm{E} \mathrm{mt}^{3}}
\end{aligned}
$$

\begin{tabular}{|c|c|c|c|}
\hline NAC: & Perfomed by: $\mathrm{QPO}$ & Date: $1 / 25-96$ & $\begin{array}{l}\text { Calculation No. 457-2003.2 } \\
\text { Revision ] }\end{array}$ \\
\hline & Checked by: $\pi S$ & $11 / 25 / 96$ & Page 20 of 39 \\
\hline
\end{tabular}

Therefore:

$$
\begin{aligned}
\mathrm{M}_{0} / \theta & =\frac{\mathrm{Emt^{3 }}}{12(\mathrm{~m}+1) \mathrm{a}} \\
& =\frac{\left(28.3 \times 10^{6}\right)(3.33)(2)^{3}}{12(3.33-1) 21.375} \\
& =1,261,471 \mathrm{in}-\mathrm{lb} / \mathrm{rad}
\end{aligned}
$$

The bottom plate is 20 times stiffer than the inner shell. The plate behavior controls the joint interface response.

The rotation of the bottom plate under lift loading is determined by treating the plate as simply supported at the radial location of the inner shell:

$\theta=\frac{3 W(m-1) a}{2 \pi E m t^{3}} \quad$ (Reference 7.4.1) 
Appendix A9.2.1 Immersion Pail Calculation 457-2003.2 (Continued)

$=\frac{3(3 \times 60,000)(3.33-1) 21.375}{2 \pi\left(28.3 \times 10^{6}\right)(3.33)(2)^{3}}$

$=0.0057$ radians .

$=0.33^{\circ}$

Applying this rotation to the end of the cylinder and solving for the edge moment. $\mathrm{M}_{0}$ :

$\mathrm{M}_{0}=\theta \times \lambda \times \mathrm{D} \quad$ (Reference 7.4.2)

$=0.0057 \times 0.454 \times 136,665$

$=354$ in $-\mathrm{lbs}$

The maximum inner shell bending stress due to $M_{0}$ is:

$$
\begin{aligned}
s_{2} & =\frac{2 \mathrm{M}_{0}}{1} \lambda^{2} \mathrm{R} \\
& =\frac{2(354)}{0.375}(0.454)^{2}(21.375) \\
& =8,318 \mathrm{psi}
\end{aligned}
$$

The bending stress due to edge shear will yield smaller stresses than those due to $\mathrm{M}_{0}$. Therefore, the maximum local bending stress due to edge bending and shear will be conservatively considered as $2 \times \mathrm{s}_{2}$ :

$$
\begin{aligned}
2 \times s_{2} & =2 \times 8318 \\
& =16,636 \mathrm{psi}
\end{aligned}
$$

\begin{tabular}{|c|c|c|c|}
\hline $\begin{array}{l}\text { NAC } \\
\text { INIERNATIONAL }\end{array}$ & Performed by: gRo & Date: & $\begin{array}{l}\text { Calculation No 457.2003.2 } \\
\text { Revision } 1\end{array}$ \\
\hline & Checked by: $R S$ & Dite/25/96 & Page 21 of 39 \\
\hline
\end{tabular}

Plate material is A240 Type 304 . The safery margin is:

Safery Margin $=1-\frac{16,636}{30,000}=0.45$

5.6.3 Inner Shell To Top Flange, Detemination of Reaction Loads

Under a two (2) point lift, a radial load, a vertical load. and a bending moment 
HNF-SD-SNF-FRD-003 Rev. 0

Appendix A9.2.1 Immersion Pail Calculation 457-2003.2 (Continued)

will be developed at the flange to lug midpoint location:

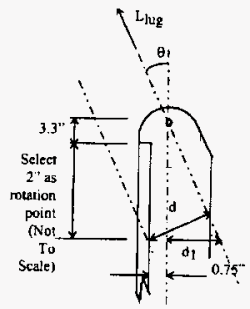

Per Section 5.4, the lift angle is $7.04^{\circ}$, and the lif load for a two (2) point lift is:

$\mathbf{L}_{\text {lug }}=90.680 \mathrm{lbs}$

Therefore

$F_{\mathrm{r}}=\mathrm{L}_{\mathrm{lug}} \times \sin \theta$

$=90,680 \times \sin \left(7.04^{\circ}\right)$

$=11,114 \mathrm{lbs}$

$F_{y}=L_{\text {lug }} \times \cos \theta$

$=90,680 \times \cos \left(7.04^{\circ}\right)$

$=89,996 \mathrm{lbs}$

$d_{1}=5.3 \times \tan \theta$

$=5.3 \times \tan \left(7.04^{\circ}\right)$

$=0.6545 \mathrm{in}$

$d_{2}=d_{1}+0.75$

$=0.6545+0.75$

$=1.4045 \mathrm{in}$

$\mathrm{d}=\mathrm{d}_{2} \mathrm{x} \cos \theta$

$=1.4045 \times \cos \left(7.04^{\circ}\right)$

$\therefore \quad 1.383$ in

\begin{tabular}{|l|l|l|}
\hline $\begin{array}{l}\text { Performed by: } \\
\text { DQO }\end{array}$ & $\begin{array}{l}\text { Date: } \\
1 / 25-96\end{array}$ & $\begin{array}{l}\text { Calculation No. 457.2003.2 } \\
\text { Revision I }\end{array}$ \\
\hline Checked by: & Date: $1 / 25 / 96$ & Page 22 of 39 \\
\hline
\end{tabular}


Appendix A9.2.1 Immersion Pail Calculation 457-2003.2 (Continued)

$$
\begin{aligned}
M & =L_{\text {lag }} \times d \\
& =90,680 \times 1.383 \\
& =125,410 \mathrm{in}-\mathrm{lbs}
\end{aligned}
$$

The section view for the inner shell flange reaction loads are:

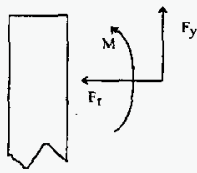

\subsubsection{Lug To Shell Double Bevel Weld Evaluation}

The shear capacity of the double bevel lug weld, $F_{\text {capacity }}$, is:

$$
\begin{aligned}
F_{\text {eapazity }} & =0.6 \times \mathrm{S}_{y} \times \text { area }_{\text {weid }} \\
& =0.6 \times 30,000 \times 22 \mathrm{in} \times 1.5 \mathrm{in} \\
& =594,000 \mathrm{lbs}
\end{aligned}
$$

Per Section 5.4, the maximum lift load for a two (2) point lif is:

$\mathbf{L}_{\text {lug }}=90.680 \mathrm{lbs}$

Safety Margin $=1 \cdot \frac{90.680}{594,000}=0.85$

\subsubsection{Maximum Longitudinal Shel] Bending Stress}

The maximum longitudinal bending stress will be determined by approximating an equivalent edge loading to be applied to the cylindrical outer shell.

The net compression reaction load at the shell flange end is:

\begin{tabular}{|l|l|l|l|}
\hline $\begin{array}{l}\text { NAC } \\
\text { INTERNationaI. }\end{array}$ & $\begin{array}{l}\text { Perfomed by } \\
\text { CRe }\end{array}$ & $\begin{array}{l}\text { Date: } \\
1 / 25-96\end{array}$ & $\begin{array}{l}\text { Calculation No 457-2003.2 } \\
\text { Revision 1 }\end{array}$ \\
\cline { 2 - 4 } & Checked by: & $1 / 25 / 96$ & Page 23 of 39 \\
\hline
\end{tabular}


Appendix A9.2.1 Immersion Pail Calculation 457-2003.2 (Continued)

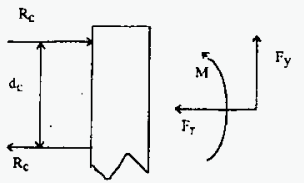

$R_{c}=\frac{M}{d_{c}}+\frac{F_{1}}{2}$

where: $d_{c}=$ moment am between flange rotation point and lower guide locations

$=24.5-2.5-[7.84-(3.29+2.5 / 2)] \cdot 2$

$=16.7 \mathrm{in}$

Therefore:

$$
\begin{aligned}
R_{c} & =\frac{125,410}{16.7}+\frac{11,114}{2} \\
& =13,067 \mathrm{lbs}
\end{aligned}
$$

The compressive load. $R_{c}$, is spread over an effective arc length of $10^{\circ}$ to determine an equivalent edge loading:

\begin{tabular}{|c|c|c|c|}
\hline $\begin{array}{l}\text { NAC } \\
\text { INIERNATIONAL }\end{array}$ & Perfomed by: geo & Date: $11-25-9$ & $\begin{array}{l}\text { Calculation No. } 457-2003.2 \\
\text { Revision I }\end{array}$ \\
\hline & Checked by: & Date: $11 / 25 / 56$ & Page 24 of 39 \\
\hline
\end{tabular}

$$
\begin{aligned}
\mathrm{R}_{\text {shell }} & =46.0 / 2 \\
& =23 \mathrm{in} \\
\mathrm{s} & =\text { arc length } \\
& =\mathrm{R}_{\text {shets } \times} \times \\
& =23 \times(10 \times \pi / 180) \\
& =4.014 \mathrm{in} \\
\mathrm{V}_{0}= & \text { edge load } \\
= & 13,067 / 4.014 \\
& =3,255 \mathrm{lbs}: \text { in }
\end{aligned}
$$


Appendix A9.2.1 Immersion Pail Calculation 457-2003.2 (Continued)

The maximum longitudinal bending stress, $s_{1}{ }^{\prime}$, per Reference 7.4.6 is:

$$
\begin{aligned}
& s_{1}^{\prime}=\frac{1.932 V_{0}}{i t^{2}} \\
& \text { where: } t=2.0 \text { in } \\
& \lambda=\sqrt{\frac{3\left(1-v^{2}\right)}{\mathrm{R}_{2}{ }^{2} \mathrm{t}^{2}}} \\
& =\sqrt[4]{\frac{3\left(1-(0.3)^{2}\right)}{(23.0)^{2}(2.0)^{2}}} \\
& =0.1895 \\
& \mathrm{~s}_{\mathrm{I}}{ }^{*}=\frac{(1.932) \times(3255)}{(0.1895) \times(2.0)^{2}} \\
& =\quad 8,296 \mathrm{psi}
\end{aligned}
$$

The maximum hoop membrane stress, $s_{2}$, per Reference 7.4.6 is:

$$
\begin{aligned}
& s_{2}=\frac{2 V_{0}}{t} i R \\
& =\frac{2(3255)}{2}(0.1895)(23.0) \\
& =\quad 14,186 \mathrm{psi}
\end{aligned}
$$

The maximum hoop bending stress, $s_{2}$, per Reference 7.4 .6 is:

$$
\begin{aligned}
s_{2}{ }^{\prime} & =\quad v \times s_{1}{ }^{\circ} \\
& =0.3 \times 8296 \\
& =2.488 \mathrm{psi}
\end{aligned}
$$

Conservatively consider the maximum stress intensity (i.e., the largest principle

\begin{tabular}{|l|l|l|}
\hline Performed by: & $\begin{array}{l}\text { Date: } \\
1 /-25 \%\end{array}$ & $\begin{array}{l}\text { Calculation No. 457.2003.2 } \\
\text { Revision 1 }\end{array}$ \\
\hline Checked by: & $\begin{array}{l}\text { Datec } \\
11 / 25 / 96\end{array}$ & Page 25 of 39 \\
\hline
\end{tabular}


Appendix A9.2.1 Immersion Pail Calculation 457-2003.2 (Continued)

stress difference) as the sum of $s_{1}{ }^{\prime}, s_{2}$ and $s_{2}{ }^{\prime}$ :

$s_{1}{ }^{\prime}+s_{2}+s_{2}{ }^{\prime}=8,296+14,186+2,488$

$=24,970 \mathrm{psi}$

Safery Margin $=1-\frac{24,970}{30.000}=0.17$

5.6.6 Maximum Circumferential Shell Bending Stress

The maximum circumferential bending stress will be determined through the use of a ring solution with diametrical radial loads applied (two point lift).

The compressive load, $R_{c}$, will be considered as a diametric load on the ring. The ring will be comprised of the flanged portion of the inner shell, eight $(8)$ inches in length and two (2) inches thick.

The ring bending moment, $\mathrm{M}$; shear load, $\mathrm{V}$; and tension load, $\mathrm{T}$, will be calculated for various locations of the ring. The flange is shaved on two (2) sides. Therefore, the critical stress locations are:

- At the maximum applied load location (i.e., the lug attachment point); and

- The minimum section location (i.e., shaved region begins $39^{\circ}$ from the lug and extends to $65^{\circ}$ from the lug)

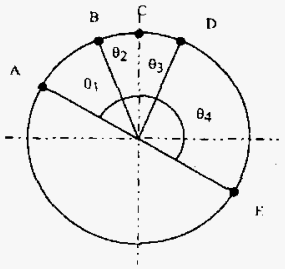

$\theta_{1}=39^{\circ}$

$\theta_{2}=13^{\circ}$

$\theta_{3}=13^{\circ}$

$\theta_{4}=180-39-13-13=115^{\circ}$

\begin{tabular}{|c|c|c|c|}
\hline A) NAC & Performed by: $Q R \theta$ & $\begin{array}{l}\text { Date: } \\
\text { //-25.96 }\end{array}$ & $\begin{array}{l}\text { Calculation No. 457.2003.? } \\
\text { Revision I }\end{array}$ \\
\hline & Checked by: ZS & Date $/ 25 / 96$ & Page 26 of 39 \\
\hline
\end{tabular}


Appendix A9.2.1 Immersion Pail Calculation 457-2003.2 (Continued)

\begin{tabular}{|c|c|c|}
\hline Lacation & Description & Angular Location " \\
\hline A & Lug Location & 0 \\
\hline B & Shave Section Start & 39 \\
\hline C & Minimum Section & 52 \\
\hline D & Shave Section End & $\mathbf{6 5}$ \\
\hline E & Lug Location & 180 \\
\hline
\end{tabular}

5.6.6.1 Maximum Circumferential Bending In Non-Shaved Areas

The properies of the flange section in the non-shaved areas are:
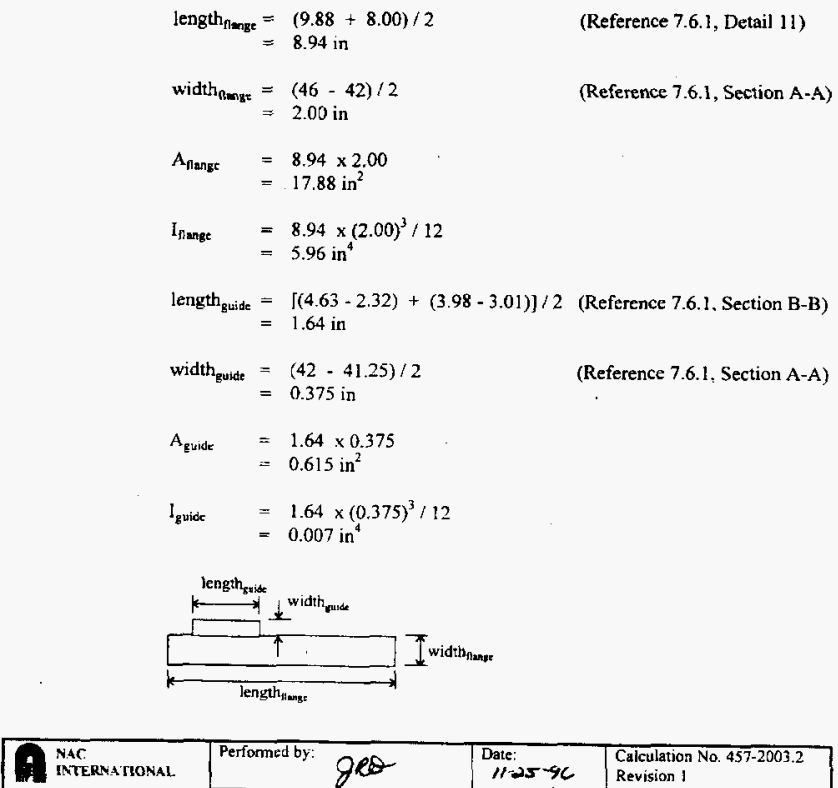

\begin{tabular}{|l|l|l|}
\hline Performed by: & Date: \\
$11-25-9 C$ & $\begin{array}{l}\text { Calculation No. 457-2003.2 } \\
\text { Revision 1 }\end{array}$ \\
\hline Checked by: & $\begin{array}{c}\text { Date } \\
11 / 25 / 96\end{array}$ & Page 27 of 39 \\
\hline
\end{tabular}


Appendix A9.2.1 Immersion Pail Calculation 457-2003.2 (Continued)

$$
\begin{aligned}
A_{\text {1ensile }} & =17.88+0.615 \\
& =18.495 \mathrm{in}^{2} \\
A_{\text {xhear }} & =2 / 3 \mathrm{~A}_{\text {tensile }} \\
& =12.33 \mathrm{in}^{2}
\end{aligned}
$$

The neutral axis is:

$$
\begin{aligned}
& n=\frac{\text { sum of all moments }}{\text { total area }} \\
& =\frac{(2 \times 8.94 \times 1)+(0.375 \times 1.64 \times(2+(0.375 / 2))}{(2 \times 8.94)-(0.375 \times 1.64)} \\
& =1.04 \text { in } \\
& \mathrm{y}_{\text {flange }}=1.04-1.00=0.04 \mathrm{in} \\
& \mathrm{y}_{\text {guide }}=(2.0+(0.375 / 2))-1.04=1.1475 \text { in } \\
& I_{n}=I_{\text {nange }}+\left(A_{\text {flange }} \times y_{\text {fange }}{ }^{2}\right)+I_{\text {guide }}+\left(A_{\text {guide }} \times y_{\text {guide }}{ }^{2}\right) \\
& =5.96+\left(17.88 \times(0.04)^{2}\right)+0.007+\left(0.615 \times(1.1475)^{2}\right) \\
& =6.806 \mathrm{in}^{4} \\
& \begin{aligned}
c_{\text {inner }} & =(2-0.375)-1.04 \\
& =1.335 \mathrm{in}
\end{aligned} \\
& =1.335 \text { in } \\
& c_{\text {outer }}=1.04 \mathrm{in} \\
& \left(\frac{c}{\mathrm{l}}\right)_{\operatorname{ma2}}=1.335 / 6.806 \\
& =0.196
\end{aligned}
$$

Per Reference 7.4.5:

$M=W R(0.3183-0.5 \sin \theta)$

\begin{tabular}{|l|l|l|l|}
\hline \multirow{2}{*}{$\begin{array}{l}\text { NAC } \\
\text { INTERNATIONial }\end{array}$} & \begin{tabular}{l} 
Peformed by: \\
\cline { 2 - 4 }
\end{tabular} & $\begin{array}{l}\text { Date: } \\
1 /-25-96\end{array}$ & $\begin{array}{l}\text { Calculation No 457-2003.2 } \\
\text { Revision 1 }\end{array}$ \\
\cline { 2 - 4 } & Checked by: & $\begin{array}{c}\text { Date } \\
11 / 25 / 76\end{array}$ & Page 28 of 39 \\
\hline
\end{tabular}


HNF-SD-SNF-FRD-003 Rev. 0

Appendix A9.2.1 Immersion Pail Calculation 457-2003.2 (Continued)

$T=0.5 \mathrm{~W}(\sin \theta)$

$V=0.5 W(\cos \theta)$

where: $\quad W=13,067 \mathrm{lbs}$

$\mathrm{R}=22 \mathrm{in}$

$\sigma_{\mathrm{t}}=$ tensile stress

$=\left(\mathrm{M} \times\left(\mathrm{c} / \mathrm{F}_{\max }\right)+\left(\mathrm{T} / \mathrm{A}_{\text {tensile }}\right)\right.$

$\sigma_{\mathrm{y}}=$ shear stress

$=V / A_{\text {shea }}$

Table 5.6.6-1

\begin{tabular}{|ccc|ccccc|}
\hline $\begin{array}{c}\text { Angle } \\
\text { (degrees) }\end{array}$ & $\begin{array}{c}\text { Angle } \\
\text { (radians) }\end{array}$ & $\begin{array}{c}\text { Moment } \\
\text { (in-lbs) }\end{array}$ & $\begin{array}{c}\text { Tension } \\
\text { (lbs) }\end{array}$ & $\begin{array}{c}\text { Shear } \\
\text { (lbs) }\end{array}$ & $\begin{array}{c}\text { Tensile } \\
\text { Stress } \\
\text { (psi) }\end{array}$ & $\begin{array}{c}\text { Shear } \\
\text { Stress } \\
\text { (psi) }\end{array}$ \\
\hline 0 & 0.000 & 91,503 & 0 & 6,534 & 17,935 & 530 \\
\hline 13 & 0.227 & $-59,168$ & $-1,470$ & 6,366 & 11,517 & 516 \\
\hline 26 & 0.454 & 28,491 & $-2,864$ & 5,872 & 5,429 & 476 \\
\hline 38 & 0.663 & 3,007 & $-4,023$ & 5,148 & 372 & 418 \\
\hline 66 & 1.152 & $-39,810$ & $-5,969$ & 2,657 & $-8,125$ & 216 \\
\hline 78 & 1.361 & $-49,094$ & $-6,391$ & 1,358 & $-9,968$ & 110 \\
\hline 90 & 1.571 & $-52,234$ & $-6,533$ & 0 & $-10,591$ & 0 \\
\hline 112.5 & 1.964 & $-41,289$ & $-6,036$ & $-2,501$ & $-8,419$ & -203 \\
\hline 135 & 2.356 & $-10,126$ & $-4,620$ & $-4,620$ & $-2,235$ & -375 \\
\hline 1575 & 2.749 & 36,510 & $-2,500$ & $-6,036$ & 7.021 & -490 \\
\hline 180 & 3.142 & $-91,518$ & 1 & $-6,533$ & 17,938 & -530 \\
\hline
\end{tabular}

5.6.6.2 Maximum Circumferential Bending In Shaved Areas

The properties of the flange section in the shaved areas are:

$$
\begin{aligned}
& \begin{aligned}
\text { length }_{\text {rlange }} & =(9.88+8.00) / 2 \quad \text { (Reference 7.6.1, Detail 11) } \\
& =8.94 \text { in }
\end{aligned} \\
& \begin{aligned}
\text { width }_{\text {llange }} & =(44.75 \cdot 42) / 2 \quad \text { (Reference } 7.6 .1, \text { Section A.A) } \\
& =1375 \mathrm{in}
\end{aligned} \\
& \mathrm{A}_{\text {tange }} \quad-8.94 \times 1.375 \\
& =12.29 \mathrm{in}^{2} \\
& I_{\text {flange }}=8.94 \times(1.375)^{3} / 12
\end{aligned}
$$

\begin{tabular}{|c|c|c|c|}
\hline $\begin{array}{l}\text { NAC } \\
\text { INTERYATIOVAL }\end{array}$ & Performed by: $g R \theta$ & $\begin{array}{l}\text { Dale: } \\
1 /-25-44\end{array}$ & $\begin{array}{l}\text { Calculation No. 457-2003.? } \\
\text { Revision! }\end{array}$ \\
\hline & Checked by: $2 S$ & Date: $11 / 25 / 96$ & Page 29 of 39 \\
\hline
\end{tabular}


Appendix A9.2.1 Immersion Pail Calculation 457-2003.2 (Continued)

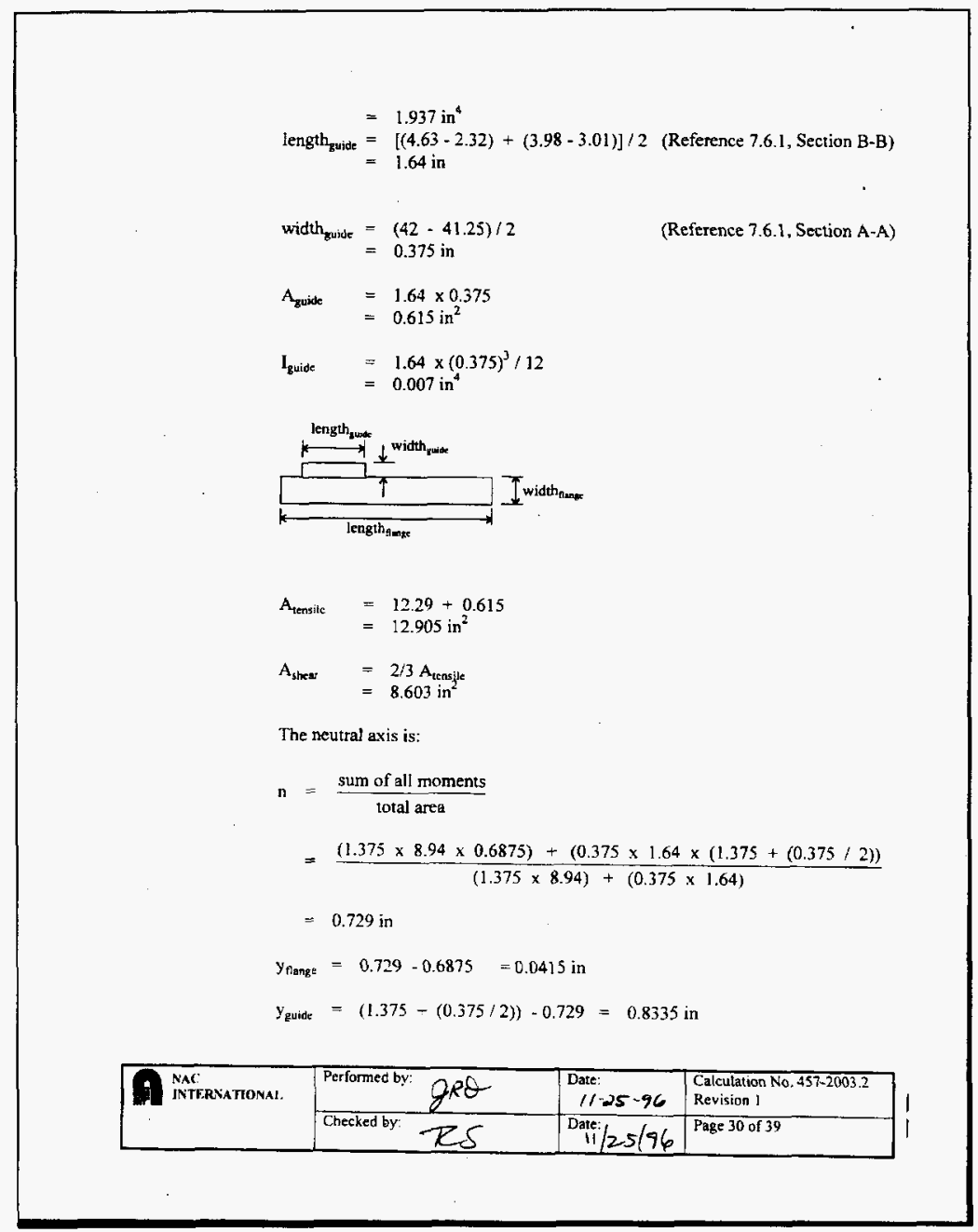


Appendix A9.2.1 Immersion Pail Calculation 457-2003.2 (Continued)

$$
\begin{aligned}
& I_{n}=I_{\text {flange }}+\left(A_{\text {flunge }} \times y_{\text {flange }}{ }^{2}\right)+I_{\text {guide }}+\left(A_{\text {puide }} \times y_{\text {guide }}{ }^{2}\right) \\
& =1.937+\left(12.29 \times(0.0415)^{2}\right)+0.007+\left(0.615 \times(0.8335)^{2}\right) \\
& =2.392 \mathrm{in}^{4} \\
& \begin{aligned}
\mathrm{c}_{\text {inner }} & =(1.375-0.375)-0.8335 \\
& =0.9165 \text { in }
\end{aligned} \\
& \mathrm{c}_{\text {outer }}=0.8335 \mathrm{in} \\
& \left(\frac{\mathrm{c}}{\mathrm{I}}\right)_{\mathrm{mux}}=0.9165 / 2.392 \\
& =0.383
\end{aligned}
$$

Per Reference 7.4.5:

$M=W R(0.3183-0.5 \sin \theta)$

$T=0.5 W(\sin \theta)$

$V=0.5 \mathrm{~W}(\cos \theta)$

where: $\quad w=13,067 \mathrm{lbs}$

$$
R=22 \text { in }
$$

$\sigma_{1}=$ tensile stress

$=\left(\mathrm{M} \times(\mathrm{c} / \mathrm{I})_{\max }\right)+\left(\mathrm{T} / \mathrm{A}_{\text {tersite }}\right)$

$\sigma_{v}=$ shear stress

$=\mathrm{V} / \mathrm{A}_{\text {shear }}$

Table 5.6.6-2

\begin{tabular}{|ccccccc|}
\hline $\begin{array}{c}\text { Angle } \\
\text { (degrees) }\end{array}$ & $\begin{array}{c}\text { Angle } \\
\text { (radians) }\end{array}$ & $\begin{array}{c}\text { Moment } \\
\text { (in-ibs) }\end{array}$ & $\begin{array}{c}\text { Tension } \\
\text { (bs) }\end{array}$ & $\begin{array}{c}\text { Shear } \\
\text { (lbs) }\end{array}$ & $\begin{array}{c}\text { Tensile } \\
\text { Stress } \\
\text { (psi) }\end{array}$ & $\begin{array}{c}\text { Shear } \\
\text { Stress } \\
\text { (psi) }\end{array}$ \\
\hline 39 & 0.68 ) & 1,044 & -4.112 & 5,077 & 81 & 412 \\
\hline 52 & 0.908 & $-21,766$ & -5.149 & 4,022 & $-4,545$ & 326 \\
\hline 65 & 1.135 & $-38,769$ & $-5,921$ & 2,761 & $-7,919$ & 224 \\
\hline
\end{tabular}

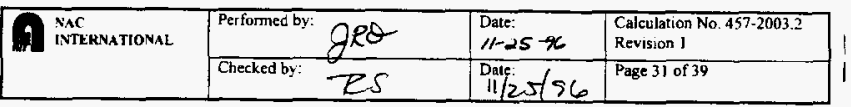


Appendix A9.2.1 Immersion Pail Calculation 457-2003.2 (Continued)

5.6.6.3 Maximum Circumferential Bending Stress, Maximum Shear Stress

The maximum circumferential bending stress from Tables 5.6.6-1 and $5.6 .6-2$ is at $180^{\circ}$, a lug location.

$\sigma_{\mathrm{t}}=17,938 \mathrm{psi}$

Safety Margin $=1-\frac{17,938}{30,000}=0.40$.

The rnaximum shear stress from Tables 5.6.6-1 and 5.6.6-2 is:

$\sigma_{v}=530 \mathrm{psi}$

The maximum shear stress is insignificant.

\subsubsection{Inner Shell Upper Flange Evaluation Under Stand Resting Conditions}

The inner shell upper flange evaluation shall be performed for both the NORMAI (4 point) and OTHER THAN NORMAL ( 2 point) conditions.

5.6.7.1 Normal Resting Condition (4 point)

The analysis of the upper flange under stand resting conditions using a normal four (4) point lift will be conducted utilizing the principle of superposition and ring equations as quoted in Reference 7.15 . The case to be analyzed involves uniform loads disposed symmetrically with respect to the trunnion axis.

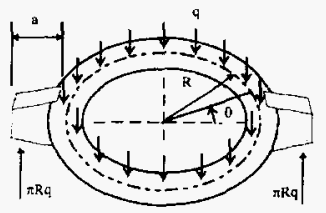

Considering Lift Condition IV, without slings and lift beam for design of pail, and a four (4) point lift factor and a load factor of three (3): $P=(57,877-400-550) \times 3 / 4=42,695$ lbs* $^{*}$.

- Use of $43.288 \mathrm{Jbs}$ from Revision 0 is conservative.

\begin{tabular}{|l|l|l|l|}
\hline \multirow{2}{*}{$\begin{array}{l}\text { Nac } \\
\text { INTERNational }\end{array}$} & \begin{tabular}{l} 
Perfomed by: $g R E$ \\
\cline { 2 - 4 }
\end{tabular} & $\begin{array}{l}\text { Date: } \\
12-2-96\end{array}$ & $\begin{array}{l}\text { Calculation No. 457-2003.2 } \\
\text { Revision 1 }\end{array}$ \\
\hline
\end{tabular}


Calculation OrRing Moment and Twist

Per Blake's Practical Stress Analysis ln Engineering Design (Reference 7.15)

Table 30.4, Page 394

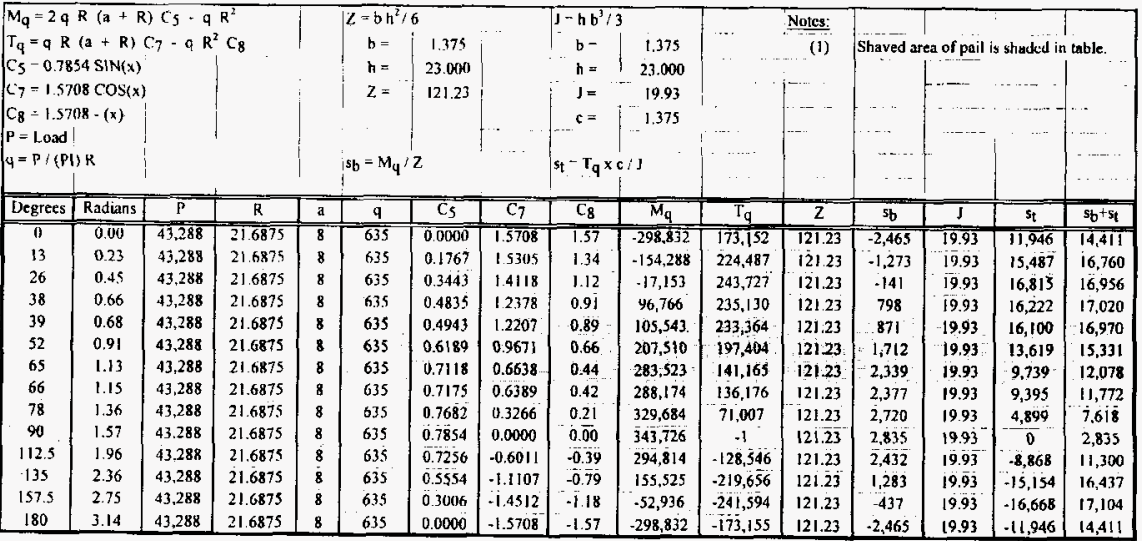

\begin{tabular}{|c|c|c|c|}
\hline \multirow[t]{2}{*}{ 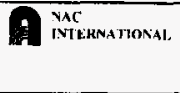 } & Performed by: & Date: $11-25-x$ & $\begin{array}{l}\text { Calculation No. } 457.2003 .2 \\
\text { Revision } 1\end{array}$ \\
\hline & Checked by: & Date: $11 / 25 / 96$ & Page 33 of 39 \\
\hline
\end{tabular}


Appendix A9.2.1 Immersion Pail Calculation 457-2003.2 (Continued)

The maximum stress intensity is:

$s_{b}+s_{t}=17,104$ psi

Safety Margin $=1-\frac{17,104}{30,000}=0.43$

5.6.7.2 Other Than Normal Resting Condition (2 point)

The analysis of the upper flange under stand resting conditions conditions using a two (2) point lif is contained in a separate calculation (Reference 7.16 ).

5.8 Lug Evaluation, Lifting Lug Region

\subsubsection{Bearing Check}

The pin bearing area is:

Bearing area $=1 \times \mathrm{D}_{\text {hole }}$

$=1.5 \times 1.69$

$=2.535 \mathrm{in}^{2}$

The bearing stress is:

Stress $=\mathrm{L}_{\text {lug }} /$ area

$=90,680 / 2.535$

$=35,771 \mathrm{psi}$

The safety margin is:

$$
\begin{aligned}
\text { Safety Margin } & =1-\left(\text { stress } /\left(1.5 \mathrm{~S}_{\mathbf{y}}\right)\right) \\
& =1-(35,771 ;(1.5 \times 50,000)) \\
& =0.52
\end{aligned}
$$

5.8.2 Net Tension

\begin{tabular}{|c|c|c|c|}
\hline NaC & Performed by: $g R Q$ & Daite: & $\begin{array}{l}\text { Calculation No.457-2003.2 } \\
\text { Revision } 1\end{array}$ \\
\hline & Checked by: & Date: $11 / 25 / 96$ & Page 34 of 39 \\
\hline
\end{tabular}

$$
\begin{aligned}
\text { Area } & =t\left(D_{\text {lug }}-D_{\text {mile }}\right) \\
& =1.5(5.0-1.69) \\
& =4.965 \mathrm{in}^{2}
\end{aligned}
$$

Since $S_{u} / 5$ is controlling: 
HNF-SD-SNF-FRD-003 Rev. 0

Appendix A9.2.1 Immersion Pail Calculation 457-2003.2 (Continued)

$\begin{aligned} \mathbf{L}_{\text {lug }} & =90680 \times(5 / 3) \\ & =151,133 \mathrm{lbs}\end{aligned}$

The stress is:

Stress $=\mathrm{L}_{\text {lug }} /($ Area $)$

$=151,133 /(4,965)$

$=30,440 \mathrm{psi}$

The safety margin is:

Safety Margin $=1-$ (stress $/ \mathrm{S}_{0}$ )

$=1-(30,440 / 80,000)$

$=0.62$

5.8.3 Shear Pull Out At 45 Degrees

$\begin{aligned} \text { Shear Area } & =t\left(R_{\text {ug }}-R_{\text {hoile }}\right) \\ & =1.5(25-0.845)\end{aligned}$

$=1.5(2.5-0.845)$

$=2.483 \mathrm{in}^{2}$

The shear stress is:

Stress $=\mathrm{L}_{\text {Hug }} / 2 \times$ (Shear Area)

$=151.133 / 2 \times(2.483)$

$=30,434 \mathrm{psi}$

The safety margin is:

Safety Margin $=1-\left(\right.$ stress $\left./\left(0.6 \mathrm{~S}_{\mathrm{u}}\right)\right)$

$=1 \cdot(30,434:(0.6 \times 80,000))$

$=0.37$

5.9 Lug Evaluation. Stand Resting Lug Region

5.9.1 Bearinu Check

The bearing area is:

The pin is $1.5^{\prime \prime} \times 1.25^{\prime \prime}$ with $1 / 8^{\prime \prime}$ chamfers. The bearing line is conservatively determined to be:

\begin{tabular}{|c|c|c|c|}
\hline $\begin{array}{l}\text { NAC: } \\
\text { INTERNATIONAL }\end{array}$ & Performed by: & $\begin{array}{l}\text { Date: } \\
1 / 25-26\end{array}$ & $\begin{array}{l}\text { Calculation No. 457-2003.2 } \\
\text { Revision I }\end{array}$ \\
\hline & Checked by: $R g$ & $11 / 25 / 96$ & Page 35 of 39 \\
\hline
\end{tabular}


Appendix A9.2.1 Immersion Pail Calculation 457-2003.2 (Continued)

$W=1.25-0.125-0.125$

$=1.0 \mathrm{in}$

Bearing area $=t \times W$

$=1.5 \times 1.0$

$=1.5 \mathrm{in}^{2}$

The bearing stress is:

Stress $=\mathrm{L}_{\text {lug }} /$ area

$=90,680 / 1.5$

$=60,453 \mathrm{psi}$

The safety margin is:

Safety Margin $=1-\left(\right.$ stress $\left./\left(1.5 \mathrm{~S}_{y}\right)\right)$

$=1-(60,453 /(1.5 \times 50,000))$

$=0.19$

5.9.2 Net Tension \& Shear

The tensile and shear stress areas are larger than that evaluated in Section 5.8 . Therefore, tension and shear stresses are aceptable by comparison to stresses in Section 5.8 .

\begin{tabular}{|c|c|c|c|}
\hline \multirow[t]{2}{*}{ INTERNATHONAL } & Performed by: $g R Q$ & $\begin{array}{l}\text { Date: } \\
1 /-25-96\end{array}$ & $\begin{array}{l}\text { Calculation No. 457-2003.2 } \\
\text { Revision I }\end{array}$ \\
\hline & Checked by: $R S$ & Dete: $11 / 25 / 96$ & Page 36 of 39 \\
\hline
\end{tabular}


Appendix A9.2.1 Immersion Pail Calculation 457-2003.2 (Continued)

\subsection{SUMMARY OF RESULTS/CONCLUSIOYS}

Summary of Stress Analysis

\begin{tabular}{|c|c|c|c|c|c|c|c|}
\hline $\begin{array}{l}\text { Drawing } \\
\text { No. }\end{array}$ & $\begin{array}{l}\text { Item } \\
\text { No. }\end{array}$ & Component & $\begin{array}{l}\text { Applied } \\
\text { Load }\end{array}$ & Design Check & $\begin{array}{l}\text { Calculated } \\
\text { Loading }\end{array}$ & Allowable & M.S. \\
\hline $457+101$ & 1 & $\begin{array}{l}\text { bottom } \\
\text { plate }\end{array}$ & $3 w$ & plate bending & $17.728 \mathrm{psi}$ & $30,000 \mathrm{psi}$ & 0.41 \\
\hline $457-101$ & 19,1 & \begin{tabular}{|l|} 
pail shell to \\
bottom \\
plate \\
interface \\
\end{tabular} & $\begin{array}{l}3 W \\
\text { pressure }\end{array}$ & $\begin{array}{l}\text { net tension } \\
\text { shell bending } \\
\& \text { shcar }\end{array}$ & $\begin{array}{l}3605 \mathrm{psi} \\
16,636 \mathrm{psi}\end{array}$ & $\begin{array}{l}30,000 \mathrm{psi} \\
30,000 \mathrm{psi}\end{array}$ & $\begin{array}{l}0.88 \\
0.45\end{array}$ \\
\hline $457-101$ & $\begin{array}{l}10 \text { to } \\
11\end{array}$ & $\begin{array}{l}\text { lug to shell } \\
\text { weld }\end{array}$ & $3 W$ & shear & $90.68 \mathrm{kips}$ & 594 kips & 0.85 \\
\hline $457-101$ & 10 & flange shell & $3 W$ & $\begin{array}{l}\text { lifting } \\
\text { longitudinal } \\
\text { bending }\end{array}$ & 24,970 psi & $30,000 \mathrm{psi}$ & 0.17 \\
\hline $4 \overline{457-107}$ & 10 & flange shell & $3 W$ & \begin{tabular}{|l|} 
lifting \\
circumferential \\
bending + \\
torsion \\
circumferential \\
shear \\
\end{tabular} & $\begin{array}{l}17,938 \mathrm{psi} \\
530 \mathrm{psi}\end{array}$ & $\begin{array}{l}30,000 \mathrm{psi} \\
18,000 \mathrm{psi}\end{array}$ & large \\
\hline $45 \overline{7-101}$ & 10 & flange shell & $3 w$ & $\begin{array}{l}\text { stand resting } \\
\text { bending + } \\
\text { torsion }\end{array}$ & $17,104 \mathrm{psi}$ & $30,000 \mathrm{psi}$ & 0.43 \\
\hline $457-101$ & I1 & lug plate & $3 w$ & $\begin{array}{l}\text { bearing: } \\
\text { lifting lug } \\
\text { location }\end{array}$ & 35,771 psi & $75,000 \mathrm{psi}$ & 0.52 \\
\hline $457-101$ & 11 & lug plate & $5 W$ & $\begin{array}{l}\text { nel tension: } \\
\text { lifting lug } \\
\text { location }\end{array}$ & $30,440 \mathrm{psi}$ & $75.000 \mathrm{psi}$ & 0.62 \\
\hline $457-101$ & 11 & lug plate & $\overline{5 W}$ & $\begin{array}{l}\text { shear pull out: } \\
\text { lifting lug } \\
\text { location }\end{array}$ & $30,434 \mathrm{psj}$ & $48,000 \mathrm{psi}$ & 0.37 \\
\hline $457-101$ & 11 & lug plate & $3 w$ & $\begin{array}{l}\text { bearing: guide } \\
\text { assembly } \\
\text { location }\end{array}$ & $60,453 \mathrm{psi}$ & $75,000 \mathrm{psi}$ & $0 . \overline{19}$ \\
\hline
\end{tabular}

The immersion pail design meets the criteria defined in Section 4.2 .

\begin{tabular}{|c|c|c|c|}
\hline NAC & Performed by: $g R \theta$ & $\begin{array}{l}\text { Date: } \\
11-25-40\end{array}$ & $\begin{array}{l}\text { Calculation No. 457.2003.2 } \\
\text { Revision I }\end{array}$ \\
\hline & Checked by: RS & Date:/25/96 & Page 37 of 39 \\
\hline
\end{tabular}


Appendix A9.2.1 Immersion Pail Calculation 457-2003.2 (Continued)

\section{REFERENCES}

7.1 Preliminary Design Analysis Report For The TN-WHC Cask and Transporation System Project 3035

Transnuclear, Inc.

7.1.1 Page A2-10.

7.1.2 Page A2-11.

7.1.3 Drawing 457-008.

7.1.4 Page A3-2

7.2 Fax from Glenn Guerra to Tom Danner dated 11-26-96, "Component Weights".

7.3 ANSI N14.6

American National Standard for Radioactive Materials

"special lifting devices for shipping containers weighing $10,000 \mathrm{lbs}(4.500 \mathrm{~kg})$ or more"

7.3.1 Section 4.2.1.1.

7.4 Roark's Formulas for Stress and Strain, 3rd edition.

7.4.1 Table X, Case 1, page 194.

7.4.2 Table XIII, Case 11, page 271.

7.4.3 Table X, Case 12, page 197.

7.4 .4 Article 62, page 230

7.4.5 Table VIII, Case 1, paye 156 .

7.4.6 Table XIII, Case 10, page 271 .

7.5 Hanford ECN 191402.

7.6 K Basin Immersion Pail Assembly TN WHC Transport Cask Drawings.

7.6. I Project 457, Drawing 101, sheet $1 / 1$.

7.6.2 Project 457, Drawing 102, sheets 1,$2 ; 2$.

7.6.3 Project 457, Drawing 103, sheets $1,2 / 2$.

7.6.4 Project 457, Drawing 112 , sheet $1,2 / 2$.

7.6.5 Project 457. Drawing 107 , sheet $1 / 1$.

7.7 ASME Boiler \& Pressure Vessel Code. 1995 edition.

7.7.1 Section II-D, page 440, Table U\& page 530, Table Y-1.

7.7 .2 Section TM-1, page 614

7.7.3 Section IJ.D, page 435. Table U \& page 500, Table $Y-1$.

7.7.4 Section III, NB-3227.1(a)

7.8 Mark's Standard Handbook for Mechanical Engineers. 7.8.1 Page 6-7.

\begin{tabular}{|c|c|c|}
\hline Performed by: $\partial \mathcal{R}$ & $\begin{array}{l}\text { Date: } \\
12-2-96\end{array}$ & $\begin{array}{l}\text { Calculation No. 457-2003.2 } \\
\text { Revision I }\end{array}$ \\
\hline Checked by: $2 S$ & Date: $12 / 2 / 96$ & Paze 38 of 39 \\
\hline
\end{tabular}


HNF-SD-SNF-FDR-003 Rev. 0

E-15166

Appendix A9.2.1 Immersion Pail Calculation 457-2003.2 (Continued)

7.9 Shigley's Mechanical Engineering Design, 1963.

7.9.1 Page 603

7.10 Specification WHC-S-0396, Revision 1, dated September, 1995.

Performance Specification for the SNF Path Forward Cask and Transportation System.

7.10.1 Page 36.

7.11 Meeting Minutes, NAC/TN. September 1996.

7.12 Design Specification 457-S-01, Revision 0.

7.13 Blodgett's Design Of Welded Structures, 1966.

7.14 AISC Manual Of Steel Construction, 9th edition.

7.15 Practical Stress Analysis In Engineering Design, Table 30.4, Page 394.

7.16 Calculation 457.2003.3, Revision 0.

\begin{tabular}{|c|c|c|c|}
\hline 9 IAC & Pefformed by: $Q R \theta$ & Date: $1 / 25: 96$ & $\begin{array}{l}\text { Calculation No. } 457-2003.2 \\
\text { Revision! }\end{array}$ \\
\hline & Checked by: RS & Date: $11 / 25 / 96$ & Page 39 of 39 \\
\hline
\end{tabular}


Appendix A9.2.2 Immersion Pail Calculation 457-2003.3

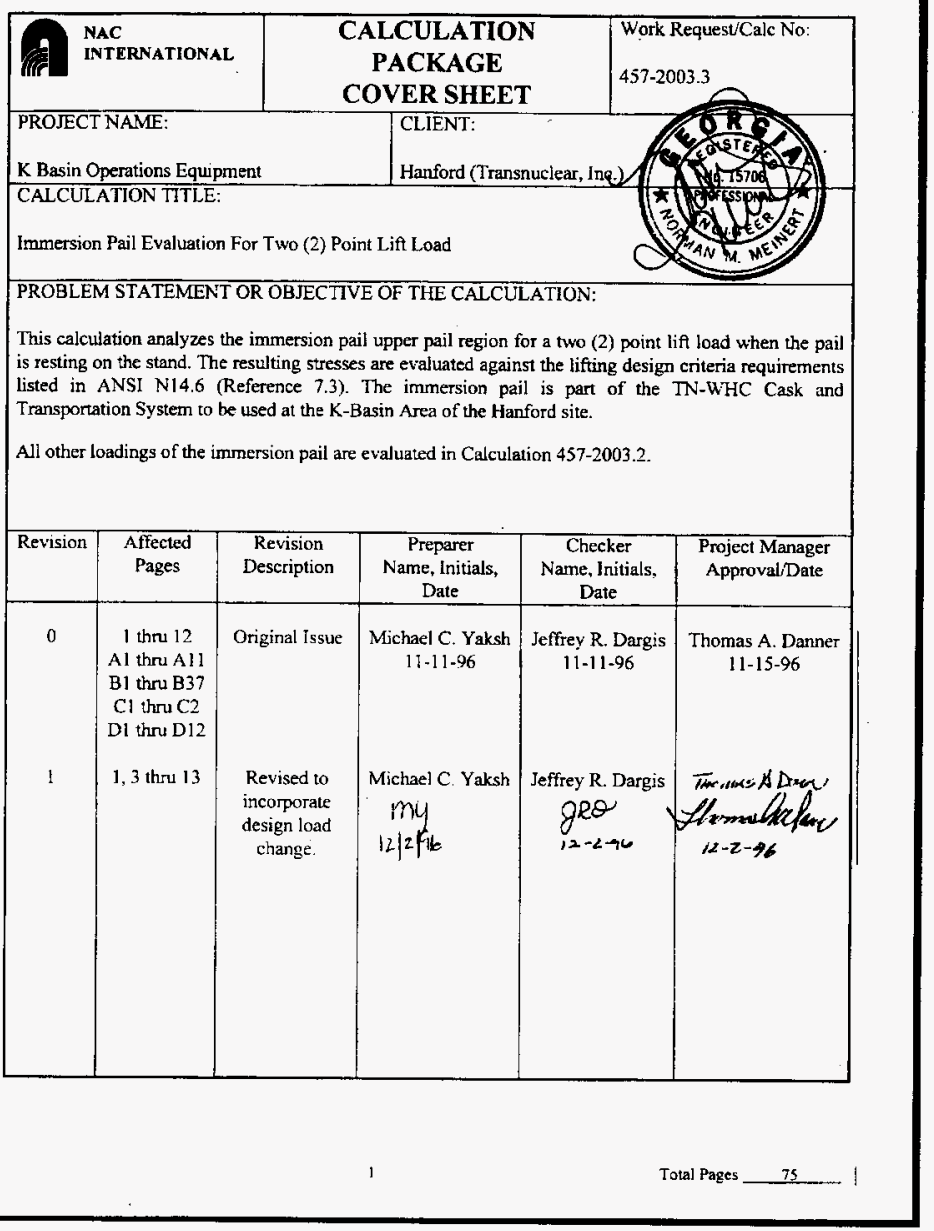


Appendix A9.2.2 Immersion Pail Calculation 457-2003.3 (Continued)

\section{INDEPENDENT DESIGN VERIFICATION CHECK SHEET}

Work Request/Calculation No: $457-2003.3$ Revision

Scope Of Analysis File: This calculation analyzes the immersion pail upper region for a two point lift load when the pail is resting on the stand. The resulting stresses are evaluated against the requirements of ANSI N14.6.

Review Methodology: Check Of Calculations

Alternate Analyses

Other (Explain)

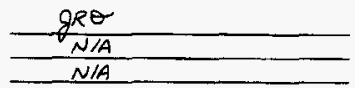

Confirm That The Work Request / Calculation Package Reviewed Includes:

1. Statement of Purpose

2. Defined Method of Analysis

3. Listing of Assumptions

4. Detailed Analysis Record

5. Statement of Conclusions / Recommendations (if applicable)

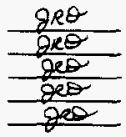

\begin{tabular}{|c|c|c|c|c|c|}
\hline \multirow[b]{2}{*}{ Step } & \multirow[b]{2}{*}{ Activitive } & \multicolumn{2}{|c|}{ Vortilleation } & \multirow[b]{2}{*}{$N / A$} & \multirow[b]{2}{*}{ Commente } \\
\hline & & $Y=$ & No & & \\
\hline 1 & $\begin{array}{l}\text { For the scope of the defined analysis: } \\
\text { A. Are the required data input complete? } \\
\text { 1. Material properties } \\
\text { 2. Geometry (drawng reference) } \\
\text { 3. Loading source term } \\
\text { If a supporting analysis is required to } \\
\text { define the load state, has it been } \\
\text { defined? } \\
\text { B. Are boundary conditicns acceptable? }\end{array}$ & & & & \\
\hline 2 & Is the method of analysis adequate for the defined scope? & & & & \\
\hline 3 & Is the worst case loading/configuration documented? & & & & \\
\hline 4 & Are the acceptance criteria defined and complete? & & & & \\
\hline 5 & Has all concutrent loading been considered? & & & & \\
\hline 6 & $\begin{array}{l}\text { Are analyses consistent with previous work for method and } \\
\text { approach? }\end{array}$ & & & & \\
\hline 7 & Are the records for input and output complete? & & & & \\
\hline 8 & Is traceability to verified sotware complete? & & & & \\
\hline$\theta$ & $\begin{array}{l}\text { Is the statement of conclusions and recommendations complete } \\
\text { and acceptable for the project and cbjectives of the defined } \\
\text { purpose? }\end{array}$ & & & & \\
\hline
\end{tabular}

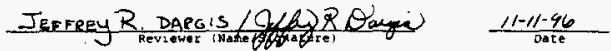

2 
Appendix A9.2.2 Immersion Pail Calculation 457-2003.3 (Continued)

\section{INDEPENDENT DESIGN VERIFICATION CHECK SHEET}

Work Request/Calculation No: 457-2003.3 Revision 1

Scope Of Analysis File; This calculation analyzes the immersion pail upper region for a two point lift load when the pail is resting on the stand. The resulting stresses are evaluated against the requirements of ANSIN14.6.

Review Methodology:

Check of Calculations

Alternate Analyses

Other (Explain)

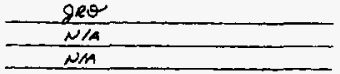

Confirm That The Work Request / Calculation Package Reviewed Includes

1. Statement of Purpose

2. Defined Method of Analysis

3. Listing of Assumptions

4. Detailed Anatysis Record

5. Statement of Conclusions / Recommendations (if applicable)

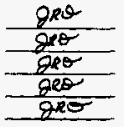

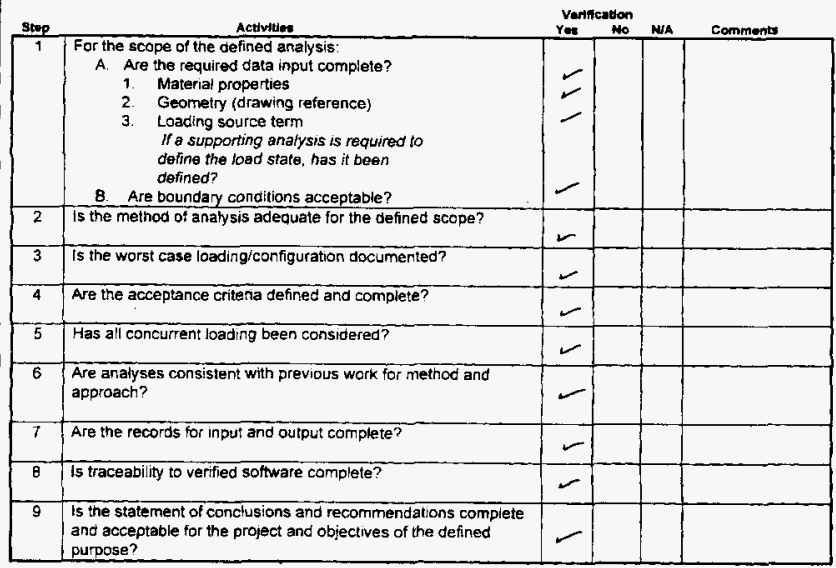

IEFfrey $R$. DARgIs (

$-\frac{12-2-96}{2 a t e}$ 
Appendix A9.2.2 Immersion Pail Calculation 457-2003.3 (Continued)

\section{TABLE OF CONTENTS}

\begin{tabular}{|c|c|}
\hline Section & Description \\
\hline- & 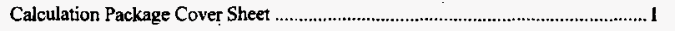 \\
\hline- & 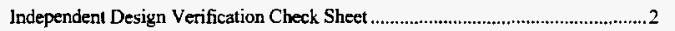 \\
\hline- & Table of Contents \\
\hline 1.0 & Synopsis of Results \\
\hline 2.0 & Introduction/Purpose \\
\hline 3.0 & 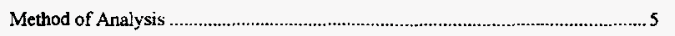 \\
\hline 4.0 & 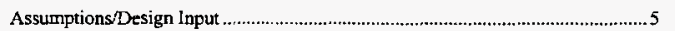 \\
\hline 5.0 & 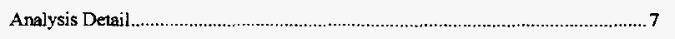 \\
\hline 6.0 & 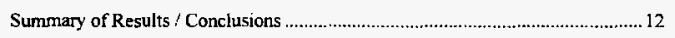 \\
\hline 70 & References.... \\
\hline
\end{tabular}

Appendix A List of Input Files for the Finite Element Model 11 total pages

Appendix B Output File for the Finite Element Model. . .37 total pages

Appendix C List of Files on 3.5" Diskene. .2 total pages

Appendix D ANSYS Software Verification .12 total pages

\begin{tabular}{|c|c|c|c|}
\hline A) NAC & Performed by: $\mathrm{mL}_{\mathrm{j}}$ & Date & $\begin{array}{l}\text { Calculation No. 457-2003.3 } \\
\text { Revisjon I }\end{array}$ \\
\hline & Checked by: gRe & $\begin{array}{l}\text { Date: } \\
12-2-4 c\end{array}$ & Page 4 of 13 \\
\hline
\end{tabular}


Appendix A9.2.2 Immersion Pail Calculation 457-2003.3 (Continued)

Summary of Stress Analysis

\begin{tabular}{|l|l|l|l|l|l|l|l|}
\hline $\begin{array}{l}\text { Drawing } \\
\text { No. }\end{array}$ & $\begin{array}{l}\text { Item } \\
\text { No. }\end{array}$ & Component & $\begin{array}{l}\text { Applied } \\
\text { Load }\end{array}$ & Design Check & $\begin{array}{l}\text { Calculated } \\
\text { Loading }\end{array}$ & Allowable & M.S. \\
\hline $457-101$ & 10 & flange shell & $3 \mathrm{~W}$ & $\begin{array}{l}\frac{\text { stand resting }}{\text { Von Mises, }} \\
\text { membrane plus } \\
\text { bending }\end{array}$ & $28,160 \mathrm{psi}$ & $30,000 \mathrm{psi}$ & 0.065 \\
\hline
\end{tabular}

The immersion pail design meets the criteria defined in Section 5.5 for a two (2) point lift load.

\subsection{INTRODUCTION / PURPOSE}

This calculation analyzes the immersion pail upper pail region for a two (2) point lif load when the pait is resting on the stand. The resulting stresses are evaluated against the lifting design criteria requirements listed in Reference 7.3. The immersion pail is part of the TN-WHC Cask and Transportation System to be used at the K-Basin Area of the Hanford site. The immersion pail design was initially evaluated in Reference 7.1. The design was revised as a result of discussions documented in Reference 7.5.

\subsection{METHOD OF ANALYSIS}

ANSYS, Revision 5.2, a structural analysis finite element program, is used to generate a $180^{\circ}$ structural model of the upper immersion pail region. input files and macros used to construct the ANSYS models and perform a linear structural analysis are contained in Appendix A. All work under this calculation designation was performed on the DEC ALPHA XL 266 computer (NAC Serial No. 02207).

\subsection{ASSUMPTIONS / DESIGN INPUTS}

\section{I Assumptions}

There are no unverified assumplions within this calculation.

\begin{tabular}{|c|c|c|c|}
\hline NAC & $\begin{array}{l}\text { Performed by: } \\
\qquad \operatorname{lng} ;\end{array}$ & Date: $12 / 2 / 4$ & $\begin{array}{l}\text { Calculation No. 457-2003.3 } \\
\text { Revision I }\end{array}$ \\
\hline & Checked by: $g e \theta$ & $\begin{array}{l}\text { Date: } \\
12 \cdot 2-96\end{array}$ & Page $5 \overline{\text { of }} 13$ \\
\hline
\end{tabular}


Appendix A9.2.2 Immersion Pail Calculation 457-2003.3 (Continued)

\subsection{Design lnputs}

\subsubsection{Design Load (Reference 7.8, Section 5.3.4)}

Load $_{\text {Design }}=57,717^{*}$ lbs for Condition IV (Full Cask At Stand Height).

- Revised load from Reference 7,9, Section 5.3.4 mimus the weight of the slings and lift beam is $57,877-400-550=56,927$ lbs. Use of Revision 0 load is conservative.

4.2.2 Iwo Point Lift Lond

$$
\begin{aligned}
\text { Load }_{\mathrm{Pai}} & =\text { Total pail load } \\
& =\text { Load }_{\text {Design }} \times \text { Load Factor } \\
& =57,717 \times 3 \\
& =173,151 \mathrm{lbs} \\
\operatorname{Load}_{2 \mathrm{PT}} & =\mathbf{L o a d}_{\mathrm{Pail}} / 2 \text { Point Lift } \\
& =173,151 / 2 \\
& =86,575 \mathrm{lbs} \\
& =86,575 \mathrm{lbs}
\end{aligned}
$$

\subsubsection{Applied Load}

The load used in the analysis, Load $_{\text {Applied, }}$ is:

$\operatorname{Load}_{\text {Applied }}=86,871 \mathrm{lbs}$

The load applied in the ANSYS analysis, $86,871 \mathrm{lbs}$, is greater than the load calculated for a two point lifi, 86,575 lbs (conservative).

\subsubsection{Upper Pail Ring Configuration}

The model was developed based on data contained on the drawing numbered "Project 457, Drawing 101, sheet 1/1" (Reference 7.6.1).

\begin{tabular}{|l|l|l|}
\hline $\begin{array}{l}\text { Perfomed by: } \\
n \cap y\end{array}$ & $\begin{array}{l}\text { Date: } \\
12 / 2 / 96\end{array}$ & $\begin{array}{l}\text { Calculation No. 457-2003.3 } \\
\text { Revision 1 }\end{array}$ \\
\hline Checked by: $g R \sigma$ & $\begin{array}{c}\text { Date: } \\
12-2-96\end{array}$ & Page 6 of 13 \\
\hline
\end{tabular}




\title{
4.2.5 Material Yield Strength
}

\author{
A240 Type 304 Stainless Steel \\ (Reference 7.7.1) \\ Fy: $\quad 30 \mathrm{ksi}$ \\ Fu: $\quad 75 \mathrm{ksi}$
}

\subsection{ANALYSIS DETAIL}

\subsection{Modeling Considerations}

This model has been developed for the sole purpose of performing a cross sectional stress analysis. The model was not intended for evaluation of stress concentrations arising from sharp discontunities.

\subsection{Material Properties}

Modulus of Elasticity, $E=27 \times 10^{8} \mathrm{psi}$

Poisson's ratio, $v \quad \approx 0.3$

\subsection{Model Desctiption}

The orgin of the model is located at the center line of the pail at the bottom surface of the model (see Figure 5-1). The centerine of the pail corresponds to the global $\mathrm{Z}$ axis. Using half symmetry, a 180 degree section of the cask is modeled.

Dimensions in the model correspond to nominal dimensions provided in Reference 7.6.1.

The pail ring model uses element SOLID45. The model was extended twenty-six (26") inches (26") from the top of the pail. This corresponds to $3.3 \sqrt{\mathrm{rt}}$, which is sufficient to represent the distribution of the load from the lugs into the pail.

The lug attachment was modeled with a spider of beam elements using element BEAM4. a three (3) dimensional heam (See Figure 5-1). The center point of the spider corresponds to the location where the pail is pinned to the support structure. This point also represents the load reaction point. The beam elements are rigid, with input values of 1000 for the cross sectional area and moments of inertia. The applied joad of $86,87 \mathrm{I} \mathrm{lbs}(1 / 2$ the total load since only $1 / 2$ of the pail was modeled) was distributed along the lower edige of the model by point mass elements (i.e.. Element Type 21, using 3D, without rotational inertia).

\begin{tabular}{|c|c|c|c|}
\hline ANTERNATIONAL & $\begin{array}{l}\text { Performed by: } \\
\qquad / 77 y\end{array}$ & $12 / 2 / 96$ & $\begin{array}{l}\text { Calculation No. } 457-2003.3 \\
\text { Revision I }\end{array}$ \\
\hline & Checked by: geo & $\begin{array}{l}\text { Date: } \\
12-2-46\end{array}$ & Page 7 of 13 \\
\hline
\end{tabular}


Appendix A9.2.2 Immersion Pail Calculation 457-2003.3 (Continued)

The method used to generate the model was to initiate a cross section in the $X Z$ plane with area elements and then rotate them about the $Z$ axis for 180 degrees. The pail outer diameter has a shaved section between $39^{\circ}$ and $65^{\circ}$. The shaved section was modeled by detaching the solid model and moving the nodes to form a flat surface (See Figure 5-1).

The input file to generate the model is contained in Appendix A. The output file corresponding to the input file is contained in Appendix B.

\subsection{Analysis Description}

Symmetrical displacement boundary conditions were applied at the plane of symmetry. $(U Y=0)$. The center point of one spider of beam elements was modeled with all displacement degrees of freedom restrained. The opposite centerpoint restrained the UY and UZ degrees of freedom only, to avoid introduction of artifical stiffness into the pail.

Only a inertia load of $1 \mathrm{G}$ was applied, since the real constants for the mass element was in pounds (not slugs).

The input for the linear static analysis is contained in the file K_PAIL.MAC, which is listed in Appendix A

To generate the model, perform the analysis, and to obtain the plots and the section data, enter ANSYS, then enter

k_pail

The output file is denoted kbasl.out and the plots are contained in the file kbasin.plt.

\subsection{Stress Criteria}

Per ANSI 14.6. the stress is not to exceed the yield stress with a safety factor of three (3). For this application, stress linearization is used to determine the stress components acting on a section. The stress components are then combined to determine the Von Mises Stress, which corresponds to a distortion cnergy stress criteria. ANSYS, upon selecting the two nodes on the surface, detemines the Von Mises stress due to membrane plus bending components.

Local stress concentrations will occur next to the beam elements. These stress concentrations are purely a result of techniques used in modeling. Local stress concentrations will also arise at the radii of the juncture of the rib and the pail.

\begin{tabular}{|c|c|c|c|c|}
\hline A VAC & Perfomed b & $m y$ & Date: $12 / 2 / 4$ & $\begin{array}{l}\text { Calculation No. 457-2003.3 } \\
\text { Revision } 1\end{array}$ \\
\hline & Checked by: & $g^{\circ}$ & $\begin{array}{l}\text { Date: } \\
12-2+16\end{array}$ & Page 8 of 13 \\
\hline
\end{tabular}


Appendix A9.2.2 Immersion Pail Calculation 457-2003.3 (Continued)

\subsection{Stress Results}

The section stresses resulting from the analysis are contained in the output file of Appendix B. A series of sectional stresses were extracted. The output file contains the stress results with the polar coordinates of the nodes forming the section. The polar system designation is:

$X$ - radial;

$Y=$ circumferenial angle (measured $+Z$ rotation); and

$Z=$ axial location (measured from the bottom of the model).

The maximium membrane plus bending Von Mises stress (SEQV) was determined to be $28,160 \mathrm{psi}$, which corresponds to a positive margin of +.065 when compared to a yield strength of 30,000 psi for A240 stainless steel.

A plot of the Von Mises stress is shown in Figure 5-2. Each circumferential divison corresponds to a $S$ degree inctement.

\begin{tabular}{|l|l|l|l|}
\hline \multirow{2}{*}{$\begin{array}{l}\text { NAC } \\
\text { INTERMATIONAL }\end{array}$} & $\begin{array}{l}\text { Performed by: } \\
\text { My }\end{array}$ & $\begin{array}{l}\text { Date: } \\
12 / 2 / \%\end{array}$ & $\begin{array}{l}\text { Calculation No. 457-2003.3 } \\
\text { Revision 1 }\end{array}$ \\
\cline { 2 - 5 } & Checked by: & $\begin{array}{l}\text { Date: } \\
12-2-96\end{array}$ & Page 9 of 13 \\
\hline
\end{tabular}


Appendix A9.2.2 Immersion Pail Calculation 457-2003.3 (Continued)

サyese $5 \cdot 1$

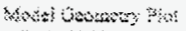

Faik- -30 ?

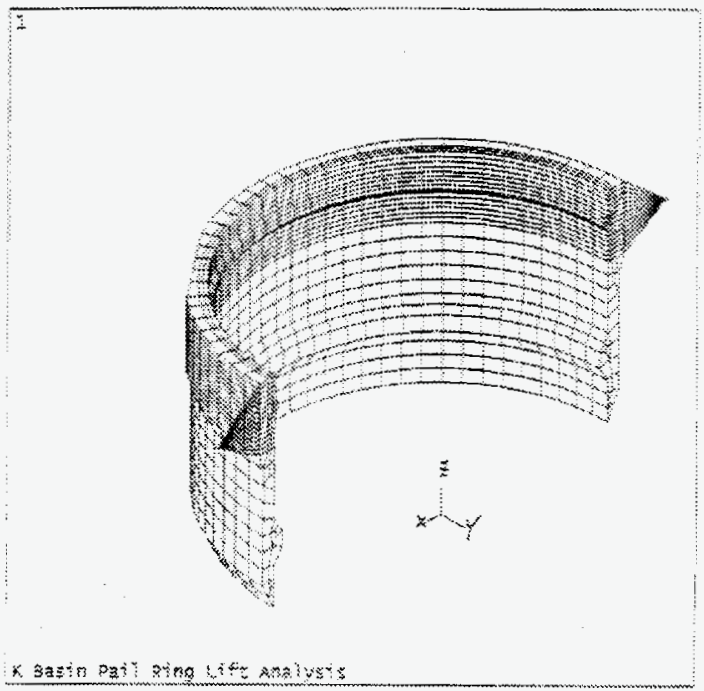

सxखs 5

Wer?

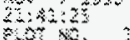

Dis

ters

$\mathrm{xy} \times \hat{b}$

स: $\cdots$

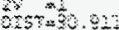

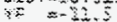

$20 \quad x$

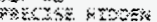

\begin{tabular}{|c|c|c|c|}
\hline \multirow[t]{2}{*}{ 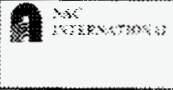 } & When sy & कि & 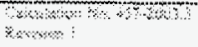 \\
\hline & "मसे : & $8 x$ & को \\
\hline
\end{tabular}




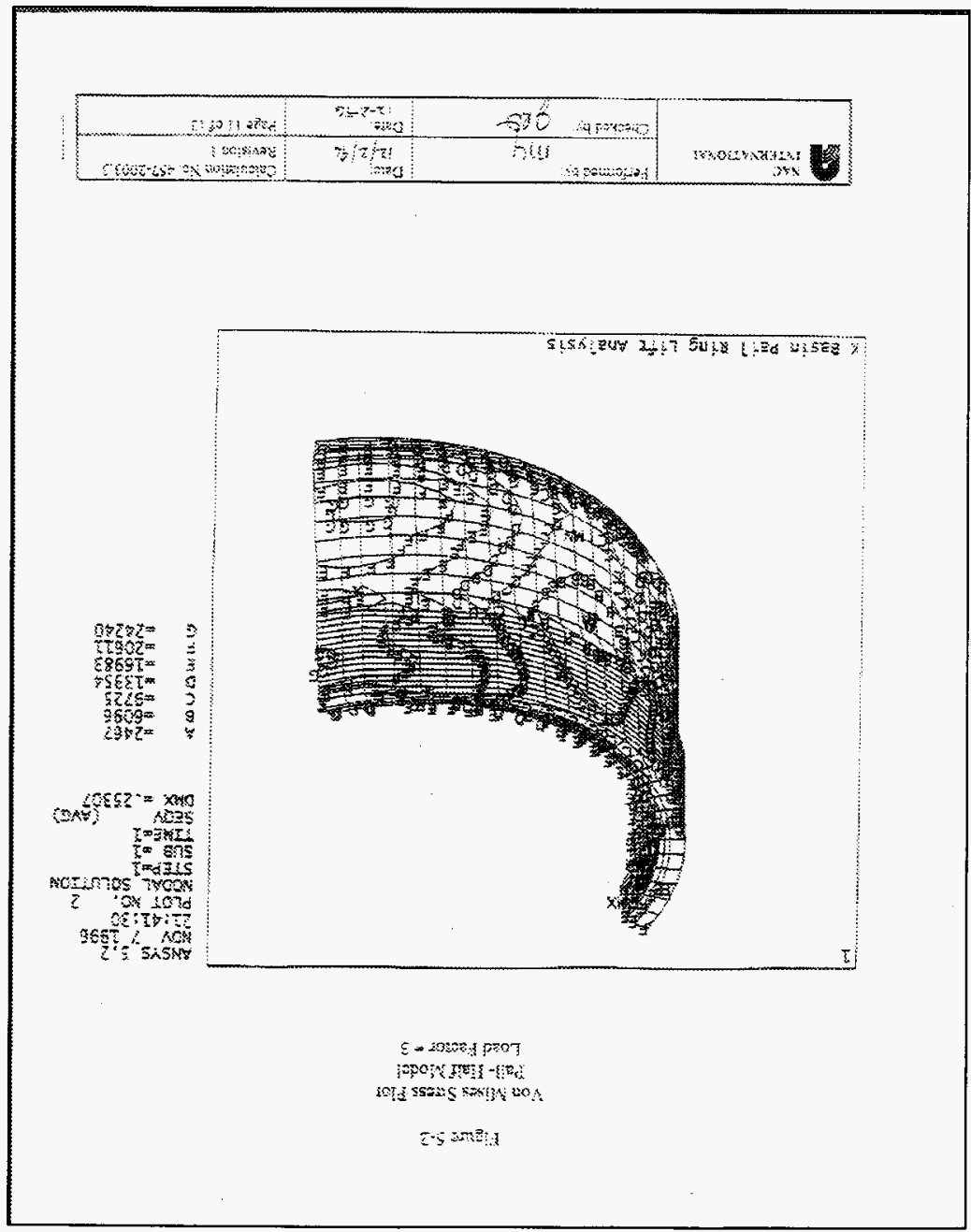

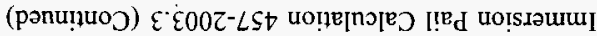

$\tau \cdot \tau \cdot 6 \forall$ x!puədd $\forall$ 
Appendix A9.2.2 Immersion Pail Calculation 457-2003.3 (Continued)

6.0 SUMMARY OF RESULTS/CONCLUSIOYS

Summary of Stress Analysis

\begin{tabular}{|l|l|l|l|l|l|l|l|}
\hline $\begin{array}{l}\text { Drawing } \\
\text { No. }\end{array}$ & $\begin{array}{l}\text { Item } \\
\text { No. }\end{array}$ & Component & $\begin{array}{l}\text { Applied } \\
\text { Load }\end{array}$ & Design Check & $\begin{array}{l}\text { Calculated } \\
\text { Loading }\end{array}$ & Allowable & M.S. \\
\hline $457-101$ & 10 & flange shell & $3 \mathrm{~W}$ & $\begin{array}{l}\frac{\text { stand resting }}{\text { Von Mises, }} \\
\text { membrane plus } \\
\text { bending }\end{array}$ & $28.160 \mathrm{psi}$ & $30,000 \mathrm{psi}$ & 0.065 \\
\hline
\end{tabular}

The immersion pail design meets the criteria defined in Section 5.5 for a two (2) point lift load.

\begin{tabular}{|c|c|c|c|c|}
\hline \multirow[t]{2}{*}{ DAC NAC } & Performed by & $\operatorname{lng}$ & Date: $12 / 2 / 96$ & $\begin{array}{l}\text { Calculation No. 457-2003.3 } \\
\text { Revision I }\end{array}$ \\
\hline & Checked by & gRO & $\begin{array}{l}\text { Date: } \\
12-2-96\end{array}$ & Page 12 of 13 \\
\hline
\end{tabular}


Appendix A9.2.2 Immersion Pail Calculation 457-2003.3 (Continued)

7.0

\section{REFERENCES}

7.1 Preliminary Design Analysis Report For The TN-WHC Cask and Transporation System Project 3035

Transnuclear, Inc.

7.3.1 Page A2-10.

7.1 .2 Page A2-11.

7.1.3 Drawing 457-008.

7.1.4 Page A3-2

7.2 Not Used.

7.3 ANSI NI 4.6

American National Standard for Radioactive Materials

"special lifting devices for shipping containers weighing $10,000 \mathrm{lbs}(4,500 \mathrm{~kg})$ or more"

7.3.1 Section 4.2.1.1.

7.4 ANSYS. Revision 5.2 User Manual

7.5 Hanford ECN 191402

7.6 K Basin lmmersion Pail Assembly TN WHC Transport Cask Drawings. 7.6.1 Project 457, Drawing 101, sheet $1 / 1$.

7.7 ASME Boiler \& Pressure Vessel Code, 1995 edition.

7.7.1 Section 11-D, page 440, Table U \& page 530, Table Y-1.

7.8 Calculation 457-2003.2, Revision 0 .

7.9 Calculation 457-2003.2, Revision 1.

\begin{tabular}{|c|c|c|c|}
\hline NAC: & Performed by: 1 hy & Dete: $/ 2 / 2 / 96$ & $\begin{array}{l}\text { Calculation No. 457-2003.3 } \\
\text { Revision ! }\end{array}$ \\
\hline & Checked by: $g R Q$ & Date: & Page 13 of 13 \\
\hline
\end{tabular}


Appendix A9.2.2 Immersion Pail Calculation 457-2003.3 (Continued)

\section{APPENDIX A}

List Of Input Files For The Finite Element Model

There a a total of 11 pages in Appendix A.

\begin{tabular}{|c|c|}
\hline \multicolumn{2}{|c|}{ Input Files } \\
\hline File Name & Description \\
\hline k_pail.mac & Main Input File \\
\hline mas21.mac & Applies The Mass Elements \\
\hline r_beam.mac & $\begin{array}{c}\text { Generates The Spider Of Beams To } \\
\text { Represem The Attaclument Lug }\end{array}$ \\
\hline
\end{tabular}

\begin{tabular}{|c|c|c|c|c|}
\hline \multirow[t]{2}{*}{$\begin{array}{l}\text { NAC } \\
\text { INTERVATLONAL }\end{array}$} & Performed by & $m_{y}$ & Date: $11-1 \mid-46$ & $\begin{array}{l}\text { Catculation No, 457-2003.3 } \\
\text { Revision } 0\end{array}$ \\
\hline & Checked by: & $8 \times 0$ & Date: $11-11-76$ & Page $A 1$ of 11 \\
\hline
\end{tabular}


Appendix A9.2.2 Immersion Pail Calculation 457-2003.3 (Continued)

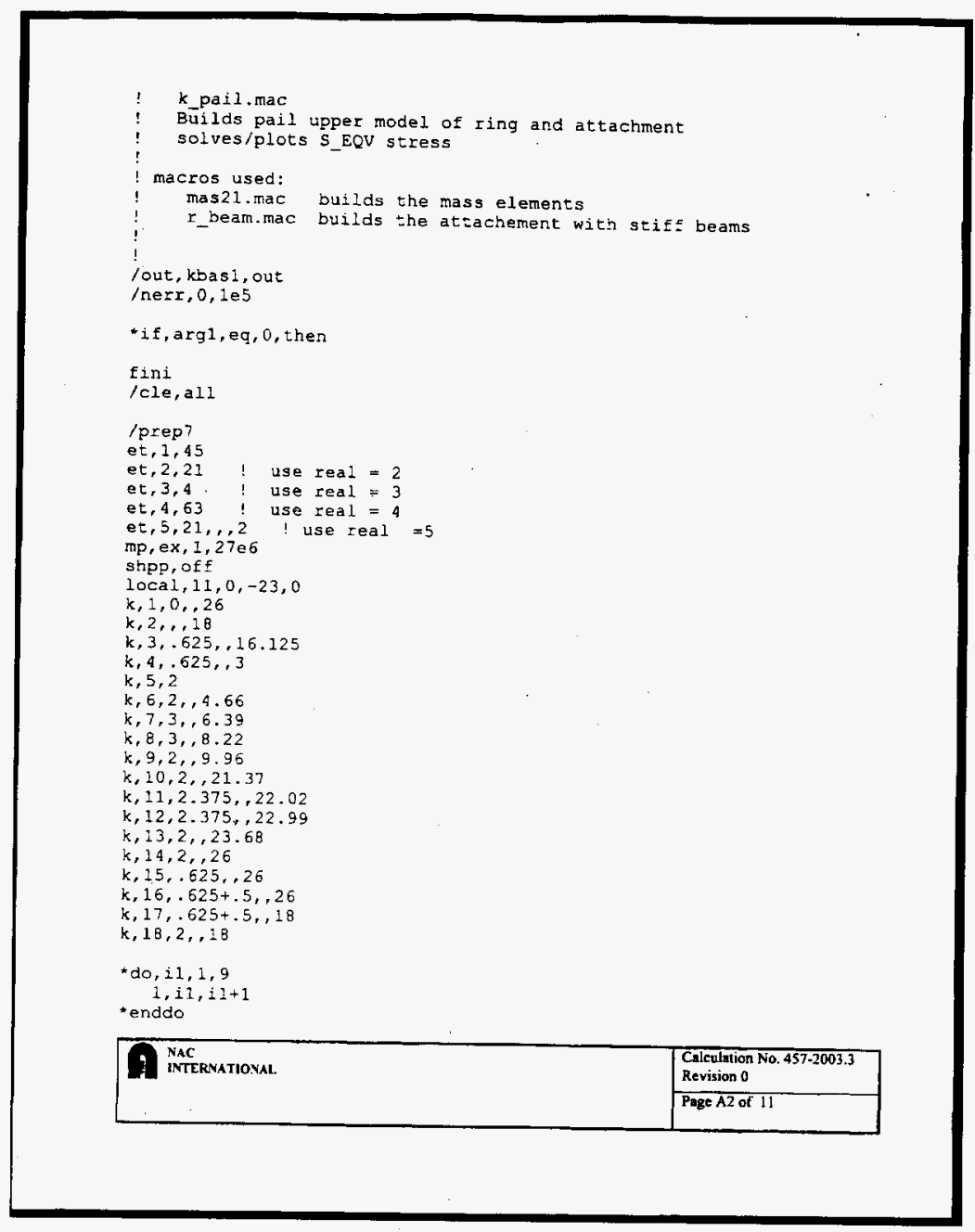


Appendix A9.2.2 Immersion Pail Calculation 457-2003.3 (Continued)

$1,14,16$

$2,16,17$

$1,17,3$

$1,1,16$

$1,18,10$

$1,10,11$

$1,11,12$

$1,13,14$

$1,9,18$

$1,17,18$

$1,12,13$

$1,2,17$

1 sel, $1,1,11,10$

lesi, all,.6

$\mathrm{cm}, c_{-13,1 i n e}$

1 sel, $,, 13,21, \mathrm{~B}$

lsel, $a, \ldots, 2,12,10$

lesi,all, , 1

cr, c_ll, I ine

1sel, , , 14, 17

lsel,a, , 19,20

lsel, $a, \ldots, 10$

$\mathrm{cm}, \mathrm{c}$ 12, Iine

lesi, ali,. 6

alis

cmsel, $u, c 11$

cmsel, $4, c_{-12}^{-12}$

lesi, all, $\overline{2}$

alls

Idel. 9

1 upper left

ksel, all

a, 1,16,17, 2

! upper right

ksel, , , 10,14

ksel, $a,, 16,18$

$1 \mathrm{slk}, 1$

al, all

! triangle transition

\section{N NAC}

INTERNATIONAI 
Appendix A9.2.2 Immersion Pail Calculation 457-2003.3 (Continued)

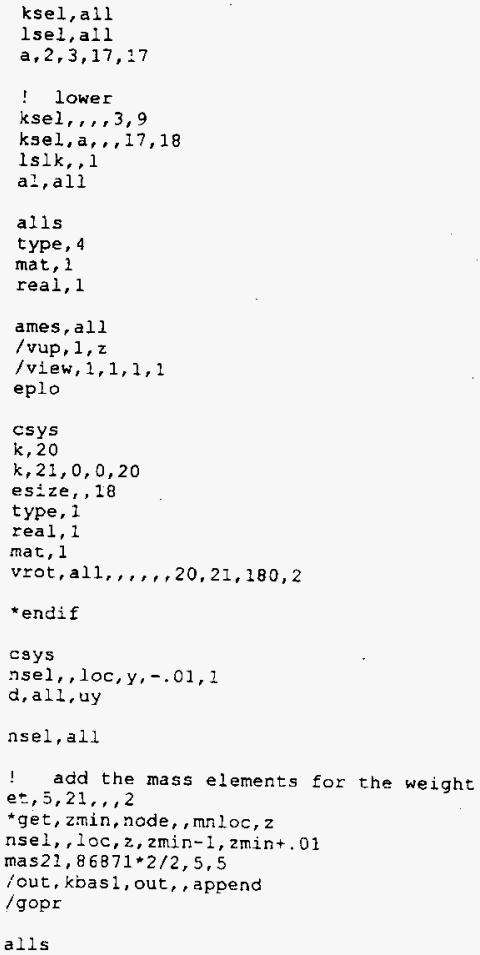


Appendix A9.2.2 Immersion Pail Calculation 457-2003.3 (Continued)

! add the beam elements for the attachments

* get, ndmx, node, , num, max

gndmx=ndmx+1

csys, 11

$n, \operatorname{gndm} x,-5.24, .21 .46$

d. gndmx, ux

d, gndmx, uy

d. gndmx, uz

$r, 3,1000,1000,1000,1000,1000$

esys

esel,al1

nsle

*get, r_o, node, , mnloc, $x$

nsel, , loc, $y,-.01,1$

nsel $, r, 100, x, r_{0} 0-.1, r_{-} 0+.01$

I beam, gndmx, 1, 3, 3

tout, kbas1, out, , append

igopr

csys

"get, $s$ x, node, gndm $x, 200, x$

* get, s_z, node, gnamx, loc, $z$

$n, \operatorname{gndm} \bar{x}+1,-5, x_{1}, s, 2$

d, gndmx +1, uy

d. gndmx +1 , u z

nsel, , loc, y, $-.01,1$

nsel,, , loc, $x,-r 0-.1,-r$ ot. 01

I_beam, gndmx $+1, \overline{1}, 3,3$

alis

modm, detach

! move the nodes for the flat

csys, 1

esel, , ename, 45

nsle

nsel, $r, 10 c, y,-41,-39$

nmod, al1, -39

nsle

* get, $r$ o, node, , mxloc, $x$

nsel, $r, 100, x, r$ o- $1, r \_0+$ I

nsel, $r, 100, y,-66,-38$

local, $12,0,0,0,0,-52$

nmod, a $11,44,75 / 2$

esel, , type, 4

edeI, all

INTERVATIONAL. 
Appendix A9.2.2 Immersion Pail Calculation 457-2003.3 (Continued)

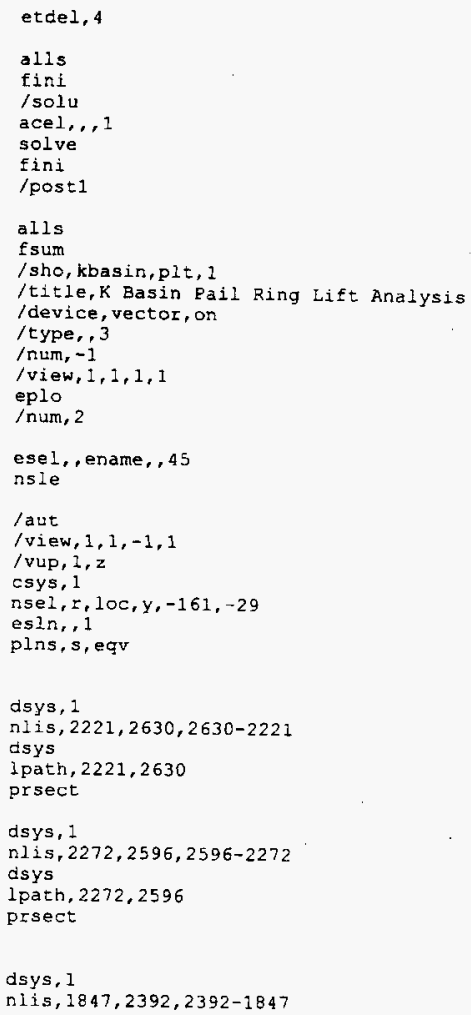


Appendix A9.2.2 Immersion Pail Calculation 457-2003.3 (Continued)

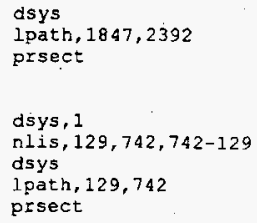


Appendix A9.2.2 Immersion Pail Calculation 457-2003.3 (Continued)

dsys, 1

nlis, $2184,2474,2474-2184$

dsys

lpath, 2184,2474

prsect

isho, $x \times x$

/sho, term

type, , 3

lout 
Appendix A9.2.2 Immersion Pail Calculation 457-2003.3 (Continued)

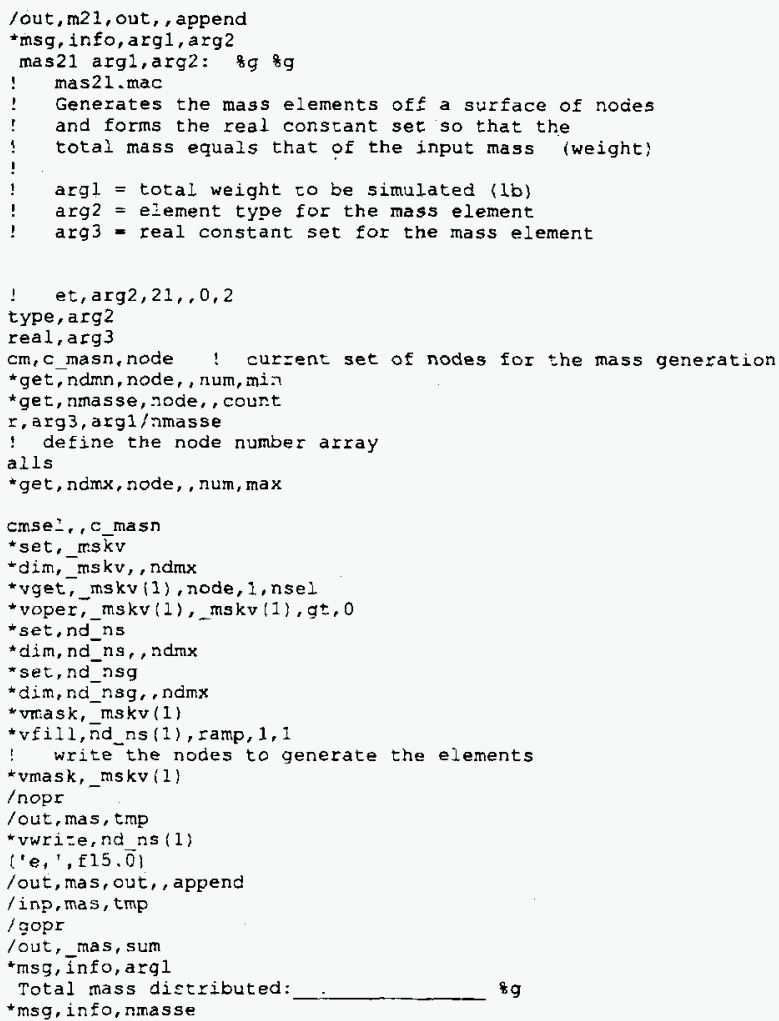

*msg, in fo, nmasse 
Appendix A9.2.2 Immersion Pail Calculation 457-2003.3 (Continued)

Number of mass elements generated: $8 i$ lout

* List, mas, sum

! parsav, all, temp,par 
Appendix A9.2.2 Immersion Pail Calculation 457-2003.3 (Continued)

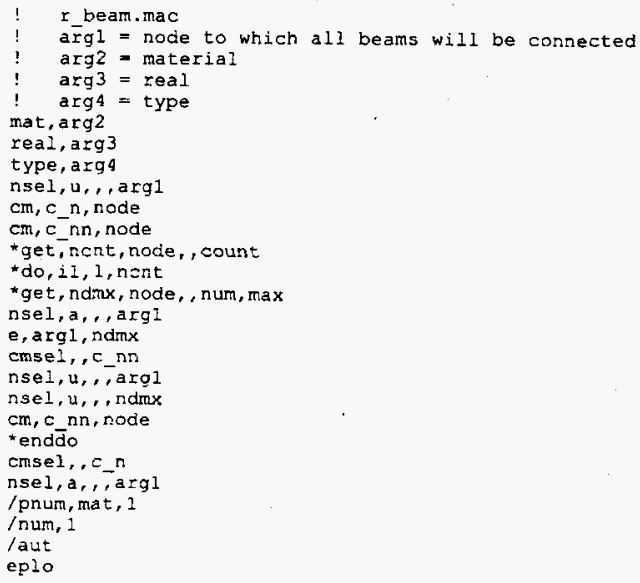


Appendix A9.2.2 Immersion Pail Calculation 457.2003.3 (Continued)

APPENDIX B

Output File for the Finite Element Model

There a a total of 37 pages in Appendix B.

\begin{tabular}{|l|c|}
\hline \multicolumn{2}{|c|}{ Output Files } \\
\hline File Name & Description \\
\hline kbas1.out & $\begin{array}{c}\text { Output File For Model / Solution / } \\
\text { Stresses }\end{array}$ \\
\hline & \\
\hline & \\
\hline
\end{tabular}

\begin{tabular}{|c|c|c|c|c|}
\hline A. VAC & Performed by & $7 n g$ & Date: $11-11-51$ & $\begin{array}{l}\text { Calculation No. 457-2003.3 } \\
\text { Revision 0 }\end{array}$ \\
\hline & Checked by: & $8 R \theta$ & Date: $11-11-96$ & Page B1 of 37 \\
\hline
\end{tabular}


Appendix A9.2.2 Immersion Pail Calculation 457-2003.3 (Continued)

\section{Computer Output Cover Sheet}

Title of Analysis: Immersion Pail Evaluation For Two (2) Point Lift Load

Work Request And Report Number: 457-2003.3, Revision 0

Program: ANSYS Version: 5.2 Originator: Michael C. Yaksh

Date of Verification: $\quad 06 / 28 / 96$

Computer Identification/NAC Control Number:

Title of Case: Deadweight (Inertia Load $=1 \mathrm{~g}$ ).

Total Number of Pages: $\quad 35$ (Not including this cover sheet).

Performed By: my Date: $\|-\|-q_{6}$

Checked By: QRQ Date: $11-11.96$ 
Appendix A9.2.2 Immersion Pail Calculation 457-2003.3 (Continued)

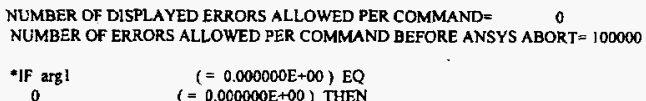

\begin{tabular}{|l|l|}
\hline Q. NAC INTENATIONAL & $\begin{array}{l}\text { Calculation No. 457-2003.3 } \\
\text { Revision 0 }\end{array}$ \\
\cline { 2 - 2 } & Page B3 of 37 \\
\hline
\end{tabular}


Appendix A9.2.2 Immersion Pail Calculation 457-2003.3 (Continued)

THREE-DIMENSIONAL MODEL

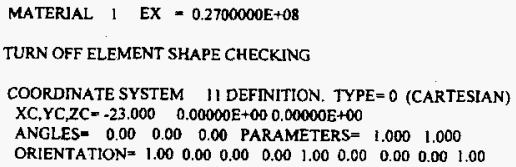

\begin{tabular}{|l|l|}
\hline NAC \\
INTERNATIONal & $\begin{array}{l}\text { Calculation No. 457-2003.3 } \\
\text { Revision 0 }\end{array}$ \\
\cline { 2 - 2 } & Page 84 of 37 \\
\hline
\end{tabular}


Appendix A9.2.2 Immersion Pail Calculation 457-2003.3 (Continued)

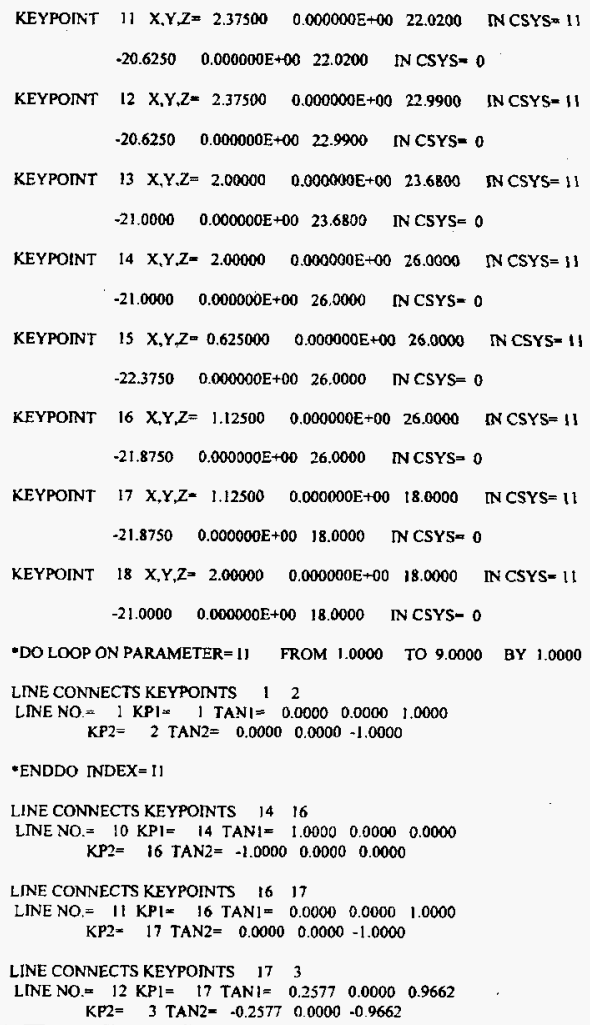


Appendix A9.2.2 Immersion Pail Calculation 457-2003.3 (Continued)

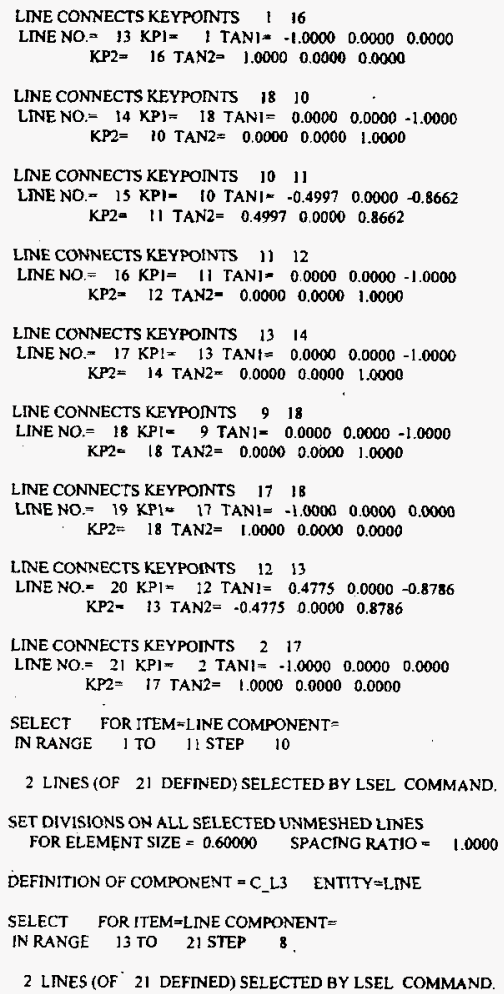

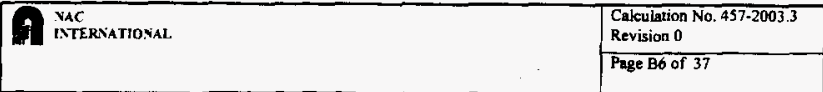


Appendix A9.2.2 Immersion Pail Calculation 457-2003.3 (Continued)

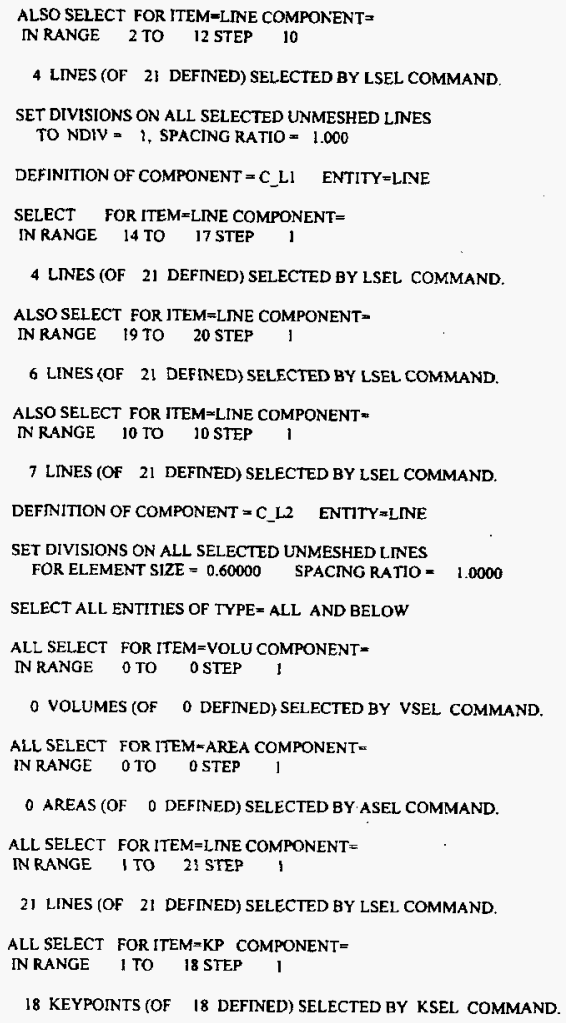

\begin{tabular}{|l|l|}
\hline INAC & Nalculation No 457-2003.3 \\
& Revision 0 \\
\hline & Page B7 of 37 \\
\hline
\end{tabular}


Appendix A9.2.2 Immersion Pail Calculation 457-2003.3 (Continued)

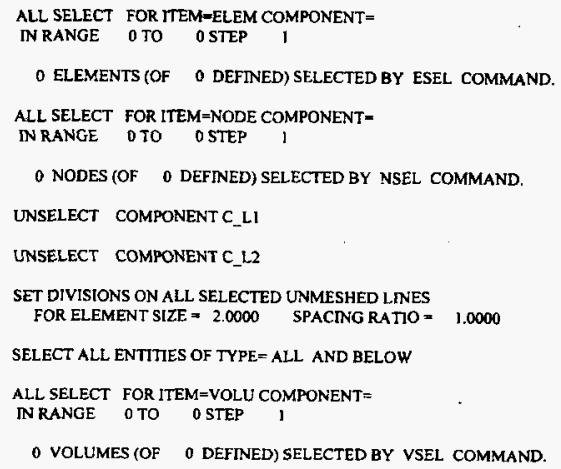


Appendix A9.2.2 Immersion Pail Calculation 457-2003.3 (Continued)

ALL SELECT FOR ITEM-KP COMPONENT=

INRANGE ITO 18 STEP I

18 KEYPONNTS (OF 18 DEFNNED) SELECTED BY KSEL COMMAND.

DEFINE AREA BY LIST OF KEYPOINTS

KEYPONT LIST = $\begin{array}{lllll}1 & 16 & 17 & 2\end{array}$

AREA NUMBER = 1

SELECT FOR ITEM=KP COMPONENT=

IN RANGE 10 TO 14 STEP 1

5 KEYPOINTS (OF 18 DEFINED) SELECTED BY KSEL COMMAND.

ALSO SELECT FOR ITEM×KP COMPONENT=

IN RANGE 16 TO 18 STEP ।

8 KEYPONTS (OF 18 DEFINED) SELECTED BY KSEL COMMAND.

SELECT ALL LINES COMPLETELY CONTAINED WITHIN KEYPOINT SET.

8 LINES (OF 20 DEFINED) SELECTED FROM

8 SELECTED KEYPONTS BY LSLK COMMAND

DEFINE AREA BY LIST OF LINES

LINE LIST = ALL SELECTED LINES

(TRAVERSED IN SAME DIRECTION AS LINE 10)

AREA NUMBER $=2$

ALL SELECT FOR ITEM-KP COMPONENT=

NRANGE 1 TO 18 STEP 1

18 KEYPONNTS (OF 18 DEFINED) SELECTED BY KSEL. COMMAND.

ALL SELECT FOR ITEM=LINE COMPONENT=

N RANGE ITO 21 STEP

20 EINES (OF 20 DEFINED) SELECTED BY LSEL COMMAND.

DEFINE AREA BY LIST OF KEYPOINTS

KEYPOLAT LIST $=\begin{array}{llll}2 & 3 & 13 & 17\end{array}$

AREA NUMBER $=3$

SELECT FOR ITEM-KP COMPONENT=

IN RANGE 3 TO 9 STEP I

7 KEYPOINTS (OF 18 DEFINED) SELECTED BY KSEL COMMAND.

INTERNATIONAL 
Appendix A9.2.2 Immersion Pail Calculation 457-2003.3 (Continued)

ALSO SELECT FOR ITEM=KP COMPONENT

IN RANGE 17 TO 18 STEP :

9 KEYPONTS (OF 18 DEFINED) SELECTED BY KSEL COMMAND

SELECT ALL LINES COMPLETELY CONTANNED WTTHIN KEYPOINT SET.

9 LINES (OF 20 DEFINED) SELECTED FROM

9 SELECTED KEYPONTS BY LSLK COMMAND.

DEFINE AREA BY LIST OF LINES

LNEE LIST = ALL SELECTED LINES

(TRAVERSED IN SAME DIRECTION AS LINE 3)

AREA NUMBER = 4

SELECT ALL ENTTTIES OF TYPE= ALL AND BELOW

ALL SELECT FOR ITEM=VOLU COMPONENT=

IN RANGE OTO OSTEP 1

0 VOLUMES (OF O DEFINED) SELECTED BY VSEL COMMAND.

ALL SELECT FOR TIEM-AREA COMPONENT=

IN RANGE I TO 4 STEP I

4 AREAS (OF 4 DEFINED) SELECTED BY ASEL COMMAND.

ALL. SELECT FOR ITEM=LINE COMPONENT =

IN RANGE I TO 21 STEP ?

20 LJNES (OF 20 DEFINED) SELECTED BY LSEL COMMAND.

ALL SELECT FOR ITEM=KP COMPONENT=

DNRANGE I TO 18 STEP 1

18 KEYPOINTS (OF 18 DEFINED) SELECTED HY KSEL COMMAND

ALL SELECT FOR ITEM=ELEM COMPONENT=

IN RANGE OTO OSTEP ।

0 ELEMENIS (OF O DEFINED) SELECTED BY ESEL COMMAND.

ALL SELECT FOR ITEM=NODE COMPONENT *

IN RANGE OTO OSTEP 1

0 NODES (OF O DEFINED) SELECTED BY NSEL COMMAND.

ELEMENT TYPE SET TO 4

MATERIAL NUMBER SET TO I 
Appendix A9.2.2 Immersion Pail Calculation 457-2003.3 (Continued)

REAL CONSTANT NUMBER= I

GENERATE NODES AND ELEMENTS N ALL SELECTED AREAS

" Meshing of area 1 in progress *"

* Mesbing of area 1 completed $" 14$ elements.

** Meshing of ares 2 in progress *"

** Initiol meshing of area 2 complere **

* AREA 2 MESHED WITH 32 QUADRILATERALS, O TRIANGLES **

** Meshing of area 2 completed ** 32 elements.

** Meshing of area 3 in progress *

-* Initial meshing of area 3 complete **

** AREA 3 MESHED WITH O QUADRILA TERALS, I TRIANGLES **

** Meshing of area 3 completed ** । elements

** Meshing of area $4 \mathrm{in}$ progress **

** Initial meshing of area 4 complete **

** AREA 4 MESHED WITH 18 QUADRILATERALS 4 TRIANGLES **

** Meshing of ares 4 completed $* * 22$ elements.

NUMBER OF AREAS MESHED $=4$

MAXIMUM NODE NUMBER = 95

MAXIMUM ELEMENT NUMBER $\quad$ - 69

VIEW UP DIRECTION FOR WINDOW 1 IS GCS Z AXIS

view point for window $\quad 1.0000 \quad 1.0000 \quad 1.0000$

PRODUCE ELEMENT PLOT N DSYS $=0$

ACTIVE COORDINATE SYSTEM SET TO 0 (CARTESIAN)

KEYPONT $20 \quad X, Y, Z=0.000000 E+00 \quad 0.000000 E+00 \quad 0.000000 E+00$ DN CSYS $=0$

KEYPONT 2] X.Y.Z $=0.000000 E+00 \quad 0.000000 E+00 \quad 20.0000 \quad$ IN CSYS $=0$

DEFAULT ELEMENT DIVISIONS PER LINE $=18$

ELEMENT TYPE SET TO 1

REAL CONSTANT NUMBER = I

MATERIAL NUMBER SET TO I

ROTATE AREAS

1. 2, 3, 4,

ABOUT THE AXIS DEFNED BY KEYPOINTS

$20 \quad 21$

Wit NaC inTERNational

Calculation No. $\overline{457-2003.3}$

Revision 0

Page BII of 37 
Appendix A9.2.2 Immersion Pail Calculation 457-2003.3 (Continued)

DEGRES OF ARC * 180.00 NUMBER OF SEGMENTS $=2$

MAXIMUM NODE NUMBER $\quad 3515$

MAXIMUM ELEMENT NUMBER $=2553$

*ENDIF

ACTIVE COORDINATE SYSTEM SET TO O (CARTESIAN)

SELECT FOR ITEM=LOC COMPONENT-Y BETWEEN-0.10000E-0! AND 1.0000 KABS- 0. TOLERANCE $=0.000000 E+00$

190 NODES (OF 3515 DEFINED) SELECTED BY NSEL COMMAND

SPECIFIED CONSTRAINT UY FOR SELECTED NODES ITO $3515 \mathrm{BY} 1$

REAL $=0.000000000 \mathrm{E}+00 \quad 1 \mathrm{MAG}=0.000000000 \mathrm{E}+00$

ALL SELECT FOR ITEM=NODE COMPONENT =

IN RANGE ITO 3515 STEP 1

3515 NODES (OF 3515 DEFINED) SELECTED BY NSEL COMMAND.

ELEMENT TYPE S IS MASS21 STRUCTURAL MASS

$\operatorname{KEYOPT}(1-12)=002200000000000$

CURRENT NODAL DOF SET IS UX UY UZ ROTX ROTY ROTZ THREE-DIMENSIONAL MODEL

"GET zmin FROM NODE ITEM=MNLOZ VALUE $=0.000000000 E+00$

SELECT FOR ITEM=LOC COMPONENT $=Z$. BETWEEN -1.0000 AND 0.10000E-01 KABS= 0. TOLERANCE- $0.000000 \mathrm{E}+00$

37 NODES (OF 3515 DEFINED) SELECTED BY NSEL COMMAND.

USE COMMAND MACRO MAS2!

ARGS* 86871. $5.0000 \quad 5.0000$

OUTPUT FILE $=\mathrm{m} 21$.out

PRINTOUT RESUMED BY /GOP

SELECT ALL ENTITIES OF TYPE= ALL AND BELOW

ALL SELECT FOR ITEM-VOLU COMPONENT=

IN RANGE 1 TO 8 STEP

8 VOLUMES (OF 8 DEFINED) SELECTED BY VSEL COMMAND.

ALL SELECT FOR ITEM=AREA COMPONENT-

IN RANGE ITO 52 STEP I

\begin{tabular}{|l|l|}
\hline NaC \\
NATERnational & $\begin{array}{l}\text { Calculation No 457.2003.3 } \\
\text { Revision 0 }\end{array}$ \\
\cline { 2 - 2 } & Page B12 of 37 \\
\hline
\end{tabular}


Appendix A9.2.2 Immersion Pail Calculation 457-2003.3 (Continued)

52 AREAS (OF 52 DEFINED) SELECTED BY ASEL COMMAND.

ALL SELECT FOR TTEM=LINE COMPONENT=

N RANGE ITO 94 STEP I

94 LINES (OF DEFINED) SELECTED BY LSEL COMMAND.

ALL SELECT FOR ITEM=KP COMPONENT-

IN RANGE 1 TO S4 STEP 1

54 KEYPOINTS (OF 54 DEFINED) SELECTED BY KSEL COMMAND.

ALL SELECT FOR ITEM=ELEM COMPONENT =

IN RANGE ITO 2590 STEP ।

2590 ELEMENTS (OF 2590 DEFNNED) SELECTED BY ESEL COMMAND.

ALL SELECT FOR ITEM-NODE COMPONENT=

IN RANGE 1 TO 3515 STEP I

3515 NODES (OF 3515 DEFINED) SELECTED BY NSEL COMMAND.

*GET ndmX FROM NODE ITEM=NUM MAX VALUE= 3515.00000

PARAMETER GNDMX $=3516.000$

ACTIVE COORDINATE SYSTEM SET TO 11 (CARTESIAN)

NODE $3516 \mathrm{KCS}-11 \quad X, Y, Z=-5.2400 \quad 0.00000 \mathrm{E}+0021.460$

SPECIFIED CONSTRAINT UX FOR SELECTED NODES 3516 TO 3516 BY ।

REAL $=0.000000000 E+00 \quad$ IMAG $=0.000000000 E+00$

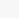

SPECIFIED CONSTRADNT UY FOR SELECTED NODES 3516 TO $3516 \mathrm{BY} \quad 1$

REAL $=0,000000000 \mathrm{E}+00 \quad \mathrm{MMAG}=0.000000000 \mathrm{E}+00$

SPECIFIED CONSTRAINT UZ FOR SELECTED NODES 3516 TO 3516 BY ।

REAL $=0.000000000 E+00$ IMAG $=0.000000000 E+00$

REAL CONSTANT SET 3 ITEMS ITO 6

$\begin{array}{llllll}1000.0 & 1000.0 & 1000.0 & 1000.0 & 1000: 0 & 0.00000 \mathrm{E}+00\end{array}$

ACTIVE COORDINATE SYSTEM SET TO O (CARTESIAN)

ALL SELECT FOR ITEM=ELEM COMPONENT =

INRANGE 1 TO 2590 STEP ।

2590 ELEMENTS (OF 2590 DEFINED) SELECTED BY ESEL COMMAND.

SELECT ALL NODES HAVING ANY ELEMENT IN ELEMENT SET.

3515 NODES (OF 3516 DEFINED) SELECTED FROM

\begin{tabular}{|l|l|}
\hline NAC INTENATIONAL & $\begin{array}{l}\text { Celeulation No. 457-2003.3 } \\
\text { Revision } 0\end{array}$ \\
\cline { 2 - 2 } & Page Bl3 of 37 \\
\hline
\end{tabular}


Appendix A9.2.2 Immersion Pail Calculation 457-2003.3 (Continued)

2590 SELECTED ELEMENTS BY NSLE COMMAND.

"GeT r_o fROM NODE TTEM-MNLOX VALUE--23.0000000

SELECT FOR ITEM=LOC COMPONENT=Y BETWEEN-0.10000E-01 AND 1.0000 KABS $=0$. TOLERANCE $\approx 0.000000 \mathrm{E}+00$

191 NODES (OF 3516 DEFINED) SELECTED BY NSEL COMMAND.

RESELECT FOR ITEM $=$ LOC COMPONENT $=X$ BETWEEN -23.100 AND -22.990

KABS $=0$. TOLERANCE $=0.000000 E \div 00$

15 NODES (OF 3516 DEFINED) SELECTED BY NSEL COMMAND

USE COMMAND MACRO R_BEAM

ARGS= $3516.0 \quad 1.0000-3.0000 \quad 3.0000$

MATERIAL NUMBER SET TO 1

REAL CONSTANT NUMBER = 3

ELEMENT TYPE SET TO 3

UNSELECT FOR ITEM NODE COMPONENT-

IN RANGE 3516 TO 3516 \$TEP I

IS NODES (OF 3516 DEFINED) SELECTED BY NSEL COMMAND.

DEFINITION OF COMPONENT $=\mathrm{C}_{-} N \quad$ ENTITY $=$ NODE

DEFINITION OF COMPONENT $=C_{-} N N \quad$ ENTTTY-NODE

"GET nent FROM NODE ITEM=COUN VALUE* 15.0000000

-DO LOOP ON PARAMETER = II FROM 1.0000 TO 15.000 BY 1.0000

-GET ndmx FROM NODE ITEM-NUM MAX VALUE= 30.0000000

ALSO SELECT FOR ITEM=NODE COMPONENT-

IN RANGE 3516 TO 3516 STEP

16 NODES (OF 3516 DEFINED) SELECTED BY NSEL COMMAND

ELEMENT 259] $3516 \quad 30 \quad 0$

SELECT COMPONENTC_NN

UNSELECT FOR ITEM-NODE COMPONENT=

IN RANGE 3516 TO 3516 STEP 1

15 NODES (OF 3516 DEFINED) SELECTED BY NSEL COMMAND. 
Appendix A9.2.2 Immersion Pail Calculation 457-2003.3 (Continued)

UNSELECT. FOR ITEM=NODE COMPONENT=
IN RANGE 30 TO 30 STEP I

14 NODES (OF 3516 DEFINED) SELECTED BY NSEL COMMAND.

DEFINITION OF COMPONENT $=C_{-}$NN ENTITY-NODE

"ENDDO INDEX=11

SELECT COMPONENTC_N

ALSO SELECT FOR ITEM=NODE COMPONENT=

IN RANGE 3516 TO 3516 STEP ।

16 NODES (OF 3516 DEFINED) SELECTED BY NSEL COMMAND.

MAT NUMBERING KEY - 1

NUMBER KEY SET TO 1 - $]$-NONE $0=$ BOTH $1=$ COLOR 2-NUMBER

AUTOMATIC SCALING FOR WTNDOW :

DISTANCE AND FOCUS PONT AUTOMATICALLY CALCULATED

PRODUCE ELEMENT PLOT IN DSYS $=0$

/OUTPUT FILE- kbasl.out

PEINTOUT RESUMED BY /GOP

ACTIVE COORDINATE SYSTEM SET TO O (CARTESIAN)

-GET s_X FROM NODE 3516 ITEM-LOC X VALUE=-28.2400000

-GET S $Z$ FROM NODE 3516 ITEM-LOC $Z$ VALUE= 21.4600000

NODE $3517 \mathrm{KCS}=0$ X Y,,$Z=28.240 \quad 0.00000 \mathrm{E}+0021.460$

SPECIFIED CONSTRAINT UY FOR SELECTED NODES 3517 TO 3517 BY 1

REAL $=0.00000000 \mathrm{E}+00$ IMAG $=0.000000000 \mathrm{E}+00$

SPECIFIED CONSTRAINT UZ FOR SELECTED NODES 3517 TO 3517 BY ।

REAL $=0.00000000 E+00 \quad I M A G=0.000000000 E-00$

SELECT FOR ITEM=LOC COMPONENT $=Y$ BETWEEN-0.10000E-01 AND 1.0000

$\mathrm{KABS}=0$. TOLERANCE $=0.00000 \mathrm{E}+00$

192 NODES (OF 3517 DEFINED) SELECTED BY NSEL COMMAND.

RESELECT FOR ITEM=LOC COMPONENT $=\mathrm{X}$ BETWEEN 22.900 AND 23.010 KABS $=0$. TOLERANCE $=0.000000 E+00$

IS NODES (OF 3517 DEFINED) SELECTED BY NSEL COMMAND.

\begin{tabular}{|c|c|}
\hline 1. NAC & $\begin{array}{l}\text { Calculation No. 457-2003.3 } \\
\text { Revision } 0\end{array}$ \\
\hline & Page B15 of 37 \\
\hline
\end{tabular}


Appendix A9.2.2 Immersion Pail Calculation 457-2003.3 (Continued)

USE COMMAND MACRO R_BEAM
ARGS $=3517.0 \quad 30000$

MATERJAL NUMBER SET TO 1

REAL CONSTANT NUMBER $=3$

ELEMENT TYPE SET TO 3

UNSELECT FOR ITEM-NODE COMPONENT-

IN RANGE 3517 TO 3517 STEP I

15 NODES (OF 3517 DEFNED) SELECTED BY NSEL COMMAND.

DEFINITION OF COMPONENT $=\mathrm{C}_{-} \mathrm{N}$ ENTITY $=$ NODE

DEFINITKON OF COMPONENT $=\mathrm{C}$ NN ENTITY-NODE

*GET nent FROM NODE ITEM-COUN VALUE $=15.0000000$

"DO LOOP ON PARAMETER= II FROM 1.0000 TO 15.000 BY 1.0000

"GET ndmX FROM NODE ITEM=NUM MAX VALUE= 1835.00000

ALSO SELECT FOR ITEM-NODE COMPONENT=

IN RANGE 3517 TO 3517 STEP ।

16 NODES (OF 3517 DEFINED) SELECTED BY NSEL COMMAND.

ELEMENT $2606 \quad 3517 \quad 1835 \quad 0$

SELECT COMPONENTC_NN

UNSELECT FOR TTEM-NODE COMPONENT=

IN RANGE 3517 TO 3517 STEP ।

I5 NODES (OF 3517 DEFINED) SELECTED BY NSEL COMMAND.

UNSELECT FOR ITEM-NODE COMPONENT=

IN RANGE 1835 TO 1835 STEP ।

14 NODES (OF 3517 DEFINED) SELECTED BY NSEL COMMAND.

DEFINITION OF COMPONENT $=C_{-}$NN ENTITY=NODE

"ENDDO TNDEX $=11$

SELECT COMPONENT C_N

ALSO SELECT FOR ITEM-NODE COMPONENT *

IN RANGE 3517 TO 3517 STEP I

\begin{tabular}{|l|l|}
\hline $\begin{array}{l}\text { NAC } \\
\text { INTERVATONAL }\end{array}$ & $\begin{array}{l}\text { Calculation No. 437-2003.3 } \\
\text { Revision 0 }\end{array}$ \\
\cline { 2 - 2 } & Page B16 of 37 \\
\hline
\end{tabular}


Appendix A9.2.2 Immersion Pail Calculation 457-2003.3 (Continued)

16 NOPES (OF 3517 DEFINED) SELECTED BY NSEL COMMAND.

MAT NUMBERTNG KEY $=$ !

NUMBER KEY SET TO $\mid-1=$ NONE $0=B O T H \quad \mid=C O L O R \quad 2-N U M B E R$

AUTOMATIC SCALING FOR WINDOW !

DISTANCE AND FOCUS PONT AUTOMATICALLY CALCULATED

PRODUCE ELEMENT PLOT IN DSYS = 0

SELECT ALL ENTITIES OF TYPE= ALI. AND BELOW

ALL SELECT FOR ITEM=VOLU COMPONENT=

NN RANGE 1 TO 8 STEP

B VOLUMES (OF 8 DEFINED) SELECTED BY VSEL COMMAND.

ALL SELECT FOR ITEM=AREA COMPONENT=

IN RANGE I TO S2 STEP I

52 AREAS (OF 52 DEFINED) SELECTED BY ASEL COMMAND.

ALL SELECT FOR ITEM=LINE COMPONENT=

IN RANGE 1 TO. 94 STEP 1

94 LINES (OF 94 DEFINED) SELECTED BY LSEL, COMMAND.

ALL SELECT FOR ITEM-KP COMPONENT=

IN RANGE 1 TO $\$ 4$ STEP I

54 KEYPOINTS (OF 54 DEFINED) SELECTED BY KSEL COMMAND.

ALL SELECT FOR TTEM=ELEM COMPONENT-

IN RANGE ITO 2620 STEP I

2620 ELEMENTS (OF 2620 DEFINED) SELECTED BY ESEL COMMAND.

ALL SELECT FOR TTEM=NODE COMPONENT=

IN RANGE ITO 3517 STEP I

3517 NODES (OF 3517 DEFINED) SELECTED BY NSEL COMMAND.

DESTROY ASSOCIATIVITY OF FINITE ELEMENT MODEL AND SOLID MODEL

NUMBER OF MESH ITEMS DISCONNECTED FROM NODES AND ELEMENTS = 154

ACTIVE COORDNATE SYSTEM SET TO 1 (CYLINDRICAL)

SELECT FOR ITEMIENAM COMPONENT=

IN RANGE 45 TO 45 STE? ।

\begin{tabular}{|l|l|}
\hline 1. NAC INTRVATIONAL & $\begin{array}{l}\text { Caiculation No. 457-2003.3 } \\
\text { Revision } 0\end{array}$ \\
\cline { 2 - 2 } & \begin{tabular}{l} 
Page B17 of 37 \\
\hline
\end{tabular} \\
\hline
\end{tabular}


Appendix A9.2.2 Immersion Pail Calculation 457-2003.3 (Continued)

2484 ELEMENTS (OF 2620 DEFINED) SELECTED BY ESEL. COMMAND.

SELECT ALL NODES HAVING ANY ELEMENT IN ELEMENT SET.

3515 NODES (OF 3517 DEFINED) SELECTED FROM

2484 SELECTED ELEMENTS BY NSLE COMMAND.

RESELECT FOR ITEM $=$ LOC COMPONENT $=Y$ BETWEEN -41.000 AND -39.000 KABS $=0$. TOLERANCE $=0.000000+00$

95 NODES (OF 3517 DEFINED) SELECTED BY NSEL COMMAND.

MODIFY ALL SELECTED NODES IN COORDINATE SYSTEM ।

NEW COORDS $=Y=-39.00$

SELECT ALL NODES HAVING ANY ELEMENT IN ELEMENT SET.

3515 NODES (OF 3517 DEFINED) SELECTED FROM

2484 SELECTED ELEMENTS BY NSLE COMMAND.

"GET $r$. FROM NODE ITEM=MXLOX $\cdot$ VALUE $=23.0000000$

RESELECT FOR ITEM $=L O C$ COMPONENT $=X$ BETWEEN 22.900 AND 24.000

$\mathrm{KABS}=0$. TOLERANCE $* 0.000000 \mathrm{E}+00$

555 NODES (OF 3517 DEFNED) SELECTED BY NSEL COMMAND.

RESELECT FOR TTEM=LOC COMPONENT $=Y$ BETWEEN -66.000 AND -38.000 KABS= 0 . TOLERANCE $=0.000000 E+\infty$

90 NODES (OF 3517 DEFINED) SELECTED BY NSEL COMMAND.

COORDINATE SYSTEM 12 DEFINITION. TYPE- O (CARTESIAN)

$X C, Y C, Z C=0.00000 E+000.00000 E+000.00000 E+00$

ANGLES $=-52.00 \quad 0.00 \quad 0.00$ PARAMETERS $=1.000 \quad 1.000$

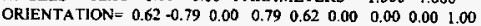

ACTIVE COORDINATE SYSTEM SET TO 12 (CARTESIAN)

MODIFY ALL SELECTED NODES IN COORDINATE SYSTEM 12

NEW COORDS $=\quad X=22.38$

SELECT FOR ITEM=TYPE COMPONENT=

IN RANGE 4 TO 4 STEP 1

69 ELEMENTS (OF 2620 DEFINED) SELECTED BY ESEL COMMAND

DELETE ALL SELECTED ELEMENTS.

DELETE ELEMENT TYPES FROM \& TO $4 \mathrm{BY} \mathrm{।}$

CURRENT NODAL DOF SET IS UX UY UZ ROTX ROTY ROTZ

\begin{tabular}{|l|l|}
\hline INAC INERYTIONAL & $\begin{array}{l}\text { Caleuletion No. 457-2003.3 } \\
\text { Revision 0 }\end{array}$ \\
\cline { 2 - 2 } & Page B18 of 37 \\
\hline
\end{tabular}


Appendix A9.2.2 Immersion Pail Calculation 457-2003.3 (Continued)

THREE-DIMENSIONAL MODEL

SELECT ALL ENTITES OF TYPE ALL AND BELOW

ALL SELECT FOR ITEM=VOLU COMPONENT-

INRANGE ITO 8 STEP 1

8 VOLUMES (OF 8 DEFINED) SELECTED BY VSEL COMMAND

ALL SELECT FOR ITEM=AREA COMPONENT=

IN RANGE ITO S2 STEP 1

52 AREAS (OF 52 DEFINED) SELECTED BY ASEL COMMAND.

ALL SELECT FOR ITEM=LINE COMPONENT=

IN RANGE 1 TO 94 STEP

94 LINES (OF 94 DEFINED) SELECTED BY LSEL COMMAND.

ALL SELECT FOR ITEM=KP COMPONENT=

IN RANGE ITO 54 STEP ।

54 KEYPOINTS (OF S4 DEFINED) SELECTED BY KSEL COMMAND.

ALL SELECT FOR ITEM=ELEM COMPONENT=

NNRANGE ITO 2620 STEP $\quad$ I

2551 ELEMENTS (OF 2551 DEFINED) SELECTED BY ESEL COMMAND.

ALL SELECT FOR ITEM-NODE COMPONENT=

IN RANGE 1 TO 35I? STEP 1

3517 NODES (OF 3517 DEFNED) SELECTED BY NSEL COMMAND.

**** ROUTINE COMPLETED **** CP $=\quad 570.773$

\#NOTE *. CP $=570.781$ TTME $=22: 00: 07$

A cotal of 353 warnings and errors written to fube err.

\#*** ANSYS SOLUTJON ROUTTNE ***.*

$A C E L=0.00000 E+000.00000 E-001.0000$

***** ANSYS SOLVE COMMAND *****

*** NOTE *** CP 570.789 TIME= 22:00:07

There is no title defined for this analysis.

** NOTE ***

CP= 571.820 TTME $=22: 00: 08$

\begin{tabular}{|l|l|}
\hline INTERNATIONAL & $\begin{array}{l}\text { Nalculation No 457.2003.3 } \\
\text { Revision 0 }\end{array}$ \\
\cline { 2 - 2 } & Page B19 of 37 \\
\hline
\end{tabular}


Appendix A9.2.2 Immersion Pail Calculation 457-2003.3 (Continued)

The model dath was checked and waming messages were found. Please review output or errors file (flube.er ) for these waming messages.

SOLUTION OPTLONS

PROBLEM DIMENSIONALITY.......... 3-D

DEGREES OF FREEDOM. .... . UX UY UZ ROTX ROTY ROTZ

ANALYSIS TYPE .............. STATIC (STEADY-STATE)

*** NOTE ***

CP. 572.055 TIME $=22: 00: 08$

Present time 0 is less than or equal to the previous time.

Time will default to $I$.

** Reordering still in progress **

"* Reordering still in progress **

$$
\text { LOAD STEP OPTIONS }
$$

LOAD STEP NUMBER. .

TIME AT END OF THE LOAD STEP. . . . . . 1,0000

NUMBER OF SUBSTEPS. . . . . . . . . 1

STEP CHANGE BOUNDARY CONDITIONS ....... NO

INERTIA LOADS $X \quad Y \quad Z$

ACEL .......... 0.00000E+000.00000E+00 1.0000

PRNT OUTPUT CONTROLS ............. NO PRINTOUT

DATABASE OUTPUT CONTROLS ...........ALL DATA WRITTEN FOR THE LAST SUESTEP

Element Fonmation Element $=10 \mathrm{Cum}$. Itet $=1 \mathrm{CP}=573.242$

Time $=1.0000$ Lasd Step 1 Substep $=1$ Equilibrium Iteration -1

Element Fornation Element= $1810 \mathrm{Cum}$. Iter $=1 \mathrm{CP}=582.734$

Timex 1.0000 Load Siep= 1 Substep- i Equilibrium lieration= I.

\section{**** CENTROID, MASS, AND MASS MOMENTS OF INERTIA *****}

CALCULATIONS ASSUME ELEMENT MASS AT ELEMENT CENTROID

TOTAL MASS - $\mathbf{8 6 8 7 1}$.

$$
\text { CENTROID MOM. OF INERTIA MOM. OF INERTIA }
$$

$X C=0.63009 E-02 \quad I X X=0.1862 E+08 \quad I X X=0.3957 E+07$

$Y C=-12.992 \quad$ IYY $=0.1969 \mathrm{E}+08 \quad$ IYY $=0.1969 \mathrm{E}+08$

$Z C=0.00000 E+00 \quad I Z Z=0.3831 E+08 \quad I Z Z=0.2365 E+08$

$\mathrm{XY}=-\mathbf{3 4 4 8 .} \quad \mathrm{IXY}=-\mathbf{0 . 1 0 5 6 \mathrm { E } + 0 5}$

$Y Y Z=0.0000 E+00 \quad I Y Z=0.0000 E+00$

$I Z X=0.0000 \mathrm{E}+00 \quad$ IZX $-0.0000 \mathrm{E}+00$

ONLY THE X-DIRECTION MASS TERMS ARE USED FOR MASS21 ELEMENTS.

\begin{tabular}{|l|l|}
\hline $\begin{array}{l}\text { NAC } \\
\text { INTER NATIONAL }\end{array}$ & $\begin{array}{l}\text { Calculation No. 457-2003.3 } \\
\text { Revision } 0\end{array}$ \\
\cline { 2 - 2 } & Page B20 of $3 \gamma$ \\
\hline
\end{tabular}


Appendix A9.2.2 Immersion Pail Calculation 457-2003.3 (Continued)

\author{
*** MASS SUMMARY BY ELEMENT TYPE *** \\ TYPE MASS \\ 586871.0
}

Range of element maximum matrix coefficients in global coordinates Maximum $=2.061031631 \mathrm{E}+10$ at element 2596

Minimum= 7637221.94 at element 1754 .

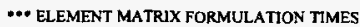
TYPE NUMBER ENAME TOTAL CP AVECP

$\begin{array}{ccccc}1 & 2484 & \text { SOLID45 } & 13.250 & 0.005 \\ 3 & 30 & \text { BEAM4 } & 0.039 & 0.001\end{array}$

537 MASS21 $\quad 0.008 \quad 0.000$

Time at end of element matrix formulation $C P=586.632813$.

Estimated number of active DOF= 10452.

Maximum wavefront $=397$.

Equation Solution Element $=500$ Cum. lter $=\mid C P=592.461$

Time= 1.0000 Load Step= I Substep= 1 Equilibrium Iteration= 1.

Equation Solution Element $=1920$ Cum. Iter $=1 \mathrm{CP}=601.836$

Time $=1.0000$ Load Step= I Substep= I Equilibrium Iteration $=1$.

Time at end of matrix triangularization $\mathrm{CP}=605.734375$.

Equation solver maximum pivot $=2.1605637 \mathrm{E}+11$ at node 3517 ROTY.

Equation solver minimum pivot- 159079.15 at node 74 UX.

Element Output EJement $=900 \mathrm{Cum}$. Iter: $=\mid \mathrm{CP}=511.555$

Timex 1.0000 Load Step- I Substep=1 Equilibrium Iteration= 1 .

*.* element result calculation times

TYPE NUMBER ENAME TOTAL CP AVE CP

$\begin{array}{ccccc}1 & 2484 & \text { SOLID45 } & 8.453 & 0.003 \\ 3 & 30 \text { BEAM4 } & 0.047 & 0.002\end{array}$

5 37 MASS21 $0.000 \quad 0.000$

-** nodal load calculation times

TYPE NUMBER ENAME TOTAL CP AVE CP

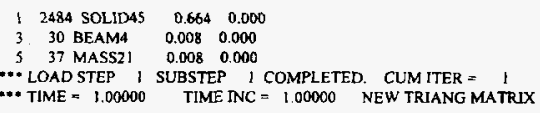

* PROBLEM STATISTICS

ACTUAL NO. OF ACTIVE DEGREES OF FREEDOM $=10452$

R.M.S. WAVEFRONT SIZE - 305.2

\begin{tabular}{|l|l|}
\hline $\begin{array}{l}\text { NAC } \\
\text { INIERNATIONAL }\end{array}$ & $\begin{array}{l}\text { Calculation No. 457-2003.3 } \\
\text { Revision 0 }\end{array}$ \\
\cline { 2 - 2 } & Page B21 of 37 \\
\hline
\end{tabular}


Appendix A9.2.2 Immersion Pail Calculation 457-2003.3 (Continued)

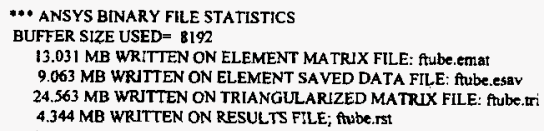


Appendix A9.2.2 Immersion Pail Calculation 457-2003.3 (Continued)

2551 ELEMENTS (OF 2551 DEFINED) SELECTED BY ESEL COMMAND.

ALL SELECT FOR ITEM-NODE COMPONENT=

IN RANGE ITO 3517 STEP I

3517 NODES (OF 3517 DEFINED) SELECTED BY NSEL COMMAND.

***** SUMMATION OF TOTAL FORCES AND MOMENTS IN GLOBAL COORDINATES *..."

$\mathrm{FX}=-0.4228059 \mathrm{E}-07$

$F Y=0.4213598 \mathrm{E}-07$

$\mathrm{FZ}=-86871,00$

$\mathrm{MX}=1128610$.

$M Y=347.3622$

$\mathrm{MZ}=-0.13 ! 2711 \mathrm{E}-05$

SUMMATION POINT $=0.00000 E+000.00000 \mathrm{E}+000.00000 \mathrm{E}+00$

DISPLAYS PUT ON PLOT FILE kbasin.plt - VECTOR MODE,

TITLE=

K Basin Pail Ring Lift Analysis

DEVICE VECTOR KEY SET TO 1

HIDDEN DISPLAY (PRECISE) IN WINDOW 1

NUMBER KEY SET TO $-1-t=N O N E$ 0-BOTH $l=$ COLOR $2=$-NUMBER

view point for window i $1.0000 \quad 1.0000 \quad 1.0000$

PRODUCE ELEMENT PLOT IN DSYS $=0$

CUMULATIVE DISPLAY NUMBER I WRITTEN TO FILE kbasin.pl - VECTOR MODE. DISPLAY TITLE-

$K$ Basin Pail Ring Lift Analysis

*** NOTE*** CP= 618.758 TIME= 22:01:01

CUMULATIVE DISPLAY NUMBER 1 WRITTEN TO FILE kbasin.pt - VECTOR MODE.

DISPLA Y TTTLE= K Basin Pail Ring Lift Analysis.

NUMBER KEY SET TO 2 - I=NONE 0-BOTH 1-COLOR 2-NUMBER

SELECT FOR ITEM-ENAM COMPONENT-

IN RANGE 45 TO 45 STEP ।

2484 ELEMENTS (OF 2551 DEFINED) SELECTED BY ESEL COMMAND.

SELECT ALL NODES HAVNG ANY ELEMENT IN ELEMENT SET.

3515 NODES (OF 3517 DEFINED) SELECTED FROM 2484 SELECTED ELEMENTS BY NSLE COMMAND

\begin{tabular}{|l|l|}
\hline NAC NARSATIONAL & $\begin{array}{l}\text { Calculation No. 457-2003.3 } \\
\text { Revision 0 }\end{array}$ \\
\cline { 2 - 2 } & Page B23 of 37 \\
\hline
\end{tabular}


Appendix A9.2.2 Immersion Pail Calculation 457-2003.3 (Continued)

AUTOMATIC SCALING FOR WINDOW 1

DISTANCE AND FOCUS PONT AUTOMATCALLY CALCULATED

view point for window $1 \quad \mathbf{1} .0000 \quad-1.0000 \quad 1.0000$

VIEW UP DIRECTION FOR WINDOW IIS GCS $Z$ AXIS

ACTIVE COORONATE SYSTEM SET TO 1 (CYLINDRICAL)

RESELECT FOR ITEM $=$ LOC COMPONENT $=Y$ BETWEEN - 161.00 AND -29.000 KABS $=0$. TOLERANCE $=0.000000+\infty$

2565 NODES (OF 3517 DEFINED) SELECTED BY NSEL COMMAND.

SELECT ONLY ELEMENTS COMPLETELY CONTAINED WITHIN NODE SET.

1821 ELEMENTS \{OF 2551 DEFINED) SELECTED FROM 2565 SELECTED NODES BY ESLN COMMAND.

DISPLAY NODAL SOLUTION. ITEM=S COMP-EQV

CUMULATIVE DISPLAY NUMBER 2 WRITTEN TO FLL kbasin.plt - VECTOR MODE. DISPLAY TITLE-

K Basin Pail Ring Lift Analysis

*.* NOTE **" CP= 623.000 TIME $=22: 01: 06$

CUMULATIVE DISPLAY NUMBER 2 WRITTEN TO FILE kbasin.plt - VECTOR MODE.

DISPLAY TTTLE= K Basin Pail Ring Lift Analysis.

DISPLAY COORDNATE SYSTEM SET TO 1 (CYLNDRICAL)

LIST ALL SELECTED NODES NN RANGE 222! TO 2630 STEP 409 DSYS= 1

$\begin{array}{ccccccl}\text { NODE } & X & Y & Z & \text { THXY } & \text { THYZ } & \text { THZXX } \\ 2221 & 23.000 & -30.000 & 22.571 & 0.00 & 0.00 & 0.00 \\ 2630 & 20.625 & -30.000 & 22.505 & 0.00 & 0.00 & 0.00\end{array}$

DISPLAY COORDINATE SYSTEM SET TO O (CARTESIAN)

DEFINE A PATH FOR SUBSEQUENT CALCULATIONS THROUGH NODES:

22212630

PRNT LINEARIZED STRESS THROUGH A SECTION DEFINED BY LPATH COMMAND. DSYS $=0$

\#.... POSTI LINEARIZED STRESS LISTING *.***

INSIDE NODE $=2221$ OUTSIDE NODE $=2630$

LOADSTEP I SUBSTEP $=1$

$T \mathrm{TME}=1.0000$. LOAD CASE $=0$

THE FOLLOWING X,Y.Z STRESSES ARE IN GLOBAL, COORDINATES.

\begin{tabular}{|l|l|}
\hline INTERNATIONAL & $\begin{array}{l}\text { Calculation No. 457-2003.3 } \\
\text { Revision 0 }\end{array}$ \\
\cline { 2 - 2 } & Page B24 of 37 \\
\hline
\end{tabular}


Appendix A9.2.2 Immersion Pail Calculation 457-2003.3 (Continued)

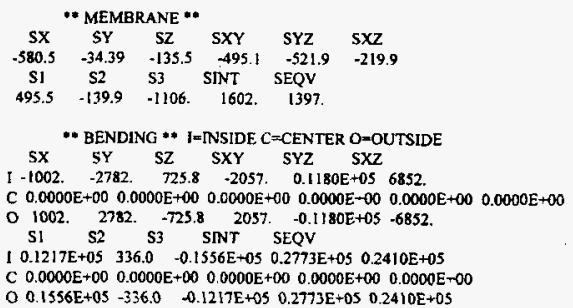

** MEMBRANE PLUS BENDING ** I=INSIDE C-CENTER O=OUTSIDE

$\begin{array}{llllll}S X & S Y & S Z & 5 X Y & S Y Z & S X Z\end{array}$

$\begin{array}{llllll}I-1583 & -2816 . & 590.3 & -2552 & 0.1128 E+05 & 6632\end{array}$

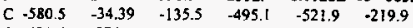

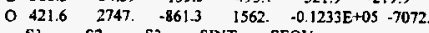

S1 $52 \quad 53 \quad$ SINT SEQV

I $0.1130 \mathrm{E}+0.5331 .6-0.1544 \mathrm{E}+05 \quad 0.2675 \mathrm{E}+05 \quad 0.2329 \mathrm{E}+0 \mathrm{~S}$

$\begin{array}{lllll}\text { C } 495.5 & -139.9 & -1106 . & 1602 . & 1397 .\end{array}$

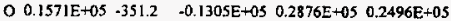

* PEAK * I=INSIDE C-CENTER O-OUTSIDE

SX SY SZ SXY SYZ SXZ

$\begin{array}{llllll}\text { I }-7.176 & -27.64 & 27.29 & 164.2 & 704.6 & 376.9\end{array}$

$\begin{array}{llllll}\text { C } 23.07 & 13.26 & -10.07 & -130.4 & -546.0 & -291.8\end{array}$

$\begin{array}{lllllll}0 & -105.5 & 115.3 & 158.7 & 118.8 & 1143 . & 611.4\end{array}$

S1 S2 S3 SINT SEQV

$\begin{array}{lllll}\text { I } 875.7 & -145.4 & \mathbf{- 7 3 7 . 8} & 1614 & 1414 .\end{array}$

$\begin{array}{lllll}\text { C } 573.9 & 127.5 & .675 .1 & 1249 . & 1096\end{array}$

0 1458. $-155.0-1135.2593$. 2268

* TOTAL " I=INSIDE C=CENTER O-OUTSIDE

$\begin{array}{llllll}5 X & S Y & S Z & \text { SXY SYZ SXZ }\end{array}$

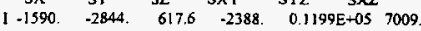

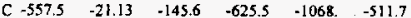

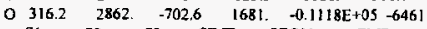

S1 S2 S3 SINT SEQV TEMP

] $0.1215 \mathrm{E}+05 \quad 172.9-0.1614 \mathrm{E}+05 \quad 0.2829 \mathrm{E}+05 \quad 0.2459 \mathrm{E}+05 \quad 0.0000 \mathrm{E}+00$

$\begin{array}{lllll}\text { C } 994.9 & -7.545 & -1712 . & 2706 . & 2370 .\end{array}$

$00.1459 E-05-503.0 \quad-0.1161 E+05 \quad 0.2621 E^{+} 05 \quad 0.2278 E+05 \quad 0.0000 E+\infty 0$

DISPLAY COORDINATE SYSTEM SET TO I (CYLINDRICAL)

LIST ALL SELECTED NODES IN RANGE 2272 TO 2596 STEP 324 DSYS $=1$

$\begin{array}{lllllll}\text { NODE } & X & Y & Z & T H X Y & T H Y Z & \text { THZX }\end{array}$

INTERAATTONAL

Calculation No. 457-2003.3

Revision 0

Page B25 of 37 
Appendix A9.2.2 Immersion Pail Calculation 457-2003.3 (Continued)

$\begin{array}{lllllll}2272 & 23.000 & -30.000 & 20.857 & 0.00 & 0.00 & 0.00 \\ 2596 & 21.000 & -30.000 & 20.808 & 0.00 & 0.00 & 0.00\end{array}$

DISPLAY COORDINATE SYSTEM SET TO O (CARTESIAN)

DEFINE A PATH FOR SUBSEQUENT CALCULATIONS THROUGH NODES:

22722596

PRINT LINEARIZED STRESS THROUGH A SECTION DEFINED BY LPATH COMMAND. DSYS= 0

$$
\begin{aligned}
& \text { *** POSTI LINEARUZED STRESS L.ISTING **** } \\
& \text { INSIDE NODE }=2272 \quad \text { OUTSIDE NODE }=\mathbf{2 5 9 6}
\end{aligned}
$$

$$
\begin{aligned}
& \text { LOAD STEP } \quad 1 \text { SUBSTEP= } \quad 1 \\
& \text { TIME }=1.0000 \quad \text { LOAD CASE }=0
\end{aligned}
$$

THE FOLLOWTNG $X, Y, Z$ STRESSES ARE IN GLOBAL COORDINATES.

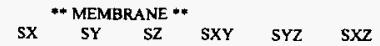

$$
\begin{aligned}
& \begin{array}{llllll}
-85.87 & 123.4 & -109.4 & 36.11 & -141.8 & 16.32
\end{array} \\
& \begin{array}{lllll}
\text { S1 } & 52 & 53 & \text { SNNT SEQV }
\end{array} \\
& \begin{array}{lllll}
192.8 & -78.97 & -185.7 & 378.6 & 338.1
\end{array}
\end{aligned}
$$

SX SY SZ SXY SYZ SXZ

$\begin{array}{lllll}1-805.1 & -2385 . & 694.7 & -1732 . & 0.1237 \mathrm{E}+05\end{array} 7148$.

C $0.0000 \mathrm{E}+00 \quad 0.0000 \mathrm{E}+00 \quad 0.0000 \mathrm{E}+00 \quad 0.0000 \mathrm{E}+00 \quad 0.0000 \mathrm{E}+00 \quad 0.0000 \mathrm{E}+00$

$\begin{array}{lllll}0 & 805.1 & 2385 & -694.7 & 1732\end{array}$

SI S2 S3 SINT SEQV

$10.1304 \mathrm{E}+05 \cdot 300.4-0.1584 \mathrm{E}+05 \quad 0.2888 \mathrm{E}+05 \quad 0.2507 \mathrm{E}+05$

C $0.0000 \mathrm{E}+00 \quad 0.0000 \mathrm{E}+00 \quad 0.0000 \mathrm{E}+00 \quad 0.0000 \mathrm{E}+00 \quad 0.0000 \mathrm{E}+00$

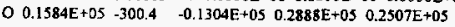

** MEMBRANE PLUS BENDING ** I=INSIDE C*CENTER O=OUTSIDE

$\begin{array}{llllll}S X & S Y & S Z & \text { SXY } & \text { SYZ } & \text { SXZ }\end{array}$

\begin{tabular}{|c|c|c|c|c|c|}
\hline & EAK * & [x[NS] & IDE C= & NTER & DUTSI \\
\hline SX & SY & $\mathrm{SZ}$ & $S X Y$ & SYZ & $S \times Z$ \\
\hline I -87.16 & 49.86 & -35.67 & 61.27 & -332.3 & -208.0 \\
\hline C 88.30 & -44.07 & 34.19 & -55.73 & 289.7 & 180.8 \\
\hline 08.675 & 58.61 & -34.08 & 82.29 & -545.0 & -343.0 \\
\hline SI & $\mathbf{S 2}$ & $\$ 3$ & SINT & SEQV & \\
\hline I 412.0 & -103.1 & .381 .9 & 793.9 & 697.6 & \\
\hline C 335.4 & 100.5 & -357.5 & 692.9 & 610,3 & \\
\hline
\end{tabular}

$\begin{array}{lllll}-2262 & 585.3 & -1696 & 0.1223 \mathrm{E}+05 & 7164\end{array}$

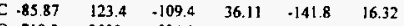

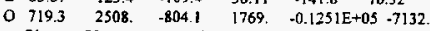

S1 S2 S3 SINT SEQV

I $0.1291 \mathrm{E}+05 \quad 238.6 \quad-0.1572 \mathrm{E}+05 \quad 0.2863 \mathrm{E}+0.05 \quad 0.2485 \mathrm{E}+05$

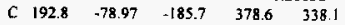

$00.1596 \mathrm{E}+05-363.8-0.1318 \mathrm{E}+05 \quad 0.2914 \mathrm{E}+05 \quad 0.2530 \mathrm{E}+05$

$$
\text { NAC } \text { IVTERNATIONAL }
$$


Appendix A9.2.2 Immersion Pail Calculation 457-2003.3 (Continued)

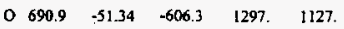

** TOTAL ** I=INSIDE C=CENTER O=OUTSIDE

$\begin{array}{llllll}\text { SX SY } & \text { SZ } & \text { SXY SYZ } & \text { SXZ }\end{array}$

J $-978.2 \quad-2212$. $549.6-1635$. $0.1190 E+056956$

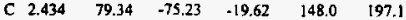

$\begin{array}{lllllll}0 & 727.9 & 2567 . & -838.2 & 1851 & -0.1306 E+05 & -7475\end{array}$

S1 S2 S3 SINT SEQV TEMP

I $0.1253 E-05 \quad 132.6 \quad-0.1531 E+05 \quad 0.2784 E+05 \quad 0.2416 E+05 \quad 0.0000 E+00$

$\begin{array}{lllll}\text { C. } 222.1 & 67.78 & -283.3 & 505.5 & 448.7\end{array}$

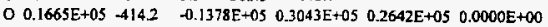

DISPLAY COORDINATE SYSTEM SET TO 1 (CYLNDRRCAL)

LIST ALL SELECTED NODES N RANGE 1847 TO 2392 STEP 545 DSYS $=1$

\begin{tabular}{|c|c|c|c|c|c|c|}
\hline DE & & & $Z$ & AXY & THYZ & X \\
\hline 1847 & 23.000 & -30.000 & 26.000 & 0.0 & $\begin{array}{ll}0 & 0.00\end{array}$ & 0.00 \\
\hline & 21.000 & -30.000 & 26.000 & 0.0 & $10 \quad 0.00$ & 0.00 \\
\hline
\end{tabular}

DISPLAY COORDINATE SYSTEM SET TO O (CARTESIAN)

DEFTNE A PATH FOR SUBSEQUENT CALCULATIONS THROUGH NODES

18472392

PRINT LINEARIZED STRESS THROUGH A SECTION DEFINED BY LPATH COMMAND. DSYS= 0

$$
\begin{aligned}
& \text {..... POSTI LINEARIZED STRESS LISTING ***** } \\
& \text { INSIDE NODE }=1847 \text { OUTSIDE NODE }=2392
\end{aligned}
$$

LOAD STEP 1 SUBSTEP= 1

TIME $=1.0000$ LOAD CASE $=0$

THE FOLLOWTNG $X, Y, Z$ STRESSES ARE IN GLOBAL COORDINATES.

$$
\begin{aligned}
& \text { "* MEMBRANE ** } \\
& \begin{array}{llllll}
S X & \text { SY SZ SXY SYZ SXZ }
\end{array} \\
& \begin{array}{llllll}
.7607 & 3503 & 363.6 & -5999 & -173.8 & -111.5
\end{array} \\
& \begin{array}{llll}
\mathrm{S} 1 & \mathrm{~S} 2 & \mathrm{~S} 3 & \mathrm{SINT} \\
6 \mathrm{SEQV}
\end{array} \\
& \text { 6126. } 364.1 \quad-0.1023 E+050.1636 E+05 \quad 0.1437 E+05 \\
& \text {-* BENDING ** j=INSIDE C=CENTER O=OUTSIDE } \\
& \begin{array}{llllll}
\text { SX } & \text { SY } & \text { SZ } & \text { SXY } & \text { SYZ } & \text { SXZ }
\end{array}
\end{aligned}
$$

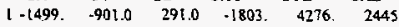

C $0.0000 \mathrm{E}+00 \quad 0.0000 \mathrm{E}+00 \quad 0.0000 \mathrm{E}+00 \quad 0.0000 \mathrm{E}+00 \quad 0.0000 \mathrm{E}+00 \quad 0.0000 \mathrm{E}+00$

$\begin{array}{lllllll}O & 1499 . & 901.0 & -291.0 & 1803 . & .4276 . & -2445 .\end{array}$

S1 S2 S3 SINT SEQV

I $4107.207 .5 \quad-6423.0 .1053 E+05 \quad 9221$.

C $0.0000 \mathrm{E}+00 \quad 0.0000 \mathrm{E}+00 \quad 0.0000 \mathrm{E}+00 \quad 0.0000 \mathrm{E}+00 \quad 0.0000 \mathrm{E}+00$

O 6423. $-207.5 \quad-4107$. $0.1053 \mathrm{E}+05 \quad 9221$.

** MEMBRANE PLUS BENDING ** I-RNSIDE C=CENTER O=OUTSIDE 


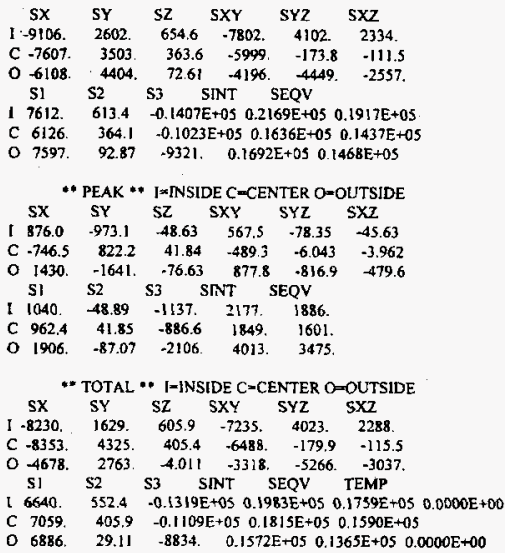

DISPLAY COORDINATE SYSTEM SET TO I (CYLINDRICAL)

LIST ALL SELECTED NODES IN RANGE 129 TO 742 STEP 613 DSYSa 1

\begin{tabular}{|c|c|c|c|c|c|c|}
\hline NODE & X & $Y$ & z & THXXY & THYZ & $T H Z X X$ \\
\hline 129 & 23.000 & -160.00 & 25.000 & 0.00 & 0.00 & 0.00 \\
\hline 74 & 21.000 & -160.00 & 26.000 & 0.00 & 0.00 & 0.00 \\
\hline
\end{tabular}

DISPLAY COORDINATE SYSTEM SET TO 0 (CARTESIAN)

DEFINE A PATH FOR SUBSEQUENT CALCULATIONS THROUGH NODES 129742.

PRINT LINEARIZED STRESS THROUGH A SECTION DEFINED BY LPATH COMMANO. DSYS= 0

**** POST! LINEARIZED STRESS LISTING ****

INSIDE NODE $=129$ OUTSIDE NODE $=742$

LOAD STEP I SUBSTEP $=$ I

TMME $=1.0000$ LOAD CASE $=0$

THE FOLLOWING $X, Y, Z$ STRESSES ARE IN GLOBAL COORDINATES.

\begin{tabular}{|l|l|}
\hline 1 NAC \\
\hline & $\begin{array}{l}\text { Calculation No. 457-2003.3 } \\
\text { Revision 0 }\end{array}$ \\
\cline { 2 - 2 } & Page B28 of 37 \\
\hline
\end{tabular}


Appendix A9.2.2 Immersion Pail Calculation 457-2003.3 (Continued)

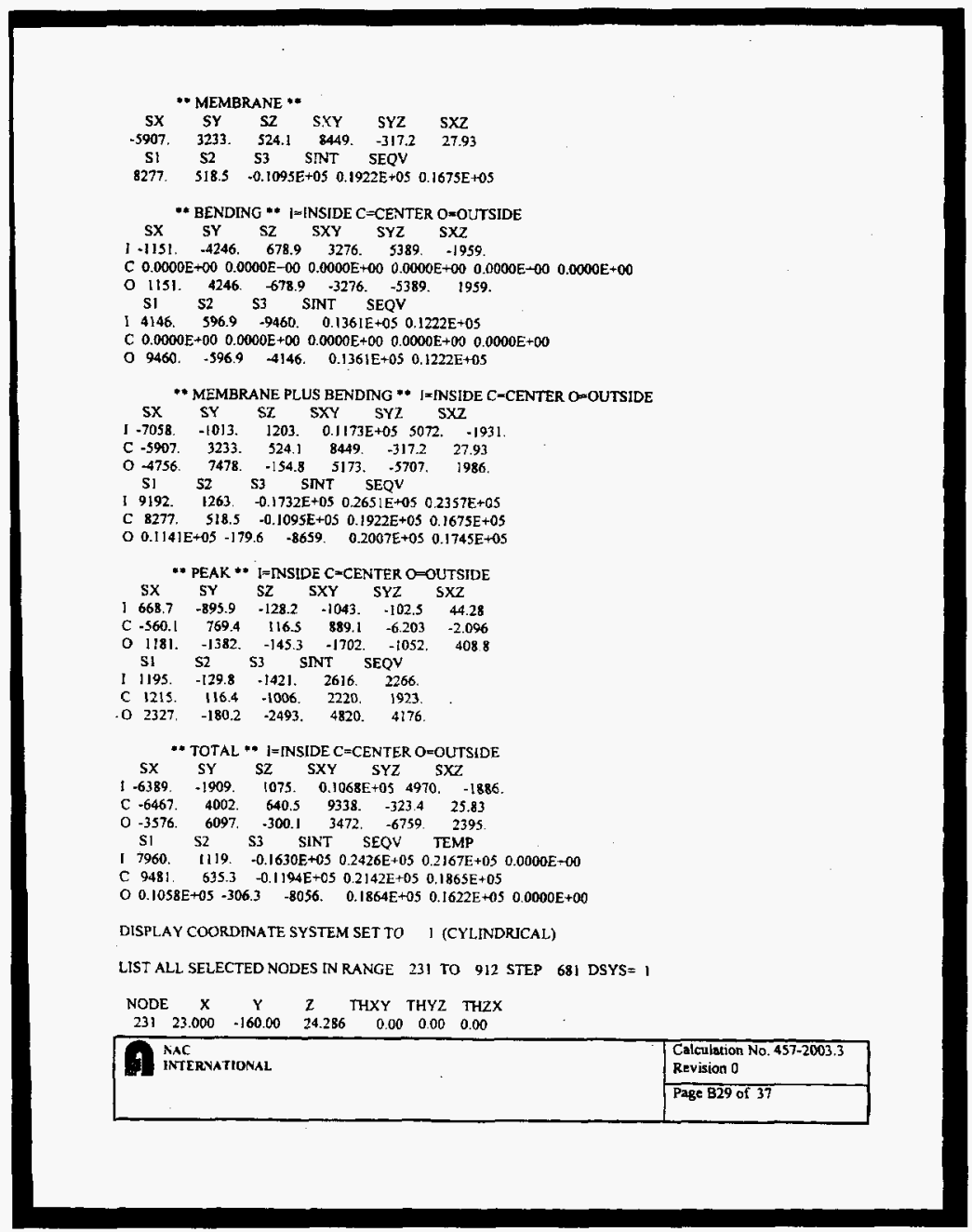


Appendix A9.2.2 Immersion Pail Calculation 457-2003.3 (Continued)

$\begin{array}{lllllll}912 & 21.000 & -160.00 & 24.260 & 0.00 & 0.00 & 0.00\end{array}$

DISPLAY COORDNATE SYSTEM SET TO O (CARTESIAN)

DEFINE A PATH FOR SUBSEQUENT CALCULATIONS THROUGH NODES:

231912

PRINT LINEARIZED STRESS THROUGH A SECTION DEFINED BY LPATH COMMAND. DSYS= 0

\#*** POSTI LINEARIZED STRESS LISTING ****

INSIDE NODE $=231$ OUTSIDE NODE $=912$

LOAD STEP I SUBSTEP = ।

TIME $=1.0000 \quad$ LOAD CASE $=0$

THE FOLLOWNG $X, Y, Z$ STRESSES ARE IN GLOBAL COORDINATES
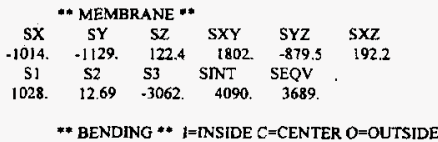

$\begin{array}{llllll}S X & 5 Y & S Z & S X Y & \text { SYZ } & \text { SXZ }\end{array}$

$\begin{array}{lllll}I .533 .2 & -5151 \text {. } 392.5 & 2619 . & 0.1423 \mathrm{E}+05 & -5183\end{array}$

C $0.0000 E+00 \quad 0.0000 \mathrm{E}+00 \quad 0.0000 \mathrm{E}+00 \quad 0.0000 \mathrm{E} \div 00 \quad 0.0000 \mathrm{E}+00 \quad 0.0000 \mathrm{E}+00$

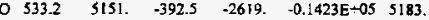

S1 S2 S3 SINT SEQV

I $0.1257 \mathrm{E}+0 \mathrm{~S} \quad 609.8 \quad-0.1847 \mathrm{E}+05 \quad 0.3104 \mathrm{E}+05 \quad 0.2712 \mathrm{E}+05$

C $0.0000 \mathrm{E}+00 \quad 0.0000 \mathrm{E}+00 \quad 0.0000 \mathrm{E}+00 \quad 0.0000 \mathrm{E}+00 \quad 0.0000 \mathrm{E}+00$

0 0.1847E-0.5 $-609.8 \quad-0.1257 \mathrm{E}+05 \quad 0.3104 E+05 \quad 0.2712 \mathrm{E}+0.5$

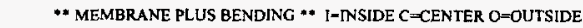

$\begin{array}{lllll}5 X & S Y & 5 Z & & \end{array}$

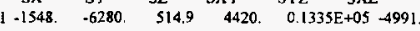

$\begin{array}{llllll}C-10 ! 4 & -1129 & 122.4 & 1802 . & -879.5 & 192.2\end{array}$

$\begin{array}{llllll}0-481.0 & 4022 . & -270.1 & -816.9 & -0.1511 E+05 & 5375 .\end{array}$

SI S2 S3 SINT SEQV

I $0.1103 E+05 \quad 367.7 \quad-0.1911 E+05 \quad 0.3014 E+05 \quad 0.2655 E+05$

$\begin{array}{ccccc}C & 1028 . & 12.69 & -3052.4090 .3689\end{array}$

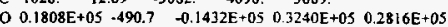

SX ** PEAK ** I=INSIDE C=CENTER O=OUTSIDE

$\begin{array}{lllll}S X & \text { SY SZ SXY SYZ SXZ }\end{array}$

$\begin{array}{llllll}35.60 & -96.44 & 6.536 & -261.1 & -410.3 & 160.0\end{array}$

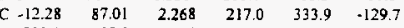

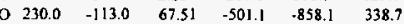

$51 \quad 52$ S3 SNT SEQV

I $541.0 \quad-117.2 \quad-478.1 \quad 1019.894 .9$

$\begin{array}{lllll}C .399 .4 & 110.0 & -432.4 & 831.8 & 731.4\end{array}$

$\begin{array}{llllll}\text { O } & 1199 . & -106.7 & -908.2 & 2108 . & 1843\end{array}$

9. NAC INTERNATIONAL

Calcularion No. 457-2003.3

Revision 0

Page $\bar{B} 30$ of 37 
Appendix A9.2.2 Immersion Pail Calculation 457-2003.3 (Continued)

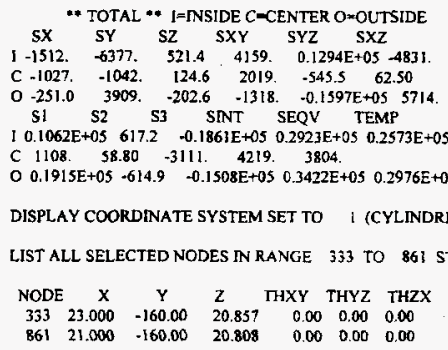

DISPLAY COORDINATE SYSTEM SET TO O (CARTESLAN)

DEFNE A PATH FOR SUBSEQUENT CALCULATIONS THROUGH NODES:

333861

PRINT LINEARIZED STRESS THROUGH A SECTION DEFINED BY LPATH COMMAND. DSYS= 0

***** POSTI LINEARIZED STRESS LISTING ****

INSIDE NODE - 333 OUTSIDE NODE = 861

LOAD STEP I SUBSTEP $=$ I

$T I M E=1.0000 \quad$ LOAD CASE $=0$

THE FOLLOWTNG $X, Y, Z$ STRESSES ARE IN GLOBAL. COORDINATES.

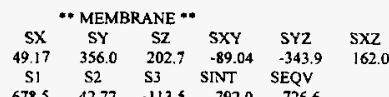

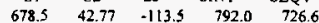

* BENDING * I-INSIDE C=CENTER O=OUTSIDE

SX SY SZ SXY SYZ SXZ

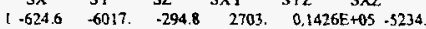

C $0.0000 \mathrm{E}+000.0000 \mathrm{E}+000.0000 \mathrm{E}+000.0000 \mathrm{E}+00 \quad 0.0000 \mathrm{E}+00 \quad 0.0000 \mathrm{E}+00$

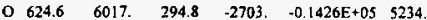

$\begin{array}{llll}S 1 & S 2 & \text { S3 SINT SEQV }\end{array}$

] $0.1186 \mathrm{E}+0.583 .7 \quad-0.1928 \mathrm{E}+05 \quad 0.3114 \mathrm{E}+05 \quad 0.2730 \mathrm{E}+05$

C $0.0000 \mathrm{E}+00 \quad 0.0000 \mathrm{E}+00 \quad 0.0000 \mathrm{E}+00 \quad 0.0000 \mathrm{E}+00 \quad 0.0000 \mathrm{E}+00$

O $0.1928 \mathrm{E}+05-483.7-0.1186 \mathrm{E}-0 \mathrm{05} \quad 0.3114 \mathrm{E}+05 \quad 0.2730 \mathrm{E}+05$

** MEMBRANE PLUS GENDNG * I=INSIDE C=CENTER O=OUTSIDE $\begin{array}{llllll}S X & S Y & S Z & S X Y & S Y Z & \text { SXZ }\end{array}$

$$
\begin{aligned}
& \text { NAC } \\
& \text { INTERNATIONAL }
\end{aligned}
$$


Appendix A9.2.2 Immersion Pail Calculation 457-2003.3 (Continued)

$\begin{array}{llllll}\text { I }-575.4 & -566 ! . & -92.14 & 2614 . & 0.1392 \mathrm{E}+05 & -5073\end{array}$

$\begin{array}{lllllll}\text { C } & 49.17 & 356.0 & 202.7 & -89.04 & -343.9 & 162.0\end{array}$

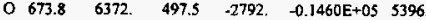

S1 S2 S3 SINT SEQV

$10.1177 \mathrm{E}+05 \quad 510.1-0.1860 \mathrm{E}+05 \quad 0.3037 \mathrm{E}+05 \quad 0.2659 \mathrm{E}+05$

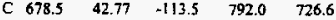

$00.1996 \mathrm{E}+05-457.0 \quad-0.1196 \mathrm{E}+05 \quad 0.3192 \mathrm{E}+05 \quad 0.2800 \mathrm{E}+05$

SX * PEAK ** I-INSIDE C=CENTER O=OUTSIDE

$\begin{array}{lllll}\text { SX } & \text { SY } & \text { SZ } & \text { SXY } & \text { SYZ } \\ 1.1038 & \text { SXZ }\end{array}$

$\begin{array}{lllll}84.7 & 59.17 & -72.21 & -403.3 & 142.6\end{array}$

$\begin{array}{llllll}C & 102.7 & -162.2 & -42.38 & 62.65 & 349.7\end{array}$

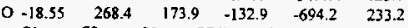

S1 S2 S3 SNN SEOV

$\begin{array}{llllll}1 & 563.0 & -115.5 & -307.4 & 870.4 & 792.1\end{array}$

$\begin{array}{llllll}\text { C } 273.2 & 110.4 & \mathbf{4 8 5 . 4} & 758.6 & 691.7\end{array}$

\begin{tabular}{llllll}
\hline & 982.8 & -69.48 & -489.7 & 1472. & 1314.
\end{tabular}

* TOTAL "* I-INSIDE C-CENTER O=OUTSIDE

$\begin{array}{llllll}S X & \text { SY } & \text { SZ } & \text { SXY } & \text { SYZ } & \text { SXZ }\end{array}$

$\begin{array}{llllll}1-679.3 & -5476 . & -32.97 & 2542 . & 0.1351 \mathrm{E}+05 & -4930\end{array}$

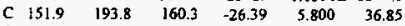

$\begin{array}{llllll}0 & 655.2 & 6641.671 .4 & -2925 & -0.1530 E+0 S & 5630\end{array}$

\$1 \$2 \$3 SNT SEQV TEMP

I $0.1146 \mathrm{E}+05393.9 \quad-0.1804 \mathrm{E}+05 \quad 0.2951 \mathrm{E}+05 \quad 0.2582 \mathrm{E}+05 \quad 0.0000 \mathrm{E}+00$

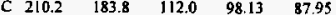

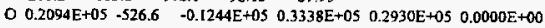

DISPLAY COORDINATE SYSTEM SET TO 1 (CYLNDRICAL)

LIST ALL SELECTED NODES NN RANGE 1367 TO 1486 STEP 119 DSYS $=1$

$\begin{array}{lccllll}\text { NODE } & X & Y & Z & \text { THXY } & \text { THYZ } & \text { THZX } \\ 1367 & 20.000 & -160.00 & 6.3900 & 0.00 & 0.00 & 0.00 \\ 1486 & 22.375 & -160.00 & 6.7500 & 0.00 & 0.00 & 0.00\end{array}$

DISPLAY COORDINATE SYSTEM SET TO O (CARTESIAN)

DEFWNE A PATH FOR SUBSEQUENT CALCULATIONS THROUGH NODES:

13671486

PRINT LINEARIZED STRESS THROUGH A SECTION DEFINED BY LPATH COMMAND. DSYS= 0

*..* POSTI LINEARIZED STRESS LISTING ****

INSIDE NODE $=1367$ OUTSIDE NODE $=1486$

LOAD STEP I SUBSTEP= ।

TIME $=1.0000$ LOAD CASE $=0$

THE FOLLOWING $X, Y Z$ STRESSES ARE IN GLOBAL COORDINATES

- MEMBRANE *.

NAC

INTERNATIONAL

Calculation No. 457-2003.3

Revision 0

Page 832 of 37 
Appendix A9.2.2 Immersion Pail Calculation 457-2003.3 (Continued)

$\begin{array}{cccccc}S X & S Y & S Z & S X Y & S Y Z & S X Z \\ 1146 . & -4760 & 860.1 & -992.8 & 658.2 & -301.0 \\ S 1 & S 2 & S 3 & \text { SINT } & \text { SEOV } & \\ 1504 . & 672.4 & -4930 . & 6433 . & 6061 .\end{array}$

* BENDING ** J=INSIDE C=CENTER $O=$ OUTSIDE

SX SY SZ SXY SYZ SXZ

I -819.4 -0.1003E+05-1645. 2069. -8455. 3117

C $0.0000 \mathrm{E}+00 \quad 0.0000 \mathrm{E}+00 \quad 0.0000 \mathrm{E}+000.0000 \mathrm{E}+00 \quad 0.0000 \mathrm{E}+00 \quad 0.0000 \mathrm{E}+00$

O $819.4 \cdot 0.1003 E+05$ 1645. -2069 . 8455. -3117

S1 S2 S3 SINT SEOV

I 4154 . $-608.0 \quad-0.1604 E+050.2020 E+050.1829 E+05$

C $0.0000 \mathrm{E}+00 \quad 0.0000 \mathrm{E}+00 \quad 0.0000 \mathrm{E}+00 \quad 0.0000 \mathrm{E}+00 \quad 0.0000 \mathrm{E}+00$

0 0.1604E+05 608.0 -4154 . D.2020E+05 D. $1829 E+05$

"* MEMBRANE PLUS BENDING *" [-INSIDE C*CENTER O-OUTSIDE

SX SY SZ SXY SYZ SXZ

J $326.6-0.1479 E+05-784.6 \quad 1276 .-7797.2816$.

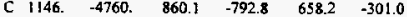

O 1966. 5274. 2505. -2862 . 9113. -3418.

S1 S2 S3 SINT SEQV

I $3940 . \quad-635.5 \quad-0.1856 \mathrm{E}+0.05 \quad 0.2250 \mathrm{E}+03 \quad 0.2059 \mathrm{E}+05$

C 1504 . $672.4-4930$. 6433: 6061 .

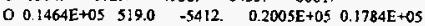

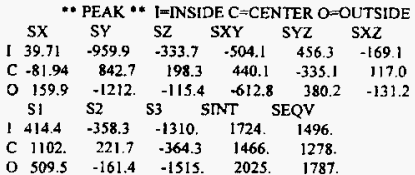

* total * I=INSIDE C=CenTER O=OUTSIDE

SX SY SZ SXY SYZ SXZ

$\begin{array}{llllll}1 & 366.3 & -0.1575 \mathrm{E}+05-1118 . & 772.2 & -7341 . & 2647\end{array}$.

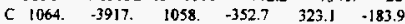

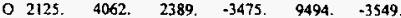

S1 S2 S3 SINT SEQV TEMP

1 3479. -1027. $-0.1896 \mathrm{E}+0 \mathrm{~S} \quad 0.2244 \mathrm{E}+0 \mathrm{~S} \quad 0.2056 \mathrm{E}+0 \mathrm{~S} \quad 0.0000 \mathrm{E}+00$

C 1289. 877.4 -3961. 5250. 5057 .

$00.1471 \mathrm{E}+05 \quad 180.8 \quad-6315 . \quad 0.2103 \mathrm{E}+05 \quad 0.1865 \mathrm{E}+05 \quad 0.0000 \mathrm{E}+00$

DISPLAY COORDINATE SYSTEM SET TO 1 (CYLINDRICAL)

LIST ALL SELECTED NODES NN RANGE 1373 TO 1492 STEF 119 DSYS $=1$

\begin{tabular}{|c|c|c|c|c|c|c|}
\hline NODE & $\mathrm{x}$ & $Y$ & $z$ & THXY & THYZ & THZXX \\
\hline 1373 & 20.000 & -130.00 & 6.3900 & 0.00 & $\begin{array}{ll}0 & 0.00\end{array}$ & 0.00 \\
\hline 1492 & 22.375 & -130.00 & 6.7500 & 0.00 & 0.00 & 0.00 \\
\hline
\end{tabular}

\begin{tabular}{|l|l|}
\hline 9 NAC \\
INTERYAJONAL & $\begin{array}{l}\text { Calculation No. 457-2003.3 } \\
\text { Revision 0 }\end{array}$ \\
\cline { 2 - 2 } & \begin{tabular}{l} 
Page B33 of 37 \\
\hline
\end{tabular} \\
\hline
\end{tabular}


Appendix A9.2.2 Immersion Pail Calculation 457-2003.3 (Continued)

DISPLAY COORDINATE SYSTEM SET TO O (CARTESIAN)

DEFINE A PATH FOR SUBSEQUENT CALCULATIONS THROUGH NODES

13731492

PRINT LINEARIZED STRESS THROUGH A SECTION DEFINED BY LPATH COMMAND. DSYS $=0$

**** POSTI LINEARIZED STRESS LISTING *****

INSIDE NODE = 1373 OUTSIDE NODE $=1492$

LOAD STEP I SUBSTEP= ।

TIME $=1.0000$ LOAD CASE $=0$

THE FOLLOWING $X, Y, Z$ STRESSES ARE IN GLOBAL COORDNATES.

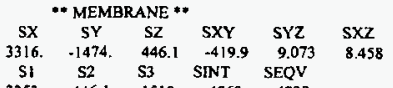

3353. $446.1 \quad-1510$. 4863 . 4238.

* BENDING * I=INSIDE C=CENTER $0=$ OUTSIDE

$\begin{array}{llllll}S X & S Y & S Z & S X Y & S Y Z & 5 X Z\end{array}$

I 5315. 552.2 1411. $-2029 . \quad-7795.9465$.

$\begin{array}{llllll}\text { C } 0.0000 \mathrm{E}+00 & 0.0000 \mathrm{E}+00 & 0.0000 \mathrm{E}+00 & 0.0000 \mathrm{E}+00 & 0.0000 \mathrm{E}+00 & 0.0000 \mathrm{E}+00\end{array}$

$0-5315$. $-552.2-1411$ 2029. 7795. -9465 .

S1 52 S3 SINT SEQV

I $0.1596 \mathrm{E}+05 \quad 509.3 \quad-9191 . \quad 0.2515 \mathrm{E}-05 \quad 0.2197 \mathrm{E}+05$

C $0.0000 \mathrm{E}+00 \quad 0.0000 \mathrm{E}+00 \quad 0.0000 \mathrm{E}+00 \quad 0.0000 \mathrm{E}+00 \quad 0.0000 \mathrm{E}+00$

0 9191. $-509.3-0.1596 E+05 \quad 0.2515 E+05 \quad 0.2197 E+05$

* MEMBRANE PLUS BENDING ** I-INSIDE C=CENTER O-OUTSIDE

SX SY SZ SXY SYZ SXZ

$\begin{array}{lllllll}\text { I } 8632 . & -921.7 & 1857 . & -2449 . & -7786 & 9474 .\end{array}$

$\begin{array}{lllllll}C & 3316 . & -1474, & 446.1 & -419.9 & 9.073 & 8.458\end{array}$

$\begin{array}{llllll}0.1999 . & -2026 . & -964.5 & 1609 & 7804 & -9457\end{array}$

\$1 \$2 S3 SNVT SEQV

$10.1770 E+05 \quad 674.1 \quad-8811 . \quad 0.2652 \mathrm{E}-0.50 .2327 \mathrm{E}+0.5$

$\begin{array}{lllll}C & 3353 . & 446.1 & -1510 . & 4863\end{array} 4238$.

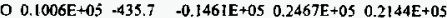

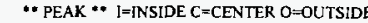

$\begin{array}{llllll}S X & S Y & S Z & \text { SXY SYZ SXZ }\end{array}$

$\begin{array}{lllllll}1 & 993.2 & -635.4 & -27.00 & 147.8 & 471.2 & -545.9\end{array}$

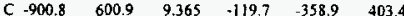

$\begin{array}{lllllll}O & 134] . & -907.5 & 19.10 & 157.7 & 430.4 & -475.4\end{array}$

S1 S2 S3 SINT SEQV

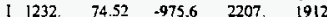

$\begin{array}{llllll}C & 818.8 & -55.31 & -1054 & 1873 . & 1623 .\end{array}$

$\begin{array}{lllll}\mathrm{O} & 1494 . & 78.64 & -1120 . & 2615\end{array} 2267$

\section{NAC}

INTERNATIONAL

Calculation No. 457-2003.3

Page $\mathrm{B34}$ of 37 
Appendix A9.2.2 Immersion Pail Calculation 457-2003.3 (Continued)

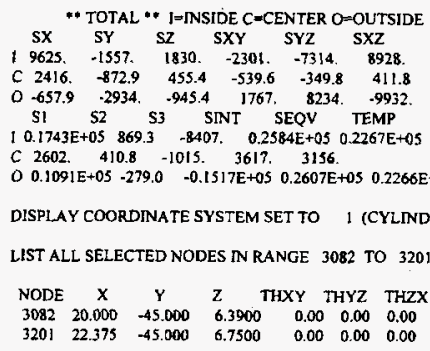

DISPLAY COORDNATE SYSTEM SET TO O (CARTESIAN)

DEFINE A PATH FOR SUBSEQUENT CALCULATIONS THROUGH NODES:

30823201

PRINT LINEARIZED STRESS THROUGH A SECTION DEFINED BY LPATH COMMAND. DSYS= 0

***** POSTI LINEARIZED STRESS LISTING ****

INSIDE NODE $=3082$ OUTSIDE NODE -3201

LOADSTEP I SUBSTEP $=1$

TIME $=1.0000$ LOAD CASE $=0$

THE FOLLOWNG $X, Y, Z$ STRESSES ARE IN GLOBAL COORDINATES

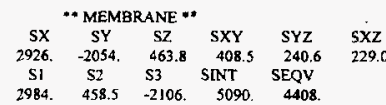

* BENDING ** j=INSIDE C=CENTER O־OUTSIDE

$\begin{array}{llllll}\text { SX } & \text { SY. } & \text { SZ } & \text { SXY } & \text { SYZ } & \text { SXZ }\end{array}$

$\begin{array}{lllllll}\text { I 3497. } & -700.0 & 926.7 & 1137 . & -9210 . & -9286 .\end{array}$

C $0.0000 \mathrm{E}+00 \quad 0.0000 \mathrm{E}+00 \quad 0.0000 \mathrm{E}+00 \quad 0.0000 \mathrm{E}-000.0000 \mathrm{E}+00 \quad 0.0000 \mathrm{E}+00$

$\begin{array}{llllll}0 & -3497 . & 700.0 & -926.7 & -1137 . & 9210\end{array} \quad 9286$

S) 52 S3 SNT SEQV

$10.1500 E+05 \quad 261.1-0.1154 E+05 \quad 0.2654 E+05 \quad 0.2303 E+05$

C $0.0000 \mathrm{E}+000.0000 \mathrm{E}+00 \quad 0.0000 \mathrm{E}+00 \quad 0.0000 \mathrm{E}+00 \quad 0.0000 \mathrm{E}+00$

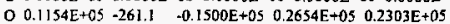

** MEMERANE PLUS BENDING ** l-INSIDE C=CENTER O=OUTSIDE

SX SY SZ SXY SYZ SXZ

$\begin{array}{llllll}\text { I } 6423 . & .2754 . & 1390 . \quad 1546 . & -8969 . & .9057 .\end{array}$

(1. NAC INTERIATIONAL


Appendix A9.2.2 Immersion Pail Calculation 457-2003.3 (Continued)

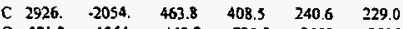

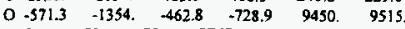

S1 S2 S3 SINT SEQV

I $0.1593 \mathrm{E}+05$ 377.1 $+0.1125 \mathrm{E}+05 \quad 0.2718 \mathrm{E}+05 \quad 0.2362 \mathrm{E}+05$

C 2984. $458.5 \quad-2106.5090 .4408$.

O $0.1235 E+05-236.5 \quad-0.1451 E+05 \quad 0.2686 E+0 S \quad 0.2328 E+05$

* PEAK * I=INIDE C=CENTER OニOUTSIDE

$\begin{array}{llllll}S X & S Y & S Z & S X Y & S Y Z & S X Z\end{array}$

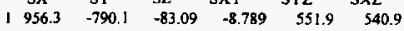

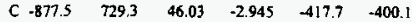

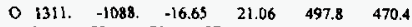

S1 S2 S3 SNNT SEQV

I 1210. $1.856 \quad-1129.2340 .2026$

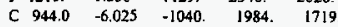

\begin{tabular}{lllll}
\hline & 1473. & 26.55 & -1293 &
\end{tabular}

* TOTAL ** I=INSIDE C-CENTER O=OUTSIDE

$\begin{array}{lllll}S X & S Y & S Z & & \end{array}$

I 7379. -3544 , 1307. 1537, $-8417, \quad-8516$,

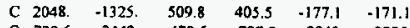

$\begin{array}{lllllll}0 & 739.6 & -2442 . & -479.5 & -707.9 & 9948 . & 9985 .\end{array}$

S1 S2 S3 SINT SEQV TEMP

I $0.1554 \mathrm{E}+05 \quad 489.1 \quad-0.1089 \mathrm{E}+05 \quad 0.2642 \mathrm{E}+05 \quad 0.2296 \mathrm{E}+05 \quad 0.0000 \mathrm{E}+00$

C 2119 . $500.0-1386$. 3505. 3038 .

$0.1318 \mathrm{E}+05-153.4-0.1521 \mathrm{E}+0.50 .2839 \mathrm{E}+05 \quad 0.2460 \mathrm{E}+05 \quad 0.0000 \mathrm{E}+00$

DISPLAY COORDNATE SYSTEM SET TO I (CYLINDRICAL)

LIST ALL SELECTED NODES IN RANGE 2184 TO 2474 STEP 290 DSYS=

$$
\begin{array}{cccccccc}
\text { NODE } & X & Y & Z & \text { THXY } & \text { THYZ } & \text { THZX } \\
2184 & 22.550 & -44.860 & 23.714 & 0.00 & 0.00 & 0.00 \\
2474 & 21.000 & -45.000 & 23.680 & 0.00 & 0.00 & 0.00
\end{array}
$$

DISPLAY COORDNATE SYSTEM SET TO O (CARTESIAN)

DEFTE A PATH FOR SUBSEQUENT CALCULATIONS THROUGH NODES

21842474

PRINT LINEARIZED STRESS THROUGH A SECTION DEFINED BY LPATH COMMAND. DSYS= 0

**** POSTI LINEARIZED STRESS LISTING *****

INSIDE NODE $=2184$ OUTSIDE NODE $=2474$

LOAD STEP I SUBSTEP

TIME $=1.0000$ LOAD CASE $=0$

THE FOLLOWTNG X,Y,Z STRESSES ARE IN GLOBAL COORDINATES.

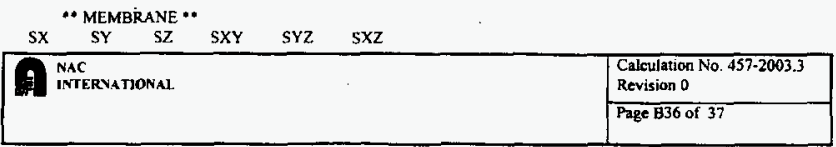


Appendix A9.2.2 Immersion Pail Calculation 457-2003.3 (Continued)

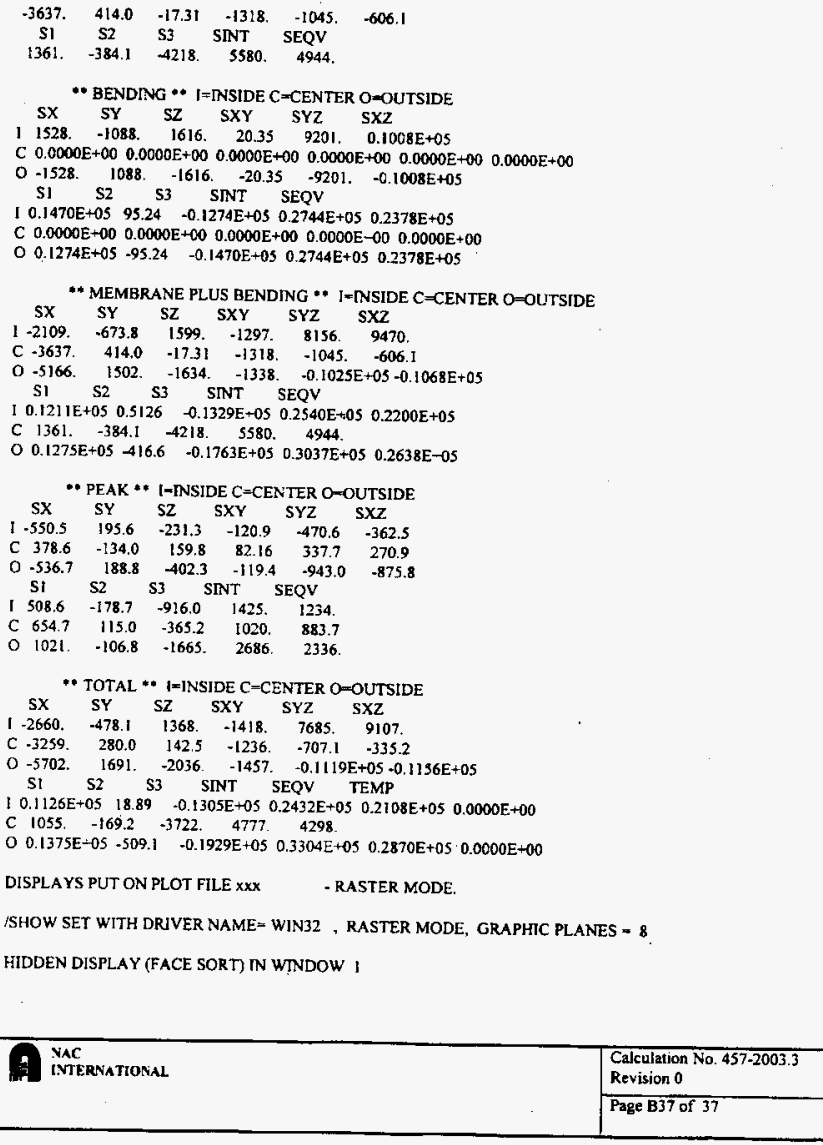


Appendix A9.2.2 Immersion Pail Calculation 457-2003.3 (Continued)

\author{
APPENDIX C \\ List of Files on 3.5" Diskette
}

There a a total of 2 pages in Appendix C.

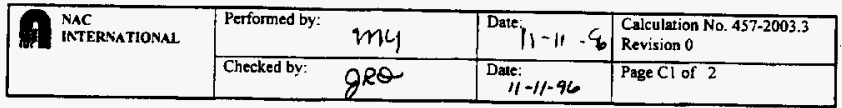


Appendix A9.2.2 Immersion Pail Calculation 457-2003.3 (Continued)

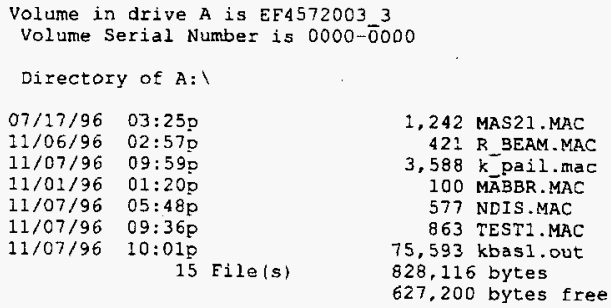


Appendix A9.2.2 Immersion Pail Calculation 457-2003.3 (Continued)

\section{APPENDIX D \\ ANSYS Software Verification}

There a a total of 12 pages in Appendix D.

Provided in Attachment D is the software verification package for ANSYS 5.2 on DEC-ALPHA XL266 Computer Serial No. 02207 . The verification test runs are listed in a table provided on page 11 . Due to the enormous number output pages, actual computer ran documentation has not been included with this appendix. Computer run documentation is maintained in fles located at NAC International in Norcross, Georgia

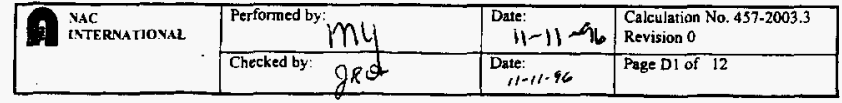


Appendix A9.2.2 Immersion Pail Calculation 457-2003.3 (Continued)

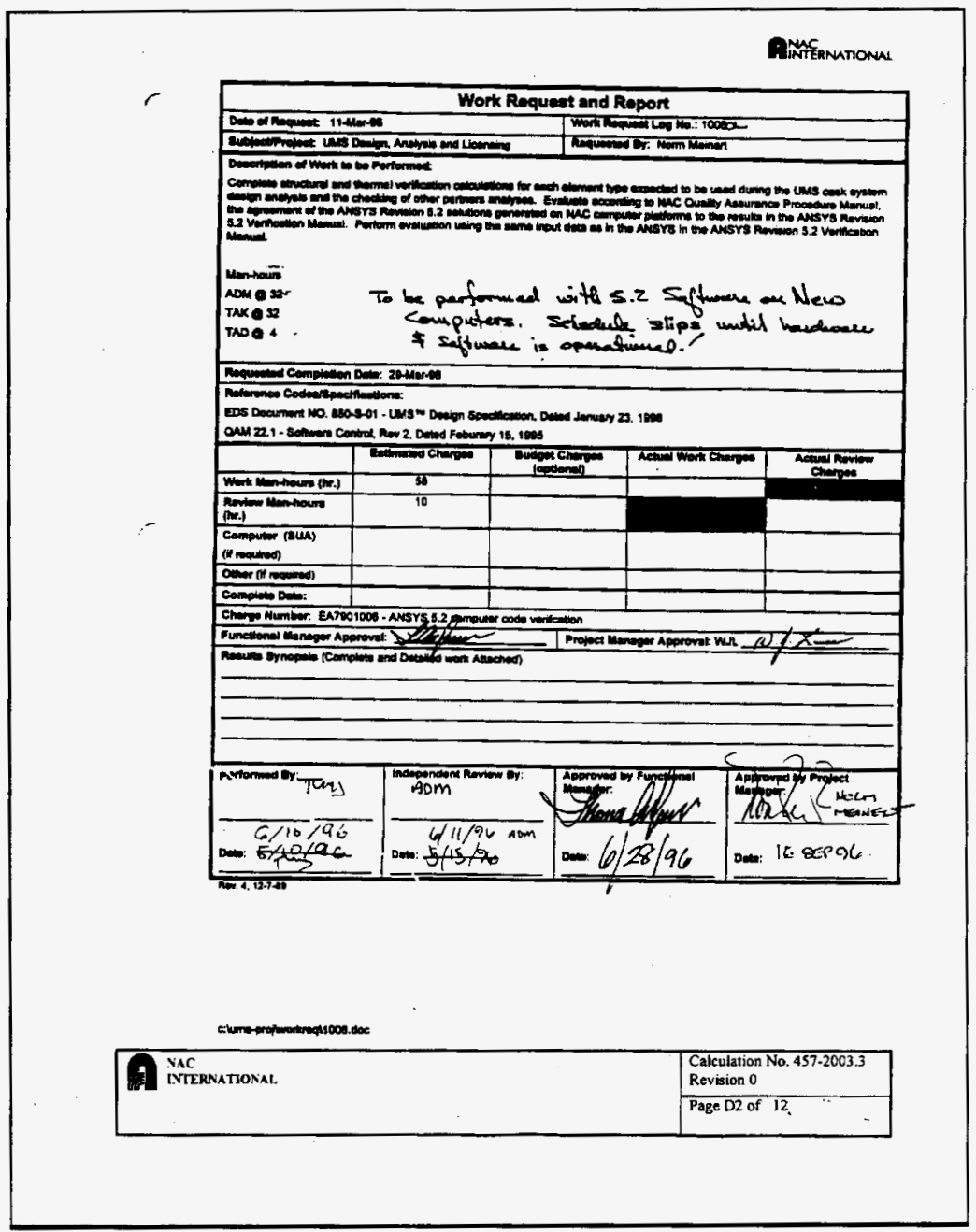


Appendix A9.2.2 Immersion Pail Calculation 457-2003.3 (Continued)

\section{DESIGN REVIEW CHECKUST}

Work Requeet Number: 10029

Scope of Analyale Flle:_ANSYS Verification

Fovlow Methodology: Check of Calculations:

Atemate Anakzis:

Others (oxplain):

Conflrm that the Work Pequent and Report lecludiea:

1. Statement of Purpose $\ldots \ldots \ldots \ldots \ldots \ldots \ldots \ldots \ldots \ldots \ldots \ldots \ldots \ldots \ldots \ldots \ldots$

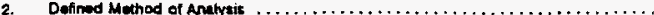

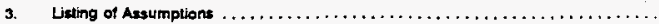

4. Detolled Anatysis Record $\ldots \ldots \ldots \ldots \ldots \ldots \ldots \ldots \ldots \ldots \ldots \ldots \ldots \ldots \ldots \ldots$

5. Shatemont of Conchusions with appropriate recommendationis as epplicable .......

Step Aetivine $\quad$ Vorifiention
Yos No N/A Commonts

1 For the seope of the defined analyses:

- Are the required input data complete?

- Material Properties

- Geomeiry (Drawing Reference)

- Loading/Source Tem

If a supporting anslysis is required to define the

load state, hus it been verifed?

- Are Boundary conditions acceotable?

2 Is the mothod of analysis adeaute tor the defined scope?

3 Is the worst case laading/configuration documented?

4 Are the aceeplanes etheris dofined and eomplate?

5 Hes all concurrant las ding bean considered?

6. Afe analyes tonsistent with previous WRR for method and approash?

7 Ave the records for tnpul and Output complate?

- Is treceability to vahidated sotwere complete?

9 Is the Statement of Conclusions and recommendations complete and accoptabile tor the project and objectives of the defined purpose?

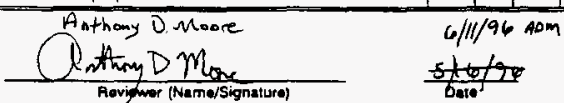

T. Th Aovifwer (Name/Signature) 
Appendix A9.2.2 Immersion Pail Calculation 457-2003.3 (Continued)

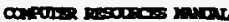

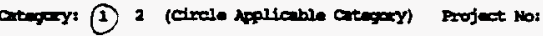

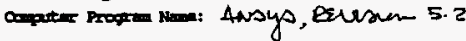

author: $\Delta$ nsys, Ine

Repanable Frograr Dnginer: $N / A$

Bardare syster (a) ware inctalled:

DeC - $\triangle 4 M A$ KL 266

(grans no orzot)

Progra Dacription:

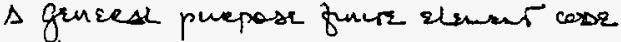

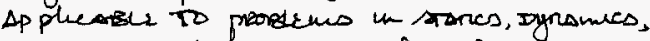

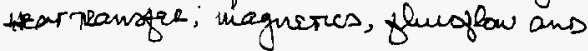
scersics.

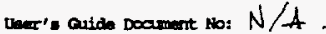

Arocound Br:

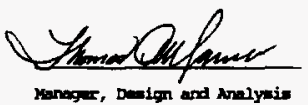




$$
\text { HNF-SD-SNF-FDR-003 Rev. } 0
$$

Appendix A9.2.2 Immersion Pail Calculation 457-2003.3 (Continued)

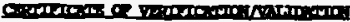

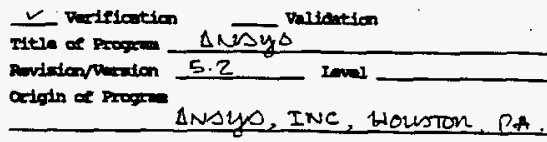

Brief Deaription of Propern

ANOYS is a gareal plepose hinir slement cone

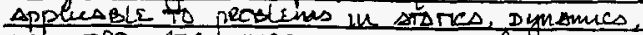

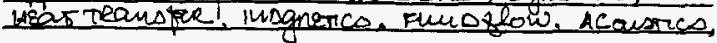

Ineted Fue which hpolichtion

Deverleas + Tberanux

Nothod ond to veley/validate Progen

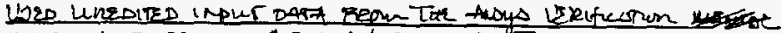

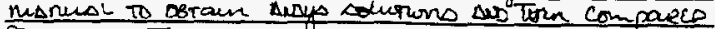

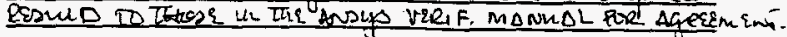

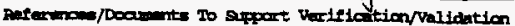

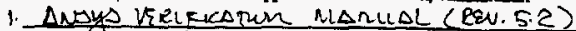

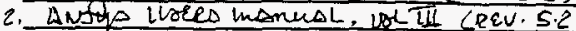

?. Whit number uma-1006

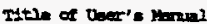
LNMYs lates manual

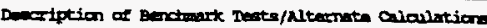
$N / A$

Peroud or The

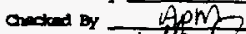
Dote $6 / 10 / 96$

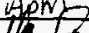

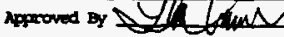

ranager, Desion and melyais.

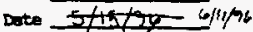
Dete $6 / 279$ 
Appendix A9.2.2 Immersion Pail Calculation 457-2003.3 (Continued)

\section{ANSYS, REVISION 5.2, COMPUTER CODE VERIFICATION ON DEC-ALPHA XI-266 COMPUTER; SERIAL NO: 02207}

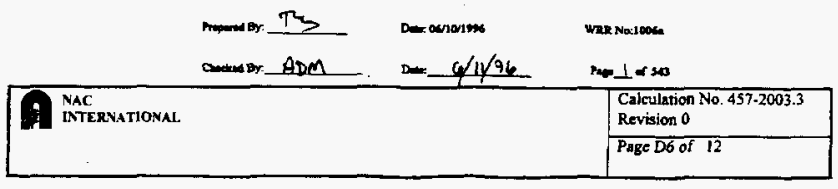


Appendix A9.2.2 Immersion Pail Calculation 457-2003.3 (Continued)

\section{TABLE OF CONTENTS}

Centents

Overview

Objective

Methodology

Result Comprison

Conclusion

Test Ceses and Result Comparison Table

References

Test Ceses Input and Output Listing

List of ANSYS Element in the Verification Procedure
Page

î̀

iii

in

iv

v

vi

vii

1

App. A

\begin{tabular}{|c|c|c|c|}
\hline & mand $\frac{T}{\text { And }}$ & 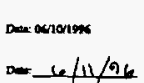 & 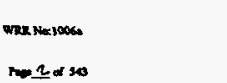 \\
\hline \multirow[t]{2}{*}{$\begin{array}{l}\text { NAC } \\
\text { INTERNATIONAL }\end{array}$} & & & $\begin{array}{l}\text { Calculation No. } 457-2003.3 \\
\text { Revision } 0\end{array}$ \\
\hline & . & & Page D7 of 12 \\
\hline
\end{tabular}


Appendix A9.2.2 Immersion Pail Calculation 457-2003.3 (Continued)

\subsection{OVERVTEW}

ANSYS, Rovision $\$ .2$ (ANSYS), an umpablithed, general purpose finite dement computer code developed by ANSYS, Ine., is arrenty instalted on two DEC-ALPHA, XL-266 computer workstutions serial mumbers 02207 and 02209 . ANSYS is delievered to NAC precompiled, with inaccessible source codzs and sufficiently thorough guidelines on installation and compther hardware requiremenes. The ANSYS code os DEC-ALPHA, XL-266 computer plation serial number 02207 is verified in this work request for structural and thermal disciplines in accordance with NAC International Quality Ateurance procedures.

\subsection{OBJECTIVE}

ANSYS, Inc. documented the verification of a wide range of ANSYS elements end cepabilities in the ANSYS Verification Merun (Ref. 1) for arightorward problems with classien or readilyavaithbie theoretical solutions. The verifiention of the program, according to ANSYS Inc, is conducted in line with writen procedure that form part of an overall Quelity Astarance program a ANSYS, Inc. The main objective of this package, therefore, is to farther verify the quality of ANSYS solutions obtained on DEC-ALPHA X工-266 computer platiorm seriel number 02207 to similar ANSYS solutions documented in the ANSYS Verification Manual (Ref. 1).

\subsection{METHODOLOGY}

A set of themal and structural elements (Appendix A) that are commonly used for struetural and heat transfer analyses in the EDS group are elected from the ANSYS Revision 5.2 element library for verification. The ANSYS Verification Mamal (Ref.1) demonstrates a wide range of ANSYS elements and capabilities in simple problems which have classical or readily obtainabie theortical solutions, and it dennonstrates, futhermore, the close agreement of the ANSYS solutions to the theoretical results i.e., texbooks or technical publications. The appitienble problems documented in the ANSYS Verification Mamual serve as the basis for additional

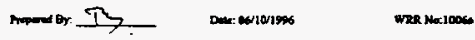

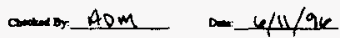

$\ln 3$ or 300

NAC INTERNATIONAL

Calculation No. 457-2003.3

Revision 0

Page D8 of 12 
Appendix A9.2.2 Immersion Pail Calculation 457-2003.3 (Continued)

verification and qualification of ANSYS capabilities for mucturl and heat transfer applications on the DEC-ALPHA XL-266 computer platform verial number 02207.

The selected ANSYS elements and capabilities that are specific to EDS enginecring applications are verified using the input dat from the ANSYS Verification Manal for all applicabie elements. The relewar inpul dona used for the rerification were extracted from the ANSTS Verification Manual arrabase (Ref. 2), and execuled for ANSYS solution without any madification to the inpuf data. The ANSYS solution obtained on the DEC-ALPA. XI266 computer platform serial mumber 02207 for selented ANSYS elements and apabitities were then compered to similar sohtions documented in the ANSYS Verification Mamul. Totel of 50 scrictural and heas transer test cases, some involving more than one element type in a single andyais pass, were examined.

\subsection{RESULT COMPARUSON}

The verification of the ANSYS code on DEC-ALPHA X1266 computer pietform serial mumber 02209 is completed and documented in work request number UMS-1006 (Ref. 3). The verification package in work request number UMS 1006 comperes andyes solutions from the DEC-ALPHA XI266 computer plasform seriel mumber 02209 to similar solutions in the ANSYS Verification Manual and presented the result in terms of percentage differences between the two ANSYS solution outputs. The DEC-ALPHA XI.266 computer piatform verial number 02207 upon which the ANSYS verification is performed in this work request is identical to the DEC. ALPHA XI.266 computer serial number 02209 upon which ANSYS is verified in work request number UMS 1006. It follows, therefore, that the ANSYS solutions obtained from DEC-ALPHA XI.265 serial tumber 02207 in this work request (WRR 1006a) must be identical to the ANSYS solution obtained from DEC-ALPHA XI266 computer serial number 02209 in work request number UMS-1006 (Ref. 3), given identical problems and hierachy of ANSYS commands in the analysis input streams. This is the corollary upon which the verification of the ANSYS code on DEC-ALPHA XZ266 computer seriaj mumber 02209 in this work request is based. In line with the asserted frollary, the verification test case and result table below shows that there is no difference in the ANSYS solution in this package for DEC-ALPHA XI266 computer platform

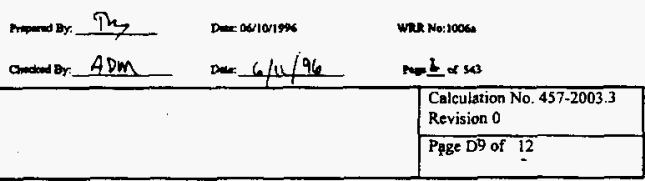


Appendix A9.2.2 Immersion Pail Calculation 457-2003.3 (Continued)

serial number 02207 and the ANSYS rolution in the andysis peckage mumber UMS-1006 for DEC-ALPHA XI.266 computer platform serial number 02209.

5.0 CONCLUSION

ANSYS, Revision 5.2 finite elemenr computer code that is aurently installed on the DECALPHA X1.266 conputer platform, eeril mumber 02207, gives solution that are in agreement with the ANSYS solution documeated in the ANSYS Verification Mnnual for all related identified NAC verification test cases.

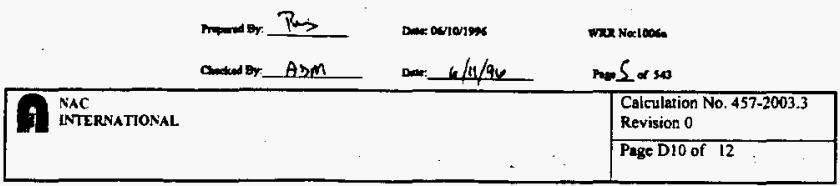


Appendix A9.2.2 Immersion Pail Calculation 457-2003.3 (Continued)

\section{VERIMCATION TEST CASES AND RESULTS TABLE}

(DEC-ALPHA XZ266 COMIUTER SERIAL * 02207

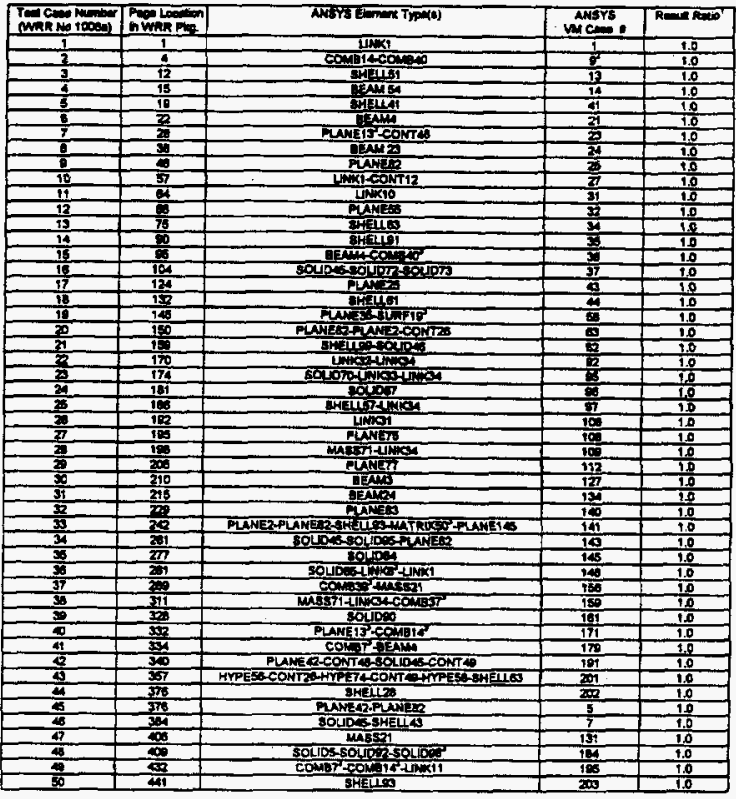

Nate:

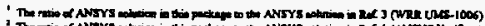

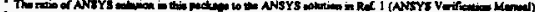

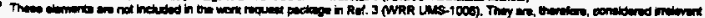

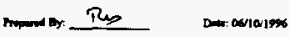

Whe No:1006e

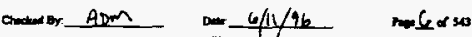


Appendix A9.2.2 Immersion Pail Calculation 457-2003.3 (Continued)

\section{REFERENCES}

1.0 ANSYS Revition 5.2 Verification Mamul, ANSYS, Inc.

2.0 DEC-ALPHA X1.266 Computer Platform; D: lansys52ldatalverif (Computer Serial No 02207)

3.0 "ANSYS Revision S.2 Verification" DEC-ALPHA XI.266 Computer, Serial No. 02209 WRR No. UMS-1006, Dated 5/2/96, Prepared By Anthony Moore.

$$
\begin{aligned}
& \text { mperses: The wer No:1006e }
\end{aligned}
$$

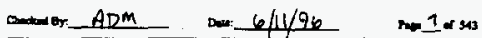


Appendix A9.2.3 Support Structure Calculation 457-2005.2

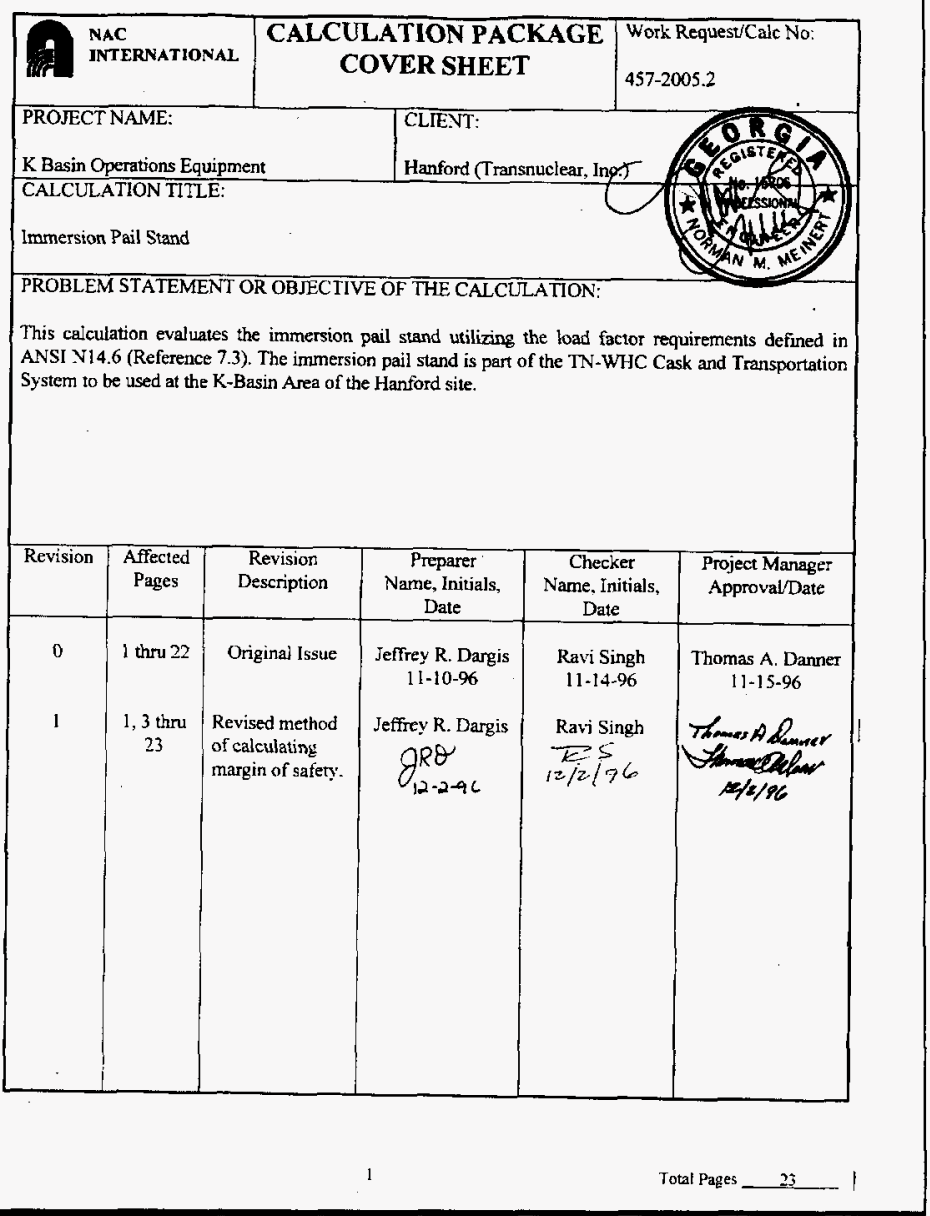


Appendix A9.2.3 Support Structure Calculation 457-2005.2 (Continued)

\section{INDEPENDENT DESIGN VERIFICATION CHECK SHEET}

Work Request/Calculation No: 457-2005.2 Revision 으

Scope Of Analysis File: This calculation evaluates the immersion pail stand stand uthizing the load factor requirements defined in ANSI N14.6.

Review Methodology: Check Of Calculations

Altemate Analyses

Other (Explain)

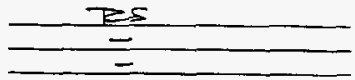

Confim That The Work Request / Calculation Package Reviewed Includes:

1. Statement of Purpose

2. Defined Method of Analysis

3. Listing of Assumptions

4. Detailed Analysis Record

5. Statement of Conclusions / Recommendations (if applicable)

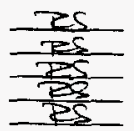

\begin{tabular}{|c|c|c|c|c|c|}
\hline Step & Activltian & & tition & & \\
\hline 1 & $\begin{array}{l}\text { For the scope of the deined analysis: } \\
\text { A. Are the required data input complete? } \\
\text { 1. Material properties } \\
\text { 2. Geometry (drawing reterence) } \\
\text { 3. Loading source term } \\
\text { If a supponting analysis is nequired to } \\
\text { define the tood state, has it boen } \\
\text { defined? } \\
\text { B. Are boundary conditions acceptable? }\end{array}$ & $\mathscr{L}$ & & $\sin$ & Comingores \\
\hline 2 & Is the method of analysis adequate for the defined scope? & $\checkmark$ & & & \\
\hline 3 & Is the worst case loadingiconfiguration documented? & $\checkmark$ & & & \\
\hline 4 & Are the acceptance criteria defined and complete? & $\checkmark$ & & & \\
\hline 5 & Has all concurrent loading been considered? & $\checkmark$ & & & \\
\hline 6 & $\begin{array}{l}\text { Are analyses consistent with previous work for method and } \\
\text { approach? }\end{array}$ & & & $\checkmark$ & $\begin{array}{l}\text { NEW CALC } \\
\text { PACKAGE. }\end{array}$ \\
\hline 7 & Are the records for input and output complete? & $\checkmark$ & & & \\
\hline 8 & Is traceability to verifed software complete? & & & $\checkmark$ & $\begin{array}{l}\text { NO SOFTWAEE } \\
\text { USED. }\end{array}$ \\
\hline 9 & $\begin{array}{l}\text { Is the statement of conclusions and recommendations complete } \\
\text { and acceptable for the project and objectives of the defined } \\
\text { purpose? }\end{array}$ & & & & \\
\hline & 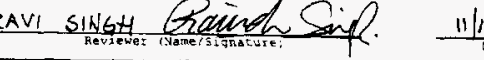 & 16 & & & \\
\hline
\end{tabular}


Appendix A9.2.3 Support Structure Calculation 457-2005.2 (Continued)

\section{INDEPENDENT DESIGN VERIFICATION CHECK SHEET}

Work Request/Caiculation No: 457-2005.2 Revision 1

Scope Of Analysis File: This calculation evaluates the immersion pail stand stand utilizing the load factor requirements defined in ANSI N14.6.

Review Methodology: Check Of Calculations

Alternate Analyses

Other (Explain)

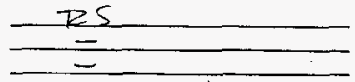

Confirm That The Work Request / Calculation Package Reviewed Includes:

1. Statement of Purpose

2. Defined Method of Analysis

3. Listing of Assumptions

4. Detailed Analysis Record

5. Statement of Conclusions / Recommendations (if applicable)

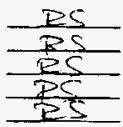

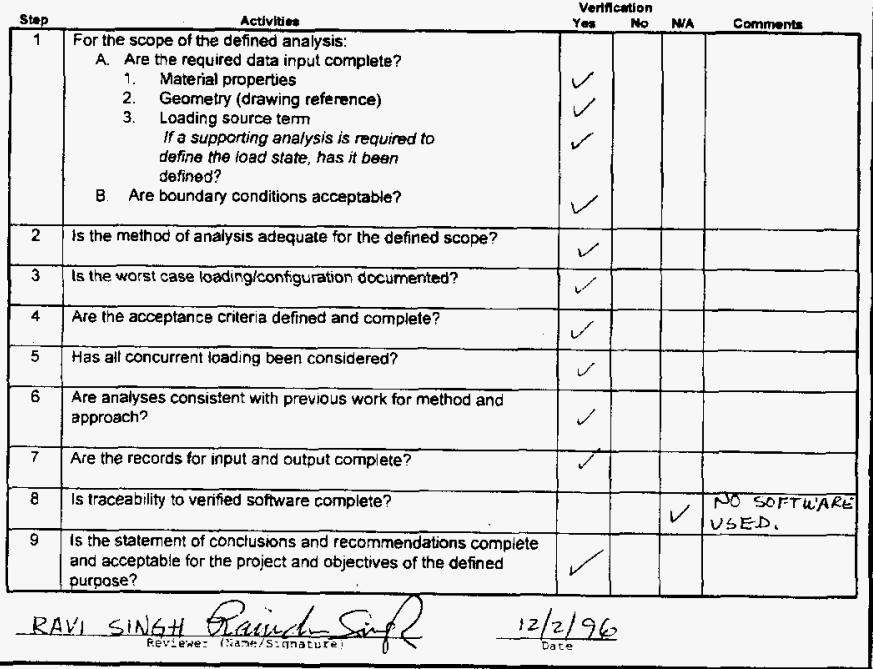

3 
Appendix A9.2.3 Support Structure Calculation 457-2005.2 (Continued)

TABLE OF CONTENTS

\begin{tabular}{|c|c|}
\hline Section & Description \\
\hline$\ldots$ & 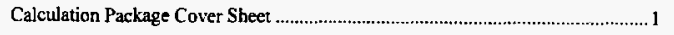 \\
\hline- & Independent Design Verification Check Sheet ... \\
\hline-- & 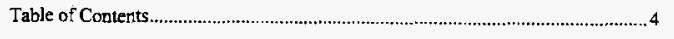 \\
\hline 1.0 & 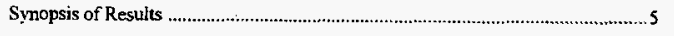 \\
\hline 2.0 & 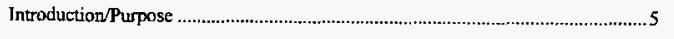 \\
\hline 3.0 & Method of Analysis \\
\hline 4.0 & 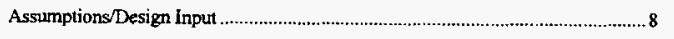 \\
\hline 5.0 & 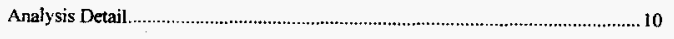 \\
\hline 6.0 & 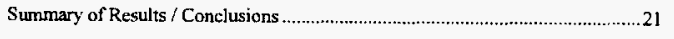 \\
\hline 7.0 & References \\
\hline
\end{tabular}

\begin{tabular}{|c|c|c|c|}
\hline VINC & Perfomed by: $g R \theta$ & $\begin{array}{l}\text { Date. } \\
11-\alpha 5-96\end{array}$ & $\begin{array}{l}\text { Calculation No. } 457-2005.2 \\
\text { Revision ? }\end{array}$ \\
\hline & Checked by: $\rightleftharpoons 5$ & $11 / 25 / 56$ & Page 4 of 23 \\
\hline
\end{tabular}


Appendix A9.2.3 Support Structure Calculation 457-2005.2 (Continued)

1.0

SYNOPSIS OF RESULTS

Summary of Stress Analysis

\begin{tabular}{|c|c|c|c|c|c|c|c|}
\hline $\begin{array}{l}\text { Drawing } \\
\text { No. }\end{array}$ & $\begin{array}{l}\text { Item } \\
\text { No. }\end{array}$ & Component & $\begin{array}{l}\text { Applied } \\
\text { Load }\end{array}$ & $\begin{array}{l}\text { Design } \\
\text { Check }\end{array}$ & $\begin{array}{l}\text { Calculated } \\
\text { Loading }\end{array}$ & Allowable & M.S. \\
\hline 457.102 & $\begin{array}{l}98, \\
99\end{array}$ & $\begin{array}{l}\text { frame stand } \\
\text { assembly }\end{array}$ & $5 \mathrm{~W}$ & buckling & 300 kips & 1,064 kips & 0.72 \\
\hline $457-102$ & $\begin{array}{l}4,7 \\
8,9 \\
15 \\
16 \\
17 \\
18\end{array}$ & tie plates & $5 \bar{W}$ & compression & $480 \mathrm{psi}$ & $694 \mathrm{psi}$ & 0.31 \\
\hline $457-102$ & 5 & comet post & $5 w$ & buckling & $72,500 \mathrm{dbs}$ & $75,000 \mathrm{lbs}$ & 0.03 \\
\hline 457.109 & 4 & lock pin & $5 W$ & double shear & $49.0 \mathrm{ksi}$ & $81.0 \mathrm{ksi}$ & 0.40 \\
\hline 457.109 & 10 & $\begin{array}{l}\text { guide } \\
\text { assembly }\end{array}$ & $5 w$ & bearing & $16.0 \mathrm{ksi}$ & $54 \mathrm{ksi}$ & $0 . \overline{70}$ \\
\hline
\end{tabular}

The immersion pail support structure design meets the criteria defined in Section 4.2.

\subsection{INTRODUCTION / PURPOSE}

This calculation evaluates the immersion pail stand utilizing the load factor requirements defined in Reference 7.3. The immersion pail stand configuration is part of the TN-WHC Cask and Transportation System to be used at the K-Basin Area of the Hanford site. The immersion pail stand design was initially evaluated in Reference 7.1.

\subsection{METHOD OF ANALYSIS}

Hand calculations using classic textbook solutions are used to structurally evaluate the immersion pail suppon structure. Per Reference 7.14 , the design considers the entire pail resting weight will be distributed to two (2) of the four (4) corner column supports.

The design will be assessed using the load factors discussed in Section 4.2. The acceptance criteria defined in Reference 7.3 limits the lifting induced tensile stresses to the lesser of:

\begin{tabular}{|c|c|c|c|c|}
\hline NAC & Performed by: & $g R$ & $\begin{array}{l}\text { Date: } \\
1-25-96\end{array}$ & $\begin{array}{l}\text { Calculation No. 457.2005.2 } \\
\text { Revision I }\end{array}$ \\
\hline & Checked by: & $E S$ & Date 25196 & Page 5 of 23 \\
\hline
\end{tabular}


Appendix A9.2.3 Support Structure Calculation 457-2005.2 (Continued)

(a) one-third material yield strength; or

(b) one-fifth the material ultimate strength.

Shear stresses will be limited to 0.6 times the tensile stress limits.

Buckling loads will be determined based on a load factor of 5.0. The critical buckling load will be determined per Reference 7.10.

The following evaluations are documented within this calculation.

- Frame Stand Buckling Evaluation;

- Tie Plate Evaluation;

- Comer Post Assembly Buckling Evaluation;

- Guide Assembly Evaluation; and

- Base Assembly - Lpper Assembly Joint Evaluation.

\subsection{Erame Stand Buckling Evaluation}

This evaluation will determine the critical buckling load for the stand assembly as a single unit, (simulianeous buckling of the entire assembly using the combined cross section of all eight tube steel columns, tie plates excluded). The buckling criteria of Reference 7.10 .2 will be used. The applied load will be $5 x$ the dead weight loading.

To evaluate net stand buckling, the following steps are performed:

- The net cross section properties are determined;

- The $\mathrm{kl} / \mathrm{r}$ parameter is determined;

- The maximum allowable section axial stress is determined

- The critical buckling load is determined: and

- The applied axial load (5W) is compared to the critical buckling load.

\subsection{Tie Plate Evaluation}

Tie plates are used to reduce the buckling potential of the comer assemblies by making the mid-section (upper-to-lower stand section joint) behave as a frame unit and to assist in the installation'fabrication of the stand

The tie plates are concentrated at the mid stand height to ensure the midpoint can be treated as an inflection point (pinned-pinned end condition, no tendency for lateral movement under load) for the comer assembly buckling evaluation (See Section 3.3).

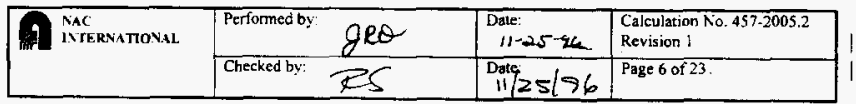


Appendix A9.2.3 Support Structure Calculation 457-2005.2 (Continued)

The following steps are performed:

- The size of the tie plates is determined;

- The required spacing between tie plates is determined:

- The tie plate design load is determined;

- The compression load on the tie plates is evaluated; and

- The tie plate welds are evaluated.

\subsection{Upper Frame Corner Post. Assemblv Buckling Evaluation}

Each comer post assembly is composed of two $4 \times 4$ tubing sections connected by comer plates. The comer plates are installed in pairs of two.

The buckling capacity is evaluated by determining the critical buckling length of the $4 \times 4$ tubing only. The length determined is then compared to the existing maximum length available to buckle in the critical mode.

The buckling length is determined by the following steps:

- The tube cross section properties are determined;

- The axial load is determined; and

- The maximum allowable load is determined and compared to the axial load

\subsection{Guide Assemblv Evaluation}

The guide assembly is comprised of a solid block element and a slide lock pin arrangement. By inspection the solid block guide and lock pin flange (with bolting) are adequate. Only the lock pin requires evaluation. For the lock pin, bearing and double shear design checks are evaluated.

\subsection{Base Assembly-Upper Assembly Joint Evaluation}

The base and upper stand assemblies are connected by a solid connector plug inserted within the $4 \times 4$ colurn tubing. The connector is full penetration welded to the base side tubing. The load path under loading conditions is through bearing at the tube steel-to-tube steel interface. The connector and its weldment serve alignment and connectivity functions only. No structural evaluation is required.

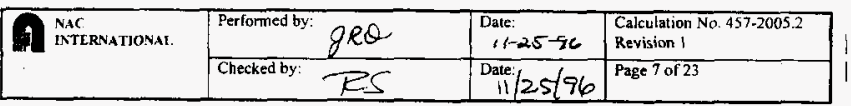


Appendix A9.2.3 Support Structure Calculation 457-2005.2 (Continued)

\subsection{ASSUMPTIONS / DESIGN INPUTS}

4.1 Assumptions

There are no unverified assumptions within this calculation.

4.2 Design Criteria

4.2.1 Tensile Stresses

Calculated tensile stress (based on load factor of 3) $\quad<S_{\text {yietit }} \quad$ (Ref. 7.3)

Calculated tensile stress (based on load factor of 5) $\quad \mathrm{S}_{\text {ulimate }}$ (Ref. 7.3)

\subsubsection{Shear Stresses}

Calculated shear stress (based on load factor of 3) $<0.6 \times \mathrm{S}_{\text {yicld }}$

Calculated shear stress (based on load factor of 5) $<0.6 \times \mathrm{S}_{\text {uttimate }}$

4.2.3 Bearing stresses

Calculated bearing stress $<1.5 \times \mathrm{S}_{y} \quad$ (Ref. 7.7.3)

4.2.4 Buckling Design

Calculated buckling load (based on load factor of 5) $<\mathrm{P}_{\mathrm{cr}}$

4.2.5 Material Properties / Stress Design Checks

All components evaluated within this WRR are made of $A 500, A 36$ or A654 carbon steel.

Poison's ratio $=0.3$

Modulus of Elasticity, $E=28.3 \times 10^{6}$ psi (Ref. 7.7)

Normalizing the material yield and ultimate strengths by the Reference 7.3 load factors yields the following (See Section 4.2.1 for load factors):

A500 GR B Carbon Stec]

(Reference 7.10.7)

F:: $\quad 46 \mathrm{ksi}$

Fu: $\quad 58 \mathrm{ksi}$

$\mathrm{F}_{\mathrm{y}} / 3=46,000 / 3=15,333 \mathrm{psi}$

$F_{v} / 5=58,000 / 5=11,600 \mathrm{psi}$

\begin{tabular}{|c|c|c|c|}
\hline $\begin{array}{l}\text { NAC } \\
\text { INIERSATIONAL }\end{array}$ & Performed by: QRQ & $\begin{array}{l}\text { Date: } \\
11-2-5-96\end{array}$ & $\begin{array}{l}\text { Calculation No. 457-2005.2 } \\
\text { Revision }\end{array}$ \\
\hline & Checked by: $\mathrm{ZS}_{S}$ & Date: $=56$ & Page $\overline{8}$ of 23 \\
\hline
\end{tabular}


Appendix A9.2.3 Support Structure Calculation 457-2005.2 (Continued)

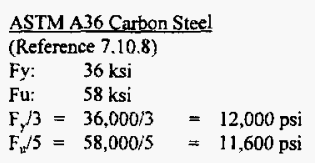

ASTM A564 Carbon Steel

Guide Pin Only

(Reference 7.15)

Fy: $\quad 105 \mathrm{ksi}$

Fu: $\quad 135 \mathrm{ksi}$

$\mathrm{F}_{\mathrm{y}} / 3=105,000 / 3=35,000 \mathrm{psi}$

$F_{u} / 5=135,000 / 5 \Rightarrow 27,000 \mathrm{psi}$

Based on the above comparisons of yield and uttimate allowables for the three (3) materials, comparing a factor (LF) of 5 against ultimate is more restrictive. Only | the limiting condition is evaluated within this WRR.

4.3 Design Conditons
Load:
$60,000 \mathrm{lbs}$
Temperature: Ambient, $100 \%$
Lifting: Two Point Lift (set) condition is design controlling per Reference 7.14.

4.4 Component Properties
4.4.1 Tube steel
(Reference 7.10.1)
size: $4 \times 4 \times 0.5$
I: $\quad 12.3$ in $^{4}$
$\mathrm{r}: \quad 1.39 \mathrm{in}$
area: $6.36 \mathrm{in}^{2}$
matl: A36

\subsubsection{Tie Plates}

size: $1 / 2$ " plate

\begin{tabular}{|l|l|l|l|}
\hline \multirow{2}{*}{$\begin{array}{l}\text { NAC } \\
\text { INTERNationil }\end{array}$} & \begin{tabular}{l} 
Performed by: gRO \\
\cline { 2 - 4 }
\end{tabular} & $\begin{array}{l}\text { Date: } \\
12-2-2\end{array}$ & $\begin{array}{l}\text { Calculation Ko. 457-2005.2 } \\
\text { Revision 1 }\end{array}$ \\
\hline
\end{tabular}


Appendix A9.2.3 Support Structure Calculation 457-2005.2 (Continued)

4.5 Elevations of the Fuel Pit (Reference 7.1)

Pit floor elevation: $\quad-25^{\prime}-9^{\prime \prime}$

Fuel floor elevation: $\quad-20 \div 9^{n}$

Load pit floor elevation: $\quad 0^{2}-0^{n}$

Top of pit wall elevation: $\quad+2^{\prime}-0^{\prime \prime}$

Water elevation: $\quad-4^{\prime}-9^{\prime \prime}$

5.0 ANALYSIS DETAIL

5.1 Frame Stand Buckling Evaluation

\subsubsection{Net Cross Section Properties}

The frame stand consists of eight (8) vertical $4 " \times 4^{\prime \prime} \times 0.5^{n}$ tube steel members. Since the $I$ and $r$ of a tube are the same for any orientation, consider the effective section as follows (Reference 7.6.2):

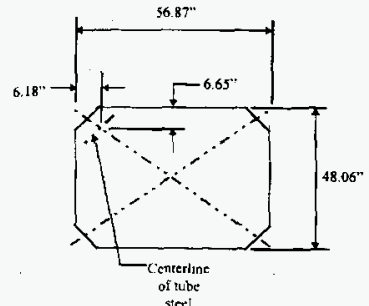

Per Reference 7.6.2:

$$
\begin{aligned}
\mathrm{L}_{1} & =56.87^{\prime \prime} \cdot\left(2 \times 6.18^{\prime \prime}\right) \\
& =44.51 \mathrm{in} \\
\mathrm{L}_{2} & =48.06^{\prime \prime}-\left(2 \times 6.65^{\prime \prime}\right) \\
& =34.76 \mathrm{in}
\end{aligned}
$$

\begin{tabular}{|c|c|c|c|}
\hline ANTERNATIONAI & Performed by: $8 R \theta$ & $\begin{array}{l}\text { Date: } \\
1 /-25-46\end{array}$ & $\begin{array}{l}\text { Calculation No. 457-2005.2 } \\
\text { Revision I }\end{array}$ \\
\hline & Checked by: $\mathrm{Ks}$ & $11 / 25 / 96$ & Page 10 of 23 \\
\hline
\end{tabular}


Appendix A9.2.3 Support Structure Calculation 457-2005.2 (Continued)

The moment of inertia of the effective section, $I$, is:

$$
\begin{aligned}
I & =\Sigma A y^{2}+\Sigma I \\
& =\left[(8 \times 6.36) \times(34.76 / 2)^{2}\right]+[8 \times(12.3)] \\
& =15,467 \mathrm{in}^{4} \\
r & =\sqrt{\frac{I}{A}} \\
& =\sqrt{\frac{15,467}{8 \times 6.36}} \\
& =17.44
\end{aligned}
$$

\section{1 .2 kij Parameter}

Due to the location of struts at each corner assembly of the upper stand, consider $k=0.8$ (Reference 7.10 .3 ). The length, 1 , is:

$$
\begin{aligned}
1 & =160^{\prime \prime}+156.5^{\prime \prime} \quad \text { (Reference 7.6.2) } \\
& =316.5^{\prime \prime} \\
& \equiv 317 \mathrm{in}
\end{aligned}
$$

\begin{tabular}{|c|c|c|c|}
\hline NAC & Performed by: $g R \theta$ & $\begin{array}{l}\text { Date: } \\
n-25-24\end{array}$ & $\begin{array}{l}\text { Calculation No. } 457.2005 .2 \\
\text { Revision I }\end{array}$ \\
\hline & Checked by: 25 & Date: $1125 / 96$ & Page 11 of 23 \\
\hline
\end{tabular}

Therefore:

$$
\begin{aligned}
k \mathrm{l} / \mathrm{r} & =0.8 \times(317) / 17.44 \\
& =14.54
\end{aligned}
$$

\subsubsection{Maximum Allowable Section Axial Stress}

Per Reference 7.10.2 and conservatively using the A36 allowabie in lieu of the A $500 \mathrm{Gr} B$ allowable (i.e., A500 Gr B has a yield strength of $42 \mathrm{ksi}$ compared to $36 \mathrm{ksi}$ for A36):
at $\mathrm{kli} r=14.54$,
F。 $\quad=20.92 \mathrm{ksi}$ 


\section{Appendix A9.2.3 Support Structure Calculation 457-2005.2 (Continued)}

\subsubsection{Critical Buckling Load}

$P_{c r}=F_{q} \times A$

$=20.920 \times(8 \times 6.36)$

$=1,064,410 \mathrm{lbs}$

5.1.5 Axial Load (5W, for buckling considerations)

Axial load $=5 \times 60,000$

$=300,000 \mathrm{lbs}$

The axial load is less than the critical buckling load, $\mathbf{P}_{\mathrm{cr}}$ :

$300,000 \mathrm{lbs}<1,064,410 \mathrm{lbs}$

The stand will not buckle as a frame assembly if the tie plate bracing is demonstrated to be adequate (Section 5.2).

\subsection{Tie Plate Evaluation}

\subsubsection{Sizing Of Tie Plates}

The frame will be braced using $1 / 2$ " tie plates on all four (4) sides. The upper frame (stand) assembly is designed with one (1) open side for placement and removal of the immersion pail.

The tie plate requirements per Reference 7.10 .9 are:

"... the end tie plates shall have a length of not less than the distance between the lines of fasteners or welds connecting them to the components of the member. Intermediate tic plates shall have a length not less than $1 / 2$ of this distance. The thickness of tie plates shall not be less than $1 / 50$ of the distance between the lines of fasteners or welds connecting them to the components of the member."

$\mathrm{I}_{1} \quad=$ maximum distance between welds of tie plates.

$=44$ in

$\mathbf{L}_{2}=$ minimum width of end tie plates

$=\mathbf{L}_{1}$

$=44$ in

\begin{tabular}{|l|l|l|l|}
\hline \multirow{2}{*}{$\begin{array}{l}\text { NAC } \\
\text { inTERNATional. }\end{array}$} & Performed by: $g R \theta$ & $\begin{array}{l}\text { Date: } \\
11-25-96\end{array}$ & $\begin{array}{l}\text { Calculation No 457-2005.2 } \\
\text { Revision I }\end{array}$ \\
\cline { 2 - 5 } & Checked by: & $\begin{array}{l}\text { Date } / 25 / 96 \\
11 / 25 / 96\end{array}$ & Page 12 of 23 \\
\hline
\end{tabular}




\section{Appendix A9.2.3 Support Structure Calculation 457-2005.2 (Continued)}

$\mathrm{L}_{3} \quad=$ minimum width of intermediate tie plates

$=1 / 2 \mathbf{L}_{1}$

$=22$ in

$\mathrm{t}=$ minimum thickness of tie plates

$=1 / 50 \mathrm{~L}$

$=0.88$ in

Tie plates used in this design are 0.5 in thick. Note that this is in violation of the requirements stated in the AlSC Code. However, the AlSC allow's exception to these requirements, provided that stress allowable reduction factors for slender compression elements are applied (See Section 5.2.4).

\subsubsection{Spacing Between Tie Plates}

The tie plate spacing requirements per Reference 7.10 .9 are:

"Compression mernbers composed of two or more shapes separated by intermittent fillers shall be connected at these fillers at intervals such that the slenderness ratio $k J / r$ of either shape, between the fasteners, does not exceed 3/4 times the governing slenderness ratio of the built-up member. The least radius of gyration $r$ shall be used in computing the slenderness ratio of each component part."

$\mathrm{kl} / \mathrm{r}_{\text {ramposine }} \Rightarrow \mathrm{kl} / \mathrm{s}$ of composite section from 5.1 .2

$=14.54$

$\mathrm{kl} / \mathrm{r}_{\text {componer } 1}=\mathbf{k l} / \mathbf{I}$ of component section

Per Reference 7.10.9

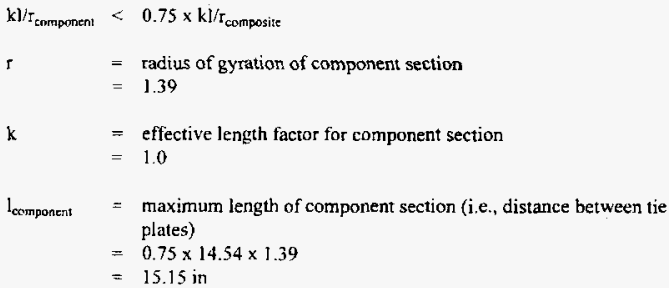

\begin{tabular}{|c|c|c|}
\hline Perfomed by ges & $\begin{array}{l}\text { Date: } \\
11-25-96\end{array}$ & $\begin{array}{l}\text { Calculation No. 457-2005.2 } \\
\text { Revision : }\end{array}$ \\
\hline Checked by: $\pi f$ & $11 / 25 / 96$ & Page 13 of 23 \\
\hline
\end{tabular}


HNF-SD-SNF-FRD-003 Rev. 0

Appendix A9.2.3 Support Structure Calculation 457-2005.2 (Continued)

Therefore, the maximum permissible spacing between tie plates is $15^{\circ}$.

\subsubsection{Tie Plate Design Load}

The tie plates are not subject to any side loads; therefore they will be designed to the load requirements of Reference 7.10.9:

"...resist a shearing stress normal to the axis of the member equal to $2 \%$ of the total compressive stress in the member."

$$
\begin{aligned}
\mathrm{W} & =\text { Load on frame. } \\
& =60,000 \mathrm{lbs} \\
\mathrm{LF} & =\text { Load factor for uitimate. } \\
& =5 \\
& =\text { Two point lift criteria reduction factor } \\
& =2 \\
\mathrm{~F}_{\text {desigrt }} & =\text { Frame Design Load } \\
& =60,000 \times 5 \times 2 \\
& =600.000 \mathrm{lbs} \\
\mathrm{F}_{\text {tie plate }} & =\text { Tie Plate Design Load } \\
& =0.02 \times \mathrm{F}_{\text {design }} \\
& =0.02 \times 600,000 \\
& =12,000 \mathrm{lbs}
\end{aligned}
$$

\begin{tabular}{|c|c|c|c|}
\hline $\begin{array}{l}\text { NAC } \\
\text { INTERNATIONAL. }\end{array}$ & Performed by: $g R \theta$ & Date: $n-25-4 c$ & $\begin{array}{l}\text { Calculation No 457-2005.2 } \\
\text { Revision I }\end{array}$ \\
\hline & Checked by: $\mathrm{ZS}$ & Date $11 / 25 / 96$ & Page 14 of 23 \\
\hline
\end{tabular}

\subsubsection{Tie Plate Evaluation}

For the evaluation of a tie plate of minimum width equal to 22 " and maximum width equal to 50", both with a thickress of 1/2", the AISC Code (Reference 7.10, page 5-36) contains the following limitations:

$$
b / t \leq \frac{95}{\sqrt{F_{y}}} \leq \frac{95}{\sqrt{36}} \leq 16
$$

For $50^{\prime \prime}$ plate:

$$
\mathrm{g} / \mathrm{t}=100
$$


Appendix A9.2.3 Support Structure Calculation 457-2005.2 (Continued)

For 22" plate:

$\mathrm{b} / \mathrm{t}=44$

Since $b / t>16$ in both cases, a stress reduction factor must be applied. Note that this falls in line with thickness conclusion in Section 5.2.1 of this calculation

The stress reduction factor is caiculated per Equation A-B5-4 of AISC Code:

when: $b / t>\frac{195}{\sqrt{\frac{F_{y}}{k_{s}}}}>\frac{195}{\sqrt{\frac{\bar{F}_{y}}{4.05}}}$

where: $\mathrm{k}_{\mathrm{c}}=4.05 /(\mathrm{h} / \mathrm{t})^{0.46}$ for $\mathrm{h} / \mathrm{t}>70$; and

$k_{c}=1.0$ for $h / t \leq 70$.

$\mathbf{h}=\mathbf{b}$

then: $Q_{s}=$ stress reduction factor

$=26,200 \mathrm{k}_{\mathrm{c}} /\left[\mathrm{F}_{\mathrm{y}} \times(\mathrm{b} / \mathrm{t})^{2}\right]$

For $50^{\text {n plate: }} \frac{\frac{195}{\sqrt{\frac{36}{4.05}}}}{\sqrt{(100)^{0+6}}}=22.7$

For 22" plate: $\frac{195}{\sqrt{\frac{36}{1.0}}}=32.5$

Since bit is greater for both size plates, for the 50 " plate

$Q_{s 56}=26,200 k_{c} /\left[F_{y} \times(b / t)^{2}\right]$

$=0.036$

For the 2?" plate.

$Q_{322}=26,200 \mathrm{k}_{\mathrm{c}} /\left[\mathrm{F}_{\mathrm{y}} \times(\mathrm{b}: \mathrm{t})^{2}\right]$

INTERNATIONAL

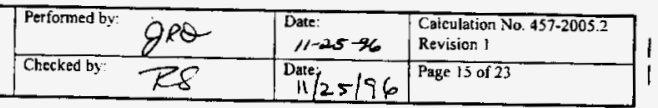


For 22" plate:

$b^{\prime} t=44$

Since $b / t>16$ in both cases, a stress reduction factor must be applied. Note that this falls in line with thickness conclusion in Section 5.2.1 of this calculation.

The stress reduction factor is calculated per Equation A-B5-4 of AISC Code:

when: $b / t>\frac{195}{\sqrt{\frac{F_{y}}{k_{c}}}}>\frac{195}{\sqrt{\frac{F_{y}}{(h / 05}}}$

where: $k_{c}=4.05 /(h / 1)^{0.46}$ for $h / t>70$; and

$k_{c}=1.0$ for $h / t \leq 70$.

$\mathbf{h}=\mathbf{b}$

then: $Q_{\mathrm{s}}=$ stress reduction factor

$=26,200 \mathrm{k}_{\mathrm{e}} /\left[\mathrm{F}_{\mathrm{y}} \times(\mathrm{b} / \mathrm{t})^{2}\right]$

For $50^{\prime \prime}$ plate: $\frac{195}{\sqrt{\frac{36}{(100)^{0.46}}}}=22.7$

For 22" plate: $\frac{195}{\sqrt{\frac{36}{1.0}}}=32.5$

Since bit is greater for both size plates, for the 50" plate

$\begin{aligned} Q_{s 50} & =26,200 k_{c} /\left[F_{y} \times(b / t)^{2}\right] \\ & =0.036\end{aligned}$

For the 22" plate:

$Q_{i 22}=26,200 \mathrm{k}_{\mathrm{c}} \cdot\left[\mathrm{F}_{\mathrm{y}} \times(\mathrm{b} / \mathrm{t})^{2}\right]$

$=0.376$

\begin{tabular}{|c|c|c|c|c|}
\hline A IAC & Performed by: & $g R$ & $\begin{array}{l}\text { Date: } \\
11-25-26\end{array}$ & $\begin{array}{l}\text { Calculation N0.457-2005.2 } \\
\text { Revision 1 }\end{array}$ \\
\hline & Checked by: & $\overrightarrow{R g}$ & $11 / 25 / 96$ & Page 15 of 23 \\
\hline
\end{tabular}


Appendix A9.2.3 Support Structure Calculation 457-2005.2 (Continued)

From Reference 7.10, page 5-101,

$\mathrm{Q}=\mathrm{Q}_{\mathrm{s} ; 0}=0.036$

$=Q_{522}=0.376$

$C_{c 50}^{\prime}=\sqrt{\frac{2 \times \pi^{2} \times E}{Q \times F}}$

$=\sqrt{\frac{2 \times \pi^{2} \times 29 \mathrm{E} 3}{0.036 \times 36}}$

$=647$

$$
\begin{aligned}
C^{\prime 22} & =\sqrt{\frac{2 \times \pi^{2} \times E}{Q \times F_{y}}} \\
& =\sqrt{\frac{2 \times \pi^{2} \times 29 E 3}{0.376 \times 36}} \\
& =205
\end{aligned}
$$

For a plate with both ends welded

$\mathrm{k}=0.65$

$1=L_{1}$

$=44$ in

$r \quad=0.289 \times 1 \quad$ (Reference 7.10)

$=0.289 \times 0.5$

$=0.1445$

\begin{tabular}{|c|c|c|c|}
\hline $\begin{array}{l}\text { NAF } \\
\text { INTERNATIONAL }\end{array}$ & Performed by: $8 R \theta$ & $\begin{array}{l}\text { Date: } \\
1 /-25-96\end{array}$ & $\begin{array}{l}\text { Calculation No. 457-2005.2 } \\
\text { Revision 1 }\end{array}$ \\
\hline & Checked by: & $11 / 25 / 96$ & Page 16 of $\overline{23}$ \\
\hline
\end{tabular}

$\mathrm{kJ} / \mathrm{r}=.65 \times 44 / .1445$

$=198$ 


\section{Appendix A9.2.3 Support Structure Calculation 457-2005.2 (Continued)}

Since $k \mathrm{l} / \mathrm{r}<\mathrm{C}_{\mathrm{C}}$, for both the $50^{\circ \prime}$ and 22 " plates:

$\mathrm{F}_{\mathbf{s 0}}=$ Stress allowable in compression, $50 "$ plate

$$
\begin{aligned}
& =\frac{Q \times\left[1-\frac{\left(\frac{k \times l}{r}\right)^{2}}{2 \times C_{c}^{2}}\right] \times F_{y}}{\frac{5}{3}+\frac{3 \times\left(\frac{k \times l}{r}\right)}{8 \times C_{c}^{\prime}}-\frac{\left(\frac{k \times l}{r}\right)^{3}}{8 \times C_{c}^{3}}} \\
& =\frac{0.036 \times\left[1-\frac{(198)^{2}}{2 \times(647)^{2}}\right] \times 36}{5}+\frac{3 \times(198)}{8 \times 647}-\frac{(198)^{3}}{8 \times(647)^{3}} \\
& =0.694 \mathrm{ksi}
\end{aligned}
$$

$\mathrm{F}_{\mathrm{a22}}$ - Stress allowable in compression, 22" plate

$$
\begin{aligned}
& =\frac{0.376 \times\left[1-\frac{(198)^{2}}{2 \times(205)^{2}}\right] \times 36}{\frac{5}{3}+\frac{3 \times(198)}{8 \times 205}-\frac{(198)^{3}}{8 \times(205)^{3}}} \\
& =3.769 \mathrm{ksi}
\end{aligned}
$$

\begin{tabular}{|c|c|c|c|}
\hline 9 IAC & Performed by: $8 R \theta$ & $\begin{array}{l}\text { Date: } \\
11-25-46\end{array}$ & $\begin{array}{l}\text { Calculation No. 457.2005.2 } \\
\text { Revision! }\end{array}$ \\
\hline & Checked by: & Date: $11 / 25196$ & Page 17 of 23 \\
\hline
\end{tabular}

The actual axial stresses are:

$$
\begin{aligned}
\sigma_{\text {axial 50 }} & =12,000 /(50 \times 0.5) \\
& =480 \mathrm{psi} \\
\sigma_{\text {axial 22 }} & =12,000 /(22 \times 0.5) \\
& =1091 \mathrm{psi}
\end{aligned}
$$

Both actual stresses are less than the allowable, therefore the plates ranging from 22 " to 50 " wide with a 1/2" thickness are acceptable. 


\section{Appendix A9.2.3 Support Structure Calculation 457-2005.2 (Continued)}

Since $\mathrm{kl} / \mathrm{r}<\mathrm{C}$, for both the 50 " and 22 " plates:

$\mathrm{F}_{\Delta 0}=$ Stress allowable in compression, 50 " plate

$$
=\frac{\mathrm{Q} \times\left[1-\frac{\left(\frac{\mathrm{kxI}}{\mathrm{r}}\right)^{2}}{2 \times \mathrm{C}_{c}^{2}}\right] \times F_{y}}{\frac{5}{3}+\frac{3 \times\left(\frac{\mathrm{k} \times \mathrm{I}}{\mathrm{r}}\right)}{8 \times \mathrm{C}_{\mathrm{c}}^{\prime}}-\frac{\left(\frac{\mathrm{kxl}}{\mathrm{r}}\right)^{3}}{8 \times \mathrm{C}_{\mathrm{c}}^{3}}}
$$$$
=\frac{0.036 \times\left[1-\frac{(198)^{2}}{2 \times(647)^{2}}\right] \times 36}{\frac{5}{3}+\frac{3 \times(198)}{8 \times 647}-\frac{(198)^{3}}{8 \times(647)^{3}}}
$$

$=0.694 \mathrm{ksi}$

$F_{a 22}=$ Stress allowable in compression, 22" plate

$$
\begin{aligned}
& =\frac{0.376 \times\left[1-\frac{(198)^{2}}{2 \times(205)^{2}}\right] \times 36}{\frac{5}{3}+\frac{3 \times(198)}{8 \times 205}-\frac{(198)^{3}}{8 \times(205)^{3}}} \\
& =3.769 \mathrm{ksi}
\end{aligned}
$$

The actual axial stresses are

$$
\begin{aligned}
\sigma_{\text {axial } 50} & =12,000 /(50 \times 0.5) \\
& =480 \mathrm{psi} \\
\sigma_{2 \times i a 1 ~ 22} & =12,000 /(22 \times 0.5) \\
& =1091 \mathrm{psi}
\end{aligned}
$$

\begin{tabular}{|c|c|c|c|}
\hline A. IAC & Performed by: $g R \theta$ & $\begin{array}{l}\text { Date: } \\
1 /-25-96\end{array}$ & $\begin{array}{l}\text { Calcuiation No. 457-2005.2 } \\
\text { Revision I }\end{array}$ \\
\hline & Checked by & Date: $11 / 25 / 96$ & Page 17 of 23 \\
\hline
\end{tabular}

Both actual stresses are less than the allowable, therefore the plates ranging from $22^{\prime \prime}$ to $50^{\circ}$ wide with a 1/2" thickness are acceptable. 
Appendix A9.2.3 Support Structure Calculation 457-2005.2 (Continued)

\subsubsection{Tie Plate Weld Evaluation}

The plates will be welded on both edges with double line fillet weids. For $50^{\prime \prime}$ plate, the weld length is $\equiv 34^{\prime \prime}$, which is twice the AISC Code requirement of $1 / 3$ the plate width $=50 / 3=16.7^{\prime \prime}$. Similarly, the $22^{\circ}$ plate weld length is $14^{\prime \prime}$, which is twice the AISC Code requirement of $22 / 3=7.3$ ".

Therefore, the weld design is adequate.

5.3 Comer Post_Assembly Buckling Evaluation

\subsubsection{Tube Cross Secction Properties}

Per Section 4.4.1:

Tube steel.

size $=4 \times 4 \times 0.5$

$1=12.3 \mathrm{in}^{4}$

I $=1.39 \mathrm{in}$

arca $=6.36 \mathrm{in}^{2}$

\subsubsection{Axial Load}

Per Reference 7.16, the maximum lift load for Condition I and IV is $<58,000 \mathrm{lbs}$. For eight tube columns, the axial load per column is:

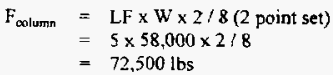

\subsubsection{Buckling Load Evaluation}

The bracing configuration was designed to ensure the stand will effectively behave as a frame assembly. However, due to the non braced section on the upper frame assembly, a localized buckling evaluation is performed. The upper / !ower frame assembly connection will serve as a pinned connection point ( $k=1.0$ applies).

Per drawing Reference 7.6.2, the maximum actual length, $L_{\text {max }}$,

$$
\begin{aligned}
L_{\cdot \operatorname{ax}} & =156 \mathrm{in} \\
& =13.0 \mathrm{ft}
\end{aligned}
$$

\begin{tabular}{|c|c|c|c|}
\hline \multirow[t]{2}{*}{ A NAC: } & Performed by $g \ell O$ & $\begin{array}{l}\text { Date: } \\
11-25-x\end{array}$ & $\begin{array}{l}\text { Calculation No. 457-2005.2 } \\
\text { Revision I }\end{array}$ \\
\hline & Checked by: RS & Date $11 / 25 / 96$ & Page 18 of 23 \\
\hline
\end{tabular}


Appendix A9.2.3 Support Structure Calculation 457-2005.2 (Continued)

Per Reference 7.10.6, with $\mathrm{kl}=1.0 \times 13=13.0$ :

The maximum allowable concentric load is $=75$ kips.

Since the applied load is less than the maximum allowable concentric load. the comer tube will not buckle and is adeçuately designec against buckling failure.

5.4 Guide Assembly Evaluation

\subsubsection{Bearing Check}

The pin bearing area is;

Consider lock pin extends $90 \%$ thru guide, therefore extension length per item 98 of Reference 7.6.4

$L=[0.9 \times(10.58-0.5$ end pin chanfer $)]-1.5$ (gap)

$=7.572 \mathrm{in}$

Pin bearing width, $\mathbf{W}$, is

$\mathrm{W}=1.5-2(0.13)$

$=1.24 \mathrm{in}$

Bearing area $=\mathrm{L} \times \mathrm{W}$

$=7.572 \times 1.24$

$=9.39 \mathrm{in}^{2}$

Therefore, the bearing stress (guide assembly is $\mathrm{A} 36$ material, pin is $17-4 \mathrm{PH}$ ) is:

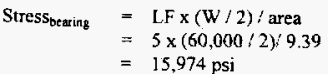

Safety Margin $=1-\left(\right.$ stress $\left./\left(1.5 \times S_{y}\right)\right)$

$=1-(16.0 /(1.5 \times 36))$

$=0.70$

\begin{tabular}{|c|c|c|c|}
\hline \multirow[t]{2}{*}{ A. NAC INERHATIONAL } & Performed by: Q & $\begin{array}{l}\text { Date: } \\
11 \rightarrow 25-26\end{array}$ & $\begin{array}{l}\text { Calculation No. 457-2005.2 } \\
\text { Revision } 1\end{array}$ \\
\hline & Checked by: 28 & Date: $11 / 25 / 36$ & Page $\overline{19}$ of 23 \\
\hline
\end{tabular}


Appendix A9.2.3 Support Structure Calculation 457-2005.2 (Continued)

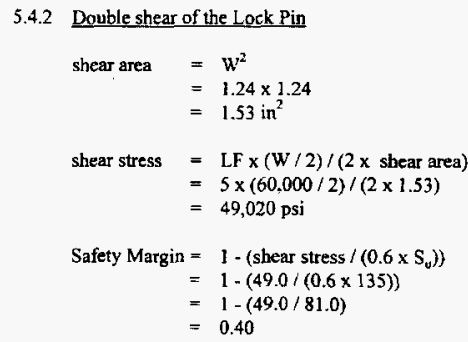

\begin{tabular}{|c|c|c|c|}
\hline $\begin{array}{l}\text { NAC } \\
\text { INIERNATIONAL. }\end{array}$ & Performed by: & $\begin{array}{l}\text { Date: } \\
1 / 25-96\end{array}$ & $\begin{array}{l}\text { Calculation Ko. 457-2005.2 } \\
\text { Revision ! }\end{array}$ \\
\hline & Checked by: & $11 / 25 / 96$ & Page 20 of 23 \\
\hline
\end{tabular}


Rev. 0

Appendix A9.2.3 Support Structure Calculation 457-2005.2 (Continued)

\subsection{SUMMARY OF RESULTS/CONCLUSIONS}

Summary of Stress Analysis

\begin{tabular}{|l|l|l|l|l|l|l|l|}
\hline $\begin{array}{l}\text { Drawing } \\
\text { No. }\end{array}$ & $\begin{array}{l}\text { Item } \\
\text { No. }\end{array}$ & $\begin{array}{l}\text { Component } \\
\text { Load }\end{array}$ & $\begin{array}{l}\text { Applied } \\
\text { Check }\end{array}$ & $\begin{array}{l}\text { Calculated } \\
\text { Loading }\end{array}$ & Allowable & M.S. \\
\hline $457-102$ & $\begin{array}{l}98, \\
99\end{array}$ & $\begin{array}{l}\text { frame stand } \\
\text { assembly }\end{array}$ & $5 \mathrm{~W}$ & buckling & $300 \mathrm{kips}$ & $1,064 \mathrm{kips}$ & 0.72 \\
\hline $\begin{array}{l}4,7, \\
8,9, \\
15, \\
16, \\
17, \\
18\end{array}$ & tie plates & $5 \mathrm{~W}$ & compression & $480 \mathrm{psi}$ & $694 \mathrm{psi}$ & 0.31 \\
\hline $457-102$ & 5 & comer post & $5 \mathrm{~W}$ & buckling & $72,500 \mathrm{lbs}$ & $75,000 \mathrm{lbs}$ & 0.03 \\
\hline $457-109$ & 4 & lock pin & $5 \mathrm{~W}$ & double shear & $49.0 \mathrm{ksi}$ & $81.0 \mathrm{ksi}$ & 0.40 \\
\hline $457-109$ & 10 & guide & $5 \mathrm{~W}$ & bearing & $16.0 \mathrm{ksi}$ & $54 \mathrm{ksi}$ & 0.70 \\
\hline
\end{tabular}

The immersion pail support structure design meets the criteria defined in Section 4.2.

\begin{tabular}{|l|l|l|l|}
\hline \multirow{2}{*}{$\begin{array}{l}\text { NaC } \\
\text { intervational }\end{array}$} & \begin{tabular}{l} 
Performed by: \\
\cline { 2 - 4 }
\end{tabular} & $\begin{array}{l}\text { Checked by: } \\
\text { Rate: }\end{array}$ & $\begin{array}{l}\text { Caiculation No. 457-2005.2 } \\
\text { Revision 1 }\end{array}$ \\
\hline
\end{tabular}


Appendix A9.2.3 Support Structure Calculation 457-2005.2 (Continued)

\subsection{REFERENCES}

7.1 Preliminary Design Analysis Report For The TN-WHC Cask and Transporation System Project 3035

Transnuclear, Inc

7.2 Not Used

7.3 ANSI N14.6

American National Standard for Radjoactive Materials

"special lifting devices for shipping containers weighing $10,000 \mathrm{lbs}(4,500 \mathrm{~kg})$ or more"

7.4 Roark's Formulas for Stress and Strain, 3rd edition.

7.5 Hanford ECN 191402

7.6 K Basin Immersion Pail Assembly TN WHC Transpont Cask Drawings.

7.6.1 Project 457, Drawing 101, sheet $1 / 1$.

7.6.2 Project 457, Drawing 102, sheets $1,2 / 2$

7.6.3 Project 457, Drawing 103, sheets 1, $2 / 2$.

7.6.4 Project 457, Drawing 109, sheet $1 / 1$.

7.7 ASME Boiler \& Pressure Vessel Code, 1995 edition.

7.7.1 Section II-D, page 99, Table $1 \mathrm{~A}$.

7.7.2 Section I-D, page 6, Table $1 \mathrm{~A}$

7.7.3 Section III, NB-3227.1(a).

7.7.4 Section II-D, page 432,496

7.8 Blodgett's Design Of Welded Structures, 1966

7.8.1 Page 3.2-16

7.8.2 Page 3.2-1

7.8.3 Page 3.2-14

7.9 Not Used.

\begin{tabular}{|c|c|c|c|}
\hline A NAT: & Performed by: $Q R$ & $\begin{array}{l}\text { Dase: } \\
1 /-25-96\end{array}$ & $\begin{array}{l}\text { Calculation No } 457-2005.2 \\
\text { Revision I }\end{array}$ \\
\hline & Checked by: TLC & $11 / 25 / 96$ & Page 22 of 23 \\
\hline
\end{tabular}


Appendix A9.2.3 Support Structure Calculation 457-2005.2 (Continued)

7.10 AlSC Manual Of Steel Construction, 9th edition.

7.10.1 Page 1.96

7.10.2 Page 3-16

7.10.3 Page 5-135

7.10.4 Page 5-37 (Section B7) and 5-42

7.10.5 Page 1-103

7.10.6 Page 3.43

7.10.7 Page 1-92

7.10.8 Page 1-7

7.10.9 Page 5-43

7.11 Not Used.

7.12 Machinery's Handbook, $22^{\text {nd }}$ Edition.

7.12.1 Page 287

7.13 Mark's Standard Handbook for Mechanical Engineers.

7.13.1 Page 5-30

7.14 Meeting Minutes, NAC/TN, September 1996.

7.15 ASTM Annual Book of ASTM Siandards Volume 01.01. Page 404

1010 Drawn Tubing.

7.16 WRR-457-2003.2

\begin{tabular}{|l|l|l|l|}
\hline \multirow{2}{*}{$\begin{array}{l}\text { NaC } \\
\text { INTERNationat }\end{array}$} & \begin{tabular}{l} 
Performed by: gRS \\
\cline { 2 - 4 }
\end{tabular} & $\begin{array}{l}\text { Date: } \\
1 / 25 \rightarrow x\end{array}$ & $\begin{array}{l}\text { Calculation No. 457-2005.2 } \\
\text { Revision 1 }\end{array}$ \\
\cline { 2 - 4 } & Checked by: & Date $/ 25 / 96$ & Page 23 of 23 \\
\hline
\end{tabular}




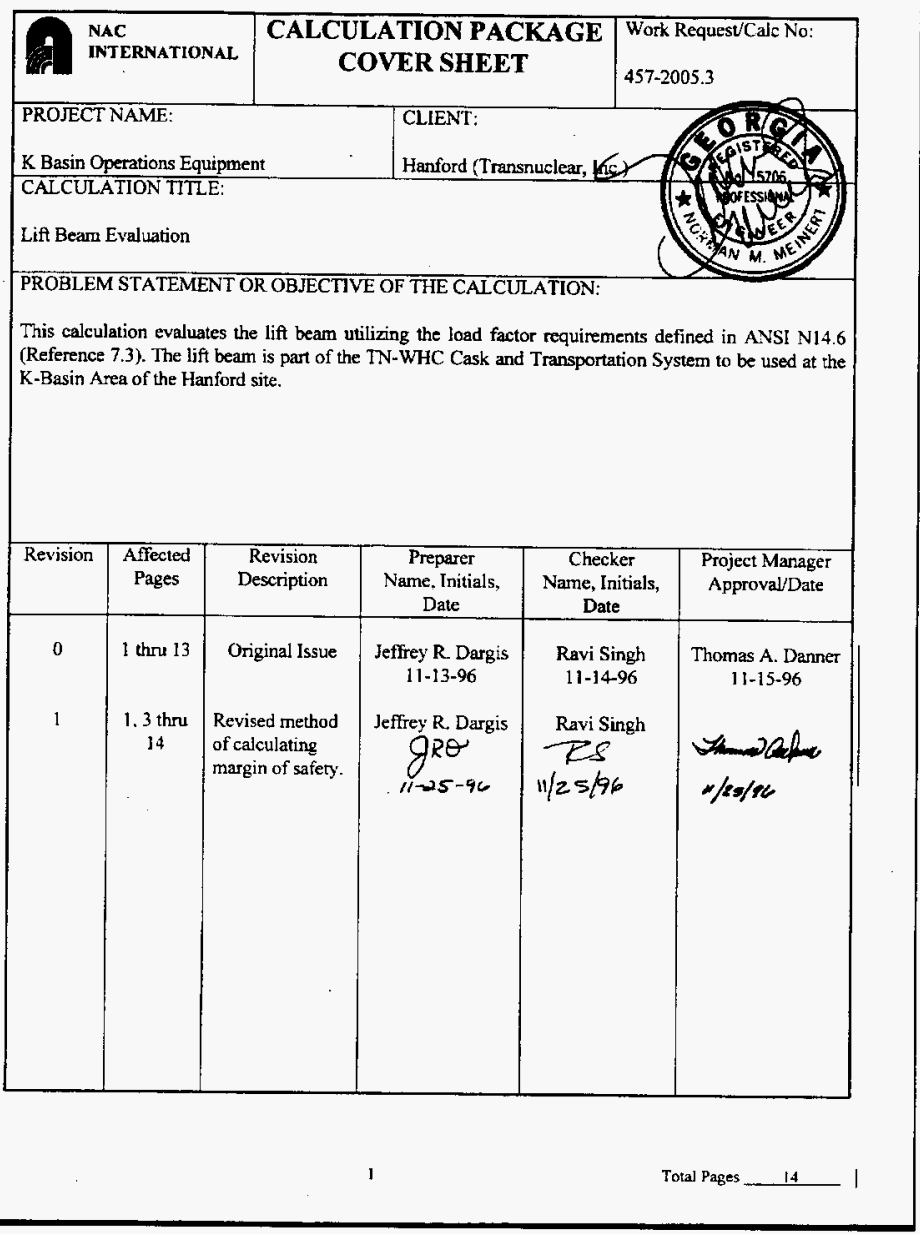


Appendix A9.2.4 Lift Beam Calculation 457-2005.3 (Continued)

\section{INDEPENDENT DESIGN VERIFICATION CHECK SHEET}

Work Request/Calculation No: 457-2005.3 Revision 0

Scope Of Analysis File: This calculation structurally evaluates the lift beam utilizing the load factor requirements defined in ANSI N14.6.

Review Methodology: Check Of Calculations

Alternate Analyses

Other (Explain)

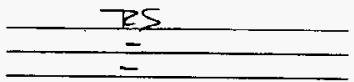

Confirm That The Work Request / Calculation Package Reviewed Includes:

1. Statement of Purpose

2. Defined Method of Analysis

3. Listing of Assumptions

4. Detailed Analysis Record

5. Statement of Conclusions / Recommendations (if applicable)

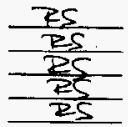

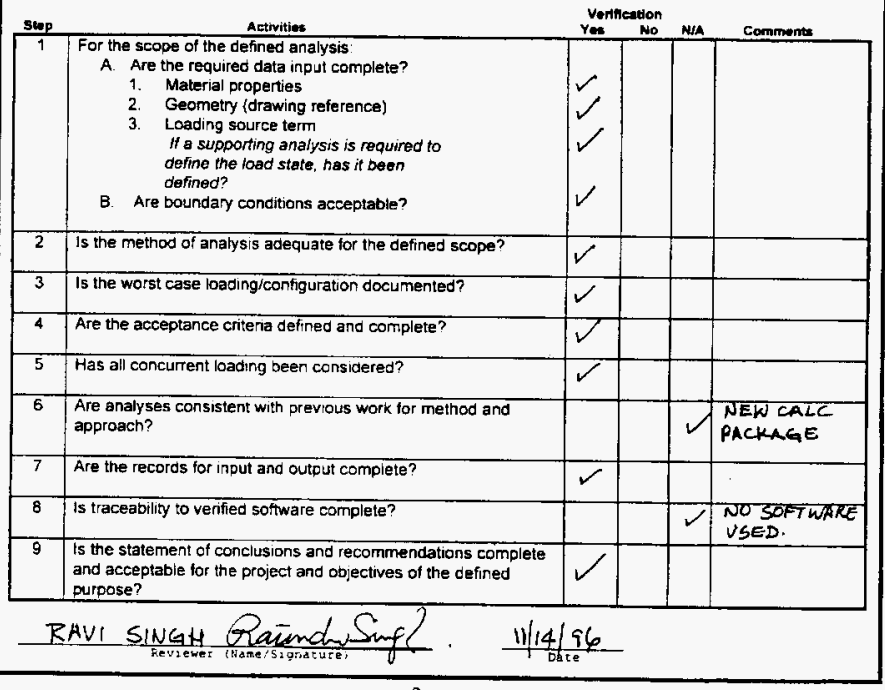


Appendix A9.2.4 Lift Beam Calculation 457-2005.3 (Continued)

\section{INDEPENDENT DESIGN VERIFICATION CHECK SHEET}

Work Request/Calculation No: 457-2005.3 Revision 1

Scope Of Analysis File: This calculation structurally evaluates the lift beam utilizing the load tactor requirements defined in ANSI N14.6.

Review Methodology: Check Of Calculations

Alternate Analyses

Other (Explain)

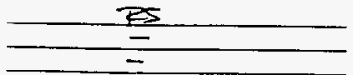

Confirm That The Work Request / Caiculation Package Reviewed Includes:

1. Statement of Purpose

2. Defined Method of Analysis

3. Listing of Assumptions

4. Detailed Analysis Record

5. Statement of Conclusions / Recommendations (if applicable)

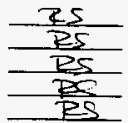

\begin{tabular}{|c|c|c|c|c|c|}
\hline Step & Activities & & $\begin{array}{l}\text { tition } \\
\text { No }\end{array}$ & N/A & Comments \\
\hline 1 & $\begin{array}{l}\text { For the scope of the defined analysis } \\
\text { A. Are the required data input complete? } \\
\text { 1. Material properties } \\
\text { 2. Geometyy (drawing reference) } \\
\text { 3. Loading source term } \\
\text { If a supporting analysis is required to } \\
\text { define the load state, has it been } \\
\text { defined? } \\
\text { B. Are boundary conditions acceptable? }\end{array}$ & $\checkmark$ & & & \\
\hline 2 & Is the method of analysis adequate for the defined scope? & 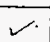 & & & \\
\hline 3 & is the worst case loading/configuration documented? & $\checkmark$ & & & \\
\hline$\overline{4}$ & Are the acceptence criteria defined and complete? & $\mathscr{V}$ & & & \\
\hline 5 & Has all concurrent loading been considered? & $\checkmark$ & & & \\
\hline 6 & $\begin{array}{l}\text { Are analyses consistent with previous work for method and } \\
\text { approach? }\end{array}$ & $\checkmark$ & & & \\
\hline 7 & Are the records for input and output complete? & $\checkmark$ & & & \\
\hline 8 & Is traceability to verifed software complete? & & & $\checkmark$ & \\
\hline$g$ & $\begin{array}{l}\text { Is the statement of conclusions and recommendations complete } \\
\text { and acceptable for the project and objectives of the defined } \\
\text { purpose? }\end{array}$ & $\checkmark$ & & & \\
\hline
\end{tabular}

BAVI SWGH Gaven Sines. $\quad 11 / 25 / 96$ 
Appendix A9.2.4 Lift Beam Calculation 457-2005.3 (Continued)

\section{TABLE OF CONTENTS}

\begin{tabular}{|c|c|}
\hline Section & Description \\
\hline- & Calculation Package Cover Sheet \\
\hline- & 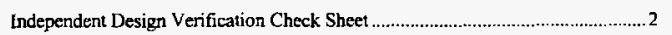 \\
\hline- & 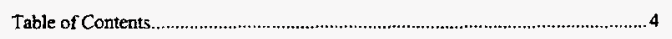 \\
\hline 1.0 & Synopsis of Results \\
\hline 2.0 & Introduction/Purpose \\
\hline 3.0 & 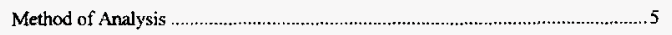 \\
\hline 4.0 & Assumptions/Design Input ................ \\
\hline 5.0 & 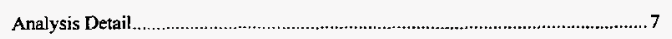 \\
\hline 6.0 & 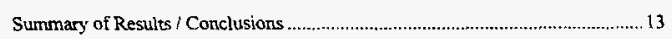 \\
\hline 7.0 & 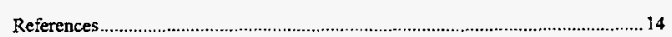 \\
\hline
\end{tabular}

\begin{tabular}{|l|l|l|l|}
\hline \multirow{2}{*}{$\begin{array}{l}\text { NAC } \\
\text { intervarjonal }\end{array}$} & Perfomed by: & $\begin{array}{l}\text { Date: } \\
11-25-76\end{array}$ & $\begin{array}{l}\text { Calculation No 457-2005.3 } \\
\text { Revision I }\end{array}$ \\
\cline { 2 - 5 } & Checked by: & $\begin{array}{c}\text { Datej } \\
1 / 25 / 96\end{array}$ & Page 4 of 14 \\
\hline
\end{tabular}


Summary of Stress Analysis

\begin{tabular}{|c|c|c|c|c|c|c|c|}
\hline $\begin{array}{l}\text { Drawing } \\
\text { No. }\end{array}$ & $\begin{array}{l}\text { Item } \\
\text { No. }\end{array}$ & Component & $\begin{array}{l}\text { Applied } \\
\text { Load }\end{array}$ & Design Check & $\begin{array}{l}\text { Calculated } \\
\text { Loading }\end{array}$ & Allowable & M.S \\
\hline $457-112$ & 1 & weidment & $\begin{array}{l}180 \text { kips } \\
\text { (plate) } \\
150 \text { kips } \\
\text { (trunnion) }\end{array}$ & $\begin{array}{l}\text { beam bending } \\
\text { beam shear } \\
\text { trunnion shear }\end{array}$ & $\begin{array}{l}25,447 \text { psi } \\
2,769 \text { psi } \\
11,933 \text { psi }\end{array}$ & $\begin{array}{l}30,000 \mathrm{psi} \\
18,000 \mathrm{psi} \\
40,200 \mathrm{psi}\end{array}$ & $\begin{array}{l}0.15 \\
0.85 \\
0.70\end{array}$ \\
\hline $457-112$ & 10 & eye plate & $180 \mathrm{kips}$ & $\begin{array}{l}\text { tension } \\
\text { bearing }\end{array}$ & $\begin{array}{l}14,400 \mathrm{psi} \\
10,650 \mathrm{psi}\end{array}$ & $\begin{array}{l}30,000 \text { psi } \\
45,000 \text { psi }\end{array}$ & $\begin{array}{l}0.52 \\
0.76\end{array}$ \\
\hline
\end{tabular}

The lift beam design meets the criteria defined in Section 4.2.

\section{INTRODUCTION / PLRPOSE}

This calculation evaluates the lift beam utilizing the load factor requirements defined in ANSI N14.6 (Reference 7.3). The lift beam is part of the TN-WHC Cask and Transportation System to be used at the K-Basin Area of the Hanford site.

\section{METHOD OF ANALYSIS}

Hand calculations using classic textbook solutions are used to structurally evaluate the lift beam.

The design will be assessed using the load factors discussed in Section 4.2. The acceptance criteria defined in Reference 7.3 limits the lifting induced tensile stresses to the lesser of:

(a) one-third material yield strength; or

(b) one-fifth the material ulimate strength.

The following evaluations are documented within this calculation.

- Design Load Development:

- Lift Beam Evaluation; and

- Pad Eye Evaluation.

\begin{tabular}{|c|c|c|c|}
\hline $\begin{array}{l}\text { NAC } \\
\text { INTERNATIONAL }\end{array}$ & Performed by: $g R$ & $\begin{array}{l}\text { Date: } \\
\text { Ifo5-46 }\end{array}$ & $\begin{array}{l}\text { Calculation No. 457-2005,3 } \\
\text { Revision ! }\end{array}$ \\
\hline & Checked by: 25 & Date: $11 / 25 / 96$ & Page 5 of 14 \\
\hline
\end{tabular}


Appendix A9.2.4 Lift Beam Calculation 457-2005.3 (Continued)

4.0

\section{ASSUMPTIONS / DESIGN INPUTS}

4.1 Assumptions

There are no unverified assumptions within this calculation.

4.2 Design Criteria

\subsubsection{Tensile Stresses}

Calculared tensile stress (based on load factor of 3) $<F_{\text {yield }}$ Calculated tensile stress (based on load factor of 5) $<F_{\text {uctumate }}$

(Ref, 7.3)

(Ref. 7.3)

\subsubsection{Shear Stresses}

Calculated shear stress (based on load factor of 3) $\quad<0.6 \times F_{\text {yield }}$ Calculated shear stress (based on load factor of 5) $\quad<0.6 \times \mathrm{F}_{\text {ulimart }}$

\subsubsection{Bearing stresses}

Calculated bearing stress $<1.5 \times F_{\text {yield }}$

\subsubsection{Material Properties / Stress Design Checks}

All components evaluated within this calculation are made of A516 GRS5 or A588 GR A carbon steel.

Poison's ratio $=0.3$

Modulus of Elasticity, $E=28.3 \times 10^{6} \mathrm{psi}$ (Ref. 7.7)

Normalizing the material yield and ultimate strengths by the Reference 7.3 load factors yields the following (See Section 4.2 .1 for load factors):

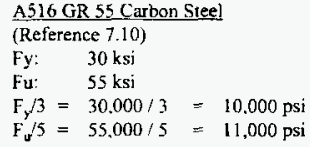

\begin{tabular}{|c|c|c|c|}
\hline $\begin{array}{l}\text { NAC } \\
\text { INTERNATHONAI }\end{array}$ & Perfomed by: $g R \theta$ & Date: & $\begin{array}{l}\text { Calculation No. 457-2005.3 } \\
\text { Revision } 1\end{array}$ \\
\hline & Checked by: $R S$ & $\begin{array}{l}\text { Date: } \\
11 / 25 / 96\end{array}$ & Page 6 of 14 \\
\hline
\end{tabular}


Appendix A9.2.4 Lift Beam Calculation 457-2005.3 (Continued)

ASTM A588 GR A Carbon Steel
(Reference 7.10)
Fy: $\quad 46 \mathrm{ksi}$
Fu: $\quad 67 \mathrm{ksi}$
Fy $/ 3=46,000 / 3=15,333 \mathrm{psi}$
Fu $/ 5=67,000 / 5=13,400 \mathrm{psi}$

Based on the comparisons of yield and Utimate allowables for the two (2) materials, comparing a factor (LF) of 3 against yield is more restrictive for A516, while using a factor (LF) of 5 against ultimate is more restrictive for A588. Only the limiting condition is evaluated within this calculation.

\subsection{ANALYSIS DETAIL}

5.1 Design Load Development:

The design loads, $\mathrm{F}$, for the lift beam and trunnion are as follows:

$$
\begin{aligned}
F_{\text {lit beam }} & =60,000 \times 3 \\
& =180,000 \mathrm{lbs} \\
F_{\text {trannion }} & =(60,000 / 2) \times 5 \\
& =150,000 \mathrm{lbs}
\end{aligned}
$$

5.2 Lift Beam Evaluation

The load is applied by the eye plate to the lift beam as follows:

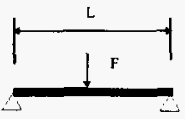

\begin{tabular}{|c|c|c|c|}
\hline A. NAC & Performed by: 8 & $\begin{array}{l}\text { Date: } \\
1 /-25-96\end{array}$ & $\begin{array}{l}\text { Calculation No. 457-2005.3 } \\
\text { Revision : }\end{array}$ \\
\hline & Checked by: $Z_{5}$ & $\begin{array}{l}\text { Date: } \\
11 / 25 / 96\end{array}$ & Page 7 of 14 \\
\hline
\end{tabular}

where:

$\mathbf{L}=36.81+((3 / 2) \times 2)$

$=34.81$ in 
Appendix A9.2.4 Lift Beam Calculation 457-2005.3 (Continued)

$$
\begin{aligned}
F & =180,000 \mathrm{lbs} \\
M & =\frac{F \times L}{4} \\
& =\frac{180,000 \times 39.81}{4} \\
& =1,791,450 \mathrm{in}-1 \mathrm{bs}
\end{aligned}
$$

The effective cross section is:

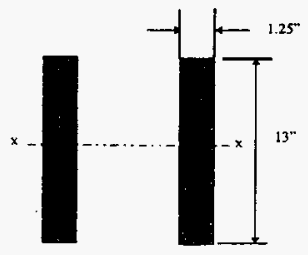

$$
\begin{aligned}
I_{x} & =\frac{2 \times b \times h^{3}}{12} \\
& =\frac{2 \times 1.25 \times 13^{j}}{12} \\
& =457.7 \mathrm{in}^{4} \\
S_{x} & =I / C \\
& =457.7 /(13 / 2) \\
& =70.4 \mathrm{in}^{3}
\end{aligned}
$$

\begin{tabular}{|l|l|l|l|}
\hline \multirow{2}{*}{$\begin{array}{l}\text { NAC } \\
\text { INIERAational }\end{array}$} & \begin{tabular}{l} 
Performed by \\
\cline { 2 - 4 }
\end{tabular} & $\begin{array}{c}\text { Date: } \\
1 / 25-96\end{array}$ & $\begin{array}{l}\text { Calculation No. 457-2005.3 } \\
\text { Revision 1 }\end{array}$ \\
\cline { 2 - 4 } & Checked by: & $\begin{array}{c}\text { Date: } \\
11 / 25 / 96\end{array}$ & Page 8 of 14 \\
\hline
\end{tabular}


Appendix A9.2.4 Lift Beam Calculation 457-2005.3 (Continued)

The maximum bending stress is:

$$
\begin{aligned}
\sigma_{b} & =M / S_{x} \\
& =1,791,450 / 70.4 \\
& =25,447 \mathrm{psi} \\
\text { MS } & =1-\frac{\sigma_{b}}{F_{y}} \\
& =1-\frac{25,447}{30,000} \\
& =0.15
\end{aligned}
$$

\begin{tabular}{|c|c|c|c|}
\hline 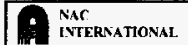 & Performed by $Q R \theta$ & $\begin{array}{l}\text { Date: } \\
1 / 25-96\end{array}$ & $\begin{array}{l}\text { Calculation No. 457-2005.3 } \\
\text { Revision ? }\end{array}$ \\
\hline & Checked by: 25 & Date $11 / 25 / 96$ & Page 9 of 14 \\
\hline
\end{tabular}

The maximum shear stress is:

$$
\begin{aligned}
\sigma_{\mathrm{v}} & =(\mathrm{F} / 2) / \mathrm{A}_{\text {ghear }} \\
& =(180,000 / 2) /(13 \times 1.25 \times 2) \\
& =2,769 \mathrm{psi} \\
\mathrm{MS} & =1-\frac{\sigma_{v}}{\left(0.6 \times \mathrm{F}_{\mathrm{y}}\right)} \\
& =1-\frac{2,769}{(0.6 \times 30.000)} \\
& =0.85
\end{aligned}
$$

The maximum shear stress for the trunnion section:

$$
\begin{aligned}
A_{v} & =\pi \times r_{\text {trunnion }}^{2} \\
& =\pi \times(2)^{2} \\
& =12.57 \mathrm{in}^{2}
\end{aligned}
$$


Appendix A9.2.4 Lift Beam Calculation 457-2005.3 (Continued)

$$
\begin{aligned}
\sigma_{v} & =F /(2 \times A) \\
& =150,000 /(12.57) \\
& =11,933 \mathrm{psi}
\end{aligned}
$$

The maximum allowable shear stress is:

$$
\begin{aligned}
F_{v} & =0.6 \times F_{u} \\
& =40,200 \mathrm{psi}
\end{aligned}
$$

The margin of safety is:

$$
\begin{aligned}
\text { MS } & =1-\frac{\sigma_{\mathrm{v}}}{\mathbf{F}_{v}} \\
& =1-\frac{11,933}{40,200} \\
& =0.70
\end{aligned}
$$

\begin{tabular}{|c|c|c|c|}
\hline INTERNATIONAL & Performed by: QPQ & $\begin{array}{l}\text { Date: } \\
1 / 25-96\end{array}$ & $\begin{array}{l}\text { Calculation No. 457.2005.3 } \\
\text { Revision 1 }\end{array}$ \\
\hline & Checked by: TS & $\begin{array}{l}\text { Date: } \\
11 / 25 / 96\end{array}$ & Page 10 of 14 \\
\hline
\end{tabular}

\subsection{Eve Plate Evaluation}

Per Reference 7.6.1, the minimum tensile area of the eye plate is

$$
\begin{aligned}
A_{1} & =5.00 \times 2.50 \\
& =12.5 \mathrm{in}^{2}
\end{aligned}
$$

The maximum tensile stress in the eye plate is:

$$
\begin{aligned}
\sigma_{1} & =\frac{F}{A_{1}} \\
\sigma_{1} & =\frac{180.000}{12.5} \\
& =14,400 \mathrm{psi}
\end{aligned}
$$


Appendix A9.2.4 Lift Beam Calculation 457-2005.3 (Continued)

The maximum allowable tensile stress is:

$F=F_{y}$

$=30,000 \mathrm{psi}$

The margin of safety is:

$$
\begin{aligned}
\text { MS } & =1-\frac{\sigma_{t}}{\mathrm{~F}} \\
& =1-\frac{14,400}{30,000}
\end{aligned}
$$

$=0.52$

The eye plate bearing area, $A_{\text {bearing }}$, is:

$A_{\text {bering }}=3.38$ in $\times 5$ in

$=16.9 \mathrm{in}^{2}$

The maximum bearing stress in the eve plate is:

$$
\begin{aligned}
\sigma_{\text {bearing }} & =\frac{F}{A_{\text {bennig }}} \\
\sigma_{\text {bearing }} & =\frac{180,000}{16.9} \\
& =10,650 \mathrm{psi}
\end{aligned}
$$

The maximum allowable bearing stress is $1.5 F_{y}$ :

$$
\begin{aligned}
F & =1.5 \times F_{y} \\
& =45,000 \mathrm{psi}
\end{aligned}
$$

\begin{tabular}{|l|l|l|l|}
\hline \multirow{2}{*}{$\begin{array}{l}\text { Nar } \\
\text { inTERvationsl. }\end{array}$} & Performed by: ORS & $\begin{array}{l}\text { Date: } \\
1 / 25-56\end{array}$ & $\begin{array}{l}\text { Calculation No 457-2005.3 } \\
\text { Revision 1 }\end{array}$ \\
\cline { 2 - 4 } & Checked by: DS & $\begin{array}{c}\text { Date } \\
11 / 25 / 96\end{array}$ & Page 11 of 14 \\
\hline
\end{tabular}


Appendix A9.2.4 Lift Beam Calculation 457-2005.3 (Continued)

The margin of safety is:

$$
\begin{aligned}
\text { MS } & =1-\frac{\sigma_{\text {mexing }}}{F} \\
& =1-\frac{10,650}{45,000} \\
& =0.76
\end{aligned}
$$

\begin{tabular}{|l|l|l|l|}
\hline \multirow{2}{*}{$\begin{array}{l}\text { NAC } \\
\text { INTER }\end{array}$} & Pefformed by: ORQ & $\begin{array}{l}\text { Date: } \\
1125-96\end{array}$ & $\begin{array}{l}\text { Calculation No. 457-2005.3 } \\
\text { Revision 1 }\end{array}$ \\
\cline { 2 - 4 } & Checked by: $R S$ & $\begin{array}{l}\text { Date: } \\
11 / 25 / 96\end{array}$ & Page 12 of 14 \\
\hline
\end{tabular}


Appendix A9.2.4 Lift Beam Calculation 457-2005.3 (Continued)

\subsection{SUMMARY OF RESULTS/CONCLLSIONS}

Summary of Stress Analysis

\begin{tabular}{|l|l|l|l|l|l|l|l|}
\hline $\begin{array}{l}\text { Drawing } \\
\text { No. }\end{array}$ & $\begin{array}{l}\text { ltem } \\
\text { No. }\end{array}$ & Component & $\begin{array}{l}\text { Applied } \\
\text { Load }\end{array}$ & Design Check & $\begin{array}{l}\text { Calculated } \\
\text { Loading }\end{array}$ & Allowable & M.S. \\
\hline $457-112$ & 1 & weldment & $\begin{array}{l}180 \mathrm{kips} \\
\text { (plate) } \\
150 \mathrm{kips} \\
\text { (tnunnion) }\end{array}$ & beam bending & $25,447 \mathrm{psi}$ & $30,000 \mathrm{psi}$ & 0.15 \\
& beam shear & $2,769 \mathrm{psi}$ & $18,000 \mathrm{psi}$ & 0.85 \\
\hline $457-112$ & 10 & eye plate & $180 \mathrm{kips}$ & $\begin{array}{l}\text { tension } \\
\text { bearing }\end{array}$ & $11,933 \mathrm{psi}$ & $40,200 \mathrm{psi}$ & 0.70 \\
\hline
\end{tabular}

The lift beam design meets the criteria defined in Section 4.2 .

\begin{tabular}{|c|c|c|c|}
\hline INTERNATIONAL & Perfomed by: $8,2 \Theta$ & $\begin{array}{l}\text { Date: } \\
1 / 25-96\end{array}$ & $\begin{array}{l}\text { Calculation No.457.2005.3 } \\
\text { Revision } 1\end{array}$ \\
\hline & Checked by: & Date: $11 / 25 / 96$ & Page 13 of 14 \\
\hline
\end{tabular}


Appendix A9.2.4 Lift Beam Calculation 457-2005.3 (Continued)

\section{REFERENCES}

7.1 Preliminary Design Analysis Report For The TN-WHC Cask and Transporation System Project 3035

Transnuctear, Inc.

$7.2 \quad$ Not Used.

7.3 ANSI N14.6

American National Standard for Radioactive Materials

"special lifting devices for shipping containers weighing $10,000 \mathrm{lbs}(4,500 \mathrm{~kg})$ or more"

7.4 Roark's Formulas for Stress and Strain, 3rd edition.

7.5 Hanford ECN 191402

7.6 K Basin Immersion Pail Assembly IN WHC Transport Cask Drawings.

7.6.1 Project 457, Drawing 112 , sheets $1,2 / 2$.

7.7 ASME Boiler \& Pressure Vessel Code, 1995 edition.

7.8 Blodgett's Design Of Welded Structures, 1966.

7.9 AISC Manual Of Steel Construction, 9th edition.

7.10 ASTM Annual Book of ASTM Standards. Volume 01.04 .

\begin{tabular}{|c|c|c|c|}
\hline 9. NAC & Performed by: $\theta$ RQ & Date: $11-25-96$ & $\begin{array}{l}\text { Calculation No. 457-2005.3 } \\
\text { Revision I }\end{array}$ \\
\hline & Checked by: $R S$ & $\begin{array}{l}\text { Date: } \\
11 / 25 / 96\end{array}$ & Page 14 of 14 \\
\hline
\end{tabular}


Appendix A9.2.5 Cold Vacuum Drying Lid Calculation 457-2001.2

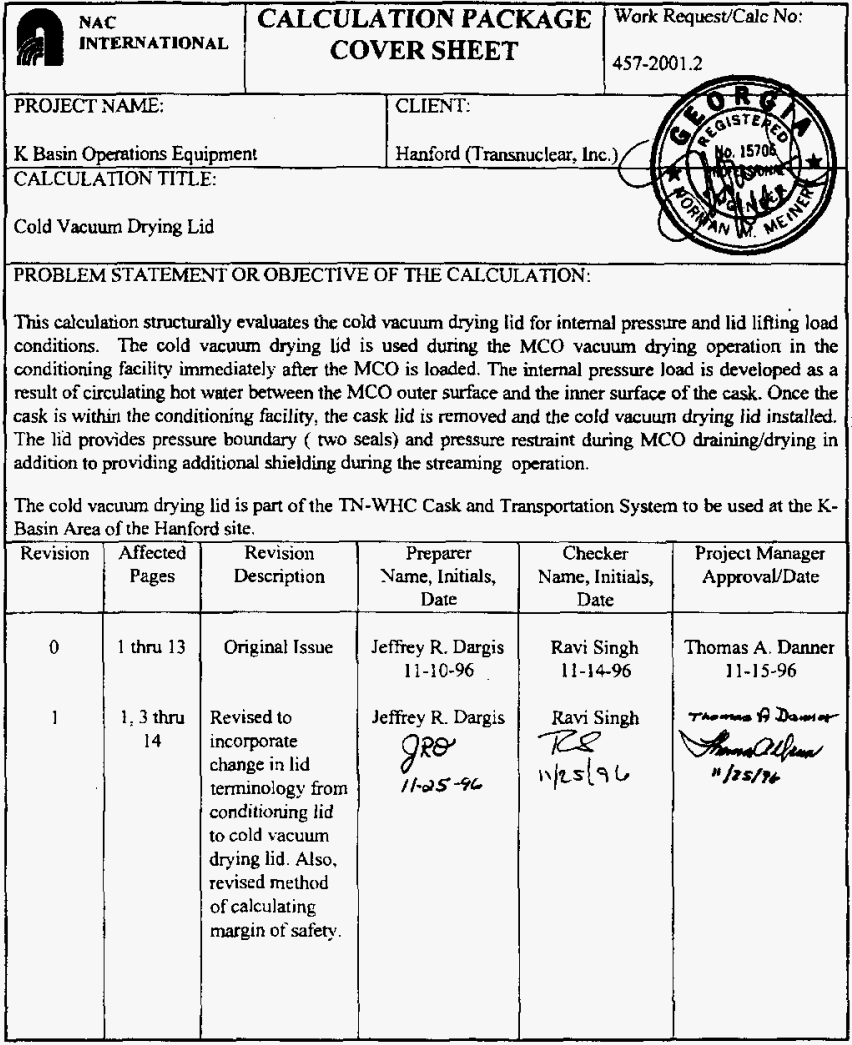


Appendix A9.2.5 Cold Vacuum Drying Lid Calculation 457-2001.2 (Continued)

\section{INDEPENDENT DESIGN VERIFICATION CHECK SHEET}

Work Request/Calculation No: 457-2001.2 Revision $\underline{0}$

Scope Of Analysis File: This calculation structurally evaluates the conditioning lid for internal pressure and lid lifting load conditions.

Review Methodology: Check Of Calculations

Alternate Analyses

Other (Explain)

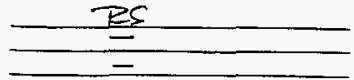

Confirm That The Work Request / Calculation Package Reviewed Includes:

1. Statement of Purpose

2. Defined Method of Analysis

3. Listing of Assumptions

4. Detailed Analysis Record

5. Statement of Conclusions / Recommendations (if applicable)

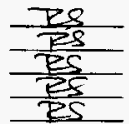

\begin{tabular}{|c|c|c|c|c|c|}
\hline Step & Activition & & o & & 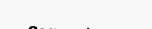 \\
\hline$\frac{\operatorname{stap}}{1}$ & $\begin{array}{l}\text { Aor the scope of the defined analysis: } \\
\text { A. Are the required data input complete? } \\
\text { 1. Material properties } \\
\text { 2. Geometry (drawing reference) } \\
\text { 3. Loading source term } \\
\text { If a supporting analysis is required to } \\
\text { define the load state, has it been } \\
\text { defined? } \\
\text { B. Are boundary conditions acceptable? }\end{array}$ & Yes & No & W/A & Comments \\
\hline 2 & is the method of analysis adequate for the defined scope? & $\sqrt{ }$ & & & \\
\hline 3 & Is the worst case loading/configuration documented? & $\checkmark$ & & & \\
\hline 4 & Are the acceptance criteria defined and complete? & $\vec{V}$ & & & \\
\hline 5 & Has all concurrent loading been considered? & & & & \\
\hline 5 & $\begin{array}{l}\text { Are anaiyses consistent with previous work for method and } \\
\text { approach? }\end{array}$ & & & $\checkmark$ & $\begin{array}{l}\text { NEW CALC } \\
\text { PACKAGE }\end{array}$ \\
\hline 7 & Are the records for input and output complete? & レ & & & \\
\hline 8 & Is traceability to verified software complete? & & & $\checkmark$ & $\begin{array}{l}\text { NO SOFTWARE } \\
\text { USED. }\end{array}$ \\
\hline 9 & $\begin{array}{l}\text { Is the statement of conclusions and recommendations complete } \\
\text { and acceptabie for the project and objectives of the defined } \\
\text { purpose? }\end{array}$ & & & & \\
\hline & 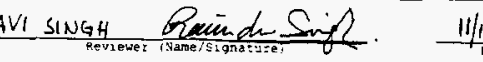 & & & & \\
\hline
\end{tabular}

2 
Appendix A9.2.5 Cold Vacuum Drying Lid Calculation 457-2001.2 (Continued)

\section{INDEPENDENT DESIGN VERIFICATION CHECK SHEET}

Work Request/Calculation No: 457-2001.2 Revision 1

Scope Of Analysis File: This calculation structurally evaluates the cold yacuum drying lid for internal pressure and lid lifting load conditions.

Review Methodology: Check Of Calculations

Alternate Analyses

Other (Explain)

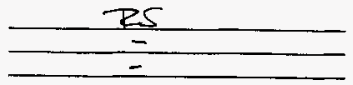

Confirm That The Work Request / Calculation Package Reviewed Inciudes:

1. Statement of Purpose

2. Defined Method of Analysis

3. Listing of Assumptions

4. Detailed Analysis Record

5. Statement of Conclusions / Recommendations (if applicable)

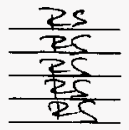

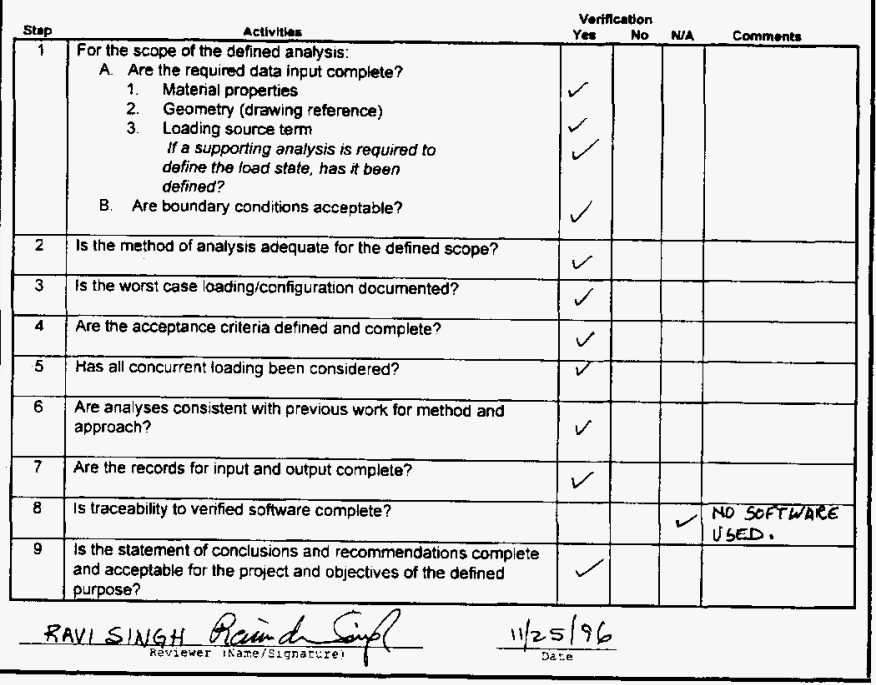

3 


\section{TABLE OF CONTENTS}

\begin{tabular}{|c|c|}
\hline Section & Description \\
\hline- & 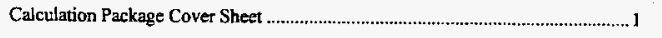 \\
\hline$\cdots$ & Independent Design Verification Check Sheet ................................................. 2 \\
\hline$\cdots$ & Table of Contents \\
\hline 1.0 & Synopsis of Results \\
\hline 2.0 & Introduction/Purpose \\
\hline 3.0 & Method of Analysis \\
\hline 4.0 & Assumptions/Design Input \\
\hline 5.0 & Analysis Detail \\
\hline 6.0 & 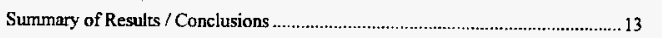 \\
\hline 7.0 & 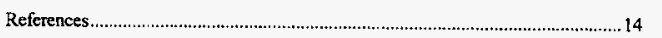 \\
\hline
\end{tabular}

\begin{tabular}{|c|c|c|}
\hline Performed by $g R \theta$ & $\begin{array}{l}\text { Date: } \\
11-\infty \$ \text { th }\end{array}$ & $\begin{array}{l}\text { Calculation No. 457-2001.2 } \\
\text { Revision I }\end{array}$ \\
\hline Checked by: $2 S$ & $\begin{array}{l}\text { Dare: } 11 / 25 / 96 \\
\text { ing }\end{array}$ & Page 4 of 14 \\
\hline
\end{tabular}


Summary of Stress Analysis

\begin{tabular}{|c|c|c|c|c|c|c|c|}
\hline $\begin{array}{l}\text { Drawing } \\
\text { No. }\end{array}$ & $\begin{array}{l}\text { Item } \\
\text { No. }\end{array}$ & Component & $\begin{array}{l}\text { Applied } \\
\text { Load }\end{array}$ & $\begin{array}{l}\text { Design } \\
\text { Check }\end{array}$ & $\begin{array}{l}\text { Calculated } \\
\text { Loading }\end{array}$ & Allowable & M.S. \\
\hline $457-106$ & 1 & lid flange & pressure & torsion & $5,031 \mathrm{psi}$ & $16.7 \mathrm{ksi}$ & 0.70 \\
\hline $457-106$ & 2 & bolt & pressure & tension & $13,590 \mathrm{psi}$ & $16.7 \mathrm{ksi}$ & 0.19 \\
\hline $457-106$ & 8 & lifting lug & $\begin{array}{l}3 \text { times } \\
\text { the } \\
\text { weight }\end{array}$ & $\begin{array}{l}\text { bearing } \\
\text { tension } \\
\text { shear }\end{array}$ & $\begin{array}{l}4,093 \text { psi } \\
4,093 \text { psi } \\
8,187 \text { psi }\end{array}$ & $\begin{array}{l}30 \mathrm{ksi} \\
30 \mathrm{ksi} \\
18 \mathrm{ksi} \\
\end{array}$ & $\begin{array}{l}0.86 \\
0.86 \\
0.55\end{array}$ \\
\hline $457-106$ & $\begin{array}{l}\text { liem } \\
8 \\
\text { to } \\
\text { Item } \\
1\end{array}$ & weld & $\begin{array}{l}3 \text { times } \\
\text { the } \\
\text { weight }\end{array}$ & shear & $620 \mathrm{psi}$ & $\begin{array}{l}\text { large } \\
\text { compared } \\
\text { to } \\
\text { actual } \\
\text { stress }\end{array}$ & large \\
\hline
\end{tabular}

Therefore the cold vacuum drying lid design meets the criteria defined in Section 4.2.

\subsection{INTRODUCTION / PURPOSE}

This calculation structurally evaluates the cold vactoum drying lid for internal pressure and lid lifting load conditions. The cold vacuum drying lid is used during the $\mathrm{MCO}$ vacuum drying operation in the conditioning facility immediately after the MCO is loaded. The internal pressure load is developed as a result of circulating hot water between the MCO outer surface and the inner surface of the cask. Once the cask is within the conditioning facility, the cask lid is removed and the cold vacuum drying lid installed. The lid is a " $Z$ " shaped ring. The lower leg of the $Z$ rests on the top of the cask. Three (3) lid bolts are used to join the cold vacuum drying lid to the cask. The upper $Z$ leg bears against the MCO top surface. The lid provides pressure boundary ( two seals) and pressure restraint during MCO draining/drying in addition to providing additional shielding during the streaming operation.

The cold vacuum drying lid is part of the TN-WHC Cask and Transportation System to be used at the K-Basin Area of the Hanford site. The cold vacuum drying lid was initially evaluated in Reference 7.1.

INTEKNA TIONAL

\begin{tabular}{|c|c|c|}
\hline Performed by & $\begin{array}{l}\text { Date: } \\
11-25-96\end{array}$ & $\begin{array}{l}\text { Calculation No. 457-2001,2 } \\
\text { Revision } 1\end{array}$ \\
\hline Checked by: & $11 / 2 \leqslant 96$ & Page 5 of 14 \\
\hline
\end{tabular}




\section{Appendix A9.2.5 Cold Vacuum Drying Lid Calculation 457-2001.2 (Continued)}

Hand calculations using classic textbook solutions are used to structurally evaluate the cold vacuum drying lid design.

The lid design will be assessed utilizing a pressure loading of 60 psi (Reference 7.10). Only the lid bolting and the upper $Z$ leg of the lid are influenced by pressure loading. The pressure induced bolt stress will be compared to the ASME Cade Class 2/3 stress allowable, $\mathbf{S}$. For upper $Z$ leg flange rotation, the pressure induced torsional stress will also be limited to $S$.

The lifting lug acceptance criteria is to limit tension and bearing stresses to the lesser of:

(a) one-third material yield strength; or

(b) one-fifth the material ultimate strength.

Shear stresses will be limited to 0.6 times the tensile stress limits.

The following evaluations are documented within this calculation:

- Lid Hold Down Evaluation; and

- Lifting Lug Assessment.

\subsubsection{Lid Hold Down Evaluation}

The lid is connected to the cask by three (3) $1-1 / 2$ 6UNC fabricated bolts. The boits attach the lower lid flange to the cask shell. The lid-to-shell contact is metal-to-metal. An $\mathrm{O}$-ring seal is used to maintain the pressure boundary between the lid and the cask. A silicone seal is also incorporated to maintain the pressure boundary between the cold vacuum drying lid and the MCO shell. The MCO seal is located on the vertical leg of the $\mathrm{Z}$ cold vacuum drying lid design. Sealing is achieved by pressurizing the silicon seal internally.

To evaluate the lid hold down, the following steps will be performed:

- The net pressure cross section and pressure load will be determined;

- The lid bolt load will be determined; and

- The maximum upper flange stress due to rotation under pressure loading will be determined.

\subsubsection{Lifting Lug Evaluation}

The lid has three (3) lifting lugs attached to the top surface of the lid. These lugs are used to install/remove the lid to/from the cask. The lug bearing stress, net tension and shear pull out will be assessed. The lifting criteria of Reference 7.3 will be used to

\begin{tabular}{|c|c|c|c|}
\hline NAC: & Performed by: gRe & 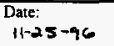 & $\begin{array}{l}\text { Calculation No. } 457.20012 \\
\text { Revision ? }\end{array}$ \\
\hline & Checked by: $R S$ & $11 / 25 / 96$ & Page 6 of 14 \\
\hline
\end{tabular}


Appendix A9.2.5 Cold Vacuum Drying Lid Calculation 457-2001.2 (Continued)

determine the adequacy of the lug design. The lug-to-lid weld will also be assessed.

\subsection{ASSUMPTIONS / DESIGN INPUTS}

\subsection{Assumprions}

There are no unverified assumptions within this calculation.

\subsection{Design Criteria}

\subsubsection{Bolt and Flange Bending Evaluation}

Calculated bolt tensile stress (due to pressure)

Calculated flange torsional stress (due to pressure)

<S (Ref. 7.7)

$<\mathrm{S}$ (Ref. 7.7)

4.2.2 Lift Lug Evaluation

Calculated tensile stress (based on load factor of 3)

Calculated tensile stress (based on load factor of 5)

Calculated shear stress (based on load factor of 3)

Calculated shear stress (based on load factor of 5)

$<0.6 \mathrm{~S}_{\text {yieid }}$

$<0.6 \mathrm{~S}_{\text {ultimate }}$

Calculated bearing stress (based on load factor of 3 )

Calculated bearing stress (based on ioad factor of 5)

$<\mathrm{S}_{\text {yield }}$

$<S_{\text {ultimatc }}$

\subsection{Design Conditions}

$$
\begin{array}{ll}
\text { Pressure: } & 60 \text { psi (Reference } 7.10) \\
\text { Temperature: } & \text { Ambient. } 100^{\circ} \mathrm{F}
\end{array}
$$

\begin{tabular}{|c|c|c|}
\hline \multirow[t]{5}{*}{ Bolts: } & \multicolumn{2}{|c|}{ (Reference 7.6.1) } \\
\hline & Size: & 1.22 " diameter \\
\hline & Material: & A.276 TP304 SS \\
\hline & Area: & $\begin{array}{l}=\pi \times D^{2} / 4 \\
=1.17 \mathrm{in}^{2}\end{array}$ \\
\hline & Threading & $1-1 / 26 \cup N C-2 A$ \\
\hline
\end{tabular}

\subsection{Component Propertics}

\begin{tabular}{|c|c|c|c|}
\hline NAC & Performed by: $g R \theta$ & $\begin{array}{l}\text { Date: } \\
11-25-96\end{array}$ & $\begin{array}{l}\text { Caleulation No. } 457-2001.2 \\
\text { Revision 1 }\end{array}$ \\
\hline & Checked by: 25 & Date $1 / 25 / 96$ & Page 7 of 14 \\
\hline
\end{tabular}

(Reference 7.6.1) 
Appendix A9.2.5 Cold Vacuum Drying Lid Calculation 457-2001.2 (Continued)

$\begin{array}{lll}\text { Lid flange: } & \text { (Reference } 7.6 .1) \\ \text { (upper leg) } & \text { Size: } & 3.5 \text { "thick } \\ & D_{\text {ing: }} & 18.48 \text { in } \\ & D_{\text {o flg }} & 31.5 \text { in } \\ & \text { Material: } & \text { ASTM A240, Type } 304\end{array}$

Lid flange: (Reference 7.6.1)

(vertical leg) Size: $\quad 11-3.5-4=3.5$ axial length

$D_{\text {i } n g: ~} \quad 25.5$ in

$\mathrm{D}_{\mathrm{o} \text { f }}$ : $\quad 31.5 \mathrm{in}$

Material: $\quad$ ASTM A240, Type 304

Lid flange: (Reference 7.6.1)

(lower leg) Size: $4.0^{\text {"n thick }}$

$D_{\mathrm{i} \theta \mathrm{g}}: \quad 25.5 \mathrm{in}$

$\mathrm{D}_{\mathrm{og}}$ : $\quad 39.81$ in

Material: $\quad$ ASTM A240, Type 304

Lift Lug: (Reference 7.6.1)

Thickness: 0.375 in

$D_{\text {lag: }} \quad 2.0$ in

$D_{\text {hile: }} \quad 1.0$ in

Materia): $\quad$ ASTM A240, Type 304

Lid Weight: $\quad W=1535 \mathrm{lbs}$. (Reference 7.6.1)

O-ring: $\quad$ Diameter: $\quad 31.8$ in (Reference 7.6.1)

4.5 Material Properties

- $\quad$ ASTM A240 (Reference 7.7.1, 7.7.2)

(Note: use SA240, Type 304 SS properties)

Fy: $30 \mathrm{ksi}$

Fu: $75 \mathrm{ksi}\left(100^{\circ}\right)$

- $\quad$ SA276 TP304 (Reference 7.5)

Fy: $30 \mathrm{ksi}$

Fu: $75 \mathrm{ksi}$

- SA240, Type 304 (Reference 7.7.4)

S: $\quad 16,700$ psi (at 200 degrees)

\begin{tabular}{|c|c|c|c|}
\hline A INTERNATIONAL & Performed by: $g R \theta$ & Date: $11-25-96$ & $\begin{array}{l}\text { Calculation No. 457-2001.2 } \\
\text { Revision } 1\end{array}$ \\
\hline & Checked by: & $\begin{array}{l}\text { Date } \\
11 / 25 / 96\end{array}$ & Page 8 of 14 \\
\hline
\end{tabular}


Appendix A9.2.5 Cold Vacuum Drying Lid Calculation 457-2001.2 (Continued)

5.0 ANALYSIS DETAIL

5.1 Lid Hold Down Evaluation

\subsubsection{I.id Bolt Pressure Load Evaluation}

The o-ring diameter is used as the pressure load development area. This area , A, is:

$$
\begin{aligned}
& A=\Pi / 4\left(D_{\theta-\mathrm{nns}}{ }^{2}\right) \\
& A=\Pi / 4\left(31.8^{2}\right) \\
& A=795 \mathrm{in}^{2}
\end{aligned}
$$

The net pressure load, F, with no reduction for the weight of the MCO is;

$$
\begin{aligned}
F & =\text { pressure } x \text { area } \\
& =60 \times 795 \\
& =47,700 \mathrm{lbs} .
\end{aligned}
$$

The lid bolt load, $\mathrm{F}_{\text {mait }}$, is;

$$
\begin{aligned}
\mathrm{F}_{\text {bolt }} & =\mathrm{F} / \text { no. bolts } \\
& =\mathrm{F} / 3 \\
& =47,700 / 3 \\
& =15,900 \mathrm{lbs} .
\end{aligned}
$$

The boit tensile stress is:

$$
\begin{aligned}
\text { Stress } & =F_{\text {bolk }} / \text { area } \\
& =15,900 / 1.17 \\
& =13,590 \mathrm{psi}
\end{aligned}
$$

The bolt al lowabie stress, $\mathrm{S}$ is:

$S=16,700$ psi

$$
\begin{aligned}
\text { Safery Margin } & =1-(\text { stress / S }) \\
& =1-(13,590 / 16,700) \\
& =0.19
\end{aligned}
$$

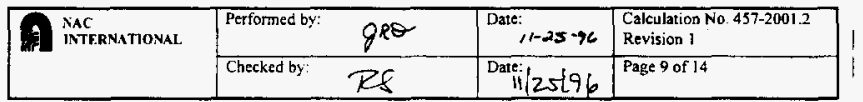


Appendix A9.2.5 Cold Vacuum Drying Lid Calculation 457-2001.2 (Continued)

\subsubsection{Lid Flange Pressure Stress Evaluation}

Conservatively, consider the pressure load is reacted at the cold vacuum drying lid upper flange. The maximum lid stress will then be developed by the rotation of the upper flange. The reaction along the inner edge of the upper flange per circumferential inch. $\mathbf{F}_{0}$, is:

$$
\begin{aligned}
F_{0} & =F /\left(2 \times \Pi \times R_{\text {j-upper flg }}\right) \\
& =47,700 /(2 \times \Pi \times(18.48 / 2)) \\
& =822 \mathrm{lbs} / \mathrm{in}
\end{aligned}
$$

The uniform torque about the upper flange center of gravity due to $F_{0}$ is

$$
\begin{aligned}
T_{0} & =F_{0} \times\left(R_{\text {crspper flg }}-R_{i-\text { upper fig }}\right) \\
& =822 \times[(31.5 / 2)-(18.48 / 2)] \\
& =5,351 \text { in-lbs } / \text { in }
\end{aligned}
$$

Per Reference 7.8.1, for a ring under distributed torque:

$\sigma_{\text {Inque }}=T_{4} \times R /(\mathrm{I} / \mathrm{c})$

where $\mathrm{R}$ is equal to the mean radius of the ring.

The effective section $\mathrm{V} / \mathrm{c}$ is:

$$
\begin{aligned}
I / \mathrm{c} & =\left(\mathrm{R}_{\text {roupper flg }}-\mathbf{R}_{\text {j-upper } \left.\mathrm{ng}_{\mathrm{g}}\right) \times \mathrm{t}^{2} / 6}\right. \\
& =((31.5 / 2)-(18.48 / 2)) \times(3.5)^{2} / 6 \\
& =13.29 \mathrm{in}^{3}
\end{aligned}
$$

\begin{tabular}{|c|c|c|c|}
\hline GI MAC & Performed by: $g R \theta$ & Date: $11-25-96$ & $\begin{array}{l}\text { Calculation No. } 457-2001.2 \\
\text { Revision } 1\end{array}$ \\
\hline & Checked by: $R g$ & Date $11 / 25196$ & Page 10 of 14 \\
\hline
\end{tabular}

The torsional stress, $\sigma_{\text {torawe }}$, is therefore:

$$
\begin{aligned}
\sigma_{\text {trrauc }} & =T_{0} \times R /(\mathrm{I} / \mathrm{c}) \\
& =5.351 \times((3 \mathrm{t} .5+18.48) / 4) / 13.29 \\
& =5.031 \mathrm{psi}
\end{aligned}
$$

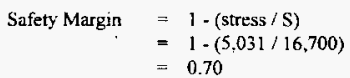


Appendix A9.2.5 Cold Vacuum Drying Lid Calculation 457-2001.2 (Continued)

\subsection{Lifting Lug Evaluation}

\subsubsection{Bearing Check}

The pin bearing area is:

Bearing area $=t \times D_{\text {hole }}$

$=0.375 \times 1.0$

$=0.375 \mathrm{in}^{2}$

To determine the most conservative load factor, the material yield and ultimate strengths will be normalized.

For SA240 Type 304 SS

$F_{y} / 3=30,000 / 3=10,000$ psi

$\mathrm{F}_{\mathrm{u}} / 5=75,000 / 5=15,000 \mathrm{psi}$

Therefore, comparing a factor (LF) of 3 against yield is more restrictive.

The bearing stress is:

$$
\begin{aligned}
\text { Stress } & =\mathrm{LF} \times(\mathrm{W} / 3 \text { lugs }) / \text { area } \\
& =3 \times(1,535 / 3) / 0.375 \\
& =4,093 \mathrm{psi}
\end{aligned}
$$

The safety margin is:

Safety Margin $=1 \cdot\left(\right.$ stress $\left./ S_{y}\right)$

$=1 \cdot(4,093 / 30,000)$

$=0.86$

5.2 .2 Kiet Tension

$$
\begin{aligned}
\text { Area } & =t\left(\mathbf{D}_{\text {lug }}-\mathrm{D}_{\text {hoie }}\right) \\
& =0.375(2.0-1.0) \\
& =0.375 \mathrm{in}^{2}
\end{aligned}
$$

\begin{tabular}{|l|l|l|}
\hline Performed by: & Date: \\
$1 /-05-96$ & $\begin{array}{l}\text { Calculation No. 457-2001.2 } \\
\text { Revision 1 }\end{array}$ \\
\hline Checked by: & $\begin{array}{r}\text { Date } 11 / 2596 \\
11\end{array}$ & Page 11 of 14 \\
\hline
\end{tabular}


Appendix A9.2.5 Cold Vacuum Drying Lid Calculation 457-2001.2 (Continued)

The tensile stress is:

Stress $=\operatorname{LF} \times(\mathbf{W} / 3$ lugs $) /$ (Area)

$=3 \times(1,535 / 3) /(0.375)$

$=4,093 \mathrm{psi}$

The safety margin is:

Safery Margin $=1-\left(\right.$ stress $\left./ S_{y}\right)$

$=1-(4,093 / 30,000)$

$=0.86$

\subsubsection{Shear Pull Out Al 45 Degrees}

$$
\begin{aligned}
\text { Shear Area } & =t\left(R_{\text {lug }}-R_{\text {hole }}\right) \\
& =0.375(1.0-0.5) \\
& =0.1875 \mathrm{in}^{2}
\end{aligned}
$$

The shear stress is:

Stress $=$ LF $\times(W / 3$ lugs $) /($ Shear Area $)$

$=3 \times(1.535 / 3$ lugs $) /(0.1875)$

$=8,187 \mathrm{psi}$

Safety Margin $\quad=1-\left(\right.$ stress $\left./\left(0.6 \mathrm{~S}_{\mathrm{y}}\right)\right)$

$=1-(8,187 ; 18,000)$

$$
=0.55
$$

\$.2.4 Lug To Lid Weld

The lug to lid is weided all-around with a 0.25 " fillet. Consider the entire lug

\begin{tabular}{|c|c|c|c|}
\hline INTERVATIONAL & Performed by: ges & $\begin{array}{l}\text { Date: } \\
\quad 11-25-46\end{array}$ & $\begin{array}{l}\text { Calculation No. 457-2001.2 } \\
\text { Revision I }\end{array}$ \\
\hline & Checked by: 2 & Date: $11 / 25 / 96$ & Page 12 of 14 \\
\hline
\end{tabular}
load is carried in shear along the vertical weld section:

$$
\begin{aligned}
A_{\text {shear }} & =2 \times 0.707 \times 0.25 * \times\left(11^{*}-4^{*}\right) \\
& =2.475 \mathrm{in}^{2} \\
\sigma_{\text {weld }} & =\mathrm{LF} \times(\mathrm{W} / \text { no. of lugs }) / \mathrm{A}_{\text {shcar }} \\
& =3 \times(1535 / 3) / 2.475 \\
& =620 \mathrm{psi}
\end{aligned}
$$

Allowabie is large compared to actual stress. Therefore, weld is adequate. 
Appendix A9.2.5 Cold Vacuum Drying Lid Calculation 457-2001.2 (Continued)

\subsection{SUMMARY OF RESULTS / CONCLUSIONS}

Summary of Stress Analysis

\begin{tabular}{|c|c|c|c|c|c|c|c|}
\hline $\begin{array}{l}\text { Drawing } \\
\text { No. }\end{array}$ & $\begin{array}{l}\text { Item } \\
\text { No. }\end{array}$ & Component & $\begin{array}{l}\text { Applied } \\
\text { Load }\end{array}$ & $\begin{array}{l}\text { Design } \\
\text { Check }\end{array}$ & $\begin{array}{l}\text { Calculated } \\
\text { Loading }\end{array}$ & Allowable & M.S. \\
\hline 457.106 & 1 & lid flange & pressure & torsion & 5,031 psi & $16.7 \mathrm{ksi}$ & 0.70 \\
\hline $457-106$ & 2 & boit & pressure & tension & $13,590 \mathrm{psi}$ & $16.7 \mathrm{ksi}$ & 0.19 \\
\hline $457-106$ & 8 & lifting lug & $\begin{array}{l}3 \text { times } \\
\text { the } \\
\text { weight }\end{array}$ & $\begin{array}{l}\text { bearing } \\
\text { tension } \\
\text { shear }\end{array}$ & $\begin{array}{l}4,093 \mathrm{psi} \\
4,093 \mathrm{psi} \\
8,187 \mathrm{psi}\end{array}$ & $\begin{array}{l}30 \mathrm{ksi} \\
30 \mathrm{ksi} \\
18 \mathrm{ksi} \\
\end{array}$ & $\begin{array}{l}0.86 \\
0.86 \\
0.55\end{array}$ \\
\hline $457-106$ & \begin{tabular}{|l|} 
Item \\
8 \\
to \\
Item \\
1 \\
\end{tabular} & weld & $\begin{array}{l}3 \text { times } \\
\text { the } \\
\text { weight }\end{array}$ & shear & $620 \mathrm{psi}$ & $\begin{array}{l}\text { large } \\
\text { compared } \\
\text { to } \\
\text { actual } \\
\text { stress } \\
\end{array}$ & large \\
\hline
\end{tabular}

Therefore the cold vacuum drying lid design meets the criteria defined in Section 4.2.

\begin{tabular}{|c|c|c|c|}
\hline A. NAC & Performed by: $g \Omega \theta$ & $\begin{array}{l}\text { Date: } \\
11-25-96\end{array}$ & $\begin{array}{l}\text { Calculation No } 457-2001.2 \\
\text { Revision ? }\end{array}$ \\
\hline & Checked by: $\quad R Q$ & Date: $11 / 25196$ & Page 13 of 14 \\
\hline
\end{tabular}


Appendix A9.2.6 Seal Lid Calculation 457-2004.2

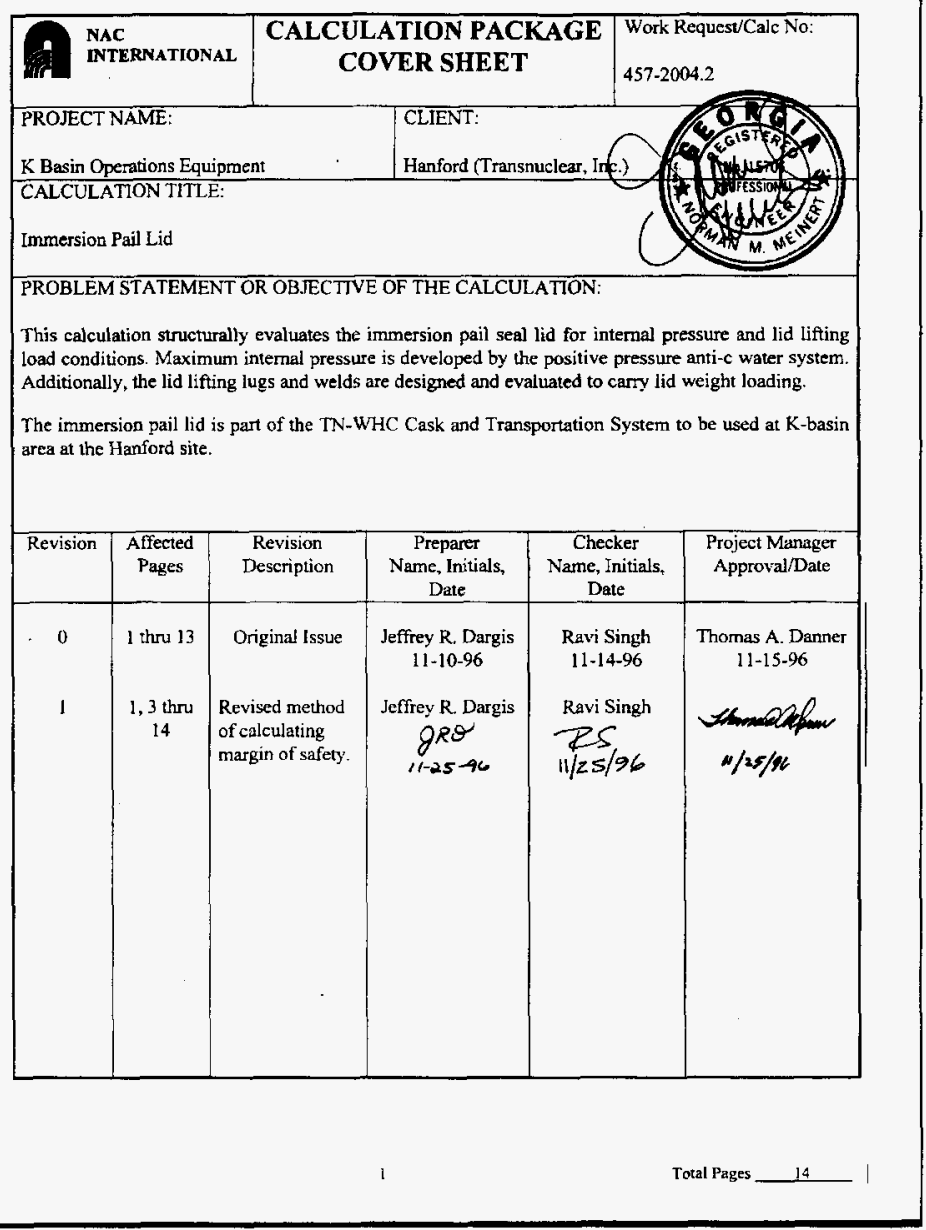


Appendix A9.2.6 Seal Lid Calculation 457-2004.2 (Continued)

\section{INDEPENDENT DESIGN VERIFICATION CHECK SHEET}

Work Request/Calculation No: 457-2004.2 Revision 0

Scope Of Analysis File: This calculation structuralky evaluates the immersion pail lid for internal pressure and lid lifting load conditions.

Review Methodology: Check of Calculations

Alternate Analyses

Other (Explain)

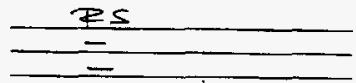

Confirm That The Work Request / Calculation Package Reviewed Includes:

1. Statement of Purpose

2. Defined Method of Analysis

3. Listing of Assumptions

4. Detailed Analysis Record

5. Statement of Conclusions / Recommendations (if applicable)

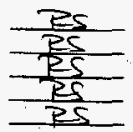

\begin{tabular}{|c|c|c|c|c|c|}
\hline stop & Acotiviten: & & ntion & 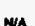 & Compents \\
\hline 1 & $\begin{array}{l}\text { For the } 5 \text { cope of the defined anahysis: } \\
\text { A. Are the required data input complete? } \\
\text { 1. Material properties } \\
2 . \text { Geometry (drawing reference) } \\
\text { 3. Loading source tem } \\
\text { If a supporting anaiysis is requined to } \\
\text { define the load state, has it been } \\
\text { defined? } \\
\text { B. Are boundary conditions acceptable? }\end{array}$ & & & & \\
\hline 2 & Is the method of analysis adequate for the defined scope? & L & & & \\
\hline 3 & Is the worst case loading/configuration documented? & $\checkmark$ & & & \\
\hline 4 & Are the acceptance criteria defined and complete? & $\checkmark$ & & & \\
\hline 5 & Has all concurrent bading been considered? & & & & \\
\hline 6 & $\begin{array}{l}\text { Are analyses consistent with previous work for method and } \\
\text { approach? }\end{array}$ & & & $\checkmark$ & $\begin{array}{l}\text { NEW CALC } \\
\text { PACKAGE }\end{array}$ \\
\hline 7 & Are the records for input and output complete? & $\checkmark$ & & & \\
\hline 8 & Is traceability to verified sottware complete? & & & $\checkmark$ & $\begin{array}{l}\text { NO SOFTWADE } \\
\text { USEDS. }\end{array}$ \\
\hline 9 & $\begin{array}{l}\text { Is the statement of conclusions and recommendations complete } \\
\text { and acceptable for the project and objectives of the defined } \\
\text { purpose? }\end{array}$ & & & & \\
\hline
\end{tabular}


Appendix A9.2.6 Seal Lid Calculation 457-2004.2 (Continued)

\section{INDEPENDENT DESIGN VERIFICATION CHECK SHEET}

Work Request/Calculation No: $457-2004.2$ Revision 1

Scope Of Analysis File: This calculation structurally evaluates the immersion pail lid for internal pressure and lid lifting load conditions.

Review Methodology: Check Of Calculations

Alternate Analyses

Other (Explain)

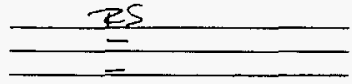

Confirm That The Work Request / Calculation Package Reviewed Includes:

1. Statement of Purpose

2. Defined Method of Analysis

3. Listing of Assumptions

4. Detailed Analysis Record

5. Statement of Conclusions / Recommendations (if applicable)

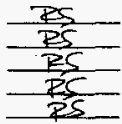

\begin{tabular}{|c|c|c|c|c|}
\hline Step & Activitiet & Yerification & $\mathrm{NA}$ & Comment \\
\hline 1 & $\begin{array}{l}\text { For the scope of the defined analysis: } \\
\text { A. Are the required data input complete? } \\
\text { 1. Material properties } \\
\text { 2. Geometry (drawing reference) } \\
\text { 3. Loading source term } \\
\text { If a supporting analysis is required to } \\
\text { define the load state, has it been } \\
\text { defined? } \\
\text { B. Are boundary conditions acceptable? }\end{array}$ & & & \\
\hline 2 & Is the method of analysis adequate for the defined scope? & & & \\
\hline 3 & Is the worst case loadingiconfiguration documented? & & & \\
\hline 4 & Are the acceptance criteria defined and complete? & & & \\
\hline 5 & Has all concurrent loading been considered? & & & \\
\hline 6 & $\begin{array}{l}\text { Are analyses consistent with previous work for method and } \\
\text { approach? }\end{array}$ & & & \\
\hline 7 & Are the records for inpul and output complete? & & & \\
\hline 8 & Is traceability to verified sottware complete? & & & $\begin{array}{l}\text { ND SOFTWARE } \\
\text { USETD. }\end{array}$ \\
\hline g & $\begin{array}{l}\text { Is the statement of conclusions and recommendations complete } \\
\text { and acceptable for the project and objectives of the defined } \\
\text { purpose? }\end{array}$ & & & \\
\hline
\end{tabular}


Appendix A9.2.6 Seal Lid Calculation 457-2004.2 (Continued)

\section{TABLE OF CONTENTS}

\begin{tabular}{|c|c|}
\hline Section & Description \\
\hline-- & 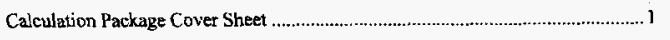 \\
\hline- & 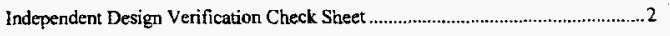 \\
\hline- & 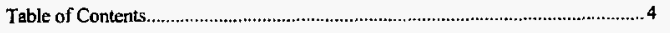 \\
\hline 1.0 & Synopsis of Results \\
\hline 2.0 & 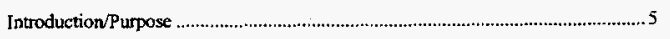 \\
\hline 3.0 & Method of Analysis \\
\hline 4.0 & 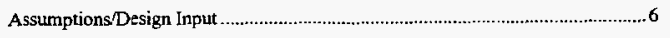 \\
\hline 5.0 & Analysis Detail \\
\hline 6.0 & 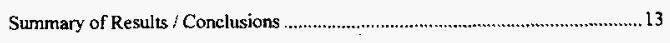 \\
\hline 7.0 & 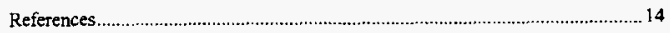 \\
\hline
\end{tabular}


Appendix A9.2.6 Seal Lid Calculation 457-2004.2 (Continued)

1.0 SYNOPSIS OF RESULTS

Summary of Stress Analysis

\begin{tabular}{|l|l|l|l|l|l|l|l|}
\hline $\begin{array}{l}\text { Drawing } \\
\text { No. }\end{array}$ & $\begin{array}{l}\text { Item } \\
\text { No. }\end{array}$ & Component & $\begin{array}{l}\text { Applied } \\
\text { Load }\end{array}$ & $\begin{array}{l}\text { Design } \\
\text { Check }\end{array}$ & $\begin{array}{l}\text { Calculated } \\
\text { Loading }\end{array}$ & Allowable & M.S. \\
\hline $457-103$ & 2 & lid flange & pressure & bending & $8,936 \mathrm{psi}$ & $25.05 \mathrm{ksi}$ & 0.64 \\
\hline $457-103$ & 20 & bolt & pressure & tension & $4,201 \mathrm{psi}$ & $16.7 \mathrm{ksi}$ & 0.75 \\
\hline $457-103$ & 3 & lifting lug & $\begin{array}{l}3 \text { times } \\
\text { the } \\
\text { weight }\end{array}$ & $\begin{array}{ll}\text { bearing } \\
\text { tension }\end{array}$ & $\begin{array}{l}1600 \mathrm{psi} \\
1600 \mathrm{psi}\end{array}$ & $30 \mathrm{ksi}$ & 0.95 \\
\hline
\end{tabular}

The immersion pail lid design meets the criteria defined in Section 4.2.

\subsection{INTRODUCTION/PURPOSE}

This calculation structurally evaluates the immersion pail seal lid for intemal pressure and lid lifting load conditions. Maximum internal pressure load is developed as by the positive pressure anti-c water system. Additionally the lid lifting lugs and welds are designed and evaluated to carry lid weight loading.

The immersion pail lid is part of the TN-WHC Cask and Transportation System to be used at K-basin area at the Hanford site. The immersion pail system was initially evaluated in Reference 7.1.

\subsection{METHOD OF ANALYSIS}

Hand calculations using classic textbook solutions are used to structurally evaluate the immersion pail lid design.

The lid design will be assessed for an intemal pressure loading of 5 psi (Reference 7.4). Only the lid bolting and adjacent flange are influenced by a pressure loading of this level. For the bolting. the pressure induced stress will be compared to the ASME Code Class 2/3 (Reference $7.7)$ bolting allowable, $S$. For flange bending. the pressure induced stress will be limited to $1.5 \mathrm{~S}$.

\begin{tabular}{|c|c|c|c|c|}
\hline (2) NAC & Performed by: & ges & $\begin{array}{l}\text { Date: } \\
1 / 25-x\end{array}$ & $\begin{array}{l}\text { Calculation No. } 457-2004.2 \\
\text { Revision 1 }\end{array}$ \\
\hline & Checked by: & $R S$ & Date; $11 / 25 / 96$ & Pape 5 of 14 \\
\hline
\end{tabular}


Appendix A9.2.6 Seal Lid Calculation 457-2004.2 (Continued)

The lifting lug acceptance criteria is to limit tension and bearing stresses to the lesser of:

(a) one-third material yield strength; or

(b) one-fifth the material ultimate strength.

Shear stresses will be limited to 0.6 times the tensile stress limits

The following evaluations are documented within this calculation:

- Lid Hold Down Evaluation; and

- Lifting Lug Assessment.

\subsection{Lid Hold Down Evaluation}

The lid is connected to the upper flange of the pail shell by four (4) $3 / 4-10$ bolts. The bolts attach the lid flange to the pail shell flange. The lid-to-pail shell contact is metalto-metal. Two (2) silicon seals are also used in the lid design. The seal at the outer lid diameter seals the lid-to-pail shell connection. The inner seal seals the lid-1o-MCO canister joint. The sealing is achieved by pressurizing the seals internally.

To evaluate the lid hold down, the following steps will be performed:

- The net pressure cross section and pressure load will be determined;

- The lid bolt load will be determined; and

- The maximum lid flange bending stress due to pressure will be determined.

\subsection{Lifting Lug Evaluation}

The lid has three (3) lifting lugs attached to the top surface of the lid. These lugs are used to install/remove the lid to/from the immersion pail. The lug bearing stress, net tension and shear pull out will be assessed. The lifting criteria of Reference 7.3 will be used to determine the adequacy of the lug design.

\section{ASSUMPTIONS / DESIGN INPUTS}

\subsection{Assumptions}

There are no unverified assumptions within this calculation.

\begin{tabular}{|l|l|l|l|}
\hline \multirow{2}{*}{$\begin{array}{l}\text { NAC } \\
\text { INTFRnational }\end{array}$} & \begin{tabular}{c} 
Performed by: gRo \\
\cline { 2 - 4 }
\end{tabular} & $\begin{array}{l}\text { Date: } \\
11-25-96\end{array}$ & $\begin{array}{l}\text { Calculation No. 457-2004.2 } \\
\text { Revision l }\end{array}$ \\
\hline
\end{tabular}


Appendix A9.2.6 Seal Lid Calculation 457-2004.2 (Continued)

\subsection{Design Criteria}

\subsubsection{Bolt and Flange Bending Evaluation}

Calculated bolt tensile stress (due to pressure) $\quad<\mathrm{S} \quad$ (Reference 7.7)

Calculated flange bending stress (due to pressure) $<1.5 \times \mathrm{S}$ (Reference 7.7)

\subsubsection{Lift Lug Evaluation}

\begin{tabular}{|c|c|}
\hline Calculated tensile stress (based on load factor of 3 ) & $<\mathrm{S}_{\text {yield }} \quad(\operatorname{Ref} .7 .3)$ \\
\hline Calculaled tensile stress (based on load factor of 5) & $<\mathrm{S}_{\text {vlumale }}(\operatorname{Ref} .7 .3)$ \\
\hline Calculated shear stress (based on load factor of 3 ) & $<0.6 \mathrm{~S}_{\text {yield }}$ \\
\hline Calculated shear stress (based on load factor of 5) & $<0.6 \mathrm{~S}_{\text {ultimate }}$ \\
\hline Calculated bearing stress (based on I & \\
\hline Calculated bearing stress (based on load factor of 5) & $<\mathrm{S}_{\text {ultimate }}$ \\
\hline
\end{tabular}

4.3 Design Conditions

Pressure: $\quad 5$ psi (Reference 7.4)

Temperature: Ambient, $100^{\circ} \mathrm{F}$

4.4 Component Properties

Eye Bolts: (Reference 7.6.3)

Size: $\quad 3 / 4-10$

Material: Commercial SS

Area: $\quad 0.334 \mathrm{in}^{2} \quad$ (Reference 7.9.1)

Threading: $3 / 4-10$ UNC -2A (Reference 7.6.3)

Supplier: McMaster-Carr

Part No. 8891 T84
Lid flange: Size: $\quad 3 / 8$ plate
$D_{i n_{g}}: \quad 41.78-(2 \times 0.07)=41.92$ in (Reference 7.6 .3 )
$\mathrm{D}_{\mathrm{o}}$ : $\mathrm{g}$ : $\quad 45.75 \mathrm{in}$
Material: $\quad$ ASTM A240, Type 304

Lift Lug: (Reference 7.6.3)

Thickness: $\quad 0.375$ in

$\mathrm{D}_{\text {lug: }} \quad 2.0 \mathrm{in}$

$\mathrm{D}_{\text {hrie: }} \quad 1.0 \mathrm{in}$

Material: ASTM A240, Type 304

\begin{tabular}{|c|c|c|c|c|}
\hline $\begin{array}{l}\text { NAC } \\
\text { INTERNATIONAL }\end{array}$ & Performed by: & geo & Date: & $\begin{array}{l}\text { Calculation No. } 457.2004 .2 \\
\text { Revision I }\end{array}$ \\
\hline & Checked by: & $-k s$ & ${ }^{11} / 25 / 96$ & Page 7 of 14 \\
\hline
\end{tabular}


Appendix A9.2.6 Seal Lid Calculation 457-2004.2 (Continued)

Lid Weight; 600 lbs. (Reference 7.6.3)

4.5 Material Propcrties

- ASTM A240

(Note: use SA240, Type 304 SS properties)

(Reference 7.7.1, 7.7.2)

Fy: $30 \mathrm{ksi}$

Fu: $75 \mathrm{ksi}\left(100^{\circ}\right)$

- SA240, Type 304

(Reference 7.7.4)

S: 16,700 psi (at 200 degrees)

- 304 SS, commercial grade

(Note: SA240. Type 304 properties used.)

5.0 ANALYSIS DETAIL

5.1 Lid Hold Down Evaluation

5.1.1 Lid Bolt Pressure Load Evaluation

The lid pressure surface area, $A$, is:

$A=\Pi / 4\left(D_{0}^{2}-D_{i}^{2}\right)$

$\mathrm{A}=\left[1 / 4\left(45.75^{2}-25.77^{2}\right)\right.$

$\Lambda=1,122 \mathrm{in}^{2}$

The net pressure load, $F$, is;

$F=$ pressure $x$ are

$=5 \times 1122$

$=5.610 \mathrm{lbs}$.

The lid bolt load, $F_{\text {boh }}$, is:

\begin{tabular}{|c|c|c|c|}
\hline INTERNATIONAL & Performed by: $g R \theta$ & $\begin{array}{l}\text { Date: } \\
11-25-46\end{array}$ & $\begin{array}{l}\text { Calculation No. 457-2004.2 } \\
\text { Revision I }\end{array}$ \\
\hline & Checked by: $R S$ & Datei/25/96 & Page 8 of 14 \\
\hline
\end{tabular}


Appendix A9.2.6 Seal Lid Calculation 457-2004.2 (Continued)

$F_{\text {mott }}=F /$ no. bolts

$=\mathrm{F} / 4$

$=5610 / 4$

$=1,403 \mathrm{lbs}$

The bolt tensile stress is

Stress $=F_{\text {balu }} /$ area

$=1403 / 0.334$

$=4,201 \mathrm{psi}$

The bolt allowable stress, $S$ is:

$S=16,700 \mathrm{psi}$

The safety margin is:

Safety Margin = $1-($ stress $/ \mathbf{S})$

$=1-(4,201 / 16,700)$

$=0.75$

\section{1 .2 Lid Flange Pressure Stress Evaluation}

The lid flange is a tab type extension from the center ring. The bending of the tab due to the pressure load is determined below.

Consider the tab as a cantilever beam of length $L$ where;

$L=0.5\left(D_{o \text { ng }}-D_{i n_{g}}\right)$

$=0.5(45.75-41.92)$

$=1.915$ in

$\equiv 2.0 \mathrm{in}$

The bending moment , $\mathrm{M}$, is (considering the bolt location at $1 / 2 \mathrm{~L}$ );

$$
\begin{aligned}
M & =F_{b o l !} \times L / 2 \\
& =1403 \times 2 / 2 \\
& =1403 \mathrm{in}-\mathrm{lbs}
\end{aligned}
$$

\begin{tabular}{|c|c|c|c|}
\hline NAC & Performed by: $\mathrm{QAO}$ & $\begin{array}{l}\text { Date: } \\
/ 1 / 25-96\end{array}$ & $\begin{array}{l}\text { Calculation No. } 457.2004 .2 \\
\text { Revision I }\end{array}$ \\
\hline & Checked by: 75 & $11 / 25 / 96$ & Page 9 of 14 \\
\hline
\end{tabular}


Appendix A9.2.6 Seal Lid Calculation 457-2004.2 (Continued)

Only four bolts are used to connect the lid. The bending moment, M, is reacted by the effective flange arc length which is estimated to be $1 / 2$ the geometric arc associated with each bolt

Therefore the bolt arc angle is;

$$
\begin{aligned}
\operatorname{arc} \angle \quad & =38^{\circ}-\left[(1.25 /(45.75 / 2)) \times\left(180^{\circ} / \Pi\right)\right] \\
& =34.87^{\circ}
\end{aligned}
$$

Therefore the effective arc angle is:

$$
\begin{aligned}
\operatorname{arc} \angle_{\text {eflestive }} & =34.87 / 2 \\
& =17.44^{\circ}
\end{aligned}
$$

The are length is:

$$
\begin{aligned}
\text { arc length } & =\mathrm{r}_{\text {bolt }} \mathrm{x} \text { arc } \angle_{\text {efreetiv }} \\
& =(44 / 2) \times\left(17.44 \times \Pi / 180^{\circ}\right) \\
& =6.7 \mathrm{in}
\end{aligned}
$$

The effective section $\mathrm{I} / \mathrm{c}$ is:

$$
\begin{aligned}
\text { I } & =b t^{3} / 12 \\
& =6.7(0.375)^{3 / 12} \\
& =0.0294 \mathrm{in}^{4} \\
\text { l/c } & =b t^{2} / 6 \\
& =6.7(0.375)^{2} / 6 \\
& =0.157 \mathrm{in}^{3}
\end{aligned}
$$

The bending stress. $\sigma_{\triangleright}$, is therefore:

$$
\begin{aligned}
\sigma_{\mathrm{b}} & =\mathrm{M} \quad \\
& =1403 \times(1 / 0.157) \\
& =8.926 \mathrm{psi}
\end{aligned}
$$

The safety margin is:

$$
\begin{aligned}
\text { Safety Margin } & =1-(\text { stress } /(1.5 \times \mathrm{S})) \\
& =1-(8.936 /(1.5 \times 16,700)) \\
& =0.64
\end{aligned}
$$

\begin{tabular}{|l|l|l|l|}
\hline \multirow{2}{*}{$\begin{array}{l}\text { NAC } \\
\text { MTERiatronal }\end{array}$} & \begin{tabular}{l} 
Performed by: $g R \theta$ \\
\cline { 2 - 4 }
\end{tabular} & $\begin{array}{l}\text { Date: } \\
11-25-76\end{array}$ & $\begin{array}{l}\text { Calculation No 457-2004.2 } \\
\text { Revision 1 }\end{array}$ \\
\cline { 2 - 4 } & Checked by: & $\begin{array}{l}\text { Datej } \\
11 / 25 / 96\end{array}$ & \begin{tabular}{l} 
Page 10 of 14 \\
\hline
\end{tabular}
\end{tabular}


Appendix A9.2.6 Seal Lid Calculation 457-2004.2 (Continued)

Conservatively computing the deflection of the flange:

$\Delta=\mathrm{F}_{\text {ball }} \times(\mathrm{L} / 2)^{3} /(3 \mathrm{E} \mathrm{I})$

$=1403 \times(2 / 2)^{3} /\left(3 \times 28.3 \times 10^{6} \times 0.0294\right)$

$=0.00056 \mathrm{in}$

The deflection of the flange is negligible.

5.2 Lifting Lug Evaluation

\subsubsection{Bearing Check}

The pin bearing area is:

Bearing area $=\mathrm{t} \times \mathrm{D}_{\text {nole }}$

$=0.375 \times 1.0$

$=0.375 \mathrm{in}^{2}$

To deternine the most conservative load factor, the material yield and uitimate strengths will be normalized.

For SA240 Type 304 SS

$F_{y} / 3=30,000 / 3=10,000$ psi

$F_{u} / 5=75,000 / 5=15,000 \mathrm{psi}$

Therefore, comparing a factor (LF) of 3 against yield is more restrictive.

The bearing stress is:

Stress $=L F \times(W / 3$ lugs $) /$ area

$=3 \times(600 / 3) / 0.375$

$=1,600 \mathrm{psi}$

The safety margin is:

Safety Margin $=\mathrm{I}-\left(\right.$ stress $\left./ \mathrm{S}_{\mathrm{y}}\right)$

$=1-(1,600 / 30,000)$

$=0.95$

\begin{tabular}{|c|c|c|c|}
\hline INTERATIONAL & Perforned by: $g R \theta$ & Date: & $\begin{array}{l}\text { Calculation No. } 457.2004 .2 \\
\text { Revision I }\end{array}$ \\
\hline & Checked by: & Date: $25 / 56$ & Page 11 of 14 \\
\hline
\end{tabular}


Appendix A9.2.6 Seal Lid Calculation 457-2004.2 (Continued)

\subsubsection{Net Tension}

$$
\begin{aligned}
\text { Area } & =t\left(D_{\text {luz }}-D_{\text {hole }}\right) \\
& =0.375(2.0-1.0) \\
& =0.375 \mathrm{in}^{2}
\end{aligned}
$$

The stress is:

$$
\begin{aligned}
\text { Stress } & =\mathrm{LF} \times(\mathrm{W} / 3 \text { lugs }) / \text { (Area) } \\
& =3 \times(600 / 3) /(0.375) \\
& =1,600 \mathrm{psi}
\end{aligned}
$$

The safety margin is:

$$
\begin{aligned}
\text { Safety Margin } & =1-\left(\text { stress } / S_{y}\right) \\
& =1-(1,600 / 30.000) \\
& =0.95
\end{aligned}
$$

5.2.3 Shear Pull Out At 45 Degrees

\begin{tabular}{|c|c|c|c|}
\hline \multirow[t]{2}{*}{ A SAC } & Performed by: $g R \theta$ & $\begin{array}{l}\text { Date: } \\
11-25-96\end{array}$ & $\begin{array}{l}\text { Calculation No. } 457.2004 .2 \\
\text { Revision ! }\end{array}$ \\
\hline & Checked by: & $11 / 25 / 96$ & Page 12 of 14 \\
\hline
\end{tabular}

$$
\begin{aligned}
\text { Shear Area } & =\mathbf{t}\left(\mathrm{R}_{\mathrm{lug}}-\mathrm{R}_{\text {hole }}\right) \\
& =0.375(1.0-0.5) \\
& =0.1875 \mathrm{in}^{2}
\end{aligned}
$$

The shear stress is:

$$
\begin{aligned}
\text { Stress } & =\mathrm{LF} \times(\mathrm{W} / 3 \text { lugs }) /(\text { Shear Area }) \\
& =3 \times(600 / 3 \text { lugs }) /(0.1875) \\
& =3.200 \mathrm{psi}
\end{aligned}
$$

The safety margin is:

$$
\begin{aligned}
\text { Safety Margin } & =1-\left(\text { stress } /\left(0.6 \times S_{y}\right)\right. \\
& =1-(3.200 /(0.6 \times 30.000)) \\
& =0.82
\end{aligned}
$$

\subsubsection{Lug to Lid Weld}

The lug to lid weld is a 0.25 " all-around weld and is negligibly loaded. Allowable is very large compared to actual stress. Therefore, weld is adequate. 
Appendix A9.2.6 Seal Lid Calculation 457-2004.2 (Continued)

\subsection{SUMMARY OF RESULTS / CONCLCSIONS}

Summary of Stress Analysis

\begin{tabular}{|l|l|l|l|l|l|l|l|}
\hline $\begin{array}{l}\text { Drawing } \\
\text { No. }\end{array}$ & $\begin{array}{l}\text { Item } \\
\text { No. }\end{array}$ & Component & $\begin{array}{l}\text { Applied } \\
\text { Load }\end{array}$ & $\begin{array}{l}\text { Design } \\
\text { Check }\end{array}$ & $\begin{array}{l}\text { Calculated } \\
\text { Loading }\end{array}$ & Allowable & M.S. \\
\hline $457-103$ & 2 & lid flange & pressure & bending & $\mathbf{8 , 9 3 6 \mathrm { psi }}$ & $25.05 \mathrm{ksi}$ & 0.64 \\
\hline $457-103$ & 20 & bolt & pressure & tension & $4,201 \mathrm{psi}$ & $16.7 \mathrm{ksi}$ & 0.75 \\
\hline $457-103$ & 3 & lifting lug & $\begin{array}{l}3 \text { times } \\
\text { the } \\
\text { weight }\end{array}$ & bearing & $1600 \mathrm{psi}$ & $30 \mathrm{ksi}$ & 0.95 \\
tension & $1600 \mathrm{psi}$ & $30 \mathrm{ksi}$ & 0.95 \\
\hline
\end{tabular}

The immersion pail lid design meets the criteria defined in Section 4.2

\begin{tabular}{|l|l|l|}
\hline Performed by: $g R \theta$ & $\begin{array}{l}\text { Date: } \\
11-25-96\end{array}$ & $\begin{array}{l}\text { Calculation No. 457-2004.2 } \\
\text { Revision } 1\end{array}$ \\
\hline Checked by: & $\begin{array}{c}\text { Date: } \\
11 / 25 / 96\end{array}$ & Page 13 of 14 \\
\hline
\end{tabular}


Appendix A9.2.6 Seal Lid Calculation 457-2004.2 (Continued)

\subsection{REFERENCES}

7.1 Preliminary Design Analysis Report For The TN-WHC Cask and Transporation System Project 3035

Transnuclear, Inc.

7.2 Not Used.

7.3 ANSI N14.6

American National Standard for Radioactive Materials

"special lifting devices for shipping containers weighing $10,000 \mathrm{lbs}(4,500 \mathrm{~kg})$ or more"

7.4 Memo to Project File From T. A. Danner, dated July 31, 1996.

7.5 Hanford ECN 191402.

7.6 K Basin Immersion Pail Assembly TN WHC Transport Cask drawings

7.6.1 Project 457 drawing 106 sheets $1,2 / 2$

7.6.2 Project 457 drawing 102 sheets $1,2 / 2$.

7.6.3 Project 457 drawing 103 sheets $1,2 / 2$.

7.6.4 Project 457 drawing 104 sheet $1 / 1$

7.7 ASME Boiler \& Pressure Vessel Code, 1995 edition.

7.7.1 Section II-D, page 530, Table Y-1

7.7.2 Section I $L-D$, page 441 , Table $U$

7.7 .3 Section II-D, page 392 Table 3

7.7 .4 Section II-D, page 432,496

7.8 Shigley's Mechanical Engineering Design. McGraw Hill, 1963, Page 603

7.9 AISC Manual Of Steel Construction, 9th edition.

7.9.1 Page 4-147.

\begin{tabular}{|c|c|c|c|}
\hline VIC & Performed by: ges & $\begin{array}{l}\text { Date: } \\
1 / 25-26\end{array}$ & $\begin{array}{l}\text { Cakculation No. 457-2004.2 } \\
\text { Revision } 1\end{array}$ \\
\hline & Checked by: T2S & Dake: $11 / 25 / 96$ & Page 14 of 14 \\
\hline
\end{tabular}


Appendix A9.2.7 Loadout Pit Work Platform 457-2007.2

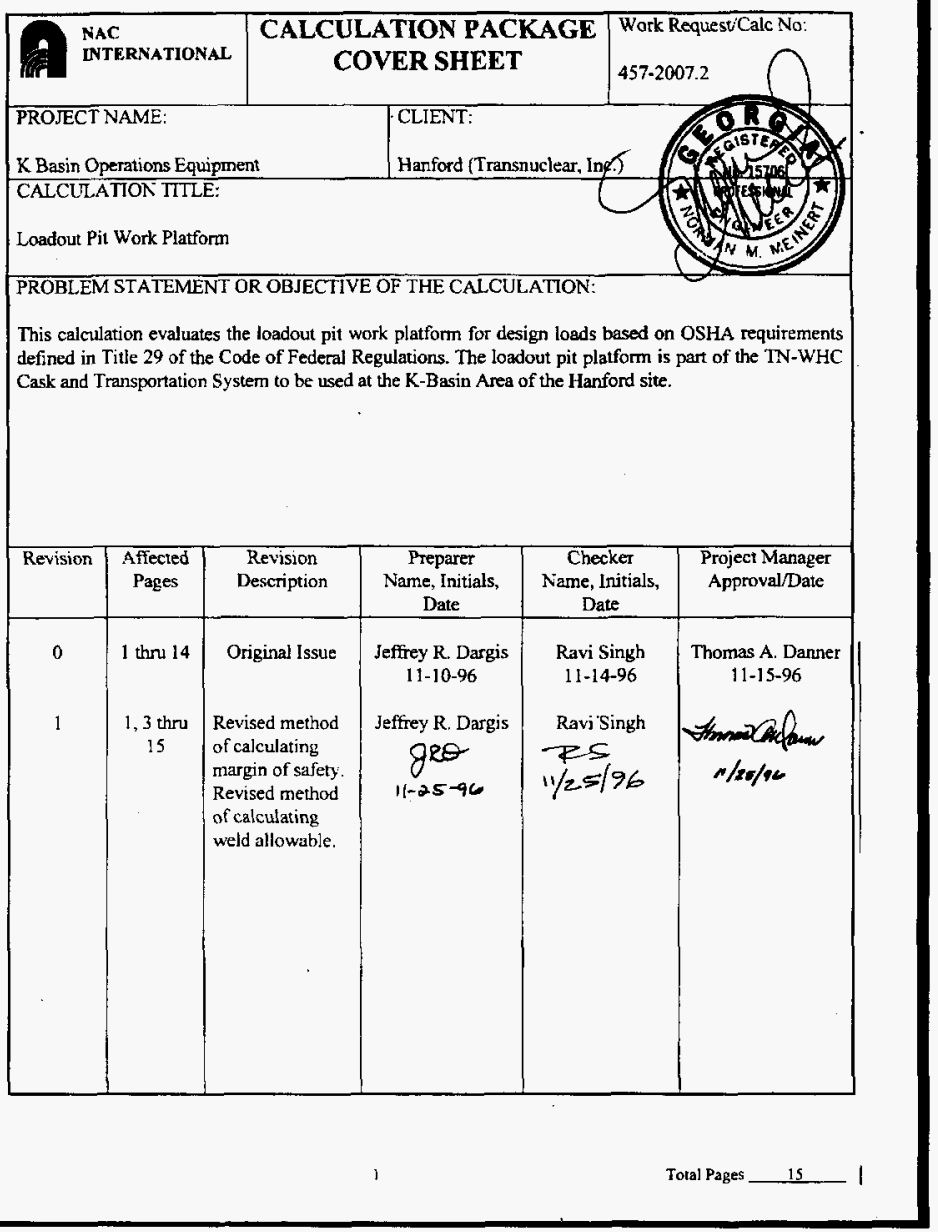


Appendix A9.2.7 Loadout Pit Work Platform 457-2007.2 (Continued)

\section{WDEPENDENT DESIGN VERIFICATION CHECK SHEET}

Work Request/Calculation No: $457-2007.2$ Revision 0

Scope Of Analysis File: This calculation evaluates the loadout pit work plattorm for design loads based on OSHA requirements defined in Title 29 of the CFR.

Review Methodology:

Check Of Calculations

Alternate Analyses

Other (Explain)

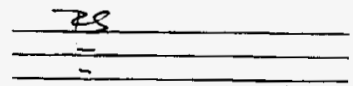

Confirm That The Work Request / Calculation Package Reviewed Inchudes:

1. Statement of Purpose

2. Defined Method of Analysis

3. Listing of Assumptions

4. Detailed Analysis Record

5. Statement of Conciusions / Recommendations (if applicable)

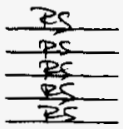

\begin{tabular}{|c|c|c|c|c|c|}
\hline Step & Acthities & & & & \\
\hline 1 & $\begin{array}{l}\text { For the scope of the defined analysis: } \\
\text { A. Are the required data input complete? } \\
\text { 1. Material properties } \\
\text { 2. Geomelry (drawing reference) } \\
\text { 3. Loading source term } \\
\text { if a supponing anslysis is nequired to } \\
\text { define the load state, nas it been } \\
\text { defined? } \\
\text { 8. Are boundary conditions acceptabte? }\end{array}$ & $\checkmark$ & & & Eoming ins \\
\hline 2 & Is the method of analysis adequate for the defined scope? & & & & \\
\hline 3 & Is the worst case loading/configuration documented? & & & & \\
\hline 4 & Are the acceptance criteria defined and complete? & & & & \\
\hline 5 & Has all concuitent loading been considered? & & & & \\
\hline 6 & $\begin{array}{l}\text { Are analyses consistent with previous work for method and } \\
\text { approach? }\end{array}$ & & & $V$ & $\begin{array}{l}\text { NEW CALC } \\
\text { PACKAGE }\end{array}$ \\
\hline 7 & Are the records for input and output complete? & & & & . \\
\hline 8 & Is traceability to verified software compiete? & & & $r$ & $\begin{array}{l}\text { NO SOFTWARE } \\
\text { VSED. }\end{array}$ \\
\hline 9 & $\begin{array}{l}\text { Is the statement of conclusions and recommendations complete } \\
\text { and acceptable for the project and objectives of the cefined } \\
\text { purpose? }\end{array}$ & & & & \\
\hline
\end{tabular}

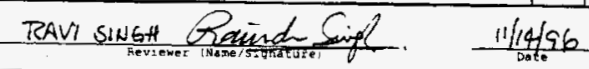


Appendix A9.2.7 Loadout Pit Work Platform 457-2007.2 (Continued)

\section{INDEPENDENT DESIGN VERIFICATION CHECK SHEET}

Work Request/Calculation No: 457-2007.2 Revision 1

Scope Of Analysis File: This calculation evaluates the loadout pit work platform for design loads based on OSHA requirements defined in Title 29 of the CFR.

Review Methodology:

Check Of Calculations

Alternate Analyses

Other (Explain)

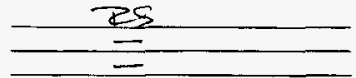

Confirm That The Work Request / Calculation Package Reviewed Includes:

1. Statement of Purpose

2. Defined Method of Analysis

3. Listing of Assumptions

4. Detailed Analysis Record

5. Statement of Conclusions / Recommendations (if applicable)

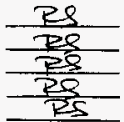

\begin{tabular}{|c|c|c|c|c|c|}
\hline Step & A tivlties & & n & th & comments \\
\hline$\frac{4}{1}$ & $\begin{array}{l}\text { For the scope of the defined analysis: } \\
\text { A. Are the required data input complete? } \\
\text { 1. Malerial properties } \\
\text { 2. Geometry (drawing reference) } \\
\text { 3. Loading source term } \\
\text { If a supporting analysis is required to } \\
\text { define the toad stats, has it bean } \\
\text { defined? } \\
\text { B. Are boundary conditions acceptabie? }\end{array}$ & $\checkmark$ & & min & 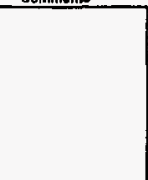 \\
\hline 2 & Is the method of analy sis adequate for the defined scope? & $\checkmark$ & & & \\
\hline 3 & Is the worst case loading/configuration documented? & & & & \\
\hline 4 & Are the acceptance criteria defined and complete? & 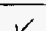 & & & \\
\hline 5 & Has all concurrent loading been considered? & $v$ & & & \\
\hline 6 & $\begin{array}{l}\text { Are analyses consistent with previous work for method and } \\
\text { approach? }\end{array}$ & & & & \\
\hline 7 & Are the records for input and output complete? & $\checkmark$ & & & \\
\hline $\bar{B}$ & Is traceability to verified sotwware complete? & & & $\checkmark$ & $\begin{array}{l}\text { ND SOFTWAEE } \\
\text { USED. }\end{array}$ \\
\hline 9 & $\begin{array}{l}\text { Is the statement of conclusions and recommendations complete } \\
\text { and acceptable for the project and objectives of the defined } \\
\text { purpose? }\end{array}$ & $\checkmark$ & & & \\
\hline & t & & & & \\
\hline
\end{tabular}


HNF-SD-SNF-FDR-003 Rev. 0

Appendix A9.2.7 Loadout Pit Work Platform 457-2007.2 (Continued)

TABLE OF CONTENTS

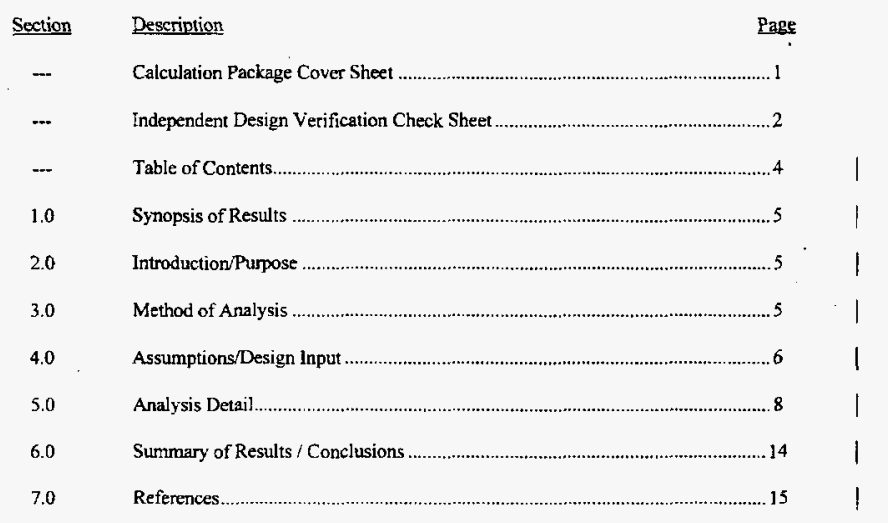

\begin{tabular}{|l|l|l|l|}
\hline \multirow{2}{*}{$\begin{array}{l}\text { NAC } \\
\text { INTERNATIONAL }\end{array}$} & \begin{tabular}{l} 
Performed by: gRS \\
\cline { 2 - 4 }
\end{tabular} & $\begin{array}{l}\text { Date: } \\
1 / 205-96\end{array}$ & $\begin{array}{l}\text { Calculation No 457-2007.2 } \\
\text { Revision 1 }\end{array}$ \\
\cline { 2 - 4 } & Checked by: & $\begin{array}{c}\text { Date } \\
11 / 25 / 96\end{array}$ & Page 4 of 15 \\
\hline
\end{tabular}


Appendix A9.2.7 Loadout Pit Work Platform 457-2007.2 (Continued)

1.0 SYNOPSIS OF RESULTS

Summary of Stress Analysis

\begin{tabular}{|c|c|c|c|c|c|c|c|}
\hline $\begin{array}{l}\text { Drawing } \\
\text { No. }\end{array}$ & $\begin{array}{l}\text { Item } \\
\text { No. }\end{array}$ & Component & $\begin{array}{l}\text { Applied } \\
\text { Load }\end{array}$ & Design Check & $\begin{array}{l}\text { Calculated } \\
\text { Loading }\end{array}$ & Allowable & M.S. \\
\hline $457-111$ & $\begin{array}{l}4,5 \\
3\end{array}$ & $\begin{array}{l}\text { brace, } \\
\text { extension, } \\
\text { brace }\end{array}$ & $2000 \mathrm{lbs}$ & $\begin{array}{l}\text { nube bending } \\
\text { shear }\end{array}$ & $\begin{array}{l}22,077 \mathrm{psi} \\
2,000 \mathrm{psi}\end{array}$ & $\begin{array}{l}27,600 \mathrm{psi} \\
18,400 \mathrm{psi}\end{array}$ & $\begin{array}{l}0.20 \\
0.89\end{array}$ \\
\hline $457-111$ & $\begin{array}{l}5 \text { to } \\
9\end{array}$ & weld & $2000 \mathrm{lbs}$ & $\begin{array}{l}\text { combined } \\
\text { shear and } \\
\text { tension }\end{array}$ & $\begin{array}{l}1,824 \\
\mathrm{lbs} / \mathrm{in}\end{array}$ & $\begin{array}{l}2,545 \\
\mathrm{lbs} / \text { in }\end{array}$ & 0.28 \\
\hline $457-111$ & $\begin{array}{l}9 \text { to } \\
7\end{array}$ & weld & 2000 lbs & weld shear & $0.133 \mathrm{ksi}$ & $14.4 \mathrm{ksi}$ & 0.99 \\
\hline
\end{tabular}

The loadout pit work platform design meets the criteria defined in Section 4.2.

\subsection{INTRODUCTION / PURPOSE}

This calculation evaluates the loadout pit work platform against the criteria requirements listed in Section 4.2. The loadout pit work platform is part of the TN-WHC Cask and Transportation System to be used at the K-Basin Area of the Hanford site. The loadout pit work platform design was initially evaluated in Reference 7.1.

\subsection{METHOD OF ANALYSIS}

Hand calculations using classic textbook solutions are used to structurally evaluate the loadout pit work platform.

The design will be assesed using the design load developed in Section 5.1. The design loading considers OSHA requirements defined in Title 29 of the Code of Federal Regulations (Reference 7.5). The acceptance criteria limits the induced loads to the requirements of the AISC Code (Reference ?.7).

The work piatform configuration consists of a tubing frame arrangement covered by steel decking. The frame is cantilevered off a wall plate and utilizes a brace for additional strength. The wall plate consists of three (3) plates welded in an inverted " $U$ " configuration for suspension of the platform from the pit beam support structure. Drawing details are provided in Reference 7.6 .

\begin{tabular}{|c|c|c|c|}
\hline 9 NAC & Perfomed by: $g R \theta$ & $\begin{array}{l}\text { Date: } \\
11-25-46\end{array}$ & $\begin{array}{l}\text { Calcutation No. } 457-2007 \text { ? } \\
\text { Revision ? }\end{array}$ \\
\hline & Checked by: & Date: $11 / 25 / 96$ & Page 5 of 15 \\
\hline
\end{tabular}


Appendix A9.2.7 Loadout Pit Work Platform 457-2007.2 (Continued)

The following evaluations are documented within this calculation.

- Design Load Development;

- Brace Evaluation;

- Extension / Brace Evaluation;

- Frame / Wall Piate Interface Weld Evaluation;

- Wall Plate Weld Evaluation; and

- Wall Plate Saddle Evaluation.

\section{ASSUMPTIONS / DESIGN INPUTS}

4.1 Assumptions

There are no unverified assumptions within this calculation.

4.2 Design Criteria

4.2.1 Loading

See Section 5.1.

4.2.2 Bending Stress Limits

Bending stress $<0.6 \times \mathrm{S}_{\mathrm{y}} \quad$ (Reference 7.7.2)

4.2.3 Shear Stress Limits

Shear suress $<0.4 \times S_{\mathrm{y}} \quad$ (Reference 7.7)

4.2.4 Weld Limits

Weld Allowable Stress Limits $<0.4 \times F_{y}$ of base metal (fillet, penetration) (Reference 7.7, AISC Table J2.5. page 5-70)

4.2.5 Platform Parameters (Reference 7,6.1)

Brace: Tube steel

$2 " \times 4 " \times 0.25^{* \prime} \times 45.50$ in long

ASTM A 500 GR B

\begin{tabular}{|c|c|c|c|}
\hline \multirow[t]{2}{*}{ VAC INFERATIONAL } & Performed by: & Date: $11-25-96$ & $\begin{array}{l}\text { Calculation No. 457-2007.2 } \\
\text { Revision I }\end{array}$ \\
\hline & Checked by: & ${ }_{11 / 25 / 96}$ & Page 6 of 15 \\
\hline
\end{tabular}


Appendix A9.2.7 Loadout Pit Work Platform 457-2007.2 (Continued)

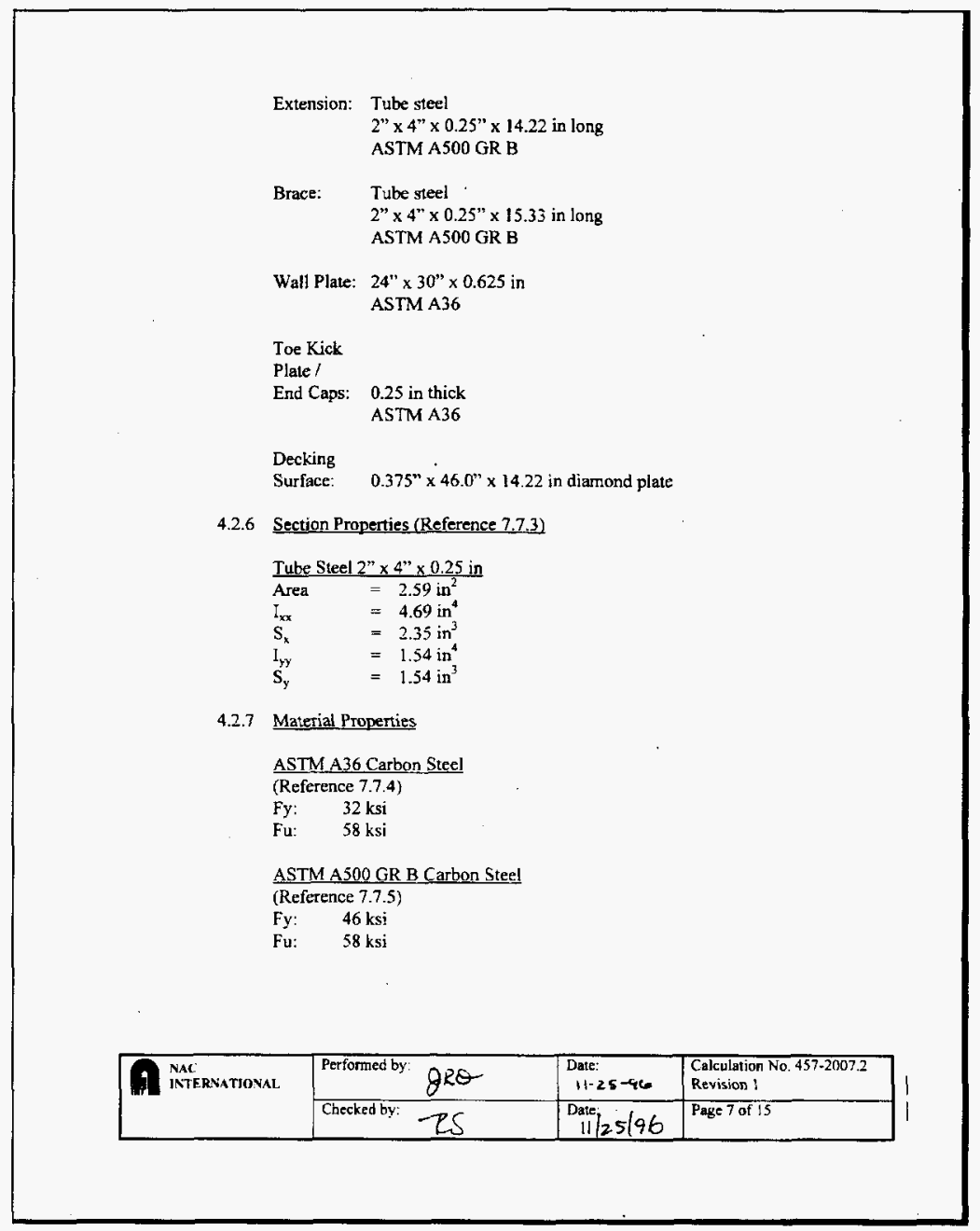


Appendix A9.2.7 Loadout Pit Work Platform 457-2007.2 (Continued)

\subsection{ANALYSIS DETAIL}

\subsection{Design Load Development}

The design load, F, is based on the larger of the following two (2) conditions:

Condition A: The heavy duty uniform loading of 29CFR1910 (Reference 7.5.2) multiplied by a load factor (LF) of four (4). The uniform distributed load, $\mathrm{p}$, is $75 \mathrm{lbs} / \mathrm{ft}^{2}$.

Condition B: Each platform section carrying a load equal to the maximum intended load multiplied by a load factor (LF) of four (4). The maximum intended load, P, shall be considered to be equal to two men, each weighing 250 lbs.

$$
\begin{aligned}
F_{A} & =p \times(\text { area }) \times L F \\
& =75 \times\left(\frac{46.0 \times 14.22}{144}\right) \times 4 \\
& =1363 \mathrm{lbs} \\
F_{B} & =P \times 4 \\
& =(2 \times 250) \times 4 \\
& =2000 \mathrm{lbs} \\
F & =\text { Greater of } F_{A} \text { or } F_{B} \\
& =2000 \mathrm{lbs}
\end{aligned}
$$

\subsection{Brace Evaluation}

The critical brace stress is located at the interface point with the extension. The notch connection will develop the full capacity of the brace (i.e., interface weld is full penetration on top and bottom, fillet on sides)

\subsubsection{Bending Stress Evaluation}

Conservatively considering the brace as a cantilevered structure fixed at the extension interface with the design load applied as a concentrated load at the platform's edge:

\begin{tabular}{|l|l|l|l|}
\hline \multirow{2}{*}{$\begin{array}{l}\text { NaC } \\
\text { INTERNATIONAL }\end{array}$} & Performed by: gRS & $\begin{array}{l}\text { Date: } \\
11-25-96\end{array}$ & $\begin{array}{l}\text { Calculation No 457-2007.2 } \\
\text { Revision 1 }\end{array}$ \\
\cline { 2 - 4 } & Checked by: 25 & $\begin{array}{c}\text { Date } \\
11 / 25 / 96\end{array}$ & Page 8 of 15 \\
\hline
\end{tabular}


Appendix A9.2.7 Loadout Pit Work Platform 457-2007.2 (Continued)

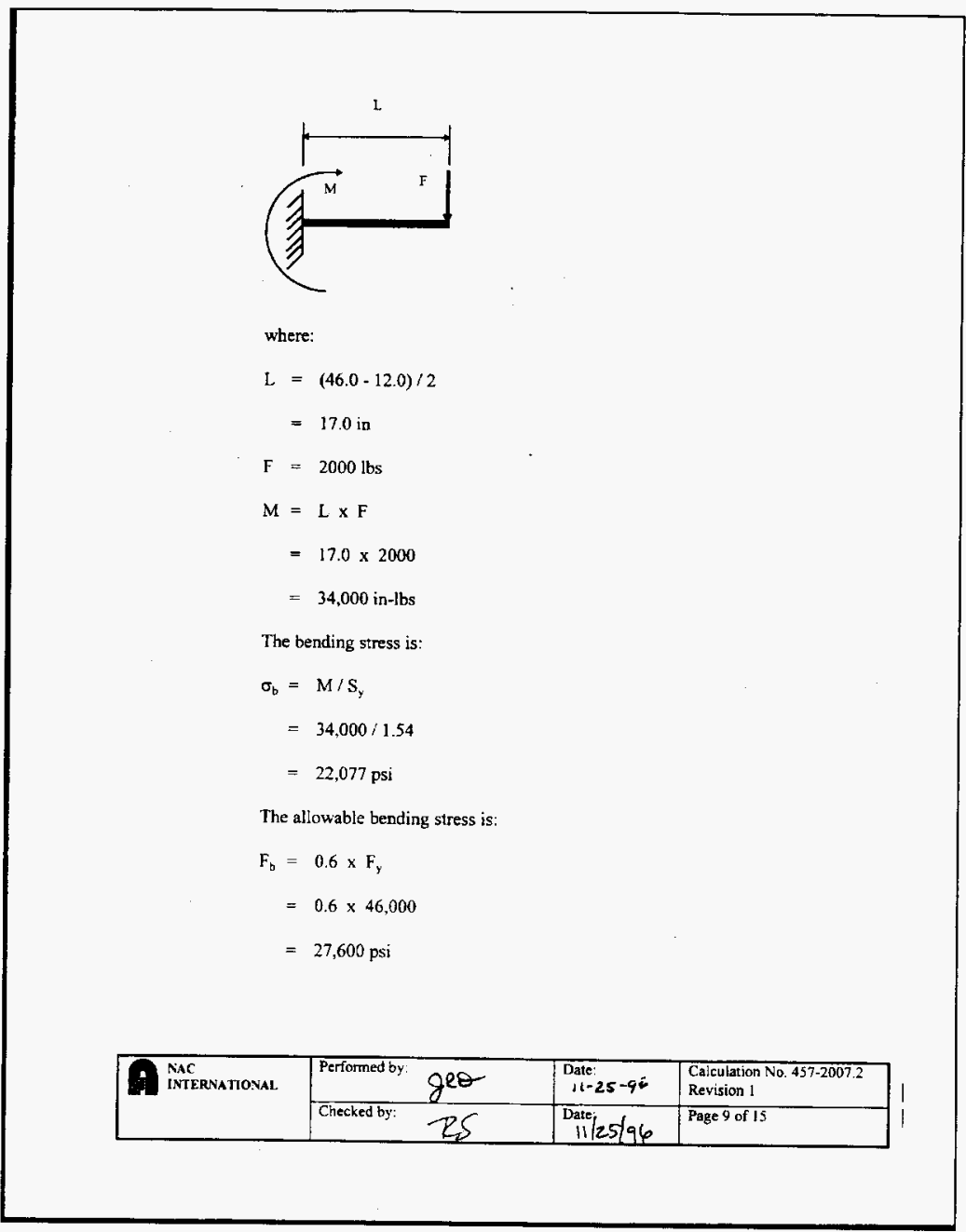


Appendix A9.2.7 Loadout Pit Work Platform 457-2007.2 (Continued)

The margin of safety is:

$$
\begin{aligned}
\mathrm{MS} & =1-\frac{\sigma_{\mathrm{b}}}{\mathrm{F}_{\mathrm{b}}} \\
& =1-\frac{22,077}{27,600} \\
& =0.20
\end{aligned}
$$

\subsubsection{Shear Stress Evaluation}

The shear stress is:

$$
\begin{aligned}
\sigma_{v} & =F /(2 \times \text { area }) \\
& =2000 /(2 \times 0.25 \times 2) \\
& =2000 \mathrm{psi}
\end{aligned}
$$

\begin{tabular}{|c|c|c|c|}
\hline $\begin{array}{l}\text { NAC } \\
\text { INTERYATHONAT. }\end{array}$ & Performed by: & $\begin{array}{l}\text { Date: } \\
11-25-96\end{array}$ & $\begin{array}{l}\text { Calculation No. 457-2007.2 } \\
\text { Revision 1 }\end{array}$ \\
\hline & Checked by: & $11 / 25 / 96$ & Page 10 of 15 \\
\hline
\end{tabular}

The allowable shear stress is:

$$
\begin{aligned}
F_{v} & =0.4 \times F_{y} \\
& =0.4 \times 46,000 \\
& =18,400 \mathrm{psi}
\end{aligned}
$$

The margin of safety is:

$$
\begin{aligned}
\mathrm{MS} & =1-\frac{\sigma_{\mathrm{v}}}{\mathrm{F}_{\mathrm{v}}} \\
& =1-\frac{2,000}{18,400} \\
& =0.89
\end{aligned}
$$


Appendix A9.2.7 Loadout Pit Work Platform 457-2007.2 (Continued)

\subsubsection{Deflection Evaluation}

Per Reference 7.4.1, the platform deflection, $\Delta$, is:

$$
\begin{aligned}
\Delta & =\frac{1}{3} \frac{F L^{3}}{(E)\left(I_{y}\right)} \\
& =\frac{1}{3} \frac{(2000)(17.0)^{3}}{\left(28.3 \times 10^{6}\right)(1.54)} \\
& =0.075 \mathrm{in}
\end{aligned}
$$

The platform deflection is insignificant.

\subsection{Extension / Brace Evaluation}

Two (2) extensions are cantilevered from the wall, each a distance $14.22^{\prime \prime}$. This is less than the distance of 17 "previously evaluated in Section 5.1 for the brace. Therefore, the results from Section 5.1 are conservative for the extension pieces. No further evaluation is required.

\subsection{Frame / Wall Plate Interface Weld Evaluation}

The kicker is welded to the wall plate via a 0.25 in all-arourd fillet.

The shear stress in the weld is:

$\sigma_{v}=\mathrm{F} / \mathrm{L}_{\text {weld }}$

where:

$$
\begin{aligned}
& \mathrm{L}_{\text {weld }}=2+2+4+4 \\
& \quad=12 \text { in (per kicker) } \\
& \text { Therefore: }
\end{aligned}
$$

\begin{tabular}{|c|c|c|c|}
\hline NAC INTERATIONAL & Performed by: gRe & $\begin{array}{l}\text { Date: } \\
11-25-96\end{array}$ & $\begin{array}{l}\text { Calculation No. 457-2007.2 } \\
\text { Revision I }\end{array}$ \\
\hline & Checked by: $\quad K S$ & $11 / 25 / 96$ & Page 11 of 15 \\
\hline
\end{tabular}

$$
\begin{aligned}
\sigma_{v} & =2000 /(2 \times 12) \\
& =83 \mathrm{lbs} / \text { in }
\end{aligned}
$$

The tensile stress in the weld is: 
Appendix A9.2.7 Loadout Pit Work Platform 457-2007.2 (Continued)

$\sigma_{\mathrm{l}}=\mathrm{M} /\left(2 \times \mathrm{S}_{\mathrm{u}}\right) \quad$ (for both kickers)

where:

$M=34,000$ in-lbs (from Section 5.2.1)

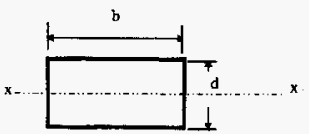

$S_{w}=b d+\frac{d^{2}}{3} \quad$ (Reference 7.10)

$=(4 \times 2)+\left[(2)^{2}: 3\right]$

$=9.33 \mathrm{in}^{2}$

Therefore:

$\sigma_{t}=34,000 /(2 \times 9.33)$

$=1822 \mathrm{lbs} / \mathrm{in}$

The resultant weld stress, $\sigma_{r}$, is:

$\sigma_{r}=\left(\sigma_{v}{ }^{2}+\sigma_{1}{ }^{2}\right)^{1 / 2}$

$=\left((83)^{2}+(1822)^{2}\right)^{1 / 2}$

$=1,824 \mathrm{lbs} / \mathrm{in}$

The allowable weld stress per Section 4.2 .4 is:

$F_{\mathrm{wr}}=0.4 \times F_{\mathrm{y}} \times \mathrm{I}_{\text {thriat }}$

$=0.4 \times 36.000 \times(0.707 \times 0.25)$

$=2,545 \mathrm{lbs} /$ in

The margin of safety is:

\begin{tabular}{|c|c|c|c|c|}
\hline INIERNATJONAL & Performed by & gRo & $\begin{array}{l}\text { Date: } \\
\quad 11-25-96\end{array}$ & $\begin{array}{l}\text { Calculation No. } 457-2007.2 \\
\text { Revision } 1\end{array}$ \\
\hline & Checked by: & ES & Date: $/ 25 / 96$ & Page 12 of 15 \\
\hline
\end{tabular}




$$
\begin{aligned}
\text { MS } & =1-\frac{\sigma_{r}}{\mathrm{~F}_{\mathrm{w}}} \\
& =1-\frac{1,824}{2,545} \\
& =0.28
\end{aligned}
$$

5.5 Wall Plate Weld Evaluation

The wall mounting plate is constructed by welding Items 7,8 , and 9 . The 9 to 7 weld stress for the full penetration weld of a 0.625 in plate is as follows:

$\sigma_{\mathrm{y}}=\mathrm{F} / \mathrm{A}_{\text {weld }}$

where:

$\mathrm{t}=$ plate thickness

$=0.625 \mathrm{in}$

I = weld length

$=24.0 \mathrm{in}$

$A_{\text {wets }}=t \times 1$

$=0.625 \times 24.0$

$=15.0 \mathrm{in}^{2}$

$\sigma_{v}=2000 / 15.0$

$=133 \mathrm{psi}$

The weld stress is insignificant compared to the weld allowable, $F_{w}$ :

$F_{w}=0.4 \times F_{y}$ (base metal) $=14,400$ psi.

5.6 Wall Plate Saddle Evaluation

The calculation perfonmed under Section 5.5 demonstrates that the saddle itse]f will develop insignificant stresses.

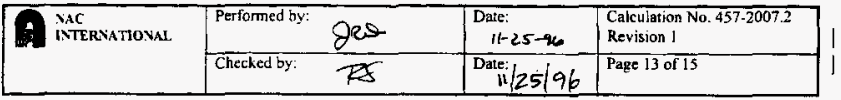


Appendix A9.2.7 Loadout Pit Work Platform 457-2007.2 (Continued)

SUMMARY OF RESULTS/CONCLUSIONS

Summary of Stress Analysis

\begin{tabular}{|l|l|l|l|l|l|l|l|}
\hline $\begin{array}{l}\text { Drawing } \\
\text { No. }\end{array}$ & $\begin{array}{l}\text { Item } \\
\text { No. }\end{array}$ & Component & $\begin{array}{l}\text { Applied } \\
\text { Load }\end{array}$ & Design Check & $\begin{array}{l}\text { Calculated } \\
\text { Loading }\end{array}$ & Allowable & M.S. \\
\hline $457-111$ & $\begin{array}{l}4,5, \\
3\end{array}$ & $\begin{array}{l}\text { brace, } \\
\text { extension, } \\
\text { brace }\end{array}$ & $2000 \mathrm{lbs}$ & tube bending & $22,077 \mathrm{psi}$ & $27,600 \mathrm{psi}$ & 0.25 \\
\hline $457-111$ & $\begin{array}{l}5 \text { to } \\
9\end{array}$ & weld & $2000 \mathrm{lbs}$ & $\begin{array}{l}\text { combined } \\
\text { shear and } \\
\text { tension }\end{array}$ & $\begin{array}{l}1,824 \\
\text { lbs } / \mathrm{in}\end{array}$ & $\begin{array}{l}2,545 \\
1 \mathrm{bs} / \mathrm{in}\end{array}$ & 0.28 \\
\hline $457-111$ & $\begin{array}{l}9 \text { to } \\
7\end{array}$ & weld & $2000 \mathrm{lbs}$ & weld shear & $0.133 \mathrm{ksi}$ & $14.4 \mathrm{ksi}$ & 0.99 \\
\hline
\end{tabular}

The loadout pit work platform design meets the criteria defined in Section 4.2.

\begin{tabular}{|c|c|c|c|}
\hline NAC & Performed by: $g^{2 \theta}$ & $\begin{array}{l}\text { Date: } \\
11 \cdot 25-96\end{array}$ & $\begin{array}{l}\text { Calculation No. 457-2007.2 } \\
\text { Revision 1 }\end{array}$ \\
\hline & Checked by: 28 & Date/25/96 & Page 14 of 15 \\
\hline
\end{tabular}




\section{REFERENCES}

7.1 Preliminary Design Analysis Report For The TN-WHC Cask and Transporation System Project 3035

Transnuclear, Inc

7.2 Not Used.

7.3 ASME Code Case N-71-16, Table 2.

7.4 Roark's Formulas for Stress and Strain, 3rd edition.

7.4.1 Table III, Case 1, page 100 .

7.5 Code Of Federal Regulations

Title 29, Part 1910.28

Safety Requirements For Scaffolding

7.5.1 Page 114, (d)8, Tubular Welded Frame Scaffolds.

7.5.2 Page 112, Table D-9.

7.6 K Basin Immersion Pail Assembly TN WHC Transport Cask Drawings.

7.6.1 Project 457, Drawing 111, sheets $1,2 / 2$.

7.7 AISC Manual Of Steel Construction, 9th edition

7.7.1 Table J2.5.

7.7.2 Page 5-45, Chapter F, Eq̣uation F1-1 (bending).

7.7.3 Page 1-103.

7.7.4 Page 1-7, Table 1 .

7.7.5 Page 1-92. Table 3.

7.7.6 Page 5-135. Table C-C2.1.

7.7.7 Page 3-52.

7.8 ASME Boiler \& Pressure Vessel Code, 1995 Edition, Section IIl, Division I.

7.9 Hanford ECN 191402

7.10 Blodgett's Design Of Welded Structures, 1966.

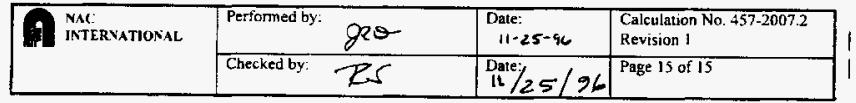


Appendix A9.2.8 Trailer Work Platform 457-2002.2

\begin{tabular}{|c|c|c|c|c|c|}
\hline a & $\begin{array}{l}\text { C } \\
\text { IERNATION }\end{array}$ & & $\begin{array}{l}\text { LCULATIC } \\
\text { ACKAGE } \\
\text { VER SHEE }\end{array}$ & $\begin{array}{l}\text { Work F } \\
457-20\end{array}$ & $\begin{array}{l}\text { Request/Calc No: } \\
02.2\end{array}$ \\
\hline $\begin{array}{l}\text { PROJECT } \\
\text { K Basin O }\end{array}$ & $\begin{array}{l}\text { NAME: } \\
\text { erations Equi }\end{array}$ & ment & $\begin{array}{l}\text { CLIENT: } \\
\text { Hanford (Tr }\end{array}$ & Isnuclear, Inc.) & \\
\hline $\begin{array}{l}\text { CALCUL } \\
\text { Transport }\end{array}$ & $\begin{array}{l}\text { TION TITLE } \\
\text { railer Work } \mathrm{P}\end{array}$ & atform & & ( & \\
\hline $\begin{array}{l}\text { PROBLEI } \\
\text { This calc } \\
\text { requireme } \\
\text { induced lo } \\
\text { The transp } \\
\text { the K-Basi }\end{array}$ & $\begin{array}{l}\text { STATEMEN } \\
\text { ation evalua } \\
\text { ts defined in } \\
\text { ds to the reqL } \\
\text { rt trailer work } \\
\text { Area of the }\end{array}$ & $\begin{array}{l}\text { T OR OBJECTIVI } \\
\text { is the transport } \\
\text { itle } 29 \text { of the Cod } \\
\text { rements of the Al } \\
\text { platform is part of } \\
\text { anford site. }\end{array}$ & $\begin{array}{l}\text { OF THE CALC } \\
\text { iler work platf } \\
\text { of Federal Regu } \\
\text { Code. } \\
\text { IN-WHC Cas }\end{array}$ & $\begin{array}{l}\text { LATION: } \\
\text { th for design loa } \\
\text { tions. The acceptar } \\
\text { and Transportation }\end{array}$ & $\begin{array}{l}\text { ds based on OSHA } \\
\text { ace criteria limits the } \\
\text { System to be used at }\end{array}$ \\
\hline Revision & $\begin{array}{l}\text { Affected } \\
\text { Pages }\end{array}$ & $\begin{array}{c}\text { Revision } \\
\text { Description }\end{array}$ & $\begin{array}{c}\text { Preparer } \\
\text { Name, Initials, } \\
\text { Date }\end{array}$ & $\begin{array}{c}\text { Checker } \\
\text { Name, Initials, } \\
\text { Date }\end{array}$ & $\begin{array}{l}\text { Project Manager } \\
\text { Approval/Date }\end{array}$ \\
\hline 0 & $\begin{array}{l}1 \text { thru } 12 \\
\text { A1 thru A4 } \\
\text { 1, } 3 \text { thru } 13\end{array}$ & $\begin{array}{l}\text { Original Issue } \\
\text { Revised method } \\
\text { of calculating } \\
\text { margin of safely. }\end{array}$ & $\begin{array}{l}\text { Ravi Singh } \\
11-11-96 \\
\text { Ravi Singh } \\
\text { R8 } \\
11 / 25196\end{array}$ & $\begin{array}{l}\text { Jeffrey R. Dargis } \\
11-11-96 \\
\text { Jeffrey R. Dargis } \\
\text { gRe } \\
11-25 \rightarrow 6\end{array}$ & 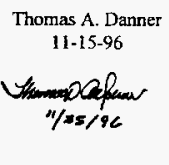 \\
\hline
\end{tabular}


Appendix A9.2.8 Trailer Work Platform 457-2002.2 (Continued)

\section{INDEPENDENT DESIGN VERJFICATION CHECK SHEET}

Work Request/Calculation No: 457-2002.2 Revision $\underline{0}$

Scope Of Analysis File: This calculation evaluates the transport trailer piatform for design loads pased on OSHA requirements defined in Trile 29 of the CFR.

Review Methodology: Check Of Calculations

Altemate Analyses

Other (Explain)

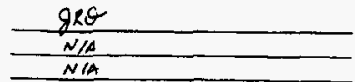

Confirm That The Work Request / Calculation Package Reviewed Includes:

1. Statement of Purpose

2. Defined Method of Analysis

3. Listing of Assumptions

4. Detailed Analysis Record

5. Statement of Conclusions / Recommendations (if applicable)

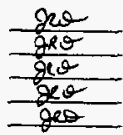

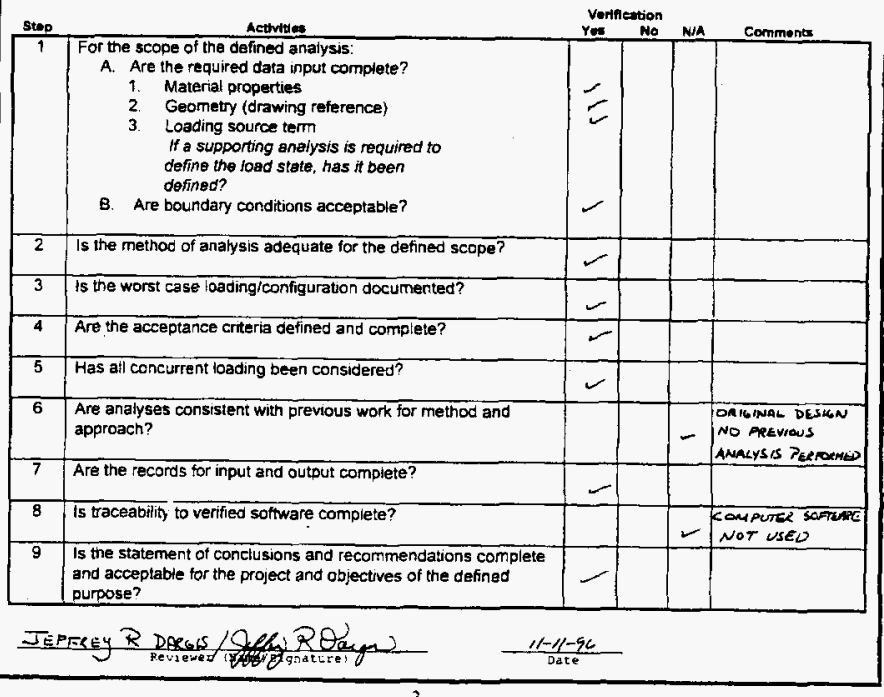


Appendix A9.2.8 Trailer Work Platform 457-2002.2 (Continued)

\section{INDEPENDENT DESIGN VERIFICATION CHECK SHEET}

Work Request/Calcutation No: 457-2002.2 Revision 11

Scope Of Analysis File: This calculation evaluates the transport trailer platform for design loads based on OSHA requirements defined in Tille 29 of the CFR.

Review Methodology: Check Of Calculations

Alternate Analyses

Other (Explain)

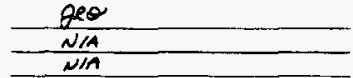

Confirm That The Work Request / Calculation Package Reviewed Includes:

1. Statement of Purpose

2. Defined Method of Analysis

3. Listing of Assumptions

4. Detailed Analysis Record

5. Statement of Conclusions / Recommendations (if applicable)

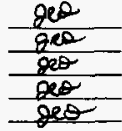

\begin{tabular}{|c|c|c|c|c|c|}
\hline$s_{-1}$ & & & tion & & \\
\hline 1 & $\begin{array}{l}\text { For the scope of the detined analysis: } \\
\text { A. Are the required data input complete? } \\
\text { 1. Materia! properties } \\
\text { 2. Geometry (drawing reference) } \\
\text { 3. Loading source term } \\
\text { If a supporting analysis is required to } \\
\text { define the ioad state, has it been } \\
\text { defined? } \\
\text { B. Are boundary conditions acceptable? }\end{array}$ & & & & \\
\hline 2 & Is the method of analysis adequate for the defined scope? & & & & \\
\hline 3 & Is the worst case loading/configuration documented? & & & & \\
\hline 4 & Are the acceptance criteria defined and complete? & & & & \\
\hline 5 & Has all concurrent toading been considered? & & & & \\
\hline$\overline{6}$ & $\begin{array}{l}\text { Are analyses consistent with previous work for method and } \\
\text { approach? }\end{array}$ & & & & \\
\hline 7 & Are the records for in put and output complete? & & & & \\
\hline 8 & Is traceability to verified sofware complete? & & & & 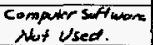 \\
\hline 9 & $\begin{array}{l}\text { Is the statement of conclusions and recommendations complete } \\
\text { and acceptable for the project and objectives of the defined } \\
\text { purpose? }\end{array}$ & & & & \\
\hline & \multicolumn{2}{|c|}{$11-25-76$} & & & \\
\hline
\end{tabular}


Appendix A9.2.8 Trailer Work Platform 457-2002.2 (Continued)

TABLE OF COYTENTS

\begin{tabular}{|c|c|}
\hline Section & Description \\
\hline- & Calculation Package Cover Sheet \\
\hline- & Independent Design Verification Check Sheet \\
\hline- & 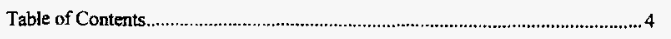 \\
\hline 1.0 & Synopsis of Results \\
\hline 2.0 & Introduction/Purpose \\
\hline 3.0 & 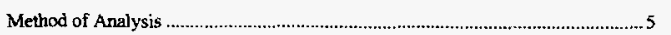 \\
\hline 4.0 & Assumptions/Design Input . \\
\hline 5.0 & 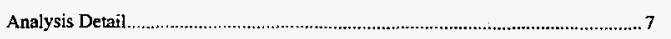 \\
\hline 6.0 & 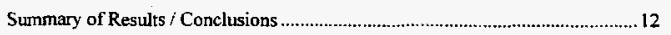 \\
\hline 7.0 & References \\
\hline
\end{tabular}

Appendix A Kee Industrial Products Load Data 4 total pages

\begin{tabular}{|l|l|l|l|}
\hline \multirow{2}{*}{$\begin{array}{l}\text { Nac } \\
\text { interiational }\end{array}$} & Performed by: ZS & $\begin{array}{l}\text { Date: } \\
11 / 25 / 96\end{array}$ & $\begin{array}{l}\text { Caiculation No. 457-2062.2 } \\
\text { Revision 1 }\end{array}$ \\
\cline { 2 - 4 } & Checked by: gRO & $\begin{array}{l}\text { Date: } \\
11-2546\end{array}$ & Page 4 of 13 \\
\hline
\end{tabular}


Appendix A9.2.8 Trailer Work Platform 457-2002.2 (Continued)

Summary of Stress Analysis

\begin{tabular}{|l|l|l|l|l|l|l|l|}
\hline $\begin{array}{l}\text { Drawing } \\
\text { No. }\end{array}$ & $\begin{array}{l}\text { Item } \\
\text { No. }\end{array}$ & Component & $\begin{array}{l}\text { Applied } \\
\text { Load }\end{array}$ & Design Check & $\begin{array}{l}\text { Calculated } \\
\text { Loading }\end{array}$ & Allowable & M.S. \\
\hline $457-105$ & 24 & channel & $125 \mathrm{lb} / \mathrm{ft}$ & bending & $8,676 \mathrm{psi}$ & $21,000 \mathrm{psi}$ & 0.59 \\
\hline $457-105$ & 26 & plank hook & $591 \mathrm{lbs}$ & bearing & $1,182 \mathrm{psi}$ & $35,000 \mathrm{psi}$ & 0.97 \\
\hline
\end{tabular}

The transport trailer work platform design meets the criteria defined in Section 4.2 .

\subsection{INTRODUCTION / PURPOSE}

This calculation evaluates the transport trailer work platform for design loads based on OSHA requirements defined in Title 29 of the Code of Federal Regulations. The transport trailer work platform is part of the TN-WHC Cask and Transportation System to be used at the K-Basin Area of the Hanford site.

\subsection{METHOD OF ANALYSIS}

Hand calculations using classic textbook solutions are used to structurally evaluate the transport trailer work platform.

The design will be assessed using the design load developed in Section 5.1. The design loading considers OSHA requirements defined in Title 29 of the Code of Federal Regulations (Reference 7.5). The acceptance criteria limits the induced loads to the requirements of the AISC Code (Refcrence 7.7).

The work platform configuration evaluated herein consists of a plank 113.45 " long and 29.29 " wide supported by $4 \times 0.180$ aluminum channels. The channels will rest on the platform pipe when in use.

The framing components of the platform are standard catalog components supplied to the requirements of Reference 7.5. Maximum unbraced lengths in the design are limited to 6' -0 " as specified in Reference 7.2 (Appendix A). Therefore, these items do not require specific stress evaluations. Drawing details are provided in Reference 7.6.1.

\begin{tabular}{|c|c|c|c|c|}
\hline $\begin{array}{l}\text { NAC } \\
\text { IVTERNATIONAT. }\end{array}$ & Performed by: & 2 & Date: $11 / 25 / 96$ & $\begin{array}{l}\text { Cakulation No. 457-2002.2 } \\
\text { Revision I }\end{array}$ \\
\hline & Checked by: & get & $\begin{array}{l}\text { Date: } \\
11-25-46\end{array}$ & Page 5 of 13 \\
\hline
\end{tabular}


Appendix A9.2.8 Trailer Work Platform 457-2002.2 (Continued)

The following evaluations are documented within this calculation.

- Design Load Development;

- Channel 4 x.180 Evaluation;

- Supporting Pipe Evaluation; and

- End Cap Evaluation.

\subsection{ASSUMPTIONS / DESIGN INPUTS}

4.1 Assumptions

There are no unverified assumptions within this calculation.

4.2 Design Ctiteria

4.2.1 Loading

See Section 5.1.

4.2.2 Stress Limits

Bending stress $<0.6 \times \mathrm{S}_{\mathrm{y}} \quad$ (Reference 7.7)

Shear stress $<0.4 \times \mathrm{S}_{\mathrm{y}} \quad$ (Reference 7.7)

4.2.3 Platform Parameters (Reference 7.6)

Channel: $\quad 4 " \times 0.180^{*} \times 113.45$ in long ASTM B308

Pipe: 1 1/2"NPS ASTM A53

4.2.4 Material Properties

ASTM B308 Aluminum

(Reference 7.3)

Fy: $\quad 35 \mathrm{ksi}$

Fu: $\quad 38 \mathrm{ksi}$

\begin{tabular}{|c|c|c|c|}
\hline Perfomed by: & & $\begin{array}{l}\text { Date: } \\
11 / 25 / 96\end{array}$ & $\begin{array}{l}\text { Calculation No. 457-2002.2 } \\
\text { Revision } 1\end{array}$ \\
\hline Checked by: & GRE & $\begin{array}{l}\text { Date: } \\
\quad 1 / 2+5-46\end{array}$ & Page 6 of 13 \\
\hline
\end{tabular}


Appendix A9.2.8 Trailer Work Platform 457-2002.2 (Continued)

\subsection{ANALYSIS DETALL}

\subsection{Design Laad Development}

The design load, $\mathrm{F}$, is the based on the larger of the following two (2) conditions:

Condition A: The light duty uniform loading of 29CFR1910 (Reference 7.5) multiplied by a load factor (LF) of four (4). The uniform distributed load, $\mathrm{p}$, is $25 \mathrm{lbs} / \mathrm{f}^{2}$.

Condition B: Each platform section carrying a load equal to the maximum intended load multiplied by a load factor (LF) of four (4). The maximum intended load, $P$, shall be considered to be equal to two men, each weighing 250 . Ibs.

$$
\begin{aligned}
F_{A} & =p \times(\text { area }) \times \mathrm{LF} \\
& =25 \times 113.45 \times 29.29 \times 4 / 144 \\
& =2308 \mathrm{lbs} \\
F_{B} & =P \times 4 \\
& =(2 \times 250) \times 4 \\
& =2000 \mathrm{lbs} \\
F & =\text { Greater of } F_{A} \text { or } F_{B} \\
& =2308 \mathrm{lbs}
\end{aligned}
$$

\begin{tabular}{|c|c|c|c|}
\hline $\begin{array}{l}\text { NAC } \\
\text { INTERNATIONAL }\end{array}$ & Performed by: $\quad 28$ & $\begin{array}{l}\text { Date: } \\
11 / 25 / 96\end{array}$ & $\begin{array}{l}\text { Calculation No. 457-2002.2 } \\
\text { Revision 1 }\end{array}$ \\
\hline & Checked by: $\quad$ gRe & Date: $/ 1-25-46$ & Page 7 of 13 \\
\hline
\end{tabular}

For the channel evaluation. an equivalent loading equal to $25 \times 4=100$ psf will be applied. Convering this to a linear loading per channel:

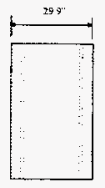

100 berite

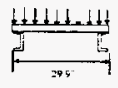

$w=100 \mathrm{psf} \times 29.9 \mathrm{in} /[(12 \mathrm{in} / \mathrm{ft}) \times 2$ channels $]=125 \mathrm{lbs} \mathrm{ft}$. 
Appendix A9.2.8 Trailer Work Platform 457-2002.2 (Continued)

\subsection{Channel Evaluation}

The channel is supported by the scaffolding pipe at each end by caps. thus developing a simply supported beam loading configuration:

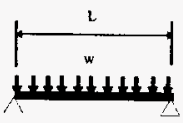

where:

$$
\begin{aligned}
\mathbf{L} & =9.45 \mathrm{ft} \\
\mathbf{w} & =125 \mathrm{lbs} / \mathrm{ft} \\
\mathbf{M} & =\frac{\mathbf{w l}^{2}}{8} \\
& =\frac{125 \times 9.45^{2}}{8} \\
& =1,395 \mathrm{ft}-\mathrm{lbs} \\
& =16.744 \mathrm{in}-\mathrm{lbs}
\end{aligned}
$$

\begin{tabular}{|c|c|c|c|}
\hline NAC & Perfomed by: $R g$ & Date: $11 / 25 / 96$ & $\begin{array}{l}\text { Calculation No. 457-2002.2 } \\
\text { Revision I }\end{array}$ \\
\hline & Checked by: $g$ ed & Date: $11-25 \%$ & Page 8 of 13 \\
\hline
\end{tabular}

The bending stress is:

$$
\begin{aligned}
\sigma_{\mathrm{b}} & =\mathrm{M} / \mathrm{S}_{\mathrm{y}} \\
& =16,744 / 1.93 \text { (use } \mathrm{C} 4 \times 5.4 \text { section modulus from Reference } 7.7 \text { ) } \\
& =8,676 \mathrm{psi}
\end{aligned}
$$

The allowable bending stress is:

$$
\begin{aligned}
F_{b} & =0.6 \times F_{y} \\
& =0.5 \times 35,000 \mathrm{psi} \\
& =21,000 \mathrm{psi}
\end{aligned}
$$


Appendix A9.2.8 Trailer Work Platform 457-2002.2 (Continued)

The margin of safety is:

$$
\begin{aligned}
\mathrm{MS} & =1-\frac{\sigma_{\mathrm{b}}}{\mathrm{F}_{\mathrm{b}}} \\
& =1-\frac{8,676}{21,000} \\
& =0.59
\end{aligned}
$$

The shear stress is:

$\sigma_{\mathrm{v}}=(\mathrm{w} \times \mathrm{L}) /(2$ channels $\mathrm{x}$ width of channel web $\mathrm{x}$ web thickness)

$=125 \times 9.45 /(2 \times 4 \times 0.18)$

$=820 \mathrm{psi}$

The allowable shear stress is:

$$
\begin{aligned}
F_{v} & =0.4 \times F_{y} \\
& =0.4 \times 35,000 \mathrm{psi} \\
& =14,000 \mathrm{psi}
\end{aligned}
$$

\begin{tabular}{|c|c|c|c|c|}
\hline NAC & Perfomed by & ES & $11 / 25196$ & $\begin{array}{l}\text { Calculation Vo. 457-2002.2 } \\
\text { Revision } 1\end{array}$ \\
\hline & Checked by: & 820 & Date: $1 /-25-96$ & Page 9 of 13 \\
\hline
\end{tabular}

The margin of safety is:

$$
\begin{aligned}
\text { MS } & =1 \cdot \frac{\sigma_{v}}{\mathrm{~F}_{v}} \\
& =1 \cdot \frac{820}{14,000} \\
& =0.94
\end{aligned}
$$


Appendix A9.2.8 Trailer Work Platform 457-2002.2 (Continued)

\subsection{Pipe Evaluation}

IOCFR29, Par 1910.28 specifies nominal two inch (2") O.D. tube for scaffolding. The drawing calls out 1.5 inch commercial pipe with a schedule 40 designation. The acceptance of the 1.5 inch pipe will be justified by comparing its sectional properties to those of the 2 inch tube.

\begin{tabular}{|c|c|c|}
\hline PROPERTIES & $2 \mathrm{NN}$ TUBE & $1.5 \mathrm{NN}$ SCH 40 PIPE \\
\hline Metal Area & $0.7087 \mathrm{in}^{2}$ & $0.799 \mathrm{in}^{2}$ \\
\hline Section Modulus & $0.314 \mathrm{in}^{3}$ & $0.326 \mathrm{in}^{3}$ \\
\hline
\end{tabular}

This comparison shows that 1.5 inch schedule 40 pipe is slightly stronger than the two inch (2") tube with a maximum wall thickness of 0.120 inches. Therefore, the 1.5 inch schedule 40 pipe will perform as well as the two inch $\left(2^{n}\right)$ tube.

The tube properties are based on 2" tube with a 0.120 inch wall thickness (Page 8-213 of Reference 7.4). The section modulus is based on the hollow circle formulas referenced on page 6-20 of Reference 7.7. The properties for 1.5 inch pipe are from page $1-93$ of Reference 7.7 .

\subsection{End Cap Evaluation}

The end cap is a 1/4 inch thick aluminum plate. Its basic loading scheme is in direct bearing.

$\sigma_{\mathbf{v}}=\mathbf{F} / \mathbf{A}_{\text {bearing }}$

where:

$\mathrm{F} \quad=\mathrm{w} \times \mathrm{L} / 2$ channels

$=125 \times 9.45 / 2$

$=591 \mathrm{lbs}$

NAC

INTERYATIONAL.

\begin{tabular}{|l|l|l|}
\hline Performed by: & $\begin{array}{l}\text { Date: } \\
11 / 25 / 96\end{array}$ & $\begin{array}{l}\text { Calculation No. 457-2002.2 } \\
\text { Revision I }\end{array}$ \\
\hline Checked by: gRo & Date: 1125 46 & Page 10 of 13 \\
\hline
\end{tabular}


Appendix A9.2.8 Trailer Work Platform 457-2002.2 (Continued)

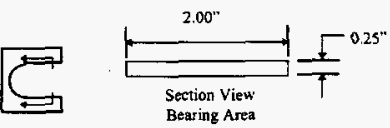

$\begin{aligned} A_{\text {bearing }} & =2 \times 0.25 \\ & =0.5 \text { inch }^{2}\end{aligned}$

The bearing stress is:

$\sigma_{b}=591 / 0.5$

$=1182 \mathrm{psi}$

The allowable bearing stress is:

$F_{b}=F_{y}$

$=35,000 \mathrm{psi}$

The margin of safety is:

$$
\begin{aligned}
\text { MS } & =1-\frac{\sigma_{\mathrm{b}}}{\mathbf{F}_{\mathrm{b}}} \\
& =1-\frac{1,182}{35,000} \\
& =0.97
\end{aligned}
$$

The plate is welded with $1 / 4$ inch fillet weld on both sides. Therefore, the weld stress is insignificant. No further evaluation is required.

\begin{tabular}{|c|c|c|}
\hline Performed by: RS & Date: $11 / 25 / 96$ & $\begin{array}{l}\text { Calculation Ko. } 457-2002.2 \\
\text { Revision i }\end{array}$ \\
\hline Checked by: & Date: $11-25-x$ & Page 11 of 13 \\
\hline
\end{tabular}


Appendix A9.2.8 Trailer Work Platform 457-2002.2 (Continued)

6.0 SUMMARY OF RESULTS/CONCLUSIONS

Summary of Stress Analysis

\begin{tabular}{|l|l|l|l|l|l|l|l|}
\hline $\begin{array}{l}\text { Drawing } \\
\text { No. }\end{array}$ & $\begin{array}{l}\text { Item } \\
\text { No. }\end{array}$ & Component & $\begin{array}{l}\text { Applied } \\
\text { Load }\end{array}$ & Design Check & $\begin{array}{l}\text { Calculated } \\
\text { Loading }\end{array}$ & Allowable & M.S. \\
\hline $457-105$ & 24 & channel & $125 \mathrm{lb} / \mathrm{ft}$ & bending & $8,676 \mathrm{psi}$ & $21,000 \mathrm{psi}$ & 0.59 \\
\hline $457-105$ & 26 & plank hook & $591 \mathrm{lbs}$ & bearing & $1,182 \mathrm{psi}$ & $35,000 \mathrm{psi}$ & 0.97 \\
\hline
\end{tabular}

The transport trailer work platform design meets the criteria defined in Section 4.2.

\begin{tabular}{|c|c|c|}
\hline Periormed by: 728 & Date: $1125 / 96$ & $\begin{array}{l}\text { Calculation No. 457.2002.2 } \\
\text { Revision I }\end{array}$ \\
\hline Checked by: $\quad g R \theta$ & Date: $/ / 2.25-90$ & Page 12 of 13 \\
\hline
\end{tabular}


Appendix A9.2.8 Trailer Work Platform 457-2002.2 (Continued)

\subsection{REFERENCES}

7.1 Preliminary Design Analysis Report For The TN-WHC Cask and Transporation System Project 3035

Transnuclear. Inc.

7.2 Kee Industrial Products.

Test Report: Ultimate Load Capacities of Set Screws.

(Provided in Appendix A)

7.3 1989 Annual Book of ASTM Standards, Volume 02.02. page 330.

7.4 Standard Handbook for Mechanical Engineers, Baumeister \& Marks, 7th edition. .

7.5 Code Of Federal Regulations

Title 29. Part 1910.28

Safety Requirements For Scaffolding

7.6 K Basin Immersion Pail Assembly TN WHC Transpor Cask Drawings.

7.6.1 Project 457, Drawing 105.

7.7 AISC Manual Of Steel Construction, 9th edition.

\begin{tabular}{|c|c|c|c|}
\hline NAC & Performed by: & Date: $11 / 25 / 96$ & $\begin{array}{l}\text { Calculation No. 457-2002. } \\
\text { Revision I } \\
\end{array}$ \\
\hline & Checked by $\quad g R \theta$ & Dale: $11-25-\pi$ & Page 13 of 13 \\
\hline
\end{tabular}


Appendix A9.2.8 Trailer Work Platform 457-2002.2 (Continued)

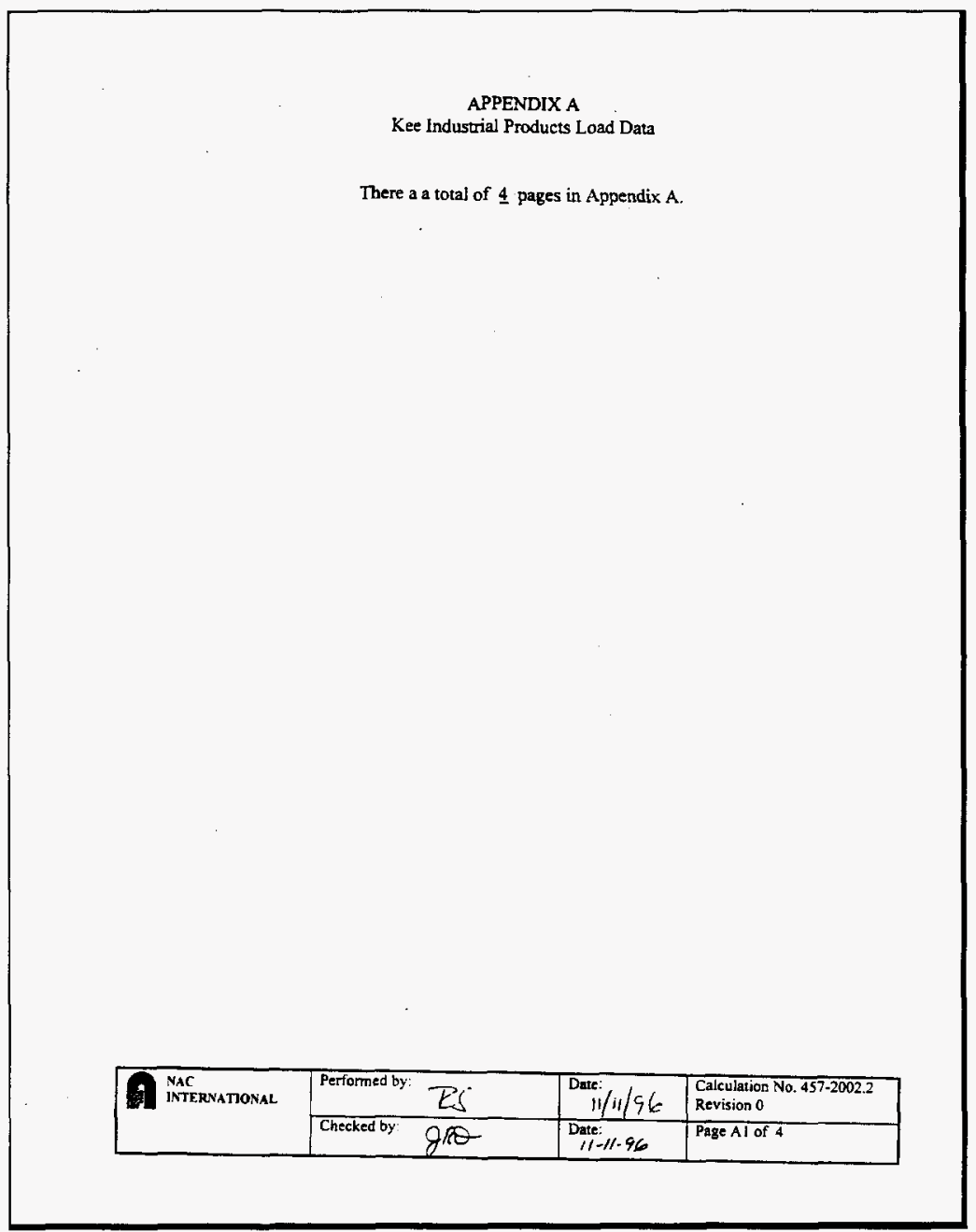




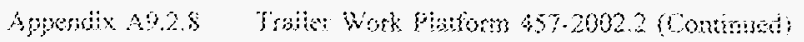

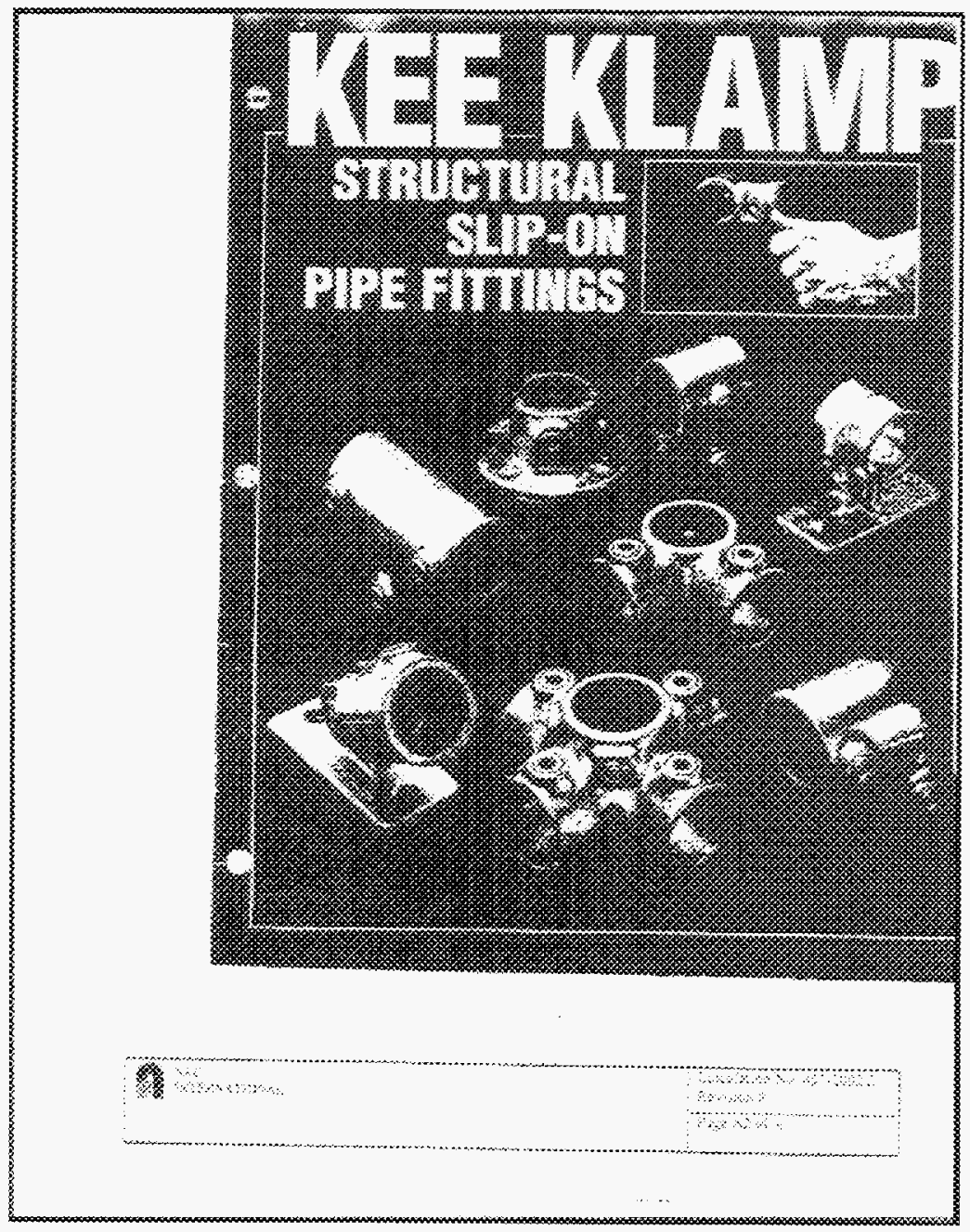


Appendx Arz

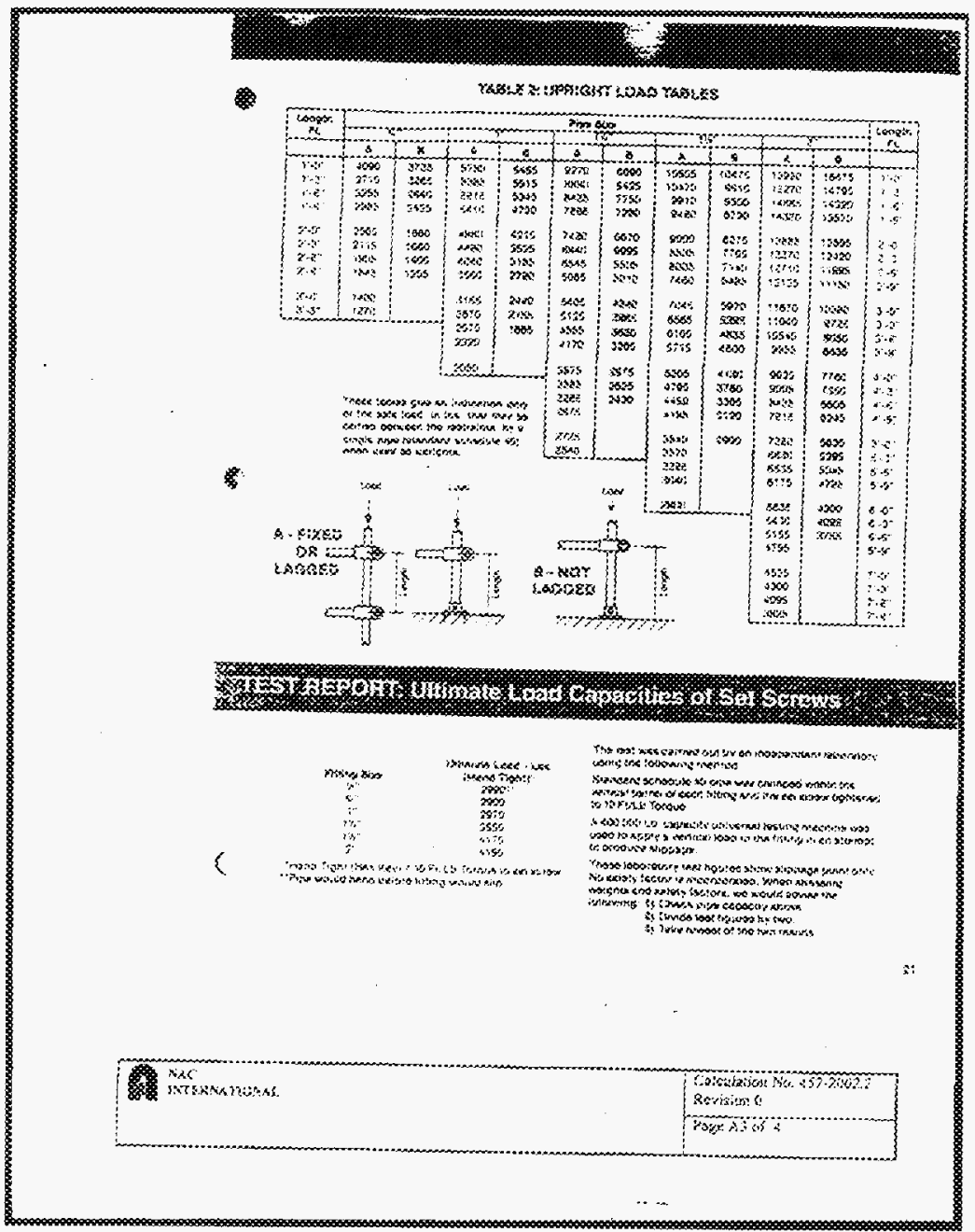




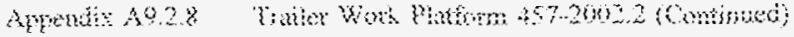

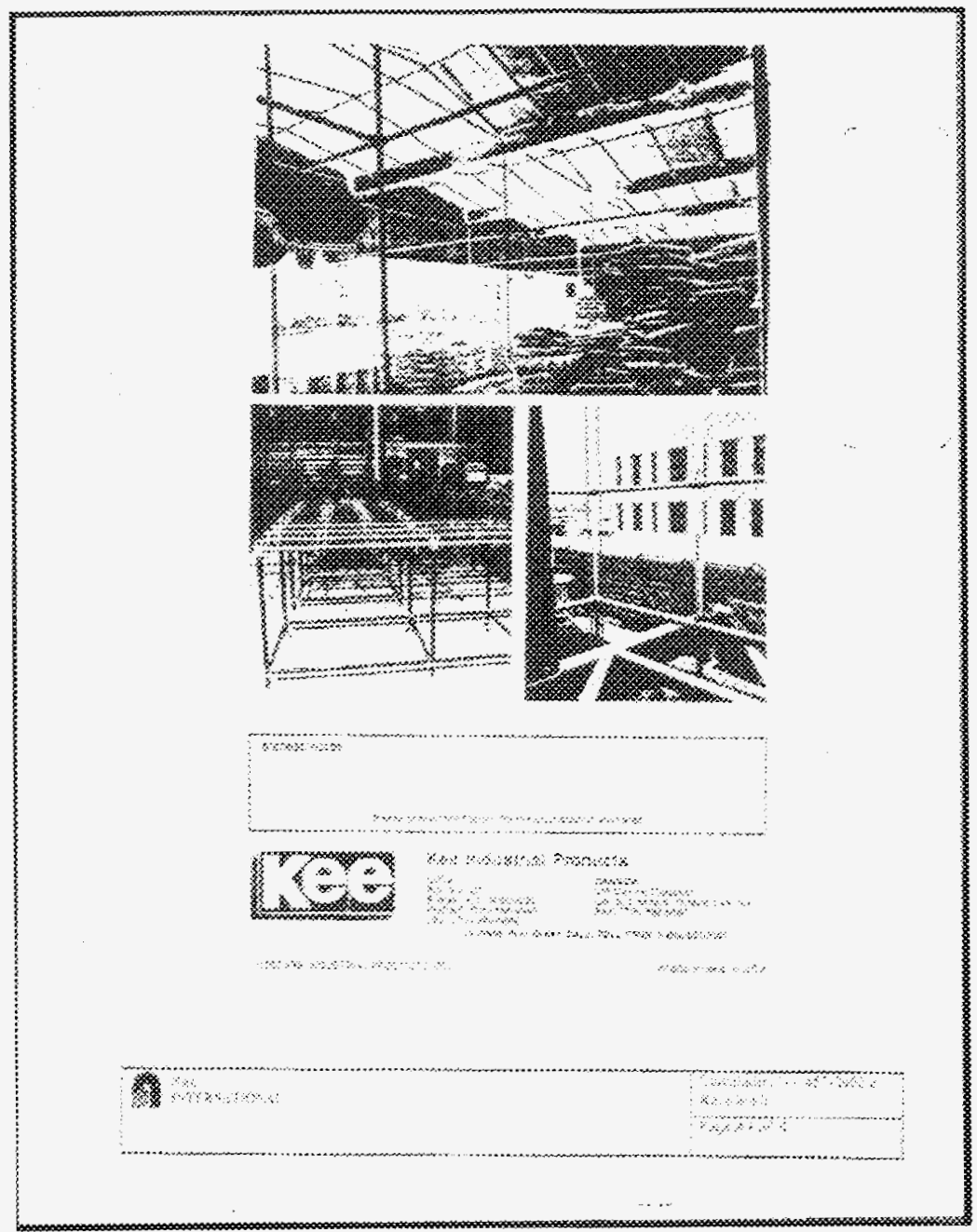




\section{PART B: PACKAGE EVALUATION}

\section{B1.0 INTRODUCTION}

\section{B1.1 SAFETY EVALUATION METHODOLOGY}

This section of the Design Analysis Report (DAR) presents the structural evaluation of the cask. This package evaluation describes the design features and presents the safety analyses which demonstrate that the cask complies with applicable requirements of the Hanford Specification ${ }^{(1)}$.

Detailed structural analyses of various cask components subjected to individual loads are provided in the Appendix B4.5C. The limiting results from these analyses are used in Section B4.3 to quantify package performance in response to the normal condition of transport load combinations and Section B4.4 to quantify package performance in response to the accident condition of transport load combinations. Tables B4.3-1 and B4.4-1 provide an overview of the performance evaluations reported in each load combination subsection for normal and accident conditions, respectively. Each subsection provides the limiting structural analysis result for the affected cask component(s) in comparison to the established design criteria. This comparison permits the minimum margin of safety for a given component subjected to a given loading condition to be readily identified. In all cases, the acceptability of the cask design with respect to established criteria and consequently with respect to the Specification performance requirements is demonstrated.

Scveral other items should be noted. In the cask, thermal stresses occur due to the effects of differential thermal expansion. When evaluating stresses, these thermal stresses are conservatively treated as primary stresses and the combined stresses due to primary loads (like pressure) and differential expansion (such as heating from $70^{\circ} \mathrm{F}$ to hot thermal conditions) are evaluated as primary stresses. In addition, some individual load cases in Appendix B4.5C were performed for the corresponding unit load $(1 \mathrm{G})$ condition (like the end drop and side drop) and are factored for the normal and accident conditions.

The stress results for the individual load case tables reported in $A$ ppendix $\mathrm{B} 4.5 \mathrm{C}$ are the maximum stresses for each individual load cases. Two or more individual load cases must be combined to determine the total stresses at the standard stress reporting locations for the various load combinations. This is accomplished using the ANSYS(2) postprocessor which algebraicly adds the stress components at each of the standard locations. The membrane stress intensity is then found from the membrane stress components and the inner and outer surface membrane plus bending stress intensities are determined from the membrane \pm bending stress components.

Figure B4.3-1 shows the selected locations on the cask body where stress results for these analyses are reported. Detailed stresses are actually available at as many locations as there are nodes in the finite element model. However, for practical considerations, the reporting of stress results is limited to those locations shown on Figure B4.3-1. These locations were selected to be 
representative of the stress distribution in the cask body with special attention given to areas subject to high stresses. The maximum stress may occur at a different location for each individual load.

The shielding analysis of the cask is performed using industry standard codes and conservative modeling assumptions. An evaluation of the shielding performance of the cask was performed assuming dry cask conditions. This evaluation is based on the maximum source term payload. The bounding source term used for the shielding evaluation is the Mark IV fuel elements, 0.95 U235 irradiation to $16 \% \mathrm{Pu}-240$, thirteen years after discharge from the $\mathrm{N}$ reactor.

The gamma and neutron analyses are performed using the one dimensional SAS1 module of SCALE-4, with the $27 \mathrm{n}-18 \mathrm{~g}$ coupled cross-section library. This uses the codes XSDRNPM and XSDOSE to calculate surface flux and translate the flux into dose rates away from the cask surface. ANSI standard flux-to-dose factors, within SCALE-4, are used for the dose calculation at the selected points. The method in which the cask and its contents are modeled for the shielding analysis and the shielding analysis results are described in the Section B3.0.

The thermal evaluation is described in Section B5.0. The ANSYS computer code is used to performed the analysis. The design input for decay heat load, ambient conditions and $\mathrm{MCO}$ geometry were obtained from the Hanford Specification.

\section{B1.2 EVALUATION SUMMARY AND CONCLUSIONS}

Appendix B4.5C provides the detailed description of the structural analyses of the cask body. That appendix describes the detailed ANSYS model used to analyze various applied loads. Tables B4.3-2 and B4.4-2 identify the individual loads (IL) analyzed which are applicable to normal and accident conditions of transport.

Detailed stresses and displacements in the ANSYS model of the cask body are obtained and stored (on magnetic tape) for every node location for each individual load case. These stored results are postprocessed to printout the stresses at the standard stress reporting locations.

Since the individual load cases are linearly elastic, their results can be scaled and superimposed as required in order to perform the normal and hypothetical accident condition load combinations.

Shielding for the cask is provided mainly by the thick-walled cask body. This provides adequate shielding for both normal and accident conditions. Figures B3.3-1 and B3.3-2 illustrate the onedimensional SAS1 models used for the analysis and Table B3.3-1 lists the compositions of the shielding materials.

The temperature distribution from the thermal analyses is used to evaluated the thermal stresses in the cask. The structural integrity of the cask is maintained during normal and accident thermal environments. 


\section{Normal Condition of Transport}

The maximum stresses due to the 1 foot free drop event are presented in Section B4.3.4.3.5. In nearly all of the load cases, the maximum stresses are less than the membrane allowable stress with large margin of safety.

The lid bolt stresses do not exceed $2 / 3$ times the yield strength during the $1 \mathrm{ft}$ drop normal conditions as shown in Section B4.3.4.3.8.

The cask body is a solid stainless steel cylinder, the thermal stresses due to differential thermal expansion are insignificant. Thermal stresses of the cask body due to the differential thermal expansion under the hot and cold environment conditions are evaluated in Section B4.3.4.2.

From the analyses presented in Section 4.3, it can be shown that the normal structural and thermal loads will not result in any structural damage of the cask and the containment function of the $\mathrm{MCO}$ will be maintained.

\section{Accident Condition of Transport}

The maximum stresses due to the 30 foot drop event are also presented in Section B4.4-4.3. In nearly all of the load cases, the maximum stresses are less than the membrane allowable stress with large margins of safety.

The lid bolt stresses do not exceed the ultimate strength during the hypothetical $30 \mathrm{ft}$ drop accidents as shown in Section B4.4.4.3.4.

During the 40 inch drop onto a 6.0 inch diameter puncture bar, the cask body may deform locally at the contact point. It has been shown by analysis (Section B4.4.4.3.5) that the cask will not be punctured and that the MCO will be confined.

The thermal stresses in the stainless steel cask body due to differential thermal expansion are insignificant. Thermal stress of the cask body due to the differential thermal expansion under the thermal accident conditions (between lid and lid bolts) is evaluated in Section B4.4.4.2.

From the analyses presented in Section 4.4, it can be shown that the accident structural and thermal loads will not impact the structural integrity of the cask, and the cask maintains the confinement function of the MCO. 


\section{HNF-SD-SNF-FDR-003 Rev. 0}

\section{References For B1.0}

1. Performance Specification For TN-WHC Cask And Transportation System, WHC-S-0396, Rev. 1, September 1995.

2. ANSYS Engineering Analysis System, User's Manual Volumes 1\&2, Rev. 5.2. 
HNF-SD-SNF-FDR-003 Rev. 0 E-15166

\section{B2.0 CONTAINMENT EVALUATION}

This Section will be written by DESH

\section{B2. 1 INTRODUCTION}

\section{B2.2 CONTAINMENT SOURCE SPECIFICATION}

\section{B2.3 NORMAL TRANSFER CONDITIONS}

B2.3.1 Conditions to be Evaluated

B2.3.2 Containment Acceptance Criteria

B2.3.3 Containment Model

B2.3.4 Containment Calculations

B2.4 ACCIDENT CONDITIONS

B2.4.1 Conditions to be Evaluated

B2.4.2 Containment Acceptance Criteria

B2.4.3 Containment Model

B2.4.4 Containment Calculations

B2.5 CONTAINMENT EVALUATION AND CONCLUSIONS

B2.6 APPENDIX 


\section{B3.0 SHIELDING EVALUATION}

\section{B3.1 INTRODUCTION}

An evaluation of the shielding performance of the TN-WHC cask was performed assuming dry cask conditions. This evaluation is based on the maximum source term payload.

The most significant shielding design features of the cask are the thick walled forged carbon steel cask body and lid. The cask body has a minimum wall thickness of 7.25 inches and a bottom thickness of 6.13 inches. A minimum cask lid thickness of 3.0 inches was used. Additional shielding is provided by the fuel assemblies, the baskets, and the MCO. The cask design does not included separate neutron shielding because of the relatively low neutron source term in the spent fuel. The method in which the cask and its contents are modeled for the shielding analysis are described in the Sections B3.3 and B3.4.

The shielding analysis of the cask is performed using industry standard codes and conservative modeling assumptions. The expected dose rates for the cask have been evaluated for the design basis fuel.

\section{B3.2 DIRECT RADIATION SOURCE SPECIFICATION}

The bounding source term used for the shielding evaluation is the Mark IV fuel elements, $0.95 \mathrm{U}$ 235 irradiation to $16 \% \mathrm{Pu}-240$, thirteen years after discharge from the $\mathrm{N}$ reactor. The source term, gamma spectra, and neutron spectra are provided in Reference 1.

\section{B3.2.1 Gamma Source}

Table B3.2-1 shows the gamma source spectra. The spectra from 0.35 to $2.25 \mathrm{MeV}$ was used since these energy levels are the primary contributors to the dose rate. The gamma source spectra provided in Reference 1 was generated from the ORIGEN2 code. However, this spectra was converted into the gamma energy groups of the SCALE $27 n-18 \gamma$ library ${ }^{(2)}$ by conserving energy. Appendix B3.7.1 details the conversion of the gamma source spectra for use in the SCALE code. 
HNF-SD-SNF-FDR-003 Rev. $0 \quad$ E-15166

Table B3.2-1 Gamma Source Spectra

\begin{tabular}{|c|c|c|}
\hline $\begin{array}{c}\text { Average Energy Level } \\
(\mathrm{MeV})\end{array}$ & Photon/sec/MCO & Fraction \\
\hline 2.25 & $8.88 \mathrm{E}+09$ & 0.00000 \\
\hline 1.83 & $6.828 \mathrm{E}+11$ & 0.00024 \\
\hline 1.495 & $1.009 \mathrm{E}+13$ & 0.00348 \\
\hline 1.165 & $2.415 \mathrm{E}+13$ & 0.00833 \\
\hline 0.9 & $3.727 \mathrm{E}+13$ & 0.01286 \\
\hline 0.7 & $9.045 \mathrm{E}+14$ & 0.31211 \\
\hline 0.5 & $1.868 \mathrm{E}+15$ & 0.64458 \\
\hline 0.35 & $5.300 \mathrm{E}+13$ & 0.01829 \\
\hline Total & $2.898 \mathrm{E}+15$ & 0.99989 \\
\hline
\end{tabular}

\section{B3.2.2 Neutron Source}

The Neutron Source for Mark IV Fuel 16\% Pu 240 at 13 years decay was also provided in Reference 2. The spontaneous fission source is $7.317 \mathrm{E}+06$ neutrons/sec/MCO with an (alpha,n) neutron source of $3.578 \mathrm{E}+06$ neutrons $/ \mathrm{sec} / \mathrm{MCO}$.

The neutron spectra for both sources were also provided in Reference 2. Similar to the gamma source spectra, this spectra must be converted into the proper neutron energy groups of the SCALE $28 \mathrm{n}-18 \gamma$ library. This is accomplished by apportioning the provided spectra into the SCALE energy groups. This analysis and conversion is detailed in Appendix B3.7.2. The spectrum used for the analysis is provided in Table B3.2-2 below. 
Rev. 0

Table B3.2-2 Neutron Spectra

\begin{tabular}{|c|c|c|c|}
\hline SCALE Group No & (alpha,n) & $\begin{array}{c}\text { Spontaneous } \\
\text { Fission }\end{array}$ & Combined \\
\hline Group 1 & & 0.02184 & 0.01463 \\
\hline Group 2 & 0.16137 & 0.22278 & 0.20251 \\
\hline Group 3 & 0.41486 & 0.22655 & 0.28869 \\
\hline Group 4 & 0.14594 & 0.12795 & 0.13389 \\
\hline Group 5 & 0.11620 & 0.15740 & 0.14380 \\
\hline Group 6 & 0.11214 & 0.15633 & 0.14175 \\
\hline Group 7 & 0.04767 & 0.08316 & 0.07145 \\
\hline Total: & 0.99818 & 0.99601 & 0.99672 \\
\hline
\end{tabular}

\section{B3.3 SUMMARY OF SHIELDING PROPERTIES OF MATERIALS}

One-dimensional SAS1 models (of the SCALE code ${ }^{(3)}$ ) were used for the gamma and neutron shielding calculations of the top, bottom, and side of the cask.

For the doses at the top and bottom of the cask, one-dimensional plane gcometry XSDRNPM models are used. The fuel region is assumed to consist of uranium dioxide, zircaloy and steel basket. These are assumed to be homogenized throughout the fuel zone. The model assumes only the active fuel region. No consideration is given to the presence of a plenum or top fitting. The configurations of the top and bottom model are shown in Figure B3.3-1.

For the gamma and neutron doses on the side of the cask, a cylindrical one-dimensional model is used in XSDRMPM as shown in Figure B3.3-2. The central fuel region is considered to consist of uranium dioxide. The fuel cladding and steel basket are included in the homogenized fuel region. The fuel region is modeled as a cylinder with the actual cavity diameter. Subsequent regions are modeled as cylindrical shells corresponding to actual dimensions.

Materials and their densities used in the XSDRNPM models are provided in Table B3.3-1. 


\section{Figure B3.3-1 Axial Models}

Axial, Cask Top - Shield Plug and Lid

\begin{tabular}{ll}
$7.62 \mathrm{~cm}$ & Lid - SS304 \\
\hline $30.48 \mathrm{~cm}$ & Shield Plug - SS304 \\
$186.29 \mathrm{~cm}$ & Fuel + Clad + Basket \\
\hline
\end{tabular}

Axial, Cask Bottom

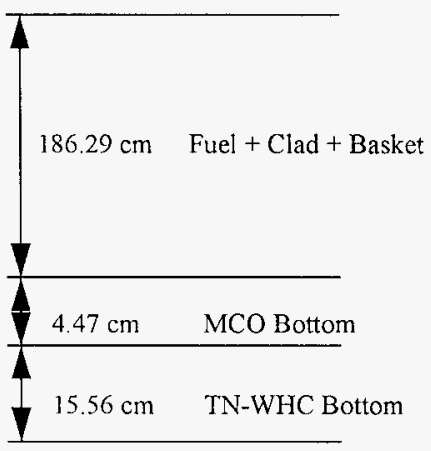


HNF-SD-SNF-FDR-003 Rev. 0

Figure B3.3-2 Radial Model

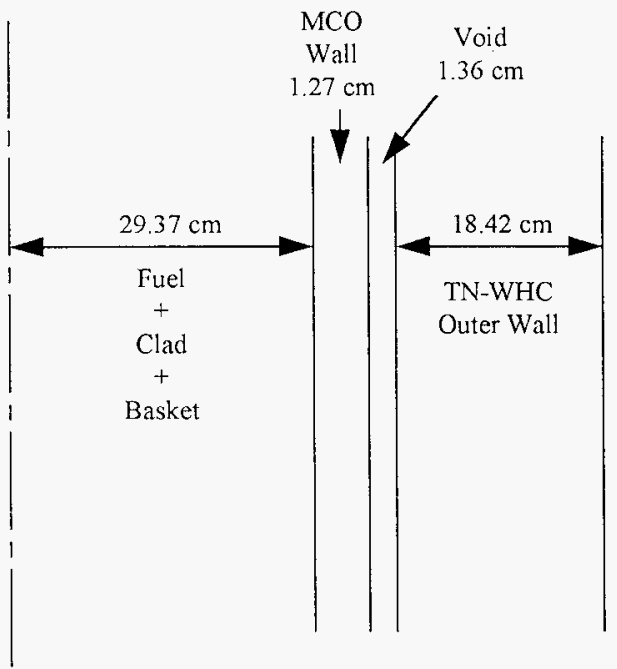


HNF-SD-SNF-FDR-003 Rev. 0

Table B3.3-1 Material Densities

\begin{tabular}{|c|c|c|}
\hline Location & Material & Density (g/cc) \\
\hline $\begin{array}{c}\text { Fuel + Clad + Basket } \\
\text { (assuming basket is 100\% } \\
\text { steel) }\end{array}$ & Uranium & 6.28 \\
\cline { 2 - 3 } & Zircaloy & 0.41 \\
\cline { 2 - 3 } & Steel (SS304) & 0.338 \\
\hline Cask Wall & Steel (SS304) & 7.92 \\
\hline Cask Lid & Steel (SS304) & 7.92 \\
\hline Shield Plug & Steel (SS304) & 7.92 \\
\hline
\end{tabular}

\section{B3.4 NORMAL TRANSFER CONDITIONS}

\section{B3.4.1 Conditions to be Evaluated}

Under normal conditions the shielding of the cask is evaluated assuming an undamaged cask and the worst case fuel.

\section{B3.4.2 Acceptance Criteria}

The shielding acceptance criteria for normal conditions for the cask is the following:

-the maximum surface dose on the accessible surface of the cask shall be less than or equal to $100 \mathrm{mrem} / \mathrm{hr}^{(2)}$; and

-the dose rate 2 meters from the surface of the cask shall be less than or equal to $10 \mathrm{mrem} / \mathrm{hr}^{(2)}$.

\section{B3.4.3 Shielding Model}

The gamma and neutron analyses are performed using the one dimensional SAS1 module of SCALE-4, with the $27 \mathrm{n}-18 \mathrm{~g}$ coupled cross-section library. This uses the codes XSDRNPM and XSDOSE to calculate surface flux and translate the flux into dose rates away from the cask surface. ANSI standard flux-to-dose factors, within SCALE-4, are used for the dose calculation at the selected points.

For the axial calculations, the DISK geometry is chosen and buckling corrections, using the cask diameter, are used to correct for a finite dimension rather than an infinite plane.

Because of the irregular bottom in the $\mathrm{MCO}$, the 3-dimensional QAD-CGGP(5) code was utilized to evaluate two models of the bottom; one with a cut-out and one without. Ratios of the 


\section{HNF-SD-SNF-FDR-003 Rev. 0}

calculated dose rates (1.32@ contact and 1.01@2 meters) were used to correct the SAS1 dose calculation for the bottom of the cask.

An additional dose rate calculation was performed to evaluate the dose rate at the drain port at the lower end of the cask. The 3-dimensional, monte carlo SAS4 module of the Scale-4 code was utilized to perform the calculation. Shielding from the quick connect coupling was conservatively neglected as shown in Figure B3.3-3.

The inputs for the SAS1 and SAS4 modules are included in Appendix B3.7.3.

\section{B3.4.4 Shielding Calculations}

Dose points, shown in Tables B3.4-1 and B3.4-1, are taken axially on the centerline of the cask and radially on the midplane.

Table B3.4-1 Summary of Maximum Dose Rates - Cask Surface (mrem/hour)

\begin{tabular}{|c|c|c|c|c|}
\hline & Sides & $\begin{array}{c}\text { Top, } \\
\text { Shield Plug }\end{array}$ & $\begin{array}{c}\text { Top, Cask Lid } \\
\text { \& Shield Plug }\end{array}$ & Bottom* $^{*}$ \\
\hline Gamma & 54.2 & 0.71 & 0.04 & 85.4 \\
\hline Neutron & 5.10 & 2.39 & 1.31 & 7.26 \\
\hline Total & 59.3 & 3.10 & 1.35 & 92.7 \\
\hline
\end{tabular}

Table B3.4-2 Summary of Maximum Dose Rates - 2 meters from the Cask Surface (mrem/hour)

\begin{tabular}{|c|c|c|c|c|}
\hline & Sides & $\begin{array}{c}\text { Top, } \\
\text { Shield Plug }\end{array}$ & $\begin{array}{c}\text { Top, Cask Lid } \\
\text { \& Shield Plug }\end{array}$ & Bottom* \\
\hline Gamma & 8.54 & 0.09 & 0.005 & 7.88 \\
\hline Neutron & 0.61 & 0.12 & 0.06 & 0.28 \\
\hline Total & 9.15 & 0.21 & 0.065 & 8.16 \\
\hline
\end{tabular}

* - on cask centerline, below MCO sump (B3.4.3)

The dose rates on the drain port cover were calculated by SAS4 to be $167 \mathrm{mrem} / \mathrm{hr} \gamma$ and 2.5 $\mathrm{mrem} / \mathrm{hr}$ neutron. At one foot from the drain port cover, the calculated dose rates were 62.7 $\mathrm{mrem} / \mathrm{hr}$ and $1.1 \mathrm{mrem} / \mathrm{hr} \gamma$ and neutron respectively. 
Figure B3.3-3 Drain Port Model

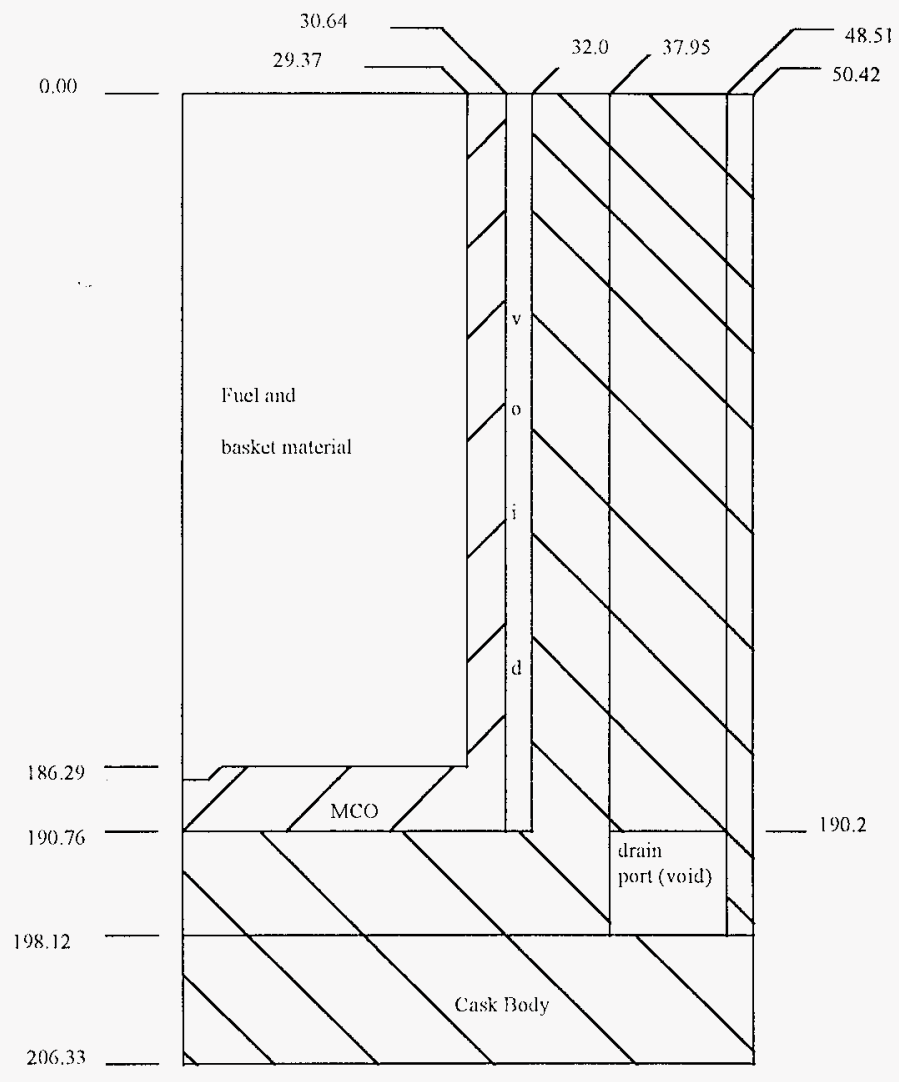




\section{B3.5 ACCIDENT CONDITIONS}

\section{B3.5.1 Conditions To Be Evaluated}

Briefly, the accidents to be evaluated are:

- Impact, the worst case credible impact for the packaging system may be simulated by a free drop of $30 \mathrm{ft}$. onto a concrete surface, the package shall impact in an orientation to cause maximum damage;

- Puncture, the worst case credible puncture is equivalent to a free drop of the packaging through a distance of 1 meter ( $40 \mathrm{in}$ ) in a position for which the maximum damage is expected; and

- Thermal, the exposure of the packaging system for not less than 30 minutes to a $800^{\circ} \mathrm{C}$ fire that has an emissivity coefficient of 0.9 .

The impact, puncture and thermal scenarios will result in no loss of shielding. Therefore, the accident dose rates are the same as the normal conditions dose rates.

\section{B3.5.2 Acceptance Criteria}

The shiclding acceptance criteria for accident conditions for the cask is the following:

- the dose rate 1 meter from the surface of the cask shall not exceed $1 \mathrm{rem} / \mathrm{hr}^{(2)}$.

\section{B3.5.3 Shielding Model}

See Section B3.4.3 shielding model for normal conditions.

\section{B3.5.4 Shielding Calculations}

See Section B3.4.4 shielding calculations for normal conditions.

\section{B3.6 SHIELDING EVALUATION AND CONCLUSIONS}

Shielding for the cask is provided mainly by the thick-walled cask body. This provides adequate shielding for both normal and accident conditions. Figures B3.3-1 and B3.3-2 illustrate the onedimensional SASI models used for the analysis and Table B3.3-1 lists the compositions of the shielding materials.

The expected dose rates were calculated using the source term provided in Reference 1 and the results of the shielding analyses are provided in Tables B3.4-1 and B3.4-2. 


\section{References For Section B3.0}

1. Engineering Change Notice, ECN-191402, SNF Project, WHC-S-0396, Rev 1

2. Document Number WHC-SD-TP-SARP-017, Preliminary Safety Analysis Report for Packaging (On-Site) Multiple Canister Overpack Cask, Draft Rev. 0, April 12, 1996.

3. Performance Specification For TN-WHC Cask And Transportation System, WHC-S-0396, Rev. 1, September 1995.

4. SCALE-4: A Modular Code System for Performing Standardized Computer Analyses for Licensing Evaluation, ORNL/NUREG/CR-0200, U.S. Nuclear Regulatory Commission, Revision 4, February 1990.

5. QAD-CGGP - A Combinatorial Geometry Version of QAD-P5A, a Point Kernel Code System for Neutron and Gamma-Ray Shiclding Calculation Using the GP Buildup Factor, CCC-495. 
B3.7 APPENDIX

\section{SHIELDING ANALYSIS}




\section{HNF-SD-SNF-FDR-003 Rev. 0}

\section{TABLE OF CONTENTS}

B3.7-1 Determination of the Gamma Source Spectra . . . . . . . . . . . . . B3.7-1

B3.7-2 Determination of the Neutron Source Spectra . . . . . . . . . . . . . B3.7-4

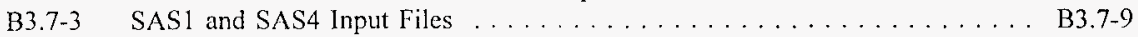




\section{B3.7-1 DETERMINATION OF THE GAMMA SOURCE SPECTRA}

The gamma source for the worst case fuel is provided in Reference 1. The gamma source spectra is shown below in Table B3.7-1.

Table B3.7-1 Photon Source for Mark IV Fuel $16 \%$ Pu 240 at 13 years decay

\begin{tabular}{|c|c|}
\hline Energy (MeV) & Photons/sec/MCO \\
\hline 0.015 & $1.67 \mathrm{E} 15$ \\
\hline 0.025 & $3.54 \mathrm{E} 14$ \\
\hline 0.038 & $3.99 \mathrm{E} 14$ \\
\hline 0.058 & $3.35 \mathrm{E} 14$ \\
\hline 0.085 & $1.85 \mathrm{E} 14$ \\
\hline 0.125 & $1.38 \mathrm{E} 14$ \\
\hline 0.225 & $1.57 \mathrm{E} 14$ \\
\hline 0.375 & $7.42 \mathrm{E} 13$ \\
\hline 0.662 & $2.33 \mathrm{E} 15$ \\
\hline 0.850 & $5.92 \mathrm{E} 13$ \\
\hline 1.250 & $3.41 \mathrm{E} 13$ \\
\hline 1.750 & $1.05 \mathrm{E} 12$ \\
\hline 2.250 & $8.88 \mathrm{E} 9$ \\
\hline 2.750 & $6.34 \mathrm{E} 8$ \\
\hline 3.500 & $8.31 \mathrm{E} 7$ \\
\hline 5.000 & $3.94 \mathrm{E} 5$ \\
\hline 7.000 & $4.50 \mathrm{E} 4$ \\
\hline 11.000 & $5.14 \mathrm{E} 3$ \\
\hline TOTAL & $6.09 \mathrm{E} 15$ \\
\hline & \\
\hline \hline
\end{tabular}

For the gamma source term, the energy levels from 0.375 to $2.25 \mathrm{MeV}$ were used since these energy levels are the primary contributors to the gamma dose. The $0.662 \mathrm{MeV}$ energy level was converted to the $0.575 \mathrm{MeV}$ energy level:

$0.662 \mathrm{MeV}=2.33 \mathrm{E} 15$ photon $/ \mathrm{sec} / \mathrm{MCO}(0.662 / 0.575)=2.68 \mathrm{E} 15$ photon $/ \mathrm{second} / \mathrm{MCO}$ 
The energy groups significant to the dose are from $0.375 \mathrm{MeV}$ to $2.50 \mathrm{MeV}$. The table below summarizes these energy groups:

Table B3.7-2 Photon Source for Mark IV Fuel $16 \%$ Pu 240 at 13 years decay

\begin{tabular}{|c|c|c|}
\hline $\begin{array}{c}\text { ORIGEN2 } \\
\text { Energy Range }\end{array}$ & $\begin{array}{c}\text { ORIGEN2 } \\
\text { Average Energy }(\mathrm{MeV})\end{array}$ & $\gamma / \mathrm{sec} / \mathrm{MCO}$ \\
\hline $0.3-0.45$ & 0.375 & $7.42 \mathrm{E}+13$ \\
\hline $0.45-0.7$ & 0.575 & $2.68 \mathrm{E}+15$ \\
\hline $0.7-1.0$ & 0.850 & $5.92 \mathrm{E}+13$ \\
\hline $1.0-1.5$ & 1.250 & $3.41 \mathrm{E}+13$ \\
\hline $1.5-2.0$ & 1.750 & $1.05 \mathrm{E}+12$ \\
\hline $2.0-2.5$ & 2.250 & $8.88 \mathrm{E}+09$ \\
\hline & Subtotal: & $2.85 \mathrm{E}+15$ \\
\hline
\end{tabular}

The energy groups from this ORIGEN2 output need to be translated into the appropriate energy groups for the SCALE $27 \mathrm{n}-18 \mathrm{~g}$ library. This is done by conserving energy. The following calculations illustrate the conversion between the ORIGEN2 energy levels to the SCALE code energy levels.

Scale Group 35, $1.66-2.0$ :

$$
\begin{aligned}
& =\frac{(2.0-1.66)}{(2.0-1.5)} *\left(\frac{1.75}{1.83}\right) * 1.05 E+12 \\
& =6.828 E+11
\end{aligned}
$$

Scale Group 36, $1.33-1.66 \mathrm{MeV}$ :

$$
\begin{aligned}
& =\frac{(1.66-1.5)}{(2.0-1.5)} *\left(\frac{1.75}{1.495}\right) * 1.05 E+12+\frac{(1.5-1.33)}{(1.5-1.0)} *\left(\frac{1.25}{1.495}\right) * 3.41 E+13 \\
& =1.009 E+13
\end{aligned}
$$

\section{B3.7-2 DETERMINATION OF THE NEUTRON SOURCE SPECTRA}

The neutron source in spent fuel is from either spontaneous fission of the actinides or from $(\alpha, n)$ reactions. For this fuel, the spontaneous fission source and $(\alpha, n)$ reactions are present. The 


\section{HNF-SD-SNF-FDR-003 ReV. 0}

E-15166

spontaneous neutron source is due primarily to six nuclides: $\mathrm{Cm}-242, \mathrm{Cm}-244, \mathrm{Cm}-246, \mathrm{Pu}-238$, $\mathrm{Pu}-240$, and $\mathrm{Pu}-242$.

From Reference 2, the following fission source is tabulated below:

Table B3.7-3 $(\alpha, n)$ and Spontaneous Fission Source for Mark IV Fuel

\begin{tabular}{|c|c|c|}
\hline Component of Source & $\begin{array}{c}\text { Source Strength } \\
\text { (neutrons/sec/MCO) }\end{array}$ & $\%$ \\
\hline$(\alpha, \mathrm{n})$ & $3.578 \mathrm{E}+06$ & $33 \%$ \\
\hline Spontaneous Fission & $7.317 \mathrm{E}+06$ & $67 \%$ \\
\hline Total & $1.090 \mathrm{E}+07$ & \\
\hline
\end{tabular}


HNF-SD-SNF-FDR-003 Rev. 0

E-15166

Table B3.7-4 Spontaneous Fission Specrta

\begin{tabular}{|c|c|}
\hline Upper Energy (MeV) & Fraction \\
\hline 0.00 & 0.00000 \\
\hline 0.10 & 0.01194 \\
\hline 0.20 & 0.01972 \\
\hline 0.30 & 0.02424 \\
\hline 0.40 & 0.02726 \\
\hline 0.50 & 0.02934 \\
\hline 0.60 & 0.03076 \\
\hline 0.70 & 0.03167 \\
\hline 0.80 & 0.03218 \\
\hline 0.90 & 0.03238 \\
\hline 1.00 & 0.03232 \\
\hline 1.20 & 0.06368 \\
\hline 1.40 & 0.06140 \\
\hline 1.60 & 0.05832 \\
\hline 1.80 & 0.05475 \\
\hline 2.00 & 0.05090 \\
\hline 2.20 & 0.04695 \\
\hline 2.40 & 0.04301 \\
\hline 2.60 & 0.03917 \\
\hline 2.80 & 0.03549 \\
\hline 3.00 & 0.03202 \\
\hline 3.20 & 0.02876 \\
\hline 3.40 & 0.02574 \\
\hline 3.60 & 0.02297 \\
\hline 3.80 & 0.02043 \\
\hline 4.00 & 0.01812 \\
\hline 4,20 & 0.01603 \\
\hline 4.40 & 0.01415 \\
\hline 4.60 & 0.01246 \\
\hline 4.80 & 0.01095 \\
\hline 5.00 & 0.00960 \\
\hline 5.50 & 0.01907 \\
\hline 6.00 & 0.01354 \\
\hline 6.50 & 0.00953 \\
\hline 7.00 & 0.00665 \\
\hline 7.50 & 0.00461 \\
\hline 8.00 & 0.00318 \\
\hline 9.00 & 0.00366 \\
\hline 10.00 & 0.00169 \\
\hline 11.00 & 0.00076 \\
\hline 12.00 & 0.00034 \\
\hline 13.00 & 0.00015 \\
\hline 14.00 & 0.00007 \\
\hline 15.00 & 0.00003 \\
\hline Total & 0.99999 \\
\hline
\end{tabular}

B3.7-4 
HNF-SD-SNF-FDR-003 ReV. 0

E-15166

Table B3.7-5 $(a, n)$ Fission Spectra

\begin{tabular}{|c|c|}
\hline Upper Fnergy (McV) & Fraction \\
\hline 0.00 & 0.00000 \\
\hline 0.10 & 0.01059 \\
\hline 0.20 & 0.01184 \\
\hline 0.30 & 0.01153 \\
\hline 0.40 & 0.01371 \\
\hline 0.50 & 0.01869 \\
\hline 0.60 & 0.02103 \\
\hline 0.70 & 0.02305 \\
\hline 0.80 & 0.02523 \\
\hline 0.90 & 0.02414 \\
\hline 1.00 & 0.01994 \\
\hline 1.10 & 0.02087 \\
\hline 1.20 & 0.02259 \\
\hline 1.30 & 0.02539 \\
\hline 1.40 & 0.02741 \\
\hline 1.50 & 0.02804 \\
\hline 1.60 & 0.02944 \\
\hline 1.70 & 0.03193 \\
\hline 1.80 & 0.03551 \\
\hline 1.90 & 0.03692 \\
\hline 2.00 & 0.03879 \\
\hline 2.10 & 0.03894 \\
\hline 2.20 & 0.04065 \\
\hline 2.30 & 0.04128 \\
\hline 2.40 & 0.03956 \\
\hline 2.50 & 0.03785 \\
\hline 2.60 & 0.03645 \\
\hline 2.70 & 0.03474 \\
\hline 2.80 & 0.03209 \\
\hline 2.90 & 0.03115 \\
\hline 3.00 & 0.02928 \\
\hline 3.10 & 0.02586 \\
\hline 3.20 & 0.02430 \\
\hline 3.30 & 0.02087 \\
\hline 3.40 & 0.01698 \\
\hline 3.50 & 0.01433 \\
\hline 3.60 & 0.01231 \\
\hline 3.70 & 0.00997 \\
\hline 3.80 & 0.00857 \\
\hline 3.90 & 0.00748 \\
\hline 4.00 & 0.00607 \\
\hline 4.10 & 0.00545 \\
\hline 4.20 & 0.00358 \\
\hline 4.30 & 0.00280 \\
\hline 4.40 & 0.00171 \\
\hline \multirow[t]{2}{*}{4.50} & 0.00109 \\
\hline & 1.00000 \\
\hline
\end{tabular}

B3.7-5 
FOR SPONTANEOUS FISSION SPECTRA

For Group 1, 6.434-20.0 MeV:

$$
\begin{aligned}
= & \left(\frac{(6.434-6.0)}{(6.5-6.0)} *\left(\frac{6.25}{11.22}\right) * 0.00953\right)+0.00665+0.00461+0.00318 \\
& +0.00366+0.00169+0.00076+0.00034+0.00015+0.00007+0.00003 \\
= & 0.02184
\end{aligned}
$$

For Group 2, $3.00-6.434$ :

$$
\begin{aligned}
= & 0.02876+0.02574+0.02297+0.02043+0.01812+0.01063+0.01415+0.01246 \\
& +0.01095+0.00960+0.01907+\left(\frac{6.434-6.00}{6.50-6.00} * 0.00953 * \frac{6.25}{4.717}\right)+0.1354 \\
= & 0.22278
\end{aligned}
$$

FOR $(\alpha, n)$ SPECTRA

For Group 2, 3.0-6.434 Mev:

$$
\begin{aligned}
& =0.02586+0.02430+0.02087+0.01698+0.01433+0.01231+0.00997 \\
& +0.00857+0.00748+0.00607+0.00545+0.00358+0.00280+0.00171+0.000109 \\
& =0.16137
\end{aligned}
$$

For Group 3, $1.85-3.0 \mathrm{MeV}$ :

$=0.02928+0.03115+0.03209+0.03474+0.03645+0.03785+0.03956+0.04128$ $+0.04065+0.03894+0.03879$

$$
\begin{aligned}
& +\frac{1.90-1.85}{1.90-1.80} * 0.03692 * \frac{1.85}{2.425} \\
= & 0.41486
\end{aligned}
$$




\section{B3.7-3 SAS1 INPUT FILES}

\section{Axial Model, Top}

$=$ SAS 1

K-BASIN FUEL 5/96 - NEUTRON WHC-ST-TP-SARP-017, GAMMA ECN 191402 27N-18COUPLE INFHOMMEDIUM

- FUEL MODELED, 6.34 MTU PER MCO - SS304 BASKET - ZIRC CLAD

$\begin{array}{llllllllll}\text { URANIUM } \quad 1 & \text { DEN=6.28 } & 1.0 & 293 & 92235 & 1.0 & 92238 & 99.0 & \text { END }\end{array}$

ZIRCALLOY 1 DEN $=0.441$ END

SS304 1 DEN $=0.338$ END

SS304 2. 1.0 END

END COMP

END

LAST

TOP MODEL 12" SHIELD PLUG ONLY

DISC REFLECTED

$\begin{array}{llllllll}1 & 130.0 & 40 & -1 & 0 & 0 & 10.8 & 2.87 \mathrm{E} 9\end{array}$

$\begin{array}{llllllll}1 & 186.29 & 40 & -1 & 0 & 0 & 10.8 & 2.878 \mathrm{E} 9\end{array}$

$\begin{array}{llll}2 & 216.77 & 31 & 0\end{array}$

END ZONE

$\begin{array}{llllllll}0.01463 & 0.20251 & 0.28869 & 0.13389 & 0.14380 & 0.14175 & 0.07145 & 26 Z\end{array}$

$\begin{array}{llllllll}0.00000 & 0.00024 & 0.00348 & 0.00833 & 0.01236 & 0.31211 & 0.64458\end{array}$

$0.01829 \quad 4 Z$

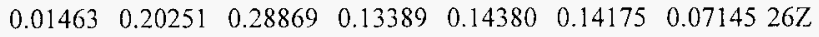

$\begin{array}{llllllll}0.00000 & 0.00024 & 0.00348 & 0.00833 & 0.01236 & 0.31211 & 0.64458\end{array}$

$0.018294 Z$

NDETEC $=4 \mathrm{DY}=103.06 \mathrm{DZ}=103.06$

READ XSDOSE

51.53

$\begin{array}{llllllll}0.0 & 2.0 & 0.0 & 100 . & 0.0 & 200 . & 0.0 & 300\end{array}$

END

$=$ SAS1

K-BASIN FUEL 5/96 - NEUTRON WHC-ST-TP-SARP-017, GAMMA ECN 191402 $27 \mathrm{~N}-18$ COUPLE INFHOMMEDIUM

- FUEL MODELED, 6.34 MTU PER MCO - SS304 BASKET - ZIRC CLAD

$\begin{array}{lllllllllll}\text { URANIUM } & 1 & \mathrm{DEN}=6.28 & 1.0 & 293 & 92235 & 1.0 & 92238 & 99.0 & \mathrm{END}\end{array}$

ZIRCALLOY 1 DEN $=0.441$ END

SS304 1 DEN=0.338 END

$\begin{array}{llll}\text { SS304 } & 2 & 1.0 & \text { END }\end{array}$ 
E-15166

END COMP

END

LAST

TOP MODEL 12 in MCO SHIELD PLUG AND 3 in TN-WHC LID

DISC REFLECTED

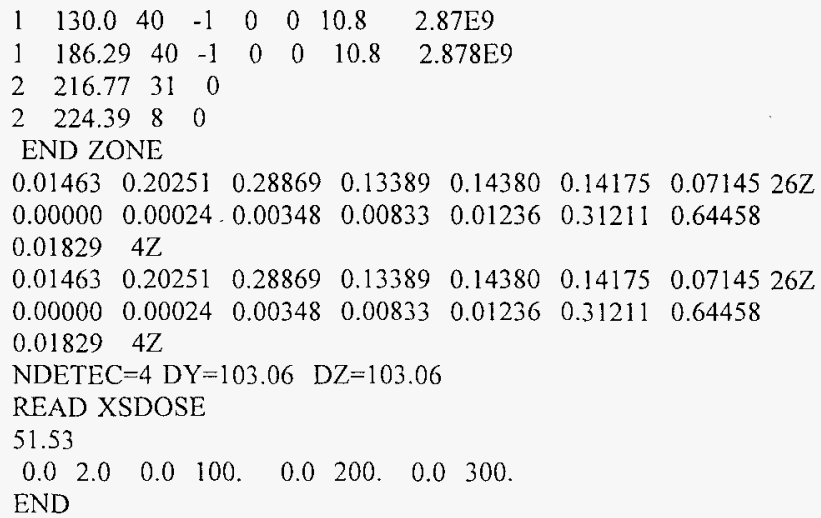

Axial Model, Bottom

$=$ SAS 1

K-BASIN FUEL 5/96 - NEUTRON WHC-ST-TP-SARP-017, GAMMA ECN 191402 $27 \mathrm{~N}-18$ COUPLE INFHOMMEDIUM

' FUEL MODELED, 6.34 MTU PER MCO - SS304 BASKET - ZIRC CLAD

$\begin{array}{llllllllll}\text { URANIUM } \quad 1 & \text { DEN=6.28 } & 1.0 & 293 & 92235 & 1.0 & 92238 & 99.0 & \text { END }\end{array}$

ZIRCALLOY 1 DEN $=0.441$ END

SS304 1 DEN=0.338 END

$\begin{array}{llll}S S 304 & 2 & 1.0 & \text { END }\end{array}$

END COMP

END

LAST

BOTTOM MODEL TN-WHC 6.125in THICK AND MCO 1.76in THICK DISC REFLECTED
$\begin{array}{lllllllll}1 & 130.0 & 40 & -1 & 0 & 0 & 10.8 & 2.87 \mathrm{E} 9\end{array}$
$\begin{array}{llllllll}1 & 186.29 & 40 & -1 & 0 & 0 & 10.8 & 2.878 \mathrm{E} 9\end{array}$
$2 \quad 190.76 \quad 4 \quad 0$ 


\section{E-15166}

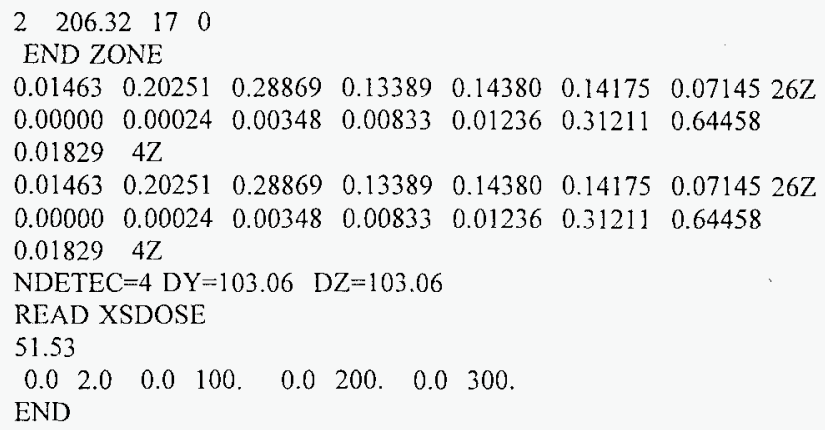

\section{Radial Model}

$=$ SAS 1

K-BASIN FUEL 5/96 - NEUTRON WHC-ST-TP-SARP-017, GAMMA ECN 191402 $27 \mathrm{~N}-18$ COUPLE INFHOMMEDIUM

' FUEL MODELED, 6.34 MTU PER MCO - SS304 BASKET - ZIRC CLAD $\begin{array}{lllllllllll}\text { URANIUM } \quad \text { I } & \text { DEN }=6.28 & 1.0 & 293 & 92235 & 1.0 & 92238 & 99.0 & \text { END }\end{array}$ 
HNF-SD-SNF-FDR-003 Rev. 0

E-15166

\section{END}

B3.7-10 


\section{SAS4 INPUT FILES}

\section{Radial Gamma}

$=$ sas 4

Westinghouse Hanford Cask 3-D Shielding Analysis 7/96

$27 \mathrm{~N}-18$ couple infhommedium

' Fuel modeled, 6.34 MTU per MCO - SS304 basket - Zr clad

uranium 1 den=6.28 $1.0 \quad 293 \quad 92235 \quad 1.0 \quad 92238 \quad 99.0$ end

zircalloy 1 den $=0.441$ end

ss304 I den $=0.338$ end

ss304 21.0 end

end comp

$\mathrm{idr}=0 \quad \mathrm{ity}=2 \quad \mathrm{izm}=4$ frd $=29.37$ end

$29.37 \quad 30.64 \quad 32.00 \quad 50.42$ end

12002 end

xend

tim $=20.0$ nst $=500$ nod $=3 \quad$ sfa $=2.898+15$ fr $2=0.7$ fr $3=0.7$ fr $4=0.7$

igo $=0$ mfu=1 nit $=800$ end

$\begin{array}{llllllll}\text { soe } 34 z & 0.00024 & 0.00348 & 0.00833 & 0.01286 & 0.31211 & 0.64458\end{array}$

$0.018294 z$ end

det $52.0 \quad 0.194 .16 \quad 81.0 \quad 0.194 .16 \quad 81.00 .206 .0$ end

gend

TN-WHC Cask Radial Gamma Dose Rate around drain port with cover(HOLE)

fue $186.29 \quad 186.30$ end

fend

cav $\begin{array}{lll}0 & 32.00 & 190.77 \text { end }\end{array}$

ins $230.64 \quad 190.76$ end

inn $2 \quad 37.95 \quad 190.80$ end

rsl $2 \quad 48.51 \quad 190.20$ and

our $2 \quad 50.42 \quad 206.33$ end

as! $2 \quad 37.95 \quad 198.12$ end

cend

end

\section{Radial Neutron}

$=\mathrm{sas} 4$ 
Westinghouse Hanford Cask 3-D Shielding Analysis 7/96 $27 \mathrm{~N}-18$ couple infhommedium

' Fuel modeled, 6.34 MTU per MCO - SS304 basket - Zr clad uranium 1 den=6.28 $1.0 \quad 293 \quad 92235 \quad 1.0 \quad 92238 \quad 99.0$ end zircalloy 1 den $=0.441$ end ss304 1 den $=0.338$ end ss304 $2 \quad 1.0$ end

end comp

idr $=0$ ity=1 izm=4 frd=29.37 end $29.37 \quad 30.64 \quad 32.00 \quad 50.42$ end

1202 end

xend

tim $=30.0$ nst $=100$ nod $=3$ sfa $=1.09+7$ fr $2=0.7$ fr $3=0.7$ fr $4=0.7$

igo $=0 \quad \mathrm{mfu}=1 \quad \mathrm{nit}=100$ end

$\begin{array}{llllllll}\text { soe } & 0.01463 & 0.20251 & 0.28869 & 0.13389 & 0.14380 & 0.14175 & 0.07145\end{array}$

$20 \mathrm{z}$ end

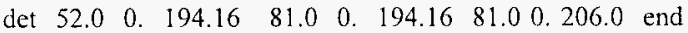

gend

TN-WHC Cask Radial Neutron Dose Rate around drain port with cover(HOLE)

fue $186.29 \quad 186.30$ end

fend

cav $0 \quad 32.00 \quad 190.77$ end

ins 230.64190 .76 end

inn 237.95190 .80 end

rs1 $2 \quad 48.51 \quad 190.20$ end

our $250.42 \quad 206.33$ end

as1 $2 \quad 37.95 \quad 198.12$ end

cend

end 


\section{B4.0 STRUCTURAL EVALUATION}

\section{B4. 1 INTRODUCTION}

This Section, including its appendices, presents the structural evaluation of the cask. This evaluation demonstrates that the cask structural design satisfies the Hanford Specification ${ }^{(1)}$.

\section{B4.2 STRUCTURAL EVALUATION OF PACKAGE}

\section{B4.2.1 Structural Design and Features}

The structural integrity of the cask under normal conditions of transport and hypothetical accident conditions specified in Hanford Specification is shown to meet the design criteria described in Sections B4.3 and B4.4. The cask is a transport packaging which consists of two major structural components: the shell or cask body assembly and the lid assembly. These components are described in Section A2.0 and are shown on drawings provided in Section A9.1, Appendix.

The shell or cask body cylinder assembly is an open ended (at the top) cylindrical unit with an integral closed bottom end. This assembly consists of a 7.31 inch thick ASME SA336 Type F304 stainless steel shell welded to a massive stainless steel bottom closure. The cask bottom is 6.13 in. thick.

The lid assembly is a 3.50 in. thick plate made of ASME SA336 Type F304 stainless steel. The lid is grooved to retain the closure seal. Two trunnions with brackets and gussets, welded to the lid, are used to lift the cask during handling. The lid, lid seal, vent and drain covers, their seals and bolts complete the packaging containment boundary. The lid assembly is bolted to the shell or body cylinder assembly with twelve (12) $1.5^{\prime \prime}$ high strength closure bolts.

The wall thickness of the outer shell, bottom plate and the lid enable the packaging to withstand the hypothetical puncture accident. The shell and lid are designed to be both strong and ductile in order to be capable of withstanding the punch loading.

Appendix B4.5A provides the impact analysis of the cask. Appendix B4.5B describes the lid bolt analysis. Appendix B4.5C presents the structural analysis of the cask body. Appendix B4.5D presents the stress analysis of the cask lifting attachment.

\section{B4.2.2 Mechanical Properties of Materials}


The mechanical properties of structural materials used in the cask is shown on Table B4.2-1 and the effects of temperature on these properties are also shown on Table B4.2-1. The materials are identified and procured by reference to ASME and corresponding ASTM specifications. The yield and ultimate strengths of the structural steels shown on Table B4.2-1 are the minimum values specified in the material specifications. The ASME design stress intensity values $\left(\mathrm{S}_{\mathrm{m}}\right)$ for Class 1 components are used to establish allowable stresses for the elastic analyses performed for the cask. Values of $\mathrm{S}_{\mathrm{m}}$ are provided on Table B4.2-1. Stress intensity limits for the various stress categories are discussed in Sections B4.3 and B4.4.

Table B4.2-1 Temperature Dependent Material Properties

\begin{tabular}{||c|c|c|c|c|c|c|c||}
\hline \hline Component & Material & $\begin{array}{c}\text { Temp. } \\
{ }^{\circ} \mathrm{F}\end{array}$ & $\begin{array}{c}\text { Ultimate } \\
\mathrm{S}_{\mathrm{u}}(\mathrm{ksi})\end{array}$ & $\begin{array}{c}\text { Yield } \\
\mathrm{S}_{\mathrm{y}}(\mathrm{ksi})\end{array}$ & $\begin{array}{c}\text { Allow. } \\
\mathrm{S}_{\mathrm{m}}(\mathrm{ksi})\end{array}$ & $\begin{array}{c}\mathrm{E} \\
\left(\mathrm{E}^{6} \mathrm{psi}\right)\end{array}$ & $\begin{array}{c}\alpha^{*} \\
\left(\mathrm{E}^{-6}\right)\end{array}$ \\
\hline $\begin{array}{c}\text { Cask Body } \\
\text { and } \\
\text { Lid }\end{array}$ & $\begin{array}{c}\text { SA-336 } \\
\text { Type F304 }\end{array}$ & 70 & 70 & 30 & 20 & 28.3 & 8.55 \\
\cline { 3 - 9 } & & 200 & 66.2 & 25 & 20 & 27.6 & 8.79 \\
\cline { 3 - 9 } & 300 & 61.5 & 22.5 & 20 & 27.0 & 9.0 \\
\cline { 3 - 9 } & 400 & 60.0 & 20.7 & 18.7 & 26.5 & 9.19 \\
\hline \multirow{2}{*}{ Lid Bolt } & $\begin{array}{c}\text { SA-479 } \\
\text { XM19 }\end{array}$ & 70 & 135 & 105 & & 28.3 & 8.87 \\
\cline { 3 - 9 } & Hot Rolled & 200 & & & & 27.6 & 9.02 \\
\cline { 3 - 9 } & & 300 & & & & 27.0 & 9.10 \\
\cline { 3 - 9 } & & 400 & & & & 26.5 & 9.14 \\
\hline
\end{tabular}

* Mean Coefficient of Thermal Expansion (in/in- $\left.{ }^{\circ} \mathrm{F}\right)$ From $70^{\circ} \mathrm{F}$ to the Indicated Temperature.

\section{B4.2.3 Chemical and Galvanic Reaction}

The materials of fabrication are summarized in section B4.2.2. All structural components are the same or similar alloys of stainless steel and therefore are not subject to chemical or galvanic interaction.

\section{B4.2.4 Size of Package and Cavity}


The basic structure of the cask is a right circular cylinder. The cask is 170.25 inches long and 39.81 inches in diameter except at the lid end where the diameter is 31.50 inches. The cask cavity has a length of 160.50 inches. The general arrangement of the cask is depicted in Figure B4.2-1. Component terminology used in this DAR is also identified on Figure B4.2-1. The closure lid end is referred to as the top with the packaging in the vertical orientation. Detailed design drawings for the cask are provided in Appendix A9.1. Table B4,2-2 summarizes the materials of construction used in the cask.

The basic components of the cask are the cask body, closure lid, and lid bolts. The cask body consists of the cylindrical shell assembly and bottom plate. The closure lid is attached to the cask body with twelve 1.5 inch diameter bolts. Two lifting trunnions with brackets and gussets are welded to the lid and are a $180^{\circ}$ apart. Two penetrations into the containment are provided to support cask operations. One is located in the lid and the other is located in the cask bottom. The maximum gross weight of the loaded cask is 57,910 pounds including a payload of 18,950 pounds. The cask is transported in the vertical orientation with the lid end facing the updirection. During transport, the cask is supported on the trailer by a tiedown system.

Table B4.2-2 Material of Construction

\begin{tabular}{|l|l|}
\hline \multicolumn{1}{|c|}{ Component } & \multicolumn{1}{c|}{ Material } \\
\hline Shell Assembly & SA-336, Type F304 \\
\hline Lid & SA-336, Type F304 \\
\hline Lid Bolt & SA-479 XM19 Hot Rolled \\
\hline Trunnion & SA-182, GR. F304 \\
\hline Brackets and Gussets & Type 304 SS \\
\hline Vent \& Drain Covers & SA-240 Type 304 \\
\hline Bolts for Vent \& Drain Covers & SA-193-B8 \\
\hline
\end{tabular}


HNF-SD-SNF-FDR-003 Rev. 0

Figure B4.2-1 General Arrangement of TN-WHC CASK

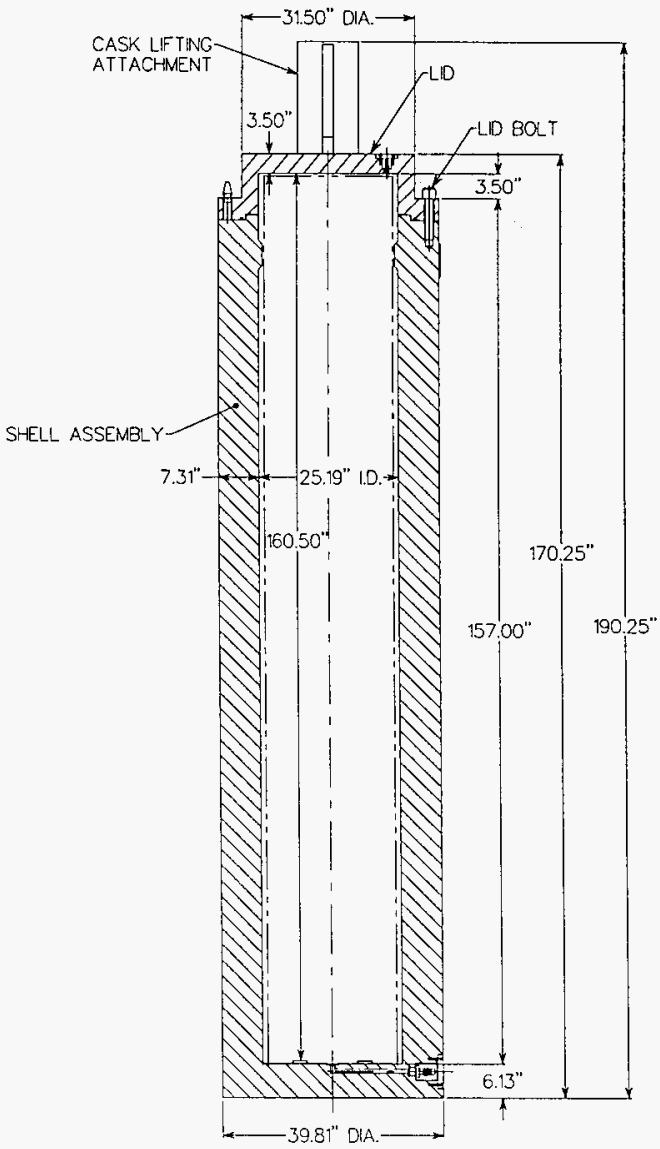




\section{B4.2.5 Weights and Center of Gravity}

The calculated gross weight of the cask in the transport configuration (including dry payload of $18,950 \mathrm{lbs}$ ) is 57,910 pounds. Approximate weights of major individual components or subassemblies are tabulated below:
Weight of Lid:
$1,890 \mathrm{Ibs}$
Weight of Shell:
$34,300 \mathrm{lbs}$
Weight of Bottom:
$2,270 \mathrm{lbs}$
Weight of Lifting Attachment:
$500 \mathrm{lbs}$
Weight of Dry MCO:
$18,950 \mathrm{lbs}$
Gross Package Weight (Dry): $\quad 57,910$ lbs

The center of gravity of the unloaded cask is located on the cylindrical axis at 82.29 inches from the outer bottom surface.

The center of gravity for a loaded cask (dry) is located on the cylindrical axis at approximately 83.6 inches from the outer bottom surface.

\section{B4.2.6 Tamper - Indicating Feature}

The tamper indicating feature is not required.

\section{B4.2.7 Positive Closure}

Positive containment closure is accomplished entirely by the bolted design. Twelve bolts are used to close the cask closure lid. Four bolts are used to close the drain port cover and the vent port cover. This extensive bolting configuration prevents unintentional opening of the containment system.

\section{B4.2.8 Cask Lifting Attachments and Tiedown System}

The detailed stress calculations of the cask lifting attachment and tiedown system are presented in Appendix $\mathrm{B} 4.5 \mathrm{D}$ and $\mathrm{B} 7.3$, respectively.

\section{B4.2.9 Brittle Fracture}

Brittle fracture of the cask body components is precluded by the choice of austenitic stainless 
steel for fabrication of the cask.

\section{B4.3 NORMAL TRANSPORT CONDITIONS}

This section describes the response of the cask to the loading conditions specified by the Hanford Specification. The design criteria established for the cask for the normal conditions of transport are described in Section 4.3.2. These criteria are selected to ensure that the package performance standards specified by the Hanford Specification is satisfied. Under normal conditions of transport there will be no loss or dispersal of radioactive contents, no significant increase in external radiation levels, and no substantial reduction in the effectiveness of the packaging.

Detailed structural analyses of various cask components subjected to individual loads are provided in the Appendices to this section. The limiting results from these analyses are used in this Section to quantify cask performance in response to the normal condition of transport load combinations selected for typical transport cask design. Table B4.3-1 provides an overview of the performance evaluations reported in each load combination subsection. Each subsection provides the limiting structural analysis result for the affected cask component(s) in comparison to the established design criteria. This comparison permits the minimum margin of safety for a given component subjected to a given loading condition to be readily identified. In all cases, the acceptability of the cask design with respect to established criteria and consequently with respect to the Hanford Specification standards is demonstrated.

The impact analysis results for the cask structure can be taken directly from the analysis in Appendix B4.5A. The structural analysis of the cask body is presented in Appendix B4.5C and covers a wide range of individual loading conditions. The stress results from the various individual loads must be combined in order to represent the stress condition in the cask body under the specified condition evaluated in this section. An explanation of the results reporting format and stress combination technique used to apply the results from Appendix $\mathrm{B} 4.5 \mathrm{C}$ is provided here.

\section{Reporting Method for Cask Body Stresses}

Appendix B4.5C provides the detailed description of the structural analyses of the cask body. That appendix describes the detailed ANSYS ${ }^{(3)}$ model used to analyze various applied loads. Table B4.3-2 identifies the individual loads (IL) analyzed which are applicable to normal conditions of transport. Some of these individual loads are axisymmetric (e.g. pressure) and others are asymmetric (e.g. free drop).

Figure B4.3-1 shows the selected locations on the cask body where stress results for these analyses are reported. Detailed stresses are actually available at as many locations as there are 


\section{HNF-SD-SNF-FDR-003 ReV. 0}

E-15166

nodes in the finite element model. However, for practical considerations, the reporting of stress results is limited to those locations shown on Figure B4.3-1. These locations were selected to be representative of the stress distribution in the cask body with special attention given to areas subject to high stresses. The maximum stress may occur at a different location for each individual load.

The stress results for the individual load case tables reported in Appendix $\mathrm{B} 4.5 \mathrm{C}$ are the maximum stresses for each individual load case. Two or more individual load cases must be combined to determine the total stresses at the standard stress reporting locations for the various load combinations. This is accomplished using the ANSYS postprocessor which algebraicly adds the stress components at each of the standard locations.

Several other items should be noted. In the cask, thermal stresses occur due to the effects of differential thermal expansion. When evaluating stresses, these thermal stresses are conservatively treated as primary stresses and the combined stresses due to primary loads (like pressure) and differential expansion (such as heating from $70^{\circ} \mathrm{F}$ to hot thermal conditions) are evaluated as primary stresses. In addition, some individual load cases in Appendix B4.5C were performed for the corresponding unit load $(1 \mathrm{G})$ condition (like the end drop and side drop) and are factored for the normal condition.

For the axisymmetric cases, the stress is constant around the circumference of the cask at each stress reporting location. For asymmetric analyses with significant differences in stress magnitudes on the extreme opposite sides of the cask (usually top and bottom for a horizontal cask) the stresses at locations on both sides are reported in separate tables.

Table B4.3-3 provides a matrix of the individual loads and how they are combined to determine the cask body stresses for the specified normal conditions of transport. The thermal stresses due to the hot and cold conditions are actually secondary stresses that could be evaluated using higher allowables than for primary stresses. They are conservatively added to the primary stresses and the combined stresses are evaluated using primary stress allowables. An " $x$ " in Table B4.3-3 indicates that the stress results for the individual load case are used directly. A quantitative number $(\mathrm{F})$ indicates the load factor applied to the individual stresses.

For the increased external pressure load combination, it is conservatively assumed that the cask cavity is at 0 psia. Since the specified load combination condition is 20 psia., the net differential pressure acting on the cask body is $20 \mathrm{psi}$. 
Table B4.3-1 Normal Conditions of Transport, TN-WHC Cask Performance Evaluation Overview

\begin{tabular}{|c|c|c|}
\hline $\begin{array}{l}\text { Loading } \\
\text { Conditions }\end{array}$ & $\begin{array}{l}\text { DAR } \\
\text { Section }\end{array}$ & Scope of Evaluation \\
\hline Thermal - Hot & $\mathrm{B} 4.3 .4 .2$ & $\begin{array}{l}\text { Cask Body Stresses due to Different Thermal } \\
\text { Expansion - Hot }\end{array}$ \\
\hline Thermal - Cold & B 4.3 .4 .2 & $\begin{array}{l}\text { Cask Body Stresses due to Different Thermal } \\
\text { Expansion - Cold }\end{array}$ \\
\hline $\begin{array}{l}\text { Maximum } \\
\text { Pressure }\end{array}$ & B 4.3.4.2 & Cask Body Stresses due to Maximum Internal pressure \\
\hline $\begin{array}{l}\text { Increased External } \\
\text { Pressure }\end{array}$ & B4.3.4.3.1 & Cask Body Stresses $($ External Pressure $=20$ psi) \\
\hline $\begin{array}{l}\text { Reduced External } \\
\text { Pressure }\end{array}$ & B 4.3 .4 .3 .2 & Cask Body Stresses (Internal Pressure $=161.2 \mathrm{psi}$ ) \\
\hline Vibration & B4.3.4.3.3 & $\begin{array}{l}\text { Cask Body Stresses for Vibration Normally Incident to } \\
\text { Transport }\end{array}$ \\
\hline Water Spray & $\mathrm{B} 4.3 .4 .3 .4$ & Negligible \\
\hline \multirow[t]{3}{*}{ Free Drop } & B4.3.4.3.5 & $\begin{array}{l}\text { Cask Body Stresses for Bottom End and Lid End } \\
\text { Drops }\end{array}$ \\
\hline & $\mathrm{B} 4.3 .4 .3 .5$ & Cask Body Stresses for Side Drop \\
\hline & $\mathrm{B} 4.3 .4 .3 .5$ & $\begin{array}{l}\text { Cask Body Stresses for C.G. Over Lid End Corner } \\
\text { Drop }\end{array}$ \\
\hline Penetration & $\mathrm{B} 4.3 .4 .3 .6$ & Negligible \\
\hline Fatigue Analysis & B4.3.4.3.7 & Fatigue Analysis Under Normal Transport Condition \\
\hline Lid Bolt Analysis & B4.3.4.3.8 & Lid Bolt Stresses - Lid End Corner Impact \\
\hline Conclusions & B4.3.4.3.9 & Summary of the Results \\
\hline
\end{tabular}


HNF-SD-SNF-FDR-003 ReV. 0

E-15166

Table B4.3-2 Normal Conditions of Transport, Individual Load Cases for Cask Body Analysis

\begin{tabular}{|c|l|c|}
\hline Load Case & \multicolumn{1}{|c|}{ Individual load Description } & $\begin{array}{c}\text { Stress Results } \\
\text { Table }\end{array}$ \\
\hline IL-1 & Bolt Preload & B4.5C-3 \\
\hline IL-2 & Thermal Stress at Hot Environment & B4.5C-11 \\
\hline IL-3 & Thermal Stress at Cold Environment & B4.5C-12 \\
\hline IL-4 & Internal Pressure & B4.5C-4 \\
\hline IL-5 & External Pressure & B4.5C-5 \\
\hline IL-6 & $\begin{array}{l}\text { 1G Down Cask Supported by Tie-Down } \\
\text { System }\end{array}$ & B4.5C-7 \\
\hline IL-7 & $\begin{array}{l}\text { Vibration Load Cask Supported by } \\
\text { Tie-Down System }\end{array}$ & B4.5C-6 \\
\hline IL-8 & One Foot End Drop on Bottom End (1G) & B4.5C-7 \\
\hline IL-9 & One Foot End Drop on Lid End (1G) & B4.5C-8 \\
\hline IL-10 & One Foot Side Drop (1G) & B4.5C-9 \\
\hline IL-11 & $\begin{array}{l}\text { One Foot C.G. Over Lid End Corner Drop } \\
\text { (1G) }\end{array}$ & B4.5C-10 \\
\hline \hline
\end{tabular}


HNF-SD-SNF-FDR-003 Rev. 0

E-15166

Table B4.3-3 Summary of Load Combinations for Normal Conditions of Transport

\begin{tabular}{|c|c|c|c|c|c|c|c|c|c|c|c|c|}
\hline \multirow[t]{2}{*}{ Load Combinations } & \multicolumn{11}{|c|}{ Applicable Individual Load } & \multirow{2}{*}{$\begin{array}{l}\text { Stress Result } \\
\text { Table No. }\end{array}$} \\
\hline & $\begin{array}{c}\text { IL- } \\
1\end{array}$ & $\begin{array}{c}\text { IL- } \\
2\end{array}$ & $\begin{array}{c}\text { IL- } \\
3\end{array}$ & $\begin{array}{l}\text { IL- } \\
4\end{array}$ & $\begin{array}{c}\text { IL- } \\
5\end{array}$ & $\begin{array}{l}\text { IL- } \\
6\end{array}$ & $\begin{array}{l}\mathrm{IL}- \\
7\end{array}$ & $\begin{array}{l}\text { IL- } \\
8\end{array}$ & $\begin{array}{c}\text { IL- } \\
9\end{array}$ & $\begin{array}{l}\text { IL- } \\
10\end{array}$ & $\begin{array}{c}\text { IL- } \\
11\end{array}$ & \\
\hline Hot Environment & $\mathrm{x}$ & $\mathrm{x}$ & & $\mathrm{x}$ & & $\mathrm{x}$ & & & & & & B4.3-5 \\
\hline Cold Environment & $x$ & & $x$ & & $\mathrm{x}$ & $x$ & & & & & & $\mathrm{~B} 4.3-6$ \\
\hline \multirow{2}{*}{$\begin{array}{l}\text { Transport } \\
\text { Vibrations }\end{array}$} & $\mathrm{x}$ & $\mathrm{x}$ & & $\mathrm{x}$ & & & $\mathrm{x}$ & & & & & B4.3-7 \\
\hline & $\mathrm{x}$ & & $x$ & & $x$ & & $\mathrm{x}$ & & & & & B4.3-8 \\
\hline \multirow[t]{2}{*}{$\begin{array}{l}1 \text { Foot Bottom End } \\
\text { Drop }(25 \mathrm{G})\end{array}$} & $\mathrm{x}$ & $\mathrm{x}$ & & $\mathrm{x}$ & & & & $\begin{array}{c}\mathrm{X} \\
\mathrm{F}= \\
25\end{array}$ & & & & B4.3-9 \\
\hline & $\mathrm{x}$ & & $\mathrm{x}$ & & $\mathrm{x}$ & & & $\begin{array}{l}x \\
F= \\
25\end{array}$ & & & & B $4.3-10$ \\
\hline \multirow[t]{2}{*}{$\begin{array}{l}1 \text { Foot Lid End Drop } \\
\qquad(25 \mathrm{G})\end{array}$} & $x$ & $x$ & & $\mathrm{x}$ & & & & & $\begin{array}{l}x \\
F= \\
25\end{array}$ & & & B4.3-11 \\
\hline & $\mathrm{x}$ & & $x$ & & $\mathrm{x}$ & & & & $\begin{array}{l}x \\
F= \\
25\end{array}$ & & & B4.3-12 \\
\hline \multirow[t]{2}{*}{$\begin{array}{l}\text { I Foot Side Drop } \\
\text { (24G) }\end{array}$} & $x$ & $\mathrm{x}$ & & $\mathrm{x}$ & & & & & & $\begin{array}{l}x \\
F= \\
24\end{array}$ & & $\begin{array}{l}\text { B4.3-13 } \\
\text { B4.3-14 }\end{array}$ \\
\hline & $x$ & & $\mathrm{x}$ & & $\mathrm{x}$ & & & & & $\begin{array}{l}x \\
F= \\
24\end{array}$ & & $\begin{array}{l}\text { B } 4.3-15 \\
\text { B } 4.3-16\end{array}$ \\
\hline \multirow[t]{2}{*}{$\begin{array}{l}1 \text { Foot Lid Corner } \\
\text { Drop (18G) }\end{array}$} & $\mathrm{x}$ & $x$ & & $x$ & & & & & & & $\begin{array}{l}x \\
F= \\
18\end{array}$ & $\begin{array}{l}\text { B4.3-17 } \\
\text { B4-3-18 }\end{array}$ \\
\hline & $x$ & & $\mathrm{x}$ & & $x$ & & & & & & $\begin{array}{l}x \\
F= \\
18\end{array}$ & $\begin{array}{l}\text { B4.3-19 } \\
\text { B4.3-20 }\end{array}$ \\
\hline
\end{tabular}




\section{B4.3.1 Conditions To Be Evaluated}

The cask will be designed to withstand each of the normal transport conditions specified in Hanford Specification as listed below:

\section{B4.3.1.1 Environmental Conditions}

The design temperature limits for the individual components, parts, and materials of the package will be determined by analyses. The analyses will be based upon the conditions listed below. The operational temperatures will be shown to not exceed the design limits.

a) Maximum heat generation rate of worst-case source (for normal conditions) plus maximum solar heat load plus maximum air temperature of $115^{\circ} \mathrm{F}$.

b) Minimum air temperature of $-27^{\circ} \mathrm{F}$ and zero heat generation rate.

\section{B4.3.1.2 Reduced External Pressure}

An external pressure of 3.5 psi absolute.

\section{B4.3.1.3 Increased External Pressure}

An external pressure of $20 \mathrm{psi}$ absolute.

\section{B4.3.1.4 Internal Pressure}

An internal working pressure of 161.2 psi will be used for stress calculations.

\section{B4.3.1.5 Vibration}

The casks will be evaluated to confirm containment integrity when exposed to normal vibration due to the transportation from the $100 \mathrm{~K}$ West and East basins to the CSB in the 200 East Area by the transport vehicle. The cask is heavy and rigid and attached directly to the trailer bed. Trailer bed shock and vibration levels are applied directly to the package. The shock and vibration design limits by truck/trailer are those stated in Hanford Specification. 


\section{B4.3.1.6 Water Spray}

The cask will be evaluated to confirm containment integrity through a water spray that simulates exposure to rainfall of approximately 2 in. $(5 \mathrm{~cm})$ per hour for at least one hour.

\section{B4.3.1.7 Free Drop}

The package will be evaluated to demonstrate containment after to a $1-\mathrm{ft}(0.3-\mathrm{m})$ free drop onto a horizontal, yielding surface. During the free drop, the MCO will not be exposed to greater than $100 \mathrm{~g}$ 's.

\section{B4.3.1.8 Penetration}

Impact of the hemispherical end of a vertical steel cylinder of 1.25 in. $(3.2 \mathrm{~cm})$ diameter and 13 $\mathrm{lb}(6.00 \mathrm{~kg})$ mass, dropped from a height of 40.00 in $(1.00 \mathrm{~m})$ onto the exposed surface of the package which is expected to be most vulnerable to puncture. The long axis of the cylinder must be perpendicular to the package surface.

\section{B4.3.2 Acceptance Criteria}

The packaging consists of two major components:

- $\quad$ Cask Body Cylinder Assembly

- Lid Assembly

The structural design criteria for these components are described below.

\section{Containment Boundary}

The containment boundary consists of the cask body cylindrical shell, bottom plate and closure flange out to the seal seating surface and the lid. The lid bolts and seals are also part of the containment boundary as is the drain and vent cover plates, bolts and seals. The containment boundary is designed to the maximum practical extent as an ASME Class I component in accordance with the rules of the ASME ${ }^{(4)}$ Boiler and Pressure Vessel Code, Section III, Subsection NB (1992 Edition). The Subsection NB guidelines for materials, design, fabrication and examination are applied to the above components as required by the Specification. 
The acceptability of the containment boundary under the applied loads is based on the following criteria:

- $\quad$ ASME Code Design Stress Intensities

- Fatigue Failure to be Precluded

- Brittle Fracture to be Precluded

- Buckling to be Prevented

The values for material properties, design stress intensities $\left(\mathrm{S}_{\mathrm{m}}\right)$ and design fatigue curves for Class 1 components given in Part D of Section II of the ASME B\&PV Code will be used for the containment boundary materials. Allowable stress levels for containment components are outlined in Table B4.3-4 of this Section.

The design properties of other materials will be based on industry-recognized specifications, or standards, or on appropriate test data.

\section{Bolting}

In the special case of bolting, the average bolt stress is limited to $2 / 3 \mathrm{~S}_{\mathrm{y}}$ and the maximum combined stress is limited to $0.9 \mathrm{~S}_{\mathrm{y}}$ for normal condition of transport.

\section{Brittle Fracture}

The containment vessel is entirely austenitic stainless steel (Type 304) which is ductile even at low temperature. Thus, brittle fracture is precluded. 
HNF-SD-SNF-FDR-003 Rev, 0

E-15166

Table B4.3-4 Containment Structure/Fastener Allowable Stress

\begin{tabular}{|c|c|c|}
\hline \multirow[t]{2}{*}{ STRESS CATEGORY } & \multicolumn{2}{|c|}{$\begin{array}{c}\text { CONTAINMENT STRUCTURE ALLOWABLE } \\
\text { STRESS } \\
\end{array}$} \\
\hline & Normal Conditions & Accident Conditions \\
\hline $\begin{array}{c}\text { Primary Membrane } \\
\text { General } \mathrm{P}_{\mathrm{m}} \\
\text { Local } \mathrm{P}_{\mathrm{L}} \\
\end{array}$ & $\begin{array}{l}\mathrm{S}_{\mathrm{m}} \\
1.5 \mathrm{~S}_{\mathrm{m}}\end{array}$ & $\begin{array}{c}\text { Lesser of : } \\
2.4 \mathrm{~S}_{\mathrm{m}} \text { or } 0.7 \mathrm{~S}_{\mathrm{u}}(1) \\
3.6 \mathrm{~S}_{\mathrm{m}} \text { or } \mathrm{S}_{\mathrm{u}}(1)\end{array}$ \\
\hline $\begin{array}{l}\text { Primary Membrane }+ \text { Bending } \\
\left(\mathrm{P}_{\mathrm{m}} \text { or } \mathrm{P}_{\mathrm{L}}\right)+\mathrm{P}_{\mathrm{b}}\end{array}$ & $1.5 \mathrm{~S}_{\mathrm{m}}$ & $\begin{array}{l}\text { Lesser of : } \\
\text { 3.6 } \mathrm{S}_{\mathrm{m}} \text { or } \mathrm{S}_{\mathrm{u}}(1)\end{array}$ \\
\hline $\begin{array}{l}\text { Range of Primary }+ \text { Secondary } \\
\left(\mathrm{P}_{\mathrm{m}} \text { or } \mathrm{P}_{\mathrm{L}}\right)+\mathrm{P}_{\mathrm{b}}+\mathrm{Q} \\
\end{array}$ & $3.0 \mathrm{~S}_{\mathrm{m}}$ & $\begin{array}{l}2 \times S_{a} \text { for } 10 \text { Cycles } \\
\text { (Code Sect. III, App. I) }\end{array}$ \\
\hline Bearing Stress & $\mathrm{S}_{\mathrm{y}}$ & $\begin{array}{l}\mathrm{S}_{\mathrm{y}} \text { for Seal Surface } \\
\mathrm{S}_{\mathrm{u}} \text { Elsewhere }\end{array}$ \\
\hline Pure Shear Stress & $0.6 \mathrm{~S}_{\mathrm{m}}$ & $0.42 \mathrm{~S}_{u}$ \\
\hline Fatigue & $\begin{array}{l}\text { Cumulative Fatigue } \\
\text { Usage Factor } \leq 1\end{array}$ & Not Applicable \\
\hline
\end{tabular}

\begin{tabular}{|c|c|c||}
\hline \multirow{2}{*}{ STRESS CATEGORY } & \multicolumn{2}{|c|}{ CONTAINMENT FASTENER ALLOWABLE } \\
& STRESS \\
\cline { 2 - 3 } & $2 / 3 \mathrm{~S}_{\mathrm{y}}$ & $\begin{array}{c}\text { Lesser of: } \\
0.7 \mathrm{~S}_{\mathrm{u}} \text { or } \mathrm{S}_{\mathrm{y}}\end{array}$ \\
\hline Average Tensile Stress & $0.4 \mathrm{~S}_{\mathrm{y}}$ & $\begin{array}{c}\text { Lesser of: } \\
0.42 \mathrm{~S}_{\mathrm{u}} \text { or } 0.6 \mathrm{~S}_{\mathrm{y}}\end{array}$ \\
\hline Average Shear Stress & $0.9 \mathrm{~S}_{\mathrm{y}}$ & $\mathrm{S}_{\mathrm{u}}$ \\
\hline Maximum Combined Stress & \\
\hline
\end{tabular}

(1) When evaluating the results from the nonlinear elastic plastic analysis for the accident conditions, the general primary membrane stress intensity, $P_{m}$. shall not exceed $0.7 \mathrm{~S}_{v}$ and the maximum primary stress intensity at any location $\left(\mathrm{P}_{\mathrm{L}}\right.$ or $\left.\mathrm{P}_{\mathrm{L}}+\mathrm{P}_{b}\right)$ shall not exceed 0.9 $\mathrm{S}_{\mathrm{u}}$. These limits are in accordance with Appendix F of Section III of the Code. 


\section{B4.3.3 Structural Model}

The cask body consists of the cylindrical shell, the bottom, the lid and the lid bolts. The elements used to model the bolts are ANSYS STIF3, beam elements. The cylindrical shell, the bottom end closure and the lid are modeled using either ANSYS STIF25 axisymmetric solid elements or ANSYS STIF42 axisymmetric solid elements. The loading applied to this type of elements may be either axisymmetric for some cases and asymmetric for other cases. The model geometry is based on Drawings H-1-81535, sheets 1 to 5 . The contact surface at the lid and cylindrical shell is modeled using separate nodes in the interfacing components. These nodes are coupled or left uncoupled for specific constraint conditions as discussed below. All the analyses were performed using the model shown in Figures B4.5C-1 and B4.5C-2. The mechanical properties for the materials in this model are the linear values described in Section B4.2.2.

\section{B4.3.4 Initial Conditions}

\section{B4.3.4.1 Environmental Heat Loading}

Section B5.0 describes the thermal analyses performed for the cask subjected to hot environment conditions. These thermal analysis results are used to support various aspects of the structural evaluations as described in the following .

\section{Maximum Temperature}

Stress allowables for packaging components are a function of component temperature. Stress allowables are based on actual maximum calculated temperatures or conservatively selected higher temperatures. Section B5.0 summarizes significant temperatures calculated for the cask subjected to hot environment conditions. These temperatures are used to establish the allowables for every normal accident (except the thermal accident which has higher temperatures) load combination evaluated in this DAR.

\section{Maximum Pressure}

For purposes of the structural analysis of containment, a value of 150 psig is used for MNOP. The structural analysis performed assuming the same outward pressure different across the cask wall as during the reduced external pressure case, $(150+14.7-3.5) 161.2$ psi. 


\section{B4.3.4.2 Maximum Thermal and Pressure Stresses}

\section{Maximum Thermal Stress - Hot Environment}

The thermal analysis of the cask is performed as described in Section B5.0. The temperature distribution from that analysis is used to perform an ANSYS structural analysis of the cask body thermal stresses.

Cask body stresses for the hot environment normal condition of transport are obtained by a combination of individual loads as summarized in Table B4.3-3. For this condition, it is assumed that the cask is in its normal transport configuration, mounted vertically on the trailer, and support by the tie-down system. Lid bolt preload effects and the dead weight loading are included.

Table B4.3-5 lists the total nodal stress intensity at each of the standard stress reporting locations. It may be noted that these stress intensities are the peak nodal stress intensities, the membrane and membrane plus bending stress intensities at the same locations are less than the peak nodal stress intensities. The maximum stress intensity calculated for this load combination is $1440 \mathrm{psi}$ which occurs at node number 94 . This stress intensity is well below the allowable membrane stress intensity of $20,000 \mathrm{psi}$, and membrane plus bending stress intensity of $30,000 \mathrm{psi}$ (at $150^{\circ} \mathrm{F}$ temperature).

\section{Maximum Thermal Stress - Cold Environment}

Containment vessel thermal stresses do occur in the cold environment due to the differential thermal expansion. The thermal stresses are determined in load case IL- 3 with results tabulated in Table B4.5C-12. The cask cavity pressure at the cold environment condition is conservatively assumed to be 0 psia. This results in a net external pressure loading of $20 \mathrm{psi}$, load case IL-5 with results in Table B4.5C-5. Again, lid bolt preload and gravity load are included for cold environment load combinations.

Brittle fracture of the cask body components is precluded by the choice of austenitic stainless steel for fabrication of the cask.

Table B4.3-6 lists the total nodal stress intensity at each of the standard stress reporting locations. It may be noted that these stress intensities are the peak nodal stress intensities, the membrane and membrane plus bending stress intensities at the same locations are less than the peak nodal stress intensities. The maximum stress intensity calculated for this load combination is $369 \mathrm{psi}$ which occurs at node number 34 . This stress intensity is well below the allowable membrane stress intensity of 20,000 psi. and membrane plus bending stress intensity of 30,000 psi. 


\section{Maximum Pressure Stresses}

For purposes of the structural analysis of containment, a value of $161.2 \mathrm{psi}$ is used. This pressure loading is analyzed using the ANSYS model of the cask body as described in Appendix B4.5C and the results are reported in Table B4.5C-4 of that Appendix. This load case and corresponding results are designated as individual load IL-4. IL-4 is used to support various load combination evaluations as listed in Table B4.3-3.

\section{B4.3.4.3 Structural Evaluations and Conclusions}

\section{B4.3.4.3.1 Increased external pressure}

Cask body stresses for the increased external pressure, 20 psia, normal condition of transport are obtained by a combination of individual loads as summarized in Table B4.3-3 (same as cold environment). The conservatively assumed minimum cask cavity pressure of 0 psia results in a net external pressure loading of 20 psi.

This load case is combined with stresses due to bolt preload, gravity and cold thermal stresses. Table B4.3-6 lists the total nodal stress intensity at each of the standard stress reporting locations. It may be noted that these stress intensities are the peak nodal stress intensities, the membrane and membrane plus bending stress intensities at the same locations are less than the peak nodal stress intensities. The maximum stress intensity calculated for this load combination is $369 \mathrm{psi}$ which occurs at node number 34 . This stress intensity is well below the allowable membrane stress intensity of 20,000 psi, and membrane plus bending stress intensity of 30,000 psi.

\section{B4.3.4.3.2 Reduced External Pressure}

Cask body stresses for the 3.5 psia ambient normal condition of transport are obtained by a combination of individual loads as summarized in Table B4.3-3 (same as hot environment). The conservatively assumed MNOP of 150 psig results in a net internal pressure loading of $(150+14.7-3.5) 161.2 \mathrm{psi}$. The lid bolt preload and gravity load are included. The thermal stress for the hot thermal condition are included in the load combination.

Table B4.3-5 lists the total nodal stress intensity at each of the standard stress reporting locations. It may be noted that these stress intensities are the peak nodal stress intensities, the membrane and membrane plus bending stress intensities at the same locations are less than the peak nodal stress intensities. The maximum stress intensity calculated for this load combination is $1440 \mathrm{psi}$ which occurs at node number 94 . This stress intensity is well below the allowable membrane stress intensity of $20,000 \mathrm{psi}$, and membrane plus bending stress intensity of $30,000 \mathrm{psi}$. 


\section{B4.3.4.3.3 Vibration}

The input loading conditions used to evaluate the cask for transport vibration are obtained from truck bed accelerations in ANSI N14.23 $3^{(5)}$. The peak inertia values used are $0.30 \mathrm{G}, 0.30 \mathrm{G}$, and $0.60 \mathrm{G}$ for the longitudinal, lateral and vertical directions, respectively. The stress due to the transport vibration load case are presented in Table B4.5C-6.

The combined stress results for this load case are provided in Tables B4.3-7 and B4.3-8. The highest nodal stress intensity is $1415 \mathrm{psi}$ which occurs at node number 94 . This stress intensity is well below the allowable membrane stress intensity of $20,000 \mathrm{psi}$, and membrane plus bending stress intensity of 30,000 psi.

\section{B4.3.4.3.4 Water Spray}

All exterior surfaces of the cask are metal and therefore not subject to soaking or structural degradation from water absorption. The water spray condition is therefore of no consequence to the cask.

\section{B4.3.4.3.5 Free Drop}

Four drop orientations are considered credible for the normal condition of transport one-foot free drop. The structural response of the cask body is evaluated for a one-foot end drop on the bottom end, lid end, one-foot corner drop on lid end and a one-foot side drop. The nodal stress intensities calculated from the finite element model analysis (Appendix B4.5C) for the above loading conditions are reported in Tables B4.5C-7, 8, 9, and 10 .

The load combinations performed to evaluate these drop events are indicated in Table B4.3-3. In all case, bolt preload effects are included. For the hot environment condition, the thermal stress load case for that temperature, the 161.2 psi pressure load case and the impact load case factored for the normal condition $G$ level are combined. For the cold temperature evaluation, the cold thermal stress case, the $20 \mathrm{psi}$ external pressure case, and the impact load case factored for the normal condition $\mathrm{G}$ level are combined.

Table B4.3-9 lists the total nodal stress intensity at each of the standard stress reporting locations for the bottom end drop under hot environment conditions. The maximum stress intensity calculated for this load combination is 2436 psi which occurs at node number 214 . This stress intensity is well below the allowable membrane stress intensity of 20,000 psi. and membrane plus bending stress intensity of 30,000 psi. 
Table B4.3-10 lists the total nodal stress intensity at each of the standard stress reporting locations for the bottom end drop under cold environment conditions. The maximum stress intensity calculated for this load combination is 1228 psi which occurs at node number 214 . This stress intensity is well below the allowable membrane stress intensity of 20,000 psi, and membrane plus bending stress intensity of 30,000 psi.

Table B4.3-11 lists the total nodal stress intensity at each of the standard stress reporting locations for the lid end drop under hot environment conditions. The maximum stress intensity calculated for this load combination is 3361 psi which occurs at node number 45 . This stress intensity is well below the allowable membrane stress intensity of $20,000 \mathrm{psi}$, and membrane plus bending stress intensity of 30,000 psi.

Table B4.3-12 lists the total nodal stress intensity at each of the standard stress reporting locations for the lid end drop under cold environment conditions. The maximum stress intensity calculated for this load combination is $3958 \mathrm{psi}$ which occurs at node number 51. This stress intensity is well below the allowable membrane stress intensity of $20,000 \mathrm{psi}$, and membrane plus bending stress intensity of 30,000 psi.

Table B4.3-13 lists the total nodal stress intensity at each of the standard stress reporting locations for the side drop under hot environment conditions (contact side). The maximum stress intensity calculated for this load combination is 3408 psi which occurs at node number 157 . This stress intensity is well below the allowable membrane stress intensity of $20,000 \mathrm{psi}$, and membrane plus bending stress intensity of 30,000 psi.

Table B4.3-14 lists the total nodal stress intensity at each of the standard stress reporting locations for the side drop under hot environment conditions (opposite contact side). The maximum stress intensity calculated for this load combination is $1691 \mathrm{psi}$ which occurs at node number 94 . This stress intensity is well below the allowable membrane stress intensity of $20,000 \mathrm{psi}$, and membrane plus bending stress intensity of 30,000 psi.

Table B4.3-15 lists the total nodal stress intensity at each of the standard stress reporting locations for the side drop under cold environment conditions (contact side). The maximum stress intensity calculated for this load combination is 3025 psi which occurs at node number 427 . This stress intensity is well below the allowable membrane stress intensity of 20,000 psi, and membrane plus bending stress intensity of $30,000 \mathrm{psi}$.

Table B4.3-16 lists the total nodal stress intensity at each of the standard stress reporting locations for the side drop under cold environment conditions (opposite contact side). The maximum stress intensity calculated for this load combination is 1551 psi which occurs at node number 157 . This stress intensity is well below the allowable membrane stress intensity of $20,000 \mathrm{psi}$, and 
membrane plus bending stress intensity of 30,000 psi.

Table B4.3-17 lists the total nodal stress intensity at each of the standard stress reporting locations for the lid end corner drop under hot environment conditions (contact side). The maximum stress intensity calculated for this load combination is 12,252 psi which occurs at node number 51. This stress intensity is well below the allowable membrane stress intensity of 20,000 psi, and membrane plus bending stress intensity of 30,000 psi.

Table B4.3-18 lists the total nodal stress intensity at each of the standard stress reporting locations for the lid end corner drop under hot environment conditions (opposite contact side). The maximum stress intensity calculated for this load combination is $10,111 \mathrm{psi}$ which occurs at node number 74 . This stress intensity is well below the allowable membrane stress intensity of 20,000 psi, and membrane plus bending stress intensity of 30,000 psi.

Table B4.3-19 lists the total nodal stress intensity at each of the standard stress reporting locations for the lid end corner drop under cold environment conditions (contact side). The maximum stress intensity calculated for this load combination is 13,796 psi which occurs at node number 51. This stress intensity is well below the allowable membrane stress intensity of $20,000 \mathrm{psi}$, and membrane plus bending stress intensity of $30,000 \mathrm{psi}$.

Table B4.3-20 lists the total nodal stress intensity at each of the standard stress reporting locations for the lid end corner drop under hot environment conditions (opposite contact side). The maximum stress intensity calculated for this load combination is 11,263 psi which occurs at node number 74. This stress intensity is well below the allowable membrane stress intensity of 20,000 psi, and membrane plus bending stress intensity of 30,000 psi.

\section{B4.3.4.3.6 Penetration}

Due to lack of sensitive external protuberances, the one meter (40 in.) drop of a 13 pound hemispherical-headed, 1-1/4 inch diameter, steel cylinder is of negligible consequence to the cask.

\section{B4.3.4.3.7 Fatigue Analysis}

The purpose of the fatigue analysis is to show quantitatively that the containment vessel stresses are within acceptable limits under normal transport conditions. The highest cyclical loading is the vibration loading. The highest nodal stress intensity under vibration loading in the containment boundary is \pm 138 psi (Table B4.5C-6, node number 133).

If we apply a stress concentration factor of 4 for the structural discontinuities, the total peak stress intensity range is \pm 552 . As shown in Figure I-9.2.2 of the ASME Code Section III, Appendix 1, austenitic steel can withstand an alternating stress of $23,700 \mathrm{psi}$ for $10^{11}$ cycles. 
Therefore the containment vessel alternating stress of $552 \mathrm{psi}$ is well below the range where fatigue failure can occur.

A separate fatigue analysis of the lid bolts is presented in Appendix B4.5B.

\section{B4.3.4.3.8 Lid Bolt Analysis}

The lid bolts are analyzed for normal condition loadings in Appendix B4.5B. The analysis is based on NUREG/CR- $6007^{(6)}$. The bolts are analyzed for the following normal conditions: operating preload, internal pressure, external pressure, temperature changes, impact loads and vibration loads.

The worst combined loading is due to a one foot end drop and 161.2 psi internal pressure. This results in a maximum stress intensity of $67,330 \mathrm{psi}$, which is below the allowable stress of 90,900 psi $\left(0.9 \mathrm{~S}_{\mathrm{y}}\right)$ at $150^{\circ} \mathrm{F}$ temperature. The fatigue analysis of the lid bolt is presented in Appendix $\mathrm{B} 4.5 \mathrm{~B}$. The analysis shows that based on 400 round trip shipments of the cask, the total usage factor is less than one.

\section{B4.3.4.3.9 Conclusion}

From the analyses presented in Section B4.3, it can be shown that the normal loads will not result in any structural damage of the cask and the containment function of the $\mathrm{MCO}$ will be maintained. 
HNF-SD-SNF-FDR-003 Rev. 0

Figure B4.3-1 Standard Stress Reporting Locations

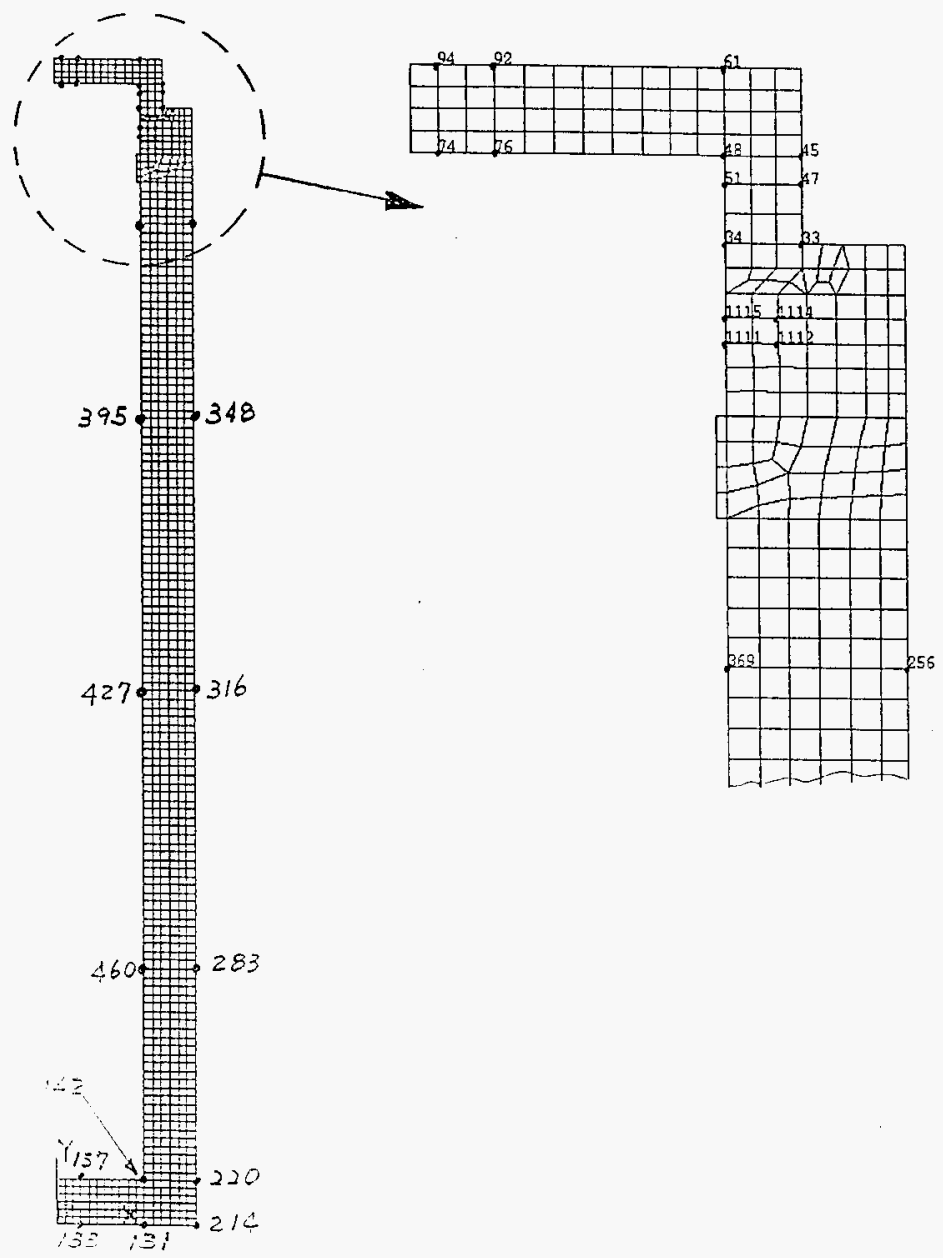


Table B4.3-5 Cask Body Stresses Under Hot Environment

(Bolt Preload, Hot Thermal,161.2 psi Internal Pressure, 1 G Gravity)

\begin{tabular}{|c|c|c|}
\hline \multicolumn{2}{|c|}{ Location } & \multirow{2}{*}{$\begin{array}{l}\text { Nodal Stress Intensity } \\
\text { (psi) }\end{array}$} \\
\hline Cask Body Component & Node Number" & \\
\hline \multirow[t]{6}{*}{ Cask Bottom } & 133 & 676 \\
\hline & 131 & 840 \\
\hline & 214 & 1300 \\
\hline & 157 & 1077 \\
\hline & 142 & 489 \\
\hline & 220 & 849 \\
\hline \multirow[t]{8}{*}{ Cask Cylinder } & 460 & 445 \\
\hline & 427 & 422 \\
\hline & 395 & 550 \\
\hline & 369 & 904 \\
\hline & 283 & 715 \\
\hline & 316 & 712 \\
\hline & 348 & 813 \\
\hline & 256 & 1004 \\
\hline \multirow[t]{4}{*}{ Cask Flange } & 1111 & 212 \\
\hline & 1112 & 503 \\
\hline & 1115 & 309 \\
\hline & 1114 & 724 \\
\hline \multirow[t]{11}{*}{ Lid } & 34 & 596 \\
\hline & 33 & 650 \\
\hline & 51 & 1296 \\
\hline & 47 & 401 \\
\hline & 74 & 975 \\
\hline & 76 & 722 \\
\hline & 48 & 911 \\
\hline & 45 & 449 \\
\hline & 94 & 1440 \\
\hline & 92 & 1288 \\
\hline & 61 & 1264 \\
\hline
\end{tabular}

* See Figure B4.3-1 for node locations. 
Table B4.3-6 Cask Body Stresses Under Cold Environment (Bolt Preload, Cold Thermal, 20 psi External Pressure, 16 Gravity)

\begin{tabular}{|c|c|c|}
\hline \multicolumn{2}{|c|}{ Location } & \multirow{2}{*}{$\begin{array}{c}\text { Nodal Stress Intensity } \\
\text { (psi) }\end{array}$} \\
\hline Cask Body Component & Node Number & \\
\hline \multirow[t]{6}{*}{ Cask Bottom } & 133 & 30 \\
\hline & 131 & 67 \\
\hline & 214 & 49 \\
\hline & 157 & 66 \\
\hline & 142 & 87 \\
\hline & 220 & 33. \\
\hline \multirow{8}{*}{ Cask Cylinder } & 460 & 70 \\
\hline & 427 & 66 \\
\hline & 395 & 67 \\
\hline & 369 & 51 \\
\hline & 283 & 51 \\
\hline & 316 & 39 \\
\hline & 348 & 28 \\
\hline & 256 & 14 \\
\hline \multirow[t]{4}{*}{ Cask Flange } & 1111 & 53 \\
\hline & 1112 & 51 \\
\hline & 1115 & 29 \\
\hline & 1114 & 241 \\
\hline \multirow[t]{11}{*}{ Lid } & 34 & 369 \\
\hline & 33 & 328 \\
\hline & 51 & 367 \\
\hline & 47 & 162 \\
\hline & 74 & 237 \\
\hline & .76 & 220 \\
\hline & 48 & 206 \\
\hline & 45 & 148 \\
\hline & 94 & 245 \\
\hline & 92 & 228 \\
\hline & 61 & 120 \\
\hline
\end{tabular}

* See Figure B4.3-1 for node locations. 
Table B4.3-7 Cask Body Stresses Under Hot Environment Vibrations

(Bolt Preload, Hot Thermal, 161.2 psi Internal Pressure, Vibrations)

\begin{tabular}{|c|c|c|}
\hline \multicolumn{2}{|c|}{ Location } & \multirow{2}{*}{$\begin{array}{c}\text { Nodal Stress Intensity } \\
\text { (psi) }\end{array}$} \\
\hline Cask Body Component & Node Number" & \\
\hline \multirow[t]{6}{*}{ Cask Bottom } & 133 & 678 \\
\hline & 131 & 853 \\
\hline & 214 & 1279 \\
\hline & 157 & 1206 \\
\hline & 142 & 483 \\
\hline & 220 & 863 \\
\hline \multirow[t]{8}{*}{ Cask Cylinder } & 460 & 433 \\
\hline & 427 & 418 \\
\hline & 395 & 550 \\
\hline & 369 & 894 \\
\hline & 283 & 713 \\
\hline & 316 & 709 \\
\hline & 348 & 826 \\
\hline & 256 & 1008 \\
\hline \multirow[t]{4}{*}{ Cask Flange } & 1111 & 211 \\
\hline & 1112 & 503 \\
\hline & 1115 & 304 \\
\hline & 1114 & 722 \\
\hline \multirow[t]{11}{*}{ Lid } & 34 & 590 \\
\hline & 33 & 651 \\
\hline & 51 & 1303 \\
\hline & 47 & 399 \\
\hline & 74 & 980 \\
\hline & 76 & .726 \\
\hline & 48 & 913 \\
\hline & 45 & 451 \\
\hline & 94 & 1415 \\
\hline & 92 & 1292 \\
\hline & 61 & 1265 \\
\hline
\end{tabular}

* See Figure B4.3-1 for node locations. 
HNF-SD-SNF-FDR-003 Rev. 0

$\mathrm{E}-15166$

Table B4.3-8 Cask Body Stresses Under Cold Environment Vibrations

(Bolt Preload, Cold Thermal, 20 psi External Pressure, Vibrations)

\begin{tabular}{|c|c|c|}
\hline \multicolumn{2}{|c|}{ Location } & \multirow{2}{*}{$\begin{array}{c}\text { Nodal Stress Intensity } \\
\text { (psi) }\end{array}$} \\
\hline Cask Body Component & Node Number" & \\
\hline \multirow[t]{6}{*}{ Cask Bottom } & 133 & 165 \\
\hline & 131 & 30 \\
\hline & 214 & 8 \\
\hline & 157 & 136 \\
\hline & 142 & 104 \\
\hline & 220 & 15 \\
\hline \multirow[t]{8}{*}{ Cask Cylinder } & 460 & 68 \\
\hline & 427 & 68 \\
\hline & 395 & 58 \\
\hline & 369 & 51 \\
\hline & 283 & 47 \\
\hline & 316 & 44 \\
\hline & 348 & 15 \\
\hline & 256 & 15 \\
\hline \multirow[t]{4}{*}{ Cask Flange } & 1111 & 59 \\
\hline & 1112 & 46 \\
\hline & 1115 & 36 \\
\hline & 1114 & 237 \\
\hline \multirow[t]{11}{*}{ Lid } & 34 & 365 \\
\hline & 33 & 328 \\
\hline & 51 & 361 \\
\hline & 47 & 160 \\
\hline & 74 & 232 \\
\hline & 76 & 216 \\
\hline & 48 & 200 \\
\hline & 45 & 145 \\
\hline & 94 & 240 \\
\hline & 92 & 224 \\
\hline & 61 & 119 \\
\hline
\end{tabular}

* See Figure B4.3-1 for node locations. 
Table B4.3-9 Cask Body Stresses Under Hot Environment 1 Foot Bottom End Drop (Bolt Preload, Hot Thermal, 161.2 psi Internal Pressure, 1 Foot Drop 25G)

\begin{tabular}{|c|c|c|}
\hline \multicolumn{2}{|c|}{ Location } & \multirow{2}{*}{$\begin{array}{c}\text { Nodal Stress Intensity } \\
\text { (psi) }\end{array}$} \\
\hline Cask Body Component & Node Number & \\
\hline \multirow[t]{6}{*}{ Cask Bottom } & 133 & 1627 \\
\hline & 131 & 1847 \\
\hline & 214 & 2436 \\
\hline & 157 & 202 \\
\hline & 142. & 888 \\
\hline & 220 & 1837 \\
\hline \multirow[t]{8}{*}{ Cask Cylinder } & 460 & 1342 \\
\hline & 427 & 1035 \\
\hline & 395 & 887 \\
\hline & 369 & 1059 \\
\hline & 283 & 1038 \\
\hline & 316 & 747 \\
\hline & 348 & 813 \\
\hline & 256 & 848 \\
\hline \multirow[t]{4}{*}{ Cask Flange } & 1111 & 253 \\
\hline & 1112 & 524 \\
\hline & 1115 & 366 \\
\hline & 1114 & 714 \\
\hline \multirow[t]{11}{*}{ Lid } & 34 & 706 \\
\hline & 33 & 599 \\
\hline & 51 & 1060 \\
\hline & 47 & 412 \\
\hline & 74 & 765 \\
\hline & 76 & 542 \\
\hline & 48 & 836 \\
\hline & 45 & 379 \\
\hline & 94 & 1147 \\
\hline & 92 & 1044 \\
\hline & L & 11 \\
\hline
\end{tabular}

* See Figure B4.j-1 for node locations. 
Table B4.3-10 Cask Body Stresses Under Cold Environment 1 Foot Bottom End Drop

(Bolt Preload, Cold Thermal, 20 psi External Pressure, 1 Foot Drop 25G)

\begin{tabular}{|c|c|c|}
\hline \multicolumn{2}{|c|}{ Location } & \multirow{2}{*}{$\begin{array}{c}\text { Nodal Stress Intensity } \\
\text { (psi) }\end{array}$} \\
\hline Cask Body Component & Node Number' & \\
\hline \multirow[t]{6}{*}{ Cask Bottom } & 133 & 923 \\
\hline & 131 & 1157 \\
\hline & 214 & 1228 \\
\hline & 157 & 1025 \\
\hline & 142 & 1199 \\
\hline & 220. & 1148 \\
\hline \multirow[t]{8}{*}{ Cask Cylinder } & 460 & 968 \\
\hline & 427 & 672 \\
\hline & 395 & 364 \\
\hline & 369 & 295 \\
\hline & 283 & 949 \\
\hline & 316 & 652 \\
\hline & 348 & 365 \\
\hline & 256 & 168 \\
\hline \multirow[t]{4}{*}{ Cask Flange } & 1111 & 66 \\
\hline & 1112 & 133 \\
\hline & 1115 & 97 \\
\hline & 1114 & 366 \\
\hline \multirow[t]{11}{*}{ Lid } & 34 & 474 \\
\hline & 33 & 347 \\
\hline & 51 & 616 \\
\hline & 47 & 205 \\
\hline & 74 & 447 \\
\hline & 76 & 412 \\
\hline & 48 & 362 \\
\hline & 45 & 221 \\
\hline & 94 & 507 \\
\hline & 92 & 472 \\
\hline & $6 !$ & 252 \\
\hline
\end{tabular}

* See Figure B4.3-1 for node locations. 


\section{HNF-SD-SNF-FDR-003 Rev. 0}

$E-15166$

Table B4.3-11 Cask Body Stresses Under Hot Environment 1 Foot Lid End Drop

(Bolt Preload, Hot Thermal, 161.2 psi Intemal Pressure, 1 Foot Drop 25G)

\begin{tabular}{|c|c|c|}
\hline \multicolumn{2}{|c|}{ Location } & \multirow{2}{*}{$\begin{array}{c}\text { Nodal Stress Intensity } \\
\text { (psi) }\end{array}$} \\
\hline Cask Body Component & Node Number" & \\
\hline \multirow[t]{6}{*}{ Cask Bottom } & 133 & 462 \\
\hline & 131 & 749 \\
\hline & 214 & 1238 \\
\hline & 157 & 972 \\
\hline & 142 & 366 \\
\hline & 220 & 871 \\
\hline \multirow[t]{8}{*}{ Cask Cylinder } & 460 & 723 \\
\hline & 427 & 1010. \\
\hline & 395 & 1432 \\
\hline & 369 & 2070 \\
\hline & 283 & 715 \\
\hline & 316 & 722 \\
\hline & 348 & 1009 \\
\hline & 256 & 959 \\
\hline \multirow[t]{4}{*}{ Cask Flange } & 1111 & 2696 \\
\hline & 1112. & 2016 \\
\hline & 1115 & 2820 \\
\hline & 1114 & 2380 \\
\hline \multirow[t]{11}{*}{ Lid } & 34. & 2533 \\
\hline & 33 & 2846 \\
\hline & 51 & 2529 \\
\hline & 47 & 3238 \\
\hline & 74 & 165 \\
\hline & 76 & 261 \\
\hline & 48 & 2951 \\
\hline & 45 & 3361 \\
\hline & 94 & 2259 \\
\hline & 92 & 2120 \\
\hline & 61 & 2377 \\
\hline
\end{tabular}

* See Figure B4.j-1 for node locations. 
HNF-SD-SNF-FDR-003 Rev. 0

E- 15166

Table B4.3-12 Cask Body Stresses Under Cold Environment 1 Foot Lid End Drop

(Bolt Preload, Cold Thermal, 20 psi External Pressure, 1 Foot Drop 25G)

\begin{tabular}{|c|c|c|}
\hline \multicolumn{2}{|c|}{ Location } & \multirow{2}{*}{$\begin{array}{c}\text { Nodal Stress Intensity } \\
\text { (psi) }\end{array}$} \\
\hline Cask Body Component & Node Number" & \\
\hline \multirow{6}{*}{ Cask Bottom } & 133 & 244 \\
\hline & 131 & 122 \\
\hline & 214 & 36 \\
\hline & 157 & 173 \\
\hline & 142 & 217 \\
\hline & 220 & 23 \\
\hline \multirow{8}{*}{ Cask Cylinder } & 460 & 349 \\
\hline & 427 & 647 \\
\hline & 395 & 928 \\
\hline & 369 & 1326 \\
\hline & 283 & 331 \\
\hline & 316 & 627 \\
\hline & 348 & 920 \\
\hline & 256 & 1136 \\
\hline \multirow[t]{4}{*}{ Cask Flange } & 1111 & 2606 \\
\hline & 1112 & 1834 \\
\hline & 1115 & 2857 \\
\hline & 1114 & 3256 \\
\hline \multirow[t]{11}{*}{ Lid } & 34 & 2445 \\
\hline & 33 & 3023 \\
\hline & 51 & 3958 \\
\hline & 47 & 3056 \\
\hline & 74 & 1048 \\
\hline & 76 & 1035 \\
\hline & 48 & 2997 \\
\hline & 45 & 2810 \\
\hline & 94. & 606 \\
\hline & 92 & 615 \\
\hline & 61 & 2300 \\
\hline
\end{tabular}

* See Figure B4.3-1 for node locations. 
Table B4.3-13 Cask Body Stresses Under Hot Environment 1 Foot Side Drop (contact side)

(Bolt Preload, Hot Thermal, 161.2 psi Internal Pressure, 1 Foot Drop 24G)

\begin{tabular}{|c|c|c|}
\hline \multicolumn{2}{|c|}{ Location } & \multirow{2}{*}{$\begin{array}{c}\text { Nodal Stress Intensity } \\
(\mathrm{psi})\end{array}$} \\
\hline Cask Body Component & Node Number ${ }^{*}$ & \\
\hline \multirow[t]{6}{*}{ Cask Bottom } & 133 & 2357 \\
\hline & 131 & 1810 \\
\hline & 214 & 1318 \\
\hline & 157 & 3408 \\
\hline & 142 & 1999 \\
\hline & 220 & 782 \\
\hline \multirow[t]{8}{*}{. Cask Cylinder } & 460 & 2882 \\
\hline & 427 & 2977 \\
\hline & 395 & 2780 \\
\hline & 369 & 2116 \\
\hline & 283 & 939 \\
\hline & 316 & 1023 \\
\hline & 348 & 898 \\
\hline & 256 & 1129 \\
\hline \multirow[t]{4}{*}{ Cask Flange } & 1111 & 1907 \\
\hline & 1112 & 830 \\
\hline & 1115 & 2217 \\
\hline & 1114 & 729 \\
\hline \multirow[t]{11}{*}{ Lid } & 34 & 1565 \\
\hline & 33 & 872 \\
\hline & 51 & 917 \\
\hline & 47 & 176 \\
\hline & 74 & 1312 \\
\hline & 76 & 946 \\
\hline & 48 & 702 \\
\hline & 45 & 59 \\
\hline & 94 & 2139 \\
\hline & 92 & 1631 \\
\hline & 61 & 1251 \\
\hline
\end{tabular}

* See Figure B4.3.1 for node locations. 
Table B4.3-14 Cask Body Stresses Under Hot Environment 1 Foot Side Drop (opp. contact side)

(Bolt Preload, Hot Thermal, 161.2 psi Internal Pressure, 1 Foot Drop 24G)

\begin{tabular}{|c|c|c|}
\hline \multicolumn{2}{|c|}{ Location } & \multirow{2}{*}{$\begin{array}{c}\text { Nodal Stress Intensity } \\
\text { (psi) }\end{array}$} \\
\hline Cask Body Component & Node Number ${ }^{*}$ & \\
\hline \multirow[t]{6}{*}{ Cask Bottom } & 133 & $\cdot 1403$ \\
\hline & 131 & 538 \\
\hline & 214. & 851 \\
\hline & 157 & 1591 \\
\hline & 142 & 560 \\
\hline & 220 & 535 \\
\hline \multirow[t]{8}{*}{ Cask Cylinder } & 460 & 1126 \\
\hline & 427 & 1219 \\
\hline & 395 & 1020 \\
\hline & 369 & 842 \\
\hline & 283 & 727 \\
\hline & 316 & 1100 \\
\hline & 348 & 931 \\
\hline & 256 & 896 \\
\hline \multirow[t]{4}{*}{ Cask Flange } & 1111 & 500 \\
\hline & 1112 & 519 \\
\hline & 1115 & 742 \\
\hline & 1114 & 635 \\
\hline \multirow[t]{11}{*}{ Lid } & 34 & 637 \\
\hline & 33. & 522 \\
\hline & 51 & 1116 \\
\hline & 47 & 343 \\
\hline & 74 & 961 \\
\hline & 76 & 967 \\
\hline & 48 & 666 \\
\hline & 45 & 378 \\
\hline & 94 & 1691 \\
\hline & 92 & 1473 \\
\hline & 61 & 1270 \\
\hline
\end{tabular}

* See Figure B4.3-1 for node locations. 
HNF-SD-SNF-FDR-003 Rev. 0

$E-15166$

Table B4.3-15 Cask Body Stresses Under Cold Environment 1 Foot Side Drop ( contact side)

(Bolt Preload, Cold Thermal, 20 psi External Pressure, 1 Foot Drop 24G)

\begin{tabular}{|c|c|c|}
\hline \multicolumn{2}{|c|}{ Location } & \multirow{2}{*}{$\begin{array}{c}\text { Nodal Stress Intensity } \\
(\mathrm{psi})\end{array}$} \\
\hline Cask Body Component & Node Number & \\
\hline \multirow[t]{6}{*}{ Cask Bottom } & 133 & 2345 \\
\hline & 131 & 946 \\
\hline & 214 & 701 \\
\hline & 157 & 3050 \\
\hline & 142 & 2303 \\
\hline & 220 & 806 \\
\hline \multirow[t]{8}{*}{ Cask Cylinder } & 460 & 2898 \\
\hline & 427 & 3025 \\
\hline & 395 & 3001 \\
\hline & 369 & 2558 \\
\hline & 283 & 1681 \\
\hline & 316 & 1761 \\
\hline & 348 & 1738 \\
\hline & 256 & 1504 \\
\hline \multirow[t]{4}{*}{ Cask Flange } & 1111 & 1948 \\
\hline & 1112 & 814 \\
\hline & 1115 & 1950 \\
\hline & 1114 & 1163 \\
\hline \multirow[t]{11}{*}{ Lid } & 34 & 1435 \\
\hline & 33 & 955 \\
\hline & $5 !$ & 1632 \\
\hline & 47 & 490 \\
\hline & 74 & 339 \\
\hline & 76 & 333 \\
\hline & 48 & 1368 \\
\hline & 45 & 615 \\
\hline & 94 & 507 \\
\hline & 92 & 229 \\
\hline & 61 & 582 \\
\hline
\end{tabular}

* See Figure B4.3-1 for node locations. 
HNF-SD-SNF-FDR-003 Rev, 0

E-15166

Table B4.3-16 Cask Body Stresses Under Cold Environment 1 Foot Side Drop (opp. contact side)

(Bolt Preload, Cold Thermal, 20 psi External Pressure, 1 Foot Drop 24G)

\begin{tabular}{|c|c|c|}
\hline \multicolumn{2}{|c|}{ Location } & \multirow{2}{*}{$\begin{array}{c}\text { Nodal Stress Intensity } \\
\text { (psi) }\end{array}$} \\
\hline Cask Body Component & Node Number & \\
\hline \multirow[t]{6}{*}{ Cask Bottom } & 133 & 1151 \\
\hline & 131 & 336 \\
\hline & 214 & 425 \\
\hline & 157 & 1551 \\
\hline & 142 & 81 \\
\hline & 220 & 315 \\
\hline \multirow[t]{8}{*}{ Cask Cylinder } & 460 & 1142 \\
\hline & 427 & 1265 \\
\hline & 395 & 1240 \\
\hline & 369 & 921 \\
\hline & 283 & 831 \\
\hline & 316 & 1221 \\
\hline & 348 & 1001 \\
\hline & 256 & 593 \\
\hline \multirow[t]{4}{*}{ Cask Flange } & 1111 & 503 \\
\hline & 1112 & 247 \\
\hline & 1115 & 471 \\
\hline & 1114 & 570 \\
\hline \multirow[t]{11}{*}{ Lid } & 34 & 396 \\
\hline & 33 & 463 \\
\hline & 51 & 566 \\
\hline & 47 & 129 \\
\hline & 74 & 618 \\
\hline & 76 & 477 \\
\hline & 48 & 503 \\
\hline & 45 & 195 \\
\hline & 94 & 1413 \\
\hline & 92 & 655 \\
\hline & -61 & 188 \\
\hline
\end{tabular}

* See Figure B4,3-1 for node locations. 
HNF-SD-SNF-FDR-003 Rev. 0 E-15166

Table B4.3-17 Cask Body Stresses Under Hot Environment 1 Foot Corner Drop (contact side)

(Bolt Preload, Hot Thermal, 161.2 psi Internal Pressure, 1 Foot Drop on lid side 18G)

\begin{tabular}{|c|c|c|}
\hline \multicolumn{2}{|c|}{ Location } & \multirow{2}{*}{$\begin{array}{c}\text { Nodal Stress Intensity } \\
\text { (psi) }\end{array}$} \\
\hline Cask Body Component & Node Number" & \\
\hline \multirow[t]{6}{*}{ Cask Bottom } & 133 & 1218 \\
\hline & 131 & 571 \\
\hline & 214 & 1073 \\
\hline & 157 & 1350 \\
\hline & 142 & 659 \\
\hline & 220 & 755 \\
\hline \multirow[t]{8}{*}{ Cask Cylinder } & 460 & 498 \\
\hline & 427 & 717 \\
\hline & 395 & 1282 \\
\hline & 369 & 2390 \\
\hline & 283 & 764 \\
\hline & 316 & 790 \\
\hline & 348 & 952 \\
\hline & 256 & 1302 \\
\hline \multirow[t]{4}{*}{ Cask Flange } & 1111 & 3912 \\
\hline & 1112 & 2796 \\
\hline & 1115 & 4161 \\
\hline & 1114 & 4203 \\
\hline \multirow[t]{11}{*}{ Lid } & 34 & 4892 \\
\hline & 33 & 4534 \\
\hline & 51 & 12252 \\
\hline & 47 & 2353 \\
\hline & 74 & 10462 \\
\hline & 76 & 8496 \\
\hline & 48 & 8284 \\
\hline & 45 & 986 \\
\hline & 94 & 5307 \\
\hline & 92 & 7955 \\
\hline & 6) & 4721 \\
\hline
\end{tabular}

* See Figure B4.3-1 for node locations. 
Rev. 0

E-15166

Table B4.3-18 Cask Body Stresses Under Hot Environment 1 Foot Corner Drop (opp. contact side)

(Bolt Preload, Hot Thermal, 161.2 psi Internal Pressure, 1 Foot Drop on lid side 18G)

\begin{tabular}{|c|c|c|}
\hline \multicolumn{2}{|c|}{ Location } & \multirow{2}{*}{$\begin{array}{c}\text { Nodal Stress Intensity } \\
(\mathrm{psi})\end{array}$} \\
\hline Cask Bodv Component & Node Number" & \\
\hline \multirow[t]{6}{*}{ Cask Bottom } & 133 & 1065 \\
\hline & 131 & 978 \\
\hline & 214 & 1423 \\
\hline & 157 & 1998 \\
\hline & 142 & 408 \\
\hline & 220 & 983 \\
\hline \multirow[t]{8}{*}{ Cask Cylinder } & 460 & 765 \\
\hline & 427 & 951 \\
\hline & 395 & 1045 \\
\hline & 369 & 1148 \\
\hline & 283 & 709 \\
\hline & 316 & 762 \\
\hline & 348 & 789 \\
\hline & 256 & 687 \\
\hline \multirow{4}{*}{ Cask Flange } & 1111 & 928 \\
\hline & 1112 & 996 \\
\hline & 1115 & 1249 \\
\hline & 1114 & 1065 \\
\hline \multirow[t]{11}{*}{ Lid } & 34 & 1693 \\
\hline & 33 & 1189 \\
\hline & 51 & 1548 \\
\hline & 47 & 961 \\
\hline & 74 & 10111 \\
\hline & 76 & 3770 \\
\hline & 48 & 1659 \\
\hline & 45 & 813 \\
\hline & 94 & 7337 \\
\hline & 92 & 3207 \\
\hline & & \\
\hline
\end{tabular}


HNF-SD-SNF-FDR-003 Rev. $0 \quad$ E-15166

Table B4.3-19 Cask Body Stresses Under Cold Environment 1 Foot Corner Drop ( contact side)

(Bolt Preload, Cold Thermal, 20 psi External Pressure, 1 Foot Drop on lid side 18G)

\begin{tabular}{|c|c|c|}
\hline \multicolumn{2}{|c|}{ Location } & \multirow{2}{*}{$\begin{array}{c}\text { Nodal Stress Intensity } \\
(\mathrm{psi})\end{array}$} \\
\hline Cask Body Component & Node Number ${ }^{*}$ & \\
\hline \multirow[t]{6}{*}{ Cask Bottom } & 133 & 1062 \\
\hline & 131 & 299 \\
\hline & 214 & 200 \\
\hline & 157 & 1283 \\
\hline & 142 & 261 \\
\hline & 220 & 99 \\
\hline \multirow[t]{8}{*}{ Cask Cylinder } & 460 & 124 \\
\hline & 427 & 354 \\
\hline & 395 & 802 \\
\hline & 369 & 1875 \\
\hline & 283 & 22 \\
\hline & 316 & 255 \\
\hline & 348 & 830 \\
\hline & 256 & 1490 \\
\hline \multirow[t]{4}{*}{ Cask Flange } & 1111 & 3829 \\
\hline & 1112 & 2645 \\
\hline & 1115 & 4199 \\
\hline & 1114 & 5087 \\
\hline \multirow[t]{11}{*}{ Lid } & 34 & 4840 \\
\hline & 33 & 4546 \\
\hline & 51 & 13796 \\
\hline & 47 & 2285 \\
\hline & 74 & 10652 \\
\hline & 76 & 4268 \\
\hline & 48 & 9060 \\
\hline & 45 & 1131 \\
\hline & 94 & 6148 \\
\hline & 92 & 9351 \\
\hline & 61 & 600 \\
\hline
\end{tabular}

* See Figure B4.3-1 for node locations. 
HNF-SD-SNF-FDR-D03 ReV. 0

E-15166

Table B4.3-20 Cask Body Stresses Under Cold Environment 1 Foot Corner Drop (opp. contact side)

(Boit Preload, Cold Thermal, 20 psi External Pressure, 1 Foot Drop on lid side 18G)

\begin{tabular}{|c|c|c|}
\hline \multicolumn{2}{|c|}{ Location } & \multirow{2}{*}{$\begin{array}{c}\text { Nodal Stress Intensity } \\
(\mathrm{psi})\end{array}$} \\
\hline Cask Body Component & Node Number" & \\
\hline \multirow[t]{6}{*}{ Cask Bottom } & 133 & 1050 \\
\hline & 131 & 166 \\
\hline & 214 & 151 \\
\hline & 157 & 1278 \\
\hline & 142 & 466 \\
\hline & 220 & 138 \\
\hline \multirow{8}{*}{ Cask Cyiinder } & 460 & 391 \\
\hline & 427 & 587 \\
\hline & 395 & 542 \\
\hline & 369 & 477 \\
\hline & 283 & 486 \\
\hline & 316 & 664 \\
\hline & 348 & 486 \\
\hline & 256 & 370 \\
\hline \multirow[t]{4}{*}{ Cask Flange } & 1111 & 860 \\
\hline & 1112 & 757 \\
\hline & 1115 & 1011 \\
\hline & 1114 & 1126 \\
\hline \multirow[t]{11}{*}{ Lid } & 34 & 1467 \\
\hline & 33 & 1163 \\
\hline & 51 & 2704 \\
\hline & -47 & 953 \\
\hline & 74 & 11263 \\
\hline & 76 & $436 \hat{i}$ \\
\hline & 48 & 2496 \\
\hline & 45 & 1003 \\
\hline & 94 & 8841 \\
\hline & 92 & 3884 \\
\hline & St & 2462 \\
\hline
\end{tabular}

* See Figure B4.3-1 for node locations. 


\section{B4.4 ACCIDENT CONDITIONS}

This section describes the response of the cask to the accident loading conditions specified by the Hanford Specification. The design criteria established for the TN-WHC for the hypothetical accident conditions are described in Section B4.4.2. These criteria are selected to ensure that the package performance standards specified by the Hanford Specification ${ }^{(1)}$ are satisfied.

The presentation of the hypothetical accident condition analyses and results is accomplished in the same manner as that used for the normal condition of transport. The detailed analyses of the various packaging components under different loading conditions are presented in the Appendices to this Section. The limiting results for the specified hypothetical accident loading conditions are taken from the Appendices and summarized here with a comparison made to the established design criteria.

Table B4.4-1 provides an overview of the performance evaluations presented in this section. Stress analysis results for the cask body and lid bolts are taken directly from the corresponding analysis appendix.

\section{Reporting Method for Cask body Vessel Stresses}

The structural analysis of the cask body was performed using an ANSYS finite element model. Stress results are reported at selected representative locations as described in Section B4.3. Because of the asymmetric characteristic of most of the hypothetical accident loads, stress results are generally reported on two opposite sides of the cask body.

Appendix $\mathrm{B} 4.5 \mathrm{C}$ provides the detailed description of the structural analyses of the cask body. That appendix describes the detailed ANSYS model used to analyze various applied loads. Table B4.4-2 identifies the individual loads (IL) analyzed using the ANSYS model which are applicable to the hypothetical accident conditions. Some of these individual loads are axisymmetric (e.g. pressure) and others are asymmetric (e.g. side impact).

Figure B4.3-1 shows the selected locations on the cask body where stress results for these analyses are reported. Detailed stresses are actually available at as many locations as there are nodes in the finite element model. However, for practical considerations, the reporting of stress results is limited to those locations shown on Figure B4.3-1. These locations were selected to be representative of the stress distribution in the cask body with special attention given to areas subject to high stresses. The maximum stress may occur at a different location for each individual load.

The stress results for the individual load case tables reported in Appendix $\mathrm{B} 4.5 \mathrm{C}$ are limited to the maximum stress components. Two or more individual load cases must be combined to determine the total stresses at the standard stress reporting locations for the various load combinations. This is accomplished using the ANSYS postprocessor which algebraicly adds the stress components at each of the standard locations. The membrane stress intensity is then found from the membrane stress components and the inner and outer surface membrane plus bending 
stress intensities are determined from the membrane \pm bending stress components.

Several other items also described in Section B4.3 should be noted. For the axisymmetric cases such as the end drop on the lid and bottom, the stress is constant around the circumference of the cask at each stress reporting location. For asymmetric analyses with significant differences in stress magnitudes on the extreme opposite sides of the cask, the stress at locations on both sides of the cask are reported in separate tables.

Table B4.4-3 provides a matrix of the individual loads and how they are combined to determine the cask body stresses for the hypothetical accident conditions. The thermal stresses due to the hot and cold conditions are actually secondary stresses that could be evaluated using higher allowables than for primary stresses. They are conservatively added to the primary stresses and the combined stresses are evaluated using the primary stress allowables. An $\mathrm{x}$ in Table B4.4-3 indicates that the stress results for the individual load case are used directly. A quantitative number ( $F$ ) indicates the load factor applied to the individual stresses.

For the minimum internal pressure load combination, it is conservatively assumed that the cask cavity is at 0 psia. The net differential pressure acting on the cask body is then 20 psi (external pressure). 
Table B4.4-1 Accident Conditions of Transport, TN-WHC Performance Evaluation Overview

\begin{tabular}{||l|c|l||}
\hline \multicolumn{1}{|c|}{$\begin{array}{c}\text { Loading } \\
\text { Conditions }\end{array}$} & $\begin{array}{c}\text { DAR } \\
\text { Section }\end{array}$ & \multicolumn{1}{|c|}{ Scope of Evaluation } \\
\hline Thermal Accident & B4.4.4.2 & $\begin{array}{l}\text { Cask Body Stresses due to Different Thermal } \\
\text { Expansion }\end{array}$ \\
\hline Maximum Pressure & B4.4.4.2 & $\begin{array}{l}\text { Cask Body Stresses due to Maximum Internal } \\
\text { pressure }\end{array}$ \\
\hline Impact & B4.4.4.3.1 & $\begin{array}{l}\text { Cask Body Stresses for Bottom End and Lid } \\
\text { End Drops }\end{array}$ \\
\cline { 2 - 4 } & B4.4.4.3.2 & Cask Body Stresses for Side Drop \\
\cline { 2 - 4 } & B4.4.4.3.3 & $\begin{array}{l}\text { Cask Body Stresses for C.G. Over Lid End } \\
\text { Corner Drop }\end{array}$ \\
\hline Lid Bolt Analysis & B4.4.4.3.4 & Lid Bolt Stresses - Lid End Corner Drop \\
\hline Puncture & B4.4.4.3.5 & $\begin{array}{l}\text { Cask Body Evaluation for 40 inch Drop onto } \\
\text { the Puncture Bar }\end{array}$ \\
\hline Conclusions & B4.4.4.3.6 & Summary of the Results \\
\hline \hline
\end{tabular}


HNF-SD-SNF-FDR-003 Rev. $0 \quad$ E-15166

Table B4.4-2 Accident Conditions of Transport, Individual Load Cases for Cask Body Analysis

\begin{tabular}{|c|l|c|}
\hline Load Case & \multicolumn{1}{|c|}{ Individual load Description } & $\begin{array}{c}\text { Stress Results } \\
\text { Table }\end{array}$ \\
\hline IL-1 & Bolt Preload & B4.5C-3 \\
\hline IL-2 & Thermal Stress at Hot Environment & B4.5C-11 \\
\hline IL-3 & Thermal Stress at Cold Environment & B4.5C-12 \\
\hline IL-4 & Internal Pressure & B4.5C-4 \\
\hline IL-5 & External Pressure & B4.5C-5 \\
\hline IL-8 & End Drop on Bottom End (1G) & B4.5C-7 \\
\hline IL-9 & End Drop on Lid End (1G) & B4.5C-8 \\
\hline IL-10 & Side Drop (1G) & B4.5C-9 \\
\hline IL-11 & C.G. Over Lid End Corner Drop (1G) & B4.5C-10 \\
\hline
\end{tabular}

* These tables are presented in Appendix B4.5C 
Table B4.4-3 Summary of Load Combinations for Accident Conditions of Transport

\begin{tabular}{|c|c|c|c|c|c|c|c|c|c|c|}
\hline \multirow{2}{*}{$\begin{array}{c}\text { Load } \\
\text { Combinations }\end{array}$} & \multicolumn{9}{|c|}{ Applicable Individual Load } & \multirow{2}{*}{$\begin{array}{c}\text { Stress } \\
\text { Result } \\
\text { Table No. }\end{array}$} \\
\hline & $\begin{array}{c}\text { IL- } \\
1\end{array}$ & $\begin{array}{l}\text { IL- } \\
2\end{array}$ & $\begin{array}{l}\text { IL- } \\
3\end{array}$ & $\begin{array}{c}\text { IL- } \\
4\end{array}$ & $\begin{array}{l}\text { IL- } \\
5\end{array}$ & $\begin{array}{c}\text { IL- } \\
8\end{array}$ & $\begin{array}{c}\text { IL- } \\
9\end{array}$ & $\begin{array}{l}\text { IL- } \\
10\end{array}$ & $\begin{array}{c}\mathrm{IL}- \\
11\end{array}$ & \\
\hline \multirow[t]{2}{*}{$\begin{array}{l}30 \text { Foot Bottom } \\
\text { End Drop (30G) }\end{array}$} & $\mathrm{x}$ & $\mathrm{x}$ & & $\mathrm{x}$ & & $\begin{array}{c}\mathrm{x} \\
\mathrm{F}= \\
30\end{array}$ & & & & B4.4-4 \\
\hline & $\mathrm{x}$ & & $\mathrm{x}$ & & $\mathrm{x}$ & $\begin{array}{l}x \\
F= \\
30\end{array}$ & & & & B4.4-5 \\
\hline \multirow[t]{2}{*}{$\begin{array}{l}30 \text { Foot Lid End } \\
\text { Drop (30G) }\end{array}$} & $\mathrm{x}$ & $\mathrm{x}$ & & $\mathrm{x}$ & & & $\begin{array}{l}x \\
F= \\
30\end{array}$ & & & B4.4-6 \\
\hline & $\mathrm{x}$ & & $\mathrm{x}$ & & $\mathrm{x}$ & & $\begin{array}{c}\mathrm{x} \\
\mathrm{F}= \\
30\end{array}$ & & & B4.4-7 \\
\hline \multirow[t]{2}{*}{$\begin{array}{l}30 \text { Foot Side Drop } \\
(40 \mathrm{G})\end{array}$} & $\mathrm{x}$ & $\mathrm{x}$ & & $\mathrm{x}$ & & & & $\begin{array}{l}x \\
F= \\
40\end{array}$ & & $\begin{array}{l}\text { B4.4-8 } \\
\text { B4.4-9 }\end{array}$ \\
\hline & $\mathrm{x}$ & & $\mathrm{x}$ & & $\mathrm{x}$ & & & $\begin{array}{l}x \\
F= \\
40\end{array}$ & & $\begin{array}{l}\text { B4.4-10 } \\
\text { B4.4-11 }\end{array}$ \\
\hline \multirow[t]{2}{*}{$\begin{array}{l}30 \text { Foot Lid } \\
\text { Corner Drop } \\
\quad(20 \mathrm{G})\end{array}$} & $\mathrm{x}$ & $\mathrm{x}$ & & $\mathrm{x}$ & & & & & $\begin{array}{c}\mathrm{x} \\
\mathrm{F}= \\
20 \\
\end{array}$ & $\begin{array}{l}\text { B4.4-12 } \\
\text { B4.4 -13 }\end{array}$ \\
\hline & $\mathrm{x}$ & & $\mathrm{x}$ & & $\mathrm{x}$ & & & & $\begin{array}{l}\mathrm{x} \\
\mathrm{F}= \\
20\end{array}$ & $\begin{array}{l}\text { B4.4-14 } \\
\text { B4.4 -15 }\end{array}$ \\
\hline
\end{tabular}


HNF-SD-SNF-FDR-003 Rev. $0 \quad$ E-15166

\section{B4.4.1 Conditions To Be Evaluated}

The cask is designed to withstand each of the accident transport conditions specified in Hanford Specification $^{(1)}$ as listed below:

\section{B4.4.1.1 Impact}

The response of the cask is evaluated for a free drop from a height of 30 feet onto an yielding surface at various orientations. The inertial loading applied to the cask components is determined Appendix B4.5A. The 30 foot drop is measured from the impact surface to the bottom of the impact limiter; the C.G. of the cask is much higher than 30 feet.

Cask body stresses are reported for the standard drop orientations of lid end and bottom end drops, side drop, center-of-gravity over lid end corner drop.

\section{B4.4.1.2 Puncture}

The worst case credible puncture incident is equivalent to a free drop of the cask through a distance of 40 in $(1 \mathrm{~m})$ in a position for which the maximum damage is expected, onto the upper end of solid, vertical, cylindrical, mild steel bar mounted on an essentially unyielding, horizontal surface. The bar must be 6 in $(15 \mathrm{~cm})$ in diameter, with the top horizontal and its edge rounded to a radius of not more than $1 / 4$ in $(6 \mathrm{~mm})$ and of a length as to cause maximum damage to the package, but not less than 8 in $(20 \mathrm{~cm})$ long.

\section{B4.4.1.3 Thermal}

An ANSYS transient thermal analysis for the 30 minute thermal accident is reported in Section B5.0. The initial condition is steady state at $115^{\circ} \mathrm{F}$ ambient with maximum decay heating. The initial steady state condition is followed by 0.5 hour severe thermal transient which is then followed by a cool-down period. The temperature through the cross section of the package at the time where individual temperatures peak ( 0.5 hours)are summarized below:

Cask inner surface temperature $=727^{\circ} \mathrm{F}$

Cask outer surface temperature $=1068^{\circ} \mathrm{F}$

Lid bolt temperature $=1068^{0} \mathrm{~F}$

\section{B4.4.2 Acceptance Criteria}

\section{Containment Boundary}

The cask shall be designed so that during accident conditions the cask maintains confinement of 
the $\mathrm{MCO}$, as demonstrated by analysis. Elastic-plastic analysis may be performed to demonstrate maintenance of confinement after the accident conditions. ASME B\&PV Code Section III, Service Level D allowables will be used for acceptance. After any accident condition, as a minimum, the containment function of the MCO shall be maintained. Allowable stress levels for containment components are outlined in Table B4.3-4 of Section 4.3.

\section{Bolting}

In the special case of bolting, the maximum combined stress is limited to $S_{u}$ for accident condition of transport.

\section{Brittle Fracture}

The containment vessel is entirely austenitic stainless steel (Type 304) which is ductile even at low temperature. Thus, brittle fracture is precluded.

\section{B4.4.3 Structural Model}

The cask body consists of the cylindrical shell, the bottom, the lid and the lid bolts. The elements used to model the bolts are ANSYS STIF3, beam elements. The cylindrical shell, the bottom end closure and the lid are modeled using either ANSYS STIF25 axisymmetric solid elements or ANSYS STIF42 axisymmetric solid elements (Puncture Analysis). The loading applied to this type of elements may be either axisymmetric for some cases and asymmetric for other cases. The model geometry is based on Drawings $\mathrm{H}-1-81535$, sheets $1,2,3,4$, and 5 provided in Section A9.1. The contact surface at the lid and cylindrical shell is modeled using separate nodes in the interfacing components. These nodes are coupled or left uncoupled for specific constraint conditions as discussed in Appendix B4.5C. All the analyses were performed using the model shown in Figures $\mathrm{B} 4.5 \mathrm{C}-1$ and $\mathrm{B} 4.5 \mathrm{C}-2$. The mechanical properties for the materials in this model are the linear values described in Section B4.2.2.

\section{B4.4.4 Initial Conditions}

\section{B4.4.4.1 Environmental Heat Loading}

Section B5.0 describes the thermal analyses performed for the cask subjected to thermal accident conditions. These thermal analysis results are used to support various aspects of the structural evaluations as described in the following .

\section{Maximum Temperature}

Stress allowables for packaging components are a function of component temperature. Stress 


\section{HNF-SD-SNF-FDR-003 Rev. $0 \quad$ E-15166}

allowables are based on actual maximum calculated temperatures or conservatively selected higher temperatures. Section B5.0 summarizes significant temperatures calculated for the cask subjected to thermal conditions. These temperatures are used to calculate the cask body thermal stress.

\section{Maximum Pressure}

For purpose of the structural analysis of containment, a value of $150 \mathrm{psi}$ is used for MNOP. The structural analysis is performed assuming the same outward pressure differences across the cask wall as during the reduced external pressure case, $(150+14.7-3.5) 161.2 \mathrm{psi}$.

\section{B4.4.4.2 Maximum Thermal and Pressure Stresses}

\section{Maximum Thermal Stress}

For the accident load combinations, the normal thermal stresses as described in Section B4.3.4.2 are used for the accident load combinations. The thermal expansion bolt stresses due to the thermal accident condition (fire condition) are presented in Appendix B4.5B.

\section{Maximum Pressure Stresses}

For purposes of the structural analysis of containment, a value of $161.2 \mathrm{psi}$ as described above is used. This pressure loading is analyzed using the ANSYS model of the cask body as described in Appendix B4.5C and the results are reported in Table B4.5C-4.

\section{B4.4.4.3 Structural Evaluations and Conclusions}

\section{B4.4.4.3.1 Bottom End and Lid End Drops}

The impact analysis of the cask shows that the maximum expected inertia loading from the 30 -foot end drop is $25 \mathrm{G}$ 's for the $90^{\circ}$ orientation.

The structural analysis of the cask body for this loading condition was performed using an inertial loading of $30 \mathrm{G}\left(90^{\circ}\right.$ bottom end drop and lid end drop). As shown in Table B4.5C-7 of Appendix B4.5C, the maximum stresses due to the bottom end drop is $1.440 \mathrm{psi}$ and $4,350 \mathrm{psi}$ for the lid end drop.

The load combinations performed to evaluate these drop events are indicated in Table B4.4-3. In all case, bolt preload effects are included. For the hot environment condition, the thermal stress load case for that temperature, the $161.2 \mathrm{psi}$ pressure load case and the impact load case factored for the accident condition $\mathrm{G}$ level are combined. For the cold temperature evaluation, the cold thermal stress case, the $20 \mathrm{psi}$ external pressure case, and the impact load case factored for the accident condition $\mathrm{G}$ level are combined. 
Table B4.4-4 lists the total nodal stress intensity at each of the standard stress reporting locations for the bottom end drop under hot environment conditions. The maximum stress intensity calculated for this load combination is 2,674 psi which occurs at node number 214 . This stress intensity is well below the allowable membrane stress intensity of 47,670 psi, and membrane plus bending stress intensity of 68,1000 psi (at $150^{\circ} \mathrm{F}$ temperature).

Table B4.4-5 lists the total nodal stress intensity at each of the standard stress reporting locations for the bottom end drop under cold environment conditions. The maximum stress intensity calculated for this load combination is 1,434 psi which occurs at node number 214 . This stress intensity is well below the allowable membrane stress intensity of $47,670 \mathrm{psi}$, and membrane plus bending stress intensity of $68,1000 \mathrm{psi}$ (at $150^{\circ} \mathrm{F}$ temperature).

Table B4.4-6 lists the total nodal stress intensity at each of the standard stress reporting locations for the lid end drop under hot environment conditions. The maximum stress intensity calculated for this load combination is 3,950 psi which occurs at node number 45 . This stress intensity is well below the allowable membrane stress intensity of $47,670 \mathrm{psi}$, and membrane plus bending stress intensity of 68,1000 psi (at $150^{\circ} \mathrm{F}$ temperature).

Table B4.4-7 lists the total nodal stress intensity at each of the standard stress reporting locations for the lid end drop under cold environment conditions. The maximum stress intensity calculated for this load combination is 4,681 psi which occurs at node number 51 . This stress intensity is well below the allowable membrane stress intensity of 47,670 psi, and membrane plus bending stress intensity of $68,1000 \mathrm{psi}$ (at $150^{\circ} \mathrm{F}$ temperature).

\section{B4 4.4.3.2 Side Drop}

The analysis of the 30 -foot side drop provided a inertial loading of $37 \mathrm{G}$ for side drop and $39 \mathrm{G}$ for slap down (Appendix 4.5A). The structural analysis of the cask body for this loading condition was performed using an inertial loading of $40 \mathrm{G}$ to envelop the worst side drop and slap down conditions. As shown in Table B4.5C-9 of Appendix B4.5C, the maximum stresses due to the side drop is 5,152 psi. This maximum stress value in the containment is much less than the allowables for general membrane stress intensity of 47,670 psi.

Table B4.4-8 lists the total nodal stress intensity at each of the standard stress reporting locations for the side drop under hot environment conditions (contact side). The maximum stress intensity calculated for this load combination is $5,085 \mathrm{psi}$ which occurs at node number 157 . This stress intensity is well below the allowable membrane stress intensity of 47.670 psi, and membrane plus bending stress intensity of $68,1000 \mathrm{psi}$ (at $150^{\circ} \mathrm{F}$ temperature).

Table B4.4-9 lists the total nodal stress intensity at each of the standard stress reporting locations for the side drop under hot environment conditions (opposite contact side). The maximum stress intensity calculated for this load combination is 2,598 psi which occurs at node number 157 . This stress intensity is well below the allowable membrane stress intensity of 47,670 psi, and 
membrane plus bending stress intensity of $68,1000 \mathrm{psi}$ (at $150^{\circ} \mathrm{F}$ temperature).

Table B4.4-10 lists the total nodal stress intensity at each of the standard stress reporting locations for the side drop under cold environment conditions (contact side). The maximum stress intensity calculated for this load combination is 5,086 psi which occurs at node number 427 . This stress intensity is well below the allowable membrane stress intensity of $47,670 \mathrm{psi}$, and membrane plus bending stress intensity of $68,1000 \mathrm{psi}$ (at $150^{\circ} \mathrm{F}$ temperature).

Table B4.4-11 lists the total nodal stress intensity at each of the standard stress reporting locations for the side drop under cold environment conditions (opposite contact side). The maximum stress intensity calculated for this load combination is 2,586 psi which occurs at node number 157. This stress intensity is well below the allowable membrane stress intensity of $47,670 \mathrm{psi}$, and membrane plus bending stress intensity of $68,1000 \mathrm{psi}$ (at $150^{\circ} \mathrm{F}$ temperature).

\section{B4.4.4.3.3 C.G. Over Corner Drop}

The response of the cask to the 30 -foot corner drop was analyzed for impact on the top or lid end corner which is the more critical cask orientation (than the bottom corner) because it is the closure end. The analysis was performed using the ANSYS model as described in Appendix B4.5C. The center-of-gravity over corner drop occurs at a drop angle of approximately $80^{\circ}$. That is, the longitudinal axis of the cask is at an angle of $80^{\circ}$ from the impact surface. The analysis (Appendix B4.5A) of the $80^{\circ}$ drop orientation resulted in maximum inertia loading of $19.7 \mathrm{G}$ (axial) along the cask longitudinal axis and $3.5 \mathrm{~g}$ transverse to the longitudinal axis at the cask CG. The maximum stress result for the 30 foot lid end corner drop is 14,964 psi.

Table B4.4-12 lists the total nodal stress intensity at each of the standard stress reporting locations for the lid end corner drop under hot environment conditions (contact side). The maximum stress intensity calculated for this load combination is 13,747 psi which occurs at node number 51. This stress intensity is well below the allowable membrane stress intensity of 47,670 psi, and membrane plus bending stress intensity of $68,1000 \mathrm{psi}$ (at $150^{\circ} \mathrm{F}$ temperature).

Table B4.4-13 lists the total nodal stress intensity at each of the standard stress reporting locations for the lid end corner drop under hot environment conditions (opposite contact side). The maximum stress intensity calculated for this load combination is 11,338 psi which occurs at node number 74. This stress intensity is well below the allowable membrane stress intensity of 47,670 psi, and membrane plus bending stress intensity of 68,1000 psi (at $150^{\circ} \mathrm{F}$ temperature).

Table B4.4-14 lists the total nodal stress intensity at each of the standard stress reporting locations for the lid end corner drop under cold environment conditions (contact side). The maximum stress intensity calculated for this load combination is $15,293 \mathrm{psi}$ which occurs at node number 51. This stress intensity is well below the allowable membrane stress intensity of $47,670 \mathrm{psi}$, and membrane plus bending stress intensity of 68,1000 psi (at $150^{\circ} \mathrm{F}$ temperature).

Table B4.4-15 lists the total nodal stress intensity at each of the standard stress reporting locations 
for the lid end corner drop under hot environment conditions (opposite contact side). The maximum stress intensity calculated for this load combination is 12,490 psi which occurs at node number 74. This stress intensity is well below the allowable membrane stress intensity of 47,670 $\mathrm{psi}$, and membrane plus bending stress intensity of $68,1000 \mathrm{psi}$ (at $150^{\circ} \mathrm{F}$ temperature).

\section{B4.4.4.3.4 Lid Bolts}

The lid bolts were analyzed in accordance with NUREG/CR-6007, Stress Analysis of Closure Bolts for Shipping Casks $^{(6)}$. This analysis is presented in Appendix B4.5B. The governing hypothetical accident load is the combination of maximum internal pressure and a near perpendicular, oblique 30-foot drop in which the lid end strikes the target first and the cask contents impact on the inner side of the lid. This drop orientation is the most severe because, based on the impact analysis (Appendix B4.5A), this drop orientation results in the highest axial inertia forces that result in the highest lid bolt tensile stresses. The results in Appendix B4.5B show that the maximum stress intensity due to tension plus shear plus bending is $72,900 \mathrm{psi}$ which is less than the allowable maximum stress intensity of $133,000 \mathrm{psi}\left(\right.$ at $150^{\circ} \mathrm{F}$ ).

The fire accident results in a maximum bolt and lid temperature of $1068^{\circ} \mathrm{F}\left(1091^{\circ} \mathrm{F}\right.$ is conservatively used for calculation). The thermal stress at the bolt due to this differential thermal expansion is $20,154 \mathrm{psi}$. The maximum combined stress including stresses due to preload and shears $49,386 \mathrm{psi}$. This stress is less than the bolt yield stress of 82,500 psi. The MCO will therefore be secure inside the cask during the fire accident.

\section{B4.4.4.3.5 Puncture}

\section{Puncture Force}

The most severe damage to the cask resulting from the puncture drop will occur on the outer center of the lid. The analysis is based on the Nelms ${ }^{(7)}$ equation. The Nelms puncture relation is given as:

$$
\begin{aligned}
& \mathrm{t}=\left(\mathrm{W} / \mathrm{S}_{u}\right)^{0.7 t} \\
& \text { Where: } \\
& \qquad \begin{array}{l}
\mathrm{t}=\text { lid thickness }=3.5^{\prime \prime} \\
\mathrm{W}=\text { package weight }=57,910 \mathrm{lbs} \\
\mathrm{S}_{u}=\text { ultimate tensile strength of the lid }=70,000 \mathrm{psi}
\end{array}
\end{aligned}
$$

The package weight that can result in puncture is:

$$
W=S_{u} t^{1 .+1}=70,000(3.5)^{1 .+1}=409,482 \mathrm{lbs}
$$


The actual package weight is $57,910 \mathrm{lbs}$, Therefore, the factor of safety for puncture resistance on the energy basis is:

$$
\text { F.S. }=409,482 / 57,910=7.07
$$

When the package contacts the puncture bar, the force applied to the package is:

$$
\begin{aligned}
& F_{1}=\text { impact force }=\sigma_{s} A_{p} \\
& \sigma_{s}=\text { dynamic flow pressure of stainless steel }=45,000 \text { psi (reference } 8 \text { ) } \\
& A_{p}=\text { area of the puncture bar }=\pi / 4(6)^{2}=28.27 \mathrm{in}^{2} \\
& F_{1}=45,000 \times 28.27=1.272 \times 10^{6} \mathrm{lbs}
\end{aligned}
$$

This force produces a cask deceleration:

$$
\mathrm{G}=\text { cask deceleration }=\mathrm{F}_{\mathrm{l}} / \mathrm{W}=1.272 \times 10^{6} / 57,910=22
$$

This deceleration is smaller to that which will occur during impact on end after the 30 foot free drop (25G). Therefore, global stresses that result from the inertial force will be smaller. The bending stress at the center of the lid will be calculated using the above finite element model.

\section{$\underline{\text { Lid Stresses }}$}

The lid stresses are computed using the 2-D model as described in Appendix B4.5C-4. The loading distribution and boundary conditions are shown on Figures B4.5C-21 and B4.5C-22. The $22 \mathrm{G}$ inertia load due to 40 " drop is applied as body acceleration. The content's inertia load is applied as equivalent pressure on the lid as follows:

$$
\begin{aligned}
& \text { Contents weight }=19,120 \mathrm{lbs} \\
& \mathrm{P}=19,120 \times 22 / \pi(12.595)^{2}=844.05 \mathrm{psi}
\end{aligned}
$$

\section{Elastic Analysis:}

ANSYS computer Code is used for the analysis. A stress run was made using the above noted loads and boundary conditions. STIF 42 (Axisymmetric) finite element was used in the analysis.

The stresses are linearized at critical sections. The maximum membrane and maximum membrane plus bending stress intensities are as follows: 


\begin{tabular}{|c|c|c|}
\hline $\begin{array}{c}\text { Location (Node number) } \\
\text { (Figure B4.5C-23) }\end{array}$ & $\mathrm{P}_{\mathrm{m}}$ (psi) & $\mathrm{P}_{\mathrm{m}}+\mathrm{P}_{\mathrm{b}}(\mathrm{psi})$ \\
\hline 84 & 12,560 & 83,280 \\
\hline 94 & 10,880 & 85,010 \\
\hline 93 & 5,275 & 70,560 \\
\hline 92 & 30,780 & 57,550 \\
\hline 91 & 25,430 & 59,960 \\
\hline
\end{tabular}

The maximum membrane stress intensity calculated for this load is 30,780 psi at node number 92 . This stress is below the allowable membrane stress intensity of 47,670 psi. The maximum membrane plus bending stress intensity is $85,010 \mathrm{psi}$ at node number 94 . This stress exceeds the allowable membrane plus bending stress intensity of $68,100 \mathrm{psi}$. Therefore, an elastic - plastic analysis was performed to recalculate the stresses.

\section{Plastic Analysis:}

A plastic analysis was performed (using the same finite element model, boundary conditions, and loads). The stress - strain properties used for the stainless steel is given in Figure B4.5C-24. The total load ( $22 \mathrm{G}$ inertia and $844.05 \mathrm{psi}$ ) was divided in eight load steps for proper converged solution.

The maximum membrane and maximum membrane plus bending stress intensities are as follows:

\begin{tabular}{|c|c|c|}
\hline $\begin{array}{c}\text { Location (Node number) } \\
\text { (Figure B4.5C-23) }\end{array}$ & $\mathrm{P}_{\mathrm{m}}$ (psi) & $\mathrm{P}_{\mathrm{m}}+\mathrm{P}_{\mathrm{b}}$ (psi) \\
\hline 84 & 2,934 & 45,030 \\
\hline 94 & 3,326 & 46,620 \\
\hline 93 & 6,889 & 42,140 \\
\hline 92 & 25,470 & 36,060 \\
\hline 91 & 26,350 & 40,780 \\
\hline
\end{tabular}

The maximum membrane stress intensity calculated for this load is 26,350 psi at node number 91 . This stress is below the allowable membrane stress intensity of 47,670 psi $\left(0.7 \mathrm{~S}_{4}\right)$. The maximum membrane plus bending stress intensity is 46,620 psi at node number 94 . This stress is below the allowable membrane plus bending stress intensity of $61,290 \mathrm{psi}\left(0.9 \mathrm{~S}_{\mathrm{u}}\right)$. The analysis shows the stresses are within the ASME Code allowables. 
HNF-SD-SNF-FRD-003 Rev. $0 \quad$ E-15166

\section{B4.4.4.3.6 Conclusions}

From the analyses presented in Section B4.4 above, it can be shown that the accident load conditions will not result in any significant structural damage of the TN-WHC cask.

The maximum combined stresses due to the 30 foot drop event are also presented in Tables B4.44 to B4.4-15. In nearly all of the load cases, the maximum stresses are less than the membrane allowable stress with a large margin of safety.

The lid bolt stresses do not exceed the ultimate strength during the hypothetical $30 \mathrm{ft}$ drop accidents as shown in Appendix B4.5B.

During the 40 inch drop onto a 6.0 inch diameter puncture bar, the packaging may deform locally above the punch. It has been shown by analysis that the package will not be punctured and that the $\mathrm{MCO}$ will be confined.

The cask body material is a stainless steel. The thermal stresses due to the differential thermal expansion are insignificant. Thermal stress of the lid bolt due to the differential thermal expansion under the thermal accident conditions is less than the yield stress.

From the analyses presented in Section 4.4, it can be shown that the accident loads will not result in any significant structural damage of the cask. The cask maintains the confinement function of the MCO. 
HNF-SD-SNF-FRD-003 Rev. $0 \quad$ E-15166

Table B4.4-4 Cask Body Stresses Under Hot Environment 30 Foot Bottom End Drop (Bolt Preload, Hot Thermal, 161.2 psi Internal Pressure, 30 Foot Drop 30G)

\begin{tabular}{|c|c|c|}
\hline \multicolumn{2}{|c|}{ Location } & \multirow{2}{*}{$\begin{array}{c}\text { Nodal Stress Intensity } \\
\text { (psi) }\end{array}$} \\
\hline Cask Body Component & Node Number" & \\
\hline \multirow[t]{6}{*}{ Cask Bottom } & 133 & 1826 \\
\hline & 131 & 2067 \\
\hline & 214 & 2674 \\
\hline & 157 & 199 \\
\hline & 142 & 1121 \\
\hline & 220 & 2067 \\
\hline \multirow[t]{8}{*}{ Cask Cylinder } & 460 & 1529 \\
\hline & 427 & 1163 \\
\hline & 395 & 957 \\
\hline & 369 & 1091 \\
\hline & 283 & 1225 \\
\hline & 316 & 874 \\
\hline & 348 & 813 \\
\hline & 256 & 817 \\
\hline \multirow[t]{4}{*}{ Cask Flange } & 1111 & 265 \\
\hline & 1112 & 536 \\
\hline & 1115 & 384 \\
\hline & 1114 & 712 \\
\hline \multirow[t]{11}{*}{ Lid } & 34 & 729 \\
\hline & 33 & 588 \\
\hline & 51 & 1012 \\
\hline & 47 & 414 \\
\hline & 74 & 722 \\
\hline & 76 & 505 \\
\hline & 48 & 821 \\
\hline & 45 & 365 \\
\hline & 94 & 1093 \\
\hline & 92 & 993 \\
\hline & 61 & 1105 \\
\hline
\end{tabular}

* See Figure B.4.3-1 For Node Locations. 
HNF-SD-SNF-FRD-003 Rev. 0 E-15166

Table B4.4-5 Cask Body Stresses Under Cold Environment 30 Foot Bottom End Drop

(Bolt Preload, Cold Thermal, 20 psi External Pressure, 30 Foot Drop 30G)

\begin{tabular}{|c|c|c|}
\hline \multicolumn{2}{|c|}{ Location } & \multirow{2}{*}{$\begin{array}{c}\text { Nodal Stress Intensity } \\
\text { (psi) }\end{array}$} \\
\hline Cask Body Component & Node Number & \\
\hline \multirow[t]{6}{*}{ Cask Bottom } & 133 & 1121 \\
\hline & 131 & 1384 \\
\hline & 214 & 1474 \\
\hline & 157 & 1225 \\
\hline & 142 & 1434 \\
\hline & 220 & 1380 \\
\hline \multirow[t]{8}{*}{ Cask Cylinder } & 460 & 1155 \\
\hline & 427 & 800 \\
\hline & 395 & 454 \\
\hline & 369 & 237 \\
\hline & 283 & 1137 \\
\hline & 316 & 780 \\
\hline & 348 & 435 \\
\hline & 256 & 200 \\
\hline \multirow[t]{4}{*}{ Cask Flange } & 1111 & 87 \\
\hline & 1112 & 151 \\
\hline & 1115 & 118 \\
\hline & 1114 & 392 \\
\hline \multirow{11}{*}{ Lid } & 34 & 496 \\
\hline & 33 & 352 \\
\hline & 51 & 669 \\
\hline & 47 & 215 \\
\hline & 74 & 491 \\
\hline & 76 & 451 \\
\hline & 48 & 396 \\
\hline & 45 & 237 \\
\hline & 94 & 562 \\
\hline & 92 & 523 \\
\hline & 61 & 280 \\
\hline
\end{tabular}

* See Figure B4.3-1 For Node Locations. 
HNF-SD-SNF-FRD-003 Rev. $0 \quad$ E-15166

Table B4.4-6 Cask Body Stresses Under Hot Environment 30 Foot Lid End Drop (Bolt Preload, Hot Thermal, 161.2 psi Internal Pressure, 30 Foot Drop 30G)

\begin{tabular}{|c|c|c|}
\hline \multicolumn{2}{|c|}{ Location } & \multirow{2}{*}{\begin{tabular}{|c} 
Nodal Stress Intensity \\
$(\mathrm{psi})$
\end{tabular}} \\
\hline Cask Body Component & Node Number* & \\
\hline \multirow[t]{6}{*}{ Cask Bottom } & 133 & 427 \\
\hline & 131 & 733 \\
\hline & 214 & 1233 \\
\hline & 157 & 944. \\
\hline & 142 & 333 \\
\hline & 220 & 875 \\
\hline \multirow[t]{8}{*}{ Cask Cylinder } & 460 & 786 \\
\hline & 427 & 1133 \\
\hline & 395 & 1161 \\
\hline & 369 & 2305 \\
\hline & 283 & 715 \\
\hline & 316 & 844 \\
\hline & 348 & 1189 \\
\hline & 256 & 1178 \\
\hline \multirow[t]{4}{*}{ Cask Flange } & 1111 & 3223 \\
\hline & 1112 & 2369 \\
\hline & 1115 & 3393 \\
\hline & 1114 & 2981 \\
\hline \multirow[t]{11}{*}{ Lid } & 34 & 2950 \\
\hline & 33 & 3384 \\
\hline & 51 & 3235 \\
\hline & 47 & 3877 \\
\hline & 74 & 33 \\
\hline & 76 & 420 \\
\hline & 48 & 3499 \\
\hline & 45 & 3950 \\
\hline & 94 & 2427 \\
\hline & 92 & 2285 \\
\hline & 61 & 2747 \\
\hline
\end{tabular}

* See Figure B4.3-1 For Node Locations. 


\section{HNF-SD-SNF-FRD-003 Rev. $0 \quad$ E-15166}

Table B4.4-7 Cask Body Stresses Under Cold Environment 30 Foot Lid End Drop Condition

(Bolt Preload, Cold Thermal, 20 psi External Pressure, 30 Foot Drop 30G)

\begin{tabular}{|c|c|c|}
\hline \multicolumn{2}{|c|}{ Location } & \multirow{2}{*}{$\begin{array}{c}\text { Nodal Stress Intensity } \\
(\mathrm{psi})\end{array}$} \\
\hline Cask Body Component & Node Number* & \\
\hline \multirow[t]{6}{*}{ Cask Bottom } & 133 & 279 \\
\hline & 131 & 139 \\
\hline & 214 & 41 \\
\hline & 157 & 203 \\
\hline & 142 & 251 \\
\hline & 220 & 27 \\
\hline \multirow[t]{8}{*}{ Cask Cylinder } & 460 & 412 \\
\hline & 427 & 769 \\
\hline & 395 & 1108 \\
\hline & 369 & 1592 \\
\hline & 283 & 395 \\
\hline & 316 & 750 \\
\hline & 348 & 1101 \\
\hline & 256 & 1364 \\
\hline \multirow[t]{4}{*}{ Cask Flange } & 1111 & 3135 \\
\hline & 1112 & 2194 \\
\hline & 1115 & 3430 \\
\hline & 1114 & 3861 \\
\hline \multirow[t]{11}{*}{ Lid } & 34 & 2861 \\
\hline & 33 & 3579 \\
\hline & 51 & 4681 \\
\hline & 47 & 3699 \\
\hline & 74 & 1212 \\
\hline & 76 & 1200 \\
\hline & 48 & 3569 \\
\hline & 45 & 3400 \\
\hline & 94 & 773 \\
\hline & 92 & 778 \\
\hline & 61 & $=285$ \\
\hline
\end{tabular}

* See Figure B4.3-1 For Node Locations 
HNF-SD-SNF-FRD-003 Rev. $0 \quad$ E-15166

Table B4.4-8 Cask Body Stresses Under Hot Environment 30 Foot Side Drop (contact side)

(Bolt Preload, Hot Thermal, 161.2 psi Internal Pressure, 30 Foot Drop 40G)

\begin{tabular}{|c|c|c|}
\hline \multicolumn{2}{|c|}{ Location } & \multirow{2}{*}{$\begin{array}{c}\text { Nodal Stress Intensity } \\
\text { (psi) }\end{array}$} \\
\hline Cask Body Component & Node Number* & \\
\hline \multirow[t]{6}{*}{ Cask Bottom } & 133 & 3921 \\
\hline & 131 & 2464 \\
\hline & 214 & 1373 \\
\hline & 157 & 5085 \\
\hline & 142 & 3503 \\
\hline & 220 & 1201 \\
\hline \multirow[t]{8}{*}{ Cask Cylinder } & 460 & 4858 \\
\hline & 427 & 5038 \\
\hline & 395 & 4825 \\
\hline & 369 & 3742 \\
\hline & 283 & 2042 \\
\hline & 316 & 2180 \\
\hline & 348 & $203 \underline{8}$ \\
\hline & 256 & 1751 \\
\hline \multirow[t]{4}{*}{ Cask Flange } & 1111 & 3213 \\
\hline & 1112 & 1339 \\
\hline & 1115 & 3525 \\
\hline & 1114 & 1198 \\
\hline \multirow[t]{11}{*}{ Lid } & 34 & 2523 \\
\hline & 33 & 1535 \\
\hline & 51 & 1431 \\
\hline & 47 & 367 \\
\hline & 74 & 1553 \\
\hline & 76 & 1092 \\
\hline & 48 & 1315 \\
\hline & 45 & 367 \\
\hline & 94 & 2628 \\
\hline & 92 & 1854 \\
\hline & 1361 & 13 \\
\hline
\end{tabular}

* See Figure B4.3-1 For Node Locations. 
Table B4.4-9 Cask Body Stresses Under Hot Environment 30 Foot Side Drop (opp. contact side)

(Bolt Preload, Hot Thermal, 161.2 psi Internal Pressure, 30 Foot Drop 40G)

\begin{tabular}{|c|c|c|}
\hline \multicolumn{2}{|c|}{ Location } & \multirow{2}{*}{$\begin{array}{c}\text { Nodal Stress Intensity } \\
(\mathrm{psi})\end{array}$} \\
\hline Cask Body Component & Node Number* & \\
\hline \multirow[t]{6}{*}{ Cask Bottom } & 133 & 1922 \\
\hline & 131 & 341 \\
\hline & 214 & 577 \\
\hline & 157 & 2598 \\
\hline & 142 & 578 \\
\hline & 220 & 326 \\
\hline \multirow[t]{8}{*}{ Cask Cylinder } & 460 & 1932 \\
\hline & 427 & 2108 \\
\hline & 395 & 1891 \\
\hline & 369 & 1209 \\
\hline & 283 & 1260 \\
\hline & 316 & 1905 \\
\hline & 348 & 1538 \\
\hline & 256 & 971 \\
\hline \multirow[t]{4}{*}{ Cask Flange } & 1111 & 819 \\
\hline & 1112 & 555 \\
\hline & 11115 & 1056 \\
\hline & 1114 & 578 \\
\hline \multirow[t]{11}{*}{ Lid } & 34 & 722 \\
\hline & 33 & 548 \\
\hline & 51 & 1001 \\
\hline & 47 & 327 \\
\hline & 74 & 973 \\
\hline & 76 & 1127 \\
\hline & 48 & 533 \\
\hline & 45 & 335 \\
\hline & 94 & 2372 \\
\hline & 92 & 1592 \\
\hline & 61 & 1269 \\
\hline
\end{tabular}

* See Figure B4.3-1 For Node Locations. 
HNF-SD-SNF-FRD-003 Rev。O E-15166

Table B4.4-10 Cask Body Stresses Under Cold Environment 30 Foot Side Drop (contact side)

(Bolt Preload, Cold Thermal, 20 psi External Pressure, 30 Foot Drop 40G)

\begin{tabular}{|c|c|c|}
\hline \multicolumn{2}{|c|}{ Location } & \multirow{2}{*}{\begin{tabular}{|c} 
Nodal Stress Intensity \\
(psi)
\end{tabular}} \\
\hline Cask Body Component & Node Number & \\
\hline \multirow[t]{6}{*}{ Cask Bottom } & 133 & 3909 \\
\hline & 131 & 1600 \\
\hline & 214 & 1169 \\
\hline & 157 & 508 \\
\hline & 142 & 3813 \\
\hline & 220 & 1335 \\
\hline \multirow[t]{8}{*}{ Cask Cylinder } & 460 & 4874 \\
\hline & 427 & 5086 \\
\hline & 395 & 5045 \\
\hline & 369 & 4297 \\
\hline & 283 & 2784 \\
\hline & 316 & 2918 \\
\hline & 348 & 2879 \\
\hline & 256 & 2498 \\
\hline \multirow[t]{4}{*}{ Cask Flange } & 1111 & 3261 \\
\hline & 1112 & 1352 \\
\hline & 1115 & 3261 \\
\hline & 1114 & 1792 \\
\hline \multirow[t]{11}{*}{ Lid } & 34 & 2389 \\
\hline & 33 & 2389 \\
\hline & 51 & 2521 \\
\hline & 47 & 769 \\
\hline & 74 & 553 \\
\hline & 76 & 526 \\
\hline & 48 & 2196 \\
\hline & 45 & 940 \\
\hline & 94 & 990 \\
\hline & 92 & 405 \\
\hline & 61. & 027 \\
\hline
\end{tabular}

* See Figure B4.3-1 For Node Locations. 
HNF-SD-SNF-FRD-003 Rev. $0 \quad$ E-15166

Table B4.4-11 Cask Body Stresses Under Cold Environment 30 Foot Side Drop (opp. contact side)

(Bolt Preload, Cold Thermal, 20 psi External Pressure, 30 Foot Drop 40G)

\begin{tabular}{|c|c|c|}
\hline \multicolumn{2}{|c|}{ Location } & \multirow{2}{*}{$\begin{array}{c}\text { Nodal } \begin{array}{c}\text { Stress Intensity } \\
(\mathrm{psi})\end{array} \\
\end{array}$} \\
\hline Cask Body Component & Node Number & \\
\hline \multirow[t]{6}{*}{ Cask Bottom } & 133 & 1917 \\
\hline & 131 & 536 \\
\hline & 214 & 702 \\
\hline & 157 & 2586 \\
\hline & 142 & 102 \\
\hline & 220 & 523 \\
\hline \multirow[t]{8}{*}{ Cask Cylinder } & 460 & 1948 \\
\hline & 427 & 2156 \\
\hline & 395 & 2112 \\
\hline & 369 & 1569 \\
\hline & 283 & 1376 \\
\hline & 316 & 2026 \\
\hline & 348 & 1659 \\
\hline & 256 & 980 \\
\hline \multirow[t]{4}{*}{ Cask Flange } & 1111 & 851 \\
\hline & 1112 & 406 \\
\hline & 1115 & 796 \\
\hline & 1114 & 797 \\
\hline \multirow[t]{11}{*}{ Lid } & 34 & 561 \\
\hline & 33 & 607 \\
\hline & 51 & 735 \\
\hline & 47 & 207 \\
\hline & 74 & 891 \\
\hline & 76 & 782 \\
\hline & 48 & 758 \\
\hline & 45 & 245 \\
\hline & 9.4 & 2355 \\
\hline & 92 & 1025 \\
\hline & 61 & 270 \\
\hline
\end{tabular}

* See Figure B4.3-1 For Node Locations. 
HNF-SD-SNF-FRD-003 Rev. $0 \quad$ E-15166

Table B4.4-12 Cask Body Stresses Under Hot Environ. 30 Foot Corner Drop (contact side)

(Bolt Preload, Hot Thermal, 161.2 psi Internal Pressure, 30 Foot Drop on lid side Corner 20G)

\begin{tabular}{|c|c|}
\hline \multirow[t]{2}{*}{ Location } & \multirow{2}{*}{$\begin{array}{rr}\text { Nodal } & \text { Stress } \\
\text { Intensity } & (\mathrm{psi}) \\
\end{array}$} \\
\hline & \\
\hline \multirow{3}{*}{ Cask Bottom } & \\
\hline & \\
\hline & \\
\hline \multirow[t]{5}{*}{ Cask Cylinder } & \\
\hline & \\
\hline & \\
\hline & \\
\hline & \\
\hline \multirow[t]{2}{*}{ Cask Flange } & \\
\hline & \\
\hline \multirow[t]{8}{*}{ Lid } & \\
\hline & \\
\hline & \\
\hline & \\
\hline & \\
\hline & \\
\hline & \\
\hline & \\
\hline
\end{tabular}

* See Figure B4.3-1 For Node Locations. 
HNF-SD-SNF-FRD-003 Rev. $0 \quad$ E-15166

Table B4.4-13 Cask Body Stresses Under Hot Environ. 30 Foot Corner Drop (opp. contact side)

(Bolt Preload, Hot Thermal, 161.2 psi Internal Pressure, 30 Foot Drop on lid side comer 20G)

\begin{tabular}{|c|c|c|}
\hline \multicolumn{2}{|c|}{ Location } & \multirow{2}{*}{$\begin{array}{c}\begin{array}{c}\text { Nodal Stress Intensity } \\
\text { (psi) }\end{array} \\
\end{array}$} \\
\hline Cask Body Component & Node Number ${ }^{\circ}$ & \\
\hline \multirow[t]{6}{*}{ Cask Bottom } & 133 & 1181 \\
\hline & 131 & 994 \\
\hline & 214 & 1441 \\
\hline & 157 & 2097 \\
\hline & 142 & 419 \\
\hline & 220 & 998 \\
\hline \multirow[t]{8}{*}{ Cask Cylinder } & 460 & 805 \\
\hline & 427 & 1012 \\
\hline & 395 & 1102 \\
\hline & 369 & 1175 \\
\hline & 283 & 708 \\
\hline & 316 & 835 \\
\hline & 348 & 786 \\
\hline & 256 & 673 \\
\hline \multirow[t]{4}{*}{ Cask Flange } & 1111 & 1026 \\
\hline & 1112 & 1061 \\
\hline & 1115 & 1363 \\
\hline & 1114 & 1166 \\
\hline \multirow[t]{11}{*}{ Lid } & 34. & 1817 \\
\hline & 33 & 1299 \\
\hline & 51 & 1806 \\
\hline & 47 & 1051 \\
\hline & 74 & 11338 \\
\hline & 76 & 4228 \\
\hline & 48 & 1897 \\
\hline & 45 & 912 \\
\hline & 94 & 8290 \\
\hline & 92 & 3588 \\
\hline & 61 & 1032 \\
\hline
\end{tabular}

* See Figure B4.3-1 For Node Locations. 
HNF-SD-SNF-FRD-003 ReV。 $0 \quad$ E-15166

Table B4.4-14 Cask Body Stresses Under Cold Environ. 30 Foot Corner Drop (contact side)

(Bolt Preload, Cold Thermal, 20 psi External Pressure, 30 Foot Drop on lid side corner 20G)

\begin{tabular}{|c|c|c|}
\hline \multicolumn{2}{|c|}{ Location } & \multirow{2}{*}{$\begin{array}{c}\text { Nodal Stress Intensity } \\
\text { (psi) }\end{array}$} \\
\hline Cask Body Component & Node Number" & \\
\hline \multirow[t]{6}{*}{ Cask Bottom } & 133 & 1180. \\
\hline & 131 & 328 \\
\hline & 214 & 222 \\
\hline & 157 & 1426 \\
\hline & 142 & 293 \\
\hline & 220 & 110 \\
\hline \multirow[t]{8}{*}{ Cask Cylinder } & 460 & 134 \\
\hline & 427 & 390 \\
\hline & 395 & 895 \\
\hline & 369 & 2084 \\
\hline & 283 & 27 \\
\hline & 316 & 285 \\
\hline & 348 & 924 \\
\hline & 256 & 1657 \\
\hline \multirow[t]{4}{*}{ Cask Flange } & 1111 & 4259 \\
\hline & 1112 & 2935 \\
\hline & 1115 & 4667 \\
\hline & 1114 & 5626 \\
\hline \multirow[t]{11}{*}{ Lid } & 34 & 5337 \\
\hline & 33 & 5048 \\
\hline & 51 & 15293 \\
\hline & 47 & 2539 \\
\hline & 74 & 11831 \\
\hline & 76 & 10274 \\
\hline & 48 & 10046 \\
\hline & 45 & 1247 \\
\hline & 94 & 6816 \\
\hline & 92 & 10367 \\
\hline & 단 & 6760 \\
\hline
\end{tabular}

* See Figure B4.3-1 For Node Locations. 
Table B4.4-15 Cask Body Stresses Under Cold Environ. 30 Foot Corner Drop (opp. contact side)

(Bolt Preload, Cold Thermal, 20 psi External Pressure, 30 Foot Drop on lid side corner 20G)

\begin{tabular}{|c|c|c|}
\hline \multicolumn{2}{|c|}{ Location } & \multirow{2}{*}{$\begin{array}{c}\text { Nodal Stress Intensity } \\
(\mathrm{psi})\end{array}$} \\
\hline Cask Body Component & Node Number & \\
\hline \multirow{6}{*}{ Cask Bottom } & 133 & 1167 \\
\hline & 131 & 187 \\
\hline & 214 & 168 \\
\hline & 157 & 1420 \\
\hline & 142 & 512 \\
\hline & 220 & 154 \\
\hline \multirow[t]{8}{*}{ Cask Cylinder } & 460 & 431 \\
\hline & 427 & 649 \\
\hline & 395 & 598 \\
\hline & 369 & 531 \\
\hline & 283 & 538 \\
\hline & 316 & 736 \\
\hline & 348 & 539 \\
\hline & 256 & 411 \\
\hline \multirow[t]{4}{*}{ Cask Flange } & 1111 & 961 \\
\hline & 1112 & 838 \\
\hline & 1115 & 1126 \\
\hline & 1114 & 1237 \\
\hline \multirow[t]{11}{*}{ Lid } & 34 & 1592 \\
\hline & 33 & 1291 \\
\hline & 51 & 2968 \\
\hline & 47 & 1048 \\
\hline & 74 & 12490 \\
\hline & 76 & 4828 \\
\hline & 48 & 2760 \\
\hline & 45 & 1103 \\
\hline & 94 & 9799 \\
\hline & 92 & 4296 \\
\hline & 61 & 2728 \\
\hline
\end{tabular}

* See Figure B+.3-1 For Node Locations. 


\section{References For Section B4.0}

1. Performance Specification For TN-WHC Cask And Transportation System, WHC-S-0396, Rev. 1, September 1995.

2. Design Criteria for the TN-WHC Cask System, E-14427, Rev. 0.

3. ANSYS Engineering Analysis System, User's Manual Volumes 1\&2, Rev. 4.4A.

4. ASME Boiler and Pressure Vessel Code, Section II, III, V \& IV, American Society of Mechanical Engineers, NY, 1992.

5. Design Basis for Resistance to Shock and Vibration of Radioactive Material Packages in Truck Transport, ANSI N14.23, American National Standards Institute, May 1980.

6. Stress Analysis of Closure Bolts for Shipping Casks, NUREG/CR-6007,

7 A Guide for Design, Fabrication, and Analyzing of Shipping Casks for Nuclear Application, Cask Designer Guide, p.18, Nelms Equation.

8. Charles R. Adams, A Comparison of Analytical Techniques for Analyzing a Nuclear-SpentFuel Shipping Cask Subjected to An end-On Impact, NUREG/CR-1018. 
HNF-SD-SNF-FRD-003 Rev. $0 \quad$ E-15166

\section{B4.5 APPENDICES}

APPENDIX B4.5A G LOAD CALCULATION

APPENDIX B4.5B LID BOLT ANALYSIS

APPENDIX B4.5C STRUCTURAL EVALUATION OF CASK BODY

APPENDIX B4.5D CASK LIFTING ATTACHMENT 
HNF-SD-SNF-FRD-003 ReV. O

E-15166

APPENDIX B4.5A

G LOAD CALCULATION

B4.5A-O 
HNF-SD-SNF-FRD-003 Rev. 0

E-15166

TABLE OF CONTENTS

Section

B4.5A-1 INTRODUCTION

B4.5A-2 GENERAL APPROACH

B4.5A-3 CASK AND CONCRETE PAD/SOIL DESCRIPTION

B $4.5 \mathrm{~A}-4$

B4.5A-5

B4.5A-6

B4.5A-7

B4.5A-8 END DROP

SIDE DROP

C.G. OVER DROP

SHALLOW ANGLE IMPACT-SLAP DOWN

REFERENCES
Page

B4.5A-1

B4.5A-1

B4.5A-2

B4.5A-4

B4.5A-8

B4.5A-13

B4.5A-21

B4.5A-25

$$
\text { B4.SA - i }
$$




\section{APPENDIX B4.5A}

\section{G LOAD CALCULATION}

\section{B4.5A-1 INTRODUCTION}

This Appendix evaluates the effects of a hypothetical drop of the cask on the concrete storage pad.

The following cases are evaluated:

- $30.0 \mathrm{ft}$. end drop onto the concrete slab.

- $30.0 \mathrm{ft}$. side drop onto the concrete slab.

- 30.0 ft. C.G. over corner drop onto the concrete slab.

- $30.0 \mathrm{ft}$. shallow angle impact (slap down) onto the concrete slab.

The impact analysis is based on methodology of EPRI NP- $4830^{(1)}$ and NP-7551 ${ }^{(2)}$. This appendix considers the mass and geometry of the cask but assumes it to be rigid compared to the concrete storage pad. The storage pad properties and the cask geometry are used to determine the pad hardness parameter. The report provides graphs that show the force on the cask as a function of storage pad hardness. Scale model drop testing at Sandia National Laboratories and full scale cask drop testing in England have recently been performed in an attempt to "benchmark" the EPRI methodology. The preliminary results of the tests (end drops) show excellent correlation with the predicted results.

\section{B4.5A-2 GENERAL APPROACH}

The EPRI reports give Force (applied to the cask) vs. Deformation (of the target) curves for different magnitudes of target hardness. The target hardness is defined as a set of parameters times the area of the impact surface. This impact area usually depends of the deformation. The following procedure is used to determine the maximum deceleration of the cask and deformation of the target:

1. A small target deformation is taken.

2. The geometry of the cask relative to the target is used to compute the impact area for the given deformation.

3. The target hardness is then computed for this target area.

4. The data in the EPRI report is used to determine the force associated with the deformation. 
5. The energy absorbed in the increments in deformation is evaluated as the area under the force vs. deformation curve for the increment in deformation.

6. The deformation is increased and step 2-5 repeated.

7. This process is continued until the absorbed energy equals to weight times drop height. This is the final solution for the force and deformation.

\section{B4.5A-3 CASK AND CONCRETE PAD/SOIL DESCRIPTION}

The geometry of the cask is shown on drawing H-1-81535 sheet 1 of 5 . The technical data used for cask and concrete slab/soil are :

$$
\begin{aligned}
& \mathrm{W}=\text { Weight of cask }=60,000 \mathrm{lbs} \\
& \mathrm{E}_{\mathrm{c}}=\text { Concrete elastic modules }=3.6 \mathrm{E} 6 \mathrm{psi} \\
& \sigma_{\mathrm{u}}=\text { Ultimate concrete strength }=4,000 \mathrm{psi} \\
& \mathrm{U}_{\mathrm{s}}=\text { Poisson's ratio of concrete }=0.17 \\
& \mathrm{~h}_{\mathrm{c}}=\text { Concrete pad thickness }=8 \text { inches } \\
& \mathrm{S}_{\mathrm{y}}=\text { Rebar yield strength }=60,000 \mathrm{psi} \\
& \mathrm{E}_{\mathrm{s}}=\text { Sub-soil modulus }=28,000 \mathrm{psi} \\
& \mathrm{U}_{\mathrm{s}}=\text { Poisson's ratio of soil }=0.49
\end{aligned}
$$

The concrete slab contains 7/8 inch diameter rebar (\#7) on 12 inch spacing top and bottom, twoway, with 2" coverage. The design criteria of the rebar are listed in the Table B4.5A-1. The ultimate moment capacity of the slab is required for the analysis. This is evaluate using standard method (reference 3, pages 85-86) and is calculated in table B4.5A-2.

Table B4.5A-1

Mechanical Properties of Rebar

\begin{tabular}{||l|l|}
\hline Rebar Size & $\# 7\left(7 / 8^{\prime \prime}\right)$ \\
\hline Spacing & $12^{\prime \prime}$ \\
\hline Rebar Dia. & $0.875^{\prime \prime}$ \\
\hline Area & $0.6 \mathrm{in}^{2}$ \\
\hline Yield Strength $-\mathrm{S}_{\mathrm{y}}$ & $60,000 \mathrm{psi}$ \\
\hline Modulus of Elasticity - E & $29 \times 10^{6} \mathrm{psi}$ \\
\hline
\end{tabular}


Table B4.5A-2

$M_{u}$ Calculation - 8" Concrete Slab With 7/8" Diameter Rebar

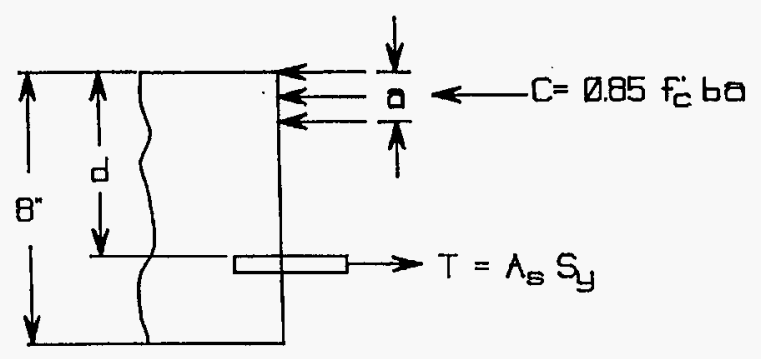

Size of Rebar $=7 / 8^{\prime \prime}$

$\mathrm{A}_{\mathrm{S}}=$ Cross Section Area of rebar $=0.6 \mathrm{in}^{2}$

$\mathrm{S}_{\mathrm{y}}=$ Rebar Yield Strength $=60,000 \mathrm{psi}$

$\mathrm{f}_{\mathrm{c}}=$ Ultimate Concrete Strength $=4,000 \mathrm{psi}$

$\mathrm{d}=$ Average Depth of Steel $=8^{\prime \prime}-2^{\prime \prime}-7 / 8^{\prime \prime}=5.125^{\prime \prime}$

By $\quad \sum \mathrm{F}=0 \quad \mathrm{C}=\mathrm{T}$

$\mathrm{A}_{\mathrm{s}} \mathrm{S}_{\mathrm{y}}=0.85 \mathrm{f}_{\mathrm{c}} \mathrm{b}$ a

$\mathrm{a}=\mathrm{A}_{\mathrm{S}} \mathrm{S}_{\mathrm{y}} / 0.85 \mathrm{f}_{\mathrm{c}} \mathrm{b}=0.6 \times 60,000 / 0.85 \times 4000 \times 12=0.8824^{\prime \prime}$

$\sum M=0$

$M_{u}=A_{S} S_{y}(d-a / 2)=0.6 \times 60,000(5.125-0.8824 / 2)=168,618 \mathrm{lbs} / \mathrm{in}$ 


\section{B4.5A-4 END DROP}

The results of the EPRI reports are presented in term of a target hardness number (S). In general this is given by:

$$
\mathrm{S}=\frac{\mathrm{M}_{\mathrm{u}} \sigma_{\mathrm{u}} \mathrm{A}}{\mathrm{W}^{2} \delta_{\mathrm{e}}}
$$

Where

$\mathrm{M}_{\mathrm{u}}=$ Ultimate moment capacity 1 foot section of slab $=168,624 \mathrm{\#} / \mathrm{in}$.

$\sigma_{u}=$ Ultimate concrete strength $=4,000 \mathrm{psi}$

$A=$ Area of impact surface.

$\mathrm{W}=$ Weight of cask $=60,000$ lbs.

$\delta_{\mathrm{e}}=$ Deflection of cask under weight of cask $(1 \mathrm{G})$.

For the end drop, the area $A=$ Area of the cask

$$
\mathrm{A}=\pi \mathrm{R}^{2}=\pi(40.57 / 2)^{2}=1292.7 \mathrm{in}^{2}
$$

The deflection $\left(\delta_{e}\right)$ is given as:

$$
\delta_{e}=\frac{W}{2 R k}\left(1-e^{-\beta R} \cos \beta R\right)
$$

Where

$$
\begin{aligned}
& k=\frac{\pi E_{s}}{1-v_{s}^{2}}=\frac{\pi(28,000)}{1-0.49^{2}}=115,758 \mathrm{psi} / \mathrm{in}
\end{aligned}
$$

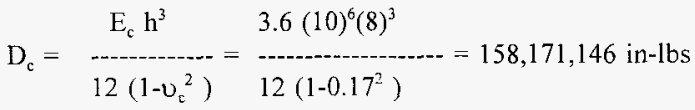

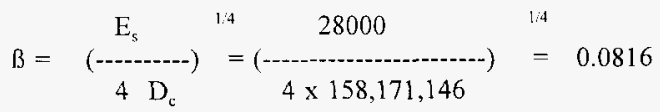




$$
\begin{aligned}
\delta_{\mathrm{e}} & =\frac{\mathrm{W}}{2 \mathrm{Rk}}\left(1-\mathrm{e}^{-\beta \mathrm{R}} \cos \beta \mathrm{R}\right)=\frac{60000}{2 \times 20.285 \times 115758}\left(\mathrm{I}-\mathrm{e}^{.0816 \times 20.285} \cos 0.0816 \times 20.285\right) \\
& =0.013^{\prime \prime}
\end{aligned}
$$

Then

$$
\mathrm{S}=\frac{\mathrm{M}_{u} \sigma_{\mathrm{u}} \mathrm{A}}{\mathrm{W} \delta_{\mathrm{c}}}=\frac{168618 \times 4000 \times 1292.7}{60000^{2} \times 0.013}=18,656
$$

The force-deformation curve is obtained by interpolating the data shown on Figure 14 of EPRI report (reference 1) and is shown on figure B4.5A-1. The peak force is $25 \mathrm{G}$ (times weight) and the displacement at the end of elastic phase is $0.39^{\prime \prime}$.

We now use energy method to compute final deformation:

Let $x=$ final deformation. Then energy absorbed by the target equals area under force vs. displacement curve to $\mathrm{x}$

$$
\begin{aligned}
& E_{a b}=W F(0.39 / 2+(x-0.39)) \\
& W=60,000 \mathrm{lbs} \\
& F=25
\end{aligned}
$$

The potential energy of the drop is

$$
\mathrm{E}_{\text {drop }}=\mathrm{W}(\mathrm{H}+\mathrm{x}) \quad \mathrm{H}=\text { drop height }
$$

For 1 foot (12") drop

$$
\begin{aligned}
& W(12+x)=W(25)(0.39 / 2+x-0.39) \\
& x=0.7031 "
\end{aligned}
$$

The total energy is

$$
\begin{aligned}
& E_{d r o p}=W(H+x)=60000(12+0.7031)=762,186 \text { in-lbs } \\
& E_{a b}=W F(0.39 / 2+x-0.39)=60000(25)(0.39 / 2+0.7031-0.39)=762,150 \text { in-lbs }
\end{aligned}
$$


For 30 foot (360") drop

$$
\begin{aligned}
& \mathrm{W}(360+\mathrm{x})=\mathrm{W}(25)(0.39 / 2+\mathrm{x}-0.39) \\
& \mathrm{x}=15.203^{\prime \prime}
\end{aligned}
$$

The total energy is

$$
\begin{aligned}
& E_{\text {drop }}=W(H+x)=60000(360+15.203)=22,512,180 \text { in-lbs } \\
& E_{a b}=W F(0.39 / 2+x-0.39)=60000(25)(0.39 / 2+15.203-0.39)=22,512,000 \text { in-lbs }
\end{aligned}
$$

The results of end drop:

1 foot Drop Peak Acceleration $=25 \mathrm{G}$

Target Displacement $=0.7031 "$

30 foot Drop Peak Acceleration $=25 \mathrm{G}$

Target Displacement $=15.2^{\prime \prime}$ 
HNF-SD-SNF-FRD-003 Rev, 0

E-15166

Figure B4.5A-1

Force vs. Displacement Curves

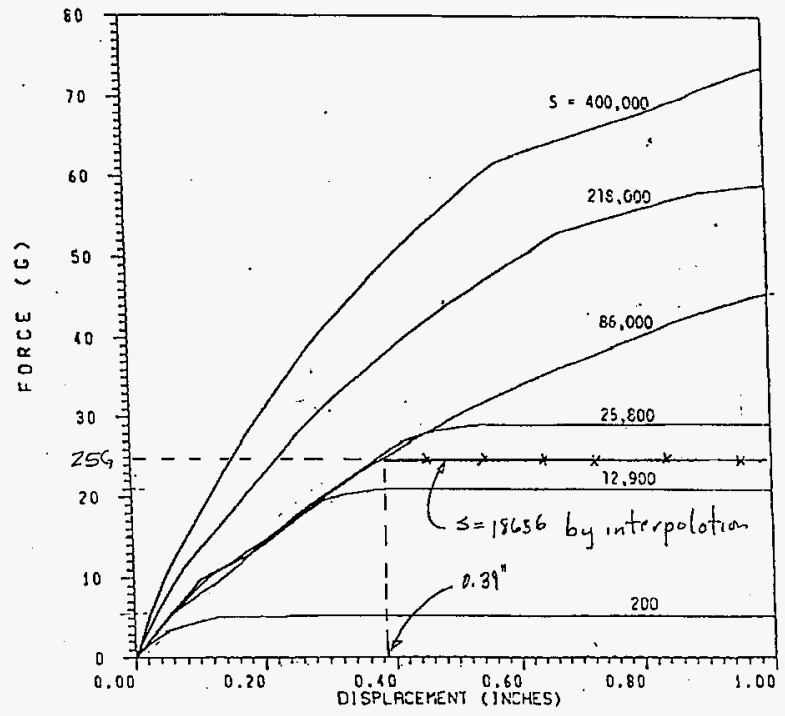




\section{B4.5A-5 Side Drop}

The side drop analysis is conducted in the same manner as for the end drop except that the expression for $\delta_{\mathrm{e}}$ varies and the target impact area changes as the depth of the penetration increase. $\delta_{\mathrm{e}}$ is given as:

$$
\delta_{e}=\frac{W B}{2 k}
$$

Where

$$
\begin{aligned}
& B=\frac{E_{s}{ }^{1 / 4}}{-E_{c} I_{c}} \\
& I_{c}=1 / 12 \mathrm{~L} \mathrm{~h}^{3}=1 / 12(168.5)(8)^{3}=7189 \mathrm{in}^{4} \\
& \beta=\left(\frac{28,000}{4 \times 3.6 \times 10^{6} \times 7189}\right)^{1 / 4}=0.0228 \\
& \mathrm{k}=\mathrm{E}_{\mathrm{s}}=28,000 \mathrm{psi} \\
& \text { W B } \\
& \delta_{\mathrm{e}}=-1 \mathrm{k}=60000 \times 0.0228 /(2 \times 28000)=0.0244^{\prime \prime} \\
& S=\frac{M_{u} \sigma_{u} A}{W^{2} B}=\frac{(168618)(4,000) A}{(60,000)^{2}(0.0244)} \\
& =7.678 \mathrm{~A}
\end{aligned}
$$

Then

The impact area $\mathrm{A}=\mathrm{L} \times \mathrm{d}$, where d depends on the deformation $\mathrm{x}$ and is shown on Table B4.5A-3. 
Table B4.5A-3

Side Drop Area Calculation

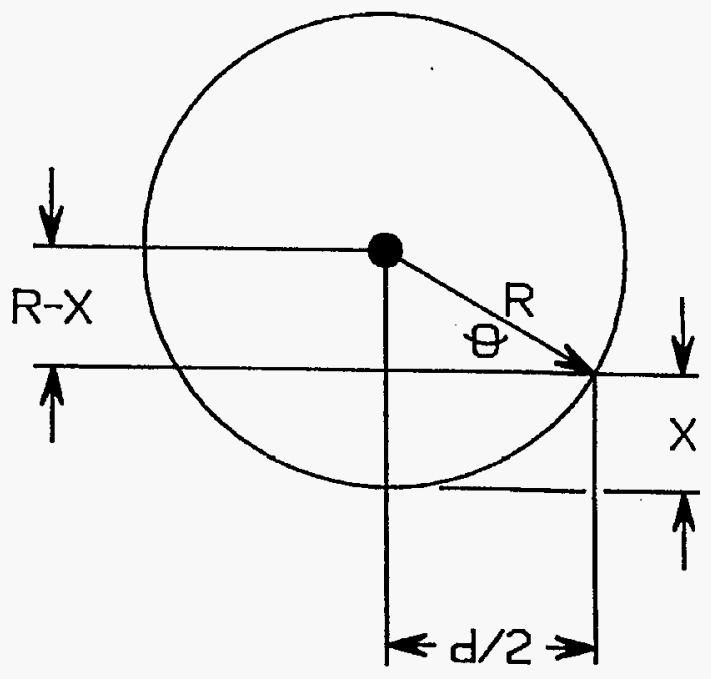

$$
\begin{aligned}
& \mathrm{SIN} \theta=(\mathrm{R}-\mathrm{X}) / \mathrm{R} \quad \theta=\mathrm{SIN}^{-1}(\mathrm{R}-\mathrm{X}) / \mathrm{R} \\
& \mathrm{R}^{2}=(\mathrm{R}-\mathrm{X})^{2}+(\mathrm{d} / 2)^{2} \\
& \mathrm{~d} / 2=\left(2 \mathrm{RX}-\mathrm{X}^{2}\right)^{1 / 2} \quad \mathrm{~d}=2\left(2 \mathrm{RX}-\mathrm{X}^{2}\right)^{1 / 2} \\
& \mathrm{~A}=\mathrm{L} \times \mathrm{d}=2 \mathrm{~L}\left(2 \mathrm{RX}-\mathrm{X}^{2}\right)^{1 / 2} \\
& \mathrm{~S}=7.678 \mathrm{~A}=7.678(2)(\mathrm{L})\left(2 \mathrm{RX}-\mathrm{X}^{2}\right)^{1 / 2}=15.356(\mathrm{~L})\left(2 \mathrm{RX}-\mathrm{X}^{2}\right)^{1 / 2}
\end{aligned}
$$


From Table B4.5A-3, $\mathrm{S}=15.356(\mathrm{~L})\left(2 \mathrm{Rx}-\mathrm{x}^{2}\right)^{1 / 2}$. Since the elastic portion of the force vs. deflection curves are shown to be unimportant, only the perfectly plastic part is used for the side drop analysis.

The following data is obtained from Figures 14 and 16 of the EPRI report (reference 1).

\begin{tabular}{||l|l|l|}
\hline S - Hardness & F - Force & Remark \\
\hline 12,900 & $21 \mathrm{G}$ & Figure 14 \\
\hline 25,800 & $29 \mathrm{G}$ & Figure 14 \\
\hline 86,000 & $48.5 \mathrm{G}$ & Figure 16 \\
\hline
\end{tabular}

These are plotted in the Figure B4.5A-2. A spread sheet solution is carried out to increment $x$, and calculate absorbed energy with solution selected at the point where absorbed energy equals $\mathrm{W}(\mathrm{H}+\mathrm{x})$.

The following steps are carried out in calculation of absorbed energy:

1. Select deformation $\mathrm{x}$

2. Calculate $\mathrm{S}, \mathrm{S}=15.356(\mathrm{~L})\left(2 \mathrm{Rx}-\mathrm{x}^{2}\right)^{1 / 2}$.

3. Calculate $\mathrm{F}, \mathrm{F}=\llbracket 18+\mathrm{S}(48.7-18) / 80000 \rrbracket \mathrm{W}$

4. Calculate energy increment $\Delta E, \Delta E=\left(x_{i}-x_{i-1}\right)\left(F_{i}+F_{i-1}\right) / 2$

5. Add $\Delta E$ to the previous energy to obtain current total energy

6. Increment $x$ until total energy equals $W(H+x)$

The spread sheet results are given on Table B4.5A-4.

\section{For 1 foot (12") drop}

Let $x=1 "$

Potential Energy $=W(H+1)=60000(12+1)=780,000$ in-lbs

$\mathrm{E}_{\text {absorbed by the target }}=1,037,026$ in-lbs (from Table A-4) $>780,000$ in-lbs

$\mathrm{G}=\mathrm{F} / \mathrm{W}=1,454,766 / 60,000=24 \mathrm{G}$ 's

For 30 foot (360") drop

Let $\mathrm{x}=12.5^{\prime \prime}$

Potential Energy $=W(H+12.5)=60000(360+12.5)=22,320,000$ in-lbs

$E_{\text {absorthed by the target }}=23,284,539 \mathrm{in}-1$ bs (from Table A-4) $>22,320,000$ in-lbs

$\mathrm{G}=\mathrm{F} / \mathrm{W}=2,195,973 / 60,000=36.6 \mathrm{G}$ 's 
HNF-SD-SNF-FRD-003 Rev 。

E-15166

Figure B4.5A-2

Force vs. Target Hardness Curve

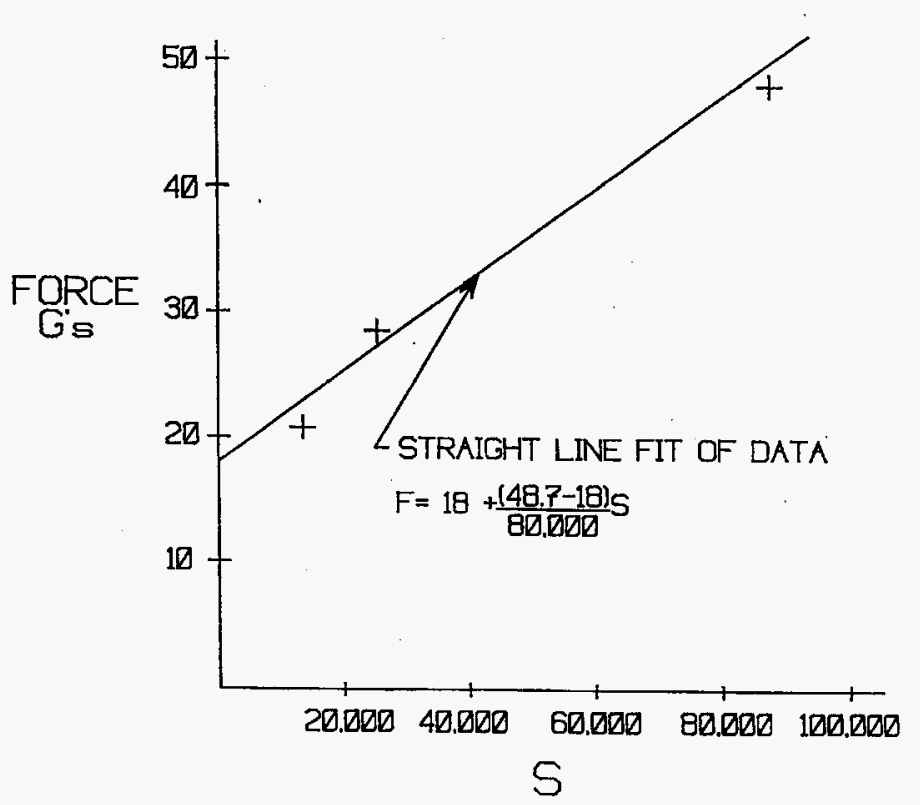


HNF-SD-SNF-FRD-003 ReV. 0

E-15166

Table B4.5A-4

Calculation of Absorbed Energy for Side Drop

\begin{tabular}{|c|c|c|c|c|c|}
\hline $\begin{array}{c}x \\
\text { (inches) }\end{array}$ & $\begin{array}{c}\mathrm{d} \\
\text { (inches) }\end{array}$ & $\bar{s}$ & $\begin{array}{l}\text { Force } \\
\text { (lbs) }\end{array}$ & $\begin{array}{c}\text { Energy Iner } \\
\text { (in-lbs) }\end{array}$ & $\begin{array}{l}\text { Toul Encrey } \\
\text { (in-lbs) }\end{array}$ \\
\hline 0 & 0.00 & 0 & 0 & & 0 \\
\hline 0.5 & 8.95 & 11,582 & $1,346,669$ & 336,667 & 336,667 \\
\hline I & 12.58 & 16,277 & $1,454,766$ & 700,359 & $1,037,026$ \\
\hline 1.5 & 15.31 & 19,808 & $1,536,084$ & 747,713 & $1,784,739$ \\
\hline 2 & 17.57 & 22,726 & $1,603,260$ & 784,836 & $2,569,575$ \\
\hline 2.5 & 19.51 & 25,243 & $1,661,218$ & 816,119 & $3,385,694$ \\
\hline 3 & 21.23 & 27.470 & $1,712,498$ & 843,429 & 4.229 .123 \\
\hline 3.5 & 22.78 & 29,473 & $1,758,614$ & 867,778 & $5,096,901$ \\
\hline 4 & 24.19 & 31,295 & $1,800,560$ & 889,794 & $5,986,695$ \\
\hline 4.5 & 25.48 & 32,965 & $1,839,027$ & 909,897 & $6,896,591$ \\
\hline 5 & 26.67 & 34,507 & $1,874,520$ & 928,387 & $7,824,978$ \\
\hline 5.5 & 27.78 & 35,936 & . $1,907,422$ & 945,485 & $8,770,463$ \\
\hline 6 & 28.80 & 37,265 & $1,938,031$ & 961,363 & $9,731,8 \geq 6$ \\
\hline 6.5 & 29.76 & 38,505 & $1,966,585$ & 976,154 & $10,707,980$ \\
\hline 7 & 30.66 & 39,665 & $1,993,277$ & 989,965 & $11,697,946$ \\
\hline 7.5 & 31.50 & 40,750 & $2,018,265$ & $1,002,885$ & $12,700,831$ \\
\hline 8 & 32.28 & 41,767 & $2,041,682$ & $1,014,987$ & $13,715,818$ \\
\hline 8.5 & 33.02 & 42,721 & $2,063,641$ & $1,026,331$ & $14,742,148$ \\
\hline 9 & 33.71 & 43,615 & $2,084,237$ & $1,036,969$ & $15,779,118$ \\
\hline 9.5 & 34.36 & 44,454 & $2,103,552$ & $1,046,947$ & $16,826,065$ \\
\hline 10 & 34.97 & 45,240 & $2,121,659$ & $1,056,303$ & $17,882,368$ \\
\hline 10.5 & 35.54 & 45,977 & $2,138,617$ & $1,065,069$ & $18,947,437$ \\
\hline 11 & 36.07 & 46,666 & $2,154,483$ & $1,073,275$ & $20,020,712$ \\
\hline 11.5 & 36.57 & 17,310 & $2,169,304$ & $1,080,9417$ & $21,101,659$ \\
\hline 12 & 37.03 & 47,910 & $2,183,122$ & $1,088,106$ & $22,189,765$ \\
\hline 12.5 & 37.46 & 48,468 & $2,195,973$ & $1,094,774$ & $23,284,539$ \\
\hline 13 & 37.86 & 48,986 & $2,207,892$ & $1,100,960$ & $24,385,505$ \\
\hline 13.5 & 38.23 & 49,464 & $2,218,908$ & $1,106,700$ & $25,492,206$ \\
\hline 14 & 38.57 & 49,904 & $2,229,046$ & $1,111,989$ & $26,604,194$ \\
\hline 14.5 & 38.89 & 50,307 & $2,238,330$ & $1,116,844$ & $27,721,038$ \\
\hline 15 & 39.17 & 50,674 & $2,246,779$ & $1,121,277$ & $28,842,315$ \\
\hline 15.5 & 39.43 & 51,006 & $2,254,412$ & $1,125,298$ & $29,967,613$ \\
\hline 16 & 39.65 & 51,303 & $2,261,246$ & $1,128,915$ & $31,096,528$ \\
\hline 16.5 & 39.86 & 51,565 & $2,267,292$ & $1,132,134$ & $32,228,662$ \\
\hline 17 & 40.03 & 51,794 & $2,272,565$ & $1,134,964$ & $33,363,626$ \\
\hline
\end{tabular}




\section{B4.5A-6 C.G. Over Corner Drop}

The C. G. over corner drop is performed in a similar manner as the side drop: Calculation of $\delta_{\mathrm{e}}$ and evaluation of the impact area as a function of the deformation into the target. Some geometry relations used to evaluate of the impact area as a function of the deformation into the target are shown in Figure B4.5A-3. Table B4.5A-5 tabulates the results of the area vs. the deformation of the target. The next quantity that is needed is the deflection, $\delta_{\mathrm{e}}$. This deflection will occur as a result of the cask resting on a small portion of the cask with the area increasing as $\delta$ increases. This is similar to the side drop condition and we use that as the basis for the evaluation. Figure B4.5A-4 illustrates this relations. From page B4.5A-8:

$$
\delta_{\mathrm{e}}=\frac{\mathrm{WB}}{-\cdot 2 \mathrm{k}}
$$

Where

$$
\begin{aligned}
& B=\frac{E_{s}{ }^{1 / 4}}{4 E_{c} I_{c}} \\
& I_{c}=1 / 12 L^{3}=1 / 12(L)(8)^{3}=42.6 \mathrm{~L} \\
& \beta=\left(\frac{28,000}{4 \times 3.6 \times 10^{6} \times I_{c}}\right]^{1 / 4}=0.21 /\left(I_{c}\right)^{3 / 4} \\
& \mathrm{k}=\mathrm{E}_{\mathrm{s}}=28,000 \mathrm{psi} \\
& \delta_{c}=W \beta / 2 \mathrm{k}=60000 \times \beta / 2 \times 28000=1.071 \beta \\
& \mathrm{L}=2 \llbracket 2 \mathrm{R} \delta_{\mathrm{e}} / \cos \theta-\delta_{\mathrm{c}}{ }^{2} / \cos ^{2} \Theta \rrbracket^{1 / 2}=2\left(173.376 \delta_{\mathrm{e}}-\delta_{\mathrm{e}}{ }^{2} / 0.0548\right)^{1 / 2}
\end{aligned}
$$

This equation is solved iteratively

\begin{tabular}{|c|c|c|c|}
\hline $\mathrm{L}_{\text {initial }}$ & $B$ & $\delta_{\mathfrak{c}}$ & $\mathrm{L}_{\text {final }}$ \\
\hline 10 & 0.0462 & 0.0495 & 5.84 \\
\hline 8 & 0.0489 & 0.0523 & 6.00 \\
\hline 6.5 & 0.0515 & 0.055 & 6.16 \\
\hline 6.2 & 0.0521 & 0.056 & 6.20 (solution) \\
\hline
\end{tabular}


From the above table, the solution of $\delta_{\mathrm{e}}=0.056$, then

$\mathrm{S}=\mathrm{M}_{\mathrm{u}} \sigma_{\mathrm{u}} \mathrm{A} / \mathrm{W}^{2} \delta_{\mathrm{e}}=168618 \times 40000 \times \mathrm{A} / 60000^{2} \times 0.056=3.346 \mathrm{~A}$

The solution is obtained on spread sheet by the following steps and is shown on Table B4.5A-5:

1. Calculate $\mathrm{S}, \mathrm{S}=3.346 \mathrm{~A}$ (reference Table $\mathrm{B} 4.5 \mathrm{~A}-5$ for value of $\mathrm{A}$ )

2. Calculate $F, F=\llbracket 18+S(48.7-18) / 80000 \rrbracket W$

3. Calculate energy increment $\Delta E, \Delta E=\left(x_{i}-x_{i-1}\right)\left(F_{i}+F_{i-1}\right) / 2$

5. Add $\Delta \mathrm{E}$ to the previous energy to obtain current total energy

6. Increment $x$ until total energy equals $W(H+x)$

For 1 foot (12") drop

Let $\mathrm{x}=1^{\prime \prime}$ (reference Table B4.5A-6)

Potential Energy $=W(H+1)=60000(12+1)=780,000$ in-lbs

$\mathrm{E}_{\text {absorbed by the target }}=812,430$ in-lbs $>780,000$ in-lbs

$\mathrm{G}=\mathrm{F} / \mathrm{W}=1,085,654 / 60,000=18.09 \mathrm{G}$ 's

\section{For 30 foot $\left(360^{\prime \prime}\right)$ drop}

Let $x=20 "$ (reference Table B4.5A-6)

Potential Energy $=\mathrm{W}(\mathrm{H}+20)=60000(360+20)=22,800,000$ in-lbs

$\mathrm{E}_{\text {absorbed by the target }}=22,865,514$ in-lbs $>22,800,000$ in-lbs

$\mathrm{G}=\mathrm{F} / \mathrm{W}=1,180,536 / 60,000=19.7 \mathrm{G}$ 's 
Figure B4.5A-3

Geometry of CG Over Corner Drop

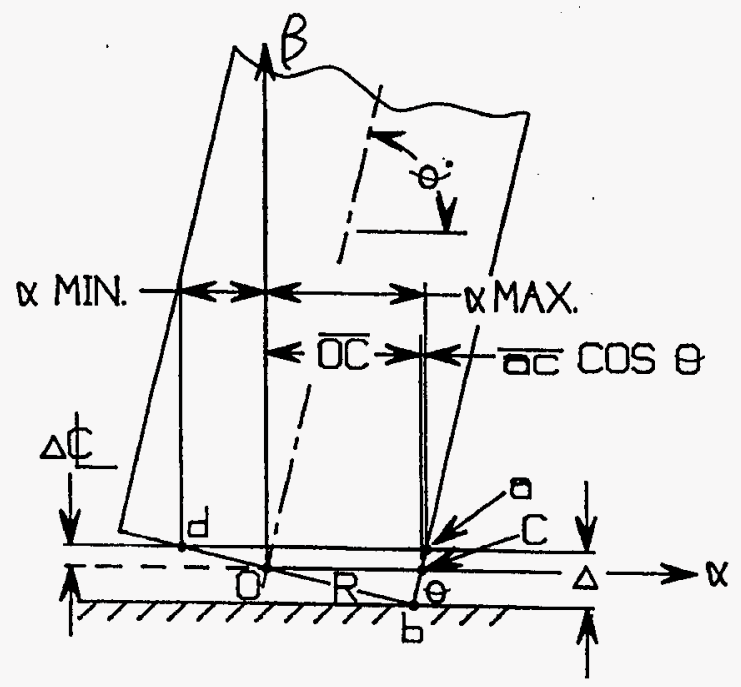

SIN $\theta=R /$ oc

$\cos \theta=b c / o c$

$\ominus=76.46^{\circ}$

$b c=o c \operatorname{Cos} \theta=R(\cos \theta / \operatorname{SIN} \theta)$

$\mathrm{ac}=\mathrm{ab}-\mathrm{bc}=\Delta / \operatorname{SIN} \theta-\mathrm{R}(\operatorname{COS} \theta / \operatorname{SIN} \theta)$

$a d=a b / \operatorname{COS} \theta=\Delta /$ SIN $\theta \operatorname{COS} \theta$

$\Delta_{s}=\Delta-\operatorname{bcSIN} \theta=\Delta-\operatorname{RCOS} \theta$

$\alpha_{\text {max }}=o c+\operatorname{acCOS} \theta=\mathrm{R} / \mathrm{SIN} \theta+\operatorname{acCOS} \theta=\mathrm{R} / \mathrm{SIN} \theta+\Delta_{\star}(\operatorname{COS} \theta / \operatorname{SIN} \theta)$

$\alpha_{\text {min }}=\operatorname{ad}-\alpha_{\max }=\Delta / \operatorname{SIN} \theta \operatorname{COS} \theta-\operatorname{R} / \operatorname{SIN} \theta-\Delta_{\star}(\operatorname{COS} \theta / \operatorname{SIN} \theta)$

$=\Delta_{\star} \quad(1 / \operatorname{SIN} \theta \operatorname{COS} \theta-\operatorname{COS} \theta / \operatorname{SIN} \theta)$ 


\section{Figure B4.5A-3 \\ Geometry of CG Over Corner Drop-Continue}

The area of the impact surface is obtained by first writing equation for intersection curves between cylinder and plane surface. We set up the following coordinate systems with origin at bottom center of cask.

By transforming coordinates:

$$
\begin{array}{ll}
\alpha=X \operatorname{XIN} \theta+Z \operatorname{COS} \theta & X=\alpha \operatorname{SIN} \theta-\beta \operatorname{COS} \theta \\
\beta=-X \operatorname{COS} \theta+Z \operatorname{SIN} \theta & Z=\alpha \operatorname{Cos} \theta+\beta \operatorname{SIN} \theta
\end{array}
$$

Equation of cylinder is $\mathrm{X}^{2}+\mathrm{Y}^{2}=\mathrm{R}^{2}$ or by transforming coordinates:

$\alpha^{2} \operatorname{SIN} \theta^{2}-2 \alpha \beta \operatorname{SIN} \theta \operatorname{COS} \theta+\beta^{2} \operatorname{COS} \theta^{2}+Y^{2}=R^{2}$

By setting the intersection of this surface with target surface, $\beta=\Delta_{\Phi}$

Then equation of intersection curve is:

$\alpha^{2} \operatorname{SIN} \theta^{2}-2 \alpha \Delta_{t} \operatorname{SIN} \theta \operatorname{COS} \theta+\Delta_{\leftarrow}{ }^{2} \operatorname{COS} \theta^{2}+\mathrm{Y}^{2}=\mathrm{R}^{2}$

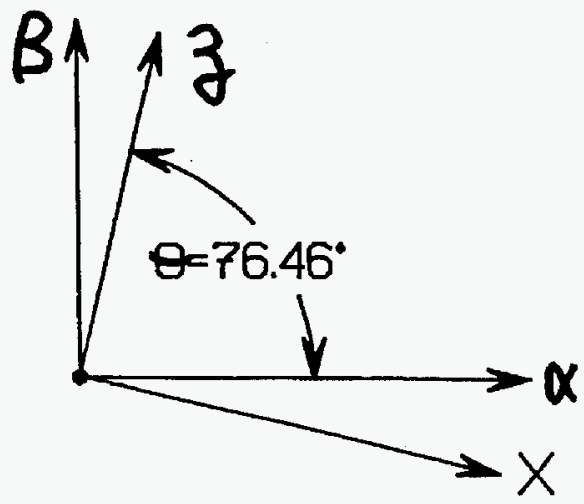




\section{HNF-SD-SNF-FRD-003 Rev. 0 \\ Figure B4.5A-3 \\ Geometry of CG Over Corner Drop-Continue}

E-15166

The area as a function of the deformation is calculated by integrating the:

$\alpha^{2} \operatorname{SIN} \theta^{2}-2 \alpha_{\star} \operatorname{SIN} \theta \operatorname{COS} \theta+\Delta_{\leftarrow}{ }^{2} \operatorname{COS} \theta^{2}+\mathrm{Y}^{2}=\mathrm{R}^{2}$

Area $A=2 \int_{\alpha_{\min }}^{\alpha_{\max }} Y \mathrm{~d} \alpha$

Where $\mathrm{Y}$ is given as above equation

This is numerically integrated using 100 divisions and the trapezoidal rule. The results are tabulated in Table B4.5A-5.

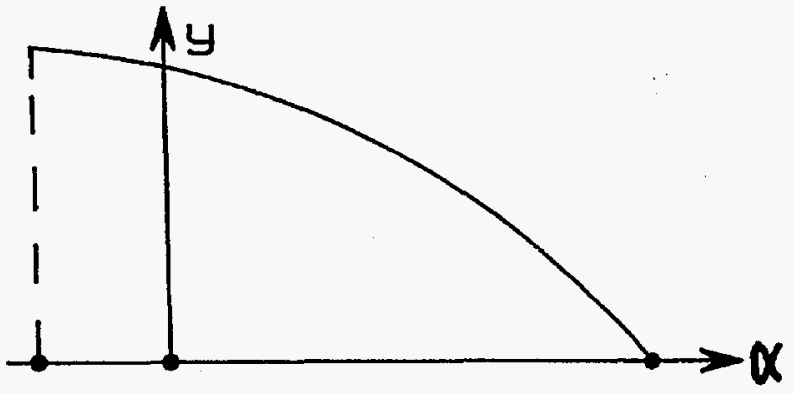


Figure B4.5A-4

CG Over Corner Drop - L Dimension Calculation

$$
(\mathrm{L} / 2)^{2}=\mathrm{R}^{2}-\left(\mathrm{R}-\delta_{\mathrm{e}} / \mathrm{COS} \theta\right)^{2} \quad \mathrm{~L}=2\left(2 \mathrm{R} \delta_{\mathrm{e}} / \operatorname{COS} \theta-\delta_{\mathrm{e}}{ }^{2} / \operatorname{Cos}^{2} \theta\right)^{1 / 2}
$$
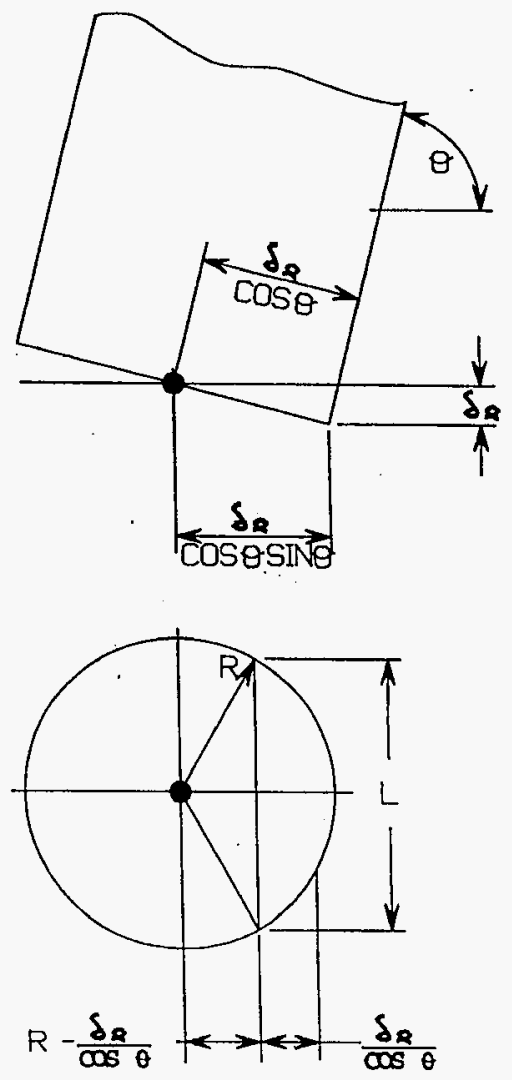
Table B4.5A-5

Area Calculation For CG Over Corner Drop

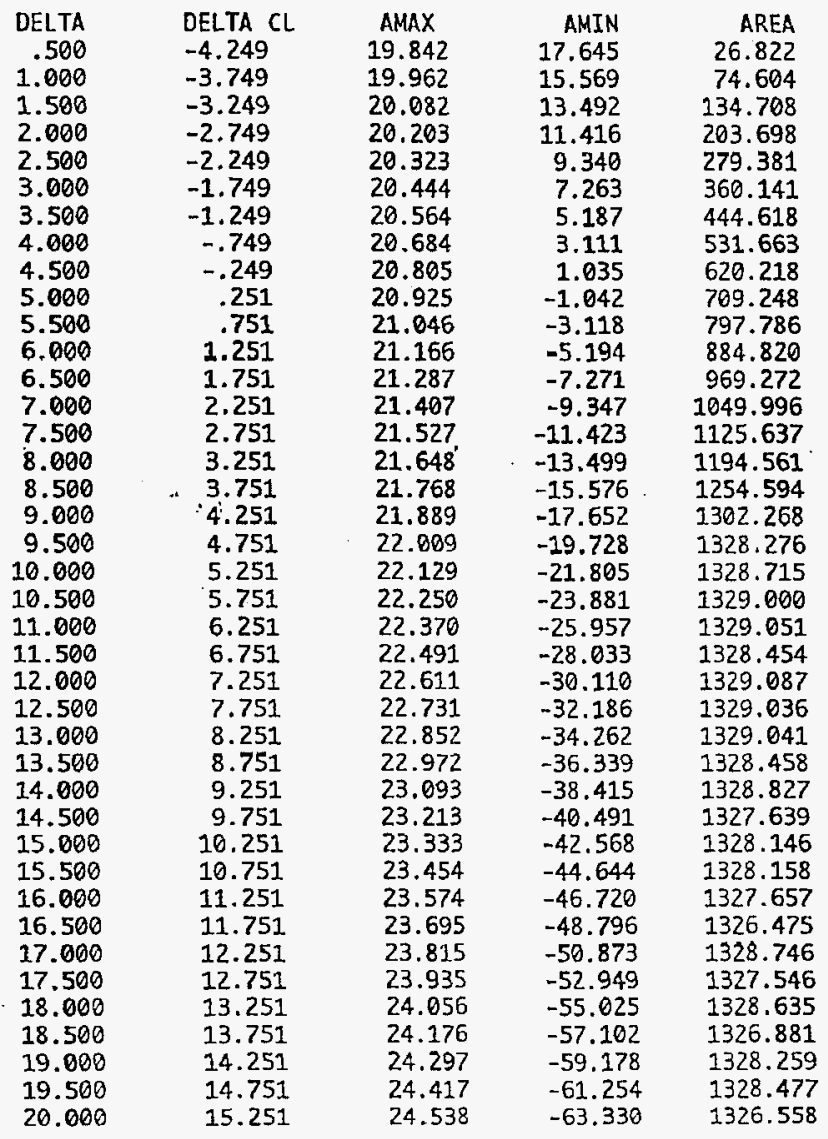


Table B4.5A-6

Calculation of Absorbed Energy for CG Over Corner Drop

\begin{tabular}{|c|c|c|c|c|c|}
\hline $\begin{array}{c}x \\
\text { (inclies) }\end{array}$ & $\begin{array}{l}\text { Area } \\
\text { (sq in) }\end{array}$ & $\bar{s}$ & $\begin{array}{l}\text { Furce } \\
\text { (las) }\end{array}$ & $\begin{array}{c}\text { Encrgy incr } \\
\text { (in-lbs) }\end{array}$ & $\begin{array}{c}\text { Total Encresy } \\
\text { (in-lbs) }\end{array}$ \\
\hline 0.5 & 26.82 & 90 & $1,082,033$ & 270.508 & 270.508 \\
\hline 1 & 74.60 & 250 & $1.085,654$ & 541.922 & 812.430 \\
\hline 1.5 & 13.7 .71 & 451 & $1,090,209$ & $543,9 \times 6$ & $1,3562,396$ \\
\hline 2 & 203.70 & 682 & $1,095,438$ & 546,412 & $1,902,807$ \\
\hline 2.5 & 279.38 & 935 & $1,101,173$ & 549.153 & $2,451,960$ \\
\hline 3 & 360.14 & 1,205 & $1,107,294$ & 552.117 & 3.004 .077 \\
\hline 3.5 & 411.62 & 1.488 & $1,113,696$ & $555,2+8$ & $3,559,32,5$ \\
\hline 4 & $\$ 31.60$ & 1,779 & $1,120,293$ & 558,497 & $4,117,822$ \\
\hline 4.5 & 620.22 & 2.075 & $1,127,004$ & 561,824 & $4,679,6-46$ \\
\hline 5 & 709.25 & 2.373 & $1.133,752$ & 565,189 & $5,241,835$ \\
\hline 5.5 & 797.79 & 2,669 & $1,140,462$ & 568,553 & $5,813,389$ \\
\hline 6 & 884.82 & 2,261 & $1,147,058$ & 571,880 & $6,385,268$ \\
\hline 6.5 & 969.27 & 3,243 & $1,153,458$ & 575,129 & $6,960,397$ \\
\hline 7 & $1,050.00$ & 3,513 & $1,159,576$ & 578,259 & $7,538,656$ \\
\hline 7.5 & $1,125.64$ & 3.766 & $\therefore 1,165,309$ & 581,221 & $8,119,877$ \\
\hline 8 & $1,194.56$ & 3,997 & $1,170,532$ & 583,960 & $8,703,837$ \\
\hline 8.5 & $1,254.50$ & 4,198 & $1,175,082$ & 586,403 & $9,290,241$ \\
\hline 9 & 1.302 .27 & 4,357 & $1,178,695$ & 588,441 & $9,878,685$ \\
\hline 9.5 & $1,328.28$ & 4,414 & $1,180,666$ & 589,840 & $10,468,525$ \\
\hline 10 & $1,328.72$ & 4,446 & $1,180,699$ & 550,341 & $11,058,866$ \\
\hline 10.5 & $1,329.00$ & 4,447 & $1,180,721$ & 590,355 & $11,6-69,221$ \\
\hline 11 & $1,329.05$ & 4,447 & $1,180,725$ & 590,361 & $12,239,583$ \\
\hline 11.5 & $1,328.45$ & 4,445 & $1,180,679$ & 590,351 & $12,829,934$ \\
\hline 12 & $1,329.09$ & 4,417 & $1.180,727$ & 590,352 & $13,420,285$ \\
\hline 12.5 & $1,329.04$ & 4,417 & $1,180,724$ & 500,363 & $14,010,648$ \\
\hline 13 & $1,329.04$ & 4,447 & $1.180,724$ & 590,362 & $14,601,010$ \\
\hline 13.5 & $1,328.46$ & 4,415 & $1,180,680$ & 590,351 & 15.191 .361 \\
\hline 14 & $1,328.83$ & 4,416 & $1,180,708$ & $590,3+7$ & $15,781,708$ \\
\hline 14.5 & $1,327.64$ & 4,442 & $1,180,618$ & 590,331 & $16,372,039$ \\
\hline 15 & $1,328.15$ & 4,414 & $1,180,656$ & 590,313 & $16,962,357$ \\
\hline 15.5 & $1,328.16$ & 4,414 & $1,180,657$ & 590,328 & $17,552,686$ \\
\hline 16 & $1,327.66$ & 4,442 & $1,180,619$ & $5 \times 0.319$ & $18,143,005$ \\
\hline 16.5 & $1,326.48$ & 4.438 & $1,180,529$ & 590.287 & $18,733,292$ \\
\hline 17 & $1,328.75$ & 4,446 & $1,180,702$ & 520,308 & $19,323,600$ \\
\hline 17.5 & $1,327.55$ & 4,442 & $1,180,611$ & 590,328 & $19,913,928$ \\
\hline 18 & $1,328.64$ & 4,446 & $1,180,693$ & 590,326 & $20,504,254$ \\
\hline 18.5 & $1,326.88$ & 4,440 & $1,180,560$ & 500,313 & $21,094,567$ \\
\hline 10 & $1,328.26$ & $4,4+4$ & $1,180,605$ & 520,306 & $21,684,873$ \\
\hline 19.5 & 1.328 .48 & 4,445 & $1,180,681$ & 590,336 & $22,275,210$ \\
\hline 20 & $1,326.56$ & 4,439 & $1,180,536$ & 590,304 & $22,865,514$ \\
\hline
\end{tabular}




\section{B4.5A-7 SHALLOW ANGLE IMPACT - SLAP DOWN}

The energy balances used for the end, side, and corner drop cases cannot be applied to the slapdown cases since the kinetic energy in the cask is now divided between vertical velocity and rotational velocity. For the first three cases, there is a simple energy transfer between the kinetic energy (due to only vertical velocity) and the energy absorbed in the target. For the slapdown case, the additional possibitily of transferring energy to the cask rotational velocity requires that a time history analysis be performed. This is possible, but the numerical procedures required are complex and one questions whether the target model presented in the EPRI report contains adequate detail for such a use. Rather slapdown data for transport casks (rigid cask with limiters impacting on a rigid target) are used to develop reasonable upper bounds for the slapdown effect.

Larger deceleration forces than found for the side drop can occur when a cask with impact limiters strikes a rigid target with the cask oriented at a small angle to the horizontal. This could occur for the problem of interest here and is discussed in this section. Some of the results found for the transport casks with limiters are discussed and results are then applied to the present problem.

When the first end of the cask impacts the target (designated end 1 for the purposes of this discussion with the other end designated end 2), the kinetic energy of the cask results from the vertical velocity of the cask. After this impact this energy is partitioned into three parts: the vertical cask velocity, the rotational velocity of the cask, and the energy absorbed by the impact limiter at this end of the cask. The vertical CG velocity of the cask decreases and the rotational velocity of the cask increases. Both of these effects reduce the vertical velocity of end 1 . However, the vertical velocity of end 2 is reduced by the decrease in cask CG velocity but increased by the increase in rotational velocity of the cask. The vertical velocity of end 2 therefore could be larger than the initial velocity of the cask, and in this case the impact force for the end 2 limiter would be larger than the force which occurs for the side drop (where the end 2 limiter impacts the target at the initial cask velocity). The magnitude of this effect and the drop angle causing the maximum slapdown effect is a function of the mass and geometric characteristics of the cask, and the stiffness characteristics of the impact limiter. A generic study of this problem for transport casks is report in reference 4 and the results of that study are presented in the Table B4.5A-7. The following definitions are given for the parameters in the table.

$\mathrm{R}_{\mathrm{l}}=$ Cask Radius

$\sigma_{\mathrm{III}}=$ Crush Strength of Limiter

$\mathrm{B}=$ Length of Limiter Along Cask axis

$\mathrm{L}=$ Cask Length

$W=$ Cask Weight

$\mathrm{A}=$ Length of Limiter Protruding Beyond End Cask

$\mathrm{H}=$ Drop Height 
HNF-SD-SNF-FRD-003 Rev. 0

Table B4.5A-7

Energy Absorbed By Second Limiter During Slapdown

\begin{tabular}{|c|c|c|c|c|c|}
\hline$R_{L} / L$ & $\sigma_{I I I} R_{L}{ }^{2} / w$ & $(B-A) / R_{L}$ & $\mathrm{H} / \mathrm{L}$ & $\begin{array}{l}\text { CRITICAL AKCXE } \\
\text { (Dogreos) }\end{array}$ & ENERGY RATIO (P) \\
\hline 0.25 & $\begin{array}{r}25 \\
50 \\
75 \\
100\end{array}$ & 0.25 & 1.75 & $\begin{array}{l}7.8 \\
3.0 \\
2.0 \\
1.5\end{array}$ & $\begin{array}{l}0.704 \\
0.721 \\
0.725 \\
0.725\end{array}$ \\
\hline 0.33 & $\begin{array}{r}25 \\
50 \\
75 \\
100\end{array}$ & 0.25 & 1.75 & $\begin{array}{l}6.9 \\
4.1 \\
2.4 \\
1.3\end{array}$ & $\begin{array}{l}0.682 \\
0.695 \\
0.702 \\
0.719\end{array}$ \\
\hline 0.50 & $\begin{array}{r}25 \\
50 \\
75 \\
100\end{array}$ & 0.25 & 1.75 & $\begin{array}{l}9.0 \\
4.4 \\
2.5 \\
2.5\end{array}$ & $\begin{array}{l}0.598 \\
0.637 \\
0.650 \\
0.650\end{array}$ \\
\hline 0.25 & $\begin{array}{r}25 \\
75 \\
100\end{array}$ & 0.5 & 1.75 & $\begin{array}{l}3.5 \\
1.0 \\
0.8\end{array}$ & $\begin{array}{l}0.693 \\
0.701\end{array}$ \\
\hline 0.33 & $\begin{array}{r}25 \\
75 \\
100\end{array}$ & ${ }_{1}^{0.5}$ & $1.75^{\circ}$ & $\begin{array}{l}0.8 \\
4.1 \\
1.0 \\
0.8\end{array}$ & $\begin{array}{l}0.705 \\
0.660 \\
0.684 \\
0.675\end{array}$ \\
\hline 0.50 & $\begin{array}{r}25 \\
75 \\
100\end{array}$ & 0.5 & 1.75 & $\begin{array}{l}4.0 \\
1.3 \\
0.9\end{array}$ & $\begin{array}{l}0.582 \\
0.603 \\
0.611\end{array}$ \\
\hline 0.25 & $\begin{array}{r}25 \\
50 \\
100\end{array}$ & 0.75 & 1.75 & $\begin{array}{l}2.2 \\
1.0 \\
0.5\end{array}$ & $\begin{array}{l}0.670 \\
0.678 \\
0.698\end{array}$ \\
\hline 0.33 & $\begin{array}{r}25 \\
75 \\
100\end{array}$ & 0.75 & 1.75 & $\begin{array}{l}2.4 \\
1.4 \\
0.6\end{array}$ & $\begin{array}{l}0.628 \\
0.634 \\
0.662\end{array}$ \\
\hline 0.50 & $\begin{array}{r}25 \\
75 \\
100\end{array}$ & 0.75 & 1.75 & $\begin{array}{l}2.8 \\
0.9 \\
0.7\end{array}$ & $\begin{array}{l}0.528 \\
0.549 \\
0.547\end{array}$ \\
\hline 0.25 & $\begin{array}{l}25 \\
75 \\
100\end{array}$ & 0.25 & 3.50 & $\begin{array}{r}15.0 \\
7.4 \\
6.0\end{array}$ & $\begin{array}{l}0.645 \\
0.686 \\
0.690\end{array}$ \\
\hline 0.50 & $\begin{array}{r}25 \\
75 \\
100\end{array}$ & 0.25 & 3.50 & $\begin{array}{l}\text { FIRST IMPAC } \\
5.6 \\
3.7\end{array}$ & $\begin{array}{c}\text { CT HORE SEYERE } \\
0.582 \\
0.598\end{array}$ \\
\hline 0.25 & $\begin{array}{r}25 \\
75 \\
100\end{array}$ & 0.75 & 3.50 & $\begin{array}{l}4.6 \\
2.1 \\
1.0\end{array}$ & $\begin{array}{l}0.638 \\
0.678 \\
0.656\end{array}$ \\
\hline 0.50 & $\begin{array}{r}25 \\
75 \\
100\end{array}$ & 0.75 & 3.50 & $\begin{array}{l}\text { FIRST IMPAC } \\
\text { FIRST IMPAC } \\
1.3\end{array}$ & $\begin{array}{c}\text { ACT MORE SEYERE } \\
\text { ACT MORE SEVERE } \\
0.502\end{array}$ \\
\hline
\end{tabular}


Table B4.5A-7 gives the critical angle for which maximum slapdown effects occur, and the energy ratio (ratio of energy absorbed by the second limiter for slapdown as compared with the side drop case). As may be seen the critical angle varies from close to 0 to 15 degree, and the maximum energy ratio equals to 0.725 . Note that the second limiter absorbs $50 \%$ of the energy for the side drop so that this maximum case indicates that the second limiter absorbs $0.725 / 0.5=$ 1.45 more energy than it would for the side drop case (a $45 \%$ increase). It was also found that the critical angle resulting in these maximum energy ratio led to the cask response where the end 1 lifted off the impact surface just before end 2 impacts the target. It can also be seen from the results in the table that the energy ratio increases as:

$$
\begin{aligned}
& \mathrm{R}_{\mathrm{L}} / \mathrm{L} \Rightarrow \text { decreases } \\
& \sigma_{11} \mathrm{R}_{\mathrm{L}}{ }^{2} / \mathrm{W} \Rightarrow \text { increases } \\
& (\mathrm{B}-\mathrm{A}) / \mathrm{R}_{\mathrm{L}} \Rightarrow \text { decreases } \\
& \mathrm{H} / \mathrm{L} \Rightarrow \text { decreases }
\end{aligned}
$$

The problem of the cask impacts to the concrete is somewhat similar to the transport casks with the energy absorption characteristics of the limiter being replaced by the energy absorption characteristics of the slab/soil target. The following parameters needed for the use of the table may be calculated directly:

$$
\begin{aligned}
& \mathrm{R}_{\mathrm{L}} / \mathrm{L}=20.285 / 168.5=0.12 \\
& \mathrm{H} / \mathrm{L}=360 / 168.5=2.14 \\
& \mathrm{~A}=0
\end{aligned}
$$

The length of the equivalent impact limiter ( B ) actually can be extend along the entire cask (as in the side drop). Since the slap down effect increases as this length decreases, the value of B is taken as equal to the width of the impact surface ( $\mathrm{d}$ defined in Table B4.5A-3). This is taken equal to 37.5" (the side drop value of $d$ at maximum values from the Table B4.5A-4). The same results are used to obtain an effective crush strength by dividing the total force of the side drop $(2,195,973 \mathrm{lbs})$ by the total impact area $(2 \times 37.5 \times 37.5)$. Therefore:

$$
\begin{aligned}
& \sigma_{I I I}=2,195,973 /(2 \times 37.5 \times 37.5)=781 \mathrm{psi} \\
& \sigma_{\text {III }} \mathrm{R}_{\mathrm{I}}{ }^{2} / \mathrm{W}=781 \times 20.285^{2} / 60,000=5.4 \\
& (\mathrm{~B}-\mathrm{A}) / \mathrm{R}_{\mathrm{L}}=(37.5-0) / 20.285=1.85
\end{aligned}
$$

The following conservative values (see above discussion of variation of energy ratio as a function of the parameters) are selected:

$$
\sigma_{\text {III }} \mathrm{R}_{\mathrm{L}}{ }^{2} \mathrm{~W}=25
$$




$$
\begin{aligned}
& (\mathrm{B}-\mathrm{A}) / \mathrm{R}_{\mathrm{L}}=0.75 \\
& \mathrm{H} / \mathrm{L}=1.75
\end{aligned}
$$

The results in the Table A- 6 then indicate the following results:

\begin{tabular}{|c|c|}
\hline $\mathrm{R}_{\mathrm{L}} / \mathrm{L}$ & Energy Ratio \\
\hline 0.25 & 0.67 \\
\hline 0.33 & 0.628 \\
\hline 0.5 & 0.528 \\
\hline
\end{tabular}

These data points fit very well with a straight line:

$$
\text { Energy Ratio }=0.815-0.575 \mathrm{R}_{\mathrm{L}} / \mathrm{L}
$$

Therefore the energy ratio for $R_{L} / L=0.12$ is equal to 0.746 . This indicates that the energy absorbed by the second end during impact could be $0.746 / 0.5=1.49$ times the energy absorbed by the side drop. If this were all taken up by an increase in force ( rather than partially by an increase in impact area) the peak deceleration would increase from the $36.6 \mathrm{G}$ for the side drop to $1.49 \times 36.6=54.4$ G.It should be noted that this deceleration would not be constant across the length of the cask but would decrease from end 2 to end 1 .

This estimate is quite conservative for the following reasons:

The parameters selected above were selected to maximize the effect. In particular the length of the impact surface (B) will increase as the cask rotate so that the most of the cask length will be engaged when the second end hits the target. The above analysis conservatively sets $\mathrm{B}=37.5$ and as shown in the following table, the energy ratio decreases as B increases.

\begin{tabular}{|c|c|}
\hline$(\mathrm{B}-\mathrm{A}) / \mathrm{R}_{\mathrm{L}}$ & Energy Ratio \\
\hline 0.25 & 0.704 \\
\hline 0.50 & 0.693 \\
\hline 0.75 & 0.670 \\
\hline
\end{tabular}

The side drop analysis shows that target hardness (S) is 7.678 times impact area. The largest impact area could be the length times diameter of the cask $(168.5 \times 40.57)=6836 \mathrm{in}^{2}$. Therefore the maximum $\mathrm{S}=52,487$. The maximum possible deceleration is equal to:

$$
\mathrm{G}=\llbracket 18+\mathrm{S}(48.7-18) / 80000 \rrbracket=\llbracket 18+52487(48.7-18) / 80000 \rrbracket=38.1 \mathrm{G}
$$

It is recommended that the maximum deceleration for the slapdown case be taken as $39 \mathrm{G}$ based on the maximum impact area. 


\section{B4.5A-8 REFERENCES}

1. "The Effects of Target Hardness on the Structural Design of Concrete Storage Pads for Spent- Fuel Casks"EPRI NP-4830, October 1986.

2. "Structural Design of Concrete Storage Pads for Spent-fuel Cask"EPRI NP-7551, August 1991.

3. "Design of Concrete Structures" By A. Nelson, Mcgraw-Hill, Inc. Eleventh Edition.

4. "Impact Loading on Waste Fuel Shipping Casks"By C.A. Miller. Transactions of the 9th International Conference on Structural Mechanics in Reactor Technology, August 1987. 
HNF-SD-SNF-FDR-003 Rev. 0

$$
E-15166
$$

\section{APPENDIX B4.5B}

LID BOLT ANALYSIS

B4.5B-O 
HNF-SD-SNF-FDR-003 Rev. 0

E-15166

\section{TABLE OF CONTENTS}

\section{Section}

B4.5B-1

B4.5B-2

B4.5B-3

B4.5B-4

B4.5B-5

B4.5B-6

B4.5B-7

B4.5B-8
INTRODUCTION

NORMAL CONDITION ANALYSIS

FATIGUE ANALYSIS

ACCIDENT CONDITION ANALYSIS

FIRE CONDITION ANALYSIS

CLOSURE FLANGE SHOULDER ANALYSIS

CONCLUSIONS

REFERENCES
Page

B4.5B-1

B4.5B-1

B4.5B-10

B4.5B-1 5

B4.5B-17

B4.5B-1 8

B4.5B-19

B4. 5B-20 


\section{APPENDIX B4.5B \\ LID BOLT ANALYSIS}

\section{B4.5B-1 INTRODUCTION}

The TN-WHC lid closure arrangement is shown in Figure B4.5B-1. The 3.5 inch thick lid is bolted directly to the end of the containment vessel body by 12 high strength stainless steel 1.5 inch diameter bolts. Close fitting alignment pins ensure that the lid is centered in the vessel.

The lid bolt is shown in Figure B4.5B-2. Note that the material is ASME SA-479, Type XM-19 Hot Rolled, which has a minimum yield strength of $105,000 \mathrm{psi}$ at room temperature. The lid closure flange and bolt arrangement is shown in Figure B4.5B-3. The bolts are designed to be preloaded at assembly to seat the seals against the 161.2 psi maximum design pressure and to withstand all normal and accident loadings without yielding. The lid bolt analysis performed below is in accordance with NUREG/CR-6007 stress analysis of Closure Bolts for Shipping Casks (Reference 1).

\section{B4.5B-2 NORMAL CONDITION ANALYSIS}

The loadings considered for normal conditions include operating preload, pressure loads, temperature, impact loads and the vibration loads.

The non-prying tensile bolt force due to the applied preload is given by the formula

$$
F_{\mathrm{a}}=\mathrm{Q} / \mathrm{KD}_{\mathrm{b}}
$$

where $Q \quad$ is the applied torque for preload, 3,565 in. Ibs.

$\mathrm{K}$ is the nut factor for empirical relation between the applied torque and the achieved preload, 0.1 , this value is based on lubricating the bolts with Neolube having a coefficient of friction of 0.03 .

$\mathrm{D}_{\mathrm{h}}$ is the minimum diameter of the closure bolt, 1.22 inch.

$F_{\text {a }}=3565 /(0.1)(1.22)=29,225 \mathrm{lbs}$

The torsional bolt moment per bolt is

$$
\mathrm{M}_{t}=0.5 \mathrm{Q}=0.5(3565)=1783 \text { in }-\mathrm{lbs}
$$

The maximum residual tensile bolt force after preload is

$$
F_{a r}=F_{a}=29,225 \mathrm{lbs}
$$


FIGURE B4.5B-1

TN-WHC LID CLOSURE
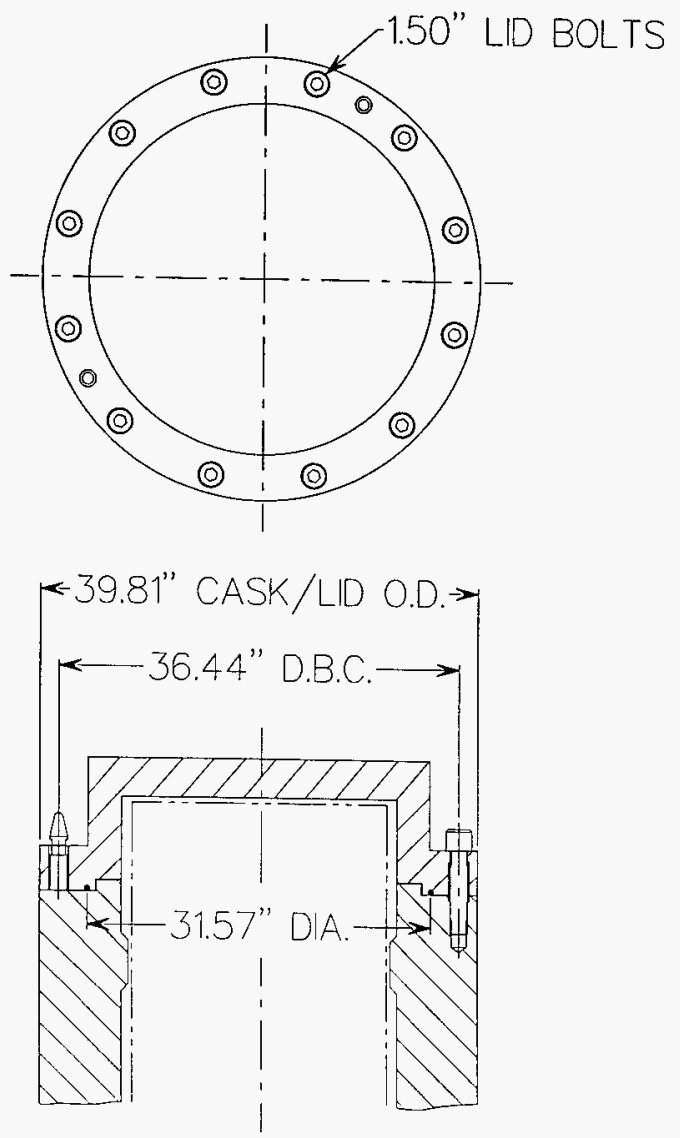
FIGURE B4.5B-2

TN-WHC LID BOLT

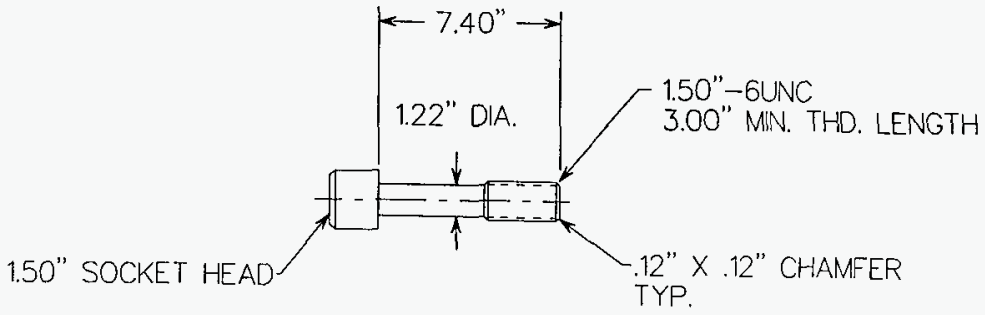

MATERIAL: SA-479, XM-19, HOT ROLLED 
FIGURE B4.5B-3

TN-WHC FLANGE AND LID BOLT ARRANGEMENT

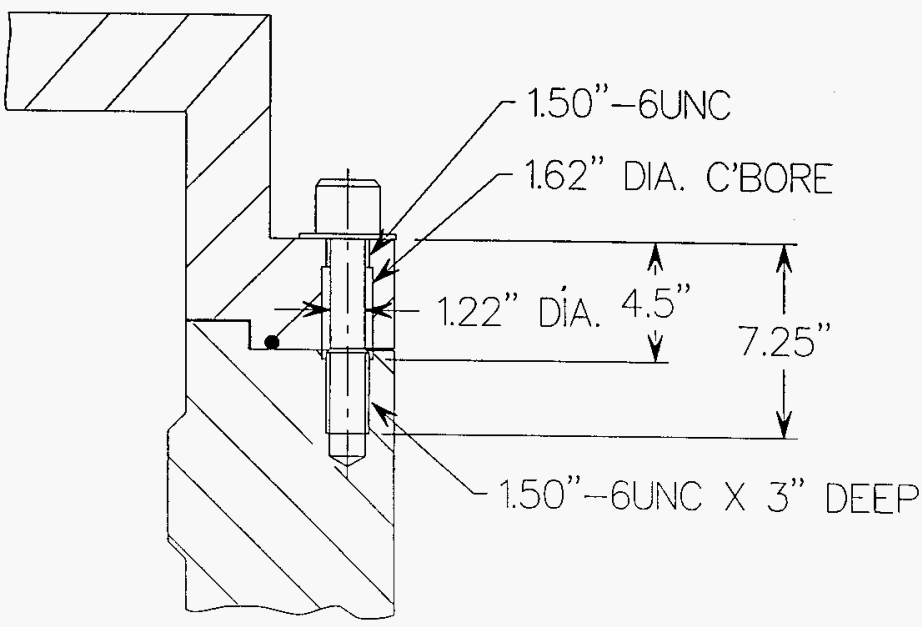


The maximum residual torsional bolt moment is

$$
\mathrm{M}_{\mathrm{tr}}=0.5 \mathrm{Q}=1783 \text { in-lbs. }
$$

The gasket seating load is negligible since o-rings are used.

The loads caused by the pressure difference between the interior and the exterior of the closure components are calculated below. The non-prying tensile bolt force is

$$
\mathrm{F}_{\mathrm{a}}=\pi\left(\mathrm{D}_{\mathrm{lg}}\right)^{2}\left(\mathrm{P}_{\mathrm{li}}-\mathrm{P}_{\mathrm{to}}\right) / 4\left(\mathrm{~N}_{\mathrm{b}}\right)
$$

where $D_{1 \mathrm{~g}}$ is the closure lid diameter at the inner seal, $31.57 \mathrm{in}$.

$P_{1 i} \quad$ is the pressure inside the closure lid and

$P_{10} \quad$ is the pressure outside the closure lid.

$\mathrm{N}_{\mathrm{b}}$ is the number of bolts, 12

The maximum differential pressure for both normal and accident conditions is $161.2 \mathrm{psi}$.

$$
\mathrm{F}_{\mathrm{a}}=\pi(31.57)^{2}(161.2) / 4(12)=10,515 \mathrm{lbs} \text {. }
$$

The increased external pressure combined with no internal pressure results is a force of $F_{a}=-1305 \mathrm{lbs}$.

The shear bolt force due to 161.2 psi differential pressure is

$$
\mathrm{F}_{\mathrm{s}}=\pi\left(\mathrm{E}_{1}\right)\left(\mathrm{t}_{\mathrm{l}}\right)\left(\mathrm{P}_{\mathrm{li}}-\mathrm{P}_{\mathrm{lo}}\right)\left(\mathrm{D}_{\mathrm{lb}}\right)^{2} / 2\left(\mathrm{~N}_{\mathrm{b}}\right)\left(\mathrm{E}_{\mathrm{c}}\right)\left(\mathrm{t}_{\mathrm{c}}\right)\left(1-\mathrm{N}_{\mathrm{ul}}\right)
$$

where

$E_{1}$ is Young's Modulus of the closure lid material, $28.3 \times 10^{6} \mathrm{psi}$

$t_{1}$ is the lid thickness, 3.5 inches

$\mathrm{P}_{\mathrm{li}}-\mathrm{P}_{\mathrm{lo}}$ is the differential pressure on the cask wall, $161.2 \mathrm{psi}$

$\mathrm{D}_{\mathrm{lb}}$ is the closure lid diameter at the bolt circle, 25.2 inches

$\mathrm{E}_{\mathrm{c}}$ is the Young's Modulus of the cask wall material, $28.3 \times 10^{6} \mathrm{psi}$

$t_{c}$ is the thickness of the cask wall, 7.3 inches

$\mathrm{N}_{\mathrm{ul}}$ is Poisson's ratio of the closure lid material, 0.3.

$$
\begin{aligned}
& \mathrm{F}_{s}=\pi\left(28.3 \times 10^{6}\right)(3.5)(36.44)^{2}(161.2) / 2(12)\left(28.3 \times 10^{6}\right)(7.31)(1-0.3) \\
& =19,167 \mathrm{lbs}
\end{aligned}
$$


The fixed edge closure lid force $\left(F_{f}\right)$ and Moment $\left(M_{f}\right)$ for the calculation of prying tensile bolt force and bending bolt moment are given by the formulas below.

$$
\begin{aligned}
& F_{f}=D_{\mathrm{lb}}\left(P_{\mathrm{li}}-P_{10}\right) / 4=36.44(161.2) / 4=1,469 \mathrm{lbs} \\
& M_{\mathrm{i}}=D_{\mathrm{lb}}{ }^{2}\left(\mathrm{P}_{\mathrm{li}}-\mathrm{P}_{\mathrm{lo}}\right) / 32=36.44^{2}(161.2) / 32=6,689 \text { in-lbs }
\end{aligned}
$$

The load caused by differential thermal expansion of the closure lid and bolt is calculated below.

$$
\begin{aligned}
& F_{a}=0.25 \pi\left(D_{b}\right)^{2}\left(E_{b}\right)\left(\alpha_{1} T_{1}-\alpha_{b} T_{b}\right) \\
& E_{b} \text { is the Young's Modulus of the bolt material, } 28.3 \times 10^{6} \mathrm{psi} \\
& \alpha_{1} \text { is the thermal expansion coefficient of the lid material, } 8.55 \times 10^{-6} \mathrm{in} / \mathrm{in}^{\circ} \mathrm{F} \\
& \mathrm{T}_{1} \text { is the temperature change of the lid. The lid can get as hot as } 150^{\circ} \mathrm{F} \text { at an ambient } \\
& \text { temperature of } 115^{\circ} \mathrm{F} \text { with maximum insolation. The lid can get as cold as }-27^{\circ} \mathrm{F} \\
& \text { in the cold environment. Assuming the lid is bolted to the cask inside at a room } \\
& \text { temperature of } 70^{\circ} \mathrm{F}, \mathrm{Tl} \text { is } 130^{\circ} \mathrm{F} \text { for the hot environment and }-97^{\circ} \mathrm{F} \text { for the cold } \\
& \text { environment. } \\
& \alpha_{b} \text { is the thermal expansion coefficient of the bolt material, } 8.87 \times 10^{-6} \text { in } / \mathrm{in}^{\circ} \mathrm{F} \\
& T_{b} \text { is the temperature change of the bolt material. This is the same as Tl. } \\
& D_{b} \text { is bolt diameter }
\end{aligned}
$$

$$
\begin{aligned}
& F_{a}=0.25 \pi(1.22)^{2}\left(28.3 \times 10^{6}\right)\left(8.55 \times 10^{6}-8.87 \times 10^{6}\right) 130 \\
& =-1377 \text { lbs for the hot environment }
\end{aligned}
$$

$$
F_{a}=-1028 \mathrm{lbs} \text { for the cold environment }
$$

There is no load caused by the thermal expansion difference between the closure lid and cask wall since they are made of the same material, and the temperature of the lid and flange are equal

The worst loading on the bolts due to a 1 foot drop is during an lid end corner drop. The lip on the cask protects the closure lid during the side drop. The non prying tensile bolt force due to a one foot lid end corner drop is

$$
F_{\mathrm{a}}=1.34(\sin \mathrm{xi})\left(\mathrm{D}_{\mathrm{lf}}\right)\left(\mathrm{W}_{1}+\mathrm{W}_{\mathrm{c}}\right)(\mathrm{ai}) / \mathrm{N}_{\mathrm{b}}
$$

where $D_{15}$ is the dynamic load factor, 1.2

$W_{1}$ is the weight of the lid, $1890 \mathrm{lbs}$

$\mathrm{W}_{\mathrm{c}}$ is the maximum weight of the cargo, $19120 \mathrm{lbs}$. 
ai is the maximum rigid body impact acceleration of the cask, $18 \mathrm{~g}$ 's

xi angle of impact

$F_{3}=1.34\left(\sin 76^{\circ}\right)(1.2)(21010)(18) / 12=49,170 \mathrm{lbs}$

The shear bolt force is not evaluated since bolts are protected in shear during impact by closureflange shoulder.

The fixed edge closure lid force $\left(F_{f}\right)$ and moment $\left(\mathrm{M}_{\mathrm{f}}\right)$ is calculated below for the $1 \mathrm{ft}$ lid end corner drop. $F_{\mathrm{f}}$ and $\mathrm{M}_{\mathrm{f}}$ are 0 for the $1 \mathrm{ft}$ side drop.

$$
\begin{aligned}
& 1.34 \mathrm{D}_{1 \mathrm{R}}(\sin x i) \mathrm{ai}\left(\mathrm{W}_{1}+\mathrm{W}_{\mathrm{c}}\right) \\
& F_{f}=\pi D_{b b} \\
& (1.34)(1.2)\left(\sin 76^{\circ}\right)(18)(21010) \\
& F_{f}= \\
& \pi(36.44) \\
& =5,155 \mathrm{lbs} \text {. }
\end{aligned}
$$

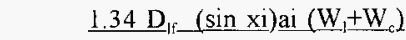

$$
\begin{aligned}
& 8 \pi \\
& (1.34)(1.2)\left(\sin 76^{\circ}\right)(18)(21010) \\
& = \\
& 8 \pi \\
& =23,477 \mathrm{Jbs} \text {. }
\end{aligned}
$$


Vibration loads are insignificant on the bolts.

The load combinations are calculated using the methodology outlined in Table 4.9 of NUREG/CR-6007.

The sum of the tensile bolt forces for operating preload and temperature is

$$
\mathrm{F}_{\mathrm{a}-\mathrm{pt}}=29225+1028=30,253 \mathrm{lbs} .
$$

The sum of the tensile forces for the remainder of the loads is

$$
\mathrm{F}_{\mathrm{a}-\mathrm{al}}=10515+49,170=59,685 \mathrm{lbs} .
$$

This is based on the 1 foot impact and internal pressure. The combined non-prying tensile bolt force is the larger of the two forces calculated above, $59,685 \mathrm{lbs}$.

\section{Combination of Prving Tensile Bolt Forces}

The maximum combined prying fixed-edge force $F_{\mathrm{f}-\mathrm{c}}$ is $1469+5155=6624 \mathrm{lbs}$ and the maximum combined prying moment $M_{i-c}$ is $6689+23477=30166$ in-lbs.

The prying tensile bolt force for the combined load is zero because there is a gap between the flange and lid at the outer edge.

Combining the non-prying and prying tensile bolt force

$$
\mathrm{F}_{\mathrm{a}-\mathrm{c}}=59,685+0=59,685 \mathrm{lbs} \text {. }
$$

The maximum average tensile stress in a lid bolt is

$$
\mathrm{S}_{\mathrm{b} \cdot \mathrm{a}}=\mathrm{F}_{\mathrm{a} \cdot \mathrm{c} / \mathrm{A}}
$$

Where $A_{t}$ is the tensile area of the bolt, $1.169 \mathrm{in}^{2}$

$$
\mathrm{S}_{\mathrm{but}}=59,685 / 1.169=51,060 \mathrm{psi}
$$

The allowable tensile stress is $2 / 3$ of the yield strength at the operating temperature of $150^{\circ} \mathrm{F}$, or $2 / 3(101,000)$ or 67,330 psi.

The shear bolt force is not evaluated since the bolts and closure lid are protected during an impact by the lip of the cask flange. The bolts are not relied upon to resist transverse shear load. The bending bolt moment is calculated below. 


$$
\mathrm{M}_{\mathrm{bb}}=\left[\pi \mathrm{D}_{\mathrm{lb}} / \mathrm{N}_{\mathrm{b}}\right]\left[\mathrm{K}_{\mathrm{b}} /\left(\mathrm{K}_{\mathrm{b}}+\mathrm{K}_{\mathrm{l}}\right)\right] \mathrm{M}_{\mathrm{f}}
$$

Where

$$
\begin{aligned}
& \mathrm{K}_{\mathrm{b}}=\left[\mathrm{N}_{\mathrm{b}} / \mathrm{L}_{\mathrm{b}}\right]\left[\mathrm{E}_{\mathrm{b}} / \mathrm{D}_{\mathrm{lb}}\right]\left[\mathrm{D}_{\mathrm{b}}{ }^{4} / 64\right] \\
&=[12 / 4.65]\left[28.3 \times 10^{6} / 36.44\right]\left[1.22^{4} / 64\right] \\
&= 0.0694 \times 10^{6} \\
& \mathrm{~K}_{1}= \mathrm{E}_{1}\left(\mathrm{t}_{\mathrm{l}}\right)^{3} / 3\left[\left(1-\mathrm{N}_{\mathrm{u}}{ }^{2}\right)+\left(1-\mathrm{N}_{\mathrm{ul}}\right)^{2}\left(\mathrm{D}_{1 \mathrm{~b}} / \mathrm{D}_{\mathrm{lo}}\right)^{2}\right]\left(\mathrm{D}_{\mathrm{lb}}\right) \\
& \mathrm{K}_{1}= 8.405 \times 10^{6} \\
& \mathrm{M}_{\mathrm{f}-\mathrm{c}}=30,166 \mathrm{in}-\mathrm{lbs} . \\
& \mathrm{M}_{\mathrm{bb}}=[\pi(36.44) / 12]\left[.0694 \times 10^{6} / 8.405 \times 10^{6}+.0694 \times 10^{6}\right][30,166] \\
&= 2,358 \text { in-lbs. }
\end{aligned}
$$

The maximum bending stress caused by the bending bolt moment is

$$
\mathrm{S}_{\mathrm{ht}}=10.186 \mathrm{M}_{\mathrm{bh}} /\left(\mathrm{D}_{\mathrm{ba}}\right)^{3}
$$

The minimum bolt diameter is equal to

$$
\begin{aligned}
& \mathbf{D}_{\mathrm{ba}}=1.22 \text { in. } \\
& \mathrm{S}_{\mathrm{bb}}=\frac{(10.186)(2358)}{(1.22)^{3}}=13,230 \mathrm{psi}
\end{aligned}
$$


HNF-SD-SNF-FDR 003 Rev, $0 \quad$ E-15166

The maximum shear stress caused by the torsional bolt moment, $M_{l}$ is

$$
\mathrm{S}_{\mathrm{bt}}=5.093 \mathrm{M}_{\mathrm{t}} /\left(\mathrm{D}_{\mathrm{ba}}\right)^{3}=5.093(3565) /(1.22)^{3}=10,000 \mathrm{psi} \leq 0.4 \mathrm{~S}_{\mathrm{y}}=40,400 \mathrm{psi}
$$

The maximum stress intensity caused by tension plus shear plus bending plus torsion is

$$
\mathrm{S}_{\mathrm{bj}}=\llbracket\left(\mathrm{S}_{\mathrm{b} 3}+\mathrm{S}_{\mathrm{bb}}\right)^{2}+4\left(\mathrm{~S}_{\mathrm{b}}\right)^{2} \rrbracket^{0.5}=\llbracket(51060+13230)^{2}+4(10000)^{2} \rrbracket^{0.5}=67,330 \mathrm{psi}
$$

This is less than the allowable maximum stress intensity of $0.9 \mathrm{~S}_{\mathrm{y}}=90,900 \mathrm{psi}$.

\section{B4.5B-3 BOLT FATIGUE ANALYSIS}

The purpose of the fatigue analysis is to show quantitatively that the fatigue damage to the bolts during normal transport conditions is acceptable. This is done by determining the fatigue usage factor for each normal transport event. From Reference 2, the shipment is to be completed in two calendar years and there are 200 shipping days per year and one shipment per shipping day. This shipping schedule translates into 400 round trip shipments. The total cumulative damage or fatigue usage for all events was conservatively determined by adding the usage factors for the individual events. The sum of the individual usage factors was checked to make certain that, for the 400 round trip shipments of the cask, the total usage factor is less than one. The following sequence of events was assumed for the fatigue evaluation.

1. Operating Preload

2. Pressure Fluctuations

3. Road Vibration

4. Test Pressure

5. Impact ( $1 \mathrm{Ft})$ End Drop

6. Handling Load

\section{Number of Load/Stress Cycles for Each Loading}

A. Test Pressure:

Proof Test: $1.5 \times$ MNOP $=241.8$ psi. This occurs once during the TN-WHC cask lifetime. (See Table B4.5B-1 for stress calculations). 


\section{HNF-SD-SNF-FDR-003 Rev. $0 \quad$ E-15166}

\section{B. $\quad$ Preload}

The number of preload cycles is two times the number of trips, or 800 . (See Table B 4.5B-1 for stress calculations).

\section{Road Vibrations}

The bolt stresses are negligible for the road vibrations.

D. 1 Foot Drop:

Assume this occurs twice in two year operating life of the cask. The stress intensity of 52,370 psi (see Table B4.5B-1 for stress derivation) is a combination of the non prying and prying tensile stress due to the one foot end drop, with a zero preload.

\section{E. Pressure and Temperature Fluctuations:}

The full internal equilibrium temperature of $150^{\circ} \mathrm{F}$ is not reached during a typical trip. However, for conservatism we assume that the temperature increases from $70^{\circ} \mathrm{F}$ to $-27^{\circ} \mathrm{F}$ during each trip (temperature increase from $70^{\circ} \mathrm{F}$ to $150^{\circ} \mathrm{F}$ does not develop tension in lid bolts). This results in a load due to differential thermal expansion coefficients, and also due to pressure change from 0 to $161.2 \mathrm{psig}$. The fatigue damage is based on 800 cycles and stress intensity range of 0 to 12,810 psi (See Table B4.5B-1 for stress calculations).

\section{F. Handling Load}

The number of handling load cycles is four times the number of trips, or 1600 . The direct stress is calculated using $3 \mathrm{G}$ load (very conservative), $60000(3) / 12(1.169)=12,840$ psi. The bending stress is $4185 \mathrm{psi}$, and total combined stress is $17,025 \mathrm{psi}$.

\section{Fatigue Evaluation - Usage Factor Calculations}

Based on the stresses and cyclic histories described above, stress histograms are plotted in Figure B4.5B-4. In this histogram, a fatigue strength reduction factor $(\mathrm{K})$ of 4.0 has been assumed in the evaluation. The stress range for each combination of events and the corresponding alternating stress are shown in Table B4.5B-2. The damage factors are computed using the fatigue curve shown in Table I-9.4 of ASME Section III Appendices. The cumulative fatigue damage factors in this case is 0.621 which is less than the limit of 1.0 . Therefore, the TN-WHC cask lid bolts satisfy the fatigue requirements. 
TABLE B4.5B-1

FORCES, MOMENTS AND STRESSES CALCULATED FOR FATIGUE EVALUATION

\begin{tabular}{|l|l|l|l|l|l|l|}
\hline $\begin{array}{l}\text { EVENT/ } \\
\text { LOAD }\end{array}$ & $\begin{array}{l}\mathrm{F}_{\mathrm{a}} \\
(\mathrm{LBS})\end{array}$ & $\begin{array}{l}\mathrm{F}_{\mathrm{f}} \\
(\mathrm{LBS})\end{array}$ & $\begin{array}{l}\mathrm{M}_{\mathrm{f}} \\
(\mathrm{IN}-\mathrm{LB})\end{array}$ & $\begin{array}{l}\mathrm{F}_{\mathrm{ap}} \\
(\mathrm{LBS})\end{array}$ & $\begin{array}{l}\tau \\
(\mathrm{PSI})\end{array}$ & $\begin{array}{c}\sigma \\
(\mathrm{PSI})\end{array}$ \\
\hline $\begin{array}{l}\text { TEST } \\
\text { PRESS. }\end{array}$ & 15,773 & 2,204 & 10,034 & 0 & $\cdots--$ & 18,062 \\
\hline $\begin{array}{l}\text { PRE- } \\
\text { LOAD }\end{array}$ & 29,225 & ---- & 3,565 & 0 & 10,000 & 32,015 \\
\hline $\begin{array}{l}\text { ONE FOOT } \\
\text { DROP }\end{array}$ & 49,170 & 5,155 & 23,477 & 0 & --- & 52,370 \\
\hline $\begin{array}{l}\text { PRESS. + } \\
\text { TEMP. }\end{array}$ & 11,543 & 1,469 & 6,689 & --- & --- & 12,810 \\
\hline $\begin{array}{l}\text { HANDLING } \\
\text { LOAD }\end{array}$ & 15,000 & ---- & 9,540 & $\cdots--$ & --- & 17,025 \\
\hline
\end{tabular}




\section{FIGURE B4.5B-4}

\section{SCHEMATIC OF STRESS HISTOGRAM AT LID BOLTS $(K=4)$}

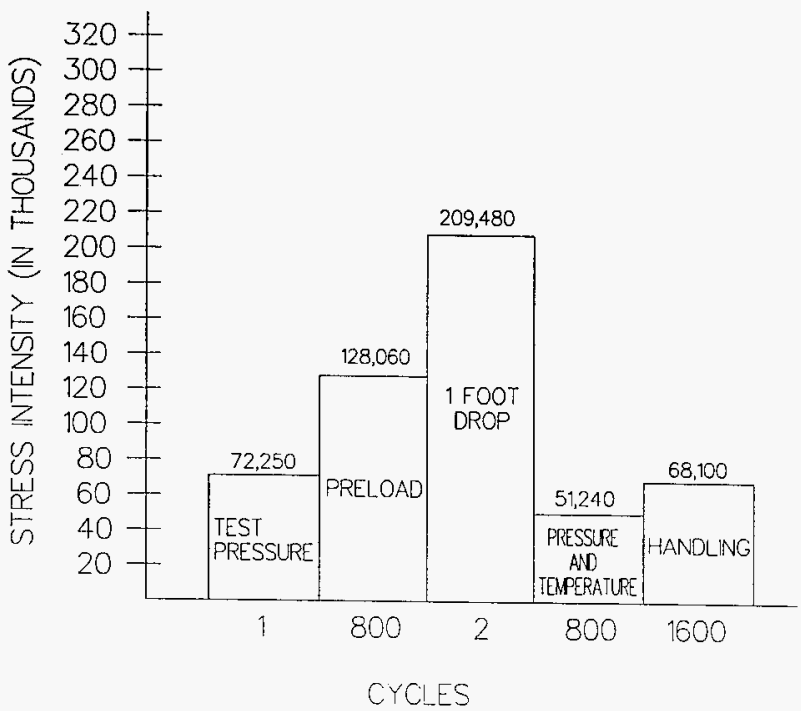


TABLE B4.5B-2

BOLT FATIGUE DAMAGE FACTORS

\begin{tabular}{|c|c|c|c|c|c|c|}
\hline \multirow{2}{*}{$\begin{array}{c}\text { EVENT } \\
\text { COMBINATIONS }\end{array}$} & \multirow{2}{*}{$\begin{array}{c}\text { STRESS } \\
\text { RANGE } \\
\text { (PSI) }\end{array}$} & \multirow{2}{*}{$\begin{array}{c}\mathrm{S}_{\mathrm{a}} \\
(\mathrm{PSI})\end{array}$} & \multirow{2}{*}{$\begin{array}{l}\text { FATIGUE } \\
\text { CURVE }\end{array}$} & \multicolumn{2}{|c|}{ CYCLES } & \multirow{2}{*}{$\begin{array}{l}\text { DAMAGE } \\
\text { FACTOR } \\
n / N\end{array}$} \\
\hline & & & & $\mathrm{n}$ & $\mathrm{N}$ & \\
\hline $\begin{array}{l}\text { TEST } \\
\text { PRESSURE }\end{array}$ & 72250 & 36125 & I-9.4 & 1 & 5000 & 0.0002 \\
\hline PRELOAD & 128060 & 64030 & $\mathrm{I}-9.4$ & 800 & 2000 & 0.4000 \\
\hline ONE FOOT DROP & 209480 & 104740 & $\mathrm{I}-9.4$ & 2 & 450 & 0.003 \\
\hline VIBRATION & 328 & 164 & $1-9.4$ & $7.2 \times 10^{8}$ & $\infty$ & 0 \\
\hline $\begin{array}{l}\text { TEMPERATURE } \\
\text { AND } \\
\text { PRESSURE }\end{array}$ & 51240 & 25620 & I-9.4 & 800 & 20000 & 0.0400 \\
\hline $\begin{array}{l}\text { HANDLING } \\
\text { LOAD }\end{array}$ & 68100 & 34050 & $\mathrm{I}-9.4$ & 1600 & 9000 & 0.178 \\
\hline TOTAL I & MAGE FA & TOR & & & & 0.621 \\
\hline
\end{tabular}




\section{B4.5B-4 ACCIDENT CONDITION ANALYSIS}

The hypothetical accident conditions considered for the bolt analysis are: impact and puncture.

The worst loading due to the 30 foot impact occurs at lid end corner drop. This results in a G loading of 20.

The nonprying tensile bolt force due to impact is:

$$
\mathrm{F}_{\mathrm{a}}=1.34\left(\mathrm{D}_{1 \mathrm{f}}\right)(\sin \mathrm{xi})\left(\mathrm{W}_{1}+\mathrm{W}_{\mathrm{c}}\right)(\mathrm{ai}) / \mathrm{N}_{\mathrm{b}}=1.34(1.2)(\sin 76)(21010)(20) / 12=54,633 \mathrm{lbs} .
$$

There is no shear bolt force due to impact, since the load is taken by the lip of the cask flange. The fixed edge closure lid force $\left(F_{f}\right)$ and moment $\left(M_{f}\right)$ are

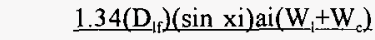

$$
\begin{aligned}
& F_{\mathrm{f}}=\pi \mathrm{D}_{\mathrm{lb}} \\
& (1.34)(1.2)(\sin 76)(20)(21010) \\
& = \\
& \pi(36.44) \\
& =5,727 \mathrm{lbs} \text {. } \\
& \mathrm{Mf}=\frac{1.34\left(\mathrm{D}_{1 \mathrm{f}}\right)(\sin \mathrm{xi}) \text { ai }\left(\mathrm{W}_{1}+\mathrm{W}_{\mathrm{a}}\right)}{8 \pi} \\
& (1.34)(1.2(\sin 76)(20)(21010) \\
& =\quad=26,085 \mathrm{in}-\mathrm{lbs} \text {. } \\
& 8 \pi
\end{aligned}
$$


The puncture accident results in an inward load on the bolts which relaxes the tensile force due to preload.

Therefore the worst accident condition for the bolts is the 30 foot lid end corner drop. This must be combined with preload, internal pressure and thermal expansion loads.

The sum of the tensile bolt forces for operating preload and temperature is $30,252 \mathrm{lbs}$. as in the normal load cases.

The sum of the non prying tensile forces from internal pressure and 30 foot drop accident is, $f_{a-c}=10515+54,630=65,148 \mathrm{lbs}$.

The combined non-prying tensile bolt force is the larger of the two forces calculated above, 65,148 lbs.

The maximum combined prying fixed edge force $\mathrm{F}_{\mathrm{f}-\mathrm{c}}$ is $5727+1469=7,196 \mathrm{lbs}$. The combined prying moment $\mathrm{M}_{\mathrm{f} \cdot \mathrm{c}}$ is $26,085+6698=32,783$ in. lbs.

The prying tensile bolt force for the combined load is zero.

Combining the non-prying and prying tensile bolt force

$\mathrm{F}_{\mathrm{a}-\mathrm{c}}=65,148+0=65,148 \mathrm{lbs}$.

The maximum average tensile stress in a lid bolt is

$\mathrm{S}_{b \mathrm{a}}=65,148 / 1.169=55,730 \mathrm{psi}$

This is less than the smaller of $0.7 \mathrm{~S}_{\mathrm{u}}$ or $0.9 \mathrm{~S}_{\mathrm{y}}$ which is $90,900 \mathrm{psi}$.

The average shear stress is due to torsion from preloading the bolts. The maximum shear stress is 10,000 psi. This is much less than the allowable stress $0.42 \mathrm{~S}_{\mathrm{y}}$ or 42,420 psi.

The stress ratio for average tensile stress is $55730 / 909000=0.613$. The stress ratio for average shear stress is $10000 / 42420$ or 0.24 .

The combined tensile and shear ratios must meet the following criterion:

$\mathrm{R}_{\mathrm{i}}^{2}+\mathrm{R}_{\mathrm{s}}^{2} \leq 1$

where $R_{r}$ is the stress ratio for average tensile stress and $R_{s}$ is the stress ratio for average shear stress ratio 
$(.613)^{2}+(.24)^{2}=.43 \leq 1$

which is acceptable.

The maximum combined fixed edge moment due to pressure and 30 foot drop, $M_{f}=6698+$ $26085=32,783$ in-lbs.

Bolt bending moment, $\mathrm{M}_{\mathrm{bt}}=[2358 / 30166] \times 32783=2562$ in-lbs.

Bolt bending stress, $\mathrm{S}_{\mathrm{bb}}=1.186 \times 2562 /(1.22)^{3}=14,372 \mathrm{psi}$

The maximum stress intensity due to tension, bending and shear is

$S_{h}=\left[(55730+14372)^{2}+4(10000)^{2}\right]^{0.5}=72,900$ psi

This stress is less than the allowable $S_{u}$ of 133,000 psi.

\section{B4.5B-5 FIRE CONDITION}

The fire accident results in a maximum bolt and lid temperature of $1068^{\circ} \mathrm{F}\left(1091^{\circ} \mathrm{F}\right.$ is conservatively used for calculation). The thermal stress caused by differential thermal expansion of the closure lid and bolt is

Thermal Stress $=\mathbf{E}_{\mathrm{b}}(\Delta \mathrm{T})\left(\alpha_{\mathrm{f}}-\alpha_{\mathrm{b}}\right)=23.5 \times 10^{6}(1091-70)(10.24-9.40) \times 10^{-6}=20,155 \mathrm{psi}$

The preload axial tensile and shear stresses are combined to obtain the maximum stress intensity The pressure load is not considered as the seal material will not be effective at this temperature.

$\mathrm{S}_{1}=\left[(20155+25000)^{2}+4(10000)^{2}\right]^{03}=49,386 \mathrm{psi}$

This stress is below the bolt material tensile strength $\left(\mathrm{S}_{\mathrm{u}}=112,500 \mathrm{psi}\right.$ at $\left.1100^{\circ} \mathrm{F}\right)$. The MCO will therefore be secure inside the cask during the fire accident.

Both the lid and lifting attachment welded to the lid are made in the same material (stainless steel), the thermal stresses due to the differential thermal expansion are insignificant. 


\section{B4.5B-6 CLOSURE FLANGE SHOULDER ANALYSIS}

Transverse lid deceleration loads are resisted by the closure flange shoulder during all normal and accident conditions. The shoulder thickness at the base has a shear area equal to

$$
\text { Shear Area }=\pi\left[(29.76)^{2}-(25.5)^{2}\right] / 4=184.9 \text { in }^{2}
$$

For conservatism, it is assumed that only a $120^{\circ}$ arc of the shoulder is effective. Effective shear is:

Effective Shear $=120 / 360(184.9)=61.6$ in $^{2}$

\section{$\underline{\text { Normal Conditions Stresses }}$}

Maximum transverse deceleration loads during normal conditions occurs during the hypothetical one foot horizontal drop. Maximum deceleration is $24 \mathrm{~g}$ 's. (Ref Appendix B4.5A)

Total Force $=24.0 \mathrm{x}$ wt of lid $=24.0(1890)=45,360 \mathrm{lbs}$.

Average Shear Stress $=45360 / 61.6=737 \mathrm{psi}$

Allowable Average Shear $=0.4 \mathrm{~S}_{\mathrm{y}}=0.4(30000)=12,000 \mathrm{psi}$

Factor of Safety $=12,000 / 737=16.3$

\section{Hypothetical Accident Conditions Stresses}

The maximum transverse deceleration load during accident conditions is $40 \mathrm{~g}$ for the horizontal 30 foot drop. The average shear stress across the shoulder is

Average shear stress $=(40 / 24) \times 737=1,228 \mathrm{psi}$

Allowable average shear $=\mathrm{S}_{\mathrm{u}} / 2=75,000 / 2=37,500 \mathrm{psi}$

Factor of Safety $=37,500 / 1,228=30.5$ 


\section{B4.5B-7 CONCLUSIONS}

The maximum average tensile stress in the lid bolts during normal conditions is 51,060 psi which is less than the allowable tensile stress of $2 / 3$ yield, or 67,300 psi. The maximum stress intensity for the bolts during normal conditions is 67,330 psi which is much less than the allowable maximum stress intensity of $90,900 \mathrm{psi}\left(0.9 \mathrm{~S}_{\mathrm{y}}\right)$.

The fatigue analysis performed shows that for 400 round trip shipments, the bolts will not fail due to fatigue. The cumulative fatigue damage factor is 0.621 which is much less than the allowable limit of 1.0 .

The maximum average tensile stress due to the accident conditions is $55,730 \mathrm{psi}$ psi, which is less than the allowable stress of $90,900 \mathrm{psi}$. The maximum stress intensity for the bolts during accident conditions is 72,900 psi which is less than the allowable stress of $133,000 \mathrm{psi}$.

During fire accident condition, bolt and flange experiences a maximum temperature of $1068^{\circ} \mathrm{F}$ and the maximum bolt stress intensity is $49,386 \mathrm{psi}$ which is below the bolt tensile strength of 112,500 psi. The lid bolts will keep the MCO secure in cask during a fire accident.

Lid shoulder stresses during both normal and accident conditions provide large margins of safety of resisting transverse lid deceleration loads. 


\section{B4.5B-8 REFERENCES}

1. NUREG/CR-6007, Stress Analysis of Closure Bolts for Shipping Casks.

2. WHC-S-0396, Rev.1," Specification for TN-WHC cask and Transportation System". 


\section{APPENDIX B4.5C \\ STRUCTURAL EVALUATION OF CASK BODY}

$B 4.5 C-O$ 
HNF-SD-SNF-FDR-003 Rev. 0 E-15166

TABLE OF CONTENTS

Section

B4.5C-1 INTRODUCTION

B4.5C-2 ANSYS ANALYSIS

B4.5C-3 LOADINGS

B4.5C-4 PUNCTURE ANALYSIS

B4.5C-5 STRESS RESULTS

B4.5C-6 REFERENCES
Page

B4.5C-1

B $4.5 \mathrm{C}-2$

B $4.5 \mathrm{C}-16$

B4.5C-32

B4.5C-39

B4.5C-53 
APPENDIX B4.5C

\section{STRUCTURAL EVALUATION OF CASK BODY}

\section{B4.5C-1 INTRODUCTION}

This appendix presents the structural analyses of the cask body including the cylindrical shell assembly and bottom assembly, and the lid. The specific methods, models and assumptions used to analyze the cask body for the various individual loading conditions specified in the Hanford Specification ${ }^{(1)}$. Stress results are reported at selected locations for each load case. Maximum stresses from this appendix are evaluated in Sections B4.3 and B4.4 where the load combinations are performed and the results evaluated against the ASME Code and design criteria described in Sections B4.3 and B4.4.

The cask body structural analyses generally use static or quasistatic linear elastic methods so that combinations of loads can be examined by superimposing the results from individual loads. The stresses and deformations due to the applied loads are generally determined using the ANSYS ${ }^{(2)}$ computer program.

The two analysis methods, described in this appendix and used to evaluate the cask body for the individual loading conditions, are:

- ANSYS Analysis - Axisymmetric and Asymmetric Loads

- Puncture Analysis

The method of combining stress results from individual load cases to evaluate the required load combinations is discussed in Section B4.3 for normal conditions of transport and Section B4.4 for hypothetical accident conditions. 


\section{B4.5C-2 ANSYS ANALYSIS}

\section{Model Description}

The cask body consists of the cylindrical shell, the bottom, the lid and the lid bolts. The elements used to model the bolts are ANSYS STIF3, beam elements. The cylindrical shell, the bottom end closure and the lid are modeled using either ANSYS STIF25 axisymmetric harmonic solid elements or ANSYS STIF42 axisymmetric solid elements (Puncture Analysis). The loading applied to this type of elements may be either axisymmetric for some cases and asymmetric for other cases. The model geometry is based on Drawings H-1-81535, sheets 1,2,3,4,5 and are provided in Section A9.1. The contact surface at the lid and cylindrical shell is modeled using separate nodes in the interfacing components. These nodes are coupled or left uncoupled for specific constraint conditions as discussed below. The lifting attachment welded to the lid changes the usual cask drop analyses on the lid end drop and corner drop. A separate analysis was done to check this impact effects. Based on this calculation, the cask and lid closure structure remain intact but the welds at the trunnion brackets and gussets fail at lower $G$ level. The bracket and gussets act like an impact limiter (reduced the $G$ force at the cask body) during the drop events. It is conservative to neglect the brackets and gussets in the cask model for calculating the globe effect of the cask body. All the analyses were performed using the model shown in Figures $\mathrm{B} 4.5 \mathrm{C}-1$ and $\mathrm{B} 4.5 \mathrm{C}-2$. The mechanical properties for the materials in this model are the linear values described in Section B4.2.2. 
Figure B4.5C-1

TN-WHC Cask Structural ANSYS Model

(2D AXISYMMETRIC)

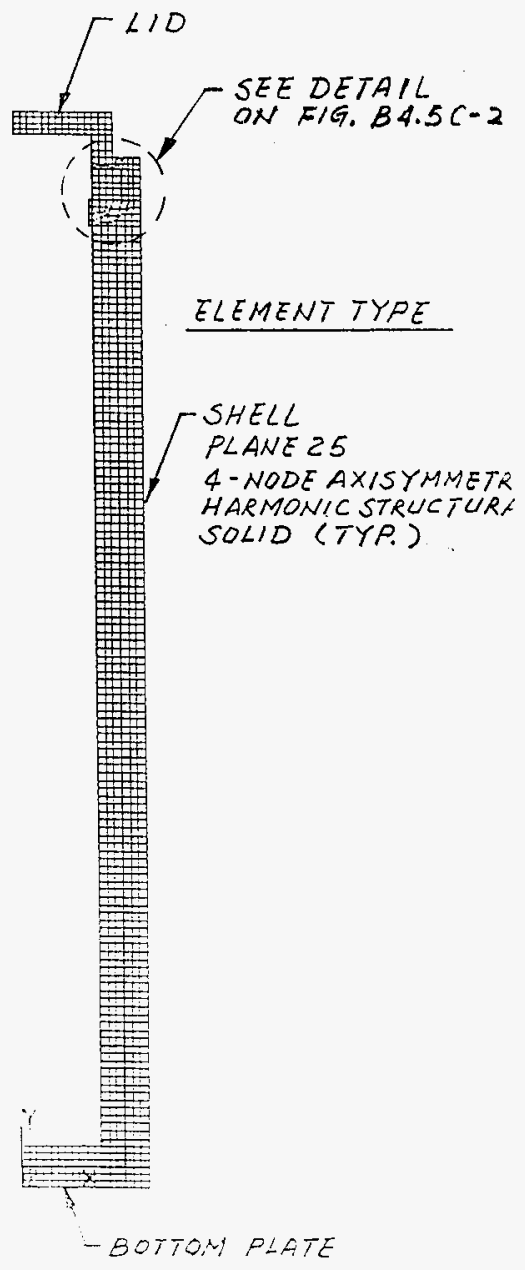

B $4.5 \mathrm{C}-3$ 
HNF-SD-SNF-FDR-003 Rev. 0

E-15166

Figure B4.5C-2

TN-WHC Cask Structural ANSYS Model (2D AXISYMMETRIC)

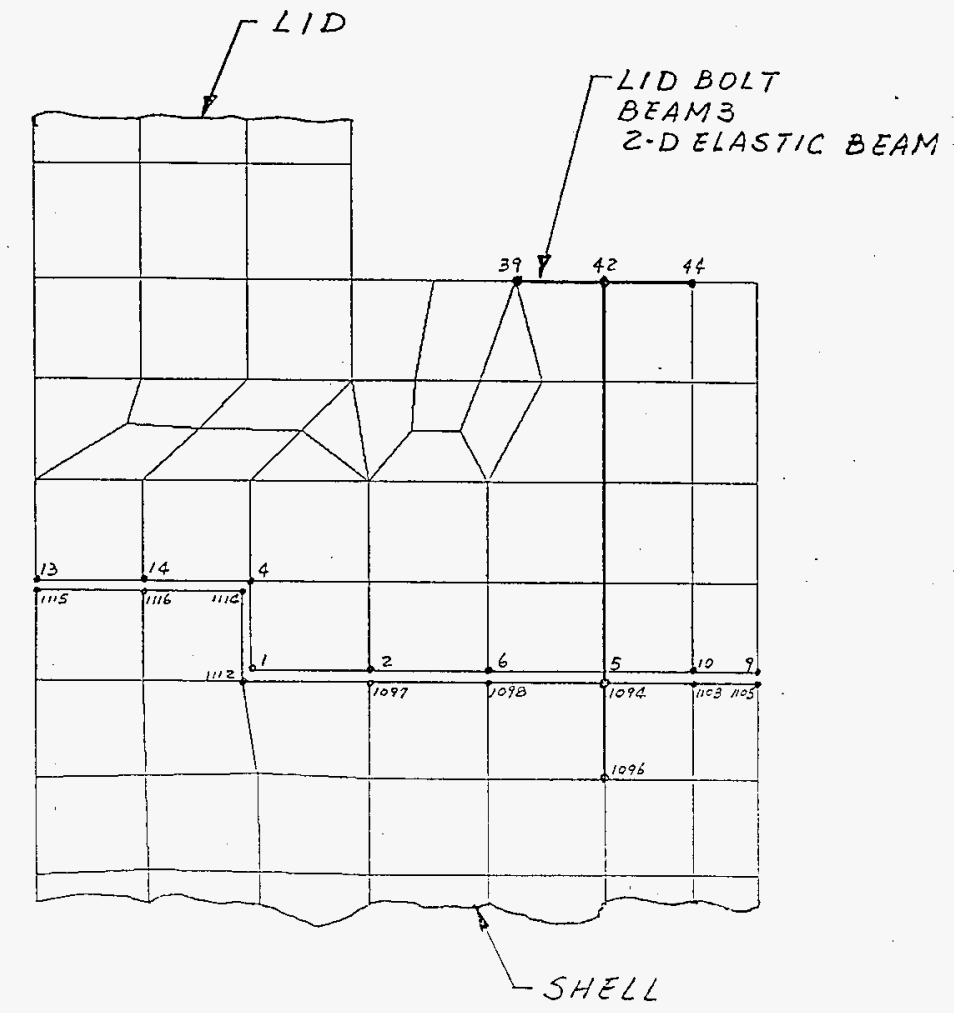

B4.5C-4 


\section{Finite Element Model Internal Constraints}

The connections between various portions of the model were made using node coupling. The bolted connection between the lid and the cask body was modeled by coupling the interfacing nodes in the radial and axial directions.

\section{Finite Element Model Boundary Conditions}

For a static finite element structural analysis the structure must be restrained in such a way that there is no rigid body motion. For the drop analyses, the dynamic equilibrium problem is solved using D'Alembert's principle, i.e. the total inertia loading is balanced by the total reaction force. In an actual dynamic event, there are no physical locations in the structure that are stationary and, unless suitable boundary conditions are selected for analysis, rigid body motion may occur. To eliminate the rigid body motion problem, the cask model was carefully restrained in such a way that no appreciable forces were developed at the restraints. When only small reactions are developed at the restrained nodes the inertia loadings and reactions are well balanced. This is true for all loading conditions analyzed.

The boundary conditions used for the different loading conditions are shown on the following figures.

Figures B4.5C-3 and 4: Boundary conditions for bolt preload, pressure and thermal

Figures B4.5C-5 and 6: Boundary conditions for bottom end drop

Figures B4.5C-7 and 8: $\quad$ Boundary conditions for lid end drop

Figures B4.5C-9 and 10: Boundary conditions for side drop

Figures B4.5C-11 and 12: Boundary conditions for corner drop 
HNF-SD-SNF-FDR-003 Rev. 0

E-15166

Figure B4.5C-3

Boundary Conditions For Bolt Preload, Pressure, and Thermal

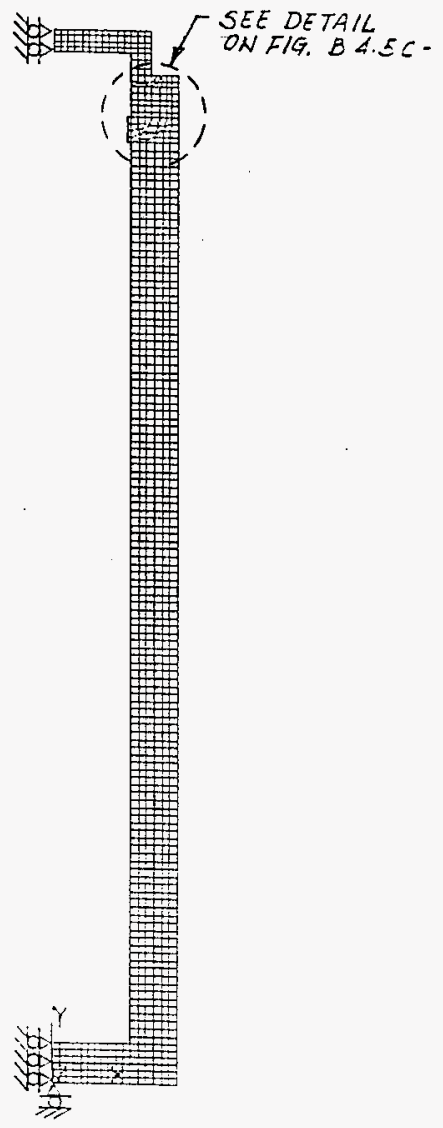

B4.5C-6 
HNF-SD-SNF-FDR-003 Rev. 0

E-15166

Figure B4.5C-4

Boundary Conditions For Bolt Preload, Pressure, and Thermal

(Lid and Lid Bolt)

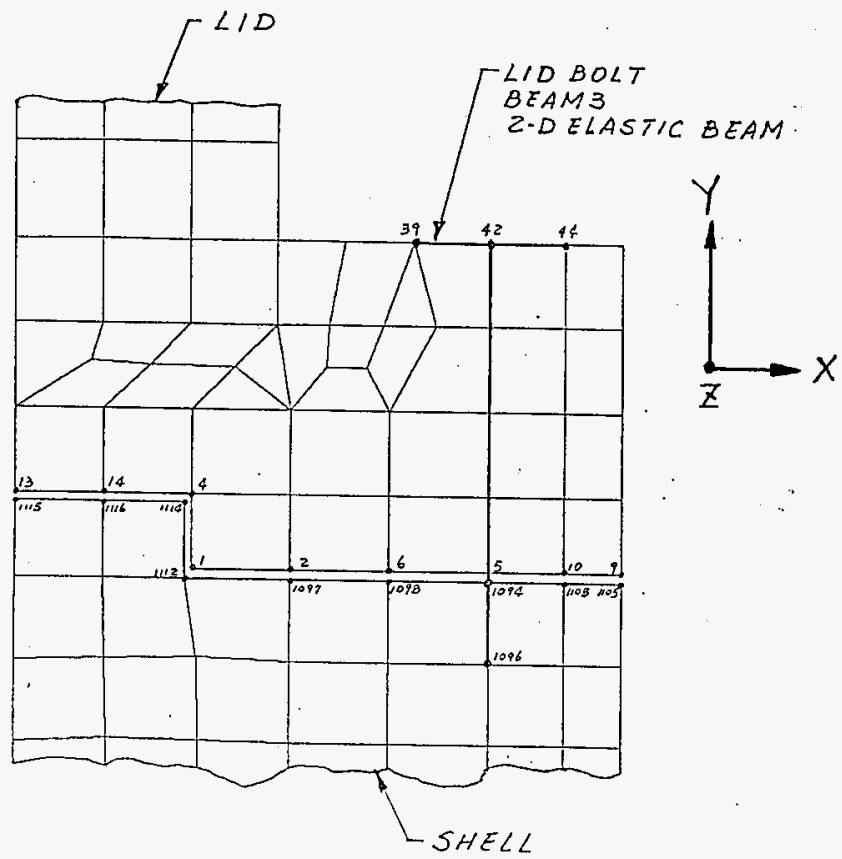

$$
\begin{aligned}
& \text { NODES COUPLED IN X DIRECTION } \\
& (4,1114)(1,1112) \\
& \text { NODES CDUPLED N Y DIRECTION } \\
& (13,115)(14,116)(4,1114)(1,1112)(2,1097) \\
& (6,1098)(5,1094)(10,1108)(9,1105) \\
& \text { NODES COUPLED W Z DIRECT1ON } \\
& (1,1112)(2,1097)(6,1098)(5,1094) \\
& (10,1108)(9,1105)
\end{aligned}
$$




\section{HNF-SD-SNF-FDR-003 Rev. 0}

E-15166

Figure B4.5C-5

Boundary Conditions For Bottom End Drop

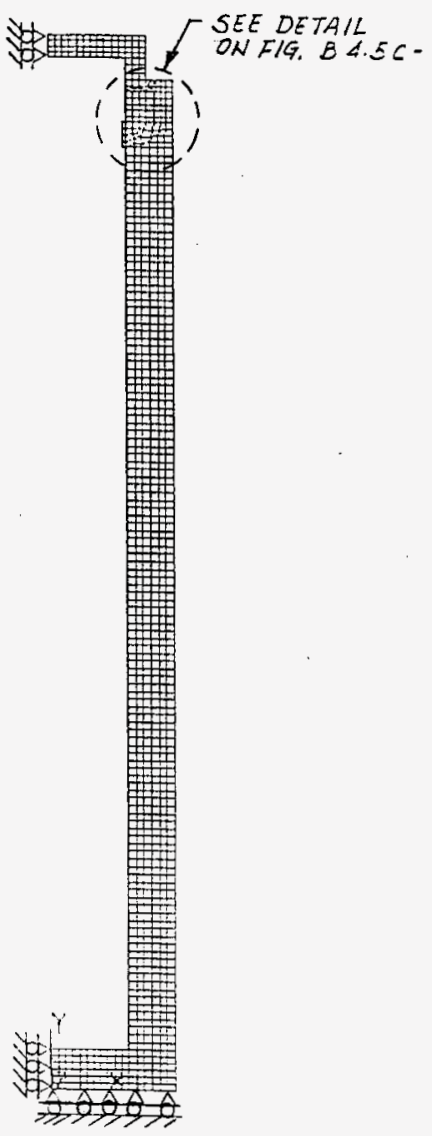


Figure B4.5C-6

Boundary Conditions For Bottom End Drop

(Lid and Lid Bolt)

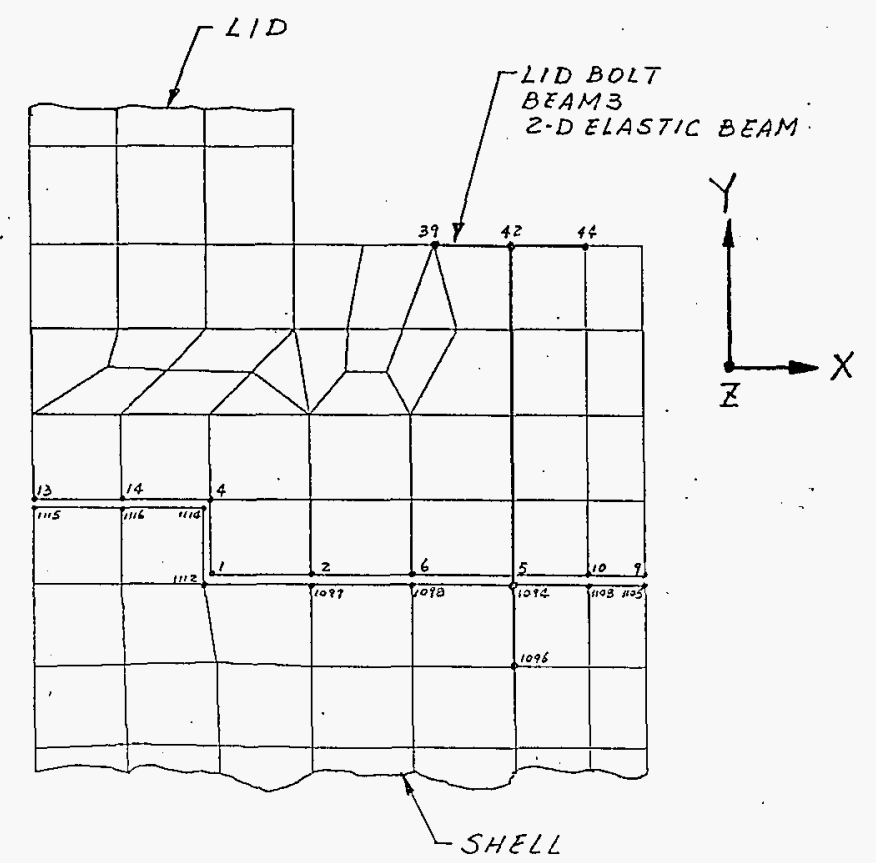

NODES COUPLED IN $\times$ DIRECTION

$(4,11 / 4)(1,1112)$

NODES COUPLED IN Y DIRECTION

$(13,1115)(14,1116)(4,1114)(1,1112)(2,1097)$

$(6,1098)(5,1094)(10,1108)(9,1105)$

NODES COUPLED IN Z DIRECTION

$(1,1112)(2,1097)(6,1098)(5,1094)$

$(10,1108)(9,1105)$ 
Figure B4.5C-7

Boundary Conditions For Lid End Drop

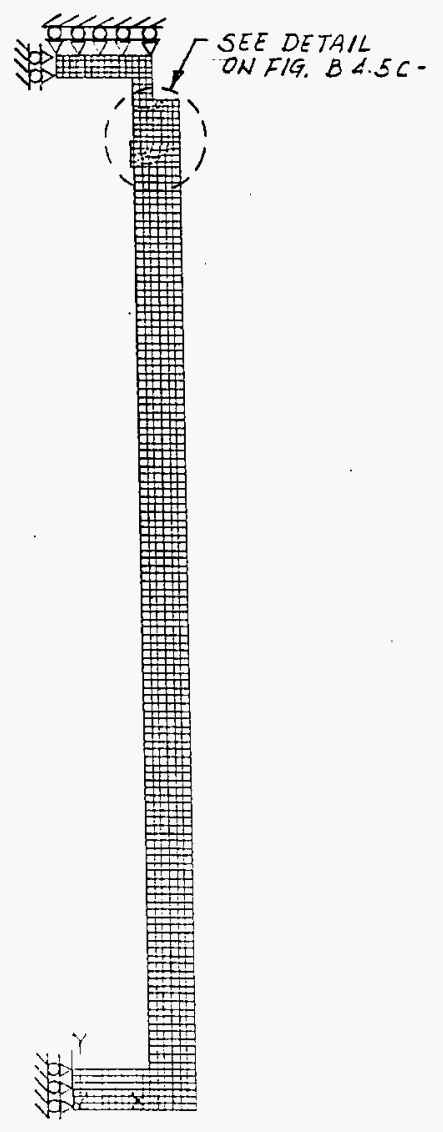


Figure B4.5C-8

Boundary Conditions For Lid End Drop

(Lid and Lid Bolt)

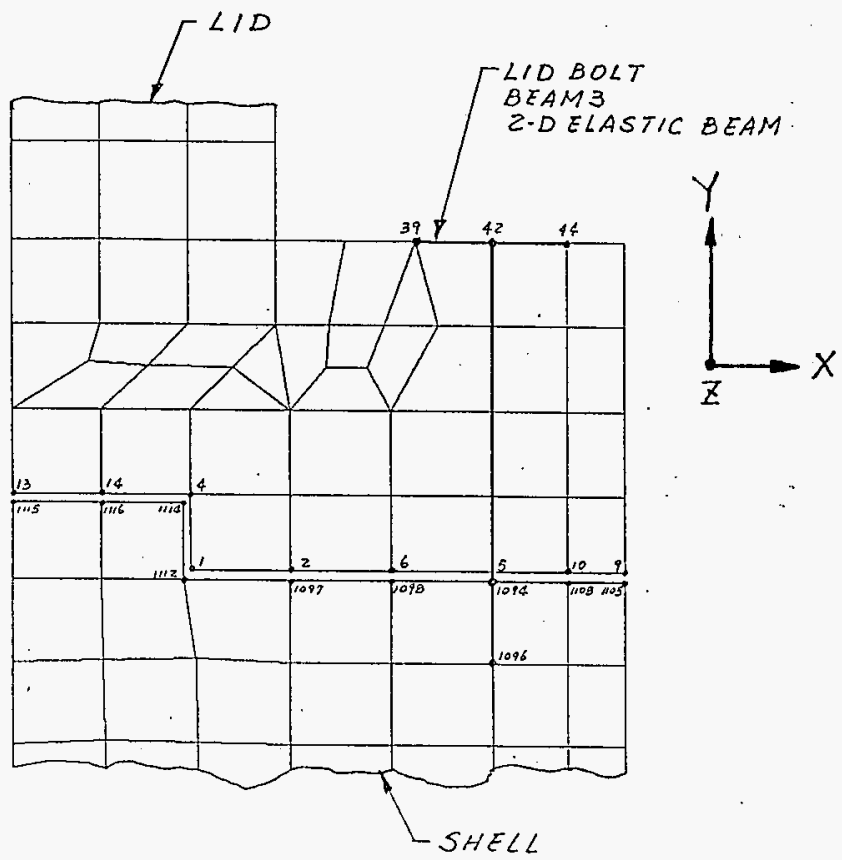

$$
\begin{aligned}
& \text { NODES COUPLED IN X DIRECTION } \\
& (4,1114)(1,1112) \\
& \text { NODES COUPLED IN Y DIRECTION } \\
& (13,1115)(14,116)(4,1114)(1,1112)(2,1097) \\
& (6,1098)(5,1094)(10,1108)(9,1105) \\
& \text { NODES COUPLED N Z D/RECTION } \\
& (1,1112)(2,1097)(6,1098)(5,1094) \\
& (10,1108)(9,1105)
\end{aligned}
$$


Figure B4.5C-9

Boundary Conditions For Side Drop

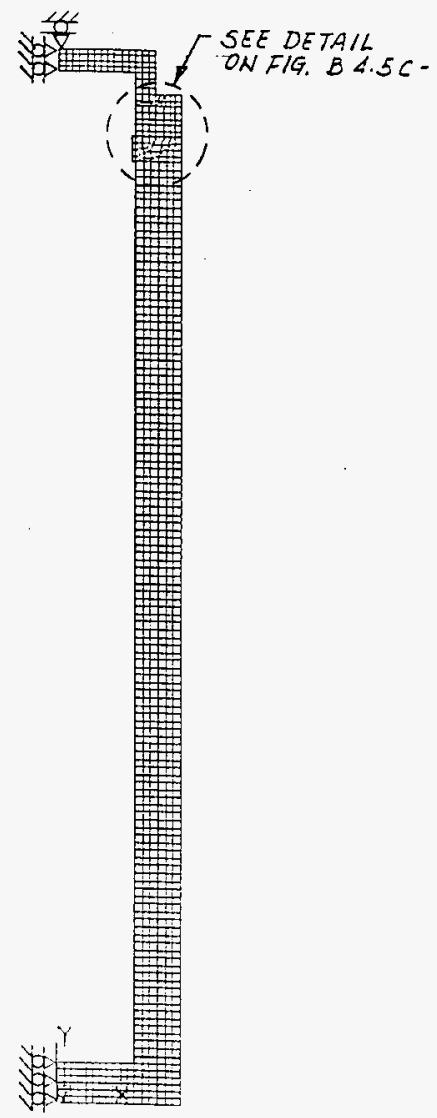


HNF-SD-SNF-FDR-003 Rev. 0

E-15166

Figure B4.5C-10

Boundary Conditions For Side Drop

(Lid and Lid Bolt)

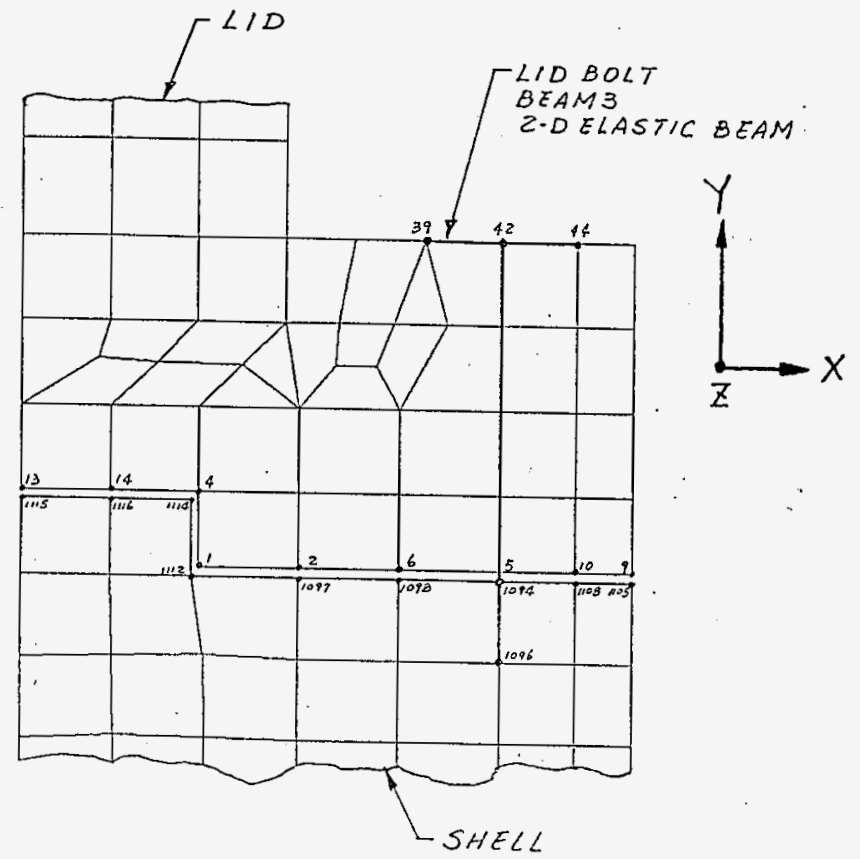

NODES COUPLED IN $\times$ DIRECTION $(4,11 / 4)(1,11 / 2)$

NODES COUPLED IN Y DIRECTION

$(13,1115)(14,1116)(4,1114)(1,1112)(2,1097)$

$(6,1098)(5,1094)(10,1108)(9,1105)$

NODES COUPLED IN Z DIRECTION

$(1,1112)(2,1097)(6,1098)(5,1094)$

$(10,1108)(9,1105)$ 
HNF-SD-SNF-FDR-003 Rev. 0

E-15166

Figure B4.5C-11

Boundary Conditions For Corner Drop

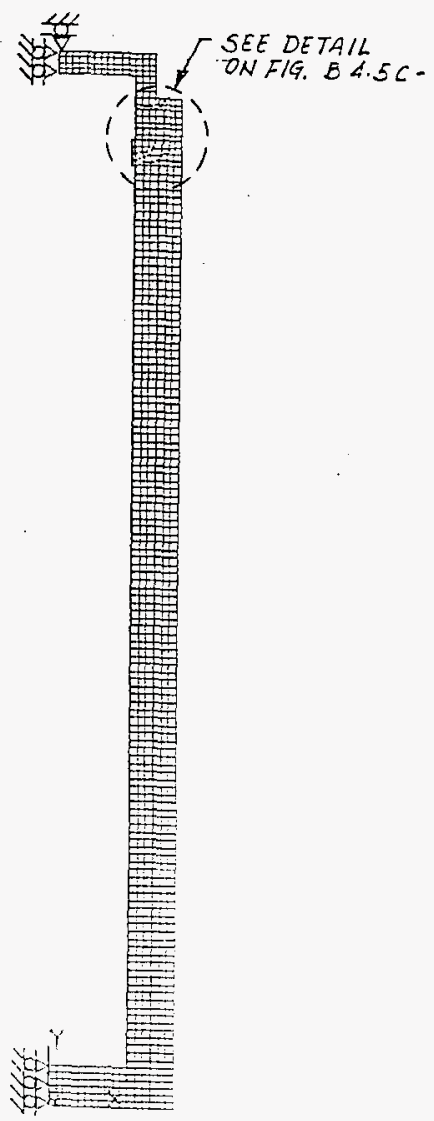


Figure B4.5C-12

Boundary Conditions For Corner Drop

(Lid and Lid Bolt)

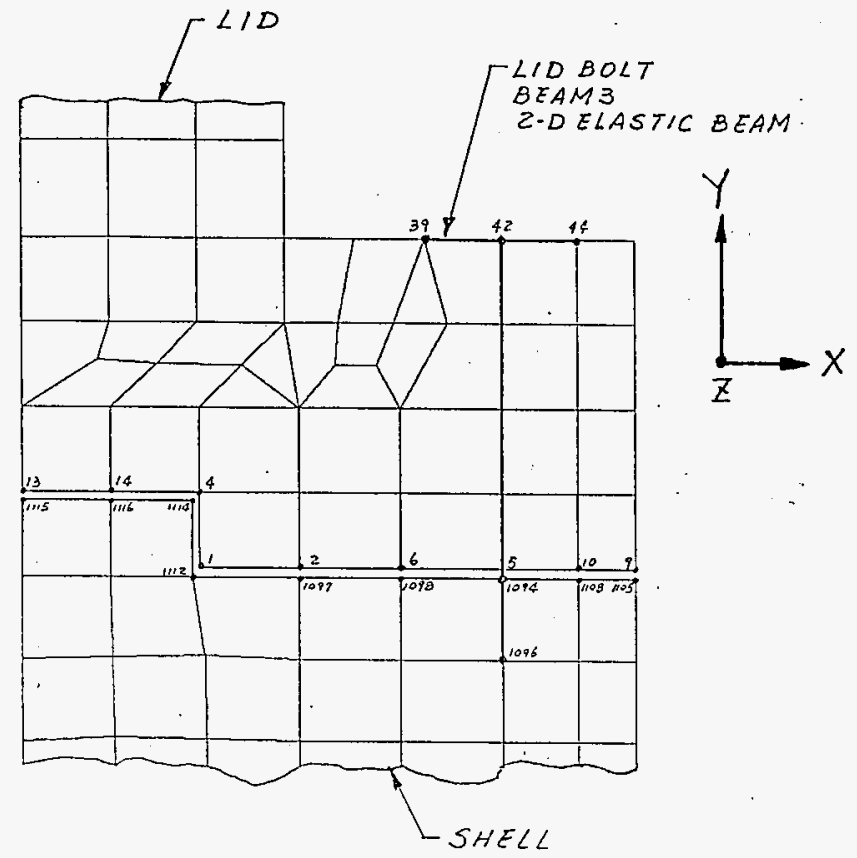

NODES COUPLED IN $\times$ DIRECTION $(4,1114)(1,1112)$

NODES COUPLED IN Y DIRECTION $(13,1115)(14,1116)(4,1114)(1,1112)(2,1097)$

$(6,1098)(5,1094)(10,1108)(9,1105)$

NODES COUPLED IN Z DIRECTION

$(1,1112)(2,1097)(6,1098)(5,1094)$

$(10,1108)(9,1105)$ 


\section{B4.5C-3 LOADINGS}

The loading conditions analyzed simulate or represent various effects due to the normal conditions of transport and hypothetical accident conditions specified in the Hanford Specification.

\section{B4.5C-3.1 Axisymmetric Loadings}

The following individual axisymmetric load cases analyzed using this ANSYS model are described in this section.
(1) Bolt preload
(2) Internal pressure loading
(3) External pressure loading
(4) 1 foot and 30 foot end drops on bottom
(5) 1 foot and 30 foot end drops on lid
(6) Thermal stresses for hot environment at $115^{\circ} \mathrm{F}$ ambient temperature
(7) Thermal stresses for minimum air temperature of $-27^{\circ} \mathrm{F}$ plus zero heat generation.
(8) Thermal accident condition

Since the individual load cases are linearly elastic, their results can be scaled and superimposed as required in order to perform the normal and hypothetical accident condition load combinations. The magnitudes of the loads used in each individual load case analysis are computed as described in the following paragraphs:

\section{Bolt Preload}

A lid seating load corresponding to 25,000 psi axial stress in the bolt is simulated by applying a prestrain of $0.000958 \mathrm{in} / \mathrm{in}$ in the bolt elements. The inputs for the ANSYS's finite element analysis are as follow:

Number of Bolts: 12

Diameter of Bolt: $11 / 2$ in.

Bolt Circle Dia: 36.44 in.

Bolt Prestrain: $\quad 0.000958$ in/in 


\section{Internal Pressure Loading}

An internal pressure of $161.2 \mathrm{psi}$ is applied to the cavity surface as shown in Figure B4.5C13.

\section{External Pressure Loading}

An external pressure of $20 \mathrm{psi}$ is applied to the outer surface of the cask body as shown in Figure B4.5C-14.

\section{1 Foot and 30 Foot End Drops on Bottom}

The analysis described in Appendix B4.5A determined the inertial loads on the cask for 1 foot and 30 foot end drops onto an yielding surface. That analysis concluded that the maximum axial decelerations are $25 \mathrm{~g}$ and $30 \mathrm{~g}$ for 1 foot and 30 foot drops respectively. A quasistatic analysis of the cask body is performed with inertial forces balanced by the impact force for 1 $\mathrm{G}$ deceleration. Since the payload or cargo is not included in the model, its loading effect is simulated as distributed pressures applied on the cask at the appropriate locations. All nodes on the outside bottom surface of the cask are fixed in the axial directions. The system of forces on the cask body is presented on Figure B4.5C-15.

Following is the derivation of the inertia load (pressure) magnitudes for the ANSYS model run:

- Weight of Cask Body:

- Weight of Cargo:

- Maximum Deceleration:

- Pressure due to cargo inertia load
$39,600 \mathrm{lb}$. (Actual wt. $=38,960 \mathrm{lb})$

$19,120 \mathrm{lb}$. (Actual wt. $=18,950 \mathrm{lb}$.)

$25 \mathrm{G}$ for 1 foot drop and $30 \mathrm{G}$ for 30 foot drop $P=19,120 / \pi(12.595)^{2}=38.366$ psi for $1 G$

$1 \mathrm{G}$ was used for the analysis. The stresses for $25 \mathrm{G}$ and $30 \mathrm{G}$ are ratioed from the $1 \mathrm{G}$ analysis results. 
HNF-SD-SNF-FDR-003 Rev. 0

E-15166

Figure B4.5C-13

Load Distribution - Internal Pressure

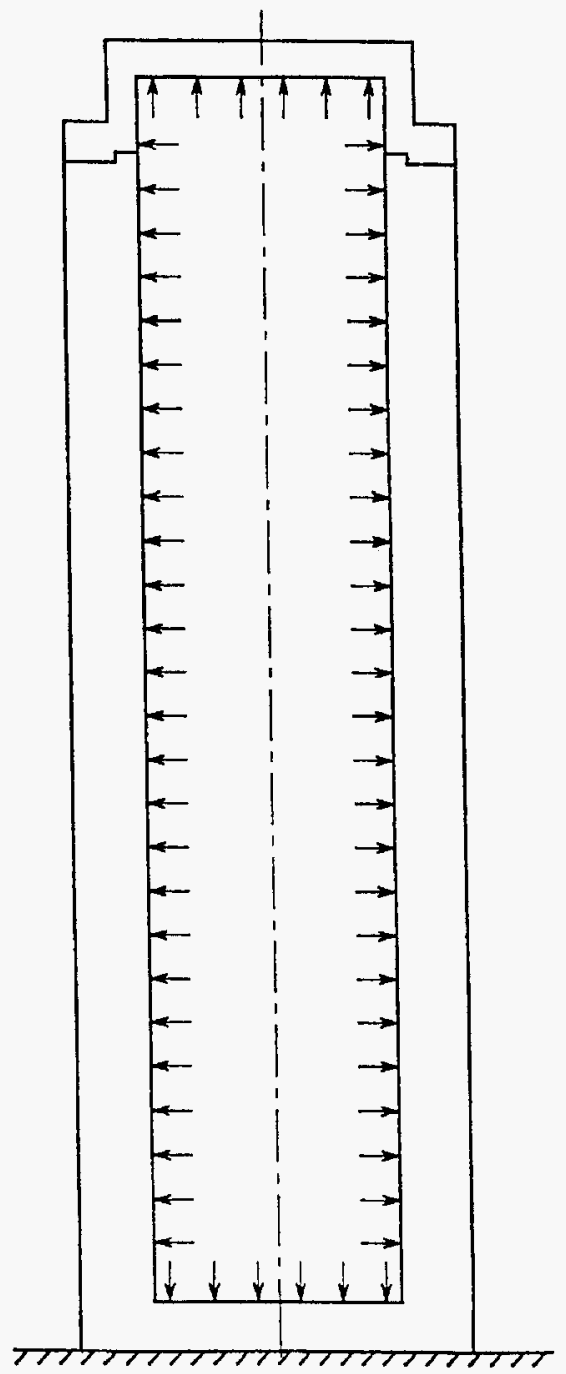

B4.5C-18 
HNF-SD-SNF-FDR-003 Rev. 0

E-15166

Figure B4.5C-14

Load Distribution - External Pressure

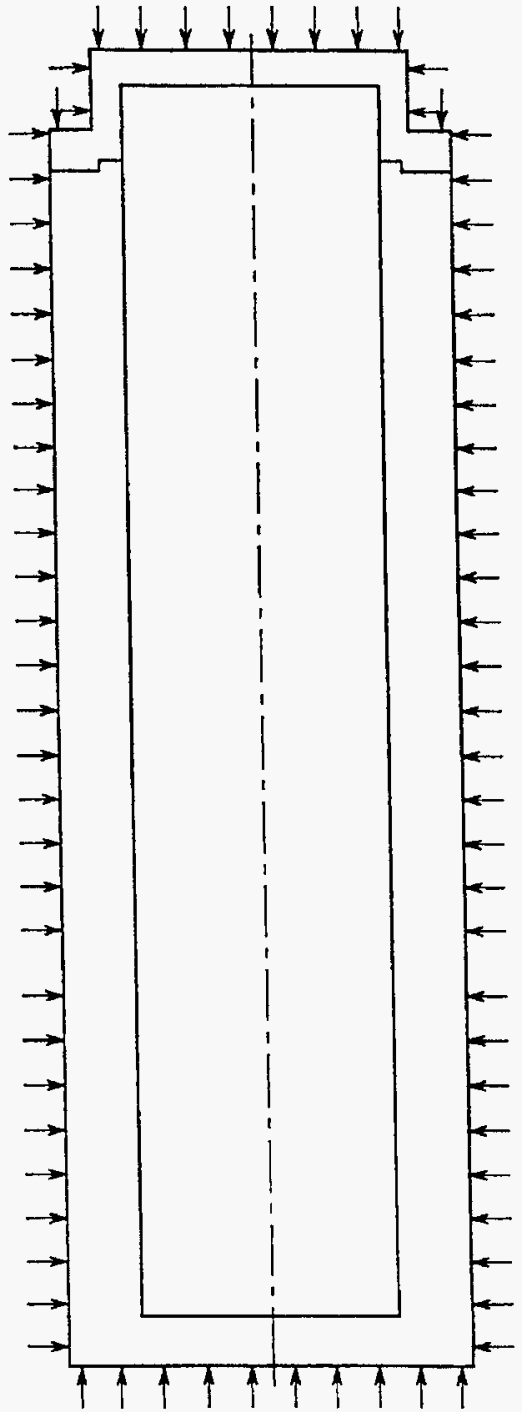

B4.5C-19 
HNF-SD-SNF-FDR-003 Rev. 0

E-15166

Figure B4.5C-15

Load Distribution - Bottom End Drop

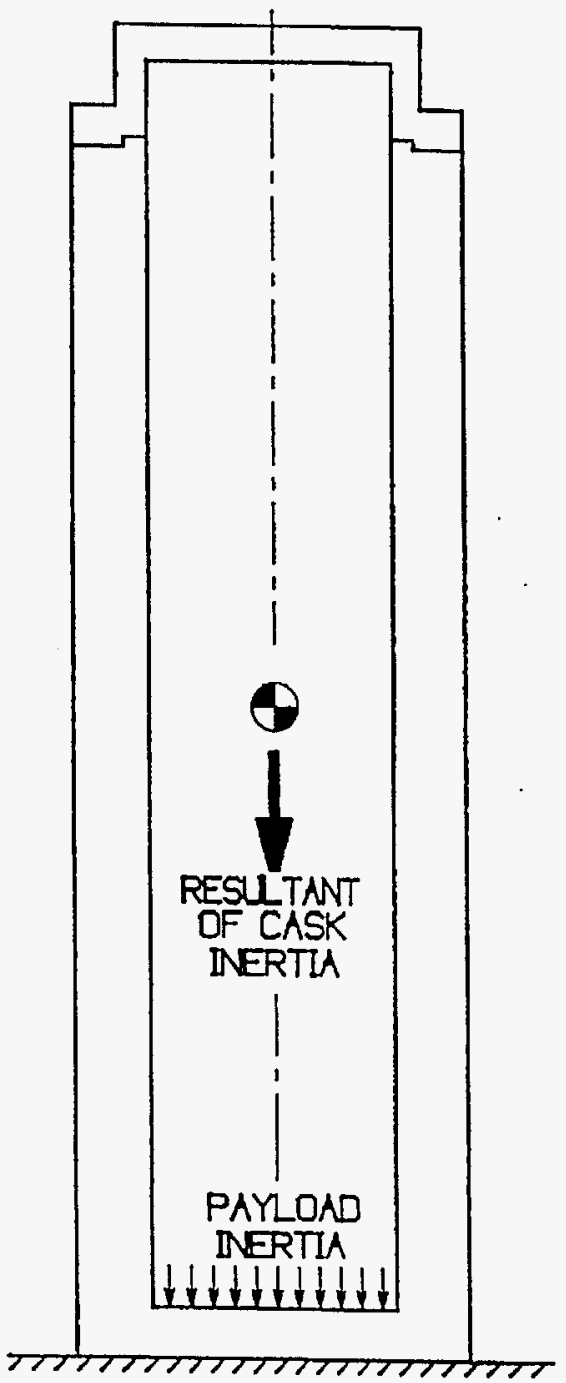

B4.5C-20 


\section{1 Foot and 30 Foot End Drops on Lid}

An analysis similar to that for the 30 foot free drop on the bottom is performed for the 1 foot and 30 foot drops on the lid. The same inertial forces are used for the lid or top impact case as for the bottom impact case. The system of forces on the cask body is presented on Figure B4.5C-16, and the derivation of the magnitudes follows:

- Weight of Cask Body:

-Weight of Cargo:

- Maximum Deceleration:

- Pressure due to cargo inertia load
$39,600 \mathrm{lb}$. $19,120 \mathrm{lb}$. $25 \mathrm{G}$ for 1 foot drop and $30 \mathrm{G}$ for 30 foot drop $\mathrm{P}=19,120 / \pi(12.595)^{2}=38.366 \mathrm{psi}$ for $1 \mathrm{G}$

$1 \mathrm{G}$ was used for the analysis. The stresses for $25 \mathrm{G}$ and $30 \mathrm{G}$ are ratioed from the $1 \mathrm{G}$ analysis results.

\section{Thermal Stress for Hot Environment Condition at $115^{\circ} \mathrm{F}$ Ambient Temperature}

The thermal analysis of the cask body is described in detail in Section B5.0 of this report. That analysis was performed to determine the temperature distribution in the cask body for the condition with maximum solar heating, maximum decay heat from MCO contents, and $115^{\circ} \mathrm{F}$ ambient air. The temperatures at the critical time-step (which resulted in the maximum temperature difference across the cask body thickness) are taken from that analysis and are used in this structural axisymmetric finite element model to calculate the cask body thermal stresses. It is assumed that there is a stress free state at $70^{\circ} \mathrm{F}$ for this case 7 below.

\section{Thermal stresses for minimum air temperature of $-27^{\circ} \mathrm{F}$ plus zero heat generation.}

In this analysis, zero heat generation is assumed with $-27^{\circ} \mathrm{F}$ ambient air temperature. Thus, the cask body will attain a uniform temperature of $-27^{\circ} \mathrm{F}$. For this temperature distribution, the cask body will mostly be stress free, except the flange region which will develop small stresses due to slightly unequal coefficients of expansion of cask lid and bolt materials.

\section{Thermal Accident Condition}

An ANSYS transient thermal analysis for the 30 minute thermal accident is reported in Section B5.0. The initial condition is steady state at $115^{\circ} \mathrm{F}$ ambient with maximum decay heating. The initial steady state condition is followed by a 0.5 hour severe thermal transient which is then followed by water quench. The temperatures through the cross section of the package at the time where individual temperatures peak $(0.5 \mathrm{hrs}$.) are summarized below:

Cask, inner surface temperature $=727^{\circ} \mathrm{F}$

Cask, outer surface temperature $=1068^{\circ} \mathrm{F}$ 


\section{HNF-SD-SNF-FDR-003 Rev. 0}

\section{E-15166}

\section{Lid Bolt temperature $=1068^{\circ} \mathrm{F}$}

The thermal expansion bolt stresses are computed at the highest bolt and flange temperature ( $1068^{\circ} \mathrm{F}$ ) in Appendix B4.5B of this report. 
Figure B4.5C-16

Load Distribution - Lid End Drop

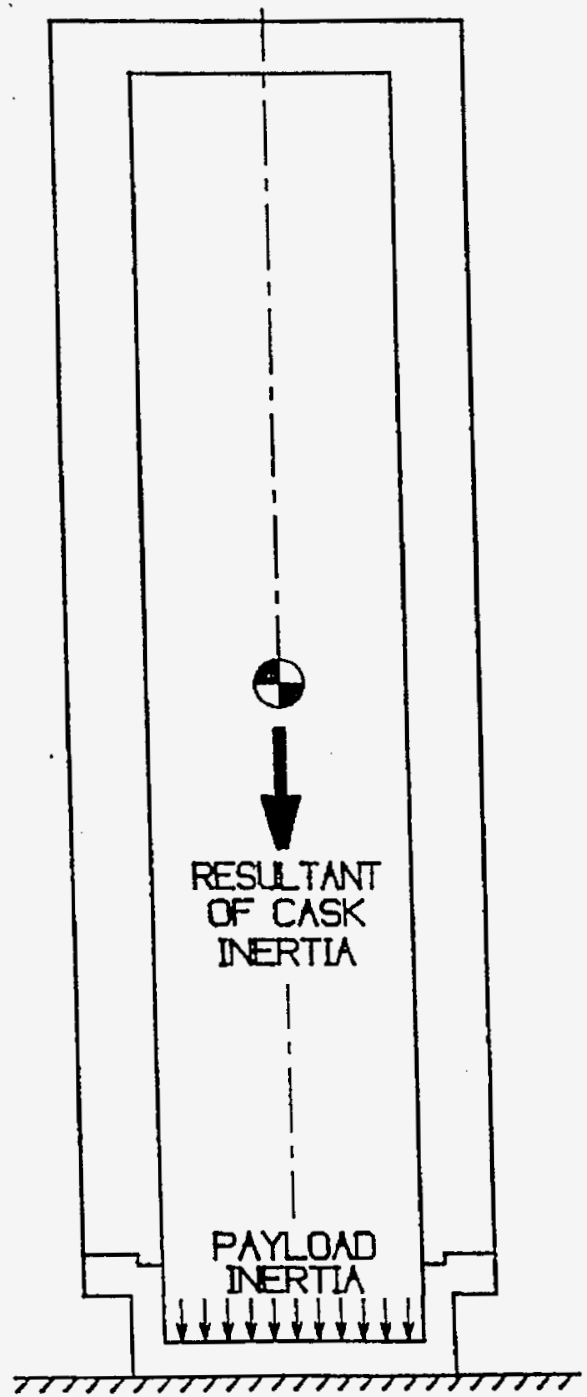

B4.5C-23 


\section{B4.5C-3.2 Asymmetric Loading}

The asymmetric loadings of the axisymmetric cask body are applied to special ANSYS harmonic elements. Each load acting on the cask is expanded into a Fourier series and is input into ANSYS as a series of load steps. Each load step contains all of the terms from the applied loads having the same mode number. The number of terms in the Fourier series required to adequately represent a load varies with the type of load (whether it is a concentrated or a distributed load) and the degree of accuracy required. In the particular case where the applied loads are distributed over a large area (i.e., 180 degrees of the cask circumference), a few terms of the series are sufficient to represent the desired loading within a few percent.

The following individual asymmetric load cases analyzed (using the same two-dimensional ANSYS model previously discussed) are described in this section.

(1) 1 foot and 30 foot side drops with the cask axis parallel to the target.

(2) 1 foot and 30 foot C.G. over top corner (lid end) drops.

\section{1 Foot and 30 Foot Side Drops}

Figure $\mathrm{B} 4.5 \mathrm{C}-17$ shows the free body diagram for the 1 foot and 30 foot free drops on the side of the packaging with all of the forces acting on the cask. These forces are assumed to vary sinusoidally around the circumference, and are:

a. The payload is assumed to be a cosine function over $90^{\circ}$ to $270^{\circ}$ range. The fourier coefficients for this function are computed in Figure B4.5C-18 using ANSYS PREP 6 . It is seen from Figure B4.5C-18 that first 3 terms of Fourier series are sufficient to define the cosine function payload.

Total force $(1 \mathrm{G})=19,120 \mathrm{lbs}$

A cosine distribution is assumed with $90^{\circ} \leq \theta \leq 270^{\circ}$

$$
\mathrm{F}=19,120=\int^{3 \pi / 2} \mathrm{P} \cos \theta \mathrm{rd} \theta \mathrm{L} \cos \theta=\mathrm{PrL} \pi / 2
$$

$\pi / 2$

$\mathrm{P}=2 \mathrm{~F} / \mathrm{rL} \pi=2(19,1200) / 12.595(160.5) \pi=6.0214 \mathrm{psi}$

This peak pressure ( $\mathrm{P}$ times fourier coefficients) is applied to the inside surface nodes. 
b. The reaction pressures due to side drop impact are assumed to be a cosine function over $162^{0}$ to $198^{\circ}$ range. This range corresponds to normal condition side drop target deformation of $1 "$. The first 15 terms are used to represent this function. The fourier coefficients are shown in Figure B4.5C-19. All loads are computed for $1 \mathrm{G}$ deceleration.

$F$, total reaction force $(1 G)=$ cask mass + payload $=38,554+19,120=57,674 \#$ say $58,000 \#$

$$
\begin{aligned}
F & =\operatorname{PrL} \int_{162^{0}}^{198^{\circ}} \cos ^{2} \theta d \theta=\operatorname{PrL}[1 / 4 \sin 2 \theta+\theta / 2] 168^{\circ} \\
& =\operatorname{PrL}(0.6084) \\
P & =58,000 / 19.905 \times 163.25 \times 0.6084=29.34 \mathrm{psi}
\end{aligned}
$$

c. The cask inertia load is developed by applying $1 \mathrm{G}$ global deceleration. Stresses for $24 \mathrm{G}$ ( 1 foot drop) and $40 \mathrm{G}$ (30 foot drop) are ratioed from $1 \mathrm{G}$ analysis results. 
HNF-SD-SNF-FDR-003 ReV. 0

E-15166

Figure B4.5C-17

Load Distribution - Side Drop

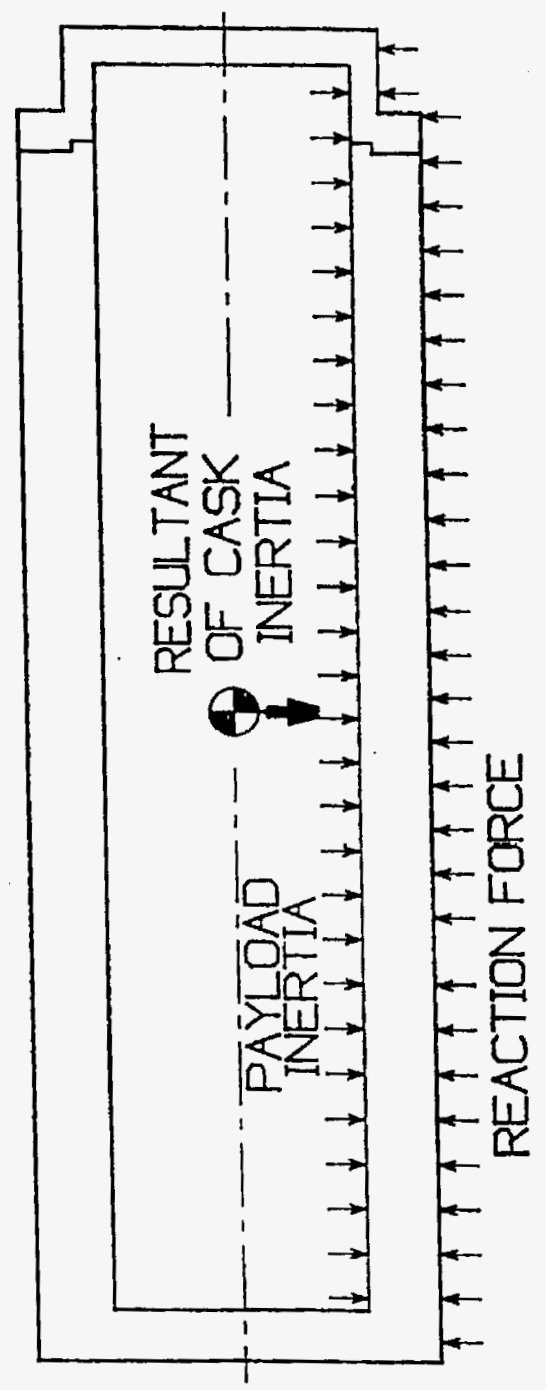


Figure B4.5C-18

Fourier Coefficients for the Function $90^{\circ} \leq \ominus \leq 270^{\circ}$

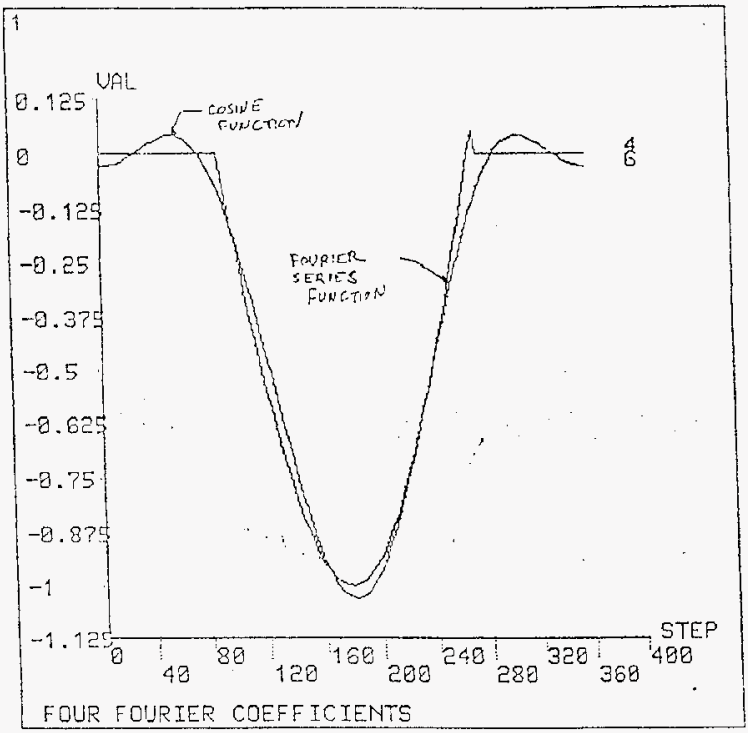

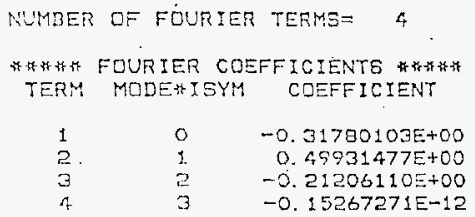


HNF-SD-SNF-FDR-003 Rev. 0

Figure $34.5 C-19$

Fourier Coefficients for the Function $162^{\circ} \leq \theta \leq 198^{\circ}$

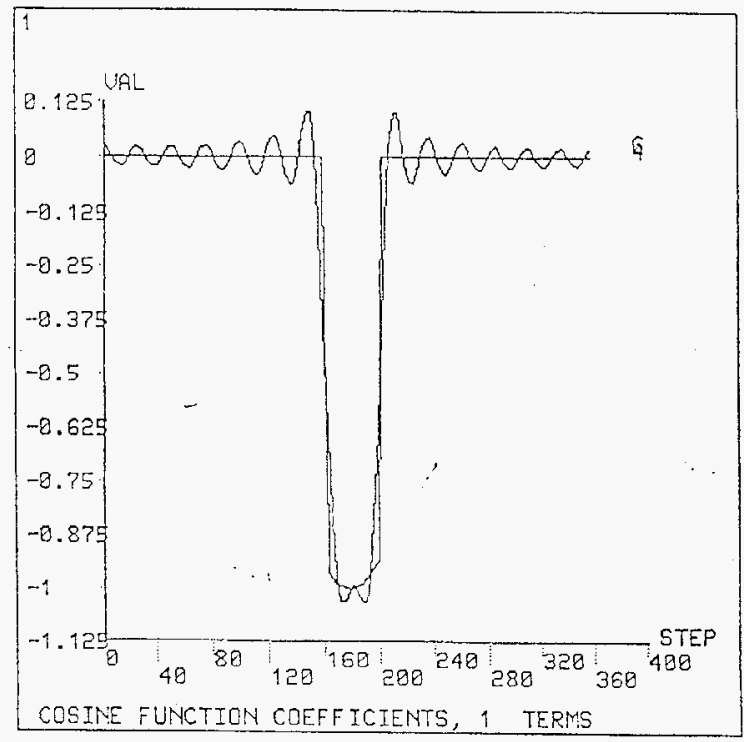

\begin{tabular}{|c|c|c|}
\hline 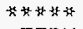 & FOURIER CD & FFICIENTS \\
\hline TERM & MODE*ISYM & COEFFICIENT \\
\hline 1 & 0 & $-0.10612053 E+00$ \\
\hline ? & 1 & $0.20825277 E+00$ \\
\hline 3 & 2 & $-0.19655742 E+00$ \\
\hline 4 & 3 & $0.17794199 E \div 00$ \\
\hline 5 & 4 & $-0.15364810 E+00$ \\
\hline 6 & 5 & o. $12527336 E+00$ \\
\hline 7 & 6 & $-0.94645397 \mathrm{E}-01$ \\
\hline$\theta$ & 7 & 0. $636793635-01$ \\
\hline . & 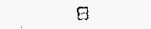 & $-0.34231121 \mathrm{E}-0:$ \\
\hline 10 & 9 & $0.795820535-02$ \\
\hline 11 & 10 & 0. $13799300 E-01$ \\
\hline 12 & 11 & $-0.30103963 E-01$ \\
\hline 13 & 12 & $0.40497913 E-01$ \\
\hline 24 & 13 & $-0.44976925 E-01$ \\
\hline 15 & 14 & 0. $43594560 E-01$ \\
\hline
\end{tabular}


HNF-SD-SNF-FDR-003 Rev. $0 \quad$ E-15166

\section{1 Foot and 30 Foot C.G. Over Top Corner (Lid End) Drops}

The crush footprint of the concrete slab was projected to the cask surface. The impact force was determined from the incrtia loading reported in Appendix $B 4.5 \AA$. The impact angle for the C.G. over corner drop is approximately $79.83^{11}$. Figure B4.5C-20 shows the free body diagram for the 1 foot and 30 foot free drops on the corner of the packaging showing all of the forces acting on the cask. These are:

(1) The crush footprint of the concrete slab was projected to the cask surface. The impact force was determined from the inertial loading reported in $A$ ppendix B4.5A. The crush force was converted into an equivalent clement surface pressure (which varies sinusoidally in both the circumferential and axial directions) which was applied normal to the surfaces. All pressures are calculated for $1 \mathrm{G}$ deceleration.

\section{Normal Pressure}

Crushing depth. $\mathrm{L}=15.0^{\prime \prime}$

$P_{\text {normai }}=2 \mathrm{~F} / \mathrm{r} L \pi$

The total length of $15.0^{\prime \prime}(1)$ is divided two lengths:

$7.0^{\prime \prime}\left(\mathrm{L}_{1}\right)$ for $15.75^{\prime \prime}$ radius and $8.0^{\prime \prime}\left(\mathrm{L}_{2}\right)$ for $19.905^{\prime \prime}$ radius

$P_{L 1}=31.969 \mathrm{psi}$

$$
P_{L 2}=22.134 \mathrm{psi}
$$

These pressures are assumed to be a cosine function over $90^{\circ}$ to $270^{\circ}$ range. The fourier coeficients for this function are computed in Figure B4.5C-18.

\section{Axial Pressure}

The axial load is distributed sinusoidally on the outer surface of the lid:

$$
F_{\text {axial }}=37,400 \times 0.984+19.120 \times 0.984=55,616 \mathrm{lbs}
$$

The computer analysis is hased on the pressure varying sinusoidally at half of the outer surface of the lid.

$$
\mathrm{P}_{\text {tuxitul }}=2 \times 55.616 / \pi \times 15.75^{2}=142.73 \mathrm{psi}
$$




\section{HNF-SD-SNF-FDR-003 ReV. 0 E-15166}

(2) The resultant body inertia force (1 G deceleration) is shown acting at the cask C.G. in Figure $34.5 \mathrm{C}-20$

(3) The cargo inertia loading was applied in two mutually perpendicular directions (one along the axis of the cask and the other perpendicular to it). The component along the axial direction was distributed uniformly over the inside surface of the lid. The other component was assumed to vary sinusoidally around the lower half of the inside surface of the inner shell. In this case, these pressures are not only varied sinusoidally around the circumference but also varied linearly with distance from the bottom inner surface $(0)$ to top inner surface (max.).

For $1 \mathrm{G}$ acceleration at $\mathrm{CG}$ :

Axial $G=1.0 \times \sin 79.83^{6}=0.984$

Normal $G=1.0 \times \cos 79.83^{\circ}=0.177$

\section{Axial Pressure}

$$
\mathrm{P}_{\mathrm{axal}}=19,120(0.984) / \pi \times 12.595^{2}=37.752 \mathrm{psi}
$$

\section{Normal Pressure}

$$
P_{\text {normal }}=2 \mathrm{~F} / \mathrm{r} L \pi
$$

The normal acceleration component variation is assumed as triangular. This triangular $\mathrm{G}$ distribution is divided in four step conservative distribution as follow:

$$
\begin{aligned}
& P_{1}=0.089 \mathrm{G}=0.536 \mathrm{psi} \\
& P_{2}=0.177 \mathrm{G}=1.065 \mathrm{psi} \\
& P_{3}=0.2745 \mathrm{G}=1.652 \mathrm{psi} \\
& P_{4}+0.372 \mathrm{G}=2.238 \mathrm{psi}
\end{aligned}
$$

These pressures are assumed to be a cosine function over $90^{\circ}$ to $270^{\circ}$ range. The fourier cocficients for this function are computed in Figure B4.5C-18.

(4) Stresses for 18 G (1 foot drop) and 20 G (30) foot drop) are ratioed from 1 G analysis results. 
Figure B4.5C-20

Load Distribution - Lid End Corner Drop

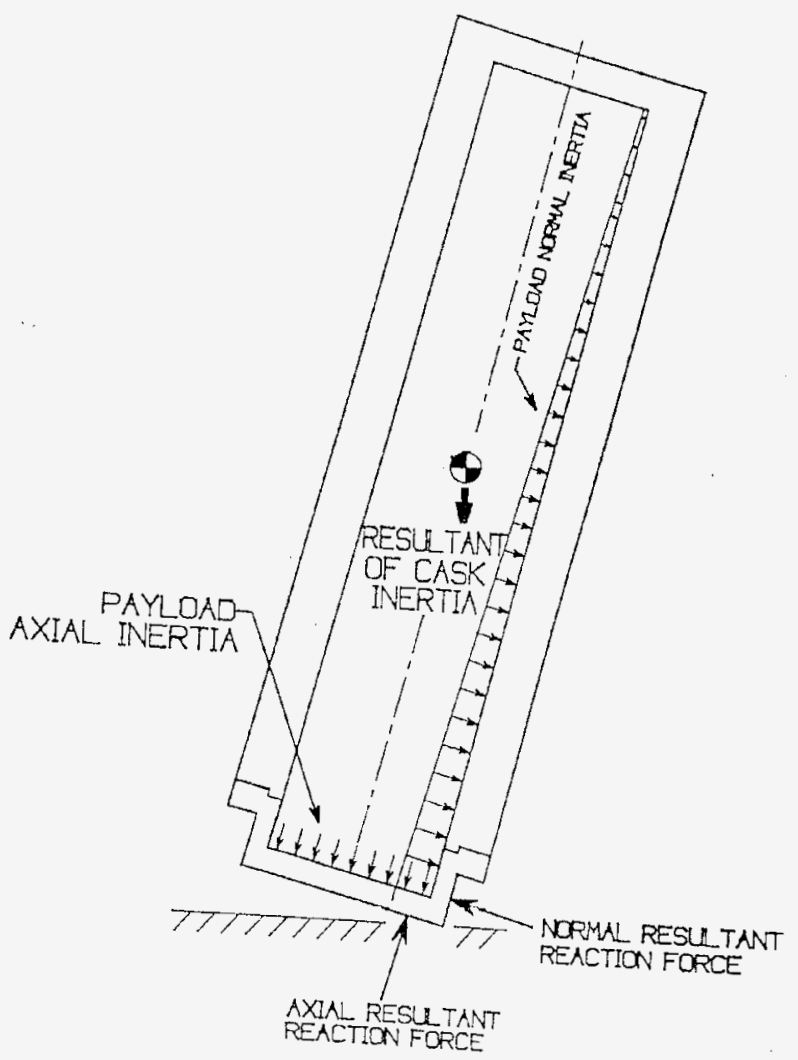




\subsection{C-4 PUNCTURE ANALYSIS}

\section{Puncture Force}

The most scvere damage to the cask resulting from the puncture drop will occur on the outer center of the lid. The analysis is based on the Nelms ${ }^{(3)}$ equation. The Nelms puncture relation is given as:

$$
\begin{aligned}
& \mathrm{t}=\left(\mathrm{W} / \mathrm{S}_{\mathrm{u}}\right)^{0,31} \\
& \text { Where: } \\
& \qquad \begin{array}{l}
\mathrm{t}=\text { lid thickness }=3.5^{11} \\
\mathrm{~W}=\text { package wcight }=57.800 \mathrm{lbs} \\
\mathrm{S}_{\mathrm{u}}=\text { ultimate tensile strength of the lid }=70,000 \mathrm{psi}
\end{array}
\end{aligned}
$$

The package weight that can result in puncture is:

$$
W=S_{11} t^{1 .+1}=70,000(3.5)^{1+1 i}=409,482 \mathrm{lbs}
$$

The actual package weight is $57.910 \mathrm{lbs}$. Therefore, the factor of safety for puncture resistance on the energy basis is:

$$
\text { F.S. }=409,482 / 57,910=7.07
$$

When the package contacts the puncture bar, the force applied to the package is:

$$
\begin{aligned}
& \mathrm{F}_{1}=\text { impact force }=\sigma, A_{p} \\
& \sigma_{\mathrm{g}}=\text { dynamic flow pressure of stainless steel }=45,000 \text { psi (reference 4) } \\
& \hat{\mathrm{p}}_{\mathrm{p}}=\text { area of the puncture bar }=\pi / 4(6)^{2}=28.27 \mathrm{in}^{2} \\
& \mathrm{~F}_{1}=45,000 \times 28.27=1.272 \times 10^{6} \mathrm{bs}
\end{aligned}
$$

This force produces a cask deceleration:

$$
\mathrm{G}=\text { cask deceleration }=F_{\mathrm{r}} / \mathrm{W}=1.272 \times 10^{6} / 57,910=22
$$

This deceleration is smaller than that which will occur during impact on end after the 30 foot free drop (25G). Therefore, global stresses that result from the inertial force will be smaller. The bending stress at the center of the lid will be calculated using the above finite element model. 
HNF-SD-SNF-FDR-003 Rev. 0 Ei-15166

\section{Lid Stresses}

The lid stresses are computed using the 2-D model as described in Section B4.5C-2. The loading distribution and boundary conditions are shown on Figures B4.5C-21 and B4.5C-22. The $22 \mathrm{G}$ inertia load due to 40 " drop is applied as bedy acceleration. The contents inertia load is applied as equivalent pressure on the lid as follow:

Contents weight $=19,120 \mathrm{lbs}$

$\mathrm{P}=19.120 \times 22 / \pi(12.595)^{2}=844.05 \mathrm{psi}$

\section{Elastic Analysis:}

ANSYS computer Code is used for the analysis. A stress run was made using the above noted londs and boundary conditions. STIF 42 (Axisymmetric) finite element was used in the analysis.

The stress are linearized at critical sections. The maximum membrane and maximum membrane plus bending stress intensities are as follow:

\begin{tabular}{|c|c|c|}
\hline $\begin{array}{c}\text { Section at Node number } \\
\text { (Figure B4.5C-23) }\end{array}$ & $\mathrm{P}_{\mathrm{m}}$ (psi) & $\mathrm{P}_{\mathrm{m}}+\mathrm{P}_{\mathrm{b}}$ (psi) \\
\hline 84 & 12.560 & 83,280 \\
\hline 94 & 10.880 & 85,010 \\
\hline 93 & 5.275 & 70,560 \\
\hline 92 & 30.780 & 57,550 \\
\hline 91 & 25.430 & 59,960 \\
\hline
\end{tabular}

The maximum membrane stress intensity caleuiated for this load is 30,780 psi at node number 92 . This stress is below the allowable membrane stress intensity of 47.670 psi. The maximum membranc plus bending stress intensity is $85,010 \mathrm{psi}$ at node number 94 . This stress exceeds the allowable membrane plus bending stess intensity of 68,100 psi (at temperature $150^{n} \mathrm{~F}$ ).

Therefore, a elastic-plastic analysis was performed to recalculate the stresses.

\section{Plastic Analysis:}

$A$ plastic analysis was performed (using the same finite element model, boundary conditions, and loads). The stress-strain propertics used for the stainless steel are given in Figure B4.5C-24. The total load (22 G incrtia and 844.05 psi) was divided in eight load steps for a proper converged solution. 
HNF-SD-SNF-FDR-003 Rev. $0 \quad$ E-15166

The maximum membrane and maxinum membrane plus bending stress intensities are as follow:

\begin{tabular}{|c|c|c|}
\hline $\begin{array}{c}\text { Section at Node number } \\
\text { (Figure B4.5C-23) }\end{array}$ & $\mathrm{P}_{\mathrm{m}}$ (psi) & $\mathrm{P}_{\mathrm{m}}+\mathrm{P}_{\mathrm{b}}$ (psi) \\
\hline 84 & 2,934 & 45,030 \\
\hline 94 & 3,326 & 46,620 \\
\hline 93 & 6,889 & 42,140 \\
\hline 92 & 25,470 & 36,060 \\
\hline 91 & 26,350 & 40,780 \\
\hline
\end{tabular}

The maximum membrane stress intensity calculated for this load is 26,350 psi at node number 91 . This stress is below the allowable membrane stress intensity of 47,670 psi $\left(0.7 \mathrm{~S}_{\mathrm{u}}\right)$. The maximum membrane plus bending stress intensity is 46,620 psi at node number 94 . This stress is below the allowable membrane plus bending stress intensity of $61.290 \mathrm{psi}\left(0.9 \mathrm{~S}_{4}\right)$. The analysis shows the stresses are within the ASME Code allowables. 
Figure B4.5C-21

Load Distribution - Puncture at Center of Lid

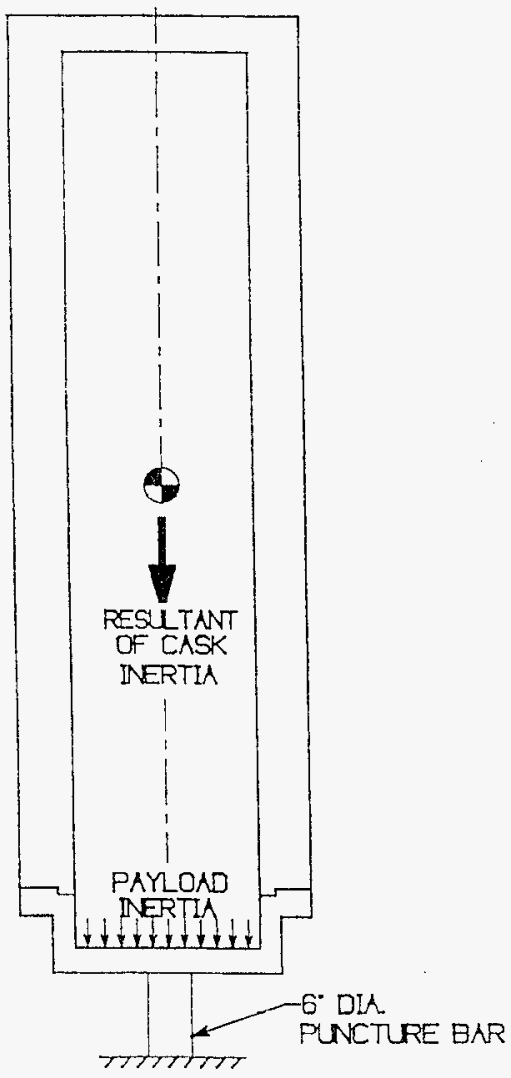


Figure $134.5 \mathrm{C}-22$

Boundary Conditions - Puncture at Center of Lid

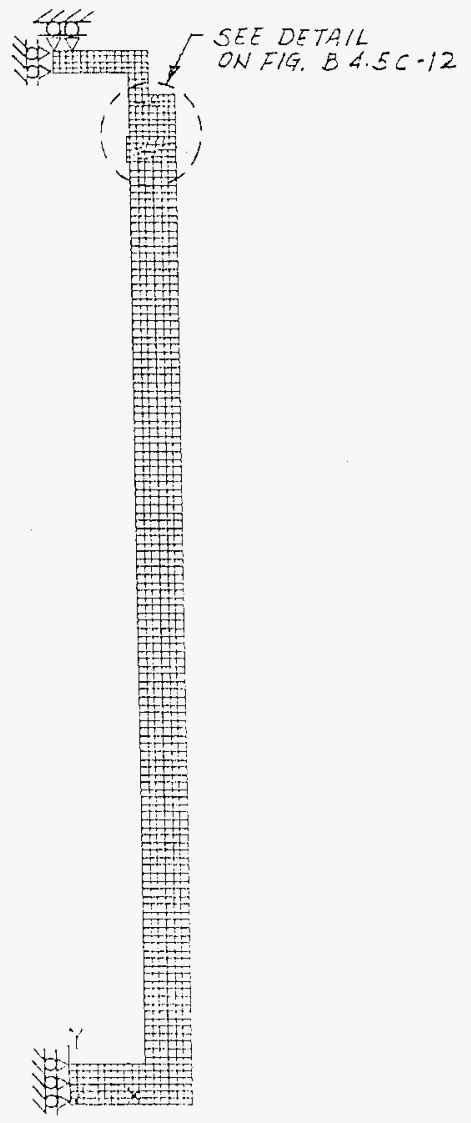


HNF-SD-SNF-FDR-003 Rev. 0 E-15166

Figure B4.5C-23

Stress Reporting Locations - Puncture at Center of Lid

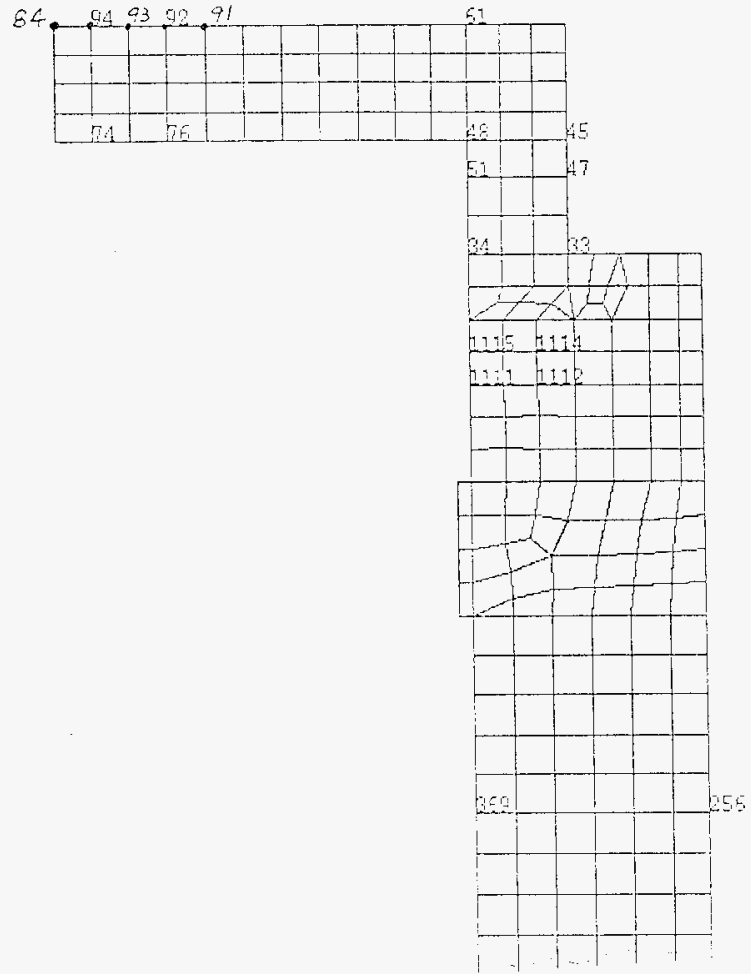


Figure B4.5C-24

Stress - Stain Curve for 304 Stainless Steel

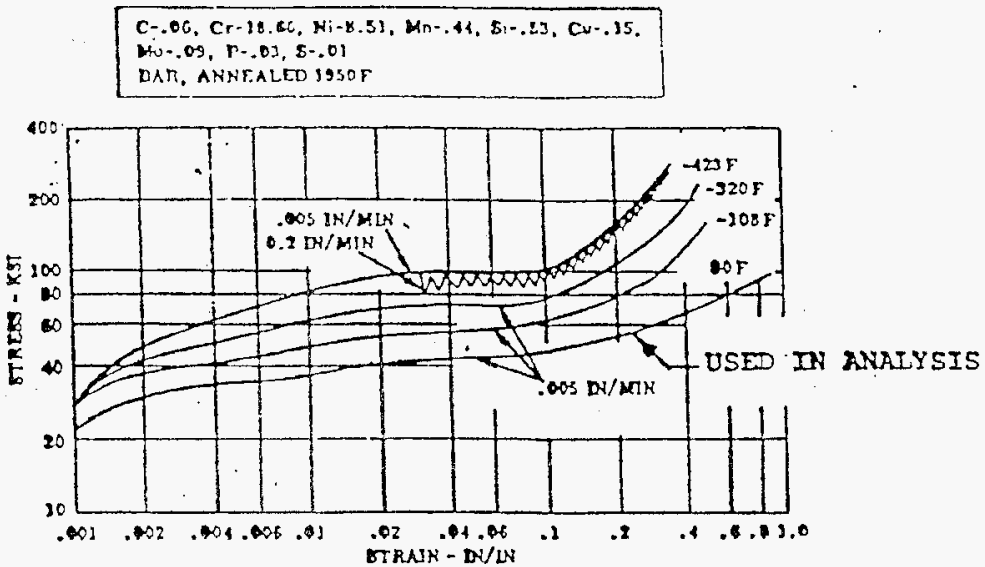

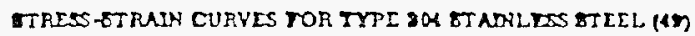




\section{B4.5C-5 STRESS RESULTS}

Detailed stresses and displacements in the ANSYS model of the cask body are obtained and stored (on magnetic tape) for every node location for ench individual load case. These stored results are postprocessed to printout the stresses at 29 standard locations on the cask body structure shown in Figure B4.5C-25. The locations sclected as shown in Figure B4.5C-25 are key points that, when carefully studied. indicate the behavior of the entire structure. The maximum stress may occur at a different location for each individual load. Since the individual lond cases are linearly elastic, their results can be scaled and superimposed as required in order to porform the normal and hypothetical accident condition load combinations.

The individual load cases analyzed are listed in Table $134.5 \mathrm{C}-2$. Linear elastic analyses were performed for all load cases. The nodal stress intensities for each of the ten load cases are reported in Tables $\mathrm{B} 4.5 \mathrm{C}-3$ to $\mathrm{B} 4.5 \mathrm{C}-12$ as listed in Table B4.5C-2. The magnitude of loads used in each individual load cose and summary of the maximum stresses are described in the Table $\mathrm{B} 4.5 \mathrm{C}-1$. There are no specific limits for individual load stresses for comparison with allowable stresses.

It should be noted that, for the axisymnetric analyses, the stress is constant around the cask at cvery location. For asymmetric analyses with significant differences in stress magnitudes on opposite sides of the cask, the stresses at locations on both sides of the cask (contacting side and side opposite contact during impact) are reported in stress tables. 
HNF-SD-SNF-FDR-003 Rev. $0 \quad$ E-15166

Figure B4.5C-25

Standard Stress Reporting Locations

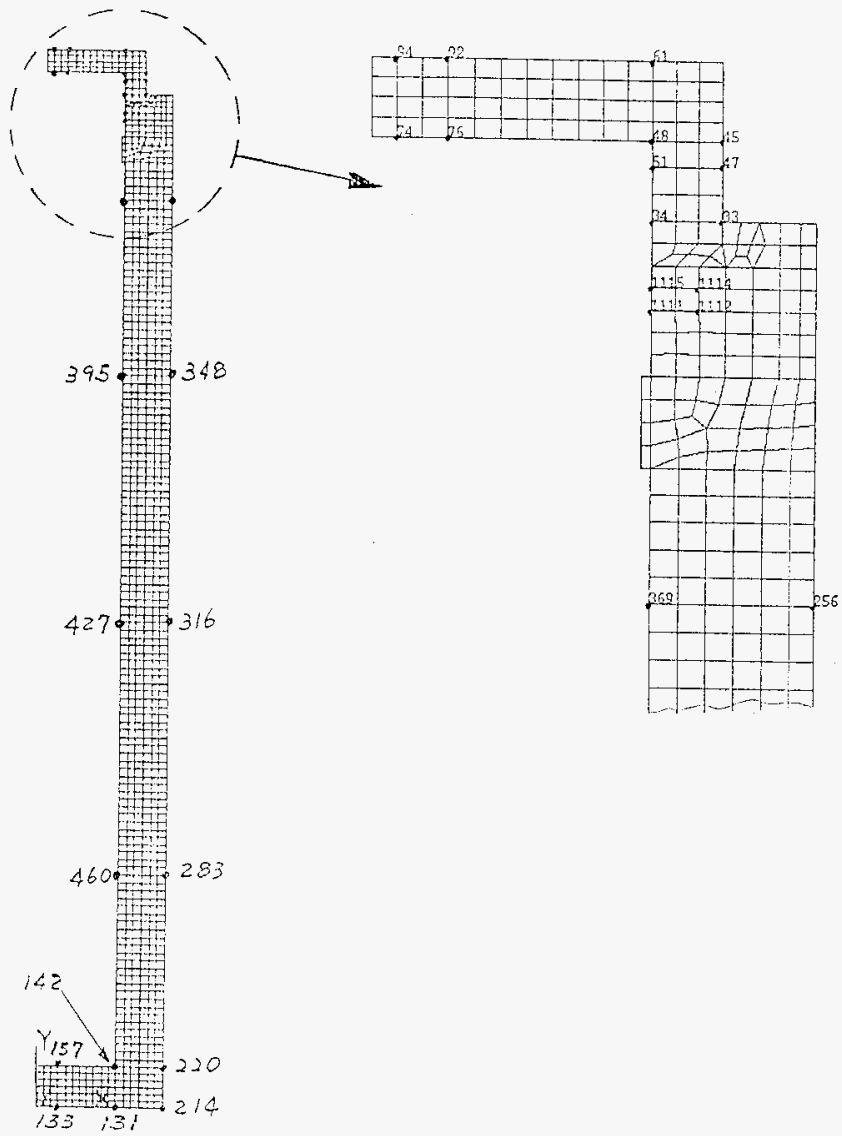


Table B4.5C-1

Cask Body Stresses - Summary of Individual Load Analyses

\begin{tabular}{|c|c|c|c|}
\hline Load Case & Loading & $\begin{array}{c}\text { Calculated Stress } \\
\text { intensity } \\
\text { (psi) }\end{array}$ & $\begin{array}{l}\text { Reference } \\
\text { Table No. }\end{array}$ \\
\hline Bolt Preload & $25,000 \mathrm{psi}$ & 313 & $\mathrm{~B} 4.5 \mathrm{C}-3$ \\
\hline Internal Pressure & $161.2 \mathrm{psi}$ & 1,675 & $\mathrm{~B} 4.5 \mathrm{C}-4$ \\
\hline External Pressure & $20 \mathrm{psi}$ & 208 & B $4.5 \mathrm{C}-5$ \\
\hline Vibration & $.6 \mathrm{G} \vee .3 \mathrm{G}, .3 \mathrm{G}$ & 138 & B $34.5 \mathrm{C}-6$ \\
\hline 1 Foot Bottom Lnd Drop & $25 G$ & 1,200 & $\mathrm{~B} 4.5 \mathrm{C}-7$ \\
\hline $\begin{array}{l}30 \text { Foot Bottom End } \\
\text { Drop }\end{array}$ & $30 \mathrm{G}$ & 1,440 & $\mathrm{~B} 4.5 \mathrm{C}-7$ \\
\hline 1 Foot Lid End Drop & $25 \mathrm{G}$ & 3,625 & $\mathrm{~B} 4.5 \mathrm{C}-8$ \\
\hline 30 Foot Lid End Drop & $30 \mathrm{G}$ & 4,350 & $\mathrm{~B} 4.5 \mathrm{C}-8$ \\
\hline $\begin{array}{l}1 \text { Foot Side Drop } \\
\text { (Envelop Slap Down) }\end{array}$ & $24 \mathrm{G}$ & 3,091 & B4.5C-9 \\
\hline $\begin{array}{l}30 \text { Foot Side Drop } \\
\text { (Envelop Slap Down) }\end{array}$ & $40 \mathrm{G}$ & 5,152 & $134.5 \mathrm{C}-9$ \\
\hline $\begin{array}{l}1 \text { Foot Lid End } \\
\text { Corner Drop }\end{array}$ & $18 \mathrm{G}$ & 13,468 & $\mathrm{~B} 4.5 \mathrm{C}-10$ \\
\hline $\begin{array}{l}30 \text { Foot Lid End } \\
\text { Corner Drop }\end{array}$ & $20 G$ & 14,964 & $\mathrm{~B} 4.5 \mathrm{C}-10$ \\
\hline Thermal Stress (Flot) & $\begin{array}{l}115^{\circ} \mathrm{F} \\
\text { Environment }\end{array}$ & 1,197 & $\mathrm{~B} 4.5 \mathrm{C}-11$ \\
\hline Thermal Stress (Cold) & $\begin{array}{c}-27^{\circ} \mathrm{F} \\
\text { Environment }\end{array}$ & 23 & B $4.5 \mathrm{C}-12$ \\
\hline Puncture & $22 \mathrm{G}$ & 46,620 & Section B4.5C-4 \\
\hline
\end{tabular}


Table B4.5C-2

Individual Load Cases For TN-WHC Cask Body Analysis

\begin{tabular}{|c|c|c|}
\hline $\begin{array}{l}\text { Load Case } \\
\text { Number }\end{array}$ & Individual Load Description & $\begin{array}{l}\text { Stress } \\
\text { Result } \\
\text { Tabie }\end{array}$ \\
\hline 1 & Bolt Prelond $\quad(25,000 \mathrm{psi})$ & $\mathrm{B} 4.5 \mathrm{C}-3$ \\
\hline 2 & Internal Pressure $\quad(p=161.2 p s i)$ & $\mathrm{B} 4.5 \mathrm{C}-4$ \\
\hline 3 & External Pressure $(p=20 p s i)$ & $\mathrm{B} 4.5 \mathrm{C}-5$ \\
\hline 4 & Vibration ( $3 G$ long., $3 G$ her. . .6G vert.) & $34.5 C-6$ \\
\hline 5 & Drop on Botton/Gravity load (1 G) & $\mathrm{B} 4.5 \mathrm{C}-7$ \\
\hline 6 & Drop on Lid End ( $1 \mathrm{G})$ & B $4.5 \mathrm{C}-8$ \\
\hline 7 & Drop on Side $(1 G)$ & $\mathrm{B} 4.5 \mathrm{C}-9$ \\
\hline 8 & Drop over Comer, Lid End (l G) & B4.5C- 10 \\
\hline 9 & Thermal Stress (hot) & B $4.5 \mathrm{C}-11$ \\
\hline 10 & Thermal Stress (cold) & $\mathrm{B} 4.5 \mathrm{C}-12$ \\
\hline
\end{tabular}


Table B4.5C-3

Cask Body Stresses For Bolt Preload $\quad(25,000$ psi)

\begin{tabular}{|c|c|c|}
\hline \multicolumn{2}{|c|}{ Location } & \multirow{2}{*}{$\begin{array}{c}\text { Nodal Stress Intensity } \\
\text { (psi) } \\
\end{array}$} \\
\hline Cask Bodv Comnonent & Node Number & \\
\hline \multirow[t]{6}{*}{ Cask Bottom } & 133 & 0 \\
\hline & 131 & 0 \\
\hline & 214 & 0 \\
\hline & 157 & 0 \\
\hline & 142 & 0 \\
\hline & 220 & 0 \\
\hline \multirow[t]{8}{*}{ Cask Cylinder } & 460 & 0 \\
\hline & 427 & 0 \\
\hline & 395 & 1 \\
\hline & 369 & 26 \\
\hline & 283 & 0 \\
\hline & 310 & 0 \\
\hline & 348 & 1 \\
\hline & 256 & 13 \\
\hline \multirow[t]{4}{*}{ Cask Flange } & 1111 & 69 \\
\hline & 1112 & 64 \\
\hline & 1115 & 50 \\
\hline & 1114 & 180 \\
\hline \multirow[t]{11}{*}{ Lid } & 34 & 313 \\
\hline & 33 & 307 \\
\hline & 51 & 236 \\
\hline & 47 & 118 \\
\hline & 74 & 93 \\
\hline & 76 & 95 \\
\hline & 48 & 128 \\
\hline & 45 & 78 \\
\hline & 94 & 24 \\
\hline & 92 & 24 \\
\hline & 61 & 12 \\
\hline
\end{tabular}

* See Figure $34.5 \mathrm{C}-25$ for the node locations 
Table 84.5C-4

Cask Body Stresses For 161.2 psi Internal Pressure

\begin{tabular}{|c|c|c|}
\hline \multicolumn{2}{|c|}{ Location } & \multirow{2}{*}{$\begin{array}{c}\text { Nodal } \begin{array}{c}\text { Stress Intensity } \\
(\mathrm{psi})\end{array} \\
\end{array}$} \\
\hline Cask Body Component & Node Number ${ }^{\circ}$ & \\
\hline \multirow[t]{6}{*}{ Cask Bottom } & 13.3 & 563 \\
\hline & 131 & 290 \\
\hline & 214 & 67 \\
\hline & 157 & 210 \\
\hline & 142 & 448 \\
\hline & 220 & 106 \\
\hline \multirow[t]{8}{*}{ Cask Cylinder } & 460 & 540 \\
\hline & 427 & 5.35 \\
\hline & 395 & 536 \\
\hline & 369 & 516 \\
\hline & 283 & 218 \\
\hline & 316 & 215 \\
\hline & 348 & 215 \\
\hline & 256 & 216 \\
\hline \multirow[t]{4}{*}{ Cask Flange } & 1111 & 410 \\
\hline & 1112 & 252 \\
\hline & 1115 & 401 \\
\hline & 11114 & 401 \\
\hline \multirow[t]{11}{*}{ Lid } & 34 & 421 \\
\hline & 33 & 293 \\
\hline & 51 & 1,569 \\
\hline & 47 & 449 \\
\hline & 74. & 1,055 \\
\hline & 76 & 932 \\
\hline & 48 & 889 \\
\hline & 45 & 108 \\
\hline & 94 & 1,675 \\
\hline & 92 & $1.55]$ \\
\hline & 61 & 85.3 \\
\hline
\end{tabular}

* See Figure B4.5C-25 for the node locations 
Table $84.5 \mathrm{C}-5$

Cask Body Stresses For 20 psi External Pressure

\begin{tabular}{|c|c|c|}
\hline \multicolumn{2}{|c|}{ Location } & \multirow{2}{*}{$\begin{array}{c}\text { Nodal Stress Intensity } \\
\text { (psi) }\end{array}$} \\
\hline Cask Bodv Component & Node Number & \\
\hline \multirow[t]{6}{*}{ Cask Bottom } & 133 & 70 \\
\hline & 131 & 36 \\
\hline & 214 & 9 \\
\hline & 157 & 27 \\
\hline & $1+2$ & 56 \\
\hline & 220 & 13 \\
\hline \multirow[t]{8}{*}{ Cask Cylinder } & 460 & 67 \\
\hline & 427 & 66 \\
\hline & 305 & 66 \\
\hline & 369 & 64 \\
\hline & 283 & 27 \\
\hline & 316 & 27 \\
\hline & 348 & 27 \\
\hline & 256 & 27 \\
\hline \multirow[t]{4}{*}{ Cask Flange } & 1111 & 51 \\
\hline & 1112 & 31 \\
\hline & 1115 & 50 \\
\hline & 1114 & 49 \\
\hline \multirow[t]{11}{*}{ Lid } & 34 & 52 \\
\hline & 33 & 36 \\
\hline & 51 & 195 \\
\hline & 47 & 56 \\
\hline & 74 & 131 \\
\hline & 76 & 115 \\
\hline & 48 & 110 \\
\hline & 45 & 75 \\
\hline & 24 & 208 \\
\hline & 92 & 193 \\
\hline & 61 & 106 \\
\hline
\end{tabular}

* See Figure B4.5C-25 for the node locations 
HNF-SD-SNF-FDR-003 Rev. 0

E-15166

Table B4.5C-6

Cask Body Stresses For Vibration Loadings $1.6 \mathrm{~g}$ vert., $.3 \mathrm{~g}$ long., $.3 \mathrm{~g}$ lat.)

\begin{tabular}{|c|c|c|c|}
\hline \multicolumn{2}{|l|}{ Location } & \multicolumn{2}{|c|}{ Nodal Stress Intensity (ps } \\
\hline Cask Body Comporent & Node & 0 Degree & 90 Degree \\
\hline \multirow[t]{6}{*}{ Cask Bottom } & 133 & 138 & 43 \\
\hline & 131 & 28 & 20 \\
\hline & 214 & 16 & 33 \\
\hline & 157 & 134 & 35 \\
\hline & 142 & 52 & 42. \\
\hline & 220 & 16 & 28 \\
\hline \multirow[t]{8}{*}{ Cask Cylinder } & 460 & 26 & 27 \\
\hline & 427 & 22 & 18 \\
\hline & 395 & 24 & 28 \\
\hline & 369 & 4 & 15 \\
\hline & 283 & $3 \times 4$ & 25 \\
\hline & 316 & 30 & 17 \\
\hline & 348 & 13 & 11 \\
\hline & 256 & 3 & 9 \\
\hline \multirow[t]{4}{*}{ Cask Flange } & 1111 & 3 & 4 \\
\hline & $-1: 12$ & 2 & 4 \\
\hline & 1115 & 4 & 4 \\
\hline & 1114 & 3 & 4 \\
\hline \multirow[t]{11}{*}{ Lid } & 34 & 2 & 6 \\
\hline & 33 & 2 & 7 \\
\hline & 51 & 4 & 8 \\
\hline & 47 & 2 & 5 \\
\hline & 74 & 8 & 6 \\
\hline & 76 & 4. & 5. \\
\hline & 48 & 2 & 7 \\
\hline & 45 & 2 & 4 \\
\hline & .24 & 7 & 7 \\
\hline & 92 & 6 & 7 \\
\hline & 61 & 5 & 4 \\
\hline
\end{tabular}

See rigtre $34.5 \mathrm{C}-25$ for the node locations 
Table B4.5C-7

Cask Body Stresses For Bottom End Drops and Gravity Load

$1 \mathrm{G}$ results, to be ratioed for 1 foot $(25 \mathrm{G})$ and 30 foot $(30 \mathrm{G})$ drops

\begin{tabular}{|c|c|c|}
\hline \multicolumn{2}{|c|}{ Location } & \multirow{2}{*}{$\begin{array}{c}\text { Nodal Stress Intensity } \\
\text { (psi) }\end{array}$} \\
\hline Cask Bodv Componen! & Node Number & \\
\hline \multirow[t]{6}{*}{ Catsk Bottom } & 133 & 40 \\
\hline & 131 & 45 \\
\hline & 214 & 48 \\
\hline & 157 & 40 \\
\hline & 142 & 47 \\
\hline & 220 & 46 \\
\hline \multirow[t]{8}{*}{ Cask Cylinder } & 460 & 37 \\
\hline & 427 & 26 \\
\hline & 395 & 14 \\
\hline & 369 & 7 \\
\hline & 283 & 37 \\
\hline & 316 & 20 \\
\hline & .48 & 14 \\
\hline & 256 & 7 \\
\hline \multirow[t]{4}{*}{ Cask Flange } & 11.11 & 4 \\
\hline & 1112 & 4 \\
\hline & 1115 & 4 \\
\hline & 1114 & 5 \\
\hline \multirow[t]{11}{*}{ Lid } & 34 & 5 \\
\hline & 33 & 4 \\
\hline & 51 & 11 \\
\hline & 47 & 2 \\
\hline & 74 & 9 \\
\hline & 76 & 8 \\
\hline & 48 & 7 \\
\hline & 45 & 3 \\
\hline & 94 & 11 \\
\hline & 92. & 10 \\
\hline & 61 & 6 \\
\hline
\end{tabular}

* Sec Figure B34.5C-25 for the node locations 
HNF-SD-SNF-FDR-003 Rev. 0

Table B4.5C-8

Cask Body Stresses For Lid End Drops

$1 \mathrm{G}$ results, to be ratioed for 1 foot $(25 \mathrm{G}$ ) and 30 toot (30 G) drops

\begin{tabular}{|c|c|c|}
\hline \multicolumn{2}{|c|}{ Location } & \multirow{2}{*}{$\begin{array}{c}\text { Nodal Stress Intensity } \\
(p s i)\end{array}$} \\
\hline Cask Body Component & Node Number" & \\
\hline \multirow[t]{6}{*}{ Cask Bottom } & 133 & 7 \\
\hline & $13 !$ & 4 \\
\hline & 214 & 1 \\
\hline & 157 & 7 \\
\hline & 142 & 7 \\
\hline & 220 & 2 \\
\hline \multirow[t]{8}{*}{ Cask Cylinder } & 460 & 13 \\
\hline & 427 & 25 \\
\hline & 395 & 36 \\
\hline & 369 & 53 \\
\hline & 283 & 13 \\
\hline & 316 & 25 \\
\hline & 348 & 36 \\
\hline & 256 & 46 \\
\hline \multirow[t]{4}{*}{ Cask Flange } & 1111 & 106 \\
\hline & 1112 & 72 \\
\hline & 1115 & 115 \\
\hline & 1114 & 121 \\
\hline \multirow[t]{11}{*}{$\operatorname{Lid}$} & 34 & 83 \\
\hline & 33 & 111 \\
\hline & 51 & 145 \\
\hline & 47 & 129 \\
\hline & 74 & 33 \\
\hline & 76 & 33 \\
\hline & 48 & 114 \\
\hline & 45 & 118 \\
\hline & 94 & 34 \\
\hline & 92 & 33 \\
\hline & 61 & -33 \\
\hline
\end{tabular}

* See Figure B.4.5C-25 for the node locations 
HNF-SD-SNF-FDR-003 Rev. 0 E-I5166

Table B4.5C-9

Cask Body Stresses For Side Drops

$1 \mathrm{G}$ results, to be ratioed for 1 foot $(24 \mathrm{G})$ and 30 foot (40 G) drops

\begin{tabular}{|c|c|c|c|}
\hline \multicolumn{2}{|l|}{ Location } & \multicolumn{2}{|c|}{ Nodal Stress Intensity (psi) } \\
\hline Cask Body Comnonent & Node Number & Contact Side & Opp. Cont. \\
\hline \multirow[t]{6}{*}{ Cask Bottom } & 13.3 & 98 & 48 \\
\hline & 131 & 41 & 12 \\
\hline & 214 & 29 & 17 \\
\hline & 157 & 127 & 65 \\
\hline & 142 & 94 & 3 \\
\hline & 220 & 33 & 13 \\
\hline \multirow[t]{8}{*}{ Cask Cylinde: } & 460 & 124 & 50 \\
\hline & 427 & 129 & 56 \\
\hline & 395 & 125 & 54 \\
\hline & 369 & 109 & 40 \\
\hline & 283 & 69 & 34 \\
\hline & 316 & 72 & 50 \\
\hline & 348 & 7 ] & 41 \\
\hline & 256 & 62 & 24 \\
\hline \multirow[t]{4}{*}{ Cask Flange } & 1111 & 82 & 22 \\
\hline & $11: 2$ & 34 & 10 \\
\hline & 1115 & 82 & 20 \\
\hline & 1114 & 39 & 14 \\
\hline \multirow[t]{11}{*}{ Lid } & 34 & 60 & 14 \\
\hline & 33 & 45 & 11 \\
\hline & 51 & 56 & 12 \\
\hline & 47 & 18 & 7 \\
\hline & 74 & 15 & 18 \\
\hline & 76 & 13 & 19 \\
\hline & 48 & 52 & 16 \\
\hline & 45 & 20 & 4 \\
\hline & 94 & 31 & 50 \\
\hline & 92 & 14 & 26 \\
\hline & 61 & -22 & 5 \\
\hline
\end{tabular}

* See Figure 34.5 C-25 for the node locations 
Table B4.5C-10

Cask Body Stresses For Comer Drops

1G results, to be ratioed for 1 font (18 G) and 30 foot (20 G) drops

\begin{tabular}{|c|c|c|c|}
\hline \multicolumn{2}{|l|}{ Location } & \multicolumn{2}{|c|}{ Nodal Stress Intensity (psi) } \\
\hline Cask Bodv Component & Node Number & Contact Side & Opp. Cont. \\
\hline \multirow[t]{6}{*}{ Cask Bottom } & 133 & 59 & 58 \\
\hline & 131 & 15 & 9 \\
\hline & 214 & 11 & 9 \\
\hline & 157 & 71 & 71 \\
\hline & $i 42$ & 16 & 23 \\
\hline & 220 & 5 & 8 \\
\hline \multirow[t]{8}{*}{ Cask Cylinder } & 460 & 6 & 20 \\
\hline & 427 & 19 & 31 \\
\hline & 395 & -46 & 28 \\
\hline & 369 & 105 & 27 \\
\hline & 283 & 3 & 26 \\
\hline & 316 & 15 & 36. \\
\hline & 348 & 47 & 26 \\
\hline & 256 & 83 & 20 \\
\hline \multirow[t]{4}{*}{ Cask Flange } & 1111 & 215 & 51 \\
\hline & $11 ! 2$ & 145 & 41 \\
\hline & 111.5 & 234 & 58 \\
\hline & 1114 & 270 & 55 \\
\hline \multirow[t]{11}{*}{ Lid } & 34 & 249 & 63 \\
\hline & 33 & 251 & 6.5 \\
\hline & 51 & 748 & 132 \\
\hline & 47 & 127 & 48 \\
\hline & 74 & 590 & 614 \\
\hline & 76 & 503 & 232 \\
\hline & 48 & 493 & 132 \\
\hline & 45 & 59 & 50 \\
\hline & 94 & 334 & 479 \\
\hline & 22 & 508 & 207 \\
\hline & $6 !$ & 332 & +33 \\
\hline
\end{tabular}

* See Finure $34.5 \mathrm{C}-25$ for the node locations 
HNF-SD-SNF-FDR-003 Rev. $0 \quad$ E-15166

Table B4.5C-11

Cask Body Stresses For Normal Hot Condition

\begin{tabular}{|c|c|c|}
\hline \multicolumn{2}{|c|}{ Location } & \multirow{2}{*}{$\begin{array}{c}\text { Nodal Stress Intensity } \\
\text { (psi) }\end{array}$} \\
\hline Cask Body Component & Node Number & \\
\hline \multirow[t]{6}{*}{ Cask Botton } & 133 & 73 \\
\hline & 131 & 632 \\
\hline & 214 & 1197 \\
\hline & 157 & 918 \\
\hline & 142 & 342 \\
\hline & 220 & 844 \\
\hline \multirow[t]{8}{*}{ Cask Cylinder } & 460 & 674 \\
\hline & 427 & 664 \\
\hline & 395 & 823 \\
\hline & 369 & 1.135 \\
\hline & 283 & 505 \\
\hline & 316 & 496 \\
\hline & 348 & 509 \\
\hline & 256 & 870 \\
\hline \multirow[t]{4}{*}{ Cask Flango } & 1111 & 506 \\
\hline & 1112 & 480 \\
\hline & 1115 & 342 \\
\hline & 1114 & 500 \\
\hline \multirow[t]{11}{*}{ Lid } & 34 & 553 \\
\hline & 33 & 396 \\
\hline & 51 & 366 \\
\hline & 47 & 255 \\
\hline & 74 & 22 \\
\hline & 76 & 273 \\
\hline & 48 & 239 \\
\hline & 45 & 148 \\
\hline & 94 & 230 \\
\hline & 92 & 236 \\
\hline & 61 & 427 \\
\hline
\end{tabular}

* See Figure B4.5C-25 for the node locations 
HNF-SD-SNF-FDR-003 Rev. $0 \quad$ E-15166

Table $\mathrm{B} 4.5 \mathrm{C}-12$

Cask Body Stresses For Normal Cold Condition

\begin{tabular}{|c|c|c|}
\hline \multicolumn{2}{|c|}{ Location } & \multirow{2}{*}{$\begin{array}{c}\text { Noda] Stress Intensity } \\
\text { (psi) }\end{array}$} \\
\hline Cask Body Component & Node Number ${ }^{*}$ & \\
\hline \multirow[t]{6}{*}{ Cask Bottom } & 133 & 0 \\
\hline & 131 & 0 \\
\hline & 214 & 0 \\
\hline & 157 & 0 \\
\hline & 142 & 0 \\
\hline & 220 & 0 \\
\hline \multirow[t]{8}{*}{ Cask Cyinder } & 460 & 0 \\
\hline & 427 & 0 \\
\hline & 305 & 0 \\
\hline & 369 & 1 \\
\hline & 283 & 0 \\
\hline & 316 & 0 \\
\hline & 348 & 0 \\
\hline & 256 & 1 \\
\hline \multirow[t]{4}{*}{ Cask Flange } & 1111 & 5 \\
\hline & 1112 & 3 \\
\hline & 1115 & 6 \\
\hline & $11: 4$ & 4 \\
\hline \multirow[t]{11}{*}{ Lid! } & 34 & 15 \\
\hline & 33 & 23 \\
\hline & 51 & 11 \\
\hline & 47 & 5 \\
\hline & 74 & 4 \\
\hline & 76 & 4 \\
\hline & 48 & 6 \\
\hline & 45 & 4 \\
\hline & 94 & 2 \\
\hline & 92 & 1 \\
\hline & 61 & 1 \\
\hline
\end{tabular}

* See Figure B4.5C-25 for the node locations 


\section{B4.5C-6 REFERENCES}

1. Performance Specification for TN-WHC Cask and Transportation System, WHC-S0396, Rev1, 1995.

2. ANSYS Engineering Analysis system, User's Manual Volumes 1 to 4, Revision 5.2

3. Cask Designers Guide " $\wedge$ Guide for the Design, Fabrication, and Operation of Shipping Casks for nuclear applications". ORNL-NSIC-68.

4. Charles R. Adams " A Comparison of Analytical Techniques for Analyzing a NuclearSpent-Fucl Shipping Cask Subjected to An end-On Impact" NUREG/CR-1018. 


\section{APPENDIX B4.5D}

\section{CASK LIFTING ATTACHMENT}

B4.5D-O 
TABLE OF CONTENTS

Section

B4.5D-1

B $4.5 \mathrm{D}-2$

B $4.5 \mathrm{D}-3$

B4.5D-4

B4.5D-5

B4.5D-6
INTRODUCTION $\ldots \ldots \ldots \ldots \ldots \ldots \ldots \ldots$. . . . . . . . .

LOADING $\ldots \ldots \ldots \ldots \ldots \ldots \ldots \ldots \ldots \ldots$ B4.5D-1

TRUNNION ANALYSIS . . . . . . . . . . . . B4.5D-1

BRACKET AND GUSSET ANALYSIS . . . . . . . B4.5D-3

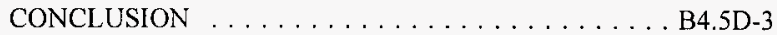

REFERENCES . . . . . . . . . . . . . B4.5D-4 


\section{APPENDIX B4.5D}

\section{CASK LIFTING ATTACHMENT}

\section{B4.5D-1 INTRODUCTION}

This appendix presents the structural analysis of cask lifting attachment. The lifting attachment consists of two trunnions which are attached to the cask lid by a set of brackets and gussets. The geometry and dimensions of lifting attachment are based on Reference 1. The details of the lifting attachment components are shown in Figure B4.5D-1. All components are made of 304 stainless steel.

Two analysis methods are used in the structural evaluation of the cask lifting attachment;

- Hand-calculations to analyze the trunnion

- $\quad$ ANSYS $^{(2)}$ finite element method to analyze the bracket, gusset and lid

\section{B4.5D-2 LOADING}

Hanford Specification ${ }^{(3)}$ requires that the lifting attachments are to be designed per ANSI N14.6 with a factor of safety of three to yield or five to ultimate strength, whichever is most restrictive Since 304 stainless steel has $30 \mathrm{ksi}$ yield and $75 \mathrm{ksi}$ ultimate strength, the factor 3 to yield is more severe. Accordingly, a factor of 3 is used on the load and the resulting stresses are compared to the yield strength.

\section{B4.5D-3 TRUNNION ANALYSIS}

Maximum Cask Weight $=60,000 \mathrm{lbs}$. (Reference 4$)$

$\mathrm{Load} /$ Trunnion $=60,000 \times 3 / 2=90,000 \mathrm{lbs}$.

(a) Stresses at Section B-B (See Figure B4.5D-2a)

Shear Stress, $\mathrm{S}_{\mathrm{s}}=90000 /\left[\pi(2)^{2}\right]=7,162 \mathrm{psi}$

Shear stress is less than the allowable $0.42 \mathrm{~S}_{\mathrm{u}}=31,500 \mathrm{psi}, \ldots \mathrm{O} . \mathrm{K}$

Moment of Inertia

$$
\mathrm{I}=\pi(2)^{4} / 4=12.57 \mathrm{in}^{4}
$$

Bending Stress

$$
\mathrm{S}_{\mathrm{b}}=\mathrm{MC} / \mathrm{I}=90,000 \times 1.505 \times 2 / 12.57=21,552 \mathrm{psi}
$$


Combined Stress Intensity

$$
\mathrm{SI}=\left[\mathrm{S}_{\mathrm{b}}{ }^{2}+4\left(\mathrm{~S}_{\mathrm{s}}\right)^{2}\right]^{0.5}=\left[21552^{2}+4(7162)^{2}\right]^{0.5}=25,878 \mathrm{psi}
$$

This stress is less than the allowable $\mathrm{S}_{\mathrm{y}}=30,000 \mathrm{psi}, \ldots \ldots$ O.K.

\section{(b) Stresses at Section A-A (Weld) (See Figure B4.5D-2b)}

Weld Thickness $=1 \mathrm{in}$.

Moment of Inertia

$$
I=\pi / 64\left[\mathrm{~d}^{4}{ }_{0}-\mathrm{d}^{4}\right]=\pi / 64\left[7^{4}-5^{4}\right]=87.18 \mathrm{in}^{4}
$$

Bending Stress

$$
\mathrm{S}_{\mathrm{b}}=\mathrm{M} \mathrm{C} / \mathrm{I}=90,000 \times 5.505 \times 3.5 / 87.18=19,890 \mathrm{psi}
$$

Shear Stress

$$
\mathrm{S}_{\mathrm{s}}=90,000 /\left(\pi / 4\left[7^{2}-5^{2}\right]=4,775 \mathrm{psi}\right.
$$

Combined Stress Intensity

$$
\mathrm{SI}=\left[19890^{2}+4(4775)^{2}\right]^{0.5}=22,065 \mathrm{psi}
$$

This stress is less than the allowable $\mathrm{S}_{\mathrm{y}}=30,000 \mathrm{psi}, \ldots \ldots$ O.K.

\section{(c) Bearing Stresses at Crane Hook Location (See Figure B4.5D-2c)}

Bearing area is based on 40 degrees (on each side of center line) contact between the hook and trunnion .

Hook Depth $=2.25$ in

Bearing Area $=2\left[2.0 \sin 40^{\circ}\right] \times 2.25=5.785 \mathrm{in}^{2}$

Bearing Stress $=90,000 / 5.785=15,560 \mathrm{psi}$

This stress is less than the allowable $\mathrm{S}_{\mathrm{y}}=30,000 \mathrm{psi} \quad \ldots \ldots$ O.K.

\section{(d) Stresses at Weld Between brackets, gussets and Lid}

The weld section properties, $\mathrm{I}=317.58 \mathrm{in}^{4}, \mathrm{e}=4.85 \mathrm{in}$.

The maximum bending moment $=90,000(5.505+4.85)=931,950 \mathrm{in}-\mathrm{lb}$

The maximum stress at bracket weld is: 
$\mathrm{F} / \mathrm{A}+\mathrm{MC} / \mathrm{I}=90000 / 9.279+931950(4.85) / 317.58=23,932 \mathrm{psi} \leq 30,000 \mathrm{psi} \ldots \ldots \ldots . . \mathrm{O} . \mathrm{K}$.

The maximum stress at gusset weld is:

$\mathrm{F} / \mathrm{A}+\mathrm{MC} / \mathrm{I}=90000 / 9.279-931950(9.4) / 317.58=-17,886 \mathrm{psi} \leq 30,000 \mathrm{psi} \ldots \ldots \ldots \ldots . \mathrm{O} . \mathrm{K}$.

\section{B4.5D-4 BRACKET, GUSSET AND LID ANALYSIS}

An ANSYS three-dimensional finite element model of bracket, gusset and lid is constructed using SOLID45 element (see Figure B4.5D-3). Since the loading and structure are symmetric, only one quarter of structure is modeled. All nodes at the lid bottom are fixed. Symmetry boundary conditions are applied at all the cut surfaces. The force of $45000 \mathrm{lbs}$. (in Z-direction) is applied as a concentrated load at the trunnion. The resulting stress intensity distribution in the model is shown in Figure B4.5D-4. The figure shows that the maximum stress intensity in bracket, gusset and lid is in the range of 10,000 to $15,000 \mathrm{psi}$. All the stresses are less than the allowable stress of 30,000 psi.

\section{B4.5D-5 CONCLUSION}

Based on the results of analyses, it is concluded that the design of the cask lifting attachment is structurally adequate to withstand the maximum lifting loads. 
Figure B4.5D-1

Cask Lifting Attachment Components
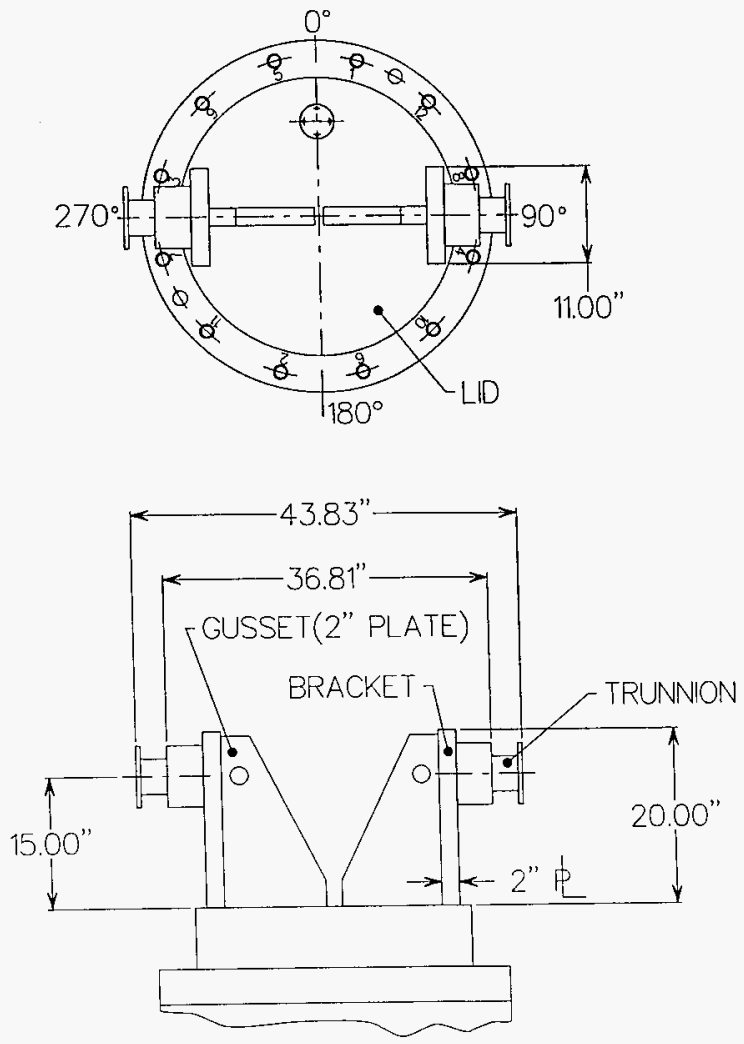
HNF-SD-SNF-FDR-003 Rev. 0

E-15166

Figure B4.5D-2

Trunnion Analysis

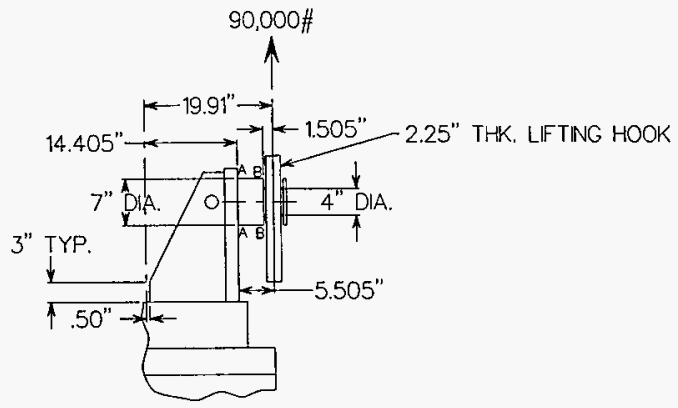

a) TRUNNION ASSEMBLY

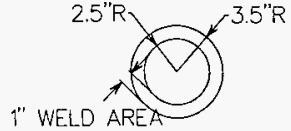

b) SECTION A-A

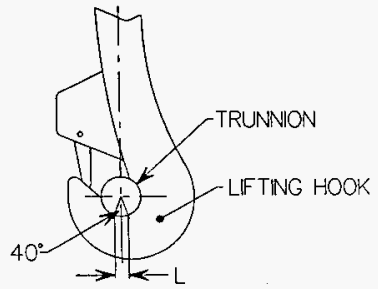

BEARING AREA $=\mathrm{L} \times$ HOOK DEPTH

c) BEARING AREA 
Figure B4.5D-3

ANSYS Finite Element Model For Cask Lifting Attachment

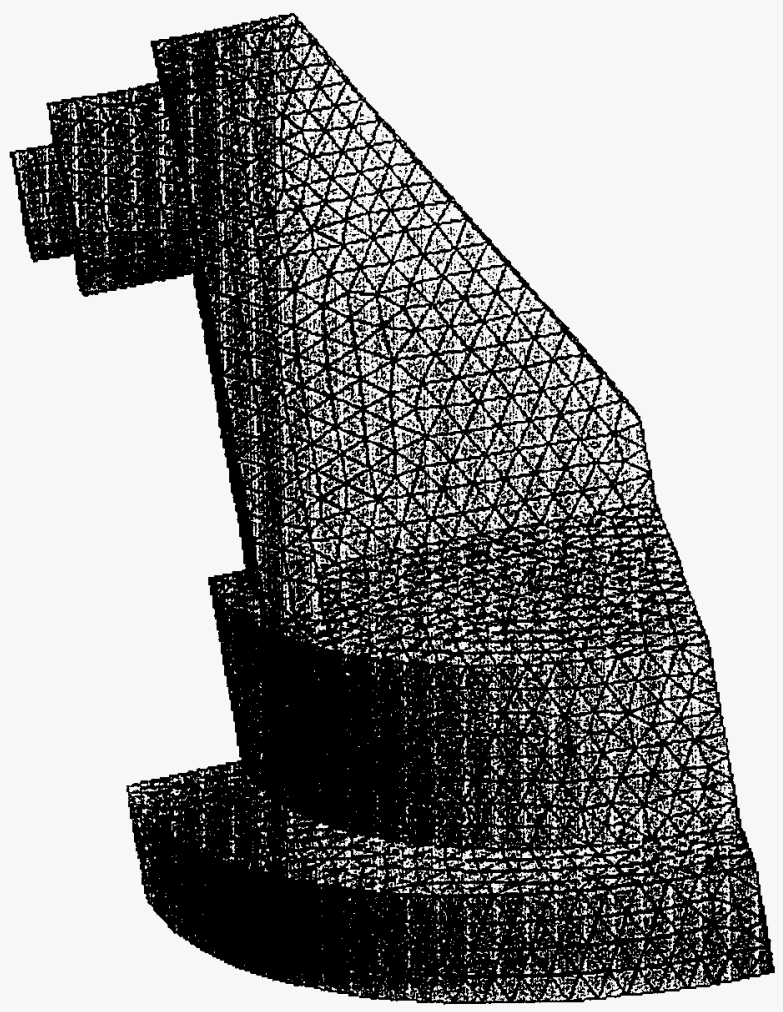


HNF-SD-SNF-FDR-003 Rev. $0 \quad$ E-15166

Figure B4.5D-4

ANSYS Finite Element Model - Nodal Stress Intensity Distribution

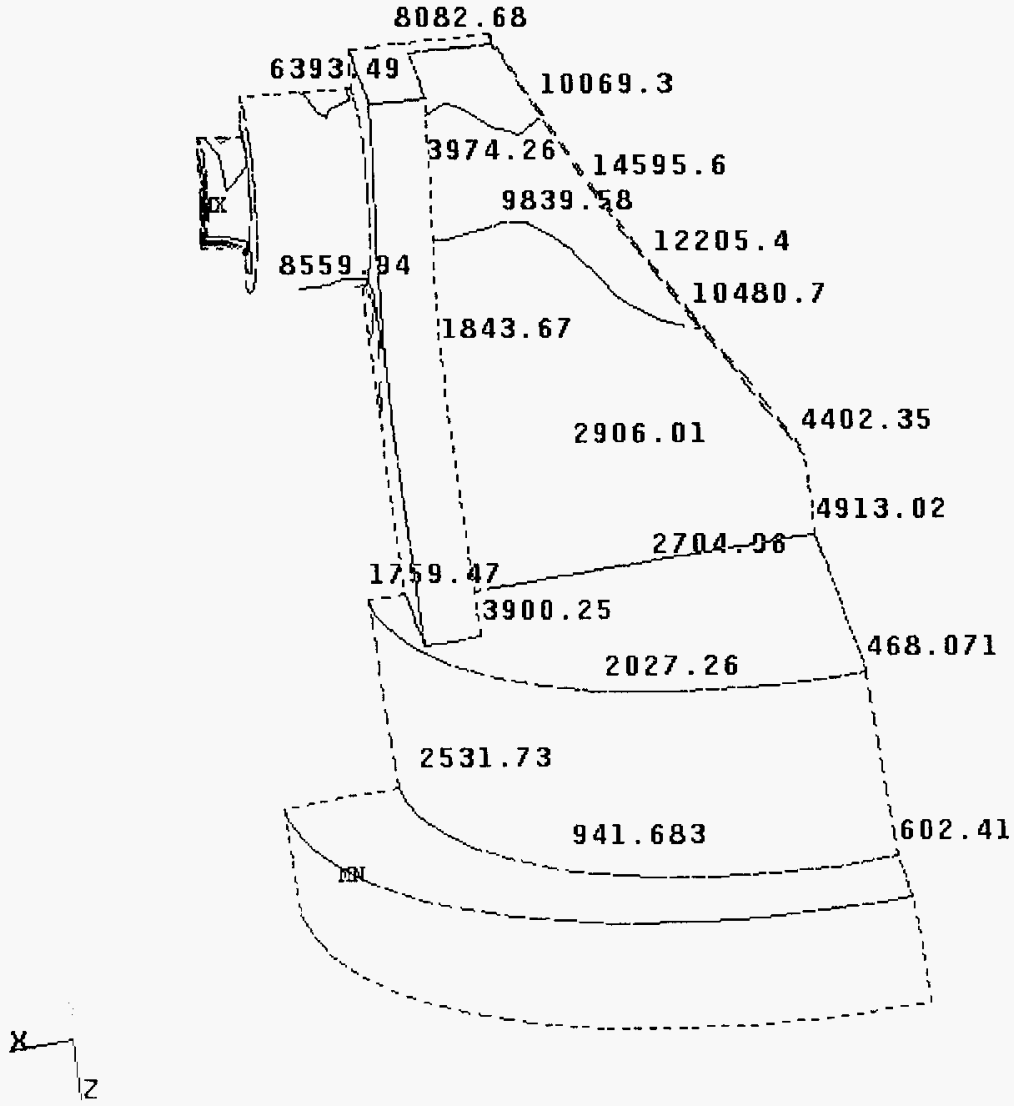




\section{HNF-SD-SNF-FDR-003 Rev. 0}

\section{B4.5D-6 REFERENCES}

1. TN-DRAWING NO. 3035-3, Rev. 4, Sheet 4 .

2. ANSYS Engineering Analysis System User's Manual, Volumes 1 to 4, Revision 5.2.

3. Specification for TN-WHC Cask and Transportation System, WHC-S-0396, Rev. 1,1995

4. TN Calculation No. 3035-4, Rev.1,"TN-WHC Weight and C.G. Calculations. 
HNF-SD-SNF-FDR-003 Rev. $0 \quad$ E-15166

\section{B5.0 THERMAL EVALUATION}

\section{B5. 1 INTRODUCTION}

The cask is designed to passively reject payload decay heat under normal conditions of transport and hypothetical accident conditions while maintaining appropriate package temperatures and pressures within specified limits. An evaluation of the cask thermal performance is presented in this chapter. Objectives of the thermal analyses performed for this evaluation are:

- Determination of package temperatures with respect to containment system material limits;

- Determination of package component temperature gradients to support calculation of thermal stresses;

- Determination of the cask cavity temperature to support containment pressurization calculations;

- Determination of the maximum MCO temperature.

The package components considered in the thermal evaluation are the cask body, the lid and the MCO. The cask body consists of a cylindrical stainless steel shell which surrounds the MCO. The lid and the bottom are fabricated from stainless steel plate material as described in Part A, Section 1.0. Temperatures calculated for the components in the package support thermal stress calculations and permit selection of appropriate temperature dependent mechanical properties used in the structural analyses. Temperatures are also calculated to demonstrate that specified limits for seal materials are not exceeded.

\section{B5.2 THERMAL SOURCE SPECIFICATION}

The thermal source term for the payload within the MCO is defined as a surface heat flux at the inside boundaries of the $\mathrm{MCO}$.

\section{B5.2.1 Normal Conditions of Transport}

The range of anticipated heat flux at the inside surfaces of the MCO under normal conditions of transport is defined in the table below. The defined sidewall surface heat flux occurs over the lower $11.5 \mathrm{ft}$ of the MCO. 


\begin{tabular}{||c|c|c|c|c||}
\hline \hline Payload & $\begin{array}{l}\text { Surface Heat } \\
\text { Flux At MCO } \\
\text { Sidewalls }\end{array}$ & $\begin{array}{l}\text { Surface Heat } \\
\text { Flux At MCO } \\
\text { Top }\end{array}$ & $\begin{array}{l}\text { Surface Heat } \\
\text { Flux at MCO } \\
\text { Bottom }\end{array}$ & Total Power \\
\hline Maximum & 12.7 Watts $/ \mathrm{ft}^{2}$ & $6.0 \mathrm{~W} / \mathrm{ft}^{2}$ & 0 & $950 \mathrm{~W}$ \\
\hline Minimum & 0 & 0 & 0 & 0 \\
\hline
\end{tabular}

\section{B5.2.2 Hypothetical Accident Conditions}

Due to possible chemical reaction of the spent fuel at elevated temperatures, the maximum surface heat flux will vary during the hypothetical accident conditions from that defined for the normal conditions of transport. This variation in surface heat flux at the inside boundaries of the $\mathrm{MCO}$ is defined in the table below. The surface heat fluxes for normal conditions are to be used in establishing the steady-state conditions preceding the hypothetical accident condition.

\begin{tabular}{||c|c|c|c|c|c||}
\hline \hline Payload & $\begin{array}{l}\text { Heat Flux at } \\
\text { Center 28 in } \\
\text { Section of } \\
\text { Sidewall }\end{array}$ & $\begin{array}{l}\text { Surface Heat } \\
\text { Flux at } \\
\text { Remainder of } \\
\text { Sidewall }\end{array}$ & $\begin{array}{l}\text { Surface Heat } \\
\text { Flux at MCO } \\
\text { Top }\end{array}$ & $\begin{array}{l}\text { Surface Heat } \\
\text { Flux at } \\
\text { MCO } \\
\text { Bottom }\end{array}$ & Total Power \\
\hline Maximum & $30 \mathrm{~W} / \mathrm{ft}^{2}$ & $12.7 \mathrm{~W} / \mathrm{ft}^{2}$ & $6.0 \mathrm{~W} / \mathrm{ft}^{2}$ & 0 & $1200 \mathrm{~W}$ \\
\hline Minimum & 0 & 0 & 0 & 0 & 0 \\
\hline
\end{tabular}

\section{B5.3 SUMMARY OF THERMAL PROPERTIES OF MATERIALS}

The thermal properties of materials used in the thermal analyses are listed below. The values are listed as given in the corresponding references. The analysis uses interpolated values for intermediate temperatures where the temperature dependency of a specific parameter is deemed significant. The interpolation assumes a linear relationship between the reported values.

Thermal radiation at the external surface of the package is considered. The external surfaces of the cask are assumed to have an emissivity of 0.85 , a typical value for weathered stainless steel surfaces $^{(1)}$. For solar absorptivity, a value of 0.5 is used. 
a. Helium (1)

Used for: Gaps between cask and MCO

\begin{tabular}{|c|c|c|c|}
\hline $\begin{array}{c}\text { Temperature } \\
\text { (deg F) }\end{array}$ & $\begin{array}{c}\text { Conductivity } \\
\text { (Btu/hr-ft-F) }\end{array}$ & $\begin{array}{c}\text { Density } \\
\text { (lb/cu. in) }\end{array}$ & $\begin{array}{c}\text { Heat Capacity } \\
\text { (Btu/lb-F) }\end{array}$ \\
\hline \hline 0 & 0.078 & $6.94 \mathrm{E}-6$ & 1.24 \\
200 & 0.097 & $4.83 \mathrm{E}-6$ & 1.24 \\
400 & 0.115 & $3.70 \mathrm{E}-6$ & 1.24 \\
600 & 0.129 & $3.01 \mathrm{E}-6$ & 1.24 \\
800 & 0.138 & $2.52 \mathrm{E}-6$ & 1.24 \\
\hline
\end{tabular}

b. Air ${ }^{(1)}$

Used for: Convection coefficients on cask surface

\begin{tabular}{|c|c|c|c|}
\hline $\begin{array}{c}\text { Temperature } \\
\text { (deg F) }\end{array}$ & $\begin{array}{c}\text { Conductivity } \\
\text { (Btu/hr-ft-F) }\end{array}$ & $\begin{array}{c}\mathrm{g} \beta \mathrm{p}^{2} / \mu^{2} \\
(1 / \mathrm{F}-\mathrm{cu} . \mathrm{ft})\end{array}$ & Prandlt No. \\
\hline \hline 100 & 0.0154 & $1.76 \mathrm{E} 6$ & 0.72 \\
200 & 0.0174 & $0.85 \mathrm{E} 6$ & 0.72 \\
--- & --- & --- & --- \\
800 & 0.0286 & $49.8 \mathrm{E} 3$ & 0.697 \\
900 & 0.0303 & $36.0 \mathrm{E} 3$ & 0.705 \\
1000 & 0.0319 & $26.5 \mathrm{E} 3$ & 0.713 \\
1500 & 0.0400 & $7.45 \mathrm{E} 3$ & 0.739 \\
\hline
\end{tabular}

Note that the analyses used air properties in the range of $100^{\circ} \mathrm{F}-200^{\circ} \mathrm{F}$, and $800^{\circ} \mathrm{F}-1500^{\circ} \mathrm{F}$ only. 

HNF-SD-SNF-FDR-003
Rev. 0
E-15166

c. Stainless Steel $(18 \mathrm{Cr}-8 \mathrm{Ni})$

Used for: MCO shell and cask body

\begin{tabular}{|c|c|c|c||}
\hline $\begin{array}{c}\text { Temperature } \\
(\operatorname{deg} \mathrm{F})\end{array}$ & $\begin{array}{c}\text { Conductivity }{ }^{(3)} \\
(\text { Btu/hr-ft-F) }\end{array}$ & $\begin{array}{c}\text { Density }{ }^{(1)} \\
(\mathrm{lb} / \mathrm{cu} . \mathrm{in})\end{array}$ & $\begin{array}{c}\text { Heat Capacity }{ }^{(1)} \\
(\mathrm{Btu} / \mathrm{lb}-\mathrm{F})\end{array}$ \\
\hline \hline 70 & 8.6 & 0.282 & 0.110 \\
100 & 8.7 & 0.282 & 0.110 \\
200 & 9.3 & 0.282 & 0.110 \\
300 & 9.8 & 0.282 & 0.110 \\
400 & 10.4 & 0.282 & 0.110 \\
500 & 10.9 & 0.282 & 0.110 \\
600 & 11.3 & 0.282 & 0.110 \\
800 & 12.2 & 0.282 & 0.110 \\
1000 & 13.2 & 0.282 & 0.110 \\
1200 & 14.0 & 0.282 & 0.110 \\
\hline
\end{tabular}

\section{B5.4 THERMAL EVALUATION FOR NORMAL TRANSFER CONDITIONS}

\section{B5.4.1 Conditions Evaluated}

For conditions normally incident to transport, the thermal analyses evaluated the package design for its ability to maintain component temperatures below the design limits. The operational temperatures will occur in conditions that have been bounded by the environmental conditions provided below:

The ambient temperatures at the Hanford Site for the peak summer month are tabulated in Table B5.4-1.

- Maximum heat generation rate of worst-case source from Section B5.2 plus maximum solar heat load (see Table B5.4-2) plus ambient air temperatures from Table B5.4-1.

- Minimum air temperature of $-27^{\circ} \mathrm{F}$ plus maximum heat generation rate from worst-case source in Section B5.2.

- Minimum air temperature of $-27^{\circ} \mathrm{F}$ and zero heat generation rate. 
Table B5.4-1 Hanford Air Temperature

\begin{tabular}{|c|c|c|c|}
\hline Time & Temperature $\left({ }^{\circ} \mathrm{F}\right)$ & Time & Temperature $\left.{ }^{\circ} \mathrm{F}\right)$ \\
\hline $12 \mathrm{a} . \mathrm{m}$. & 82 & 2 p.m. & 111 \\
\hline 2 a.m. & 78 & 4 p.m. & 115 \\
\hline 4 a.m. & 75 & 6 p.m. & 113 \\
\hline 6 a.m. & 74 & 8 p.m. & 100 \\
\hline 8 a.m. & 85 & 10 p.m. & 89 \\
\hline 10 a.m. & 97 & 12 p.m. & 82 \\
\hline 12 p.m. & 103 & & \\
\cline { 1 - 2 } & & &
\end{tabular}

Table B5.4-2 Maximum Solar Radiation Received (Btu/hr- $\mathrm{ft}^{2}$ )

\begin{tabular}{|c|c|c|c|c|c|c|c|c|c|}
\hline \multirow{2}{*}{ Time } & \multicolumn{8}{|c|}{ Vertical surfaces facing } & \multirow{2}{*}{$\begin{array}{c}\text { Horizontal } \\
\text { surface facing } \\
\text { up }\end{array}$} \\
\hline & $\mathrm{N}$ & $N E$ & $E$ & $\mathrm{SE}$ & S & SW & W & $\mathrm{NW}$ & \\
\hline 4 a.m. & 0 & 0 & 0 & 0 & 0 & 0 & 0 & 0 & 0 \\
\hline 6 a.m. & 57 & 192 & 211 & 105 & 17 & 17 & 17 & 17 & 64 \\
\hline 8 a.m. & 35 & 173 & 268 & 208 & 42 & 32 & 32 & 32 & 127 \\
\hline 10 a.m. & 42 & 56 & 177 & 213 & 126 & 45 & 42 & 42 & 281 \\
\hline 12 noon & 45 & 45 & 49 & 120 & 167 & 120 & 49 & 45 & 314 \\
\hline 2 p.m. & 42 & 42 & 42 & 45 & 126 & 213 & 177 & 56 & 281 \\
\hline 4 p.m. & 35 & 32 & 32 & 32 & 42 & 208 & 268 & 173 & 127 \\
\hline $6 \mathrm{p} . \mathrm{m}$. & 57 & 17 & 17 & 17 & 17 & 105 & 211 & 192 & 64 \\
\hline 8 p.m. & 0 & 0 & 0 & 0 & 0 & 0 & 0 & 0 & 0 \\
\hline
\end{tabular}




\section{B5.4.2 Acceptance Criteria}

Several thermal design criteria have been established for the cask.

- Containment of radioactive material is a major design requirement for the cask. Therefore, seal temperatures must be maintained within specified limits to satisfy the required containment criteria under normal conditions. The operating temperature range recommended by the potential manufacturer $\left(\mathrm{Parker}^{(4)}\right)$ of the butyl seals is $-75^{\circ} \mathrm{F}$ to $250^{\circ} \mathrm{F}$. This range applies to all containment boundary seals used in the cask closure lid and containment penetrations.

- Maximum normal operating outside surface temperature of the cask shall be less than $180^{\circ} \mathrm{F}$ in maximum air temperature and in the shade.

- Maximum temperatures of containment structural components must not adversely affect the containment function.

- Maximum temperatures of the package must not adversely affect the shielding function.

- The maximum MCO shell temperature allowed under normal conditions of transport shall be $167^{\circ} \mathrm{F}$.

In general, all the thermal criteria are associated with maximum temperatures. The ability of the containment system structural materials to function properly under the lowest service temperature conditions is discussed in Section 4.3.

\section{B5.4.3 Thermal Model}

A two-dimensional axisymmetric finite element computer model of the TN-WHC cask and payload (MCO) was used to model the thermal performance of the cask. The ANSYS computer program ${ }^{(5)}$ was utilized for the analyses. This program is a large scale, general purpose finite element code which can perform steady-state and transient thermal analyses using linear and nonlinear material properties.

The thermal model represents the TN-WHC cask standing vertically. The model includes the cask and the MCO shell. The interior region of the MCO is not modeled, as per reference 2 . The areas between the MCO and the cask were modeled with helium, which is the assumed fill gas. Heat transfer between the MCO and the cask was conservatively assumed to be via gaseous conduction only. The MCO is assumed to be centered in the cask. Figure B5.4.3-1 shows the thermal model.

The bottom of the cask does not receive any heat from the sun and it is in good thermal contact with the transporter when it is being transported. From the perspective of heat transfer, the transporter will behave like a fin, which will allow the cask to conduct heat through its bottom 
and into the environment. The magnitude of this effect is difficult to quantify, as it depends on the emissivity and absorptivity of the transporter coatings, the materials, and the geometry of the transporter. Therefore, the analysis conservatively assumed that the bottom of the cask was an adiabatic surface.

The ANSYS axisymmetric thermal solid element, PLANE55, was used to model the MCO, the cask, and the helium between the two. At the surface of the cask, two dimensional surface effect elements, SURF19, were overlaid on the mesh. The solar radiation data given in Table B5.4-2 was applied to these elements, after accounting for the solar absorptivity of the cask surface. The data for the vertical surfaces was averaged circumferentially so that it could be applied to the axisymmetric model.

The decay heat of the MCO contents was simulated by applying an elemental heat flux on the inside of the sidewalls and top of the $\mathrm{MCO}$ of $12.7 \mathrm{~W} / \mathrm{ft}^{2}$ and $6.0 \mathrm{~W} / \mathrm{ft}^{2}$, respectively, as per section B5.2.1.

The cask rejects heat passively through its outer surfaces. This was simulated by applying a convection load directly on the outer face of the PLANE55 elements on the surface of the cask. A effective heat transfer coefficient for the convection load was determined for each of the twelve time ranges given in Table B5.4-1. The effective heat transfer coefficient, $h_{e f f}$, depends on natural convection and radiation at the cask surface, and varies with the ambient temperature, $\mathrm{T}_{\mathrm{amb}}$, the surface temperature, $\mathrm{T}_{\mathrm{s}}$, and the properties of air, which are evaluated at the average temperature, $\left(\mathrm{T}_{\mathrm{s}}+\mathrm{T}_{\mathrm{amb}}\right) / 2$. An environmental view factor of 1.0 and an environmental emissivity of 1.0 are assumed. The relationship is given below:

$$
\begin{aligned}
& h_{\text {eff }}=h_{\text {conv }}+h_{\text {rad }} \\
& h_{\text {conv }}=C_{1} k\left[\operatorname{Pr}\left(T_{s}-T_{a m b}\right)\left(g \beta \rho^{2} / \mu^{2}\right)\right]^{1 / 3} \\
& h_{\text {rad }}=\sigma_{b} \text { e }\left[T_{s}^{4}-T_{a m b}^{4}\right] /\left(T_{s}-T_{a m b}\right)
\end{aligned}
$$

where, $\quad C_{1}=0.130$ for vertical cylindrical surfaces ${ }^{(1)}$, and 0.156 for horizontal surfaces facing up $^{(1)}$

$\mathrm{e} \quad=0.85$ for weathered stainless stee ${ }^{(1)}$

\section{B5.4.4 Thermal Analysis}

A transient analysis was performed to account for the time varying nature of the solar heat load. The model was solved in steady-state mode with $8 \mathrm{AM}$ ambient temperature and insolation data to generate the initial conditions for the transient analysis. The $8 \mathrm{AM}$ conditions were chosen as a good approximation of the average ambient temperature and insolation that the cask experiences. The transient run began with midnight ambient temperature and insolation data and stepped changed the ambient temperature and insolation data every two hours for two full cycles (48 hours). 
Figure B5.4.4-1 shows the temperature history across the cask (radially) at the hottest MCO node and the outer surface of the cask at this axial location. This location is approximately centered between the cask lid and bottom. Comparison of the temperatures of the model at $\mathrm{N}$ hours and $\mathrm{N}+24$ hours shows very little change, which demonstrates that the initial conditions for the transient analysis were appropriately chosen. Note that there is a phase lag between the two curves, which is due to the thermal inertia of the system.

Figure B5.4.4-2 shows the temperature distribution of the $\mathrm{MCO}$ and cask at the time that the maximum temperature of the $\mathrm{MCO}$ is reached. This occurs at 9:17 PM. Referring to Figure B5.4.4-1, the maximum cask temperature at the same axial location occurs at 8 PM. This is consistent with the behavior expected based on an examination of the ambient temperature and insolation data of Tables B5.4-1 and B5.4-2. The difference in time between the peak MCO temperature and the peak adjacent cask surface temperature is due to the thermal inertia of the system, as noted above.

Figure B5.4.4-3 shows the temperature distribution of the cask at the time that the maximum temperature of the cask is reached. This occurs at 2:00 PM, when solar insolation on the top of the cask has been at its maximum value for two hours (from noon to $2 \mathrm{PM}$ ).

\section{B5.4.4.1 Internal Temperatures}

The maximum MCO temperature under normal conditions of transport is $155^{\circ} \mathrm{F}$. This is less than the design limit of $167^{\circ} \mathrm{F}$. The minimum temperature is $-27^{\circ} \mathrm{F}$.

The maximum cask temperature under normal conditions of transport is $140^{\circ} \mathrm{F}$. This is less than the seal temperature limit of $250^{\circ} \mathrm{F}$. The minimum temperature is $-27^{\circ} \mathrm{F}$, which above the minimum seal temperature of $-75^{\circ} \mathrm{F}$. The containment and shielding functions of the stainless steel cask and the seals are not compromised at the temperature range calculated.

\section{B5.4.4.2 Maximum Surface Temperatures}

The normal conditions of transport maximum surface temperature is $140^{\circ} \mathrm{F}$. This is less than the temperature limit of $180^{\circ} \mathrm{F}$.

\section{B5.5 THERMAL EVALUATION FOR ACCIDENT CONDITIONS}

\section{B5.5.1 Conditions Evaluated}

The hypothetical fire accident condition is defined as the exposure of the cask for 30 minutes to a $1475^{\circ} \mathrm{F}$ fire that has an emissivity coefficient of 0.9 . The surface absorptivity of the package is the greater of the anticipated absorptivity or 0.8 . The surface convective heat transfer for the package is assumed to be natural convection. The decay heat of the $\mathrm{MCO}$ during the thermal accident is defined in section B5.2.2. The package is actively cooled following the 30 minutes fire. The active cooling consists of quenching the outer package surfaces using a water spray 
from a fire hose. The quenching water flows at 125 gallons per minute for 45 minutes, followed by a flow rate of $50 \mathrm{gpm}$ for an additional 100 minutes. The temperature of the quenching water is $85^{\circ} \mathrm{F}$. Thereafter normal conditions are assumed to prevail.

\section{B5.5.2 Acceptance Criteria}

The following acceptance criteria have been established:

- The MCO shell temperature may exceed the temperature limit for the normal conditions of transport for a time period not to exceed 180 minutes following the fire.

- The maximum $\mathrm{MCO}$ shell temperature should not be significantly above $252^{\circ} \mathrm{F}$ to minimize gas generation.

- There is no requirement that the cask maintain containment of the MCO during the thermal accident. This means that the cask seals may exceed their operational temperature limits during the thermal accident. However, the cask must maintain confinement of the MCO within the cask at all times.

\section{B5.5.3 Thermal Model}

The thermal model that was developed for the normal conditions of transport was reused with a single modification; the SURF 19 elements on the outer surface of the cask were removed because there are no solar insolation loads during the thermal accident. The analysis assumed that the bottom of the cask was adiabatic during the establishment of the initial conditions, the fire, and the quench.

During the establishment of the initial conditions, the decay heat of the MCO contents is represented as the normal conditions of transport decay heat flux. During and after the 30 minute fire, the decay heat of the MCO contents was simulated by applying an elemental heat flux of 30 $\mathrm{W} / \mathrm{ft}^{2}$ on the center $28^{\prime \prime}$ section of the MCO sidewall, $12.7 \mathrm{~W} / \mathrm{ft}^{2}$ on the remainder of the sidewall, and $6.0 \mathrm{~W} / \mathrm{ft}^{2}$ on the top of the $\mathrm{MCO}$.

During establishment of the initial conditions, the cask rejects heat passively through its outer surfaces. This was simulated by applying a convection load directly on the outer face of the PLANE55 elements on the surface of the cask. A effective heat transfer coefficient for the convection load was determined for an ambient temperature of $115^{\circ} \mathrm{F}$. The effective heat transfer coefficient, $h_{\mathrm{eff}}$, depends on natural convection and radiation at the cask surface, and varies with the surface temperature, $T_{s}$, and the properties of air, which are evaluated at the average temperature, $\left(\mathrm{T}_{\mathrm{s}} \div \mathrm{T}_{\mathrm{amb}}\right) / 2$. An environmental view factor of 1.0 and an environmental emissivity of 1.0 are assumed. The relationships are as given in $\mathrm{B} 5.4 .3$, with $\mathrm{T}_{\text {amb }}=115^{\circ} \mathrm{F}=575^{\circ} \mathrm{R}$.

During the 30 minute fire, heat transfer between the cask and the environment is a combination of natural convection and radiation. The absorptivity of the cask for radiation from a $1475^{\circ} \mathrm{F}$ fire is taken to be 0.8. The natural convection relationship is the same as that given in B5.4.3. The 
radiative heat transfer coefficient is given below:

$$
h_{\mathrm{rad}}=\sigma_{\mathrm{b}} \mathrm{e}_{\mathrm{c}}\left[\mathrm{T}_{\mathrm{s}}^{4}-\mathrm{e}_{\mathrm{f}}\left(1475^{\circ} \mathrm{R}\right)^{4}\right] /\left(\mathrm{T}_{\mathrm{s}}-1475^{\circ} \mathrm{R}\right) \quad \text { (with } \mathrm{T}_{\mathrm{s}} \text { in }{ }^{\circ} \mathrm{R} \text { ) }
$$

where, $\quad \mathrm{e}_{\mathrm{c}}=0.8$

$\mathrm{e}_{\mathrm{f}}=0.9$

The post-fire active cooling, or quench, was modeled as a convection load on the surface of the cask. The analysis assumed an effective heat transfer coefficient of $50 \mathrm{Btu} / \mathrm{hr}-\mathrm{ft}^{2}-^{\circ} \mathrm{F}$ at $85^{\circ} \mathrm{F}$, increasing linearly with temperature to $2000 \mathrm{Btu} / \mathrm{hr}-\mathrm{ft}^{2}-{ }^{\circ} \mathrm{F}$ at $212^{\circ} \mathrm{F}$, and constant at $2000 \mathrm{Btu} / \mathrm{hr}$ $\mathrm{ft}^{2}-{ }^{\circ} \mathrm{F}$ for temperatures greater than $212^{\circ} \mathrm{F}^{(1)}$.

\section{B5.5.4 Thermal Analysis}

A transient analysis was performed to account for the time varying nature of the loading. The package was assumed to be initially at steady state using the maximum ambient temperature of $115^{\circ} \mathrm{F}$, the normal conditions of transport decay heat rates as defined in Section B5.2.2, and no solar insolation. The solution to the model with these loading conditions formed the initial conditions for the accident condition analysis.

The accident conditions MCO decay heat loading together with the $1475^{\circ} \mathrm{F}$ fire's effective heat transfer coefficient was imposed on the model for 30 minutes. This was followed by the accident conditions MCO decay heat loading combined with the active cooling heat transfer coefficient for 145 minutes.

Figure B5.5.4-1 shows thermal model, depicting the cross-sectional locations that appear in the following two figures. Figure B5.5.4-2 shows the temperature history of a cross-section of the model at the location that the MCO reaches its maximum temperature. Figure B5.5.4-3 shows the temperature history of the top corner cross-section. The maximum cask temperature lies along this cross-section.

Figure B5.5.4-4 shows the temperature distribution of the $\mathrm{MCO}$ and cask at time zero. This is the starting point for the transient analysis.

Figure B5.5.4-5 shows the temperature distribution of the $\mathrm{MCO}$ and cask at the end of the fire. The helium has been removed from the plot for clarity. The maximum cask temperature occurs at this time.

Figure B5.5.4-6 shows the temperature distribution of the $\mathrm{MCO}$ and cask at the time that the maximum temperature of the MCO is reached. Again, the helium has been removed for clarity. This occurs approximately 54 minutes after the end of the fire.

\section{B5.5.5.1 Internal Temperatures}

The maximum MCO temperature during the accident conditions is $256^{\circ} \mathrm{F}$ and is within the 
temperature range of $250^{\circ} \mathrm{F}$ to $260^{\circ} \mathrm{F}$.

During accident conditions (start of fire to end of water quench), the maximum MCO shell temperature exceeds the $167^{\circ} \mathrm{F}$ normal temperature limit for less than 180 minutes, and meets the acceptance criteria.

\section{B5.5.5.2 Maximum Surface Temperatures}

The maximum cask surface temperature occurs at the end of the fire. The cask reaches a temperature of $1068^{\circ} \mathrm{F}$ in the upper corner - see Figure B5.5.4-5. The cask maintains confinement of the $\mathrm{MCO}$ at these temperatures, thereby meeting the design requirements with regards to confinement.

\section{B5.6 THERMAL EVALUATION AND CONCLUSIONS}

The thermal evaluation for normal conditions of transport concludes that the TN-WHC design meets all the applicable requirements. The maximum temperatures calculated using conservative assumptions are relatively low $\left(140^{\circ} \mathrm{F}\right)$. The minimum temperature is $-27^{\circ} \mathrm{F}$. This temperature range has no adverse effect on the performance of the cask components (including seals). During accident conditions, the temperatures calculated in the cask components will not compromise the structural integrity of the cask. The cask will continue to maintain its confinement function. The MCO temperatures remain below $260^{\circ} \mathrm{F}$ during fire accident conditions. 
HNF-SD-SNF-FDR-003 Rev. $0 \quad$ E-15166

Figure B5.4.3-1 Normal Gonditions Thermal Model

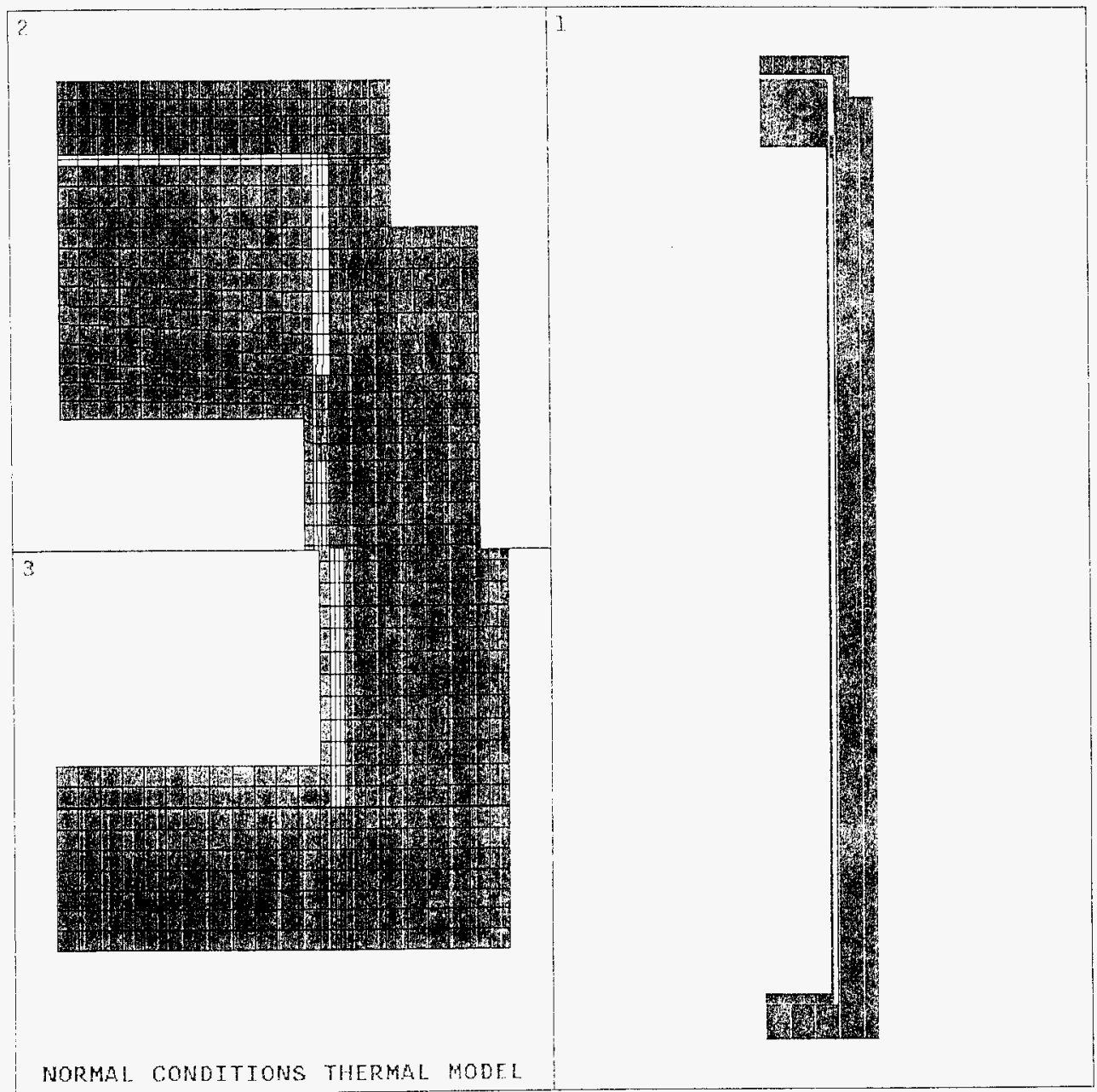


Figure B5.4.4-1 Normal Condhions Temperature History at Hottest MCO location

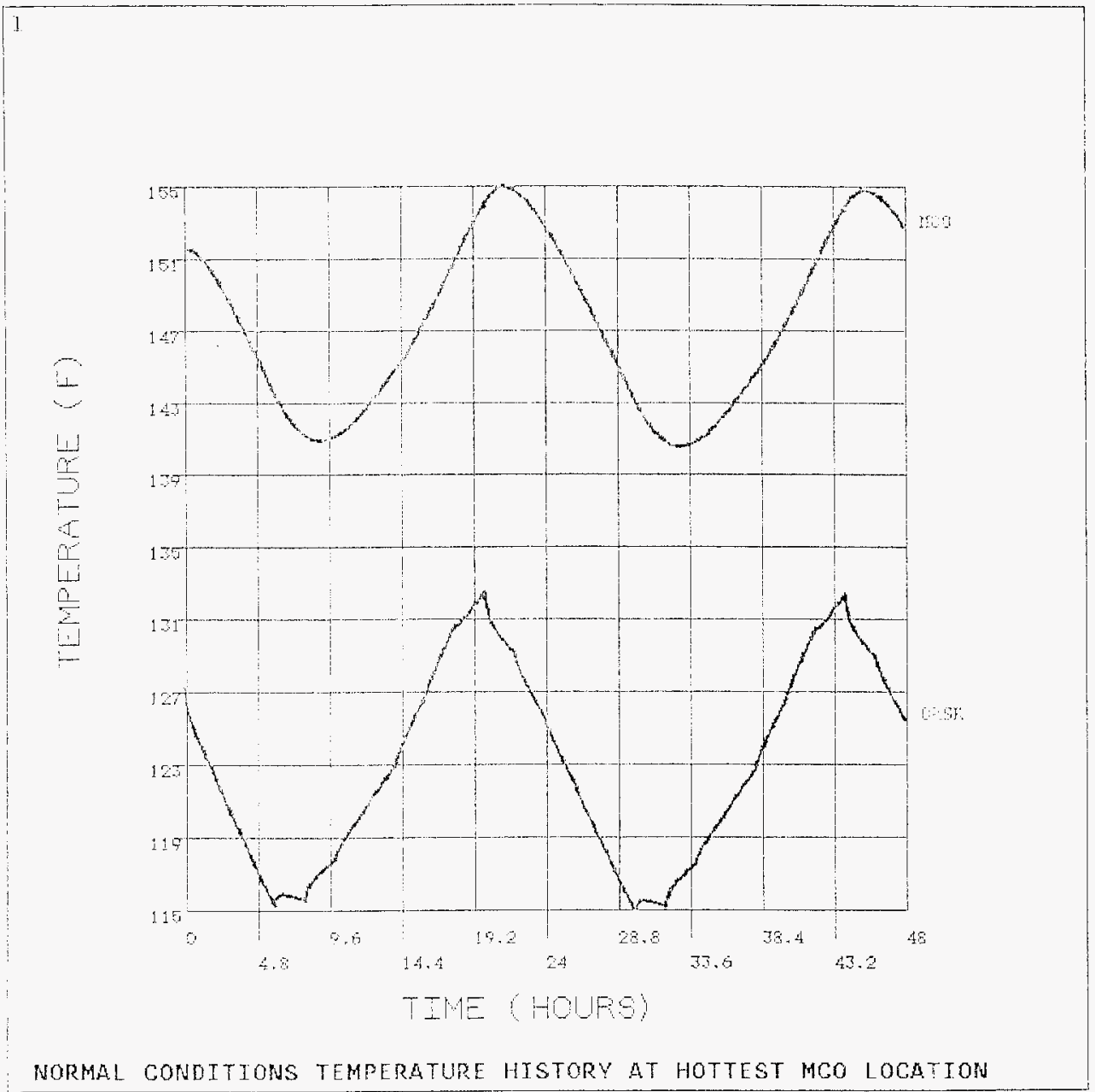




\section{HNF-SD-SNF-FDR-003 \\ Rev. 0

Figure B5.4.4-2 Normal Conditions MCO and Cask Temp. Distribution - Maximum MCO Temperature

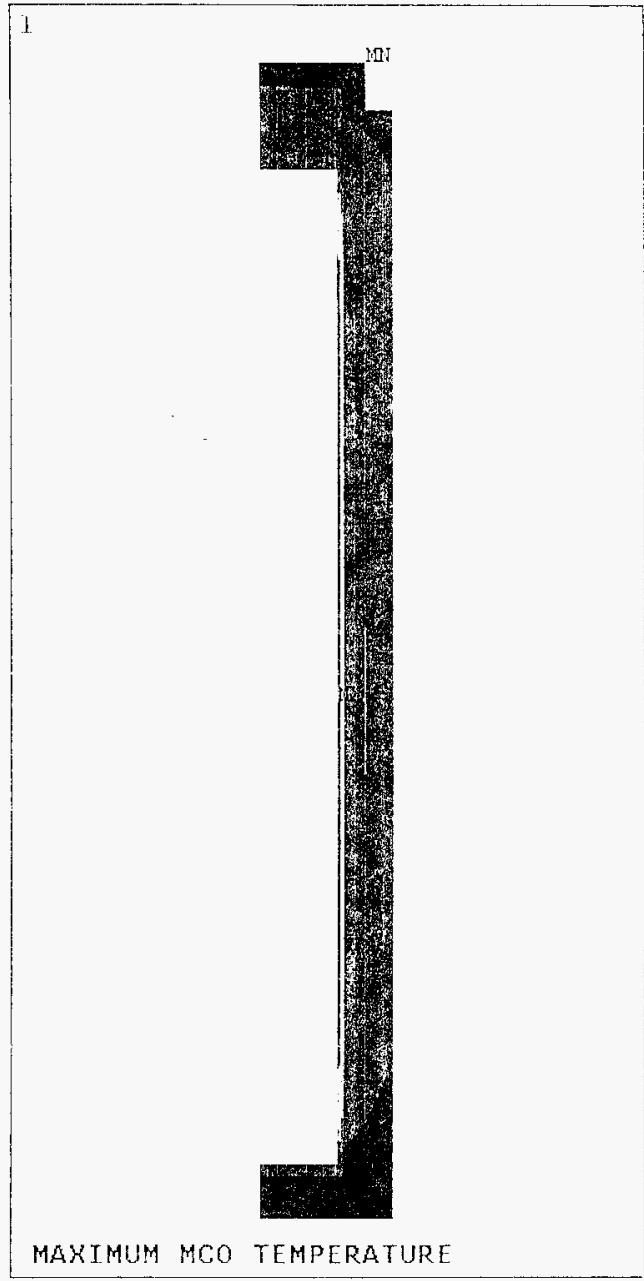

ANSYS 5.2

OCT $30 \quad 1996$

I $0: 37: 53$

NODAL SOLUTION

TIME $=21.287$

TEMP

SMN $=124.924$

SMK $=154.931$

124.929

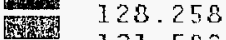

rats 131.592

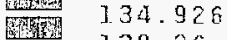

138.26

141.595

144.928

$\square \quad 148.263$

151.597

Hase 154.931 
HNF-SD-SNF-FDR-003 ReV. 0 E-15166

Figure B5.4.4.3 Normal Conditions Cask Temperature Distribution - Maximum Cask Temperature

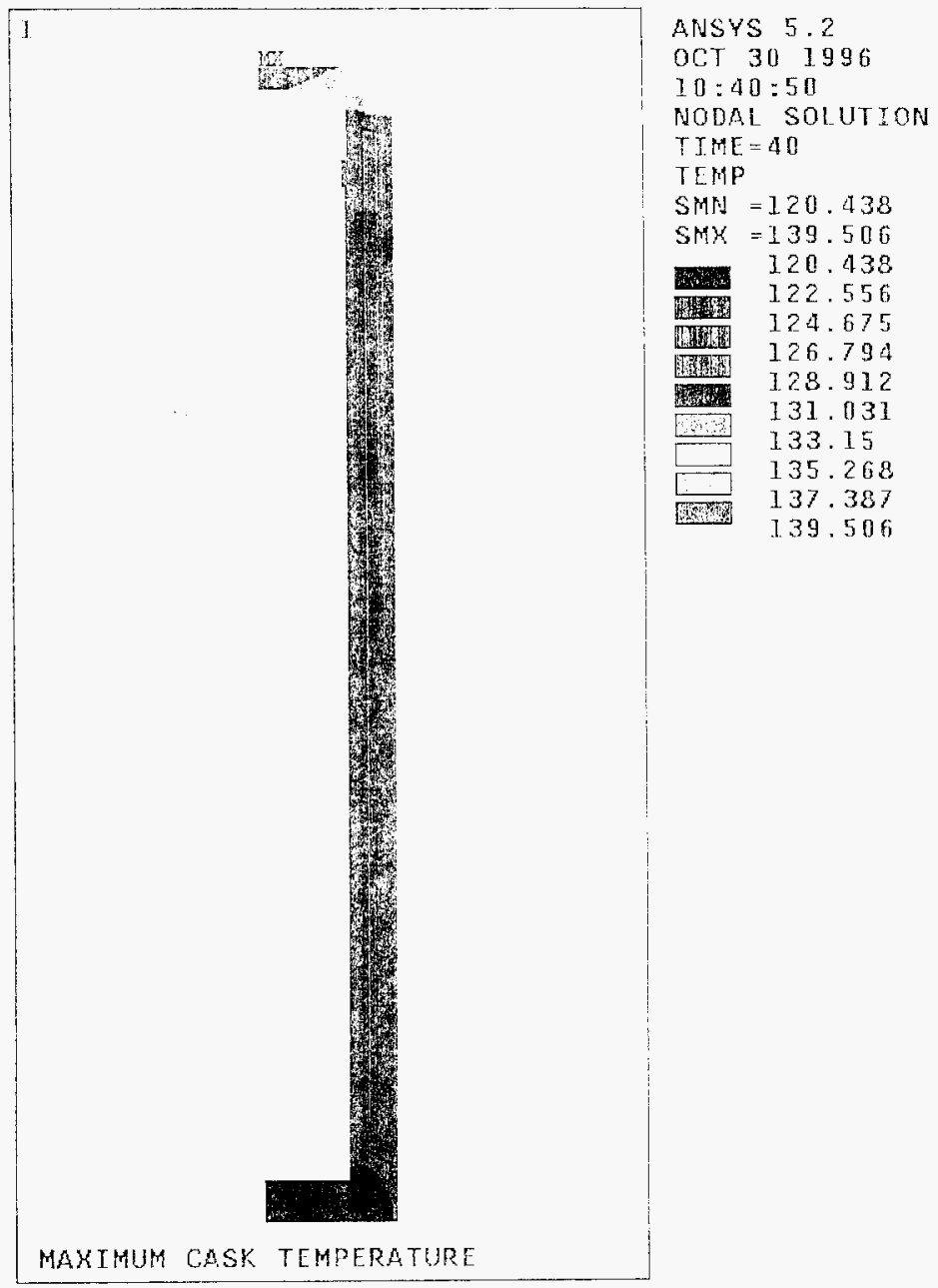




\section{HNF-SD-SNF-FDR-003 Rev. $0 \quad$ E-15166}

Figure B5.5.4-1 Accident Conditions Thermal Model

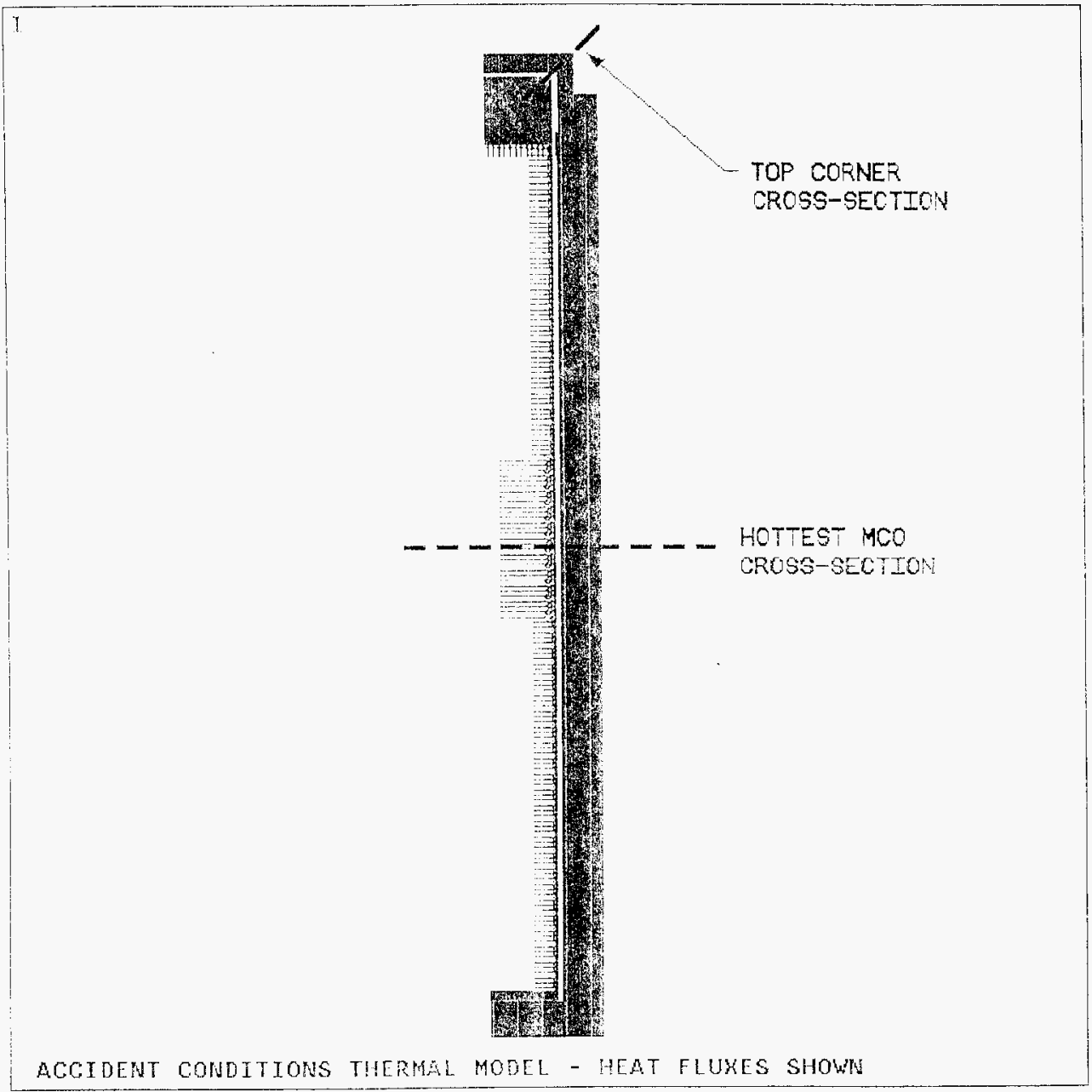


Figure B5.5.4-2 Accident Conditions Temperature History at Hottest MCO Location

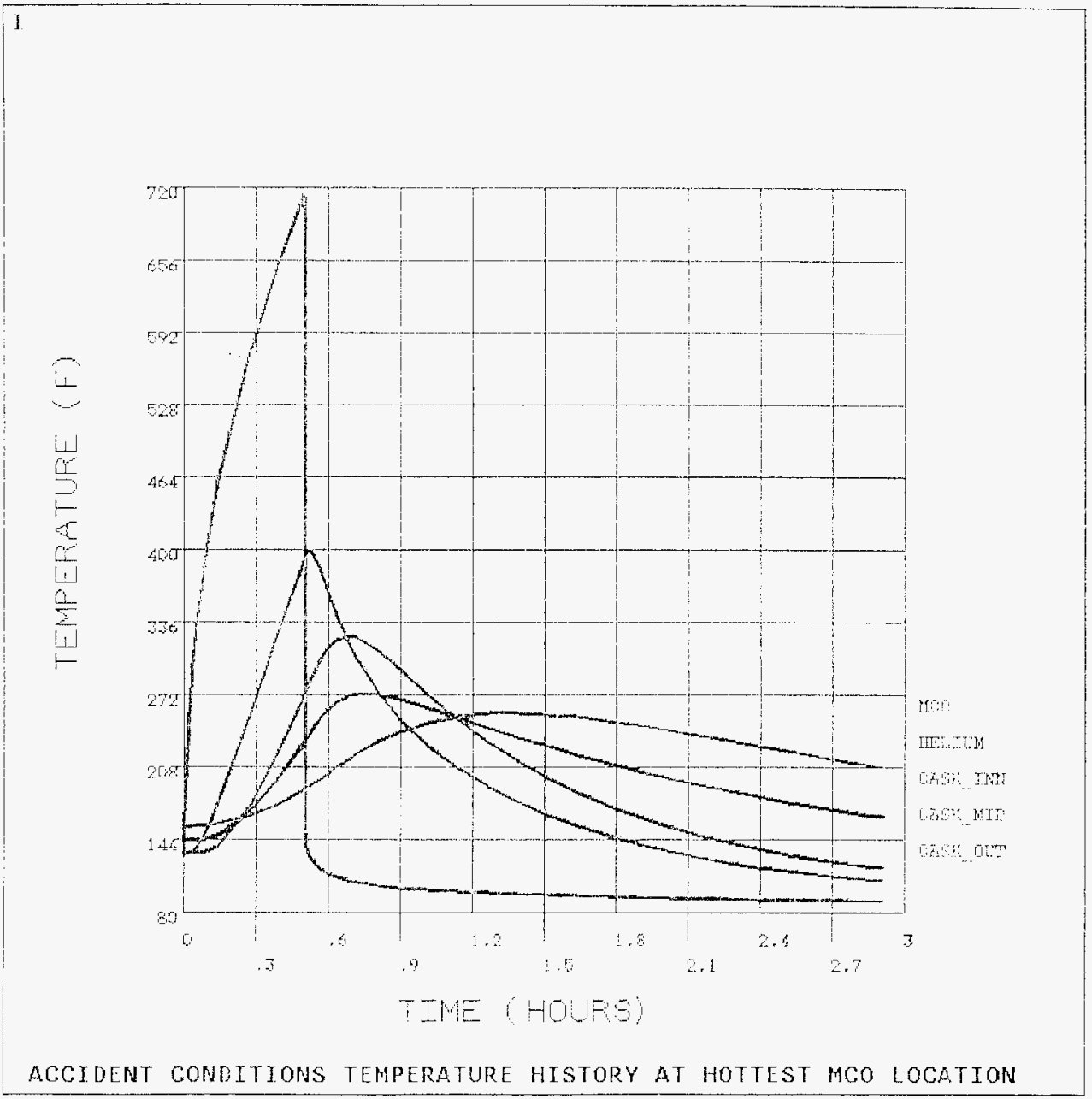


HNF-SD-SNF-FDR-003 Rev. 0 E-15166

Figure B5.5.4.3 Accident Conditions Temperature History at Top Comer Hottest Cask)

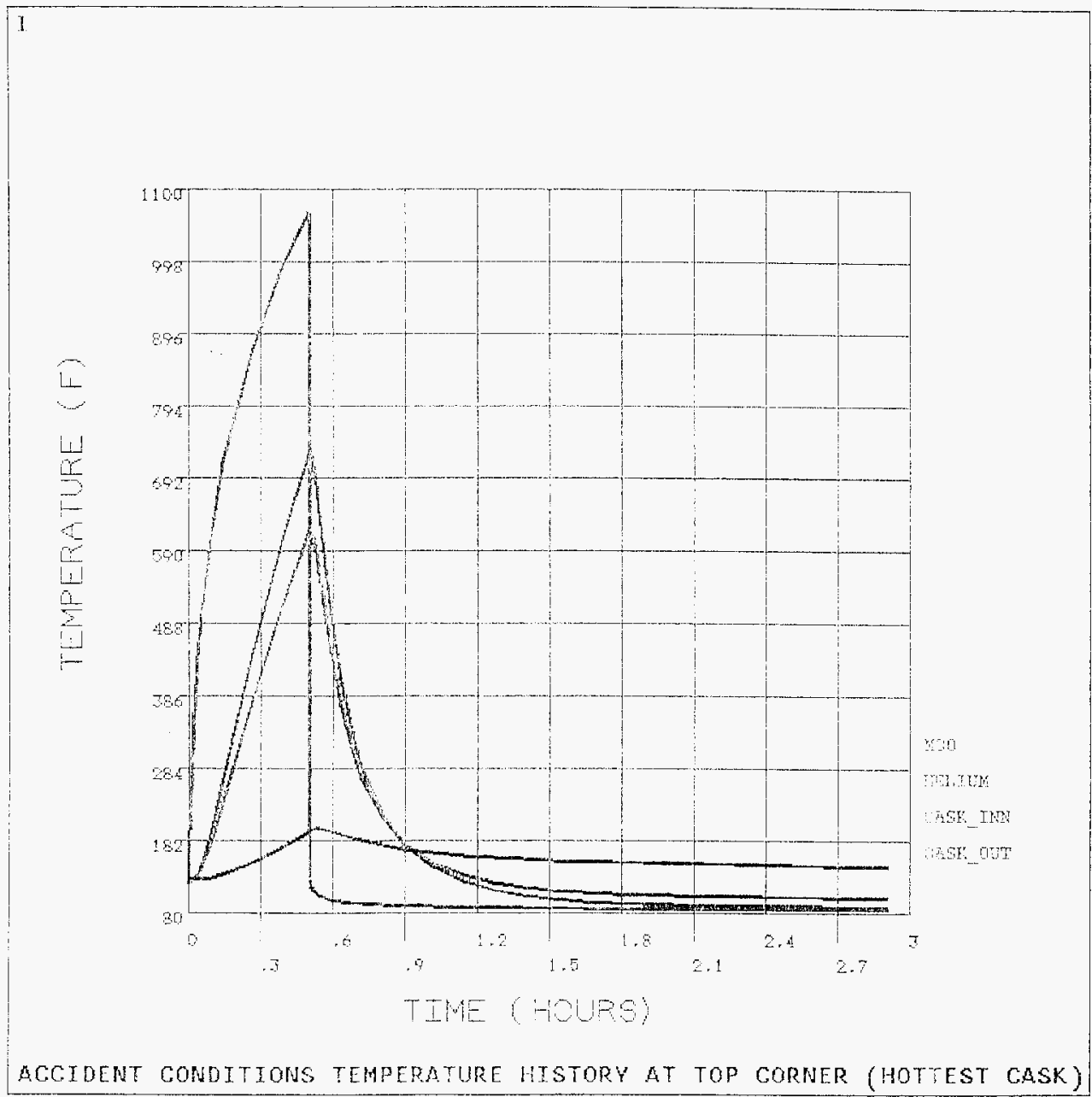


HNF-SD-SNF-FDR-003 Rev. $0 \quad$ E-15166

Figure B5.5.4-4 Accident Conditions MCO and Cask Temp. Distribution - Initial Conditions

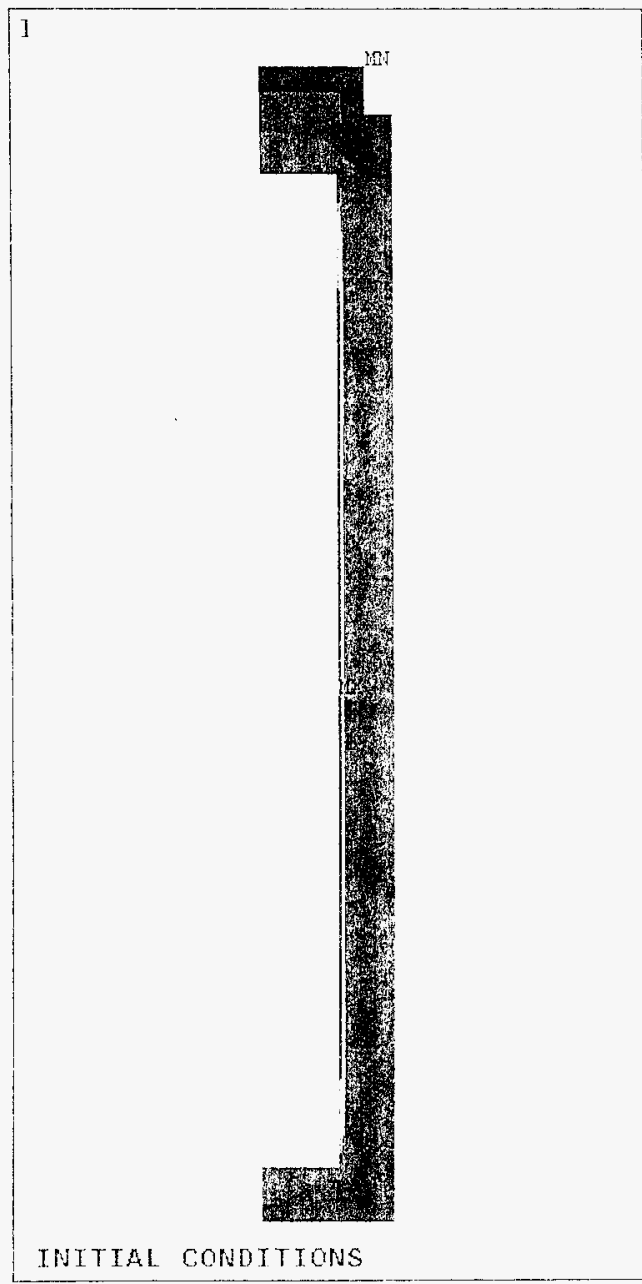

ANSYS 5.2

OCT $30 \quad 1996$

$10: 02: 06$

NODNL SOLUTION

TIME $=.100 \mathrm{E}-05$

TEMP

$S M N=121.437$

$\sin x=153.883$

$121 \cdot 437$

125.042

1.28.647

132.252

135.857

139.463

143.068

$\square \quad 140.673$

17. 150.273

153.823 
Figure B5.5.4-5 Accident Conditions MCO and Cask Temp. Distribution - End of Fire

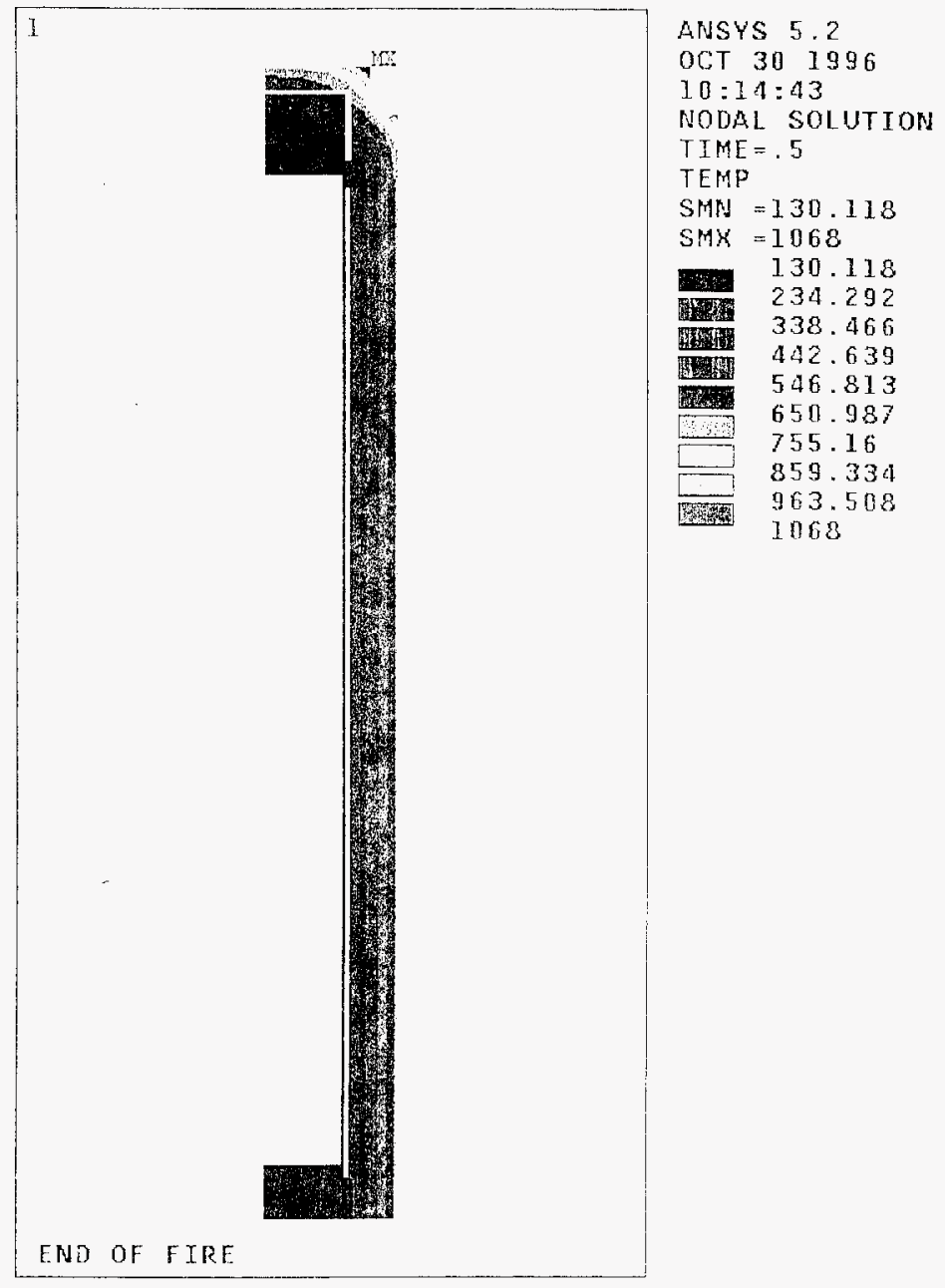


HNF-SD-SNF-FDR-003 Rev. $0 \quad$ E-15166

Figure B5.5.4-6 Accident Conditions MCO and Cask Temp. Distribution - Hottest MCO

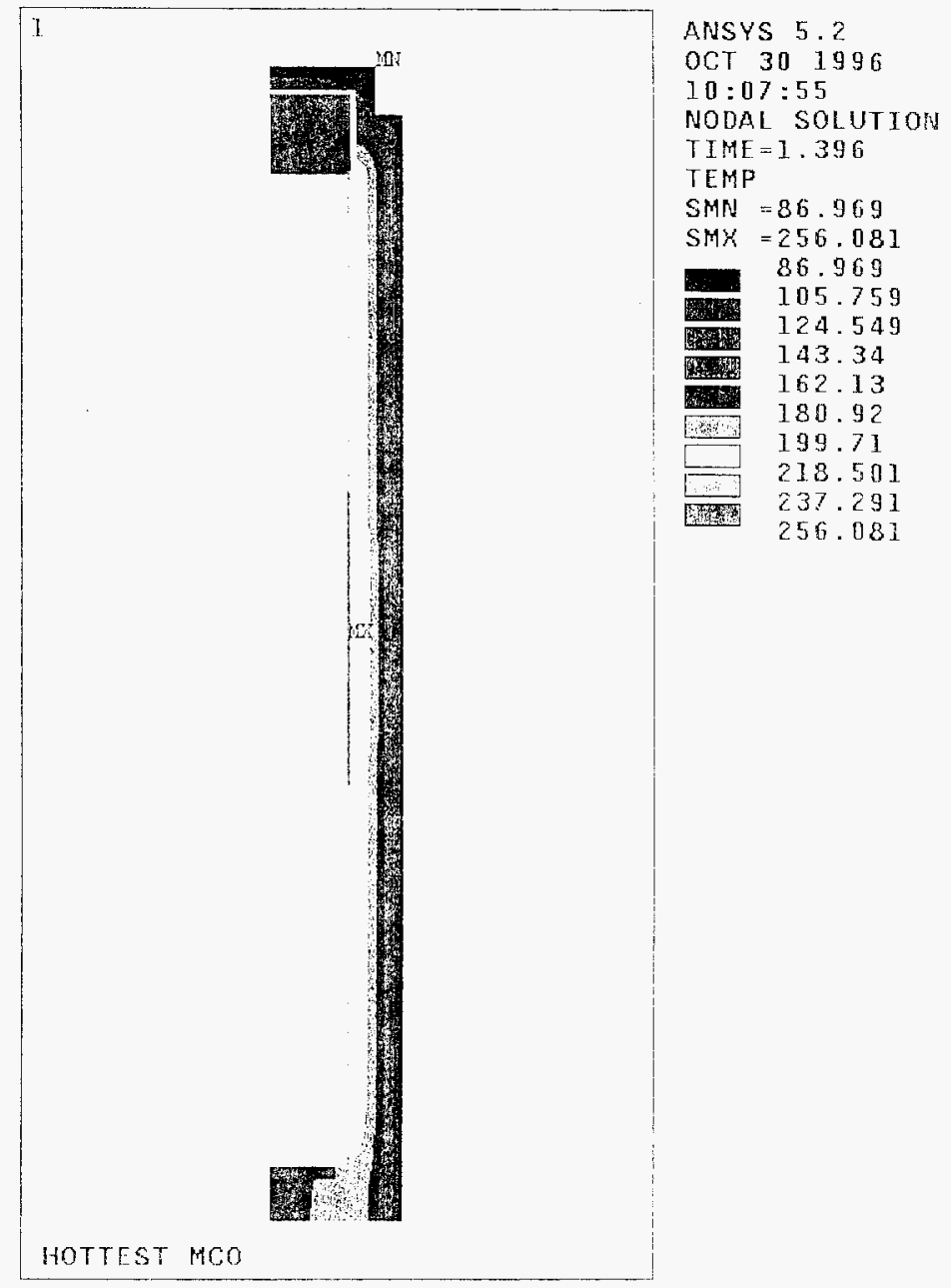




\section{References For Section B5.0}

1. Kreith, et. al., Principles of Heat Transfer, 3rd Edition, Harper and Row Publishers, 1973.

2. Performance Specification For TN-WHC Cask And Transportation System, WIC-S-0396, Rev. 1, September 1995, and WIIC ECN 191402 dated May 22. 1996.

3. ASME Boiler and Pressure Vessel Code, Section 111, Appendices, 1992 Ed.

4. Parker O-Ring Handbook, ORD 5700, Parker Seal Group, KY, 1992.

5. Ansys Engineering Analysis System, Lser’s Manuals for ANSYS Rev. 5.2, Ansys, Inc, Houston, PA; 1995. 
HNF-SD-SNF-FDR-003 Rev. 0 E-15166

\section{B6.0 PRESSURE AND GAS GENERATION EVALUATION}

B6. 1 GAS GENERATION

To be provided by DESH.

B6.2 PACKAGE PRESSURE

The maximum internal working pressurc for the cask is 150 psig (1033 $\mathrm{kPa}$ ) as required by the WIIC Specification. The stress due to this pressure is evaluated in Section B4.0.

B6.3 APPENDIX 
HNF-SD-SNF-FDR-003 Rev. 0 E-15166

\section{B7.0 PACKAGE TIEDOWN SYSTEM EVALUATION}

\section{B7.1 SYSTEM DESIGN}

The basic components of the system ire:

- Frames and Beams

- Cask Tiedown Device

- Cask Hold Doun Device

- Cask Suppori Device

The eight frames. two cross beams and two support braces (including the weld attachments of the vertical support (rames to the support pads of the trailer) are the major components of the tiedown system. The loads from the cask are transmitted by the frames to the trailer. The cask tiedown device consists of two rotating flanges, six tiedown bolts, and two hold down pins. The cask support device consists of one cylindrical cup, bottom plate, and fourteen (14) attachment bolts. A detailed design drawing for the eask tiedown system is provided in Section $A 9.1$.

The cask is supported by the support device (cylindrical cup) at the bottom and secured by the tiedown device (two rotating langes) at the top. The two rotating flanges can be opened and closed (by removing the six tiedown bolts and rotated from the hold down pins) for loading and unloading the cask. Figure B7.1-1 shows the opened and closed positions of the rotating flanges of the cask ticdown device. The cask is transported in the vertical orientation with the lid end facing up.

\section{B7.2 ATTACHNENTS AND RATINGS}

\section{B7.2.1 Design Load Factors}

The tiedown system is used to secure the cask to the trailer during transportation, and is designed to meet the requirements of Hanford Specificution"1). The tiedown systems is capable of resisting the forces for road, as described below:

Tabie B7.2-1 Load Factors for Tiedown Systems

\begin{tabular}{|c|c|c|c|}
\hline Mode & Longitudinal & Lateral & Vertical \\
\hline Rond & $+2 \mathrm{G}$ & $\pm 1 \mathrm{G}^{*}$ & $3 \mathrm{G}$ down. 2G ip \\
\hline
\end{tabular}

* A lateral load factor $1.5 \mathrm{G}$ is used for stress calculation of the tiedown system, reference to Appendix B7.3 for detailed ioading information. 


\section{HNF-SD-SNF-FDR-003 \\ Rev. 0 \\ E-15166}

\section{B7.2.2 Design Weight}

The loading condition used for designing the tiedown system includes the total weight of the loaded cask. $60000 \mathrm{lbs}$, and weight of tiedown system, $5000 \mathrm{lbs}$, for a total design weiglt of 65000 lbs.

\section{B7.2.3 Design Loadings}

In the transport configuration (Figure B7.2-1), the tiedown loads from the cask are transmitted through the cask hold down devices and the cask surfaces in contact with the tiedown system, to the ticdown system. As described above, the total loading used for designing the tiedown system is $65,000 \mathrm{lbs}$. The $2 \mathrm{G}$ vertical load is shared by the four cask hold down devices and transmitted to the cask tiedown device as shown on Figure B7.2-2. The $3 \mathrm{G}$ vertical down load is supported by the cask support device which is directly wolded to the trailer main beams. Figure B7.2-3 shows this loading condition. The $1.5 \mathrm{G}$ load component which is transverse to the direction of travel is shared between the cask tiedown device and cask support device as shown on Figure B7.2-4. The $2 \mathrm{G}$ loxi component applied in the direction of travel is also shared between the cask tiedown device and cask support device as shown on Figures B7.2-5 and B7.2-6. Figure B7.2-7 shows $1 \mathrm{G}$ static load with deflection.

\section{B7.2.4 Summary and Conclusions}

Detailed stress analysis of the tiedown system is presented in Appendix B7.3. A summary of crifical stresses is presented in Table B7.2-2 and stresses are compared with the allowables. All the allowables are based on ASMF Code, Section III. Subscetion NF (NF-3322). All the stresses are within the allowables. The interaction between compression and bonding are evaluated using Equations 20 and 21 of $\mathrm{NF}-3322$, and interaction between tension and bending are using Equation 21 of NF-3322. Table B7.2-3 shows the results of this calculation. Based on the results of the analysis, it is concluded that the ticdown system is structurally safe for the specified loads. 
Table B7.2-2 Summary of Tiedown System Stress Evaluation

\begin{tabular}{|c|c|c|}
\hline Component & $\begin{array}{l}\text { Max. Calculated Stress } \\
\text { (nsi) }\end{array}$ & $\begin{array}{l}\text { Allowable } \\
\text { Stress } \\
\text { (psi) }\end{array}$ \\
\hline Frame- Lcegs & $\begin{array}{l}\text { Max. Direct }=5,073 \text { (Compression) } \\
\text { Max. Bending }=14,477\end{array}$ & $\begin{array}{l}26,400 \\
29,040\end{array}$ \\
\hline Cross Beam & $\begin{array}{l}\text { Max. Direct }=828 \text { (Tension) } \\
\text { Max. Bending }=15,852\end{array}$ & $\begin{array}{l}26,400 \\
29,040\end{array}$ \\
\hline Welds at Bottom Leg & $\begin{array}{l}\text { Max. Tension }=10,224 \\
\text { Max. Shear }=5,707\end{array}$ & $\begin{array}{l}26,400 \\
17,600\end{array}$ \\
\hline Tiedown device-Clamp & $\begin{array}{l}\text { Max. Direct }=2,446(\text { Tension }) \\
\text { Max. Bending }=20,513\end{array}$ & $\begin{array}{l}23,400 \\
25,740\end{array}$ \\
\hline Bolt & $\begin{array}{l}\text { Max. Shear }=23,460 \\
\text { Max. Tension }=36,700\end{array}$ & $\begin{array}{l}40,400 \\
60,600\end{array}$ \\
\hline $\begin{array}{l}\text { Cusk Hold Down Device } \\
\text {-Arm }\end{array}$ & $\begin{array}{l}\text { Max. Tension }=11,430 \\
\text { Vix. Bending }=43,200 \\
\text { Vax. Shear }=11,933\end{array}$ & $\begin{array}{l}43,960 \\
64,480 \\
39,080\end{array}$ \\
\hline -Pin-1.5"Dia. & $\begin{array}{l}\text { Max. Bending }=18,420 \\
\text { Max. Shear }=8,490\end{array}$ & $\begin{array}{l}66,660 \\
40,400\end{array}$ \\
\hline -Bracket & $\begin{array}{l}\text { Bearing }=16,000 \\
\text { Tensile }=6000 \\
\text { Shear }=4,570\end{array}$ & $\begin{array}{l}39,000 \\
17,550 \\
15,600\end{array}$ \\
\hline $\begin{array}{l}\text { Cask Support Device } \\
\text {-Plate }\end{array}$ & Mux. Bending $=18,429$ & 19,400 \\
\hline - Bolt & Max. Shear $=24,560$ & 40,400 \\
\hline
\end{tabular}


HNF-SD-SNF-FDR-003 Rev. 0 E-15166

Table B7.2-3

Interaction Equation Evaluations

\begin{tabular}{|l|l|l|}
\hline Component & $\begin{array}{l}\text { Max. Axial Compression and Bending } \\
(\text { NF-3322.1. Equation 20 \& 21) }\end{array}$ & $\begin{array}{l}\text { Max. Axial Tension and Bending } \\
\text { (NF-3322.1. Equation 21) }\end{array}$ \\
\hline Frame Leg & 0.522 & 0.65 \\
\hline Tiedown Clamp & & 0.902 \\
\hline Hold Down Am & & 0.807 \\
\hline
\end{tabular}


HNF-SD-SNF-FDR-003 ReV. 0

Figure B7.1-1 TN-WHC Cask Tiedown System - Opened and Closed Positions
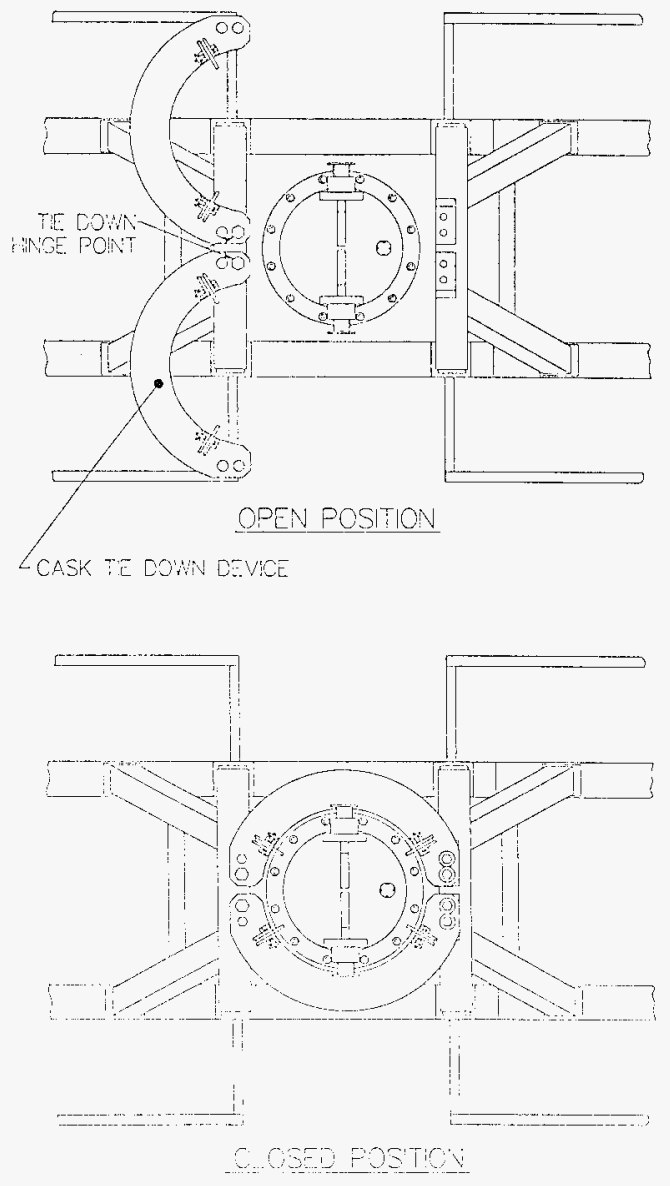


\section{HNF-SD-SNF-FDR-003 Rev. 0}

E-15166

Figure B7.2-1 TN-WHC Cask Transport Configuration
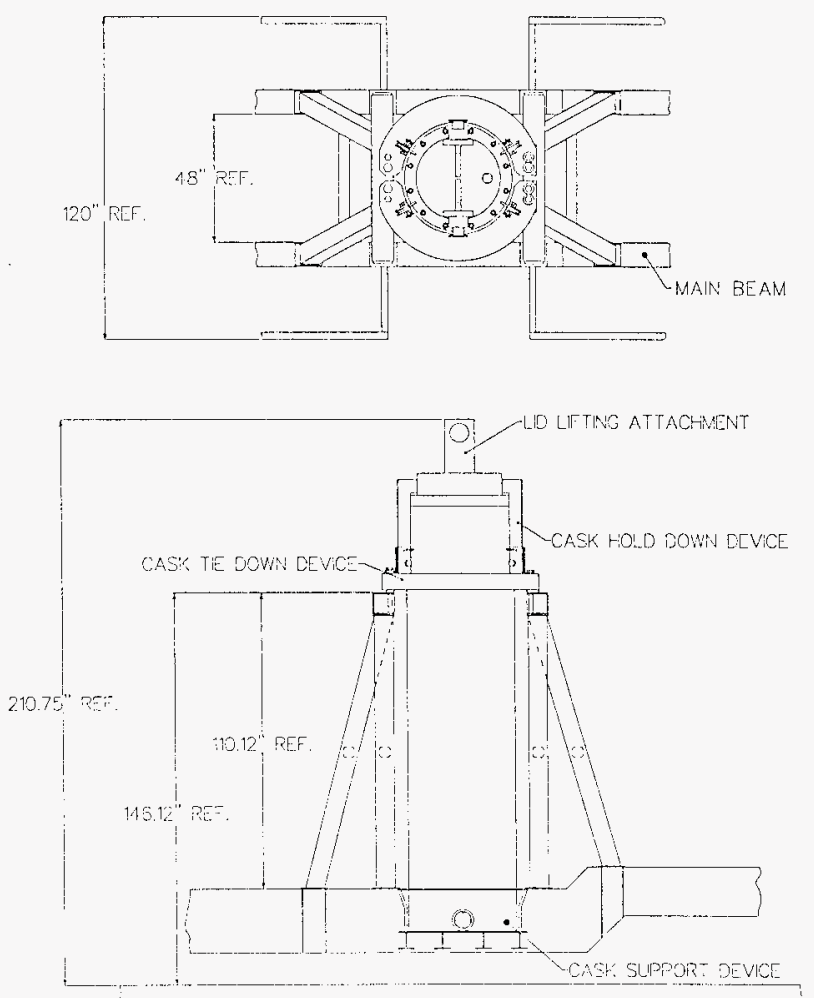
Figure B7.2-2 Tiedown Loading Condition - 2G Vertical Up

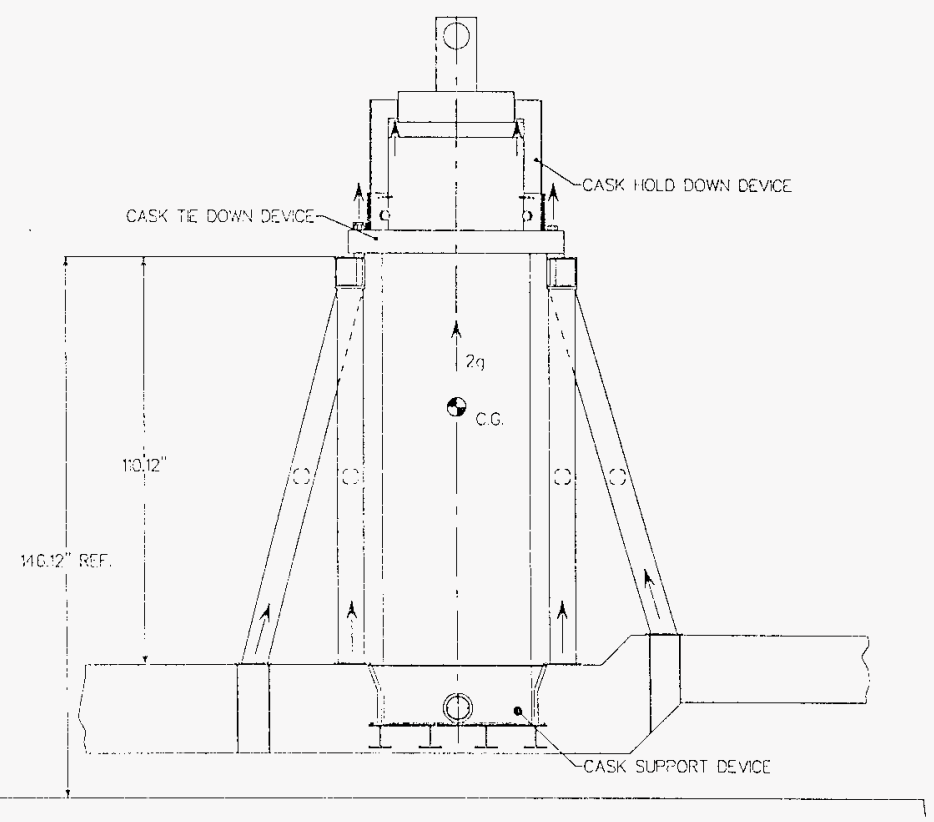


Figure B7.2-3 Tiedown Loading Condition - 3G Down

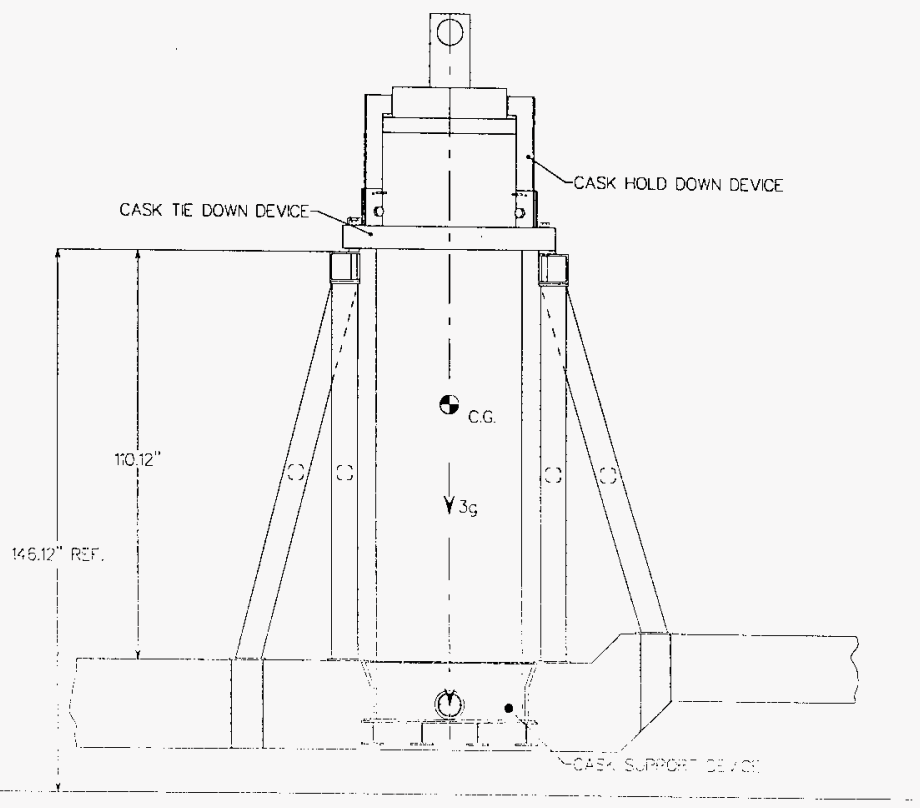


Figure B7.2-4 Tiedown Loading Condition - 1.5G Lateral

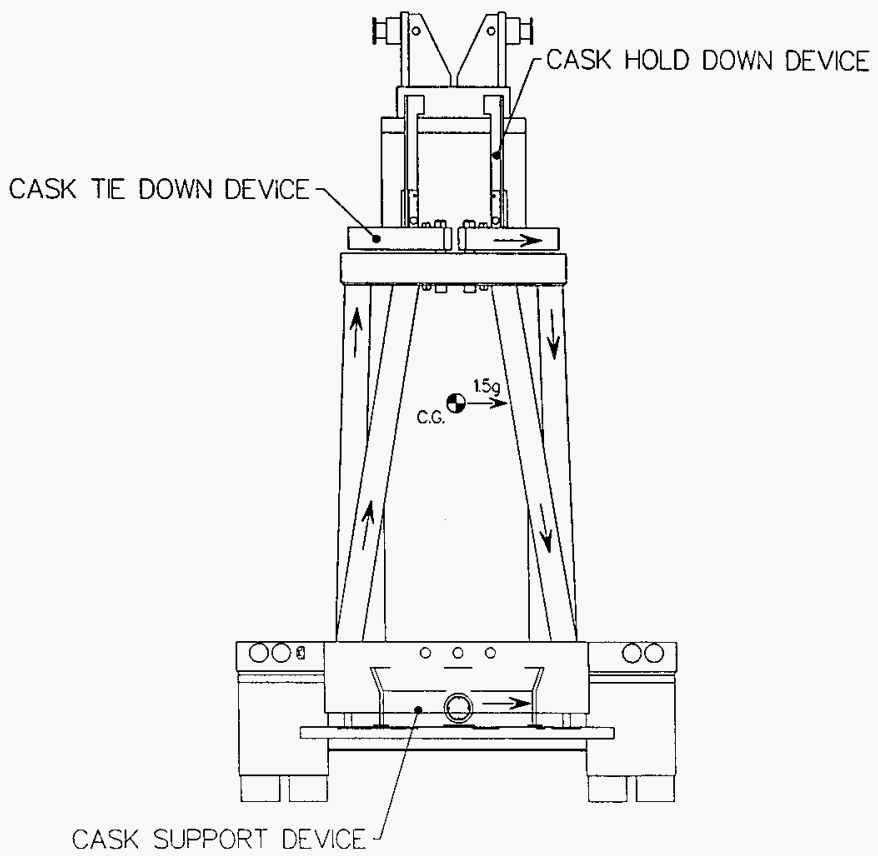


Figure B7.2-5 Tiedown Loading Condition - 2G Longitudinal (fore)

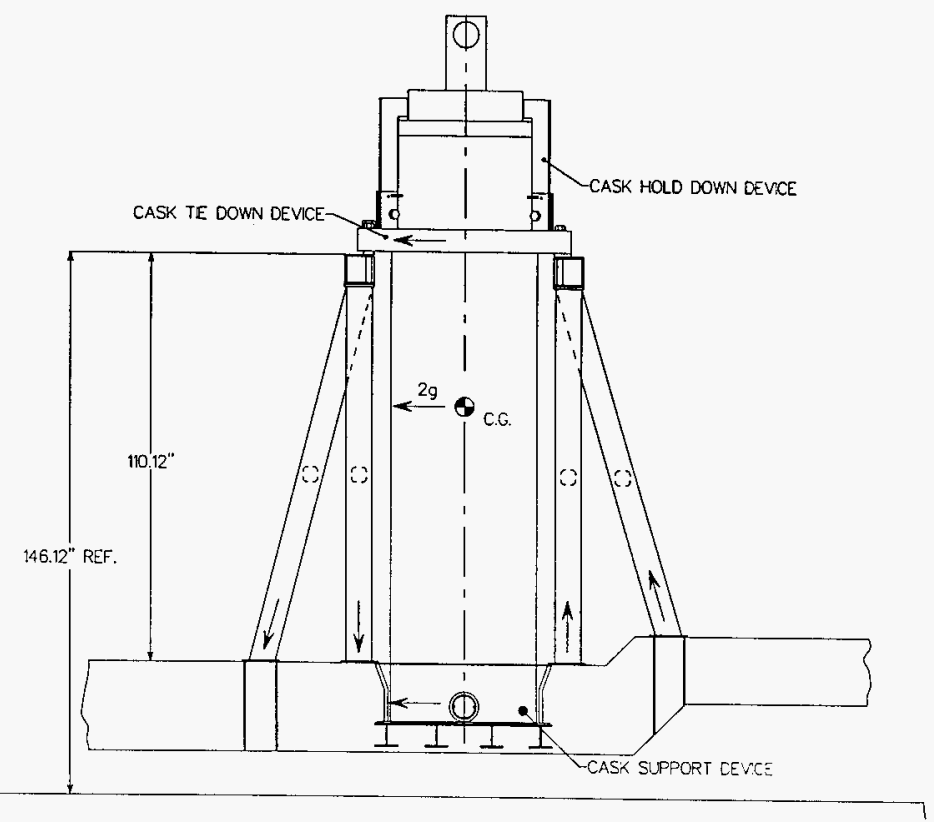


Figure B7.2-6 Tiedown Loading Condition - 2G Longitudinal (aft)

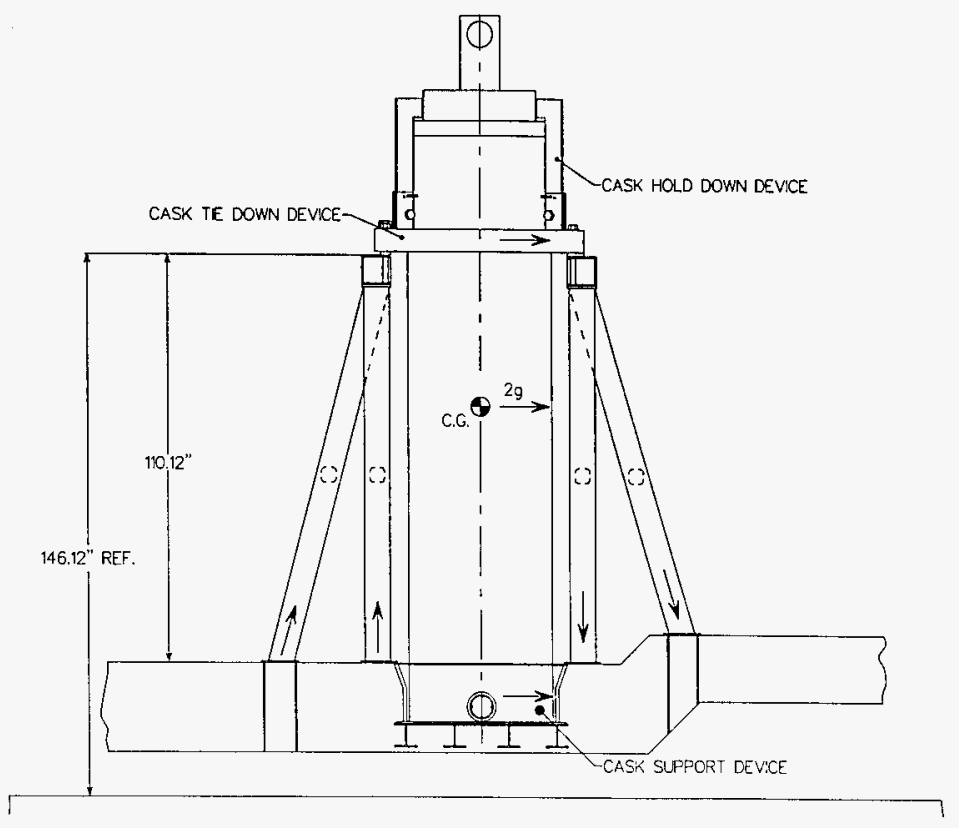


Figure B7.2-7 Tiedown Loading Condition - 1G Static Deflection

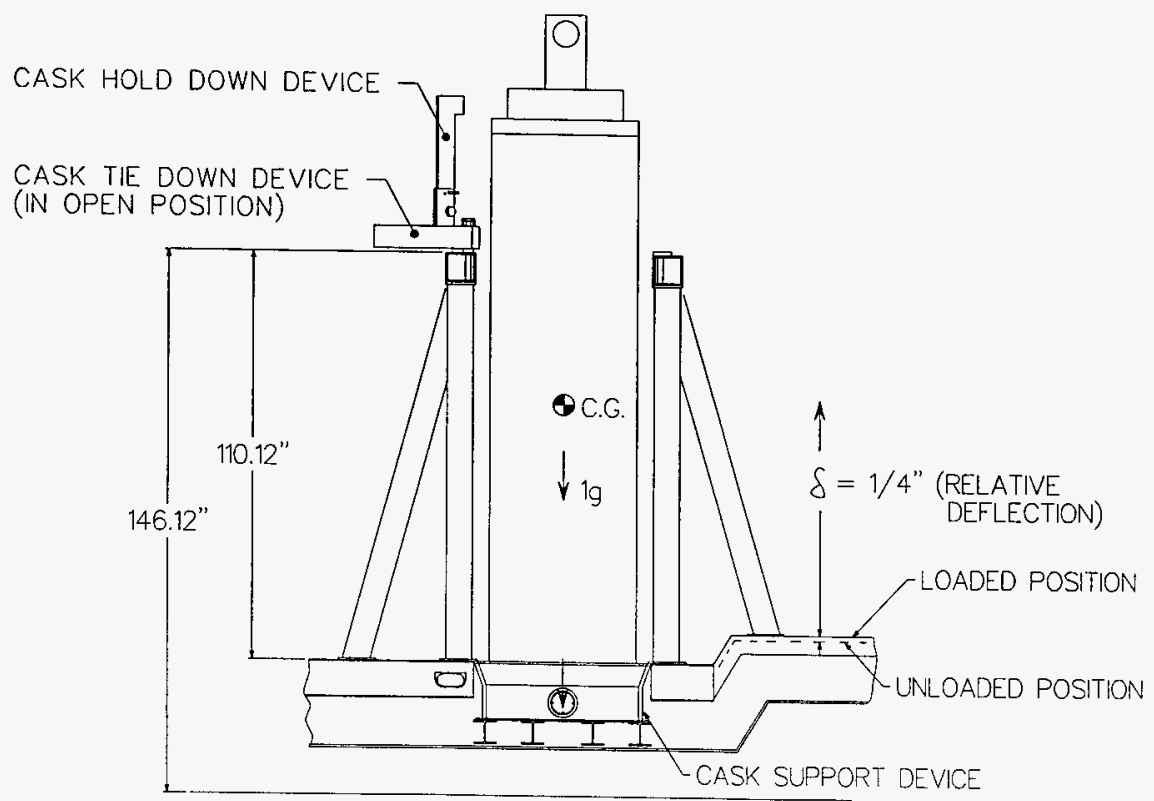




\section{References For Section B7.0}

1. Performance Specification For TN-WHC Cask And Transportation System, WHC-S-0396, Rev. 1, September 1995. 
HNF-SD-SNF-FDR-003 ReV. 0

E-15166

APPENDIX B7.3

CASK TIEDOWN SYSTEM 
TABLE OF CONTENTS

Section

B7.3-1

B7.3-2

B7.3-3

B7.3-4

B7.3-5

B7.3-6

B7.3-6

B7.3-7
INTRODUCTION

MECHANICAL PROPERTIES OF MATERIALS

WEIGHT AND CENTER-OF-GRAVITY

LOADINGS

ANALYSES

DESIGN CRITERIA FOR TIEDOWN SYSTEM

SUMMARY AND CONCLUSIONS

REFERENCES
Page

B7.3-1

B7.3.1

B7.3-1

B7.3-1

B7.3-6

B7.3-23

B7.3-25

B7.3-28 
APPENDIX B7.3

CASK TIEDOWN SYSTEM

\section{B7.3-1 INTRODUCTION}

The purpose of the tiedown system is to restrain the cask during transportation by road. The tiedown attachments have to resist the forces from the road transportation as described in Hanford Specification ${ }^{(1)}$. The detailed tiedown system design is given in Drawing No. H-1-81539, Sheets 1 to 3 and its operation is described in Section B7.0 of this report. This appendix presents the structural analysis of the tiedown system design for the prescribed loadings. The analysis of the tiedown system components is conducted using a combination of computer finite element techniques and hand-calculations. The terminology of the components, as used in this analysis, is shown in Figure B7.3-1.

\section{B7.3-2 MECHANICAL PROPERTIES OF MATERIALS}

The mechanical properties of structural materials used in the tiedown system components are shown on Table B7.3-1. The effect of an elevated temperature of $150^{\circ} \mathrm{F}$ on yield strength is also shown on Table B7.3-1. The yield and ultimate strengths of the structural steels shown on Table B7.3-1 are the minimum values specified in the material specifications. A modulus of $29 \times 10^{6}$ psi for steel and $10 \times 10^{6} \mathrm{psi}$ for aluminum is used in the analyses.

\section{B7.3-3 WEIGHT AND CENTER-OF-GRAVITY}

The approximate weight of each tiedown system major component is given on Table B7.3-2. The estimated weight of entire tiedown system is about $5000 \mathrm{lbs}$. The center of gravity of the loaded cask on the trailer is approximately $104.27^{\prime \prime}$ above the ground.

\section{B7.3-4 LOADING}

The tiedown system is used to secure the cask to the trailer against the transportation loads. These loads are summarized in Table B7.3-3. The stress analysis of tiedown system components is conducted based on these loadings.

It may be also pointed out that each structural component of the tiedown system is not substantially effected by each load in Table B7.3-3. Stress analyses are conducted only for the loadings from this table which are applicable to that component. 
HAF-SD-SNF-FRD-003 Rev. 0

E-15166

Figure B7.3-1

Tiedown System Components

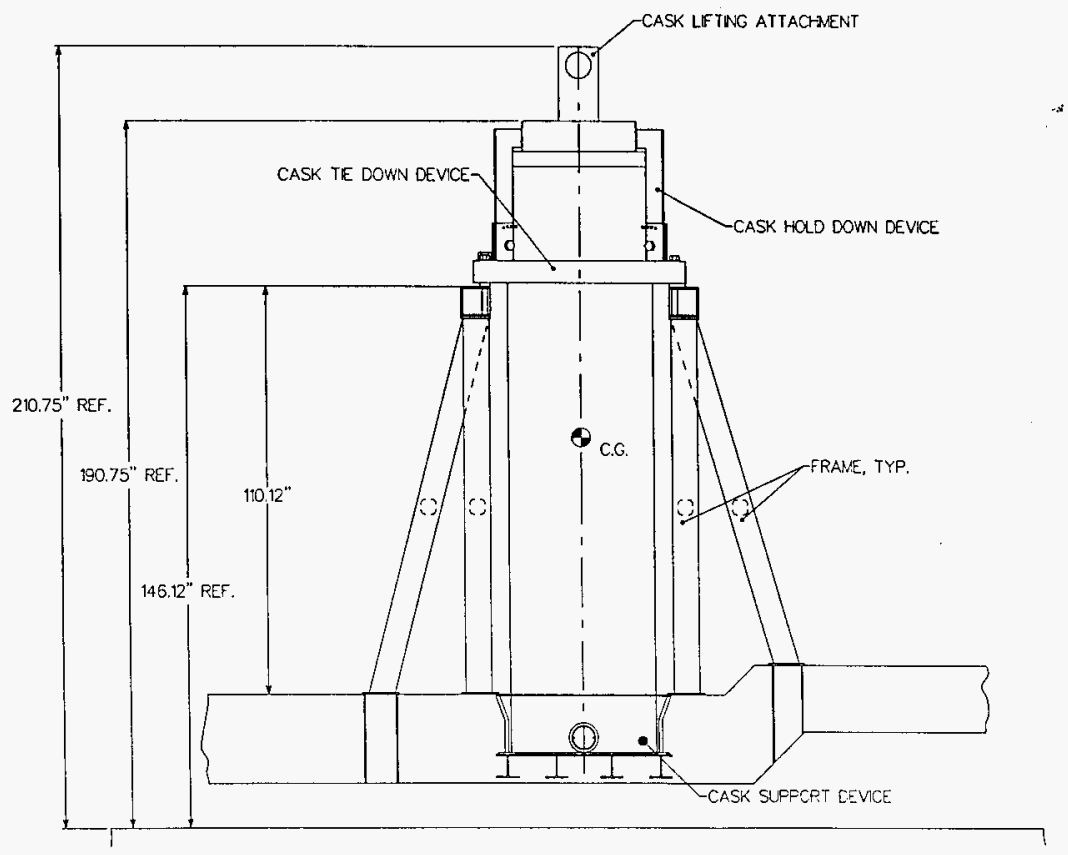


HNF-SD-SNF-FRD-003 Rev. O

Table B7.3-1

Material Properties of The Tiedown System

\begin{tabular}{|l|l|l|l||}
\hline Component & Material & $\begin{array}{l}\text { Properties @ } \\
70^{\circ} \mathrm{F}(\mathrm{ksi})\end{array}$ & $\begin{array}{l}\text { Properties @ 1500 F } \\
\text { (ksi) }\end{array}$ \\
\hline Frames & A500 Gr.B & $\begin{array}{l}\mathrm{S}_{\mathrm{y}}=46 \\
\mathrm{~S}_{\mathrm{u}}=58\end{array}$ & $\mathrm{~S}_{\mathrm{y}}=44$ \\
\hline Cross Beam & A500 Gr.B & $\begin{array}{l}\mathrm{S}_{\mathrm{y}}=46 \\
\mathrm{~S}_{\mathrm{u}}=58\end{array}$ & $\mathrm{~S}_{\mathrm{y}}=44$ \\
\hline $\begin{array}{l}\text { Tie Down Device - } \\
\text { Clamp } \\
\text { (0.31" plate) }\end{array}$ & $\begin{array}{l}\text { B209-6061T6 } \\
\text { Aluminum }\end{array}$ & $\begin{array}{l}\mathrm{S}_{\mathrm{y}}=40 \\
\mathrm{~S}_{\mathrm{u}}=45\end{array}$ & $\mathrm{~S}_{\mathrm{y}}=39$ \\
\hline $\begin{array}{l}\text { Hold Down Device } \\
\text { (0.75"plate) }\end{array}$ & $\begin{array}{l}\text { A-514 Gr.B } \\
\text { (T1, Type A) }\end{array}$ & $\begin{array}{l}\mathrm{S}_{\mathrm{y}}=100 \\
\mathrm{~S}_{\mathrm{u}}=110\end{array}$ & $\mathrm{~S}_{\mathrm{y}}=97.7$ \\
\hline $\begin{array}{l}\text { Support Device } \\
\text { (0.75" plate) }\end{array}$ & $\begin{array}{l}\text { 6063-T6 } \\
\text { Aluminum }\end{array}$ & $\begin{array}{l}\mathrm{S}_{\mathrm{y}}=31 \\
\mathrm{~S}_{\mathrm{u}}=35\end{array}$ & $\mathrm{~S}_{\mathrm{y}}=29.4$ \\
\hline $\begin{array}{l}\text { Bolts for Support } \\
\text { Device }\end{array}$ & A-193-B7 & $\mathrm{S}_{\mathrm{y}}=105$ & $\mathrm{~S}_{\mathrm{y}}=101$ \\
\hline Pins & $\begin{array}{l}\text { A-479 XM19 } \\
\text { Hot Rolled } \\
\text { Stainless Steel }\end{array}$ & $\begin{array}{l}\mathrm{S}_{\mathrm{y}}=125 \\
\mathrm{~S}_{\mathrm{u}}=135\end{array}$ & $\mathrm{~S}_{\mathrm{y}}=101$ \\
\hline $\begin{array}{l}\text { Bolts for Tiedown } \\
\text { device }\end{array}$ & $\begin{array}{l}\text { A-479 XM19 } \\
\text { Hot Rolled } \\
\text { Stainless Steel }\end{array}$ & $\begin{array}{l}\mathrm{S}_{\mathrm{y}}=105 \\
\mathrm{~S}_{\mathrm{u}}=135\end{array}$ & $\mathrm{~S}_{\mathrm{y}}=101$ \\
\hline
\end{tabular}


Table B7.3-2

Estimated Weight of The Tiedown System Components

\begin{tabular}{|c|c|}
\hline Component & Estimated Weight - lbs. \\
\hline Frames (8) $\quad$ TS $7 " \times 7 " \times 0.5^{\prime \prime}$ & 2,986 \\
\hline $\begin{array}{l}\text { Stiffening Braces (4) } \\
\text { TS 4"x 4"x } 0.5 "\end{array}$ & 351 \\
\hline $\begin{array}{l}\text { Cross Beams }(2) \\
\text {-TS } 8 \text { "x } 8 \text { "x } 0.625^{\prime \prime} \\
\text {-Plates } 61^{\prime \prime} \times 8^{\prime \prime} \times 0.625^{\prime \prime}\end{array}$ & 789 \\
\hline $\begin{array}{l}\text { Tie Down Device } \\
\text { - Clamps }(2) \\
\text { - Brackets }(8)\end{array}$ & 175 \\
\hline $\begin{array}{l}\text { Hold Down Device } \\
\text { - Arms (4) }\end{array}$ & 174 \\
\hline Support t Device (1) & 388 \\
\hline Bolts, Pins & 120 \\
\hline Total Estimated Weight & $\begin{array}{c}4983 \\
(\approx 5000)\end{array}$ \\
\hline
\end{tabular}




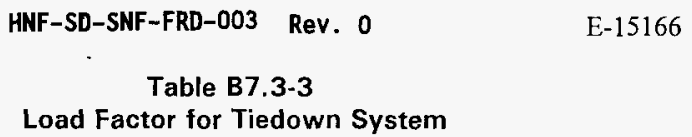

\begin{tabular}{||l|l|l|l|l||}
\hline Case & $\begin{array}{c}\text { Load } \\
\text { Condition }\end{array}$ & Longitudinal & Lateral & Vertical \\
\hline $\mathrm{A}$ & $\begin{array}{l}\text { Tiedown } \\
\text { System }\end{array}$ & $2 \mathrm{G}$ & $1 \mathrm{G}$ & $\begin{array}{l}3 \mathrm{G} \text { down } \\
2 \mathrm{G} \text { up }\end{array}$ \\
\hline $\mathrm{B}$ & Trailer & $\begin{array}{l}2 \mathrm{G} \text { fore } \\
1.5 \mathrm{G} \text { aft }\end{array}$ & $1.5 \mathrm{G}$ & $\begin{array}{l}2.5 \mathrm{G} \text { down } \\
2.0 \mathrm{G} \text { up }\end{array}$ \\
\hline $\mathrm{C}$ & $\begin{array}{l}\text { Design Load } \\
\text { Factors for } \\
\text { Tiedown } \\
\text { System }\end{array}$ & $\begin{array}{l}2 \mathrm{G} \text { fore } \\
2 \mathrm{G} \text { aft }\end{array}$ & $1.5 \mathrm{G}$ & $\begin{array}{l}3 \mathrm{G} \text { down } \\
2 \mathrm{G} \text { up }\end{array}$ \\
\hline
\end{tabular}




\section{B7.3-5 ANALYSIS}

The tiedown system has been analyzed by using a combination of computer finite element and hand-calculation methods. For the purpose of structural analyses, the tiedown system is divided in the following main components (see Figure B7.3-1):

Frame and Cross Beams

Cask Tie down Device

Cask Hold down Device

Cask Support Device

The method of analysis, critical loads, boundary condition and results of analysis of each component are described below:

\section{a) Frame and Cross Beams}

This component was analyzed by using ANSYS Finite Element Computer Code ${ }^{(2)}$. The finite element model of frames, cross beams and stiffening braces is presented in Figures B7.3-2 (finite element nodes) and B7.3-3 (finite element elements). 3-dimensional beam finite element (BEAM4) was used in the construction of the model. The beam real constants are shown in Figure B7.3-4. It may be pointed out that the cask tiedown device clamp in this frame model was simulated by the beam elements. The beam real constants are given in Table B7.3-4. The analyses were conducted for the load case C of Table B7.3-3. The G loads in this table were converted to forces at C.G. by multiplying the load factor with cask weight of $60,000 \mathrm{lbs}$. These forces at C.G. were transferred to the appropriate model nodes. The inertia forces due to the frame weight were accounted for by applying the appropriate accelerations. The typical applied boundary condition and forces in each load case are presented in Figures B7.3-5 to B7.3-8. The detailed resulting forces, stresses and displacements in the model are available in computer output files. A summary of maximum stresses in frame legs and cross beams for each loading is shown in Tables B7.3-5 to B7.3-8. 
HNF-SD-SNF-FRD-003 ReV. 0

E-15166

Figures B7.3-2

ANSYS Finite Element Model - Frame (nodes)

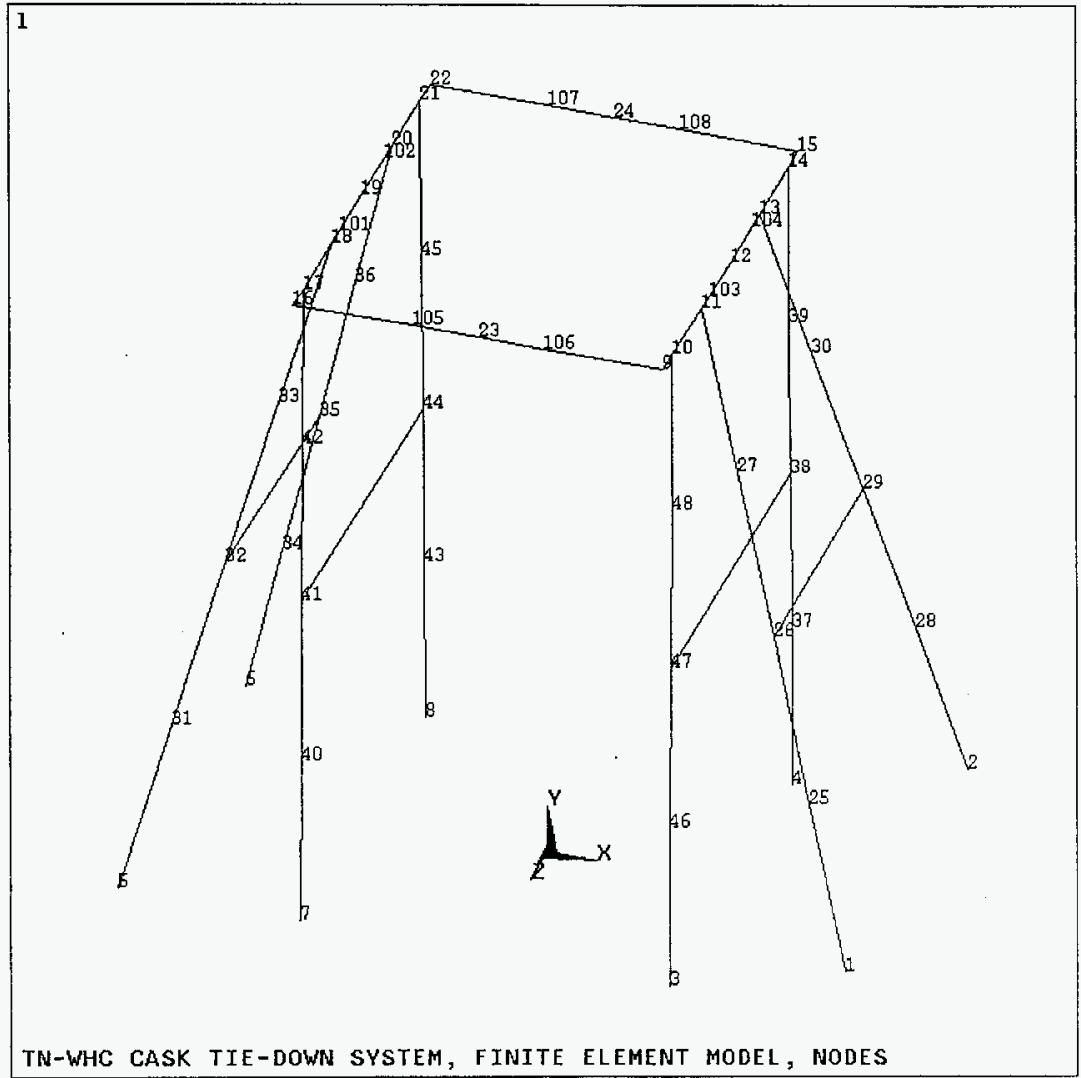


HNF-SD-SNF-FRD-003 Rev. $0 \quad$ E-15166

Figures B7.3-3

ANSYS Finite Element Model - Frame (elements)

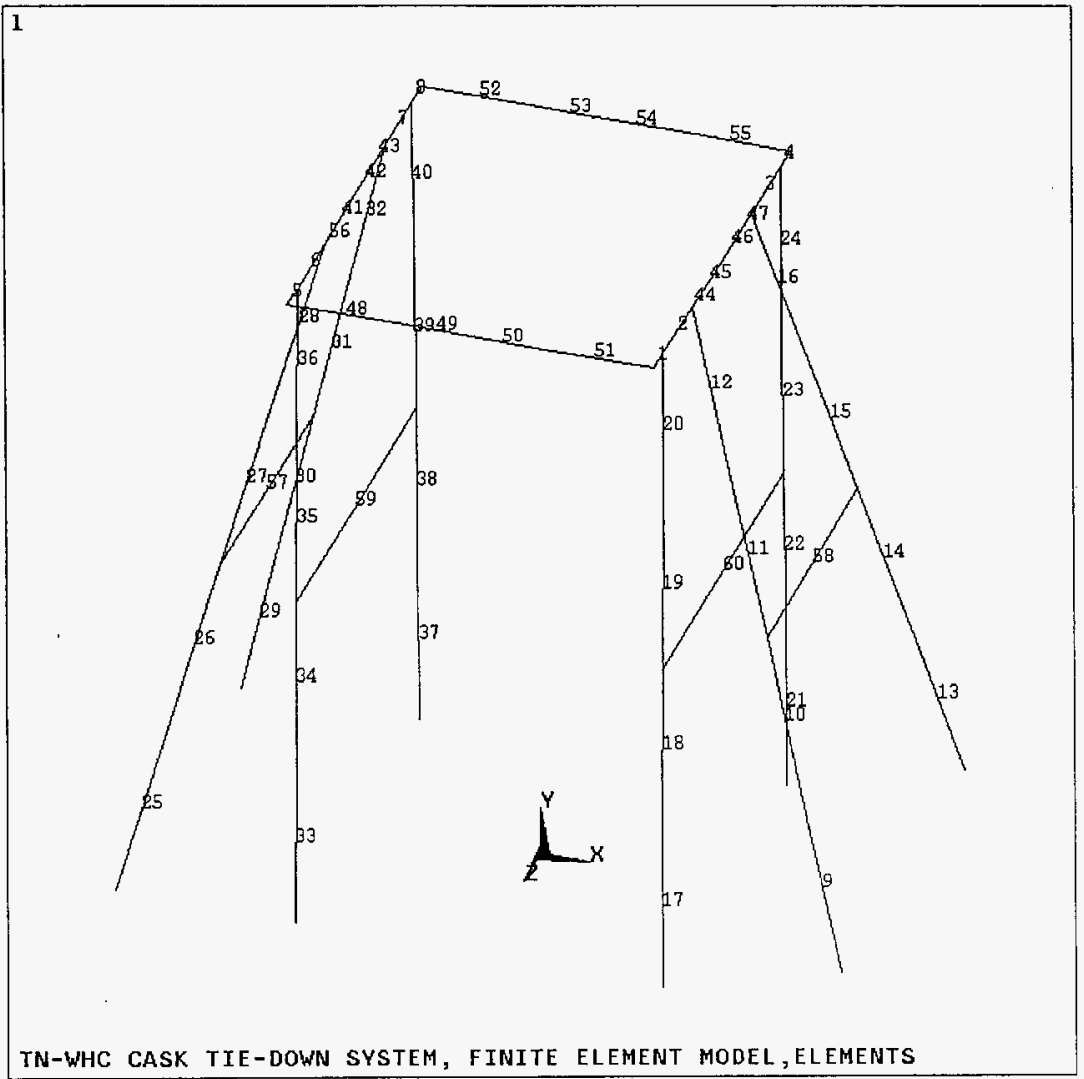


HNF-SD-SNF-FRD-003 Rev. 0 D-15166

Figures B7.3-4

ANSYS Finite Element Model - Frame (real constants)

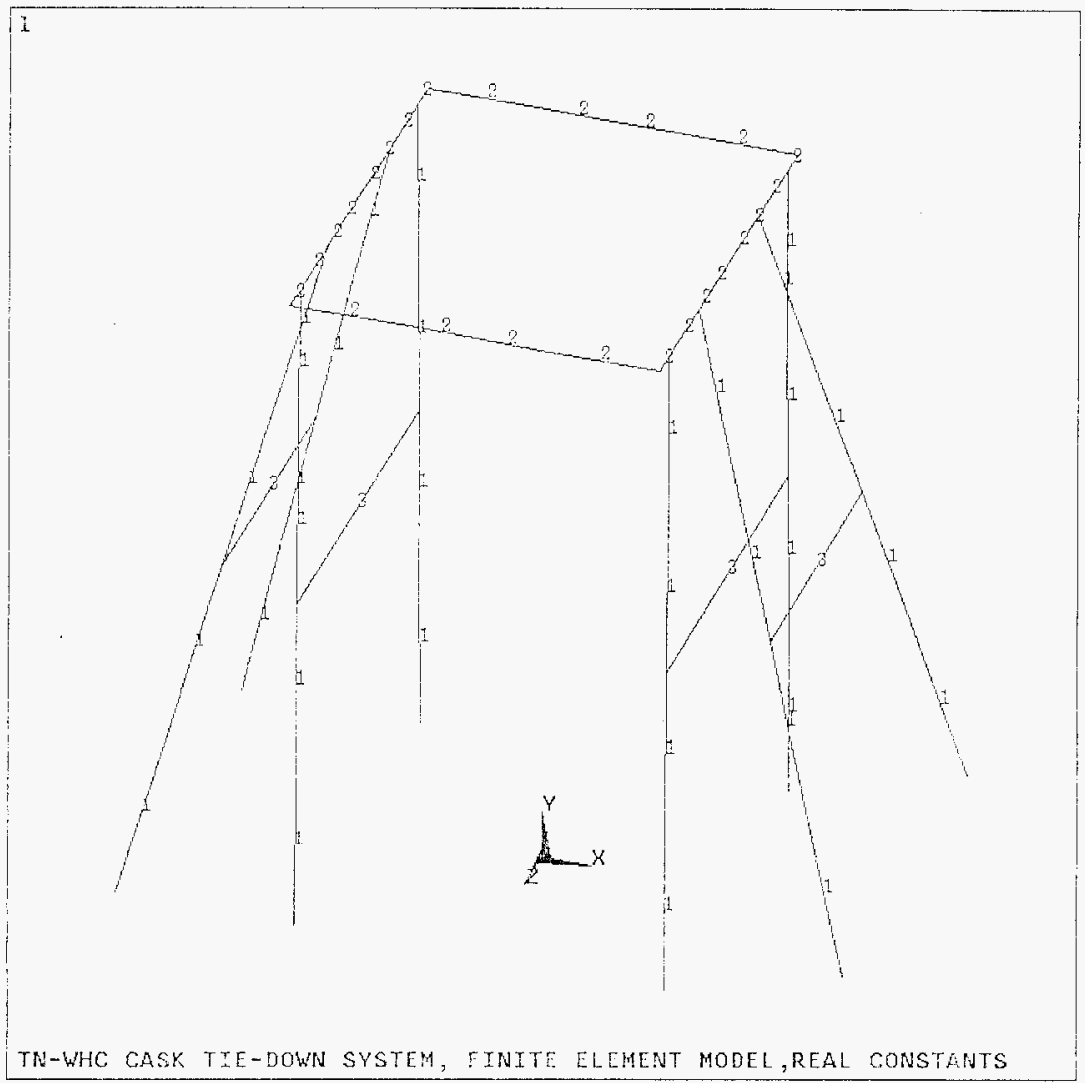


Figure B7.3-5

Frame Model - Loading \& Eoundary Conditions for Longitudinal $2 \mathrm{G}$ (Forel Load Analysis

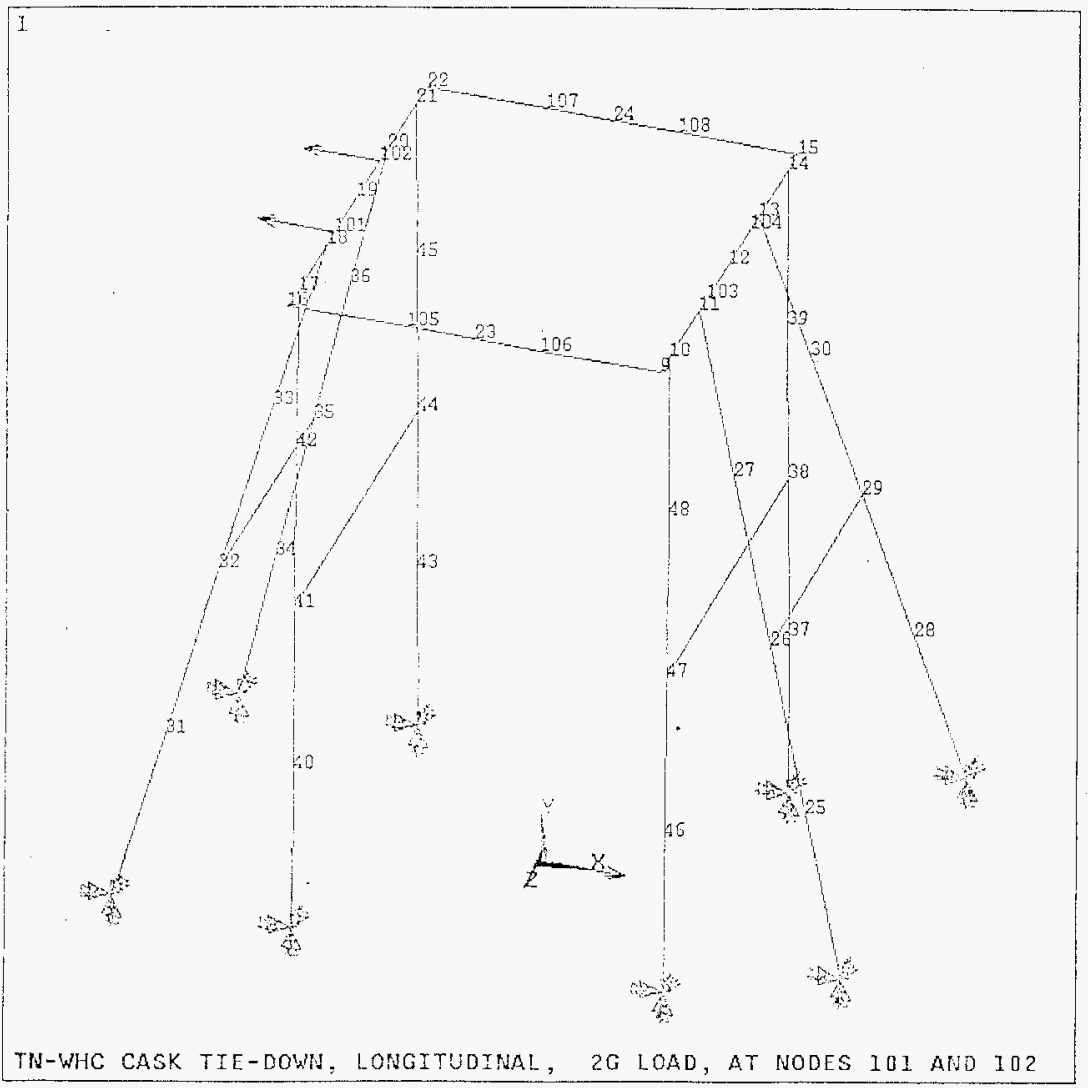


Figure $67.3-6$

Frame Mode! - Loading \& Boundary Conditions for Longitudinal 26 (aft) Load Analysis

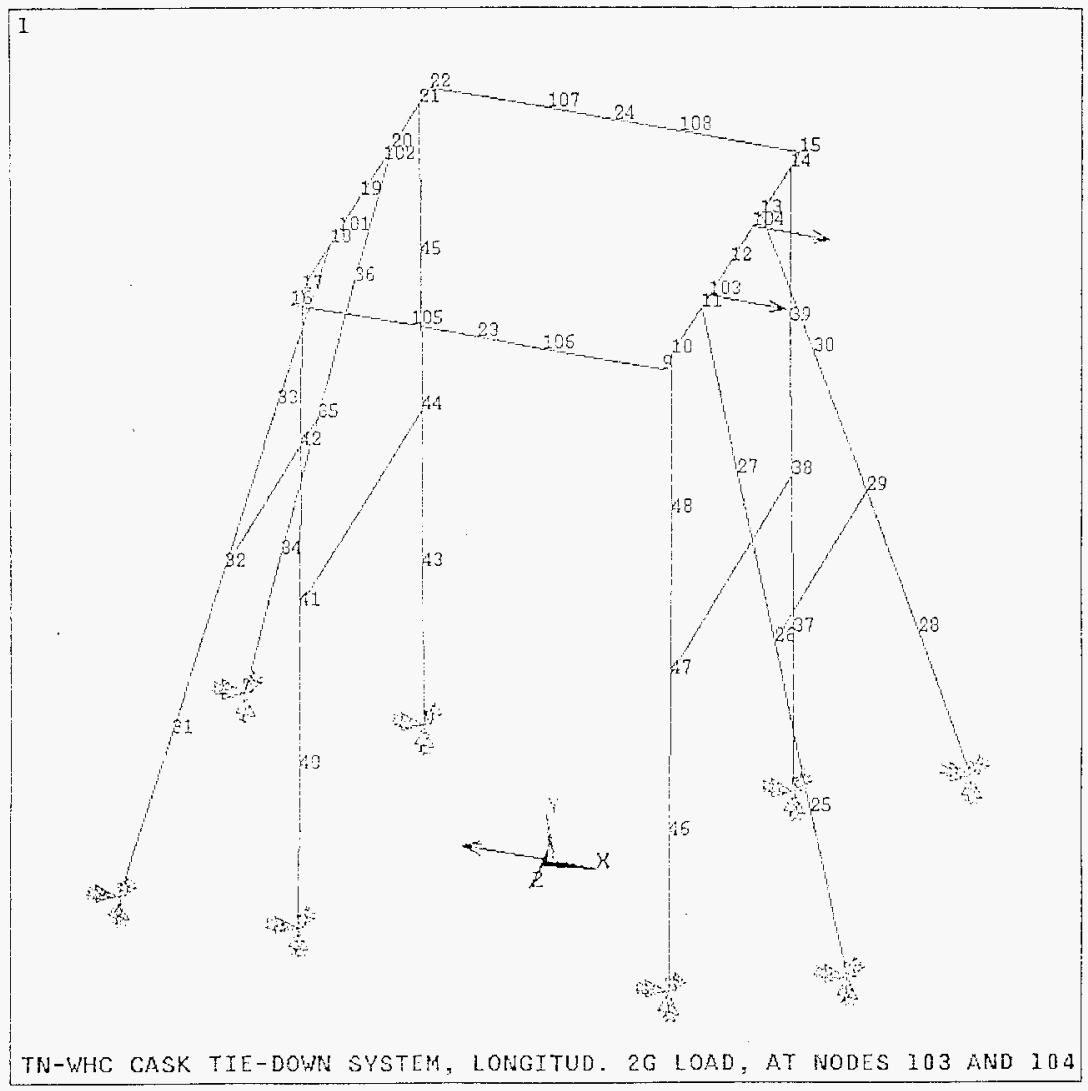


Figure 87.3-7

Frame Model - Loading \& Boundary Concitions for Lateral 1.5G Load Analysis

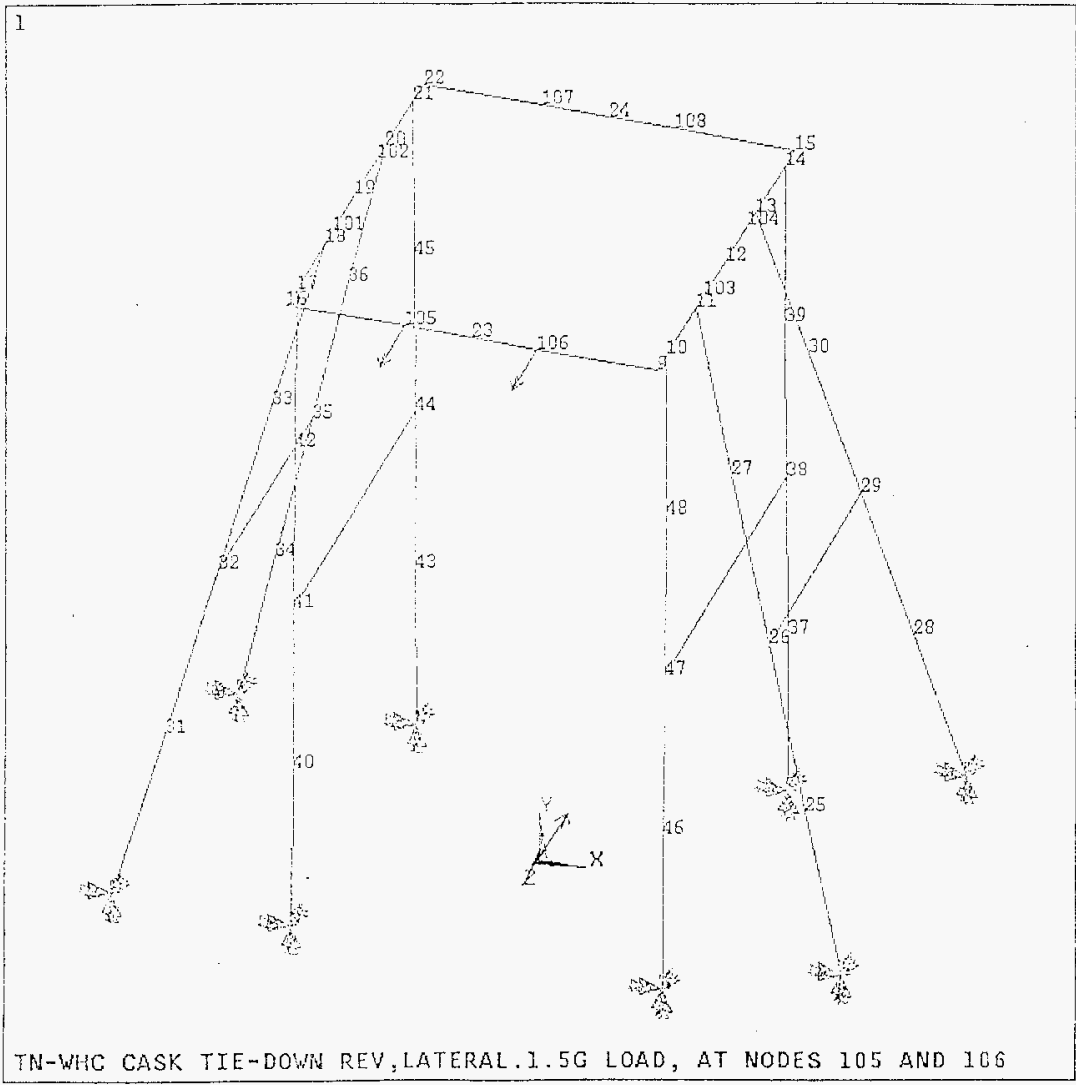


Figure $37.3-3$

Frame Model - Loading \& Boundary Conditions for Up $2.0 \mathrm{G}$ Load Analysis

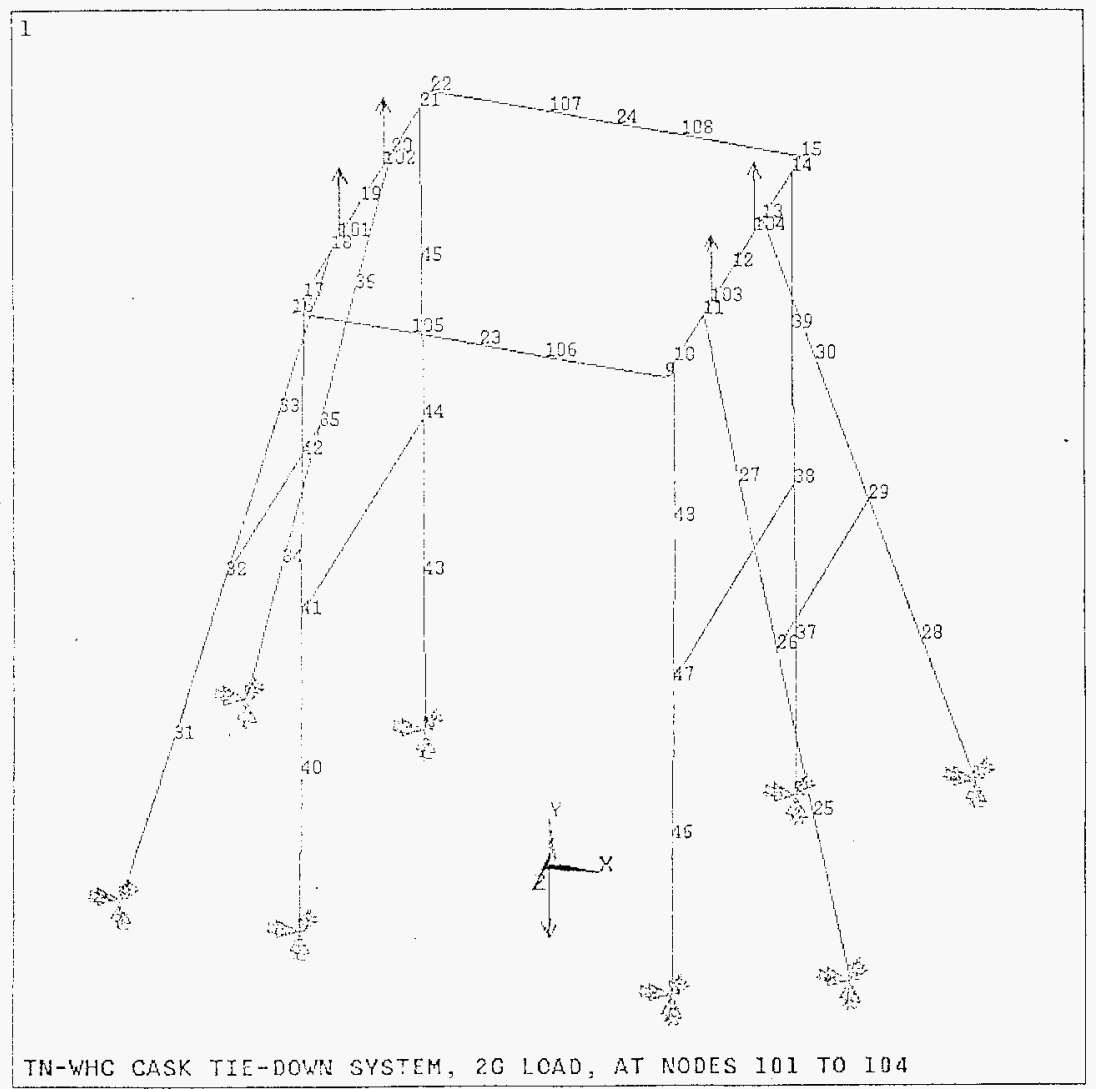


HNF-SD-SNF-FRD-003 Rev. 0

E- 15166

Table B7.3-4.

Frame Finite Element Mocie! - Bearn Real Constants

\begin{tabular}{|c|c|c|c|c|}
\hline $\begin{array}{l}\text { Real } \\
\text { Constant } \\
\text { Numbir }\end{array}$ & Section & $\begin{array}{l}\text { Arco } \\
\text { (iig)? }\end{array}$ & $\begin{array}{c}\text { Moment of } \\
\text { Inertia } \\
(\mathrm{in})^{t}\end{array}$ & $\begin{array}{l}\text { Depth } \\
\text { (iir) }\end{array}$ \\
\hline 1 & TS $-7^{11} \times 7^{11} \times 0.5^{\prime \prime}$ & 12.4 & 84.6 & 70 \\
\hline 2 & $\begin{array}{l}\text { IS- } 8 " \times 8 " \times 0.625 " \\
\text { with reinforcencnt phate } \\
8 " \text { wde anci } 0.625^{\prime \prime} \text { wick }\end{array}$ & 17.4 & 179.7 & 80 \\
\hline 3 & $\operatorname{TS}-4^{\prime \prime} \times 4 " \times 0.5^{\prime \prime}$ & 6.36 & 12.3 & +0 \\
\hline
\end{tabular}


Tabie $57.3-5$

Frame Members Stresses - Loading Longitudinal $2 \mathrm{G}$ (fore) (Fx at nodes 101 and 102 )

\begin{tabular}{|c|c|c|c|c|c|c|}
\hline $\begin{array}{l}\text { Leg } \\
\text { (Element) }\end{array}$ & $\begin{array}{l}\text { Locution } \\
\text { Node) }\end{array}$ & $\begin{array}{l}\text { Dircet } \\
\text { Stress } \\
(p s i) \\
\end{array}$ & $\begin{array}{c}\text { Bonding } \\
\text { Stoss- } \\
y-a x s \\
\text { (nsi) }\end{array}$ & $\begin{array}{l}\text { Bending } \\
\text { Stecss- } \\
z-\text { axis } \\
\text { (psi) }\end{array}$ & $\begin{array}{c}\text { Max. } \\
\text { Combined } \\
\text { Stress } \\
\text { (psi) }\end{array}$ & $\begin{array}{l}\text { Min. } \\
\text { Combined } \\
\text { Stress } \\
\text { (psi) }\end{array}$ \\
\hline $\begin{array}{c}1 \\
(9)\end{array}$ & 1 & -4562 & 7605 & 542 & 12709 & -3586 \\
\hline $\begin{array}{c}2 \\
(16)\end{array}$ & 2 & -4562 & 7605 & 542 & 12709 & -3586 \\
\hline $\begin{array}{c}3 \\
(20) \\
\end{array}$ & 10 & -3451 & 6566 & 6604 & 9718 & -6620 \\
\hline $\begin{array}{c}4 \\
(24) \\
\end{array}$ & 14 & -3451 & 6566 & 6604 & 9718 & -6620 \\
\hline $\begin{array}{c}5 \\
(28)\end{array}$ & 18 & -4664 & 4990 & 4344 & 4680 & $-: 4007$ \\
\hline $\begin{array}{c}6 \\
(32)\end{array}$ & 20 & -4664 & 4900 & 4344 & 4680 & $-\because 4007$ \\
\hline $\begin{array}{c}7 \\
(36)\end{array}$ & 17 & 3641 & $683:$ & 7317 & 17788 & 10506 \\
\hline $\begin{array}{c}8 \\
(40) \\
\end{array}$ & 21 & 3641 & 6831 & 7317 & 17788 & 10506 \\
\hline $\begin{array}{c}\text { Beam } \\
(41)\end{array}$ & 19 & 81 & 7711 & 7553 & 15344 & -5183 \\
\hline
\end{tabular}


HNF-SD-SNF-FRD-003 Rev. 0

Table B7.3-6

Frame Members Stresses - Loading Longitudinal 2G (aft) (Fx at nodes 103 and 104 )

\begin{tabular}{|c|c|c|c|c|c|c|}
\hline $\begin{array}{l}\text { Jeeg } \\
\text { (Element) }\end{array}$ & $\begin{array}{l}\text { Location } \\
\text { (Node) }\end{array}$ & $\begin{array}{l}\text { Direct } \\
\text { Stress } \\
\text { (psi) }\end{array}$ & $\begin{array}{l}\text { Benling } \\
\text { Stress- } \\
y \text { - axis } \\
\text { (psi) }\end{array}$ & $\begin{array}{c}\text { Bending } \\
\text { Stress- } \\
z-\text { axis } \\
\\
\text { (psi) }\end{array}$ & $\begin{array}{c}\text { Max. } \\
\text { Combined } \\
\text { Stress } \\
\\
\text { (psi) } \\
\end{array}$ & $\begin{array}{l}\text { Min. } \\
\text { Combined } \\
\text { Strcss } \\
\\
\text { (osi) }\end{array}$ \\
\hline $\begin{array}{c}1 \\
(12)\end{array}$ & 11 & -5073 & 5605 & 5079 & 5610 & -15757 \\
\hline $\begin{array}{c}2 \\
(16) \\
\end{array}$ & 13 & -5073 & 5605 & 5079 & 5610 & -15757 \\
\hline $\begin{array}{c}3 \\
(20) \\
\end{array}$ & 10 & 4001 & 6516 & 7061 & 18479 & -10477 \\
\hline $\begin{array}{c}4 \\
(24)\end{array}$ & 14 & 4001 & $65: 6$ & 7961 & 18479 & -10477 \\
\hline $\begin{array}{c}5 \\
(28) \\
\end{array}$ & 16 & 4050 & 4615 & 5050 & 13724 & -5606 \\
\hline $\begin{array}{c}6 \\
(32) \\
\end{array}$ & 20 & 4059 & 4615 & 5050 & 13724 & -5606 \\
\hline $\begin{array}{c}7 \\
(36) \\
\end{array}$ & 17 & -3075 & 6741 & 5932 & 9598 & -15748 \\
\hline $\begin{array}{c}8 \\
(40) \\
\end{array}$ & 21 & -3075 & 6741 & 5932 & 9598 & -15748 \\
\hline $\begin{array}{c}\text { Bcam } \\
(45)\end{array}$ & 12 & 64 & 8280 & 7031 & 15375 & -15247 \\
\hline
\end{tabular}


HNF-SD-SNF-FRD-003 Rev. 0

E-15166

Table B7.3-7

Frame Nembers Stresses - Loading Lateral $1.5 \mathrm{G}$

( $F z$ at nodes 105 and 106 )

\begin{tabular}{|c|c|c|c|c|c|c|}
\hline $\begin{array}{c}\text { Leg } \\
\text { (Element) }\end{array}$ & $\begin{array}{l}\text { Location } \\
\text { (Node) }\end{array}$ & $\begin{array}{l}\text { Direct } \\
\text { Stress } \\
\text { (psi) }\end{array}$ & $\begin{array}{c}\text { Bending } \\
\text { Stress- } \\
y-\text { axis } \\
\text { (psi) }\end{array}$ & $\begin{array}{c}\text { Bending } \\
\text { Stress- } \\
z-\text { axis } \\
\text { (psi) }\end{array}$ & $\begin{array}{l}\text { Max. } \\
\text { Combined } \\
\text { Stress } \\
\text { (psi) }\end{array}$ & $\begin{array}{l}\text { Min. } \\
\text { Conbined } \\
\text { Stress } \\
\text { (psi) }\end{array}$ \\
\hline $\begin{array}{c}1 \\
(9)\end{array}$ & 1 & -3969 & 103 & 9143 & 5276 & -13216 \\
\hline $\begin{array}{c}2 \\
(16)\end{array}$ & 13 & 3837 & 185 & 9633 & 13654 & .5982 \\
\hline $\begin{array}{c}3 \\
(19) \\
\end{array}$ & 3 & 265 & 163 & 7740 & 8168 & -7639 \\
\hline $\begin{array}{c}4 \\
(21)\end{array}$ & 4 & -505 & 230 & 7862 & 7588 & -8598 \\
\hline $\begin{array}{c}5 \\
(25) \\
\end{array}$ & 5 & -3615 & 322 & 8748 & 5453 & -12685 \\
\hline 6 & 20 & 3490 & 30 & 9245 & 12765 & -5786 \\
\hline $\begin{array}{c}7 \\
(33)\end{array}$ & 7 & 100 & 234 & 8384 & 8719 & -8518 \\
\hline $\begin{array}{c}8 \\
(37) \\
\end{array}$ & 8 & -348 & 98 & 8508 & 8258 & -8954 \\
\hline $\begin{array}{l}\text { Beam } \\
(44)\end{array}$ & 11 & 828 & 10436 & 5416 & 16679 & -15025 \\
\hline
\end{tabular}


Table B7.3-8

Frame Members Stresses - Loading Up 2.0G

(Fy at nodes 101, 102,103 and 104)

\begin{tabular}{|c|c|c|c|c|c|c|}
\hline $\begin{array}{c}\operatorname{Leg} \\
\text { (Element })\end{array}$ & $\begin{array}{l}\text { Location } \\
\text { (Nodic) }\end{array}$ & $\begin{array}{l}\text { Direct } \\
\text { Stress } \\
\text { (psi) }\end{array}$ & $\begin{array}{c}\text { Bending } \\
\text { Stress- } \\
y-\text { axis } \\
\text { (psi) }\end{array}$ & $\begin{array}{c}\text { Bending } \\
\text { Stress- } \\
z-a x i s \\
\text { (psi) }\end{array}$ & $\begin{array}{l}\text { Max. } \\
\text { Combincd } \\
\text { Stress } \\
\text { (psi) }\end{array}$ & $\begin{array}{l}\text { Min. } \\
\text { Combined } \\
\text { Stress } \\
\text { (psi) }\end{array}$ \\
\hline $\begin{array}{c}1 \\
(12)\end{array}$ & 11 & 1694 & 89 & 1787 & 3570 & -182 \\
\hline $\begin{array}{c}2 \\
(16) \\
\end{array}$ & 13 & 1694 & 89 & 1787 & 3570 & -182 \\
\hline $\begin{array}{c}3 \\
(20) \\
\end{array}$ & 10 & 855 & 58 & 2284 & 3197 & -1486 \\
\hline $\begin{array}{c}4 \\
(24) \\
\end{array}$ & 14 & 855 & 58 & 2284 & 3197 & -1486 \\
\hline $\begin{array}{c}5 \\
(28) \\
\end{array}$ & 18 & 1765 & 52 & 1633 & 3449 & 80 \\
\hline $\begin{array}{c}6 \\
(32) \\
\end{array}$ & 20 & 1765 & 52 & 1633 & 3449 & 80 \\
\hline $\begin{array}{c}7 \\
(36) \\
\end{array}$ & 17 & 797 & 100 & 2164 & 3061 & -1467 \\
\hline $\begin{array}{c}8 \\
(40) \\
\end{array}$ & 21 & 797 & 100 & 2164 & 3061 & -1467 \\
\hline $\begin{array}{l}\text { Beam } \\
(46)\end{array}$ & 12 & 328 & 3103 & 1386 & 4828 & -4172 \\
\hline
\end{tabular}




\section{HNF-SD-SNF-FRD-003 Rev. 0}

b) Cask Tic down Device

The cask tic down device consists of clamps. hinge pins, bolts and pad for hold down brackets. The clamp was analyzed by using ANSYS Finite Element Computer Code. Tho 3-dimensional bean finite clement (BENM.4) was used in the construction of the model. The leading and boundary conditions for the $2 \mathrm{G}$ longitudinal, $1.5 \mathrm{G}$ lateral and $2 \mathrm{G}$ up are presented in Figures B7.3-9, B7.3-10 and B7.3-7 i, respectively. The critical stresses are presented in Table

37.3-10. The stresses in hinge pin. bolts and pad were hand-calculated. These stresses are also summarized in Pabie B7.3-10. 
Figure B7.3-9

Clamp Model - Loading \& Boundary Conditions for Longitudinal $2 G$ Load Analysis

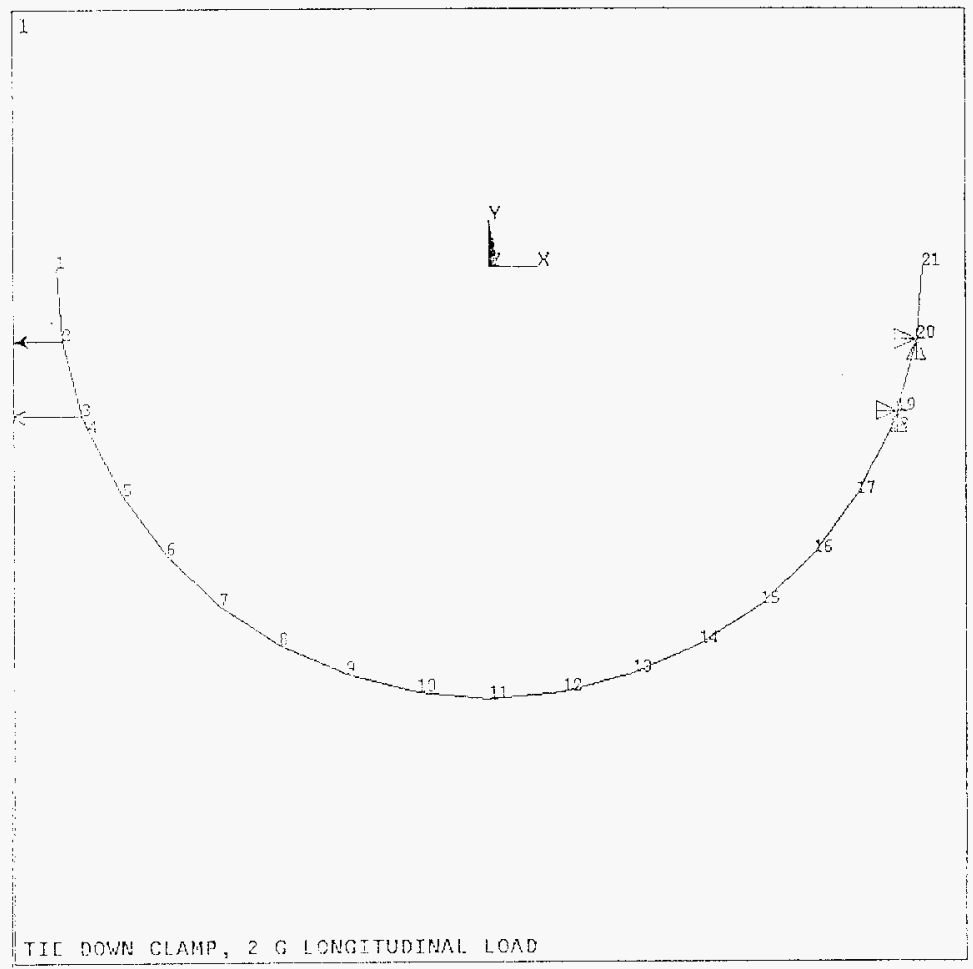


Figure B7.3-10

Clamp Model - Loading \& Boundary Conditions for Lateral 1.5G Load Analysis

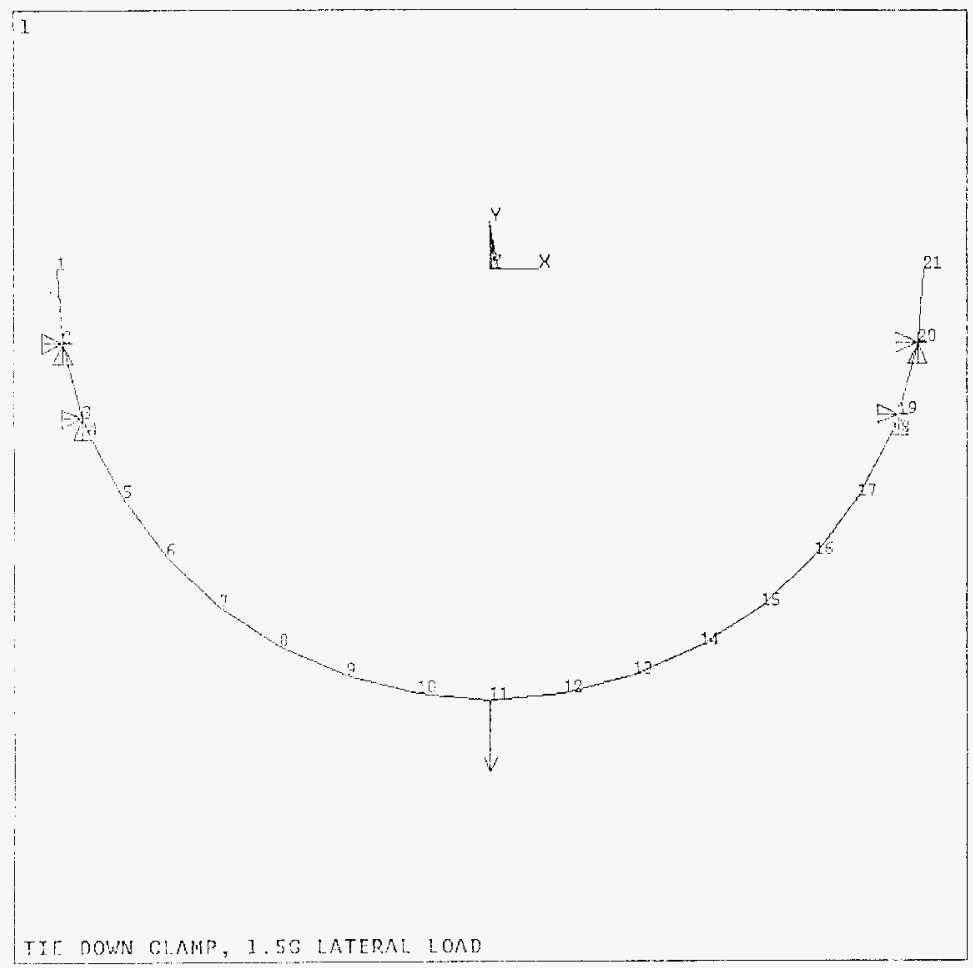


Figure B7.3-11

Clamp Model - Loading \& Boundary Conditions for Up 2.0G Load Analysis

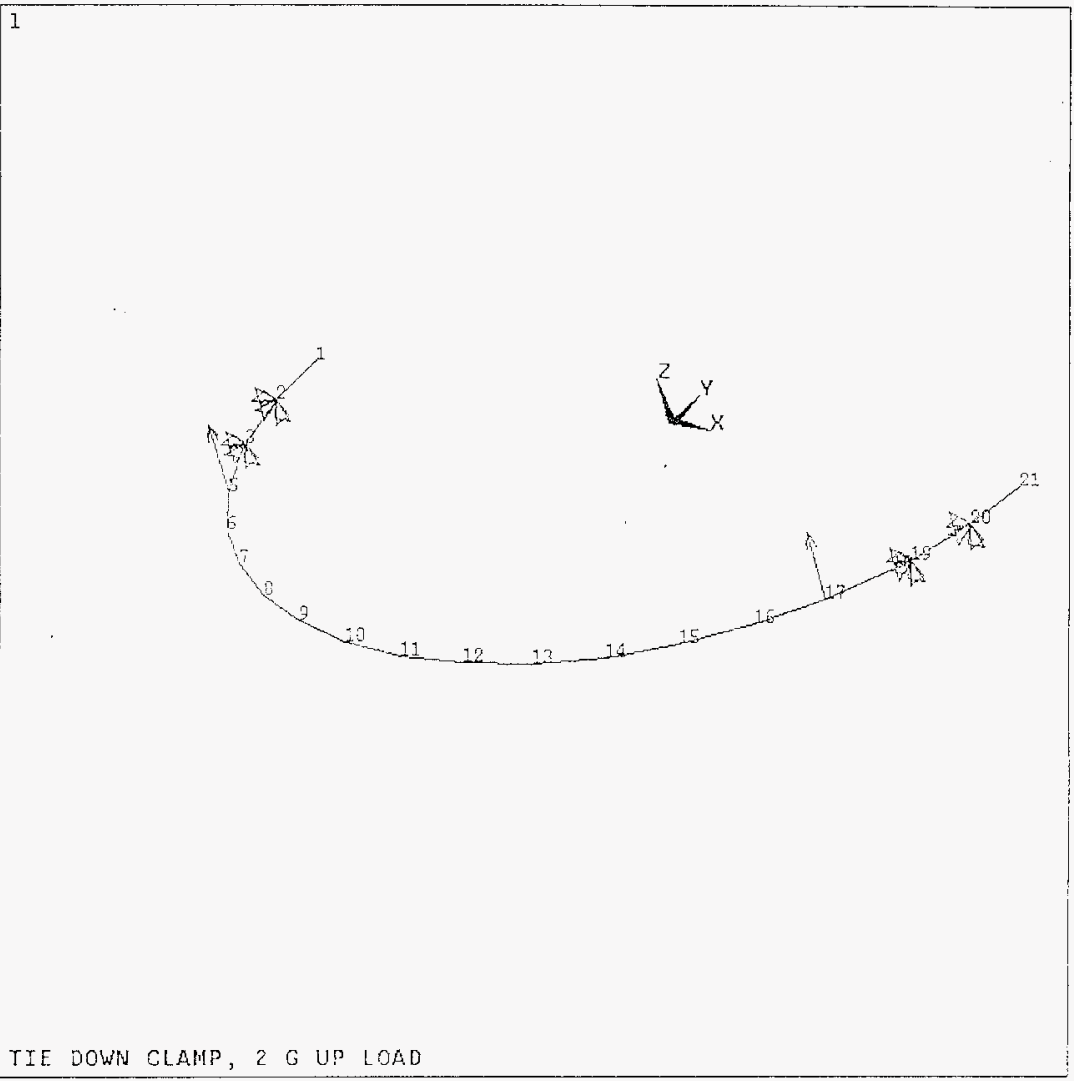


c) Cask Hold down Device

The cask hold down device consists of hold down arms, brackets and bolts. The analysis was conducted using hand calculations. The only load that is applicabie for these components is $2 \mathrm{G}$ upward. The resulting stresses are summarized in Table B7.3-10.

d) Cask Support Device

The cask support device consists of support plate, cup and bolts. The analysis was condreted using ANSYS Finite Element Computer Code. The two end of plate are bolted to the bottom plate which is welded to the support beams, the support beans are welded to the trailer main beams. The plates was modeled using 3-dimensional shell elements. The supporting beams were modeled with 3-dimentional beam elements. The cup was not modeled. The $3 \mathrm{G}$ downward load was applied to the cup area us a distributed pressure. The maximun stress intensity in the plate is 18,429 psi. The bolt stresses were hand calculated for a combined loading due to 20 longitudinal and 1.50 lateral accelerations. The maximum shear stross in the bolts is 24,560 psi. All the plate and bot stresses are within the allowabs, as shown in Tabie B7.3-10.

\section{B7.3-6 DESIGN CRITERIA FOR TIEDOWN SYSTEM}

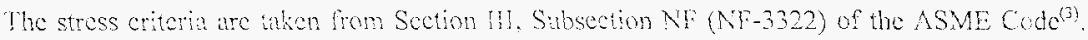
There are several types of structual criteria discussed in Subsection NF-3322. These criteria, are to cnsure stability under compessive loading, to ensure stability under bending and to prevent falure under combind loaling. Tuble B7.3-9 lists the stress limits of the materials based on this criterial. 
HNF-SD-SNF-FRD-003 Rev. 0

F-15166

Table $87.3-9$

Stress Criteria for the Tiedown System

\begin{tabular}{|c|c|c|c|c|c|c|}
\hline \multirow[t]{2}{*}{ Material } & \multirow[t]{2}{*}{$\begin{array}{c}S_{\vdots} \\
\text { (ksi) }\end{array}$} & Tension & Bending & Shear & Compression & $\begin{array}{l}\text { Interaction } \\
\text { Equation }\end{array}$ \\
\hline & & $0.65^{*}$ & $0.66 \mathrm{~S}_{\mathrm{y}}$ & $0.4 \mathrm{~S}_{y}$ & $\begin{array}{l}\mathrm{NP}-3322.1 \\
\mathrm{EQ} .4 \text { or } 5\end{array}$ & $\begin{array}{l}N F-3322.1 \\
E Q .20 \text { or } 21\end{array}$ \\
\hline$\triangle 500$ Gr. B & 44 & 26.4 & 29.04 & 17.6 & & \\
\hline A.514 Gr. B & 97.7 & 58.62 & 64.48 & 39.08 & & \\
\hline $6061-16$ & 39 & 23.4 & 25.74 & 15.6 & & \\
\hline $6063-\mathrm{T} 6$ & 29.4 & 17.64 & 19.4 & 11.76 & & \\
\hline A193-B7 & $10:$ & 60.6 & 66.66 & 40.4 & & \\
\hline $\begin{array}{l}\text { A479-XM19 } \\
\text { Hot Rolled }\end{array}$ & 101 & 606 & 66.66 & 40.4 & & \\
\hline
\end{tabular}

* For pin-connecied members, using $0.45 \mathrm{~S}$, 


\section{B7.3-7 SUMMARY AND CONGLUSIONS}

A summary of critical stresses is presented in Table B7.3-10 and stresses are compared with the allowables. Nil stresses are within the allowables. The interaction between compression and bending are evaluated using Equations 20 and 21 of NF-3322, and interaction between tension and bending are using Equation 21 of NF-3322. Table B7.3-11 shows the results of the caleulations. Based on the results of the analyses, it is concluded that tiedown system is structurally safe for the specified loads. 
HNF-SD-SNF-FRD-003 Rev. 0

E. -15166

Table 87.3-10

Summary - Stress Evaluation

\begin{tabular}{|c|c|c|}
\hline Component & $\begin{array}{l}\text { Max Calculated Stress } \\
\text { (psi) }\end{array}$ & $\begin{array}{l}\text { Allowabie } \\
\text { Stress } \\
\text { (psi) }\end{array}$ \\
\hline Frame- Taegs & $\begin{array}{l}\text { Max. Direct }=5,073 \text { (Compression) } \\
\text { Max. Bending }-14,477\end{array}$ & $\begin{array}{l}26,400 \\
29,040\end{array}$ \\
\hline Cross Bcam & $\begin{array}{l}\text { Max. Direct }=828(\text { Tension }) \\
\text { Max. Bending }=15,852\end{array}$ & $\begin{array}{l}26,400 \\
29,040\end{array}$ \\
\hline Welds at Bottom Leg & $\begin{array}{l}\text { Max. Tension }-19,224 \\
\text { Max. Shear }=5,707\end{array}$ & $\begin{array}{l}26,400 \\
17,600\end{array}$ \\
\hline Tledown device-Clamp & $\begin{array}{l}\text { Max. Direct }=2,446 \text { (Tension) } \\
\text { Max. Bending }=20,513\end{array}$ & $\begin{array}{l}23,400 \\
25,740\end{array}$ \\
\hline Bolt & $\begin{array}{l}\text { Max. Shcar }=23,460 \\
\text { Max. Tension }=36,700\end{array}$ & $\begin{array}{l}40,400 \\
60,600\end{array}$ \\
\hline $\begin{array}{l}\text { Cask Hold Down Derice } \\
\text {-Arm }\end{array}$ & $\begin{array}{l}\text { Max. Tension }=11,430 \\
\text { Max. Pending }=43,200 \\
\text { Max. Shear }=11,933\end{array}$ & $\begin{array}{l}43,960 \\
64,480 \\
39,080\end{array}$ \\
\hline -pin-1.5"Dia. & $\begin{array}{l}\text { Max. Bending }-18.420 \\
\text { Max. Shaar }=8.490\end{array}$ & $\begin{array}{l}66,660 \\
40,400\end{array}$ \\
\hline -Bracket & $\begin{array}{l}\text { Bcaring }=16,000 \\
\text { Tensic }=6000 \\
\text { Shcar }=4.570 \\
\end{array}$ & $\begin{array}{l}39,000 \\
17,550 \\
15,500\end{array}$ \\
\hline $\begin{array}{l}\text { Cask Support Device } \\
\text {-Plate } \\
\text { - Bolt }\end{array}$ & $\begin{array}{l}\text { Max. Bending }=18,429 \\
\text { Max. Shear }=34,560\end{array}$ & $\begin{array}{l}19,400 \\
40,400\end{array}$ \\
\hline
\end{tabular}


HNF-SD-SNF-FRD-003 Rev. 0

E-15166

Table 87.3-11

interaction Equation Evaluations

\begin{tabular}{|l|l|l|}
\hline Component & $\begin{array}{l}\text { Max. Axial Compression and Bending } \\
(\mathrm{NF}-3322.1, \text { Equation 20 \& 21) }\end{array}$ & $\begin{array}{l}\text { Max. Axial Tension and Bending } \\
(\mathrm{NF}-3322.1, \text { Equation 21) }\end{array}$ \\
\hline Frame Leg & 0.522 & 0.65 \\
\hline Tiedown Clamp & & 0.902 \\
\hline Hold Down Arm & & 0.807 \\
\hline
\end{tabular}




\section{HNF-SD-SNF-FRD-003 Rev. 0}

B7.3-7 REFERENCES

1. WHC-S-0396. Revision !, "Specincation For TN-WHC Cask and Transportation System" 1995.

2. ANSYS User's Manual, Revision 5.2. Volumes 1 to 4.

3. ASME Boiler and Pressume Wessel Code 1992, Section III. Subsection NF. 


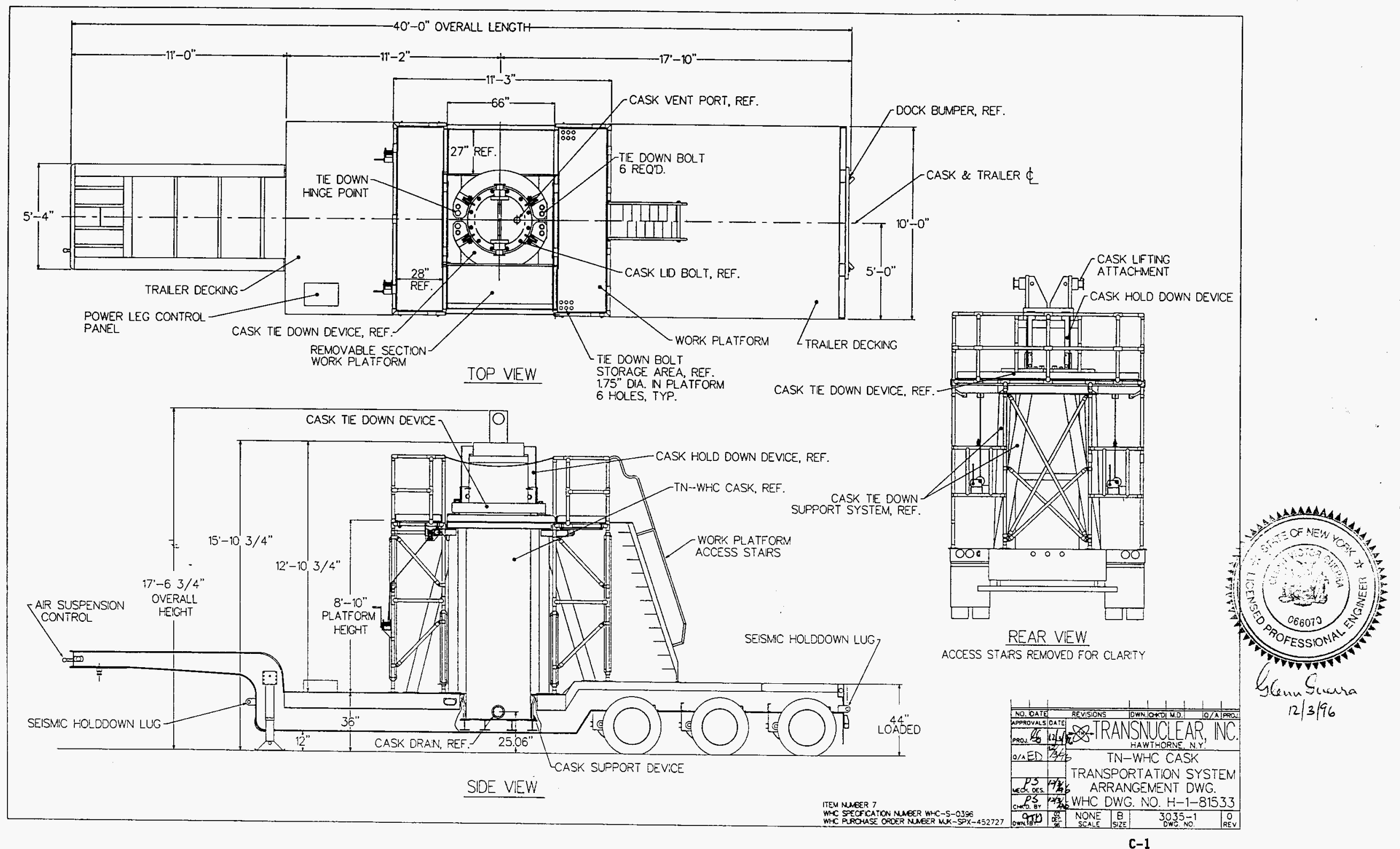




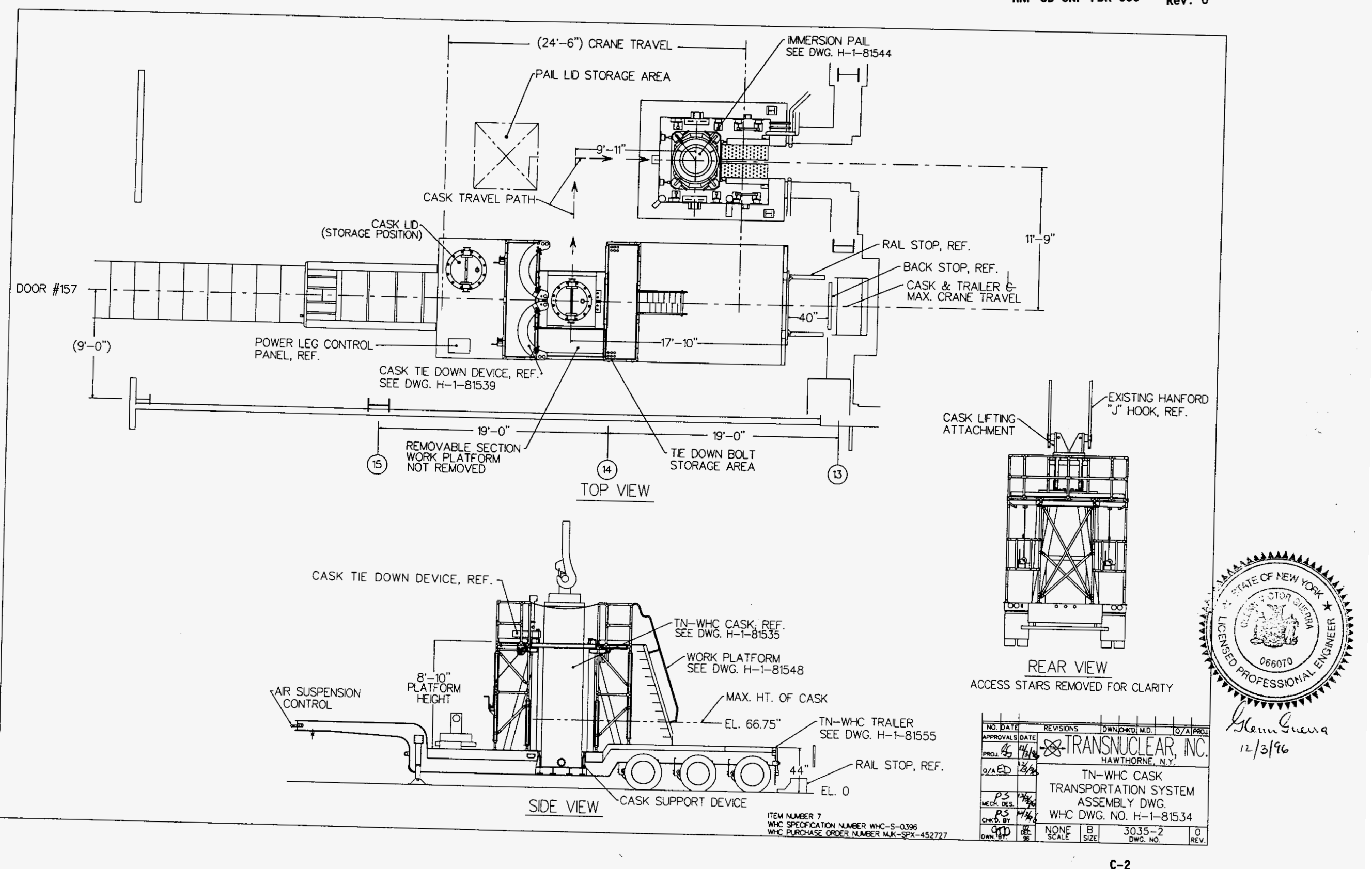




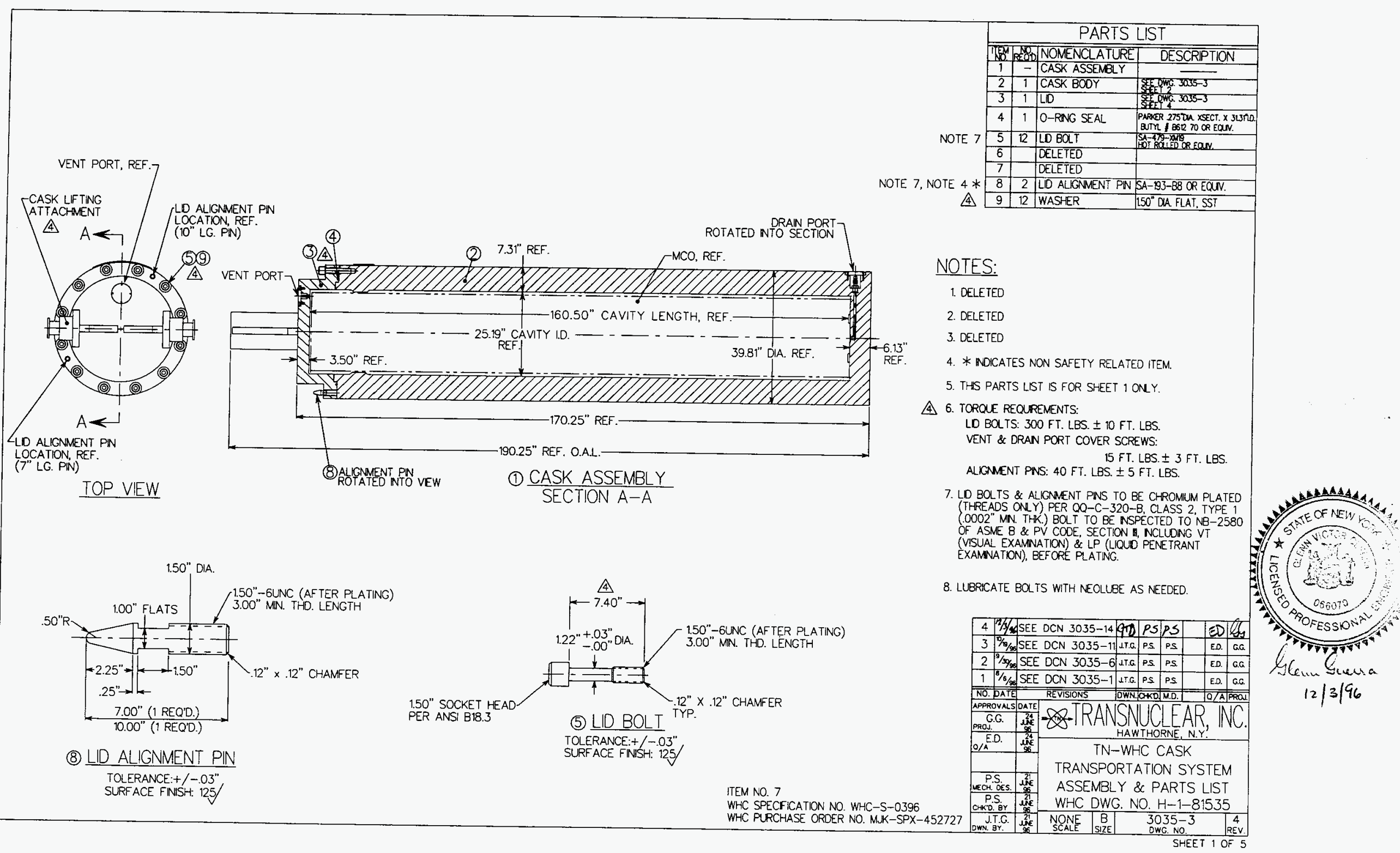




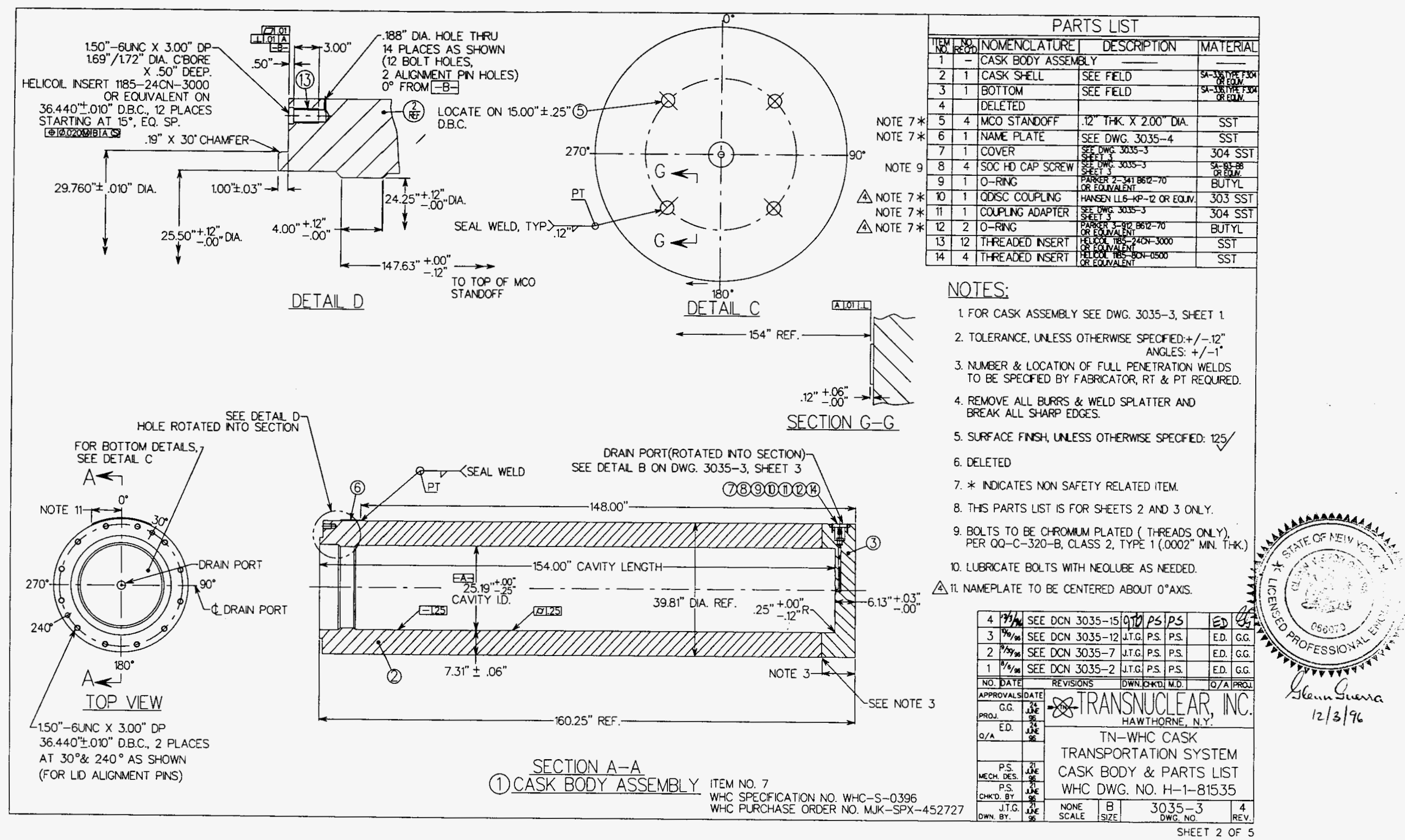



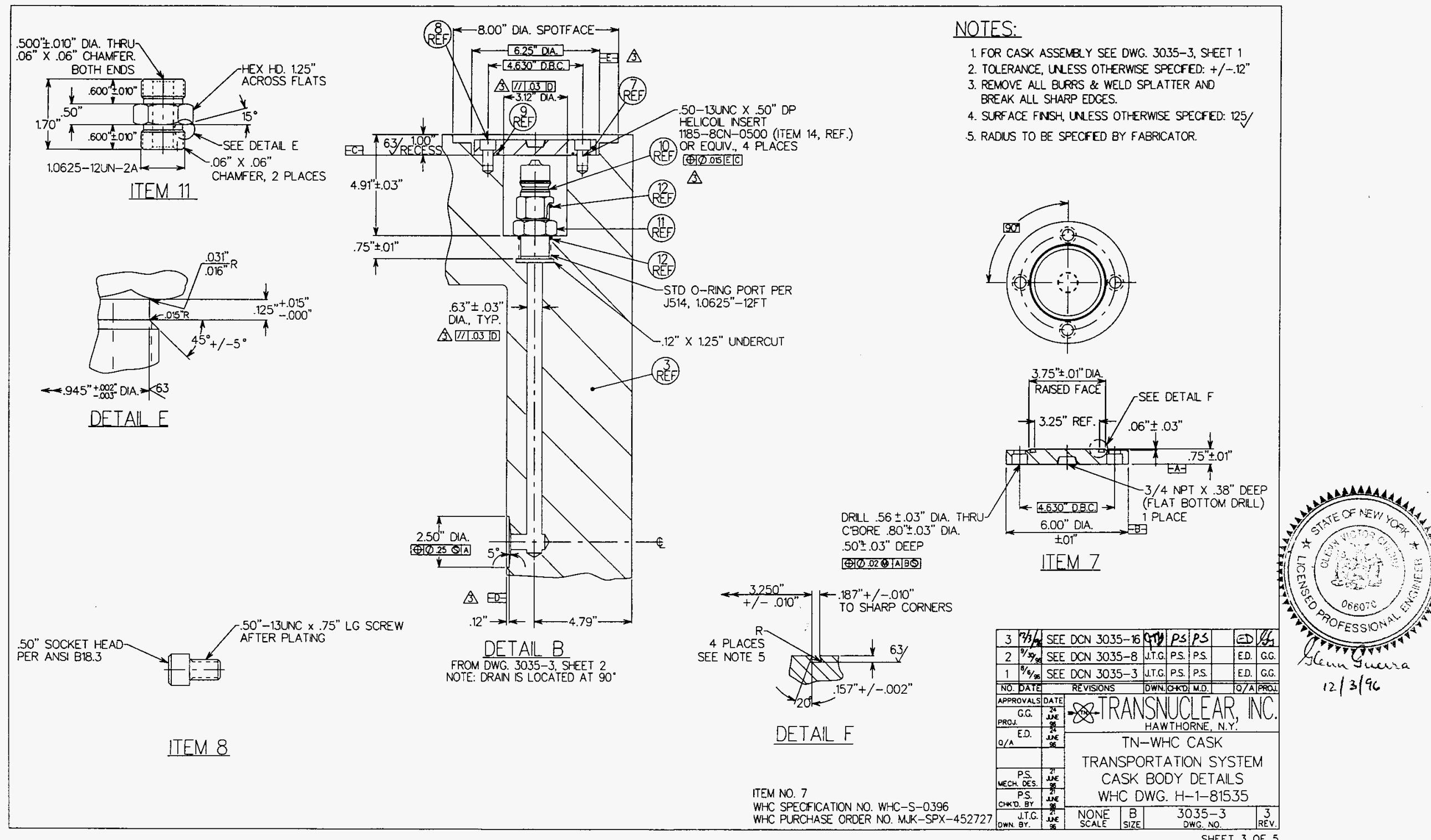


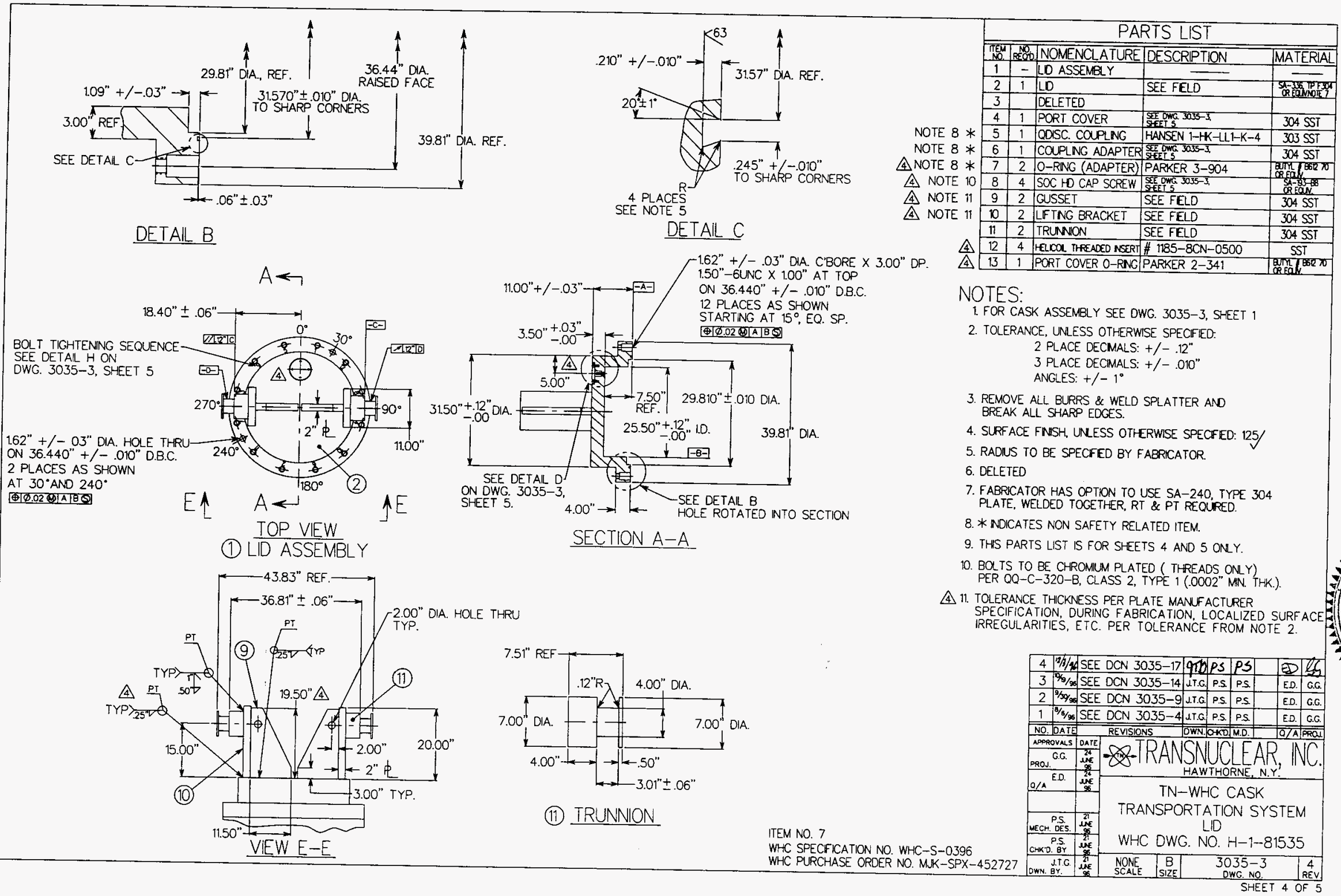




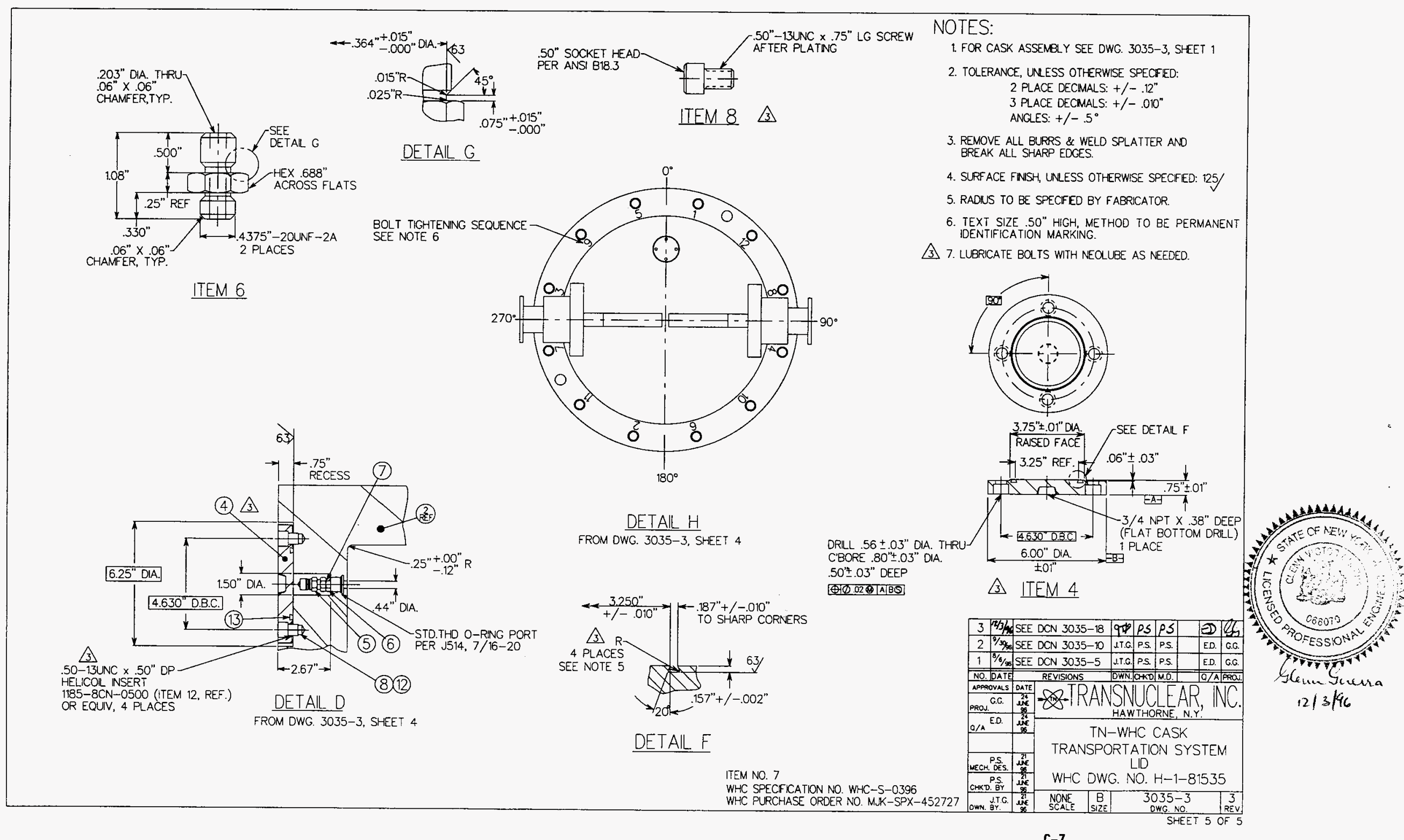




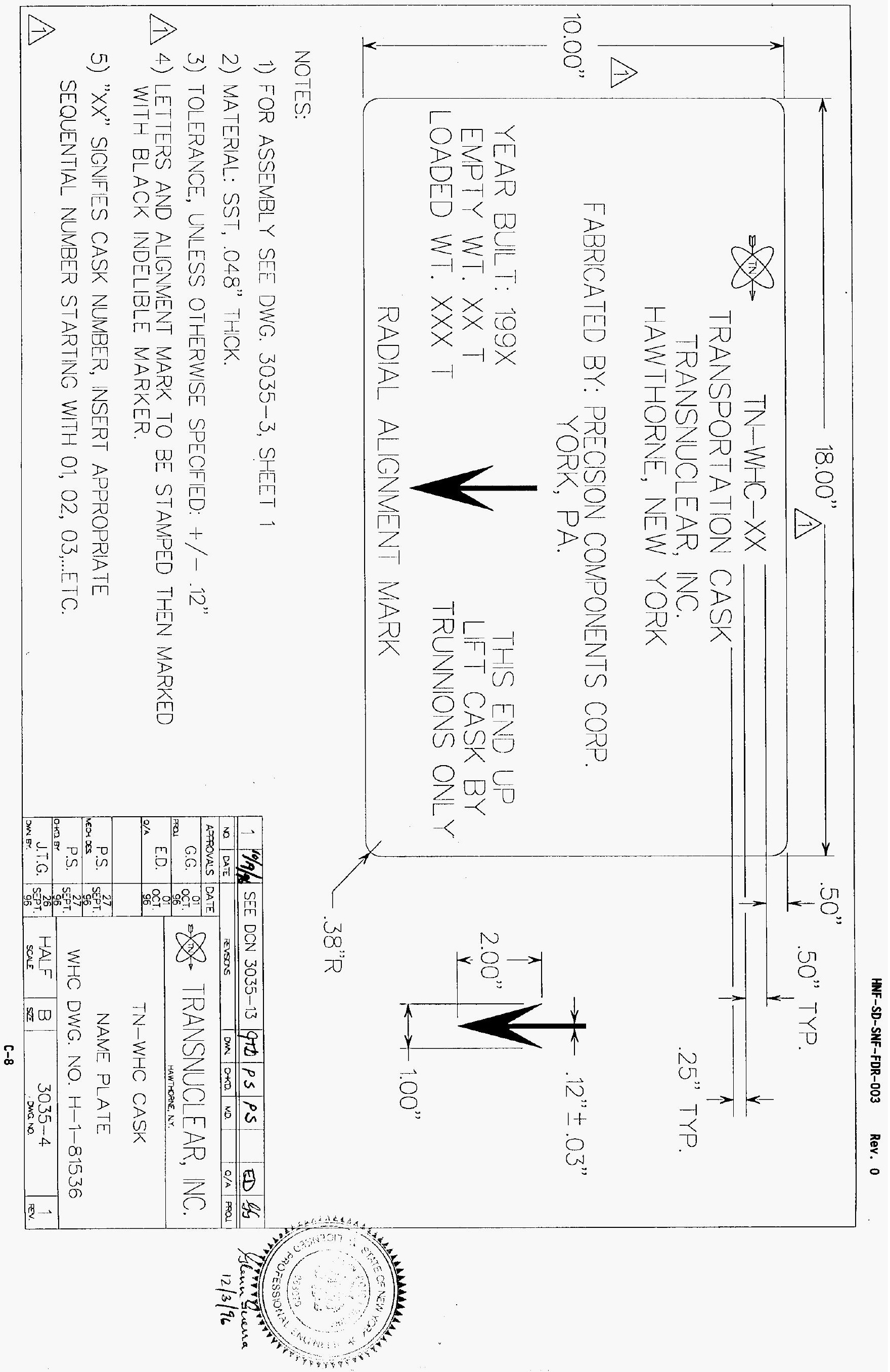




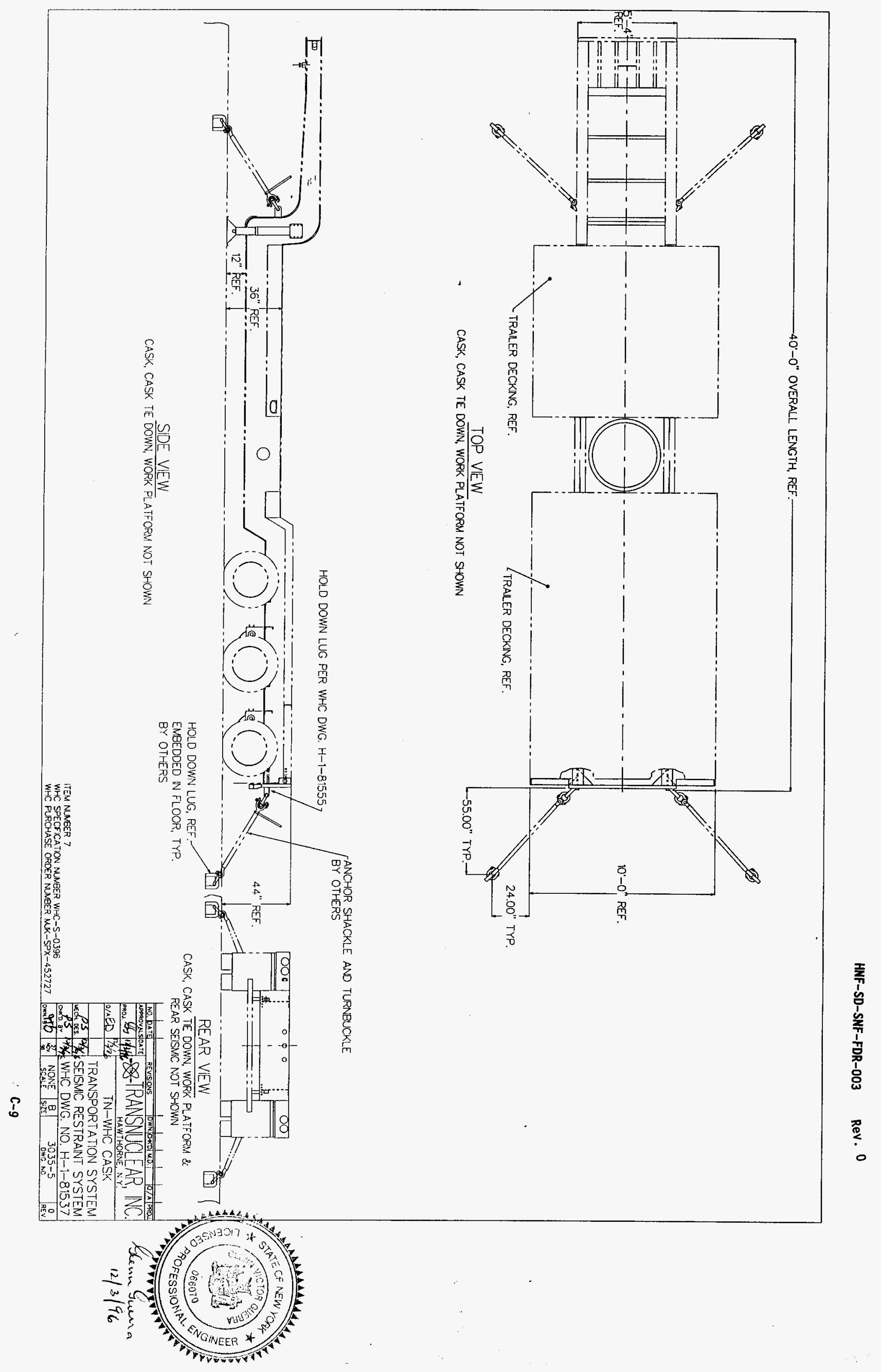



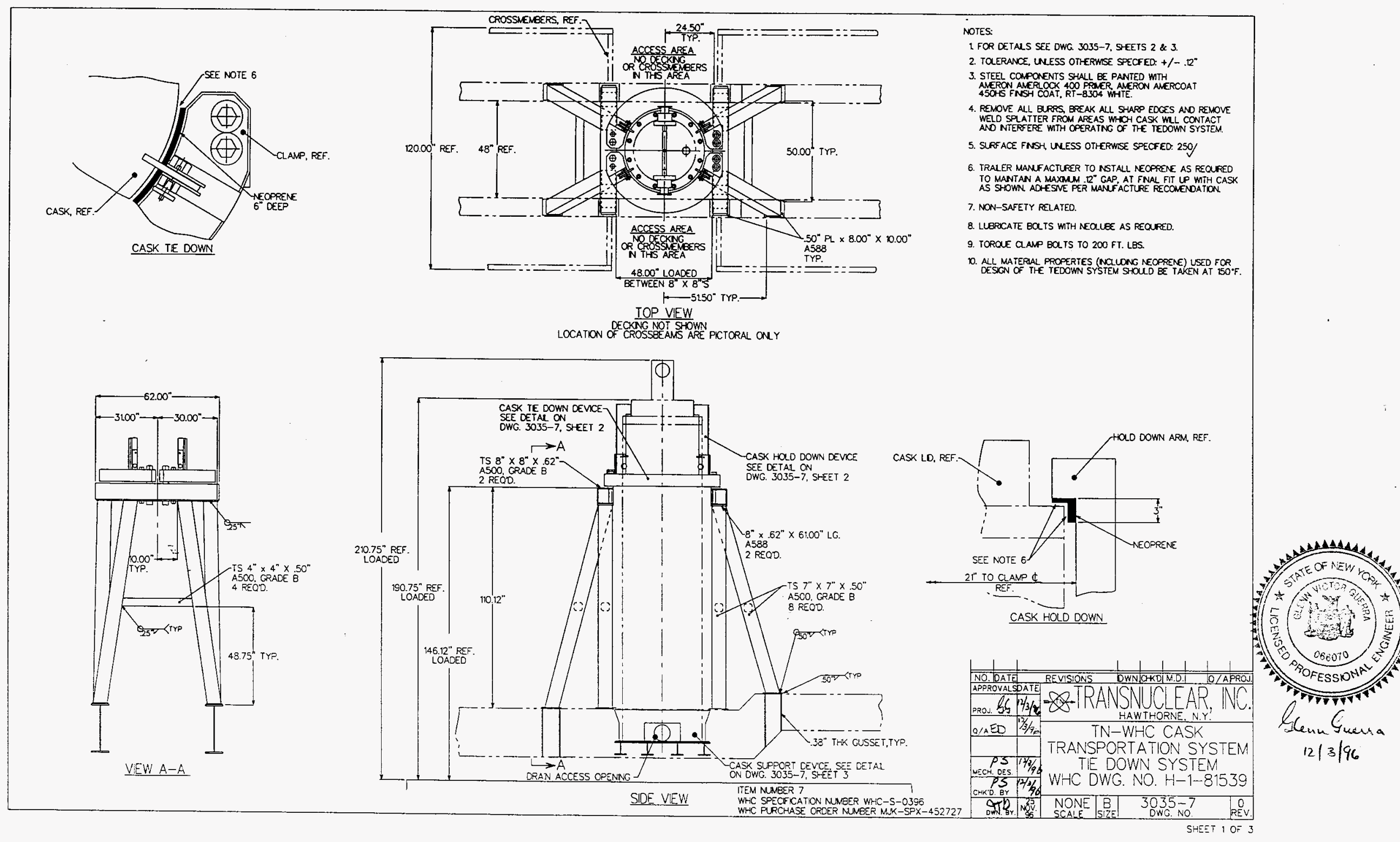


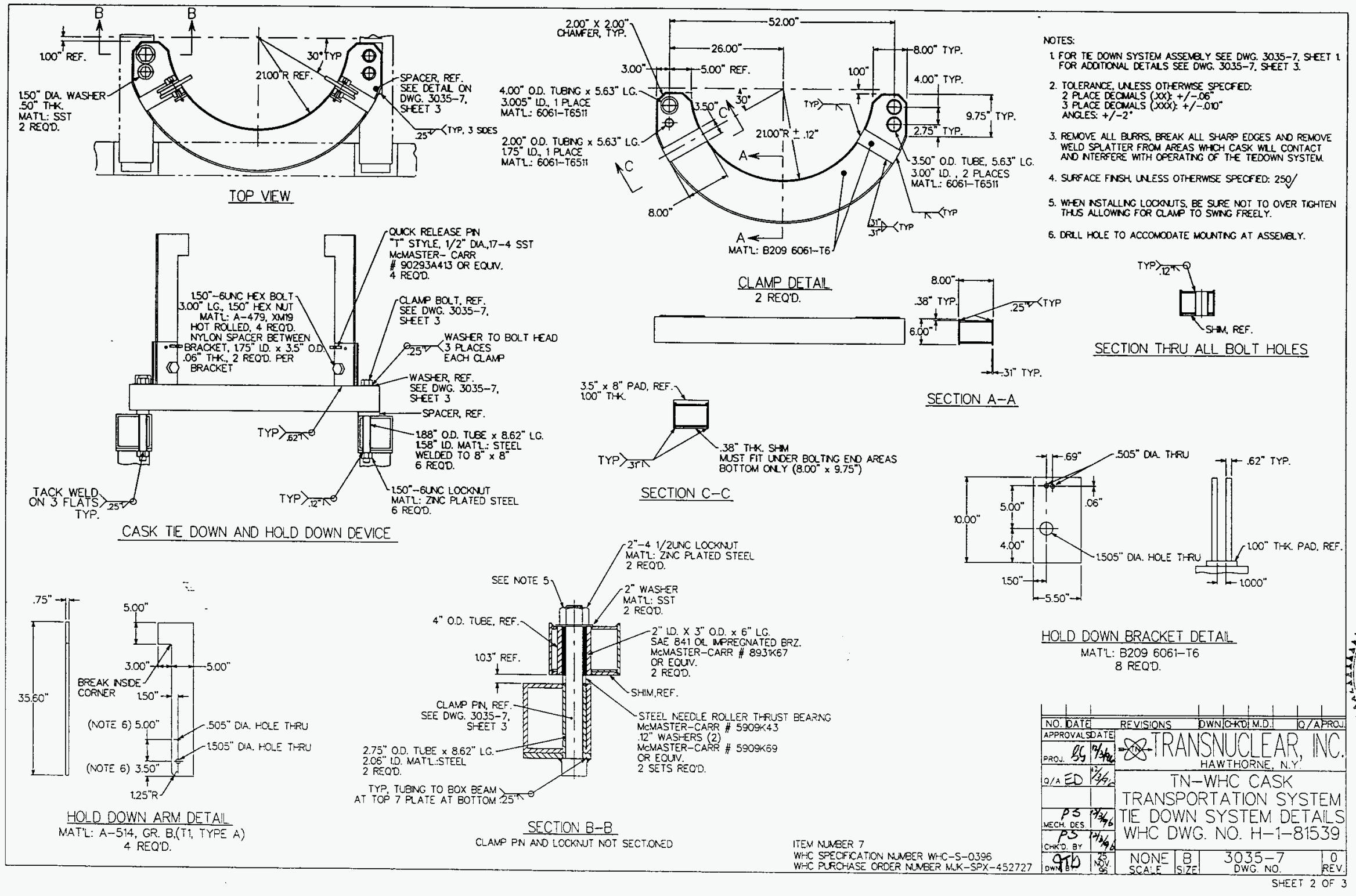


HNF-SD-SNF-FDR-003 Rev. 0
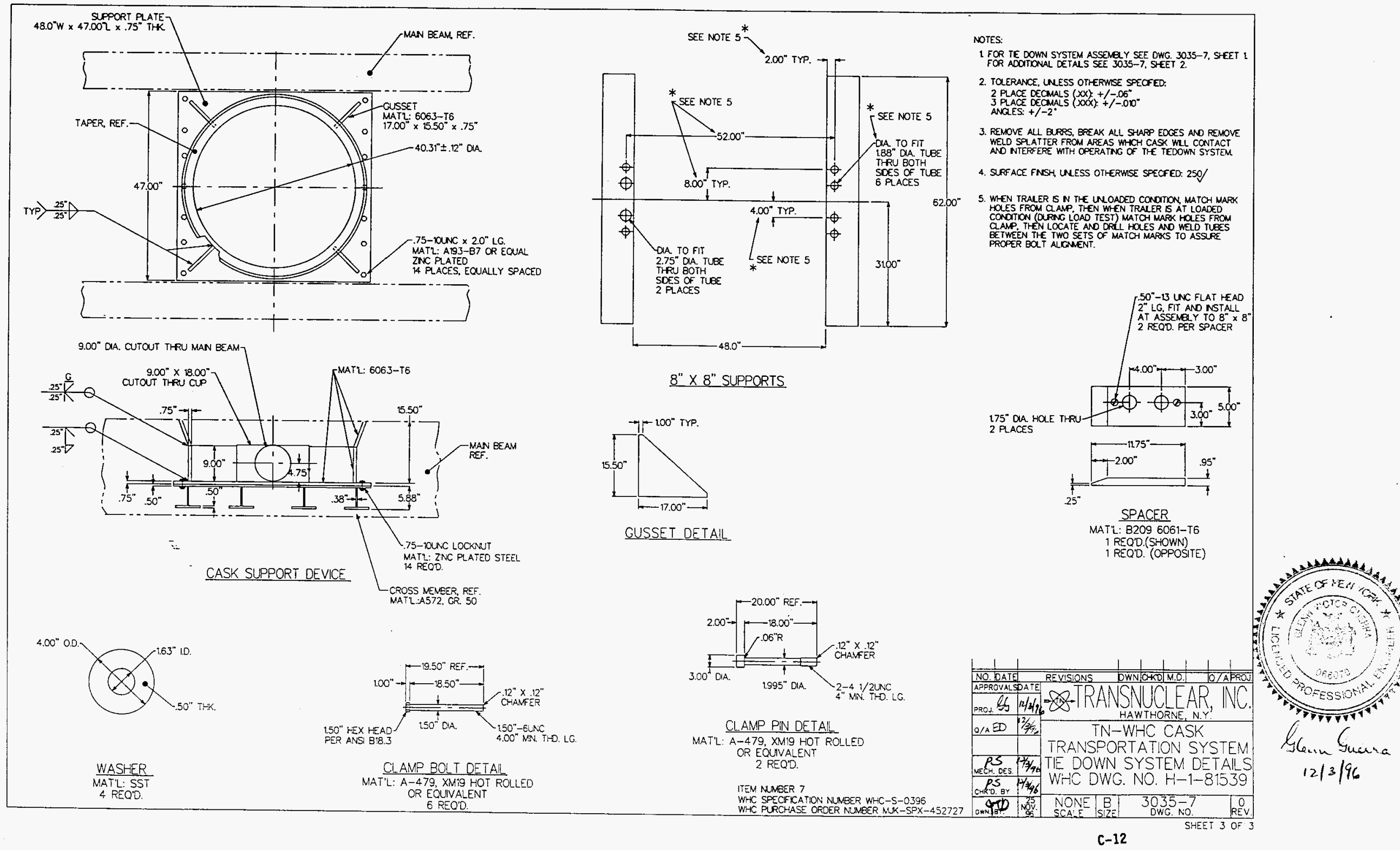


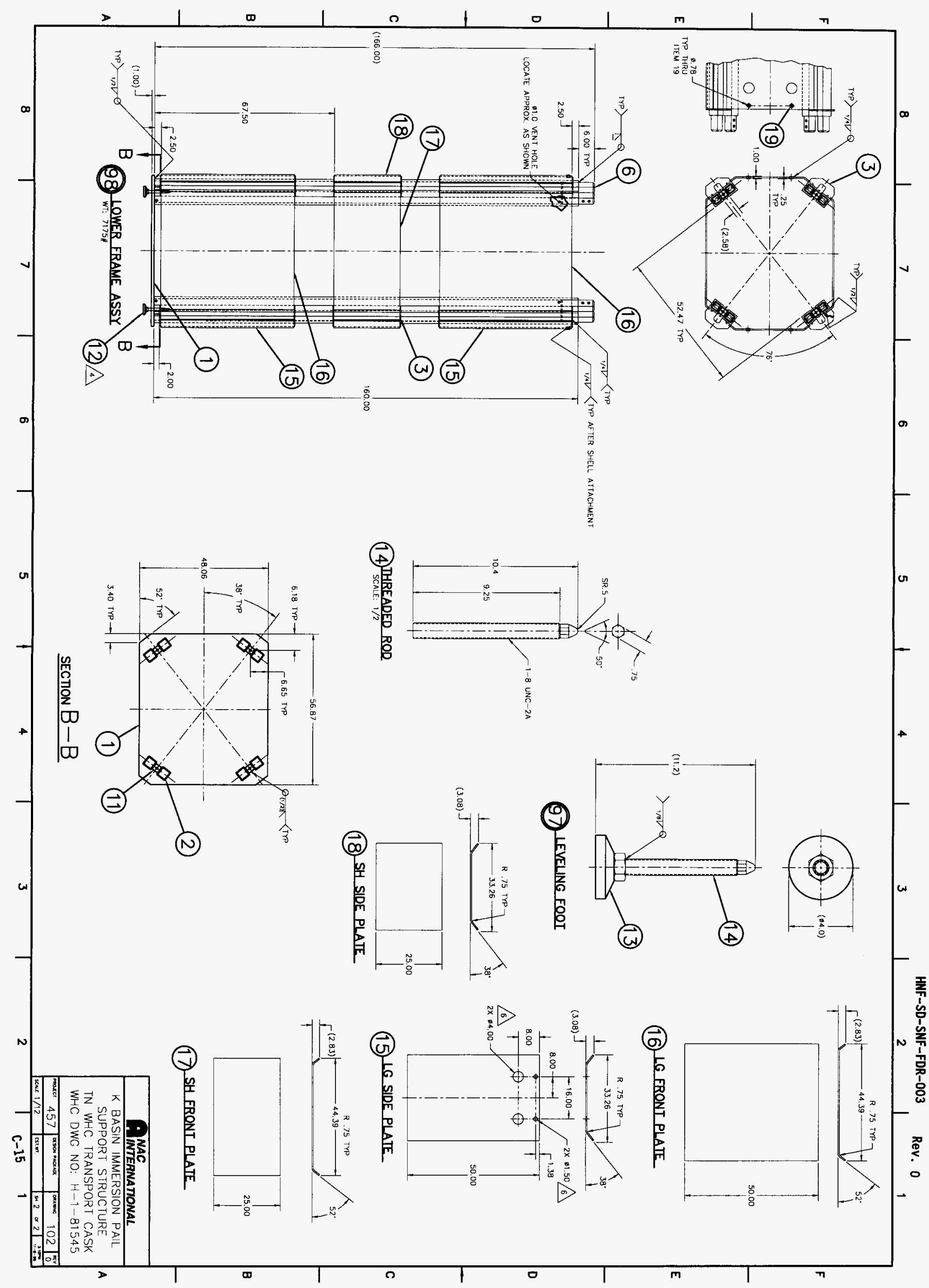




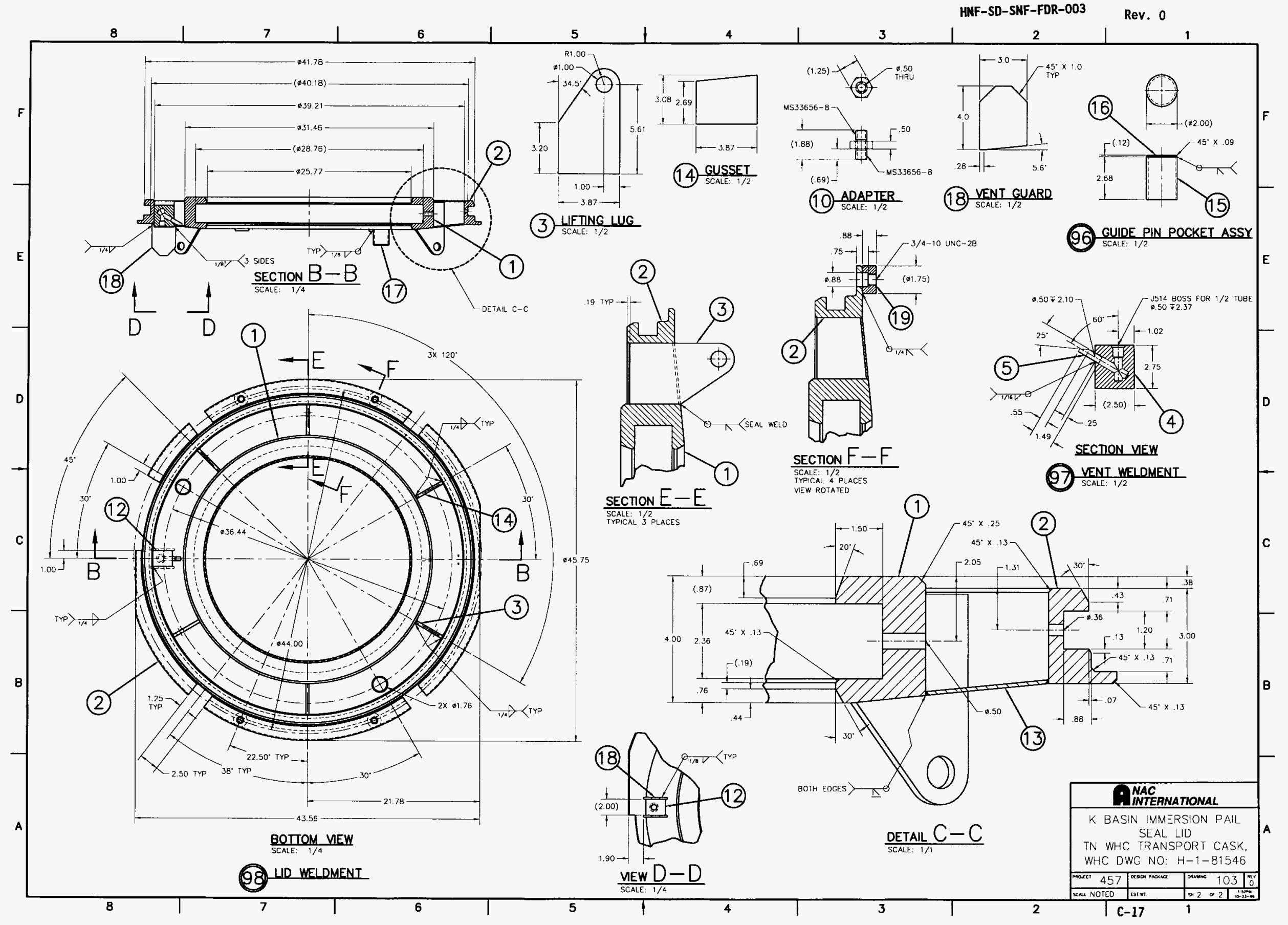




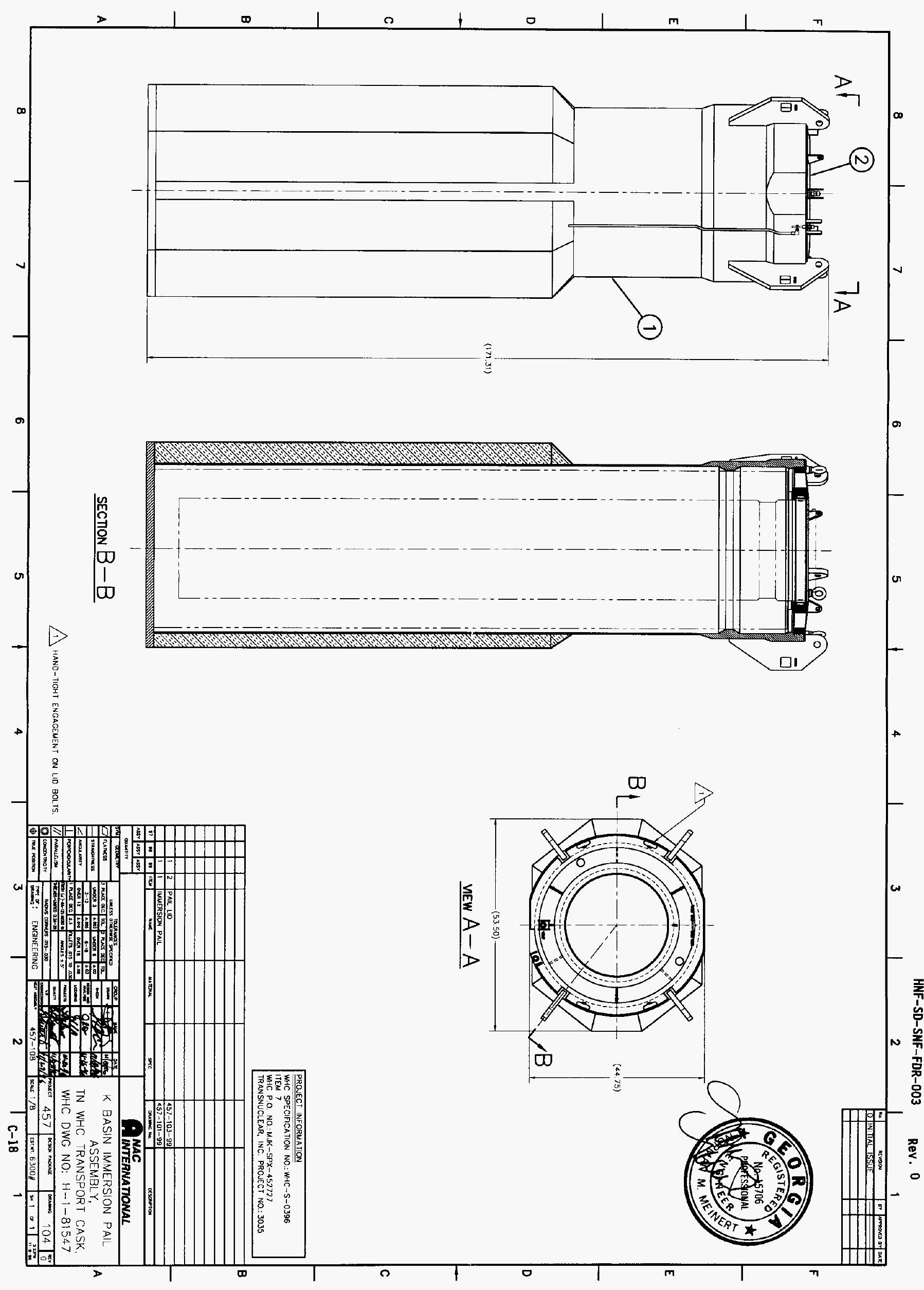




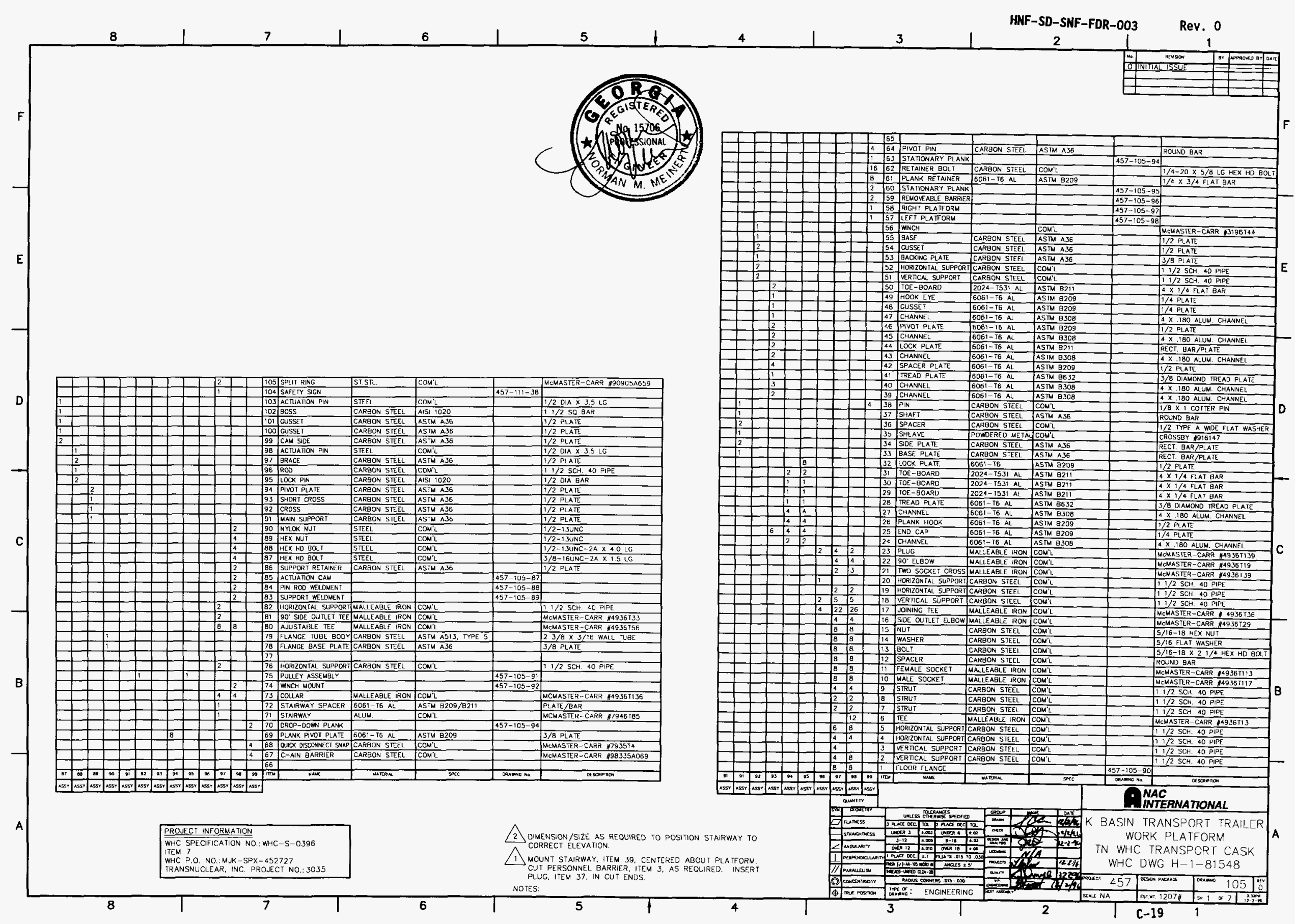




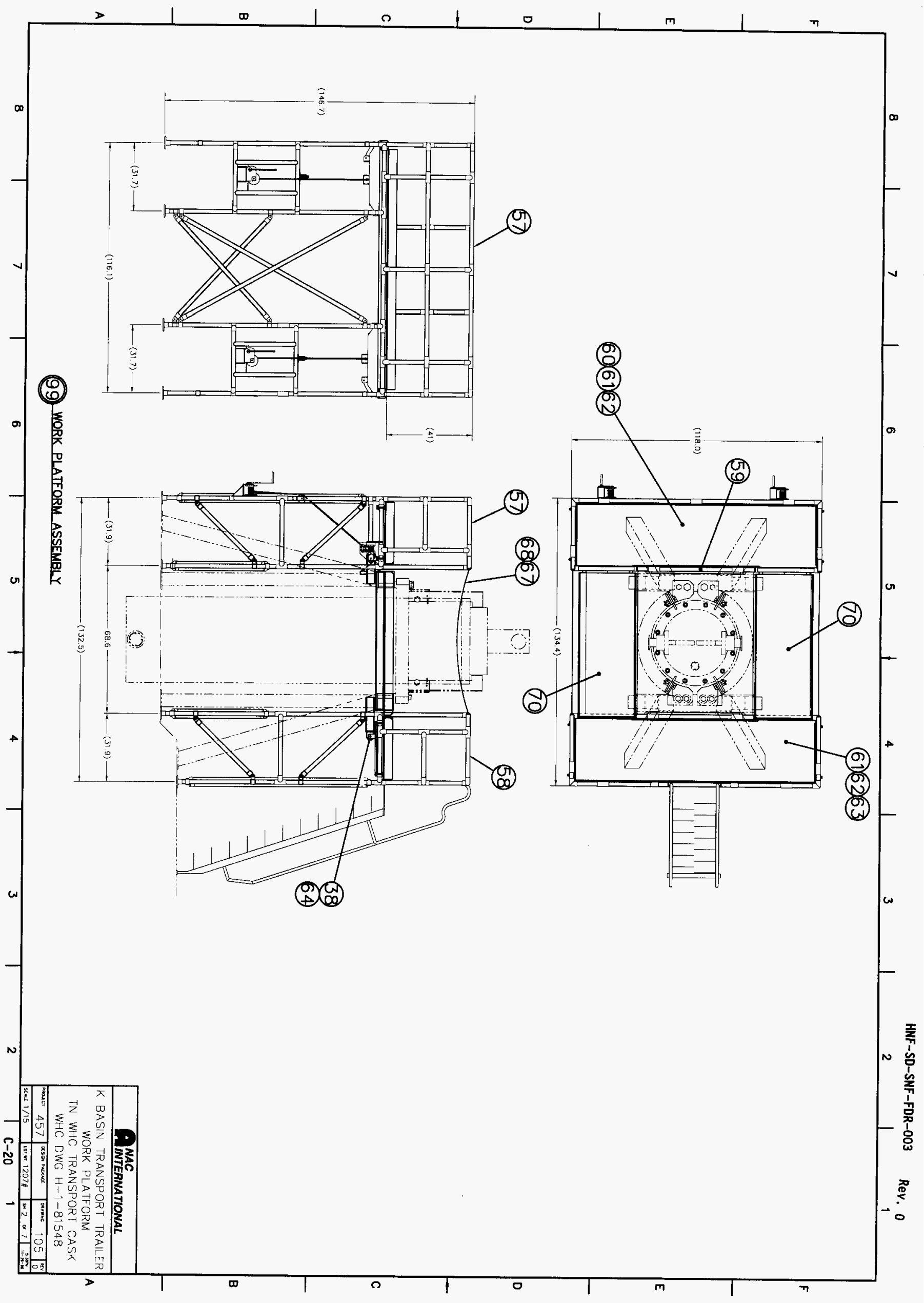




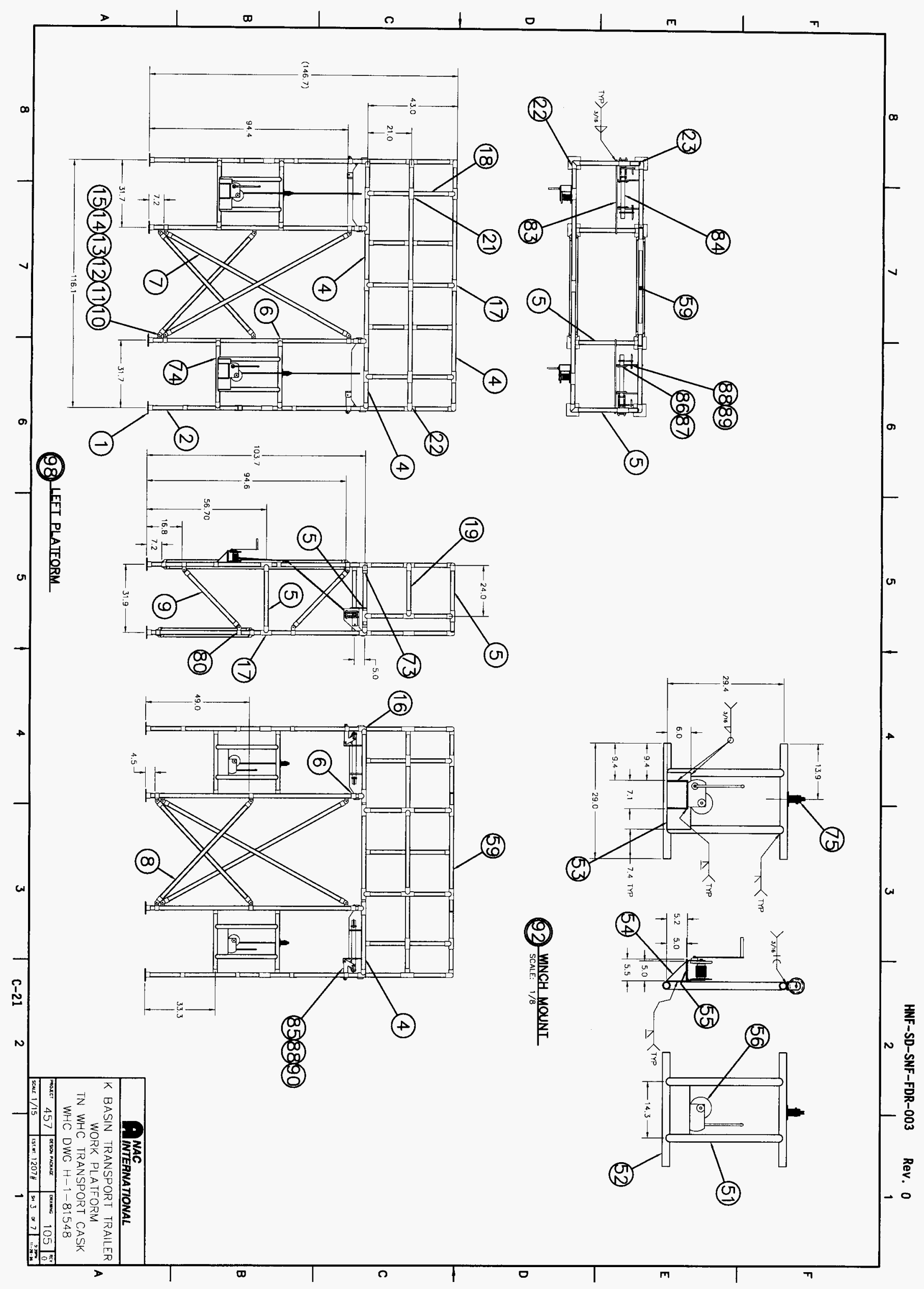




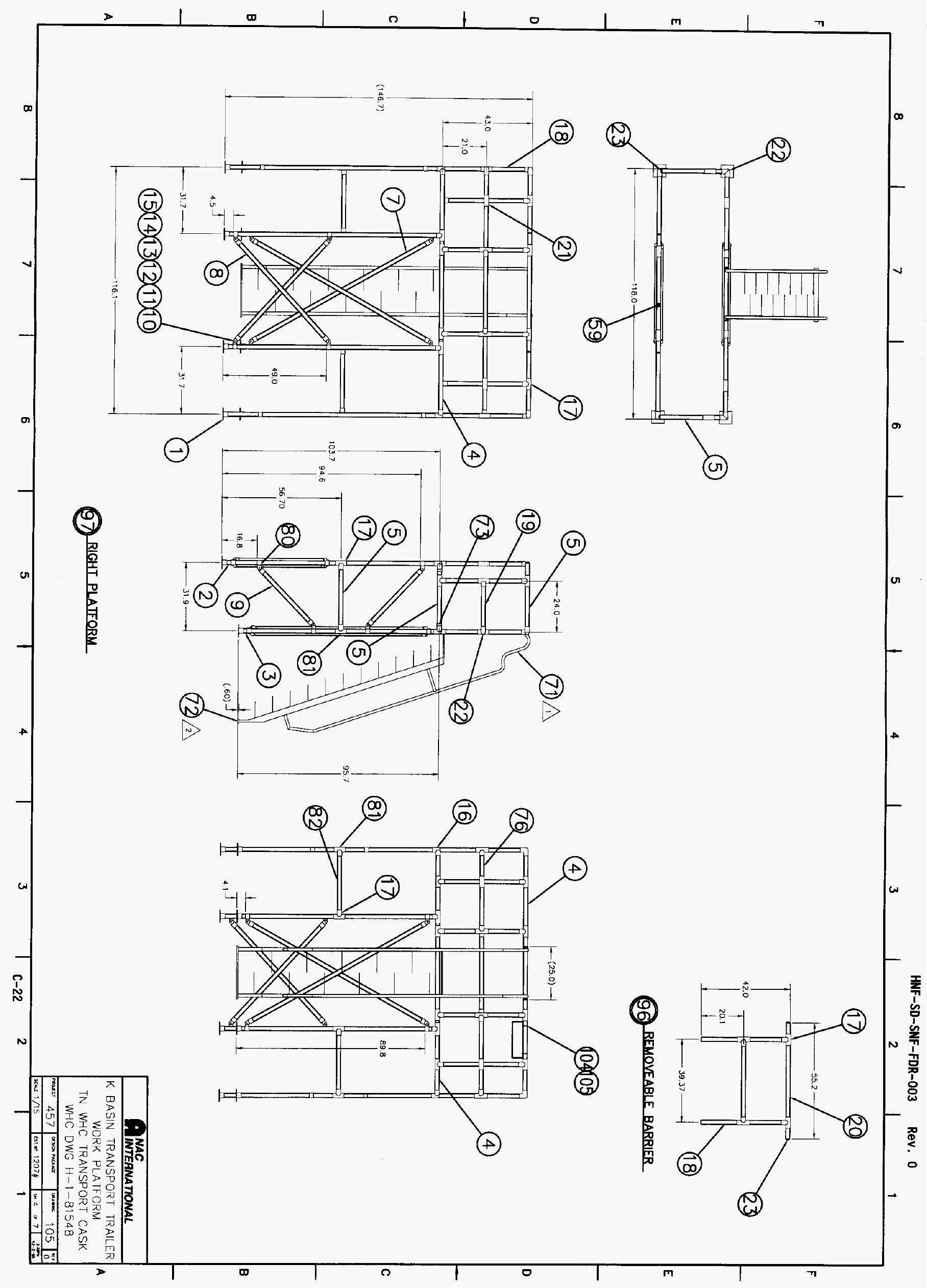




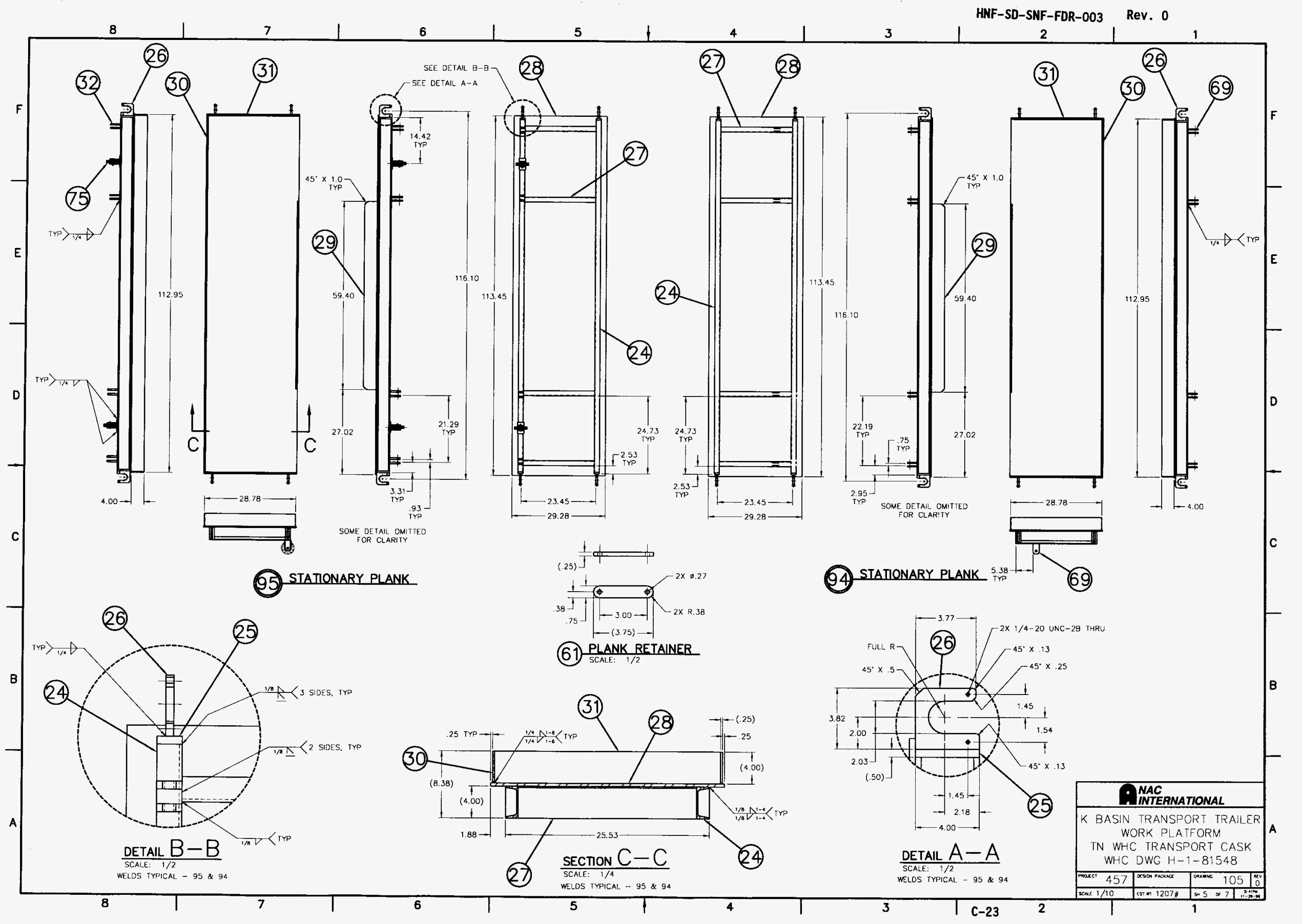




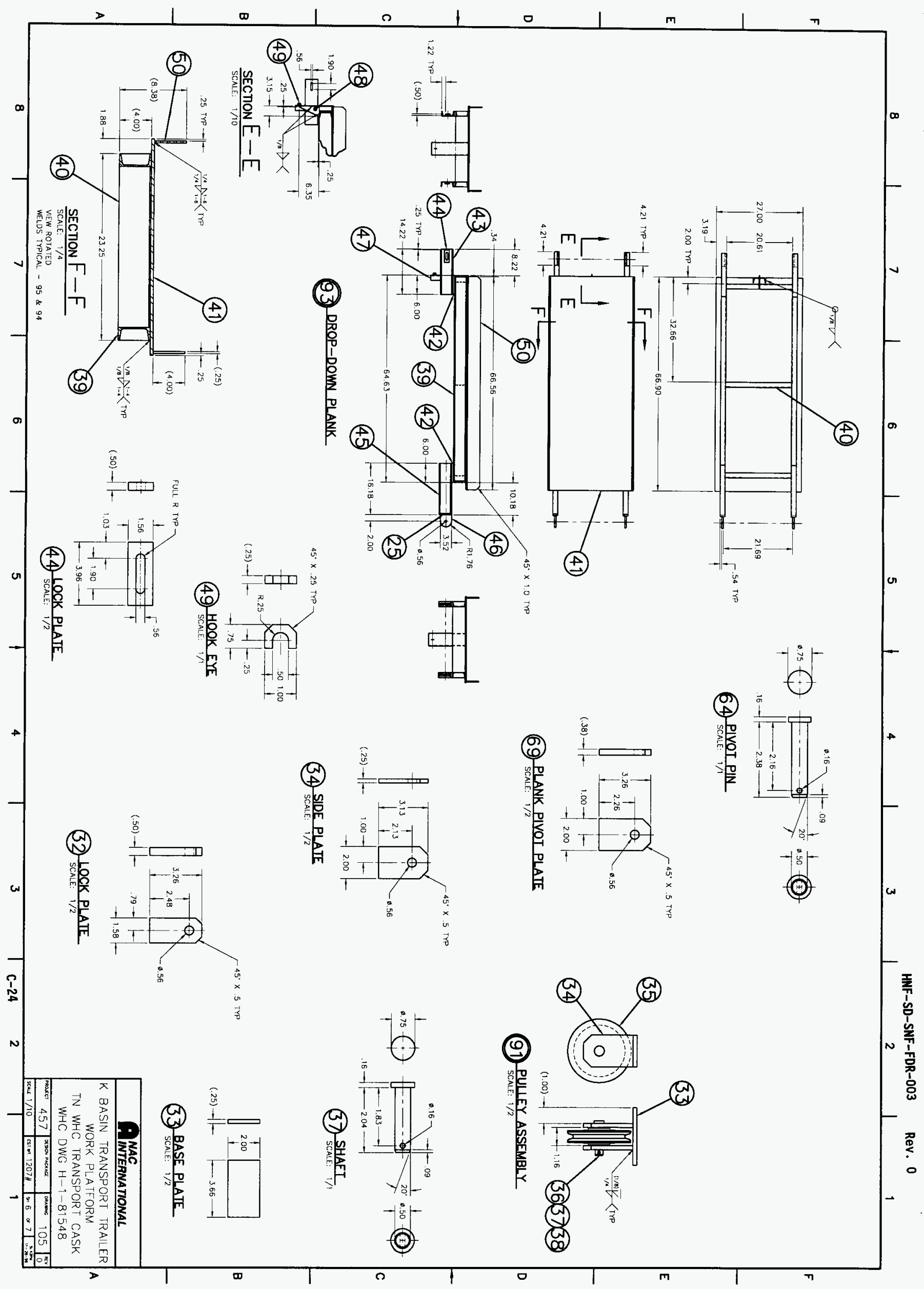




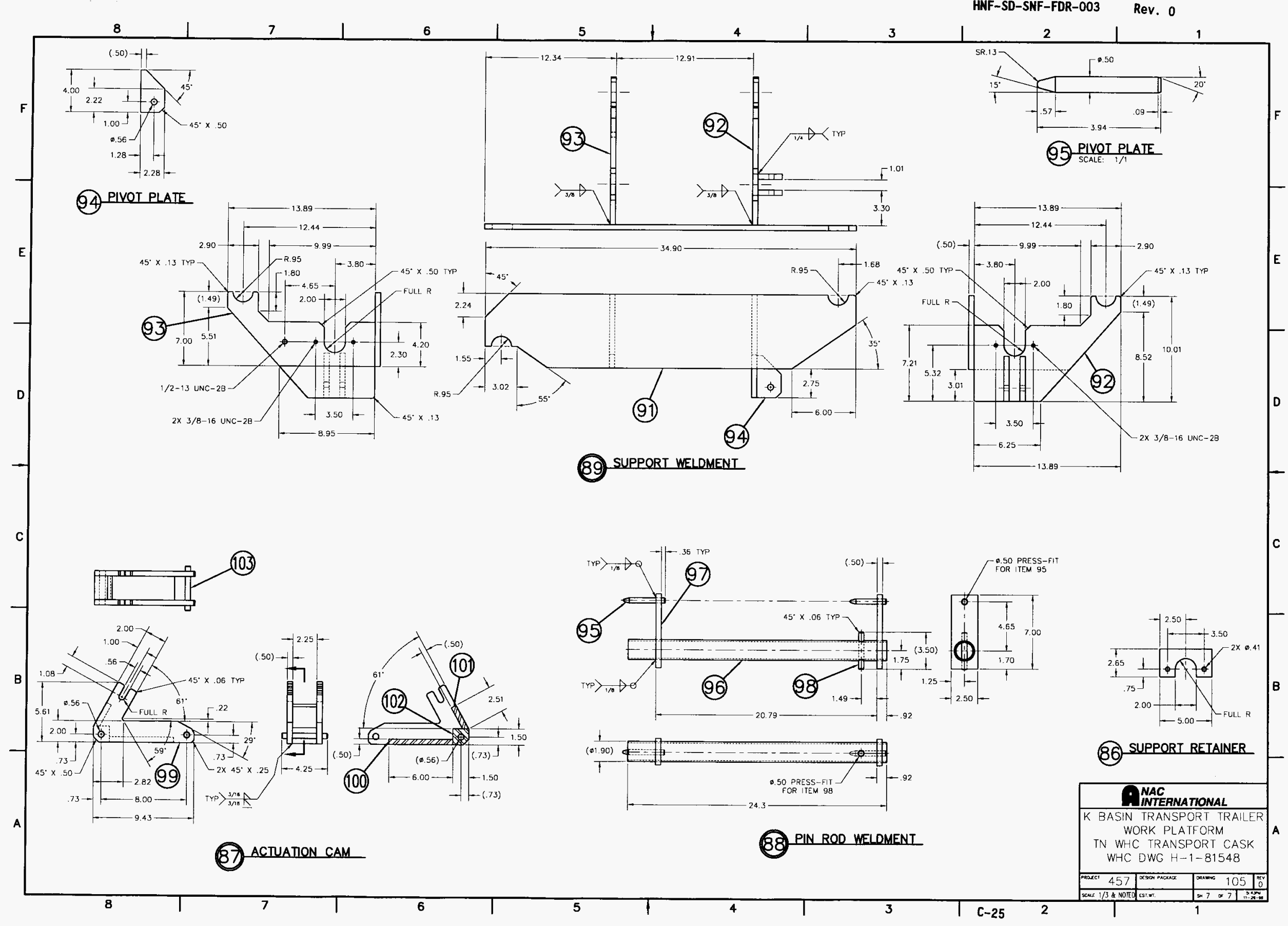




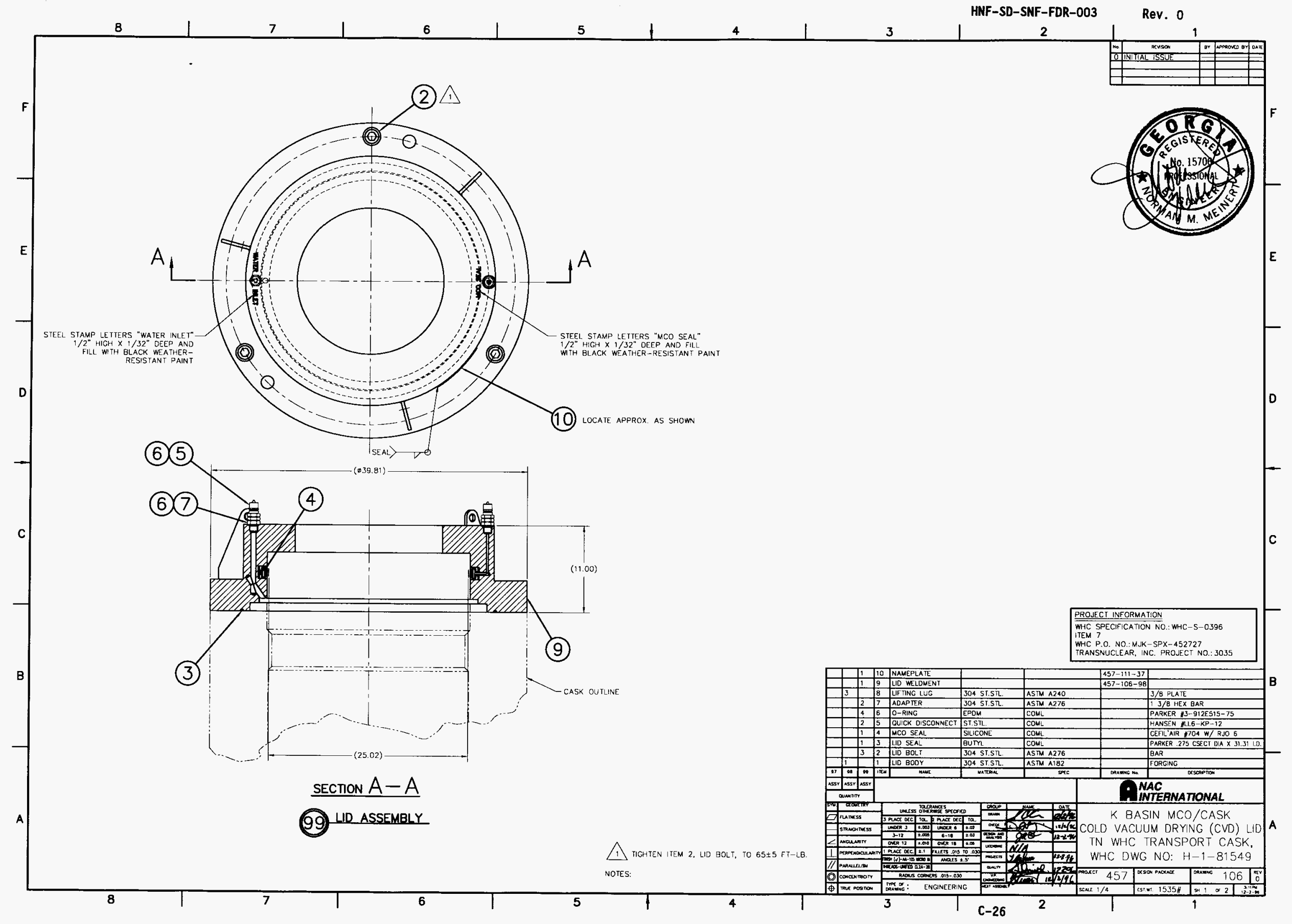




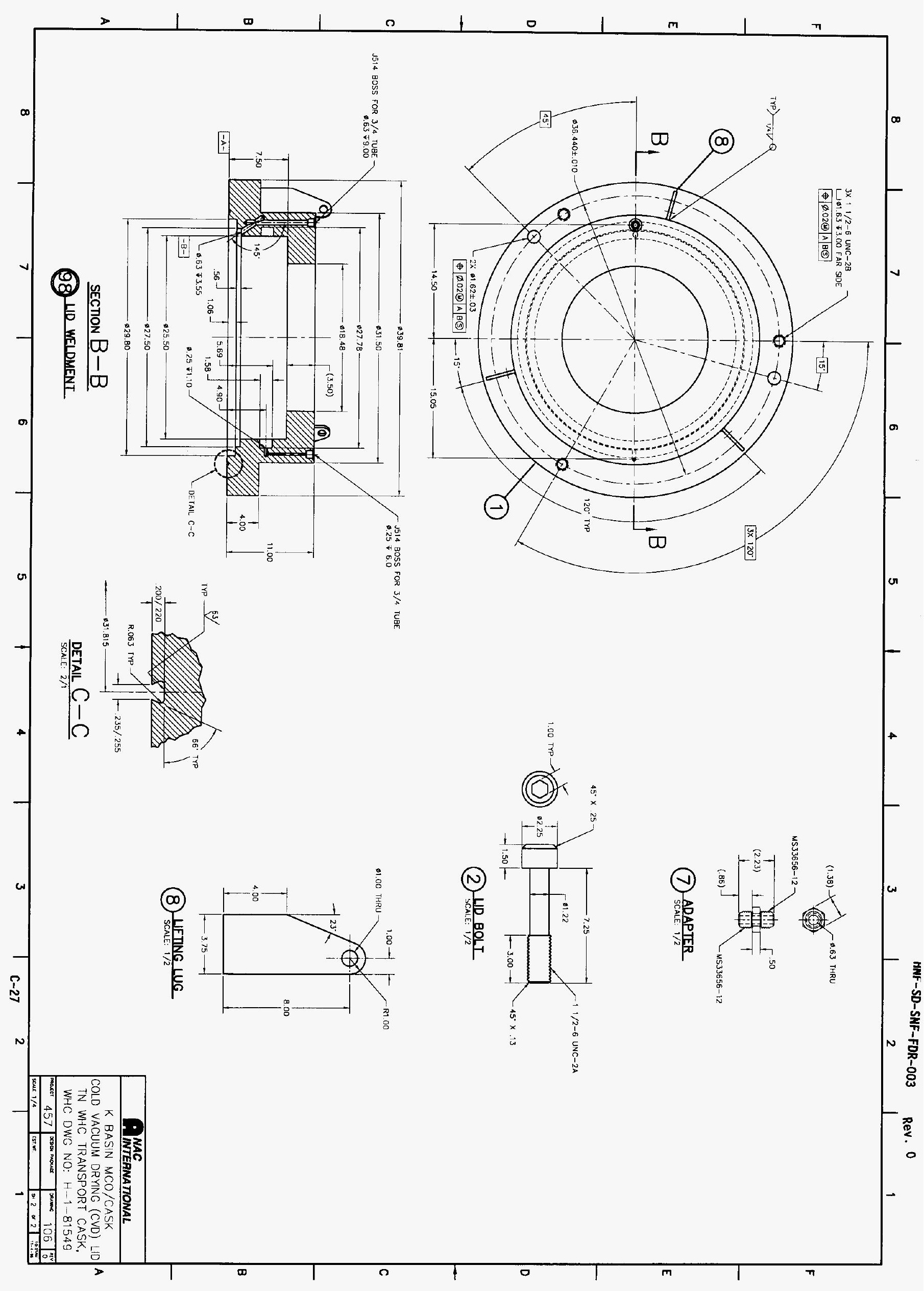




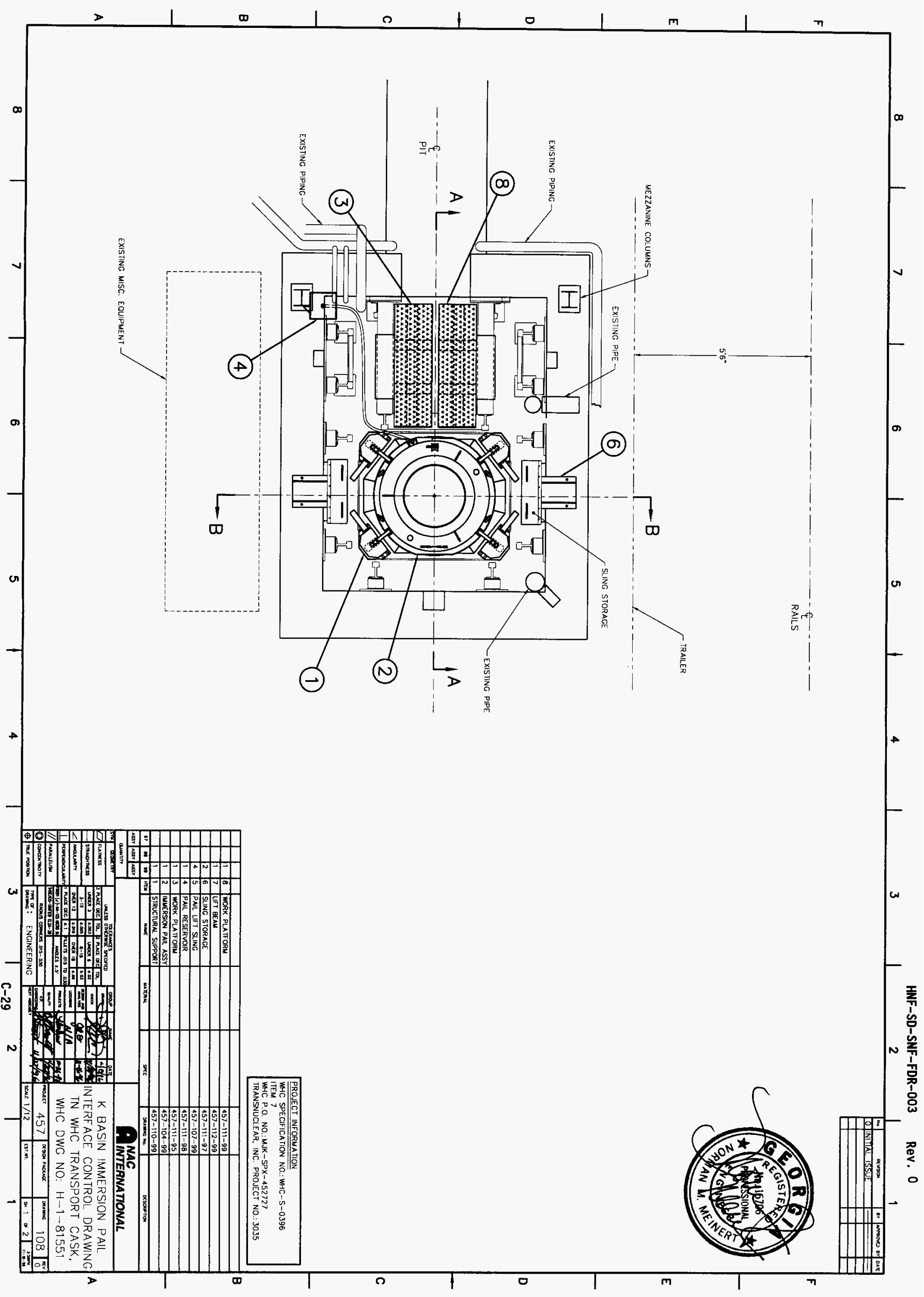




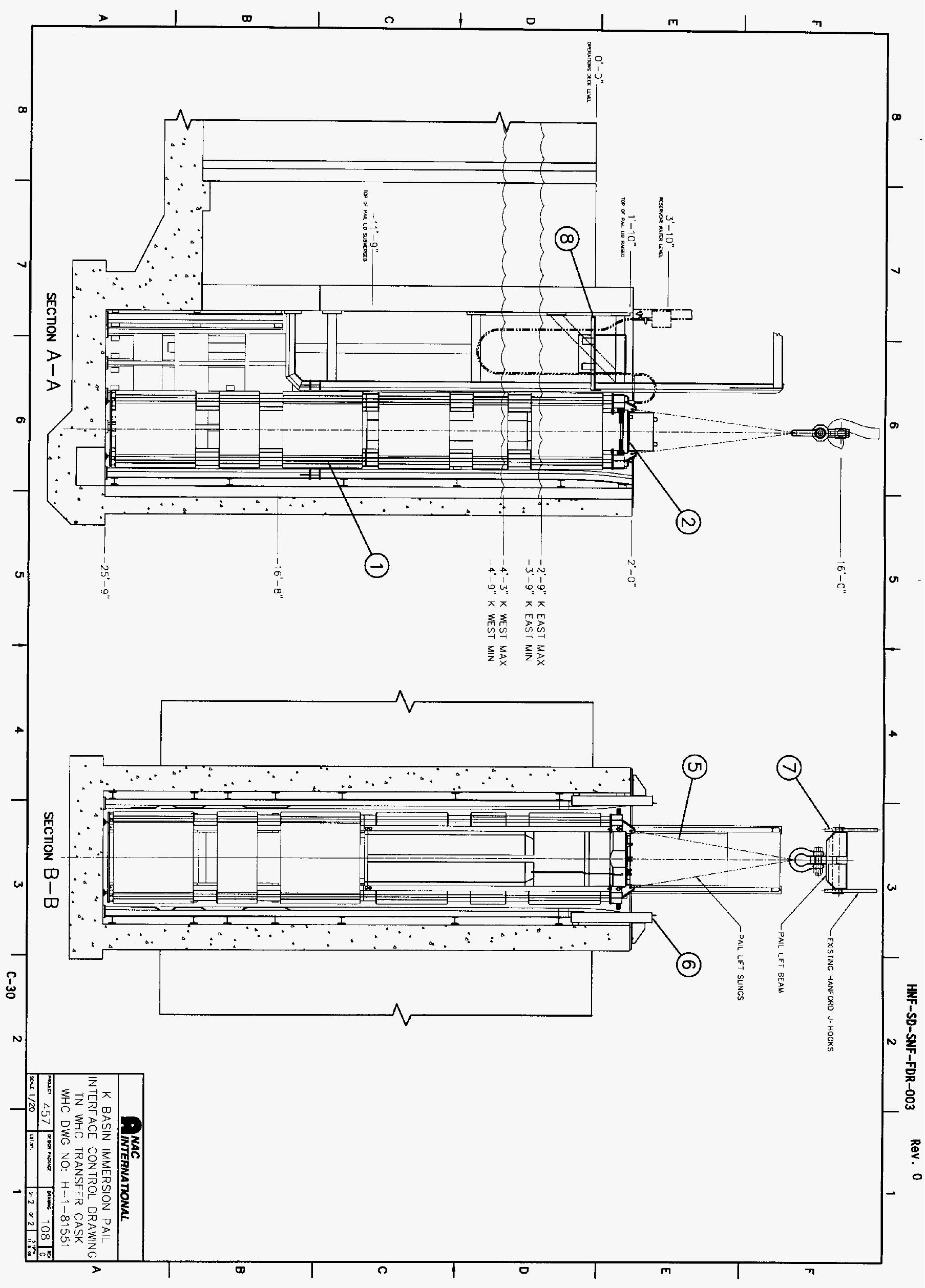




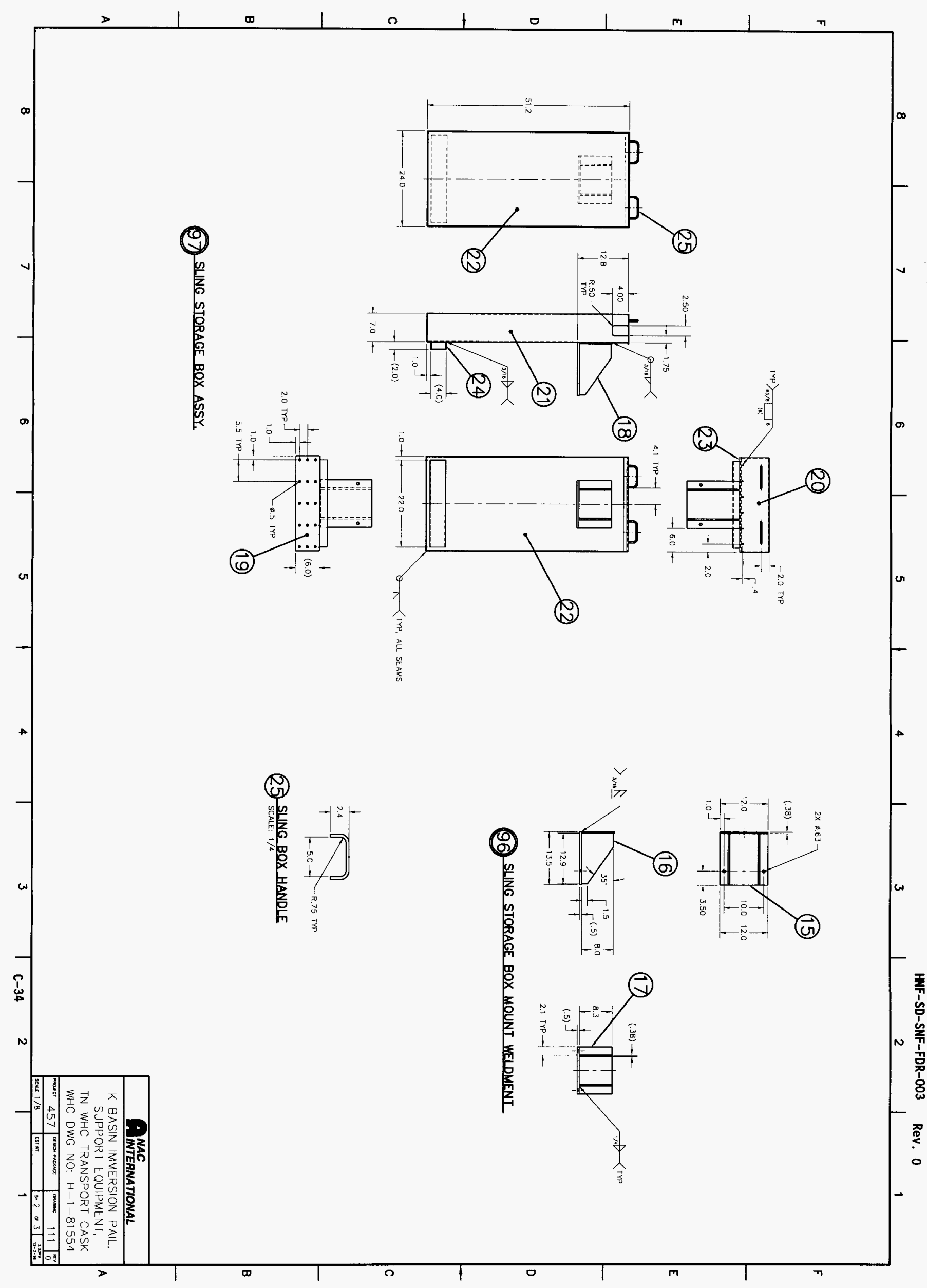




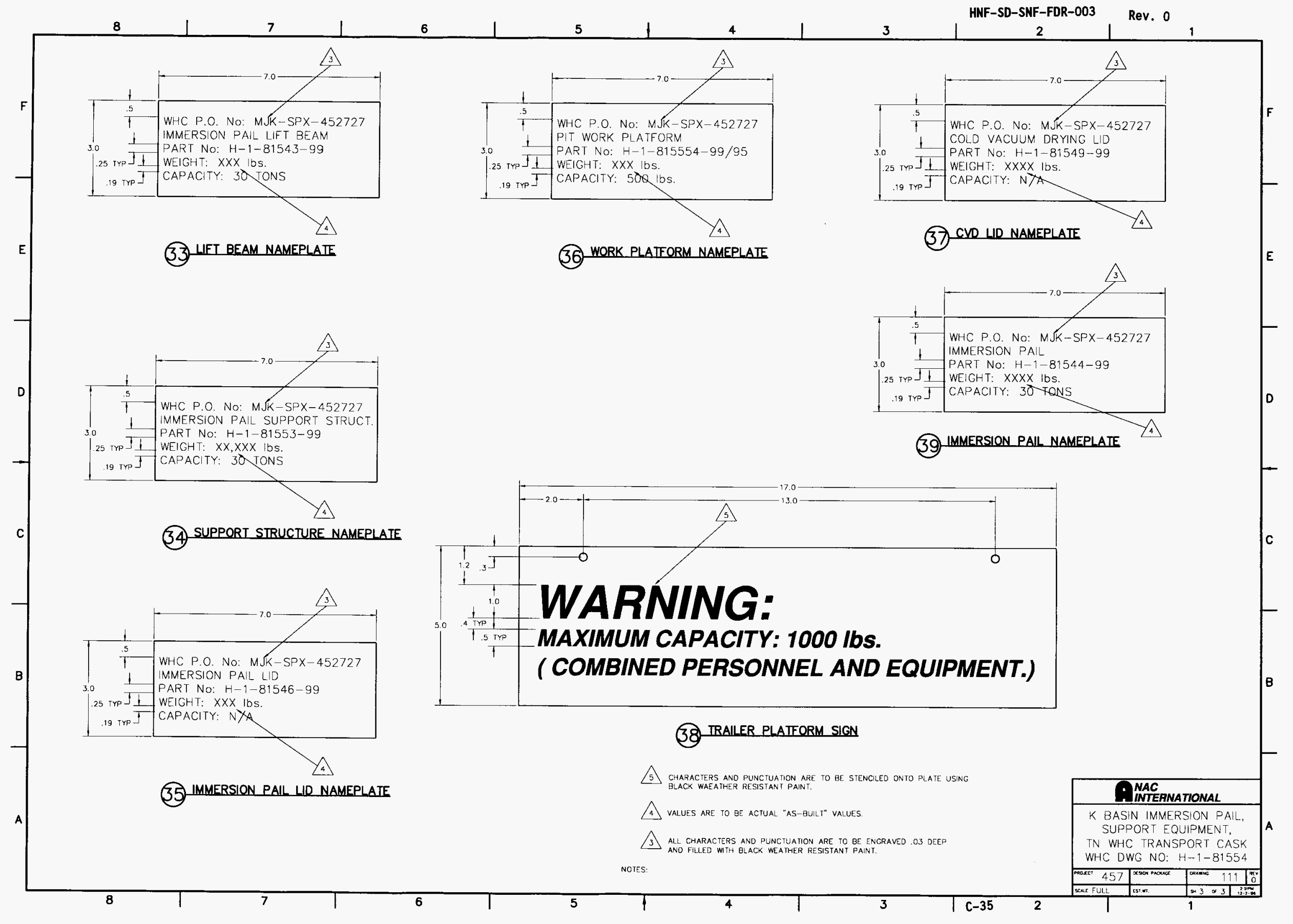




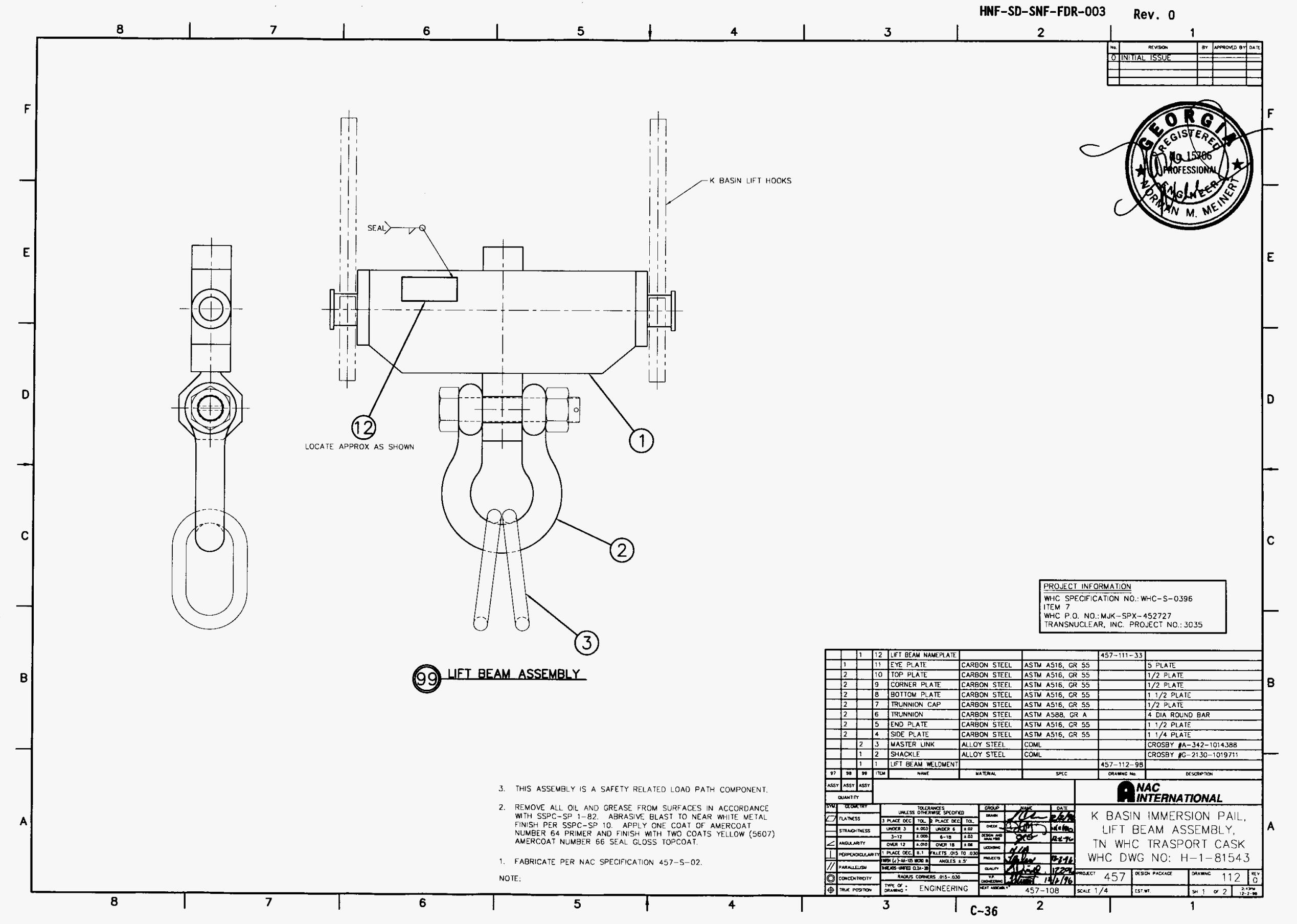




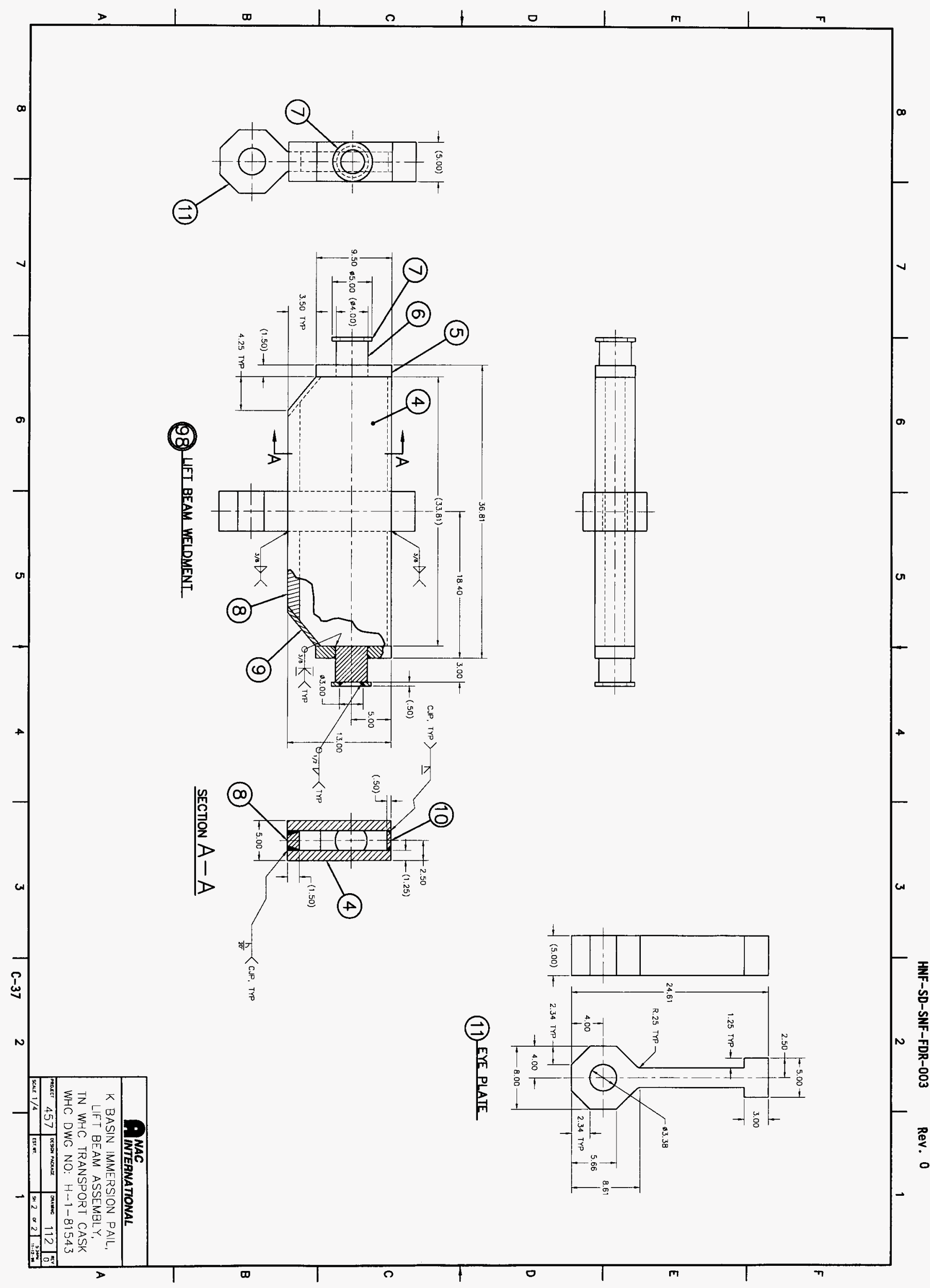




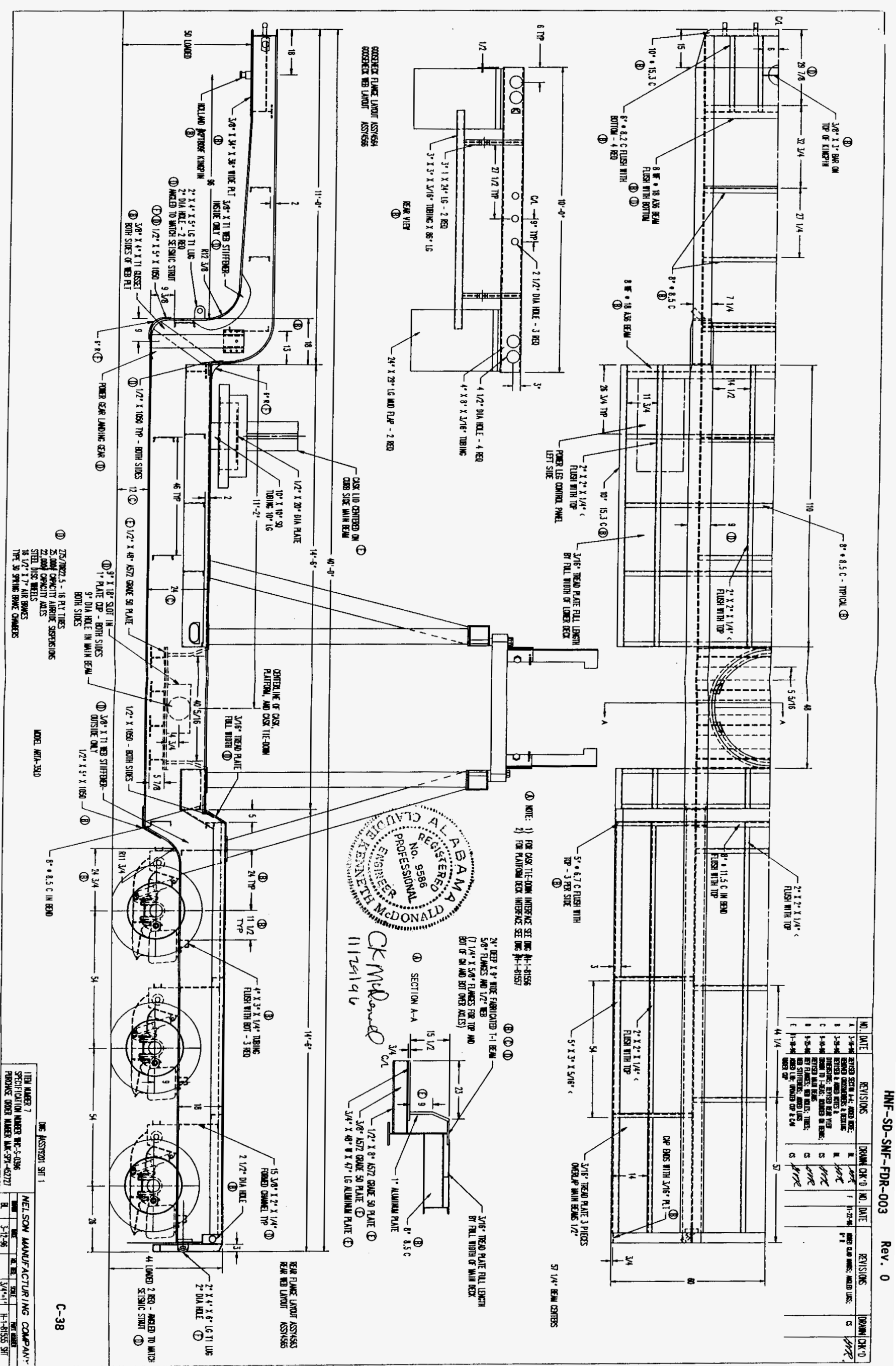




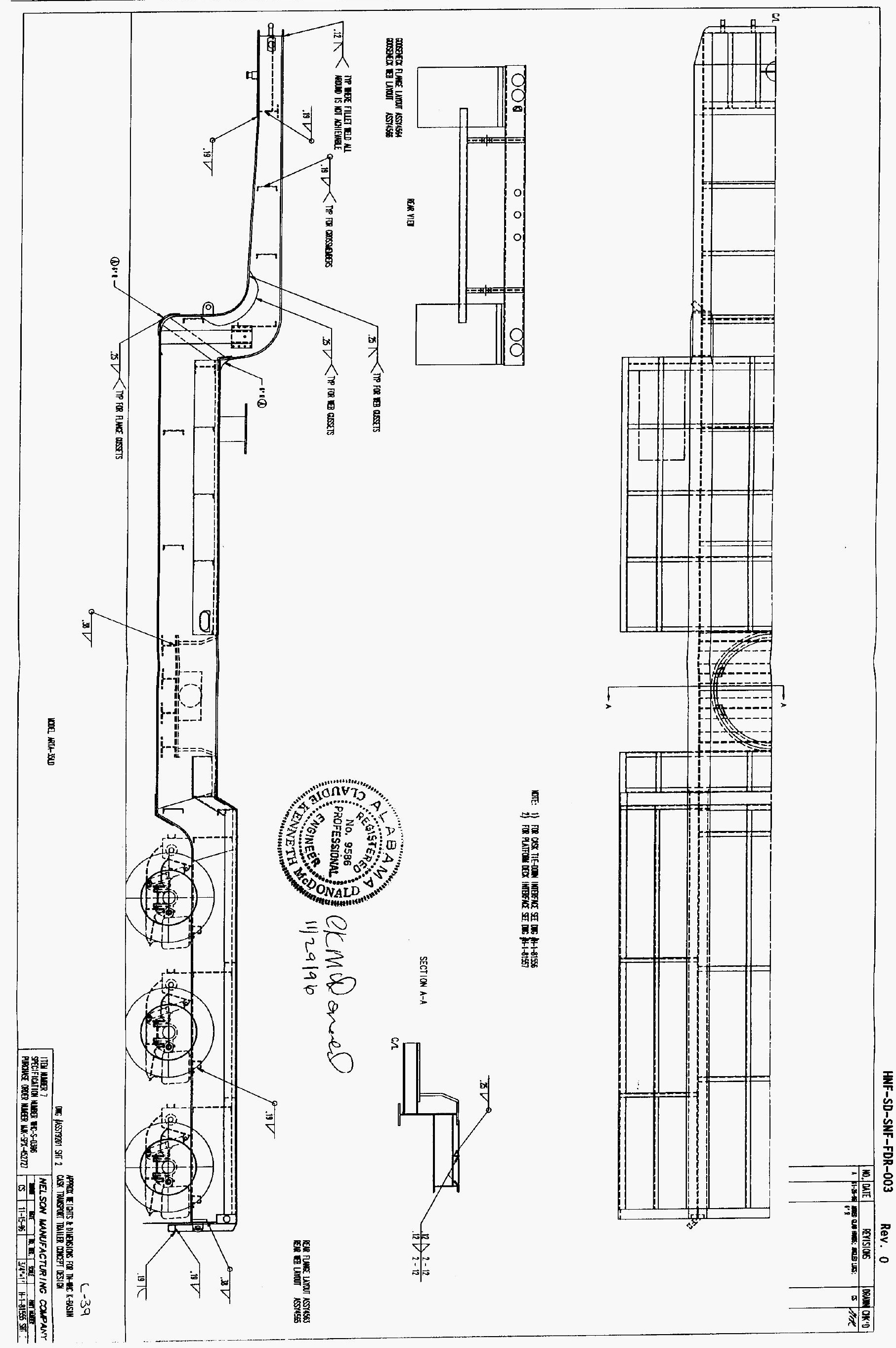




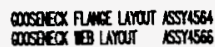

\section{2xop II 11}

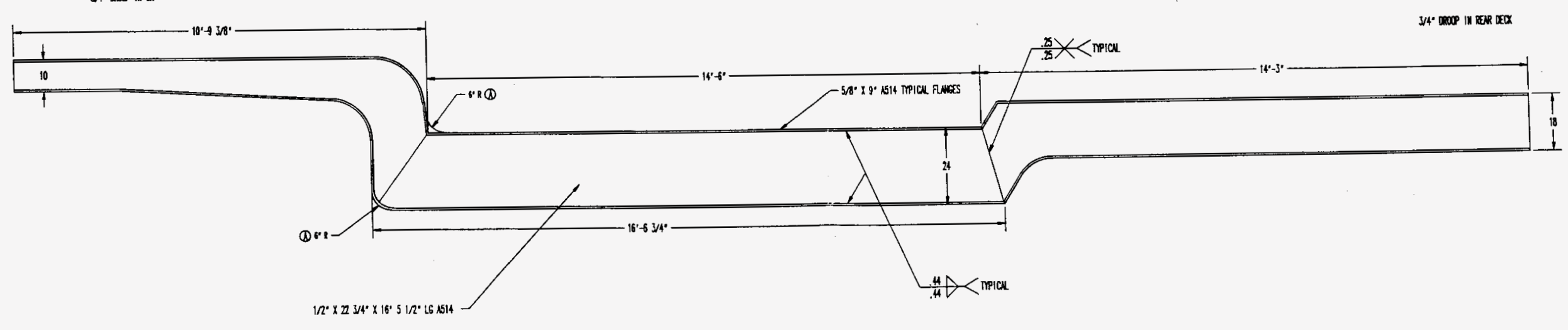

$12^{\circ} \times 73314^{\circ} \times 16^{\prime} 51 \Omega^{\circ} 16 \$ 1514$

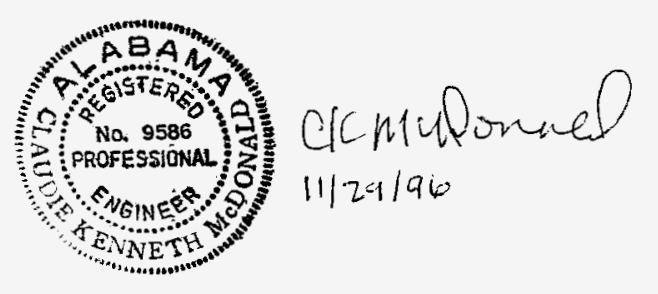


DISTRIBUTION SHEET

\begin{tabular}{|c|c|c|c|c|c|}
\hline \multirow{2}{*}{$\begin{array}{l}\text { To } \\
\text { Distribution }\end{array}$} & \multirow{2}{*}{\multicolumn{3}{|c|}{$\begin{array}{l}\text { From } \\
\text { SNF Storage Projects }\end{array}$}} & \multicolumn{2}{|l|}{ Page 1 of 1} \\
\hline & & & & \multicolumn{2}{|l|}{ Date $2 / 5 / 97$} \\
\hline \multirow{2}{*}{\multicolumn{4}{|c|}{ Project Title/Work Order }} & \multicolumn{2}{|c|}{ EDT No. 620085} \\
\hline & & & & \multicolumn{2}{|l|}{ ECN No. NA } \\
\hline Name & MSIN & $\begin{array}{l}\text { Text } \\
\text { With All } \\
\text { Attach. }\end{array}$ & Text Only & $\begin{array}{l}\text { Attach./ } \\
\text { Appendix } \\
\text { Only }\end{array}$ & $\begin{array}{l}\text { EDT/ECN } \\
\text { Only }\end{array}$ \\
\hline $\begin{array}{l}\text { K. E. Ard } \\
\text { G. D. Bazinet } \\
\text { S. A. Brisbin } \\
\text { D. M. Chenault } \\
\text { W. S. Edwards } \\
\text { L. H. Goldmann } \\
\text { C. R. Hoover } \\
\text { J. J. Irwin } \\
\text { A. T. Kee } \\
\text { T. D. Merkling } \\
\text { C. B. Loftis } \\
\text { R. W. Rasmussen } \\
\text { C. A. Thompson } \\
\text { Central Files }\end{array}$ & $\begin{array}{l}X 3-85 \\
\text { B4-55 } \\
\text { R3-86 } \\
\text { R3-86 } \\
\text { G1-11 } \\
\text { R3-86 } \\
\text { G1-50 } \\
\text { HO-34 } \\
\text { R3-86 } \\
\text { X3-79 } \\
\text { S7-41 } \\
\text { R3-86 } \\
\text { R3-85 } \\
\text { A3-88 } \\
\end{array}$ & $x$ & & & $\begin{array}{l}X \\
X \\
X \\
X \\
X \\
X \\
X \\
X \\
X \\
X \\
X \\
X \\
X\end{array}$ \\
\hline mogit Zules & $R 3-11$ & $x$ & & & \\
\hline
\end{tabular}

